San Jose State University

SJSU ScholarWorks

Master's Theses

Master's Theses and Graduate Research

2007

\title{
Intercomparison of lower stratospheric performance of global reanalyses
}

Fred S. Snively

San Jose State University

Follow this and additional works at: https://scholarworks.sjsu.edu/etd_theses

\section{Recommended Citation}

Snively, Fred S., "Intercomparison of lower stratospheric performance of global reanalyses" (2007). Master's Theses. 3392.

DOI: https://doi.org/10.31979/etd.xp32-he7v

https://scholarworks.sjsu.edu/etd_theses/3392

This Thesis is brought to you for free and open access by the Master's Theses and Graduate Research at SJSU ScholarWorks. It has been accepted for inclusion in Master's Theses by an authorized administrator of SJSU ScholarWorks. For more information, please contact scholarworks@sjsu.edu. 


\title{
INTERCOMPARISON OF LOWER STRATOSPHERIC PERFORMANCE OF GLOBAL REANALYSES
}

\author{
A Thesis \\ Presented to \\ The Faculty of the Department of Meteorology
}

San Jose State University

In Partial Fulfillment

of the Requirements for the Degree

Master of Science

by

Fred S. Snively

May 2007 
UMI Number: 1445266

Copyright 2007 by

Snively, Fred S.

All rights reserved.

\section{INFORMATION TO USERS}

The quality of this reproduction is dependent upon the quality of the copy submitted. Broken or indistinct print, colored or poor quality illustrations and photographs, print bleed-through, substandard margins, and improper alignment can adversely affect reproduction.

In the unlikely event that the author did not send a complete manuscript and there are missing pages, these will be noted. Also, if unauthorized copyright material had to be removed, a note will indicate the deletion.

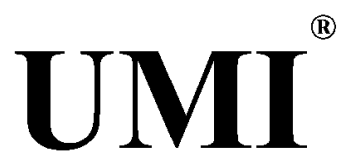

UMI Microform 1445266

Copyright 2007 by ProQuest Information and Learning Company.

All rights reserved. This microform edition is protected against unauthorized copying under Title 17, United States Code.

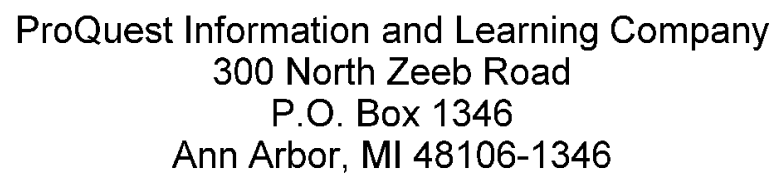


(C) 2007

Fred S. Snively

ALL RIGHTS RESERVED 
APPROVED FOR THE DEPARTMENT OF METEOROLOGY

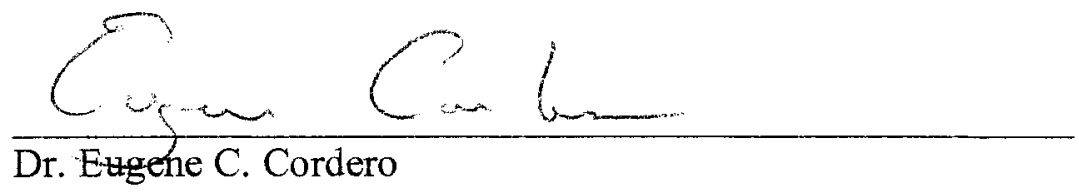

Dr. Eugene C. Cordero
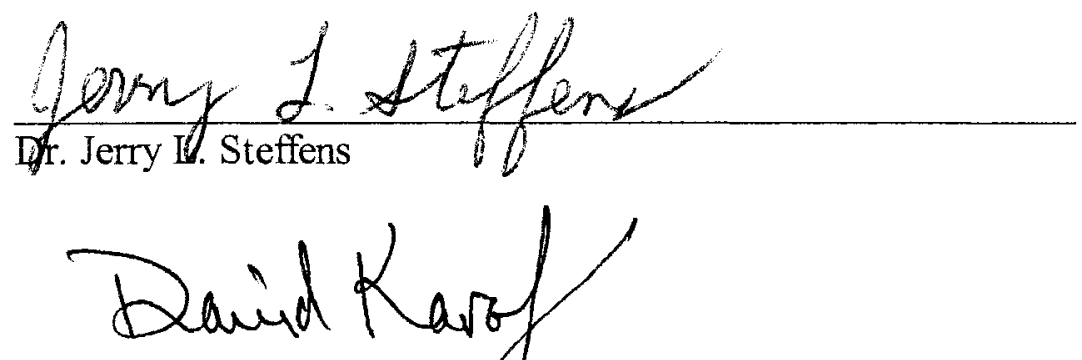

Dr. David Karoly, Universify of Oklahoma

APPROVED FOR THE UNIVERSITY

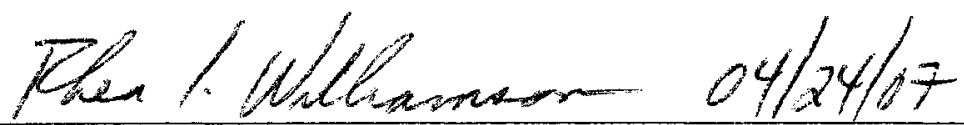




\title{
ABSTRACT \\ INTERCOMPARISON OF LOWER STRATOSPHERIC PERFORMANCE OF GLOBAL REANALYSES
}

\author{
by Fred S. Snively
}

This thesis selects two major global reanalyses, NCEP-NCAR and ERA-40, from five candidates, based on duration of coverage and on design parameters and performance evaluations in the literature for the domain from 100 to $10 \mathrm{hPa}$. Fourdimensional fields of average monthly temperature, zonal wind speed, and meridional wind speed from these two reanalyses are intercompared from 1958 through 2001 and compared with commensurate data where available. The thesis concludes with the identification, based on the intercomparison and comparisons, of a climatology suitable for the evaluation of global climate model performance in the lower stratosphere.

Temperature results of both reanalyses can be recommended for climatological application in most time periods and spatial regions, but not all. Extratropical wind speed results are effectively equivalent in the two reanalyses. However, meridional wind speed results differ dramatically and no recommendation is possible without further study. 


\section{ACKNOWLEDGEMENTS}

I first wish to acknowledge the current and former faculty of the SJSU Meteorology Department: Drs. Scot Rafkin, Jerry Steffens, Kevin Perry, Alison Bridger, Jindra Goodman, Douglas Sinton, Robert Bornstein, Eugene Cordero, and Thomas Rickenbach (in order of our first contact), as well as Dr. Alejandro Garcia (SJSU Physics) and Drs. William Brenkow and Lawrence Breaker of Moss Landing Marine Laboratories. It has been a wonderful nine years working with them; they helped provide the foundation for this thesis.

Next, I am indebted to the members of my thesis committee: Dr. Eugene Cordero (Chair) and Dr. Jerry Steffens of SJSU, and Dr. David Karoly of the Univ. of Oklahoma. Eugene originally suggested intercomparison of reanalyses as a thesis subject and has guided the project strategically and tactically during our weekly meetings and by his thorough reviews of numerous drafts. His enthusiasm and sense of humor have made the creation of this thesis a very pleasurable task. He allowed me to have, in Richard Feynman's words, "the pleasure of finding things out." I appreciate Dr. Steffens' willingness to serve on my committee in spite of his usual heavy teaching load. I will sleep better at night knowing that he has reviewed and approved of the statistical exercises contained in the thesis. When Dr. Karoly agreed to serve on my committee, he surely was not expecting to review a document of the size of this thesis. His comments constituted a true review.

In addition to the review and guidance of my committee, the thesis has benefited significantly from personal communication with the following experts who graciously 
and rapidly responded with thorough answers to sometimes probing questions posed by an unknown graduate student:

\begin{tabular}{|c|c|}
\hline Philip Arkin & ESSIC, Univ. of Maryland \\
\hline Wesley Ebisuzaki & NCEP, NOAA \\
\hline Masao Kanamitsu & $\begin{array}{l}\text { Scripps Institute of Oceanography, UCSD } \\
\text { (formerly NCEP, NOAA) }\end{array}$ \\
\hline Carl Mears & Remote Sensing Systems \\
\hline Masami Sakamoto & Japanese Meteorological Agency \\
\hline Dian Seidel & Air Resources Laboratory, NOAA \\
\hline Adrian Simmons & ECMWF \\
\hline Richard Swinbank & UK Met Office \\
\hline
\end{tabular}

This thesis also benefits from Dr. Romeo L. Moruzzi, my revered control systems professor at Worcester Polytechnic Institute during 1959-62. From his example, I learned the art of system engineering.

The most satisfying credit accrues to my wife, Evelyn Miller. Her technical review was often as intense as the committee's; when she didn't understand, she wanted to know why the material did not enable her to understand. Her editorial and stylistic comments were unassailable, coming as they did from the author of the SJSU Outstanding Graduate Thesis of 2006. In addition, she provided endless encouragement and support and was usually reasonably gracious when I did not follow her advice.

This thesis is dedicated to Mr. Michael Voss. The Meteorology Department computer facilities have been so reliable during the last nine years, at least as reliable as any in my $32+$ year professional career, that one tends never to think of the individual who created and maintains them with unfailing calm and good humor. Mike has responded expeditiously to my every request as a graduate student and as an instructor. Mike - this one's for you! 


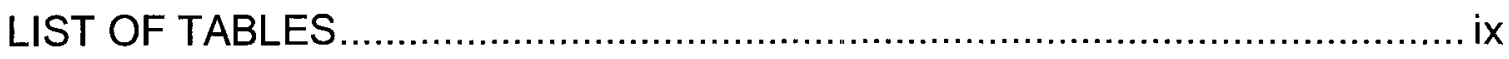

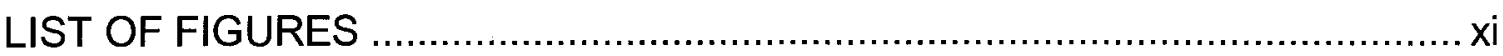

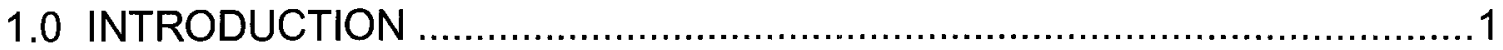

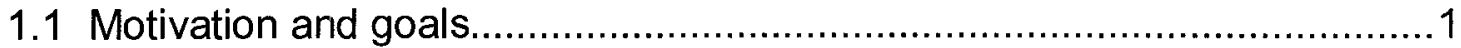

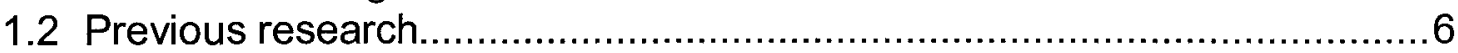

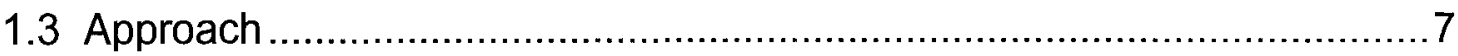

2.0 REANALYSIS AND THE STRATOSPHERIC DATA SETS ......................10

2.1 Meteorological reanalysis - Introduction and general considerations ......10

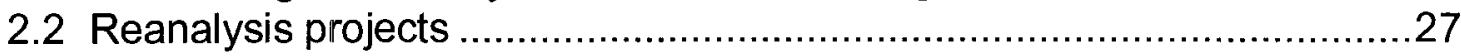

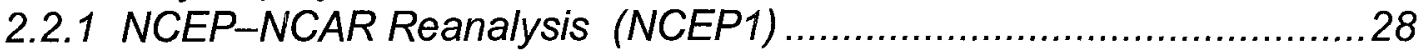

2.2.2 NCEP-DOE AMIP-I/ Reanalysis (R-2) (NCEP2) ............................ 38

2.2.3 ECMWF ERA-15 Reanalysis (ERA-15) .....................................40

2.2.4 ECMWF ERA-40 Reanalysis (ERA-40) .......................................46

2.2.5 Japanese Re-Analysis 25 Years (JRA-25) ...................................53

2.2.6 Reanalysis project and coverage timelines...................................58

2.2.7 Reanalysis and supporting data pressure levels............................61

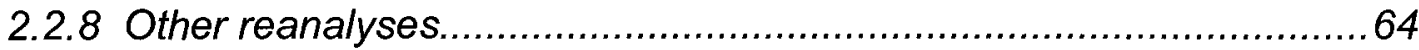

2.2.9 Selection of reanalyses for intercomparison .................................67

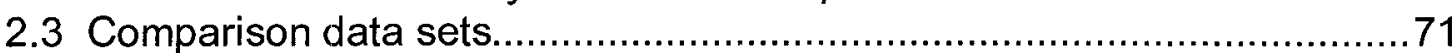

2.3.1 UKMO Stratospheric Analysis (METO) ....................................... 71

2.3.2 Freie Universität Berlin (FUB) ................................................... 72

2.3.3 Radiosonde Atmospheric Temperature Products for Assessing

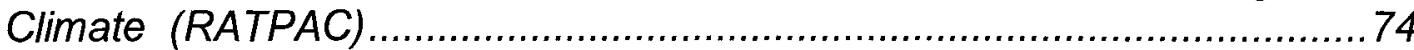

2.3.4 Satellite Temperature Lower Stratosphere (TLS)..........................77

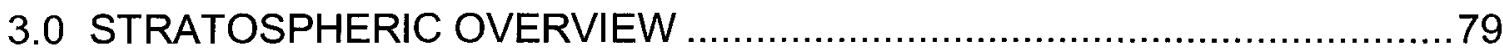

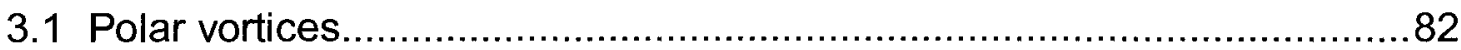

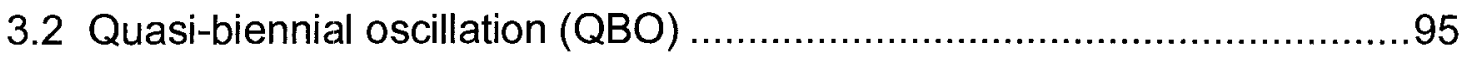

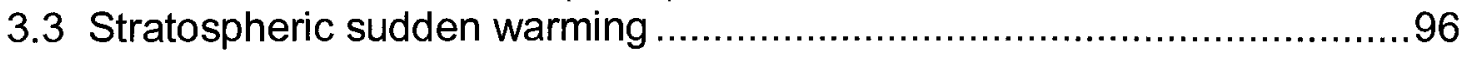

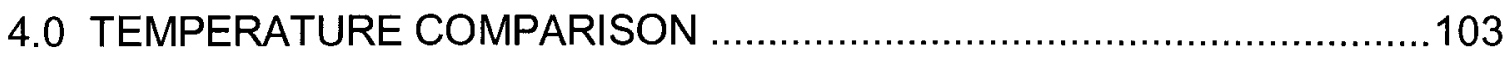

4.1 Height - latitude structure of monthly temperature differences ..............105

4.2 Temperature anomaly time series ................................................146

4.3 Pressure surface temperature anomalies and differences ....................159

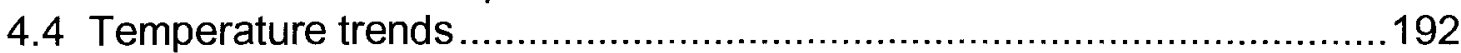

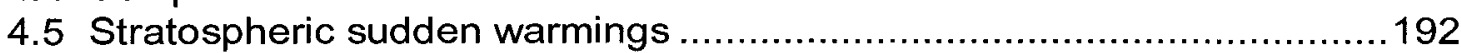

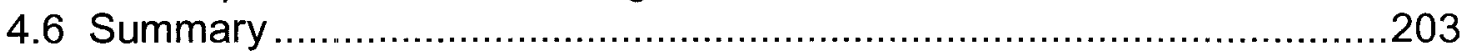

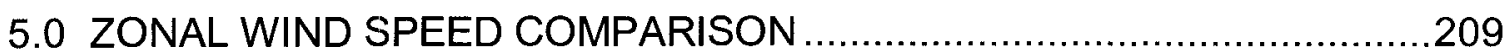

5.1 Height - latitude structure of monthly zonal wind speed differences .......209

5.2 Zonal wind speed anomaly time series............................................238

5.3 Longitudinal distribution of zonal wind speed anomalies ......................251 
5.4 Location, speed, and linear trends of maximum polar wind speeds .......267

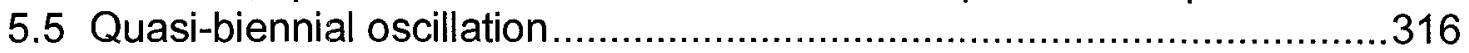

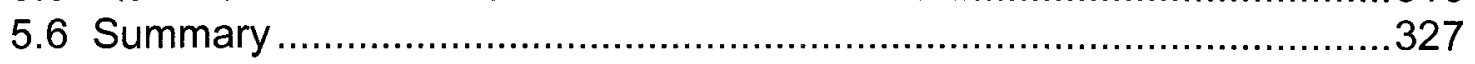

6.0 MERIDIONAL WIND SPEED COMPARISON.....................................331

6.1 Height - latitude structure of monthly meridional wind speeds...............332

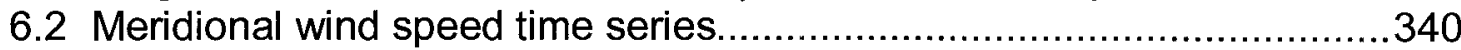

6.3 Meridional wind speed time-latitude structure …................................352

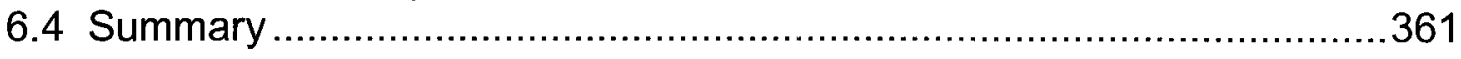

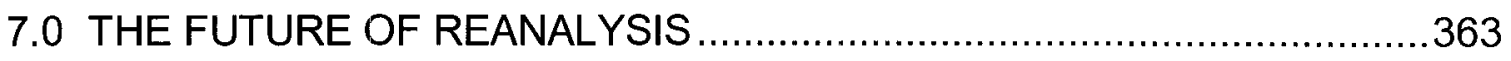

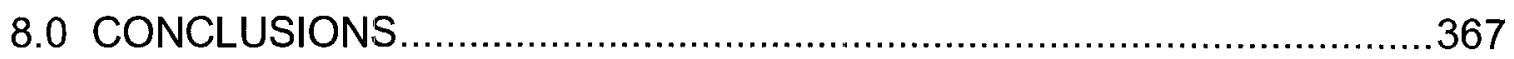

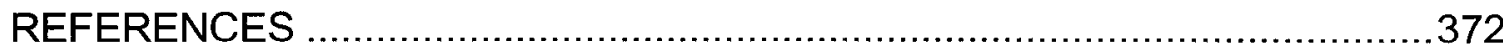

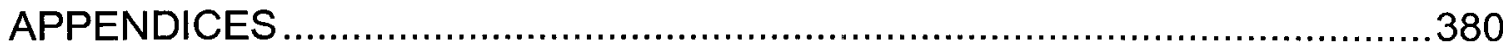

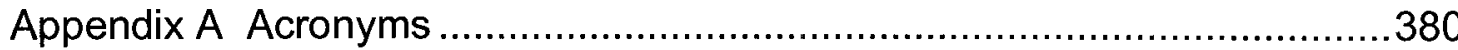

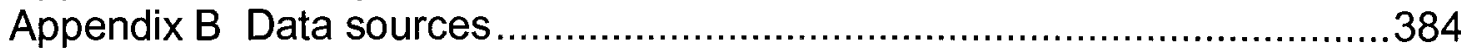

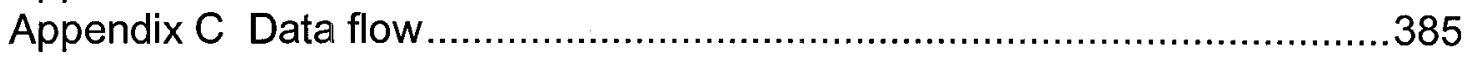

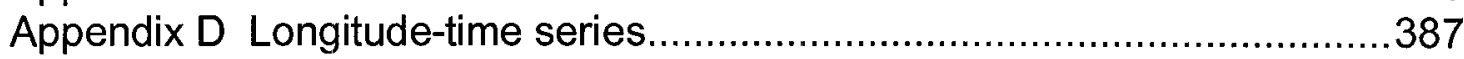

Appendix E Frequency-domain filtering of time series...............................390 


\section{LIST OF TABLES}

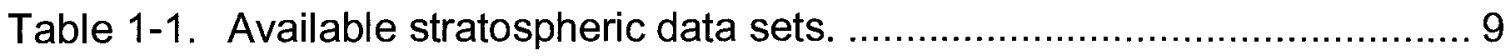

Table 2-1. NCEP1 characteristics. ........................................................... 31

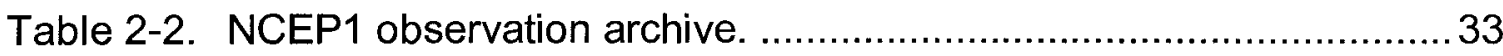

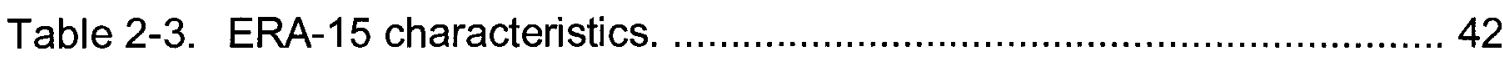

Table 2-4. Use of satellite temperature data in ERA-15.............................. 43

Table 2-5. ERA-15 data sources. .......................................................... 44

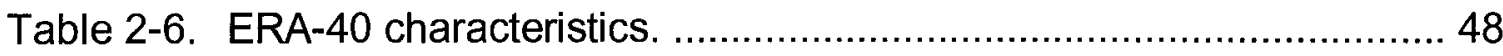

Table 2-7. JRA-25 characteristics. ...................................................... 56

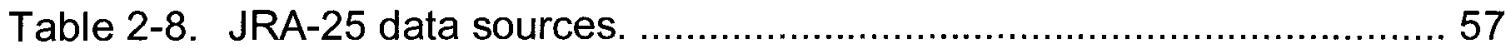

Table 2-9. Comparison of NCEP1 and ERA-40 characteristics. ..................... 70

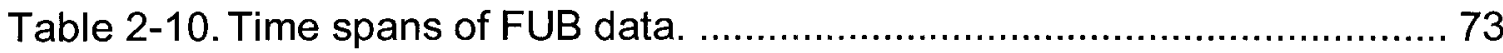

Table 3-1. General nature of polar vortices. ................................................ 94

Table 3-2. Stratospheric sudden warming events. ......................................100

Table 4-1. Maximum temperature differences $(K)$ and their locations for cases presented in Figs. 4-1 through 4-6 and Figs. 4-7 through

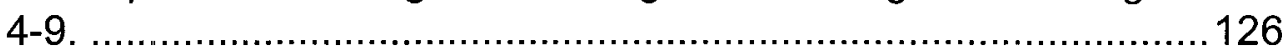

Table 4-2. Comparison of Section 4.1 reanalysis temperature difference

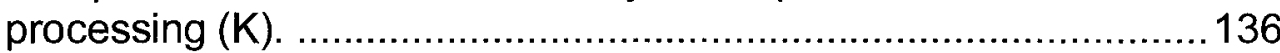

Table 4-3. Unfiltered maximum, filtered maximum, mean, and unfiltered standard deviation of temperature difference time series $(K)$ for subplots of Figs. 4-31 and 4-32.

Table 4-4. Mean (and standard deviation) of maxima and minima temperature anomalies from longitudinal mean and their differences (1980-2001) (K).

Table 4-5. Temperature differences between NCEP1 and ERA-40 reanalyses and TLS $(K)$. 192

Table 4-6. Character and symbol definitions in Table 4-7. ...........................195

Table 4-7. Stratospheric sudden warming events. .................................... 196

Table 4-8. Characterization of SSW by NCEP1 and ERA-40 reanalyses. ...... 197 
Table 4-9. Percentages of warmings of known SSW events detected with WMO definition and with temperature or wind speed reversals and percentages of false detections.

Table 5-1. Maximum zonal wind speed differences $\left(\mathrm{m} \mathrm{s}^{-1}\right)$ and their locations for cases presented in Figs. 5-1 through 5-9.

Table 5-2. Comparison of Section 5.1 reanalysis zonal wind speed difference processing.

Table 5-3. Unfiltered maximum, filtered maximum, mean, and unfiltered standard deviation of zonal wind speed difference time series $\left(\mathrm{m} \mathrm{s}^{-1}\right)$ for subplots of Figs. 5-25 and 5-26.

Table 5-4. Total vortex counts and mean vortex latitudes for Northern and Southern Hemispheres in NCEP1 and ERA-40 reanalyses. 275

Table 5-5. Ratio of ERA-40 to NCEP1 maximum standard deviations. 320

Table 6-1. Difference in mean meridional wind speeds $\left(\mathrm{m} \mathrm{s}^{-1}\right)$ : NCEP1 minus ERA-40 1958-2001 and (1979-2001).

Table 6-2. Rms difference in NCEP1 and ERA-40 meridional wind speeds $\left(\mathrm{m} \mathrm{s}^{-1}\right)$ 1958-2001 and (1979-2001).

Table 6-3. Correlation of NCEP1 and ERA-40 meridional wind speeds $\left(\mathrm{m} \mathrm{s}^{-1}\right)$ 1958-2001 and (1979-2001).

Table 6-4. QBO variance present in meridional wind speeds 1958-2001. .... 345

Table 8-1. Recommended reanalysis temperature usage. 369 


\section{LIST OF FIGURES}

Fig. 2-1. Differences in 15-year means of NCEP1 (upper) and ERA-40 (lower) monthly- and zonally-averaged temperatures. 1960-74 means are subtracted from 1985-99 means. Temperature differences in $\mathrm{K}$.

Fig. 2-2. Rms differences of NCEP1 minus ERA-40 monthly- and zonallyaveraged temperatures for years 1960-74 (upper) and years 1985-99 (middle). NCEP1 minus ERA-40 differences for years 1958-99 minus differences for years 1960-74 (lower). Rms temperature differences in $\mathrm{K}$.

Fig. 2-3. Rms differences of NCEP1 minus ERA-40 monthly- and zonallyaveraged zonal wind speeds for years 1960-74 (upper) and years 1985-99 (lower). NCEP1 minus ERA-40 differences for years 1985-99 minus differences for years 1960-74 (lower). Rms wind speed differences in $\mathrm{m} \mathrm{s}^{-1}$.

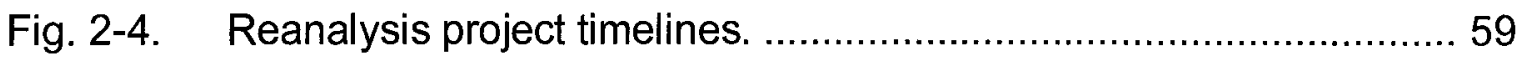

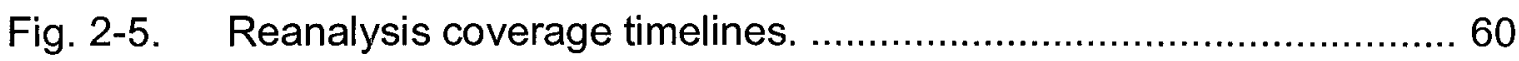

Fig. 2-6. Available data pressure levels (left/black) and model pressure levels (right/red) in the reanalyses and comparison data sets. Available pressure levels between 100 and $10 \mathrm{hPa}$ are bolded. See text for discussion of "TLS" diagram.

Fig. 2-7. Steps in the development of the RATPAC-A data set. .................. 77

Fig. 3-1. Midlatitude mean temperature profile based on U.S. Standard Atmosphere (1976).

Fig. 3-2. Meridional structure of zonal climatology for December, January, and February (DJF): zonal wind speeds $\left(\mathrm{m} \mathrm{s}^{-1}\right)$ (upper), horizontal temperature gradients $\left(\mathrm{K} \mathrm{deg}^{-1}\right)$ (middle), temperatures $(\mathrm{K})$ (lower) [lon: mean of $0^{\circ}-356.25^{\circ}$, lat: $86.25^{\circ} \mathrm{S}-86.25^{\circ} \mathrm{N}$, press: $1000-0.316 \mathrm{hPa}$, time: monthly mean of Jan 1992-Dec 1999].

Fig. 3-3. Same as Fig. 3-2 except climatology for March, April, and May (MAM).

Fig. 3-4. Same as Fig. 3-2 except climatology for June, July, and August (JJA).

Fig. 3-5. Same as Fig. 3-2 except climatology for September, October, and November (SON). 
Fig. 3-6. Eight-year pressure level (height) vs. latitude structure of zonal climatology: mean zonal wind speeds $\left(\mathrm{m} \mathrm{s}^{-1}\right)$ (upper), standard deviation of zonal wind speeds $\left(\mathrm{m} \mathrm{s}^{-1}\right)$ (lower) [lon: mean of $0^{\circ}-356.25^{\circ}$, lat: $86.25^{\circ} \mathrm{S}-86.25^{\circ} \mathrm{N}$, press: $1000-0.316 \mathrm{hPa}$, time: mean of Jan 1992-Dec 1999].

Fig. 3-7. Eight-year pressure level (height) vs. latitude structure of zonal climatology: mean temperatures (K) (upper), standard deviation of temperatures $(\mathrm{K})$ (lower) [lon: mean of $0^{\circ}-356.25^{\circ}$, lat: $86.25^{\circ} \mathrm{S}-86.25^{\circ} \mathrm{N}$, press: $1000-0.316 \mathrm{hPa}$, time: monthly mean of Jan 1992-Dec 1999].

Fig. 3-8. Height vs. time contour of QBO, westerly winds (red), easterly winds (blue), contour interval $5 \mathrm{~m} \mathrm{~s}^{-1}$ ERA-40 [lon: mean of $0^{\circ}-356.25^{\circ}$, lat: $0^{\circ}$, press: $120-2.8 \mathrm{hPa}$, time: $\left.1980-1989\right]$. 96

Fig. 3-9. Geopotential height reanalysis of major midwinter warming event of 22 February 1979, NCEP2: Heights in decameters, contour interval: 16 decameters [lon: $0^{\circ}-357.5^{\circ}$, lat: $0^{\circ}-90^{\circ} \mathrm{N}$, press: $10 \mathrm{hPa}$, time: daily mean 22 February 1979]. 102

Fig. 4-1. Mean, standard deviation, rms, and maxima of time series of monthly- and zonally-averaged temperature differences $(\mathrm{K})$ : NCEP1 minus ERA-40 [lon: mean of $0^{\circ}-357.5^{\circ}$, lat: $90^{\circ} \mathrm{S}-90^{\circ} \mathrm{N}$, press: $100-10 \mathrm{hPa}$, time: Jan 1958-Dec 2001]. ... 111

Fig. 4-2. Same as Fig. 4-1 except time: Jan 1958-Dec 1978. ................... 112

Fig. 4-3. Same as Fig. 4-1 except time: Jan 1979-Dec 2001. ................... 113

Fig. 4-4. Same as Fig. 4-1 except time: Jan 1992-Dec 1999. .................... 114

Fig. 4-5. Same as Fig. 4-1 except NCEP1 minus METO, lat: 87.5 ${ }^{\circ}-87.5^{\circ} \mathrm{N}$, and time: Jan 1992-Dec 1999.

Fig. 4-6. Same as Fig. 4-5 except ERA-40 minus METO. ......................... 116

Fig. 4-7. Mean, standard deviation, rms, and maxima of longitude-time series of monthly-averaged temperature differences $(K)$ : NCEP1 minus ERA-40 [lon: mean of $0^{\circ}-357.5^{\circ}$, lat: $90^{\circ} \mathrm{S}-90^{\circ} \mathrm{N}$, press: $100-10 \mathrm{hPa}$, time: Jan 1958-Dec 2001]. ... 120

Fig. 4-8. Same as Fig. 4-7 except time: Jan 1958-Dec 1978. ................... 121

Fig. 4-9. Same as Fig. 4-7 except time: Jan 1979-Dec 2001.................... 122 
Fig. 4-10. Standard deviation, rms, and maxima of longitude-time series (Fig. 4-7) minus time series of monthly- and zonally-averaged (Fig. 4-1) temperature differences (K): NCEP1 minus ERA-40 [lon: mean of $0^{\circ}-357.5^{\circ}$, lat: $90^{\circ} \mathrm{S}-90^{\circ} \mathrm{N}$, press: $100-10 \mathrm{hPa}$, time: Jan 1958-Dec 2001].

Fig. 4-11. Same as Fig. 4-10 except longitude-time series is Fig. 4-8 and zonally-averaged time series is Fig. 4-2, and time: Jan 1958Dec 1978.

Fig. 4-12. Same as Fig. 4-10 except longitude-time series is Fig. 4-9 and zonally-averaged time series is Fig. 4-3, and time: Jan 1979Dec 2001.

Fig. 4-13. Mean, standard deviation, rms, and maxima of longitude-time series of monthly-averaged temperature differences with all variance with period less than 13 months removed before differencing (K): NCEP1 minus ERA-40 [lon: mean of $0^{\circ}-357.5^{\circ}$, lat: $90^{\circ} \mathrm{S}-90^{\circ} \mathrm{N}$, press: $100-10 \mathrm{hPa}$, time: Jan $1958-$ Dec 2001].

Fig. 4-14. Same as Fig. 4-13 except time: Jan 1958-Dec 1978. 131

Fig. 4-15. Same as Fig. 4-13 except time: Jan 1979-Dec 2001. 132

Fig. 4-16. Standard deviation, rms, and maxima of filtered longitude-time series (Fig. 4-13) minus unfiltered longitude-time series (Fig. 4-7) temperature differences (K): NCEP1 minus ERA-40 [lon: mean of $0^{\circ}-357.5^{\circ}$, lat: $90^{\circ} \mathrm{S}-90^{\circ} \mathrm{N}$, press: $100-10 \mathrm{hPa}$, time: Jan 1958-Dec 2001].

Fig. 4-17. Same as Fig. 4-16 except filtered longitude-time series is Fig. 4-14, unfiltered longitude-time series is Fig. 4-8, and time: Jan 1958-Dec 1978.

Fig. 4-18. Same as Fig. 4-16 except filtered longitude-time series is Fig. 4-15, unfiltered longitude-time series is Fig. 4-9, and time: Jan 1979-Dec 2001.

Fig. 4-19. Standard deviation of monthly- and zonally-averaged temperatures for DJF and MAM seasons $(K)$, all variance less than 13 months removed: [lon: mean of $0^{\circ}-357.5^{\circ}$, lat: $90^{\circ} \mathrm{S}-90^{\circ} \mathrm{N}$, press: $100-1 \mathrm{hPa}$, time: Jan 1979-Dec 1999]. ..... 140

Fig. 4-20. Same as Fig. 4-19 except JJA and SON seasons. 141 
Fig. 4-21. Standard deviation of monthly- and zonally-averaged seasonal temperatures as a function of pressure level $(\mathrm{K})$, all variance less than 13 months removed: [lon: mean of $0^{\circ}-357.5^{\circ}$, lat: see text, press: 100-1 hPa, time: Jan 1979-Dec 2001]. Significance of individual traces discussed in text.

Fig. 4-22. Three-year moving standard deviation of South Pole $\left(90^{\circ} \mathrm{S}-\right.$ bold) and Southern Hemisphere $\left(90^{\circ} \mathrm{S}-30^{\circ} \mathrm{S}\right.$ - dashed) temperature (K) (all variance with period less than 13 months removed by frequency-domain filtering): [lon: mean of $0^{\circ}-357.5^{\circ}$, lat: as stated, press: $10 \mathrm{hPa}$, time: Jan 1958-Dec 2001].

Fig. 4-23. Same as Fig. 4-22 except North Pole $\left(90^{\circ} \mathrm{N}\right.$ - bold) and Northern Hemisphere $\left(30^{\circ} \mathrm{N}-90^{\circ} \mathrm{N}\right.$ - dashed).

Fig. 4-24. Anomaly time series of zonally-averaged monthly-averaged temperatures $(K)$ (all variance with period less than 13 months removed by frequency-domain filtering): [lon: mean of $0^{\circ}-357.5^{\circ}$, lat: as stated, press: $10 \mathrm{hPa}$, time: Jan 1958-Dec 2001].

Fig. 4-25. Anomaly time series of monthly- and zonally-averaged temperatures (all variance with period less than 13 months removed by frequency-domain filtering): [lon: mean of $0^{\circ}-357.5^{\circ}$; lat: as stated; press: $100 \mathrm{hPa}$; time: Jan 1958-Dec 2005 (NCEP1), Jan 1958-Dec 2001 (ERA-40), Jan 1992-Dec 1999 (METO), Jan 1958-Dec 2005 (RATPAC)]

Fig. 4-26. Same as Fig. 4-25 except press: $70 \mathrm{hPa}$................................ 150

Fig. 4-27. Same as Fig. 4-25 except press: $50 \mathrm{hPa}$.................................. 151

Fig. 4-28. Same as Fig. 4-25 except press: $30 \mathrm{hPa}$................................. 152

Fig. 4-29. Same as Fig. 4-25 except press: $20 \mathrm{hPa}$ and RATPAC data not available.

Fig. 4-30. Same as Fig. 4-25 except press: $10 \mathrm{hPa}$ and RATPAC data not available.

Fig. 4-31. Time series of monthly- and zonally-averaged temperature differences (see text) and 12-month moving rms: NCEP1 minus ERA-40: [lon: mean of $0^{\circ}-357.5^{\circ}$, lat: as stated, press: as stated, time: Jan 1958-Dec 2001].

Fig. 4-32. Same as Fig. 4-31 except pressure levels. 
Fig. 4-33. Mean Northern Hemisphere temperature anomaly from longitudinal mean (K): NCEP1 [lon: $0^{\circ}-357.5^{\circ}$, lat: $0^{\circ}-90^{\circ} \mathrm{N}$, press: as stated, time: monthly means from Jan 1958Dec 2005]. 162

Fig. 4-34. Mean Southern Hemisphere temperature anomaly from longitudinal mean (K): NCEP1 [lon: $0^{\circ}-357.5^{\circ}$, lat: $90^{\circ} \mathrm{S}-0^{\circ}$, press: as stated, time: monthly means from Jan 1958Dec 2005].

Fig. 4-35. December Northern Hemisphere monthly-averaged temperature anomaly from longitudinal mean $(\mathrm{K})$. Longitude (upper) and latitude (middle) location of maximum and minimum anomalies and magnitudes of anomalies (lower). [lon: $0^{\circ}-357.5^{\circ}$, lat: $0^{\circ}-90^{\circ} \mathrm{N}$, press: $10 \mathrm{hPa}$, time: Dec monthly average 1958-2005 (NCEP1), 1958-2001 (ERA-40)].

Fig. 4-36. October Southern Hemisphere monthly-averaged temperature anomaly from longitudinal mean (K). Longitude (upper) and latitude (middle) location of maximum and minimum anomalies and magnitudes of anomalies (lower). [lon: $0^{\circ}-357.5^{\circ}$, lat: $90^{\circ} \mathrm{S}-0^{\circ}$, press: $10 \mathrm{hPa}$, time: Oct monthly average 1958 2005 (NCEP1), 1958-2001 (ERA-40)].

Fig. 4-37. Standard deviation of temperature anomaly longitude-time series $(\mathrm{K})$. [lon: $0^{\circ}-357.5^{\circ}$, lat: $90^{\circ} \mathrm{S}-90^{\circ} \mathrm{N}$, press: 100-10 hPa, time: Jan 1980 through Dec 2001].

Fig. 4-38. Mean, standard deviation, and maxima of time series of monthlyaveraged temperature differences (K): NCEP1 minus ERA-40 [lon: $0^{\circ}-357.5^{\circ}$, lat: $90^{\circ} \mathrm{S}-90^{\circ} \mathrm{N}$, press: $100 \mathrm{hPa}$, time: Jan 1958-Dec 2001].

Fig. 4-39. Same as Fig. 4-38 except press: 70 hPa. .................................. 178

Fig. 4-40. Same as Fig. 4-38 except press: 50 hPa. ................................. 179

Fig. 4-41. Same as Fig. 4-38 except press: $30 \mathrm{hPa}$................................. 180

Fig. 4-42. Same as Fig. 4-38 except press: $20 \mathrm{hPa}$.................................. 181

Fig. 4-43. Same as Fig. 4-38 except press: $10 \mathrm{hPa}$................................ 182

Fig. 4-44. Seidel et al. (2004) and RSS weighting factors vs. pressure level. 
Fig. 4-45. Mean, standard deviation, and maxima of time series of monthlyaveraged temperature differences (K): NCEP1 with RSS weighting minus TLS. [lon: $2.5^{\circ}-357.5^{\circ}$, lat: $80^{\circ} \mathrm{S}-80^{\circ} \mathrm{N}$, press: $250-10 \mathrm{hPa}$, time: Jan 1979-Dec 2001].

Fig. 4-46. Same as Fig. 4-45 except ERA-40. ........................................... 187

Fig. 4-47. Mean monthly- and zonally-averaged temperature differences (K). [lon: $2.5^{\circ}-357.5^{\circ}$, lat: $80^{\circ} \mathrm{S}-80^{\circ} \mathrm{N}$, press: $250-10 \mathrm{hPa}$, time: Jan 1979-Dec 2001].

Fig. 4-48. Same as Fig. 4-47 except standard deviation (LTS: Longitudetime series, ZTS: Zonally-averaged time series).

Fig. 4-49. Time series of NCEP1 and ERA-40 with RSS and SEA weighting minus TLS time series in latitude bands (all variance with period less than 13 months removed). [lon: $2.5^{\circ}-357.5^{\circ}$, lat: as stated, press: $250-10 \mathrm{hPa}$, time: Jan 1979-Dec 2001].

Fig. 4-50. Application of temperature detection algorithm to NCEP1 monthly- and zonally-averaged temperatures. Downward red triangles represent detections, upward black triangles and their labels indicate SSW events from the climatology of Table 4-7. [lon: mean of $0^{\circ}-357.5^{\circ}$, lat: $85^{\circ} \mathrm{N}-90^{\circ} \mathrm{N}$, press: $10 \mathrm{hPa}$, time: Jan 1976-Dec 1989].

See text and Table 4-6 for definition of characters and symbols. ... 199

Fig. 5-1. Mean, standard deviation, rms, and maxima of time series of monthly- and zonally-averaged zonal wind speed differences $\left(\mathrm{m} \mathrm{s}^{-1}\right)$ : NCEP1 minus ERA-40 [lon: mean of $0^{\circ}-357.5^{\circ}$, lat: $90^{\circ} \mathrm{S}-90^{\circ} \mathrm{N}$, press: $100-10 \mathrm{hPa}$, time: Jan 1958-Dec 2001]. ... 213

Fig. 5-2. Same as Fig. 5-1 except time: Jan 1958-Dec 1978. ................... 214

Fig. 5-3. Same as Fig. 5-1 except time: Jan 1979-Dec 2001.................... 215

Fig. 5-4. Same as Fig. 5-1 except time: Jan 1992-Dec 1999. .................... 216

Fig. 5-5. Same as Fig. 5-1 except NCEP1 minus METO, [lat: $87.5^{\circ} \mathrm{S}-87.5^{\circ} \mathrm{N}$, and time: Jan 1992-Dec 1999]. 217

Fig. 5-6. Same as Fig. 5-5 except ERA-40 minus METO. ........................... 218

Fig. 5-7. Mean, standard deviation, rms, and maxima of longitude-time series of differences in monthly-averaged zonal wind speed $\left(\mathrm{m} \mathrm{s}^{-1}\right)$ : NCEP1 minus ERA-40 [lon: mean of $0^{\circ}-357.5^{\circ}$, lat: $90^{\circ} \mathrm{S}-90^{\circ} \mathrm{N}$, press: $100-10 \mathrm{hPa}$, time: Jan 1958-Dec 2001]. ....222

Fig. 5-8. Same as Fig. 5-7 except time: Jan 1958-Dec 1978. 223 
Fig. 5-9. Same as Fig. 5-7 except time: Jan 1979-Dec 2001.

Fig. 5-10. Standard deviation, rms, and maxima of longitude-time series (Fig. 5-7) minus time series of differences in monthly- and zonally-averaged (Fig. 5-1) zonal wind speed $\left(\mathrm{m} \mathrm{s}^{-1}\right)$ : NCEP1 minus ERA-40 [lon: mean of $0^{\circ}-357.5^{\circ}$, lat: $90^{\circ} \mathrm{S}-90^{\circ} \mathrm{N}$, press: $100-10 \mathrm{hPa}$, time: Jan 1958-Dec 2001]. .225

Fig. 5-11. Same as Fig. 5-10 except longitude-time series is Fig. 5-8 and zonally-averaged time series is Fig. 5-2, and time: Jan 1958Dec 1978. 226

Fig. 5-12. Same as Fig. 5-10 except longitude-time series is Fig. 5-9 and zonally-averaged time series is Fig. 5-3, and time: 1979-2001. 227

Fig. 5-13. Mean, standard deviation, rms, and maxima of longitude-time series of monthly-averaged zonal wind speed differences with all variance with period less than 13 months removed before differencing $\left(\mathrm{m} \mathrm{s}^{-1}\right)$ : NCEP1 minus ERA-40 [lon: mean of $0^{\circ}-357.5^{\circ}$, lat: $90^{\circ} \mathrm{S}-90^{\circ} \mathrm{N}$, press: $100-10 \mathrm{hPa}$, time: Jan 1958-Dec 2001].

Fig. 5-14. Same as Fig. 5-13 except time: 1958-1978. ............................. 232

Fig. 5-15. Same as Fig. 5-13 except time: 1979-2001 ............................... 233

Fig. 5-16. Standard deviation, rms, and maxima of filtered longitude-time series (Fig. 5-13) minus unfiltered longitude-time series (Fig. 5-7) zonal wind speed differences $\left(\mathrm{m} \mathrm{s}^{-1}\right)$ : NCEP1 minus ERA-40 [lon: mean of $0^{\circ}-357.5^{\circ}$, lat: $90^{\circ} \mathrm{S}-90^{\circ} \mathrm{N}$, press: 100-10 hPa, time: Jan 1958-Dec 2001].

Fig. 5-17. Same as Fig. 5-16 except filtered longitude-time series is Fig. 5-14, unfiltered longitude-time series is Fig. 5-8, and time: 1958-1978.

Fig. 5-18. Same as Fig. 5-16 except filtered longitude-time series is Fig. 5-15, unfiltered longitude-time series is Fig. 5-9, and time: 1979-2001.

Fig. 5-19. Anomaly time series of monthly- and zonally-averaged zonal wind speed (all variance with period less than 13 months removed by frequency-domain filtering): [lon: mean of $0^{\circ}-357.5^{\circ}$; lat: as stated; press: $100 \mathrm{hPa}$; time: Jan 1958-Dec 2005 (NCEP1), Jan 1958-Dec 2001 (ERA-40), Jan 1992-Dec 1999 (METO)]. 
Fig. 5-20. Same as Fig. 5-19 except press: $70 \mathrm{hPa}$ and for equatorial series all variance with period less than 36 months removed by frequency-domain filtering.

Fig. 5-21. Same as Fig. 5-19 except press: $50 \mathrm{hPa}$ and for equatorial series all variance with period less than 36 months removed by frequency-domain filtering.

Fig. 5-22. Same as Fig. 5-19 except press: $30 \mathrm{hPa}$ and for equatorial series all variance with period less than 36 months removed by frequency-domain filtering.

Fig. 5-23. Same as Fig. 5-19 except press: $20 \mathrm{hPa}$ and for equatorial series all variance with period less than 36 months removed by frequency-domain filtering.

Fig. 5-24. Same as Fig. 5-19 except press: $10 \mathrm{hPa}$ and for equatorial series all variance with period less than 36 months removed by frequency-domain filtering.

Fig. 5-25. Time series of monthly- and zonally-averaged zonal wind speed differences (see text) and 12-month moving rms: NCEP1 minus ERA-40: [lon: mean of $0^{\circ}-357.5^{\circ}$, lat: as stated, press: as stated, time: Jan 1958-Dec 2001].

Fig. 5-26. Same as Fig. 5-25 except pressure levels. .................................. 249

Fig. 5-27. Mean Northern Hemisphere zonal wind speed variation from longitudinal mean $\left(\mathrm{m} \mathrm{s}^{-1}\right)$ : NCEP1 [lon: mean of $0^{\circ}-357.5^{\circ}$, lat: $90^{\circ} \mathrm{S}-90^{\circ} \mathrm{N}$, press: as stated, time: variation: stated month, monthly mean within: Jan 1980-Dec 2005]. 253

Fig. 5-28. Mean Southern Hemisphere zonal wind speed variation from longitudinal mean $\left(\mathrm{m} \mathrm{s}^{-1}\right)$ : NCEP1 [lon: mean of $0^{\circ}-357.5^{\circ}$, lat: $90^{\circ} \mathrm{S}-90^{\circ} \mathrm{N}$, press: as stated, time: variation: stated month, monthly mean within: Jan 1980-Dec 2005].

Fig. 5-29. Standard deviation of zonal wind speed anomaly longitude-time series $\left(\mathrm{m} \mathrm{s}^{-1}\right)$. [lon: $0^{\circ}-357.5^{\circ}$, lat: $90^{\circ} \mathrm{S}-90^{\circ} \mathrm{N}$, press: 100-10 hPa, time: Jan 1980-Dec 2001].

Fig. 5-30. Mean, standard deviation, and maxima of time series of monthlyaveraged zonal wind speed differences $\left(\mathrm{m} \mathrm{s}^{-1}\right)$ : NCEP1 minus ERA-40 [lon: $0^{\circ}-357.5^{\circ}$, lat: $90^{\circ} \mathrm{S}-90^{\circ} \mathrm{N}$, press: $100 \mathrm{hPa}$, time: Jan 1958-Dec 2001].

Fig. 5-31. Same as Fig. 5-30 except press: $70 \mathrm{hPa}$...................................... 262

Fig. 5-32. Same as Fig. 5-30 except press: $50 \mathrm{hPa}$..................................2 263 


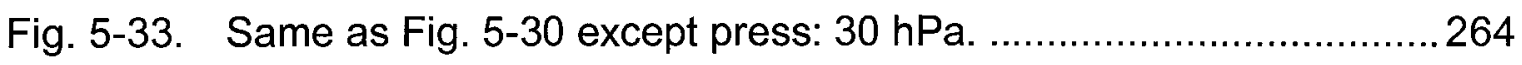

Fig. 5-34. Same as Fig. 5-30 except press: $20 \mathrm{hPa}$.................................. 265

Fig. 5-35. Same as Fig. 5-30 except press: $10 \mathrm{hPa}$.................................26

Fig. 5-36. Latitude (upper) and pressure level (lower) locations of monthly maxima of monthly- and zonally-averaged northern polar vortex wind speeds [lon: mean of $0^{\circ}-357.5^{\circ}$, lat: $45^{\circ} \mathrm{N}-80^{\circ} \mathrm{N}$, press: $30-10 \mathrm{hPa}$, time: Jan 1958-Dec 2001].

Fig. 5-37. Latitude (upper) and pressure level (lower) locations of monthly maxima of monthly- and zonally-averaged southern polar vortex wind speeds [lon: mean of $0^{\circ}-357.5^{\circ}$, lat: $45^{\circ} \mathrm{S}-80^{\circ} \mathrm{S}$, press: $30-10 \mathrm{hPa}$, time: Jan 1958-Dec 2001].

Fig. 5-38. Mean, mean \pm one standard deviation and maximum/minimum monthly- and zonally-averaged northern polar vortex wind speeds at $60^{\circ} \mathrm{N}, 10 \mathrm{hPa}$ (left) and within latitudes and pressure levels (right): [lon: mean of $0^{\circ}-357.5^{\circ}$, lat: $45^{\circ} \mathrm{N}-80^{\circ} \mathrm{N}$, press: $30-10 \mathrm{hPa}$, time: Jan 1958-Dec 2001].

Fig. 5-39. Mean, mean \pm one standard deviation and maximum/minimum monthly- and zonally-averaged southern polar vortex wind speeds at $60^{\circ} \mathrm{N}, 10 \mathrm{hPa}$ (left) and maximum latitudes and pressure levels (right): [lon: mean of $0^{\circ}-357.5^{\circ}$, lat: $45^{\circ} \mathrm{S}-80^{\circ} \mathrm{S}$, press: $30-10 \mathrm{hPa}$, time: Jan 1958-Dec 2001] .... 274

Fig. 5-40. Monthly- and zonally-averaged northern polar vortex zonal wind speeds and linear regression trends (blue: 1958-2001, green: 1958-78, red: $1979-2001$ ). [lon: mean of $0^{\circ}-357.5^{\circ}$, lat: as stated, press: as stated, time: Jan 1958-Dec 2001].

Fig. 5-41. Same as Fig. 5-40 except southern polar vortex zonal wind speeds. 279

Fig. 5-42. 1979-2001 linear regression trends and 5\% confidence intervals for monthly- and zonally-averaged polar vortex zonal wind speeds - blue: NCEP1, green: ERA-40, red: NCEP1 minus ERA-40. [lon: mean of $0^{\circ}-357.5^{\circ}$, lat: as stated, press: as stated, time: 1979-2001]. 280

Fig. 5-43. Logical arrangement of figures. 283

Fig. 5-44. November NH monthly- and zonally-averaged zonal wind speed and temperature and 1979-2001 temperature trend (5\% and $10 \%$ statistical significance limits shown in green): [lon: mean of $0^{\circ}-357.5^{\circ}$, lat: $45^{\circ} \mathrm{N}-90^{\circ} \mathrm{N}$, press: $100-10 \mathrm{hPa}$, time: 1979-2001]. 
Fig. 5-45. November NH monthly- and zonally-averaged horizontal temperature gradient and its 1979-2001 trend, zonal wind speed and its $1979-2001$ trend $(5 \%$ and $10 \%$ statistical significance limits shown in green): [lon: mean of $0^{\circ}-357.5^{\circ}$, lat: $45^{\circ} \mathrm{N}-90^{\circ} \mathrm{N}$, press: $100-10 \mathrm{hPa}$, time: $\left.1979-2001\right]$. 290

Fig. 5-46. Same as Fig. 5-44 except December. ......................................... 291

Fig. 5-47. Same as Fig. 5-45 except December. ....................................... 292

Fig. 5-48. Same as Fig. 5-44 except January. ............................................ 293

Fig. 5-49. Same as Fig. 5-45 except January. ........................................... 294

Fig. 5-50. Same as Fig. 5-44 except February. ……................................. 295

Fig. 5-51. Same as Fig. 5-45 except February. ........................................ 296

Fig. 5-52. October and November NH monthly- and zonally-averaged temperature and zonal wind speed 1979-2001 trends vs. latitude (blue: NCEP1, green: ERA-40, bold: mean trend, light lines: $5 \%$ statistical significance): [lon: mean of $0^{\circ}-357.5^{\circ}$, lat: $45^{\circ} \mathrm{N}-90^{\circ} \mathrm{N}$, press: $100-10 \mathrm{hPa}$, time: 1979-2001] ........... 297

Fig. 5-53. Same as Fig. 5-52 except December and January. ..................... 298

Fig. 5-54. Same as Fig. 5-52 except February and March. .......................... 299

Fig. 5-55. June SH monthly- and zonally-averaged zonal wind speed and temperature and 1979-2001 temperature trend (5\% and $10 \%$ statistical significance limits shown in green): [lon: mean of $0^{\circ}-357.5^{\circ}$, lat: $90^{\circ} \mathrm{S}-45^{\circ} \mathrm{S}$, press: $100-10 \mathrm{hPa}$, time: 1979-2001].

Fig. 5-56. June SH monthly- and zonally-averaged horizontal temperature gradient and its 1979-2001 trend, zonal wind speed and its 19792001 trend $(5 \%$ and $10 \%$ statistical significance limits shown in green): [lon: mean of $0^{\circ}-357.5^{\circ}$, lat: $90^{\circ} \mathrm{S}-45^{\circ} \mathrm{S}$, press: 100-10 hPa, time: 1979-2001]........................................... 301

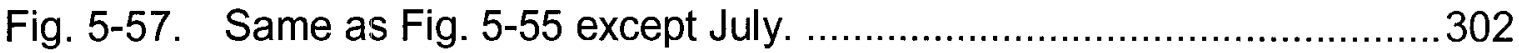

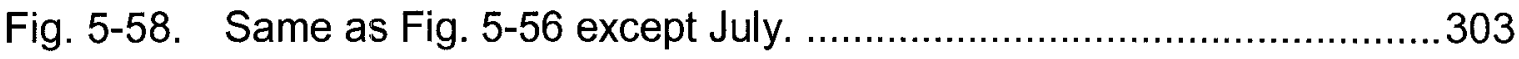

Fig. 5-59. Same as Fig. 5-55 except August. .............................................. 304

Fig. 5-60. Same as Fig. 5-56 except August. ................................................ 305

Fig. 5-61. Same as Fig. 5-55 except September. ..................................... 306

Fig. 5-62. Same as Fig. 5-56 except September. ...................................... 307 
Fig. 5-63. May and June SH monthly- and zonally-averaged temperature and zonal wind speed 1979-2001 trends vs. latitude (blue:

NCEP1, green: ERA-40, bold: mean trend, light lines: $5 \%$ statistical significance): [lon: mean of $0^{\circ}-357.5^{\circ}$, lat: $90^{\circ} \mathrm{S}-45^{\circ} \mathrm{S}$, press: $100-10 \mathrm{hPa}$, time: $\left.1979-2001\right]$................. 308

Fig. 5-64. Same as Fig. 5-63 except July and August. ................................ 309

Fig. 5-65. Same as Fig. 5-63 except September and October. ..................... 310

Fig. 5-66. NH monthly- and zonally-averaged 1979-2001 temperature trends $\left(\mathrm{m} \mathrm{s}^{-1}\right.$ decade $\left.^{-1}\right) \quad 5 \%$ and $10 \%$ statistical significance limits shown in green: [lon: mean of $0^{\circ}-357.5^{\circ}$, lat: $45^{\circ} \mathrm{N}-90^{\circ} \mathrm{N}$, press: $100-10 \mathrm{hPa}$, time: 1979-2001].

Fig. 5-67. Same as Fig. 5-66 except Southern Hemisphere with lat: $90^{\circ} \mathrm{S}-45^{\circ} \mathrm{S}$. 313

Fig. 5-68. NH monthly- and zonally-averaged 1979-2001 zonal wind speed trends $\left(\mathrm{m} \mathrm{s}^{-1}\right.$ decade $\left.^{-1}\right) \quad(5 \%$ and $10 \%$ statistical significance limits shown in green): [lon: mean of $0^{\circ}-357.5^{\circ}$, lat: $45^{\circ} \mathrm{N}-90^{\circ} \mathrm{N}$, press: $30-10 \mathrm{hPa}$, time: $\left.1979-2001\right]$.

Fig. 5-69. Same as Fig. 5-68 except Southern Hemisphere with lat: $90^{\circ} \mathrm{S}-45^{\circ} \mathrm{S}$.

Fig. 5-70. NCEP1 monthly- and zonally-averaged equatorial zonal wind speeds (bold blue line) and FUB (light black line) equatorial data set. Rms difference computed for pressure levels shown and $70 \mathrm{hPa}$ (not shown). [NCEP1: Ion: mean of $0^{\circ}-357.5^{\circ}$, lat: $0^{\circ}$, press: as noted, time: Jan 1958-Dec 1999; FUB: see text].

Fig. 5-71. ERA-40 monthly- and zonally-averaged equatorial zonal wind speeds (bold green line) and FUB (light black line) equatorial data set. Rms difference computed for pressure levels shown and $70 \mathrm{hPa}$ (not shown). [ERA-40: lon: mean of $0^{\circ}-357.5^{\circ}$, lat: $0^{\circ}$, press: as noted, time: Jan 1958-Dec 1999; FUB: see text].

Fig. 5-72. Standard deviation of monthly- and zonally-averaged zonal wind speeds for DJF and MAM seasons $\left(\mathrm{m} \mathrm{s}^{-1}\right)$ all variance less than 13 months removed: [lon: mean of $0^{\circ}-357.5^{\circ}$, lat: $90^{\circ} \mathrm{S}-90^{\circ} \mathrm{N}$, press: $100-1 \mathrm{hPa}$, time: Jan 1958-Dec 2001]. 321 
Fig. 5-73. Standard deviation of monthly- and zonally-averaged zonal wind speeds for JJA and SON seasons $\left(\mathrm{m} \mathrm{s}^{-1}\right)$ all variance less than 13 months removed: [lon: mean of $0^{\circ}-357.5^{\circ}$, lat: $90^{\circ} \mathrm{S}-90^{\circ} \mathrm{N}$, press: $100-1 \mathrm{hPa}$, time: Jan 1958-Dec 2001]. 322

Fig. 5-74. Spectral analysis of NCEP1 (bold blue) and ERA-40 (bold green) equatorial zonal wind speeds and the FUB (black) equatorial data set. [NCEP1 and ERA-40: Ion: mean of $0^{\circ}-357.5^{\circ}$, lat: $0^{\circ}$, press: as stated, time: monthly means from Jan 1958-Dec 1999; FUB: see text].

Fig. 5-75. Phase plots of NCEP1 and ERA-40 equatorial zonal wind speeds plotted with respect to FUB equatorial data set winds. [lon: mean of $0^{\circ}-357.5^{\circ}$, lat: $0^{\circ}$, press: as stated, time: Jan 1958-Dec 1999]. 326

Fig. 6-1. Climatology of monthly- and zonally-averaged meridional wind speed $\left(\mathrm{m} \mathrm{s}^{-1}\right)$ : NCEP1: [lon: mean of $0^{\circ}-357.5^{\circ}$, lat: $90^{\circ} \mathrm{S}-90^{\circ} \mathrm{N}$, press: $100-10 \mathrm{hPa}$, time: seasonal means from Jan 1979-Dec 2001].

Fig. 6-2. Climatology of monthly- and zonally-averaged meridional wind speed $\left(\mathrm{m} \mathrm{s}^{-1}\right)$ : ERA-40: [lon: mean of $0^{\circ}-357.5^{\circ}$, lat: $90^{\circ} \mathrm{S}-90^{\circ} \mathrm{N}$, press: $100-10 \mathrm{hPa}$, time: seasonal means from Jan 1979-Dec 2001].

Fig. 6-3. Means of monthly- and zonally-averaged meridional wind speeds $\left(\mathrm{m} \mathrm{s}^{-1}\right)$ : NCEP1 and ERA-40: [lon: mean of $0^{\circ}-357.5^{\circ}$, lat: $90^{\circ} \mathrm{S}-90^{\circ} \mathrm{N}$, press: $100-10 \mathrm{hPa}$, time: as stated].

Fig. 6-4. Mean (upper), standard deviation, rms, and maximum (lower) of monthly- and zonally-averaged meridional wind speed differences $\left(\mathrm{m} \mathrm{s}^{-1}\right)$ : NCEP1 minus ERA-40: [lon: mean of $0^{\circ}-357.5^{\circ}$, lat: $90^{\circ} \mathrm{S}-90^{\circ} \mathrm{N}$, press: $100-10 \mathrm{hPa}$, time: Jan 1958-Dec 2001].

Fig. 6-5. Time series of monthly- and zonally-averaged meridional wind speed (all variance with period less than 13 months removed by frequency-domain filtering): [lon: mean of $0^{\circ}-357.5^{\circ}$; lat: as stated; press: $100 \mathrm{hPa}$; time: Jan 1958-Dec 2005 (NCEP1), Jan 1958-Dec 2001 (ERA-40)].

Fig. 6-6. Same as Fig. 6-5 except press: $70 \mathrm{hPa}$.....................................347

Fig. 6-7. Same as Fig. 6-5 except press: $50 \mathrm{hPa}$................................... 348

Fig. 6-8. Same as Fig. 6-5 except press: $30 \mathrm{hPa}$.................................... 349

Fig. 6-9. Same as Fig. 6-5 except press: $20 \mathrm{hPa}$................................... 350 
Fig. 6-10. Same as Fig. 6-5 except press: $10 \mathrm{hPa}$.

Fig. 6-11. Time-latitude structure of monthly- and zonally-averaged meridional wind speed for NCEP1 (left) and ERA-40 (right). Contours $0.1 \mathrm{~m} \mathrm{~s}^{-1}$ : [lon: mean of $0^{\circ}-357.5^{\circ}$; lat: as stated; press: $100 \mathrm{hPa}$; time: Jan 1958-Dec 2005 (NCEP1), Jan 1958-Dec 2001 (ERA-40)]. 355

Fig. 6-12. Same as Fig. 6-11 except press: $70 \mathrm{hPa}$ 356

Fig. 6-13. Same as Fig. 6-11 except press: $50 \mathrm{hPa}$. 357

Fig. 6-14. Same as Fig. 6-11 except press: $30 \mathrm{hPa}$. 358

Fig. 6-15. Same as Fig. 6-11 except press: $20 \mathrm{hPa}$. 359

Fig. 6-16. Same as Fig. 6-11 except press: $10 \mathrm{hPa}$. 360

Fig. C-1. Data Flow. 386

Fig. D-1. Three-dimensional zonally-averaged data set. 388

Fig. D-2. Time series for one pressure-level - latitude point in a threedimensional zonally-averaged data set. 388

Fig. D-3. Longitude-time series for one pressure level - latitude point in a three-dimensional data set. 389

Fig. E-1. NCEP1 monthly temperature anomaly time series at $10 \mathrm{hPa}$, latitude-weighted from $90^{\circ} \mathrm{S}$ to $30^{\circ} \mathrm{S}$ and corresponding annual calendar-year averages.

Fig. E-2. Variance spectrum of NCEP1 monthly temperature anomaly time series of Fig. E-1. 393

Fig. E-3. Variance spectrum of NCEP1 monthly temperature anomaly time series of Fig. E-1 and spectrum after notch filtering at 12, 6,4 , and 3 month periods (upper) and after removal of periods less than 13 months (lower).

Fig. E-4. Time series from notch (upper) and low-pass (lower) filtering (Fig. E-2) and annual calendar-year averages from Fig. E-1. 395

Fig. E-5. Variance spectra of anomaly time series of zonally- and monthlyaveraged temperatures: NCEP1 and ERA-40 (upper), ratios (middle), and differences (lower) of their spectra [lon: mean of $0^{\circ}$ to $357.5^{\circ}$, lat: $90^{\circ} \mathrm{S}$ to $30^{\circ} \mathrm{S}$, press: $10 \mathrm{hPa}$, time: Jan 1958 to Dec 2001]. 
Fig. E-6. Variance spectra of anomaly time series of monthly- and zonally-averaged temperatures: NCEP1 and ERA-40 (upper), ratios (middle), and differences (lower) of their spectra [lon: mean of $0^{\circ}$ to $357.5^{\circ}$, lat: $30^{\circ} \mathrm{N}$ to $90^{\circ} \mathrm{N}$, press: $100 \mathrm{hPa}$, time: Jan 1958 to Dec 2001]. 


\subsection{INTRODUCTION}

\subsection{Motivation and goals}

In 1988 Lennart Bengtsson and Jagadish Shukla observed that then-available global atmospheric data sets were not suitable for analyses of global climate change. These data sets captured the initial conditions used daily for numerical weather forecast models, and also contained valuable descriptions of dynamic and physical atmospheric processes and of the general circulation. However, continuing improvements in the forecast models and in data assimilation systems prevented production of the consistent and homogeneous long-term time series required for valid analyses of global climate change. Bengtsson and Shukla (1988) predicted that data assimilation systems employing optimal interpolation and normal mode initialization would reach "an apparent plateau in their evolution ... within one or two years." This anticipated maturation of data assimilation was another essential motivation for their proposal that

a comprehensive analysis of global observations based on a fourdimensional data assimilation system with a realistic physical model should be undertaken to integrate space and in situ observations to produce internally consistent, homogeneous, multivariate data sets for the earth's climate system. The concept is equally applicable for producing data sets for the atmosphere, the oceans, and the biosphere, and such data sets will be quite useful for studying global climate change.

They concluded,

for this reason we suggest that a reanalysis project be initiated to reanalyze a ten-year period 1979 to 1988 using state-of-the-art data assimilation systems including a model of the highest possible resolution. The project should commence in 1989 or 1990 with the objective to produce a full data set within a period of about two years. 
Trenberth and Olsen (1988) compared data sets of global analyses and forecasts produced by the United States National Meteorological Center (NMC) and the European Centre for Medium-Range Weather Forecasts (ECMWF) from 1979 to 1988 in an attempt to establish a global climate record. Although generally successful, their work was handicapped by changes in the analysis processes and forecast models of the two centers. Buried deep in their concluding remarks is their argument for reanalyses.

Consequentially a strong case can be made for a reanalysis of the original data, preferably enhanced with further observations (either delayed or nonreal time) that did not arrive before the operationally imposed cutoff, using the same state-of-the-art analysis system.

Their paper appeared one month before Bengtsson and Shukla (1988) in the same journal, the Bulletin of the American Meteorological Society (BAMS). Clearly, reanalysis was an idea whose time had come. The initial planning and development of the first reanalysis project by NMC began within a year (Kalnay et al. 1996) and ECMWF's first reanalysis program was begun in 1993 (Gibson et al. 2006). (NMC became the Environmental Modeling Center of the National Centers for Climate Prediction (NCEP) in 1995.) As arguably the preeminent meteorological organizations in the world, these two organizations could be expected to take the lead in reanalyses. Bengtsson and Shukla (1988) was clearly a message of intent as much a proposal; at that time Bengtsson was the director of ECMWF and Shukla director of the Center for OceanLand-Atmosphere Interactions (COLA) and a professor at the University of Maryland, approximately 10 miles from NMC.

A meteorological reanalysis is a retroactive record of global atmospheric parameters based on the assimilation of all available observational data, some of which 
may not have been available for forecasts in real time. Stable configurations, adapted from operational numerical weather prediction and assimilation systems, that remain unchanged for the duration of the production of the reanalysis have produced multi-decade reanalyses of all available observational data. A succinct summary of reanalysis by Randel et al. $(2002,2004)$ : "the use of numerical forecast models to predict first-guess fields, and incorporate observations by optimal data assimilation" is quite similar to that of Gibson et al. (2006): "Thus continuous data assimilation over a long period is really equivalent to running the forecast model as a global circulation model and relaxing toward the observations and forcing fields at six hourly intervals."

Reanalysis results are a unique asset in the study of global climate change; they contain the values of a large number of atmospheric variables, both observed and derived, with horizontal resolutions of at least $2.5^{\circ} \times 2.5^{\circ}$, with at least 28 pressure levels from the surface through the lower stratosphere, at time intervals as short as six hours, and for periods as long as 49 years. Thus, data describing the behavior of all significant atmospheric variables in the lower $99 \%$ (by mass) of the atmosphere for almost the past half century are available.

However, these atmospheric histories are not perfect. The processing time required to generate multi-decade data sets limits horizontal and vertical resolution. While model and assimilation systems are by definition frozen, the observing systems that supply the data to be assimilated change with time. Errors in these data, in its calibration, and the weighting of numerous data sources in the assimilation process result 
in differences among reanalyses. Finally there are human, procedural, and computational errors.

An intercomparison is “a mutual comparison of corresponding parts" (Webster's Revised Unabridged Dictionary (C) 1996, 1998). This thesis intercompares two of the five major reanalyses to identify a stratospheric climatology that may be employed in the evaluation of global climate models (GCMs), such as those submitted in support of the Fourth Assessment Report of the Intergovernmental Panel on Climate Change (IPCC). The IPCC issued Assessment Reports in 1990, 1995, and 2001 and a fourth is being released in 2007. IPCC Assessment Reports "form the standard scientific reference for all those concerned with climate change and its consequences" (IPCC 2001). They contain the comprehensive scientific consensus of the climate change community. In addition to supporting the current IPCC assessment report, GCMs form the bases of other analyses and process studies such as the World Meteorological Organization (WMO) ozone assessments.

Underlying the assessment of $21^{\text {st }}$ century climate change in the Fourth Assessment Report will be the simulations produced by some of the twenty GCMs submitted for this purpose. For the first time, most of these GCMs directly couple ocean and atmosphere without flux adjustments. These simulations attempt to model the $20^{\text {th }}$ century climate based on known levels of natural and anthropogenic forcing and also predict the $21^{\text {st }}$ century climate. Accurate modeling of the $20^{\text {th }}$ century climate is the best indicator of accurate $21^{\text {st }}$ century predictions. 
Understandably, one of the foci of the IPCC Fourth Assessment Report is evaluation of $20^{\text {th }}$ century model performance and $21^{\text {st }}$ century model projections in the troposphere. However, the coupling between stratospheric and tropospheric weather and climate processes and, in particular, the influence of stratospheric conditions on the troposphere, has been established; its relevance and implications grow with continuing research, some of which is identified below:

- Baldwin and Dunkerton $(1999,2001)$ have shown downward propagation of anomalies in the midlevel stratosphere to the troposphere.

- Thompson and Solomon (2002) correlate the trend of increased strength of the Southern Hemisphere lower stratosphere polar vortex, due to photochemical ozone losses, with the significant tropospheric trend of a cooling Antarctic plateau and a warming subpolar region.

- Thompson et al. (2002) demonstrate a statistically significant impact on Northern Hemisphere winter weather from the low-frequency variability of the stratospheric circulation and a modulation of this impact by the phase of the quasi-biennial oscillation (QBO). They argue that weather prediction models must include appropriate stratospheric dynamics.

- Black (2002) provides observational evidence that "variations in the [Northern Hemisphere] winter stratospheric polar vortex can directly influence surface climate" and also suggests that "a proper simulation of tropospheric climate requires an adequate representation of stratospheric dynamical and physical processes."

- Coughlin and Tung (2001) used 150-year Northern Hemisphere surface temperatures filtered by the northern annular mode (NAM) to show that the QBO signal can be traced with statistical significance from the upper stratosphere to the extratropical surface.

- Chan (1995) shows that western North Pacific tropical cyclone activity is related to the stratospheric QBO.

- Hsu and Yung (1999) found evidence of the QBO in tropospheric methane.

- Nathan and Cordero (2007) derive an ozone-modified refractive index that relates stratospheric ozone distribution to stratosphere-troposphere communication, specifically, to vertically propagating planetary waves. 
At the 2003 SPARC Workshop on the Role of the Stratosphere in Tropospheric Climate, 43 invited papers were presented on this subject (Gillett et al. 2006). Another workshop on this subject will take place in September 2007.

Thus, the tropospheric performance of global climate models supporting the IPCC assessment and other activities identified above is dependent on their representation of the stratosphere. This dependency is also crucial in efforts to improve these models. Validation of the $20^{\text {th }}$ century stratospheric performance of these models requires the use of the longest running and most representative atmospheric reanalyses available. Since none of these reanalysis results is without defects, assessments of their stratospheric applicability are presented in Sections 4 through 6 of this thesis, which intercompare reanalysis outputs and also compare these outputs with commensurate independent data where such data is available.

\subsection{Previous research}

Randel et al. (2002) produced an intercomparison of middle atmosphere climatologies for the years 1992-97 that included evaluations of the following reanalysis products: temperature, mean zonal wind speed, and mean zonal heat and momentum fluxes. This work was later abstracted as Randel et al. (2004). Pawson and Fiorino (1998a, 1998b, 1999) compared the tropical stratospheric performance of the NCEP1 and ERA-15 reanalyses (acronyms introduced in Section 1.3). The GCM-Reality Intercomparison Project for SPARC (GRIPS) (Pawson et al. 1999) assessed the performance of 13 middle-atmosphere climate models; ERA-15 data was employed to 
represent climatological "observations." Manney et al. (2003) demonstrated the differences in Northern Hemisphere stratospheric data sets, including one reanalysis. Manney et al. (2005) evaluated the descriptions of the 2002 Antarctic winter and warming in ten stratospheric data sets, including three reanalyses.

To date there is no published detailed evaluation of the performance of global reanalysis products over extended periods of time in the stratosphere above $100 \mathrm{hPa}$. Such an evaluation is the subject of this thesis.

\subsection{Approach}

This thesis intercompares the stratospheric performance of two of the five major global atmospheric reanalyses with the aid of comparison data to identify a realistic climatology for use in the evaluation of the efficacy of IPCC models, as well as models employed in the WMO ozone assessments, and other stratospheric analyses.

Global meteorological reanalysis is introduced and the general characteristics of reanalysis are discussed in Section 2. This section continues with descriptions and comparisons of the relevant characteristics of the five major global reanalysis projects:

NCEP1 United States National Centers for Environmental Prediction (NCEP) and National Center for Atmospheric Research (NCAR) (NCEP-NCAR): reanalysis data from 1948 and continuing

NCEP2 United States National Centers for Environmental Prediction and Department of Energy Atmospheric Model Intercomparison Project (NCEP-DOE AMIP-II Reanalysis (R-2)): reanalysis data from 1979 and continuing

ERA-15 European Centre for Medium-Range Weather Forecasts (ECMWF): reanalysis data 1979-93

ERA-40 ECMWF: reanalysis data 1957-2002 
JRA-25 Japan Meteorological Agency (JMA) and the Central Research Institute of Electric Power Industry (CRIEPI): reanalysis data from 1979 and continuing

The section also discusses the rationale for selection of two of these (NCEP1 and ERA-40) for intercomparison. The section concludes with the description of four comparison data sets:

METO United Kingdom Meteorology Office (UKMO) daily stratospheric analyses

FUB Institut für Meteorologie of the Freie Universität Berlin Northern Hemisphere temperature and geopotential height analyses

RATPAC NOAA Radiosonde Atmospheric Temperature Products for Assessing Climate large-scale regional mean time series based on expert-analyzed radiosonde data and independent of satellite and reanalysis data

TLS Stratospheric temperature data from Channel 4 of the Microwave Sounding Unit (MSU) and Channel 9 of the Advanced Microwave Sounding Unit (AMSU), carried on eleven NOAA polar-orbiting spacecraft, merged to form the TLS (Temperature Lower Stratosphere) data set by Remote Sensing Systems (RSS) of Santa Rosa, CA.

Section 3 presents an overview of stratospheric climatology.

Sections 4, 5, and 6 compare the temperature, zonal wind speed, and meridional wind speed results, respectively, of two reanalyses. Monthly mean data is employed throughout this thesis. Reanalyses are compared over the full 44-year commensurate time span of reanalysis data; for this purpose, monthly-averaged data is quite adequate. High frequency, e.g., daily, intercomparisons of general variability and phenomena such as transient eddy heat flux and stratospheric sudden warming are beyond the scope of this thesis. Further, three of the four comparison data sets contain only monthly mean data and the fourth, RATPAC, only annual data. Monthly data for a single pressure level, 
with $2.5^{\circ} \times 2.5^{\circ}$ horizontal resolution, for the 48 years from 1958 through 2005 , consume approximately 23.7 MB of storage. The size of a file of daily data of these same dimensions would be approximately $8.7 \mathrm{~GB}$ !

Table 1-1 indicates the full calendar year data available for each parameter compared. The acronyms in this table are defined above and are discussed further in Sections 2.2 and 2.3 .

Table 1-1. Available stratospheric data sets.

\begin{tabular}{|l|c|c|c|c|c|c|}
\cline { 2 - 7 } \multicolumn{1}{c|}{} & \multicolumn{2}{c|}{ REANALYSES } & \multicolumn{3}{c|}{ COMPARISON DATA SETS } \\
\cline { 2 - 7 } \multicolumn{1}{c|}{} & NCEP1 & ERA-40 & METO & FUB & RATPAC & TLS \\
\hline \multirow{2}{*}{ Temperature } & $1958-$ & $1958-$ & $1992-$ & $1964-$ & $1958-$ & $1979-$ \\
& 2006 & 2001 & 1999 & 2001 & 2006 & 2006 \\
\hline \multirow{2}{*}{ Zonal Wind } & $1958-$ & $1958-$ & $1992-$ & & & \\
& 2006 & 2001 & 1999 & & & \\
\hline \multirow{2}{*}{ Meridional Wind } & $1958-$ & $1958-$ & & & & \\
& 2006 & 2001 & & & & \\
\hline
\end{tabular}

Conclusive intercomparison of only two reanalyses requires additional independent data to determine the more accurate of the two. The four comparison data sets are used wherever possible to provide additional independent assessments. The literature, most notably the intercomparisons of eight stratospheric climatologies in the SPARC Intercomparison of Middle Atmosphere Climatologies (Randel et al. 2002, 2004), adds useful independent perspective and evaluations in limited, specific circumstances.

Section 7 addresses the future of reanalysis. Section 8 contains a summary and the conclusions of this thesis. Material referenced in this thesis is identified in Section 9. Finally, a set of five appendices present related supporting material. 


\subsection{REANALYSIS AND THE STRATOSPHERIC DATA SETS}

Section 2.1 provides an introduction to meteorological reanalysis and discusses the general nature of reanalysis. Section 2.2 introduces the five major global reanalyses; it discusses their philosophy, design, and the evaluation of their stratospheric performance in the research literature. Four additional reanalyses that are not applicable to the analyses of this thesis are briefly described to demonstrate the breadth of the field. Section 2.2 concludes with the selection of the two reanalyses that will be intercompared in Sections 4 through 6 of this thesis.

To support the reanalysis intercomparison, four additional data sets of lesser temporal or spatial extent are employed. They are introduced and discussed in Section 2.3.

\subsection{Meteorological reanalysis - Introduction and general considerations}

This section provides an introduction to meteorological reanalysis and discusses the general nature of reanalysis. A meteorological reanalysis is a retroactive record of global atmospheric parameters based on the assimilation of all available observational data, some of which may not have been available for forecasts in real time.

A meteorological reanalysis is a series of retrospective forecasts (Kalnay et al. 1996):

- Produced for an extended reanalysis period (typically decades) 
- Based on all recoverable and applicable observational data (surface land synoptic, rawinsonde, surface marine, aircraft, satellite, and others), some of which were not available in real time, from many countries, organizations, and agencies, quality controlled and data managed by an extensive "data preparation" or "data archive" system

- Employing a state-of-the-art forecast system consisting of a data assimilation system and forecast model (typically a global operational system used with minimal modification) that are kept unchanged over the reanalysis period (and may contain additional quality control features)

- Resulting in output data sets of the analyzed and derived variables for the user community

Only the four organizations identified in Section 1.3 have undertaken the Herculean task of producing global, multi-decade reanalyses of the atmosphere. They have produced or are producing reanalyses that are currently available for use in the evaluation of GCM output. The output data created by these reanalyses range in duration from 15 to 59 years. 
The five reanalysis projects and their acronyms employed in this thesis are:

1. NCEP-NCAR Reanalysis

2. NCEP-DOE AMIP Reanalysis (R-2)

3. ECMWF ERA-15

4. ECMWF ERA-40

5. Japanese Re-Analysis Project 25 Years "JRA-25"
"NCEP1"

"NCEP2"

"ERA-15"

"ERA-40"

Three-dimensional variational assimilation (3DVAR) is the basic methodology used in four of the five global reanalysis projects considered in this thesis. The ECMWF ERA-15 reanalysis used Optimal Interpolation (OI). More general than OI with respect to observations, 3DVAR supports nonlinear relationships between observations and analysis variables. These relationships may be functions of more than one analysis variable, and observations may simultaneously affect the analysis of several variables (Andersson et al. 1998).

Three-dimensional data assimilation is accomplished in a continuous cycle consisting of a series of three conceptual steps (Kalnay et al. 1996; Kanamitsu 1989; Lorenc et al. 2000):

1. Based on the most recent analysis, a short-term forecast (typically six hours) using a comprehensive atmospheric or coupled ocean-atmospheric model with full physics produces a first guess for step 2. This forecast is sometimes termed the "background field."

2. The difference between the background field and all observational data for the time interval of interest, weighted as a function of statistical uncertainty, is used to form a cost function that is minimized with three-dimensional variational analysis (3DVAR). This minimization is subject to dynamical balance constraints, i.e., mass and wind. The results of this step are "optimal analysis increments." 
3. The set of analysis increments from the second step are used to modify the background field and form the analysis field that becomes the initial field for the next forecast, i.e., step 1 of the next iteration.

In four-dimensional variational analysis (4DVAR), a background field is established as above. A cost function is then generated that (1) creates an optimum fit to all observations in a (typically) 6-hour interval by integrating the forecast model and observations over that interval while (2) also minimizing the difference between the timevarying fields and the evolving forecast. The forecast that minimizes the cost function is captured at the midpoint of the assimilation interval (ECMWF 2006b;

Andersson et al. 1998). A significant disincentive to 4DVAR is its computational cost; incremental 4DVAR at ECMWF and Meteo-France used for operational forecasts reportedly runs five to ten times longer than 3DVAR (NCAR/MMM 2006).

Although a global, multi-decade reanalysis is a Herculean task, the Hercules in these cases are state-of-the-art computing systems. The staff of one reanalysis project (NCEP1) estimated a production rate of approximately 1.2 reanalysis months each 24-hour computing day (allowing for analytical runs to evaluate changes in observing systems and reruns to correct database problems discovered after processing begins). At this processing rate there is minimal opportunity for "the detailed human scrutiny that operational output normally receive" (Kalnay et al. 1996).

Human contribution to a reanalysis effort is in the software life-cycle of planning, design, development, integration, and testing of system components for data preparation, assimilation, and distribution as well as actual production. Especially critical in reanalyses are the identification, acquisition, pre-processing, and quality control (at 
multiple steps in the process) of data, some of which may not have been available at the time of the original operational forecast. One project archive description (ERA-40) lists roughly 16 "data suppliers" and 64 "data sources / types" from other countries, organizations, and agencies in addition to that center's own extensive data sources (Kållberg et al. 2006). Uppala et al. (2005) point out that not only must reanalysis systems properly prepare and assimilate current operational data; they are required to do the same with data from older and perhaps obsolete observational systems. "From memory, I believe we generated a total of more than 50 (and probably a bit more than 60 ) terabytes by the whole assimilation process. Input observational data accounted for a few terabytes - mostly the satellite radiances" (A. J. Simmons 2006, personal communication).

Reanalysis output may be grouped into three categories (Kistler et al. 2001):

1. Type A variables; those that are strongly influenced by observed data and thus are in the most reliable category:

Upper air temperature, zonal wind speed in the extratropics, geopotential height

2. Type B variables; those that are influenced by both observations and the model:

Meridional wind speed, zonal wind speed in the Tropics, moisture variables, surface parameters

3. Type $\mathrm{C}$ variables; those that are completely determined by the model within the constraints of other observations:

Precipitation, surface fluxes, heating rates

The comparisons in this thesis consider three higher reliability variables:

- Upper air temperature Type A 
- Zonal wind speed

Type A (midlatitudes), Type B (Tropics)

- Meridional wind speed Type B

Upper air temperature observations include radiosonde, rocketsonde, and satellite radiance data. Radiosonde data is the only direct source of wind observations in the stratosphere and then only in the lower regions. However, the multivariate nature of 3DVAR described above infers wind information from radiosonde temperature observations and radiance data from sensors on polar-orbiting spacecraft. Thus the characterization of zonal wind speeds as "Type A" by Kistler et al. (2001) may be valid in the troposphere and lower stratosphere, but in the remainder of the stratosphere reanalysis wind speeds are the result of data assimilation and subject to its uncertainties.

The scope and duration of reanalysis projects make them valuable to the scientific community but also render them vulnerable to errors and limitations. Data may be incomplete, inconsistent, and/or inaccurate; these conditions may vary independently in any of the four dimensions. The forecast model and assimilation system may contain errors and/or suffer from limitations of parameterization and/or resolution. Finally, there are state-of-the-art limitations imposed by computing technology and modeling techniques. 
Data assimilation systems are "frozen" in a known, tested, and documented configuration prior to the beginning of a reanalysis to eliminate apparent change due to system changes. Databases are also as complete as possible at this time. However, the decades encompassed by current multi-decade reanalyses were decades of quantum leaps in observing system technology, capability, and impact, especially on reanalysis.

Kistler et al. (2001) identify "three major phases" in the evolution of global observing system technology:

- Early years from the 1940 s to the International Geophysical Year in 1957 when the first upper-air observations were established

- Modern rawinsonde network from 1958 to 1978

- Modern satellite era from 1979 to the present

From 1957 to 2002 geographical and temporal radiosonde coverage declined.

However, because accuracy improved, the number of measurements accepted by the ECMWF assimilation system increased from 1979-90 to 1991-2001

(Uppala et al. 2005).

The remote sensing satellite system whose data was introduced into NCEP1 and ERA-40 in late 1978 to begin the modern satellite era was TOVS, the Television InfraRed Observation Satellite (TIROS) Operational Vertical Sounder. However, Bromwich and Fogt (2004) identify a slightly earlier system, VTPR (Vertical Temperature Profile Radiometer), whose data was introduced into both NCEP1 and ERA-40 reanalyses about the beginning of 1973. The VTPR data had a measurable impact on NCEP1's $100 \mathrm{hPa}$ reanalyses at four Antarctic coastal stations beginning in 1973 (as demonstrated in Figs. 4(d) and 5(d) of Marshall 2002). Thus, the modern 
satellite era had an unheralded precursor for six years although Li et al. (1999) state that without extensive reprocessing such as was done for ERA-40, "VTPR retrievals are not of sufficient quality to be usable before 1975."

Uppala et al. (1999) identify six "main epochs" based on satellite sensor capabilities:

- No satellite data

- VTPR

- TOVS and CMW

- TOVS, CMW, and SSM/I

- TOVS, CMW, SSM/I, and ERS

- TOVS, CMW, SSM/I, ERS, and ATOVS 1998 to the present
Mid-1957 through 1971

1972 through 1978

1979 to the present

1987 to the present

1991 to the present

Definitions of above acronyms not previously defined are:

CMW Cloud Motion Winds (obtained from differential cloud positions in sequential satellite images)

SSM/I Special Sensor Microwave/Imager

ERS Earth Resources Satellite

ATOVS Advanced Television InfraRed Observation Satellite (TIROS)

Operational Vertical Sounder

Therefore, it is obvious that although reanalysis systems remained unchanged, the observing systems changed dramatically. "The introduction of satellite data in 1979 resulted in a significant change in the climatology, especially above $200 \mathrm{hPa}$ and south of $50^{\circ} \mathrm{S}$, suggesting that the climatology based on the years $1979-$ present day is most reliable" (Kistler et al. 2001) Also, because of the absence of an adequate global radiosonde network before the 1957-58 International Geophysical Year, the pre-1958 era should be regarded as the "least reliable period, especially for the Southern Hemisphere" 
(Kistler et al. 2001). Although the NCEP1 reanalysis output begins in 1948, no work reported in journal articles reviewed during the preparation of this thesis uses data before January 1958.

Figure 2-1 illustrates the pressure level vs. latitude structure of the difference of zonally-averaged monthly temperatures averaged over the 15-year intervals 1960-74 and 1985-99 for both the NCEP1 and ERA-40 reanalyses. The earlier period is subtracted from the latter. This figure graphically demonstrates the "significant change in the climatology, especially above $200 \mathrm{hPa}$ and south of $50^{\circ} \mathrm{S}$ " (Kistler et al. 2001) that resulted from the introduction of satellite data in 1979. Lesser change is also evident in both reanalyses in the northern polar regions above $20 \mathrm{hPa}$ and in NCEP1 at the equatorial tropopause. The upper plot of Fig. 2-1 recreates Fig. 33b of Randel et al. (2002). 

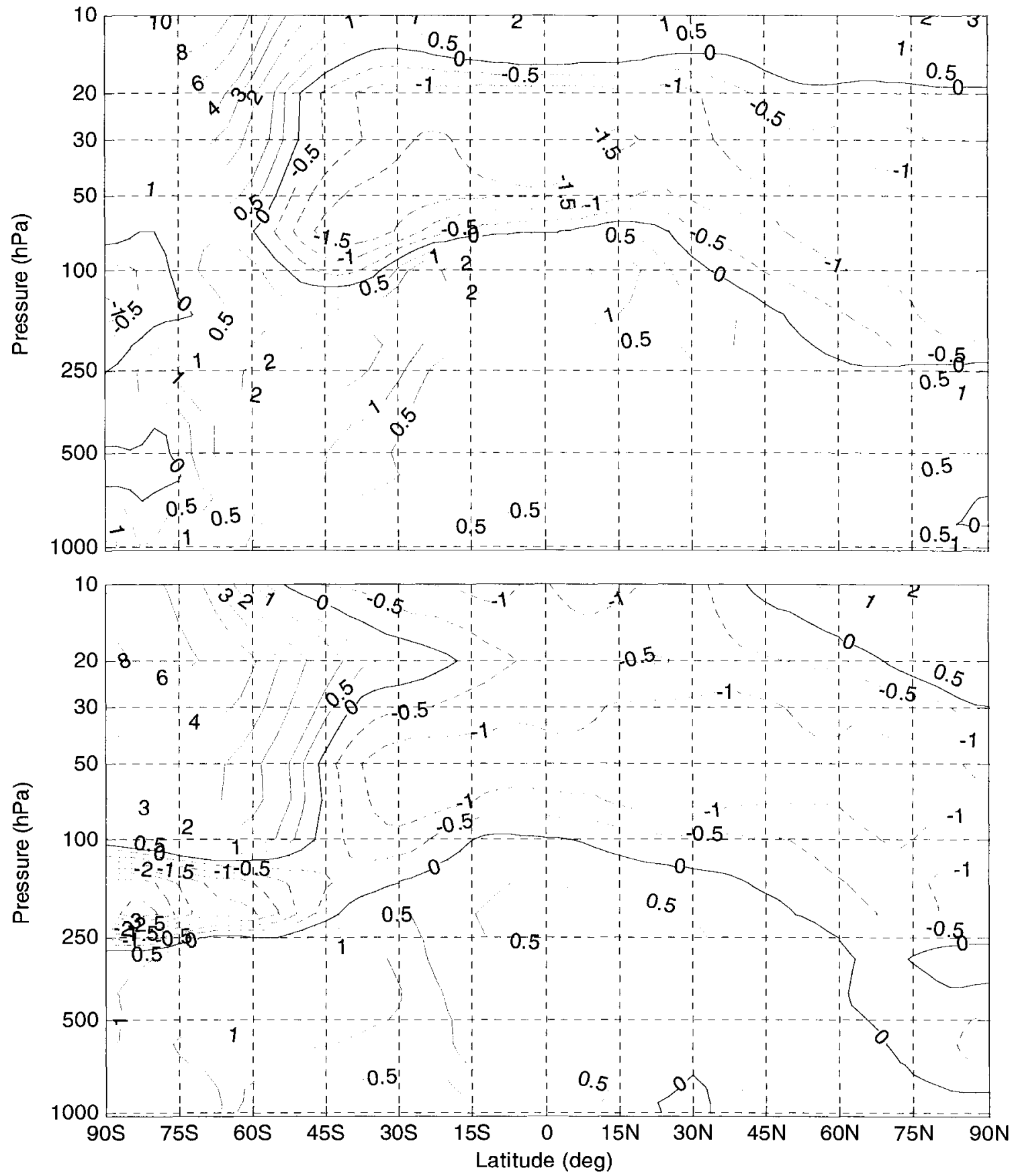

Fig. 2-1. Differences in 15-year means of NCEP1 (upper) and ERA-40 (lower) monthlyand zonally-averaged temperatures. 1960-74 means are subtracted from 1985-99 means. Temperature differences in $K$. 
Reanalysis climatologies that span two or more of the phases that Kistler et al. identified above can never be "corrected" for changes in their fields due to observing system changes; the data will never exist! Kistler et al. (2001) state that "However, because of changes in the observing system, estimation of trends with the reanalysis is not recommended," but then suggest to the users of reanalyses a number of rather obvious techniques they might employ to preserve the validity of research that must span these change points:

- Check the types of observations in time and space (surface, rawinsonde, satellite, etc.) employed in the reanalysis and evaluate the implications for the research at hand.

- "Compute trends for the periods before and after 1979 separately."

- Compare trends for more than one reanalysis. (This step is necessary but not sufficient to insure reliability since significant amounts of data are shared among several or all of the major reanalysis projects.)

Sterl (2004) summarizes the impact of the quantity of observations on reanalysis results:

A reanalysis is effectively a model run constrained by observations. If only few observations are available, the constraint is weak and the model essentially produces its own intrinsic variability. With an increasing amount of observations available, the model is more and more forced to follow the observed variability rather than its own intrinsic one.

Gibson et al. (2006), reflecting on the results of an extensive sensitivity study of prognostic cloud models for ERA-15, stated that "many aspects of a re-analysis are to a high degree defined by the assimilating forecast model, and particularly by its physics." 
Figure 2-2 illustrates this situation as outlined below:

1. The difference between NCEP1 and ERA-40 monthly- and zonally-averaged temperature data is computed for the years 1960-74, before the modern satellite era.

2. Root-mean-square (rms) values of the temperature difference are computed and presented in the upper subplot.

3. Steps 1 and 2 are repeated for the years 1985-99, during the modern satellite era, and presented in the middle subplot.

4. The 1985-99 rms results are subtracted from the 1960-74 rms results and presented in the lower subplot.

This figure graphically demonstrates that the climatologies inherent in the models of the two reanalysis systems do produce their "own intrinsic variability" in the absence of constraining observations. Comparison of the upper two subplots shows, and the lower subplot emphasizes, that the differences in the two reanalyses are significantly larger in the absence of satellite temperature observations above $200 \mathrm{hPa}$ and south of $50^{\circ} \mathrm{S}$, the region identified by Kistler et al. (2001). In the stratospheric Southern Hemisphere with its minimal radiosonde network, satellite temperature observations serve as the constraint. The (1) areas of increased temperature at 200 and $30 \mathrm{hPa}$ adjacent to the South Pole and (2) those at $100 \mathrm{hPa}$ spanning the Equator in the middle subplot (1985-99) are consequences of known problems in (1) ERA-40 and (2) NCEP1 temperature results, respectively, and are discussed in Sections 2.2.4 (ERA-40) and 2.2.1 (NCEP1). 

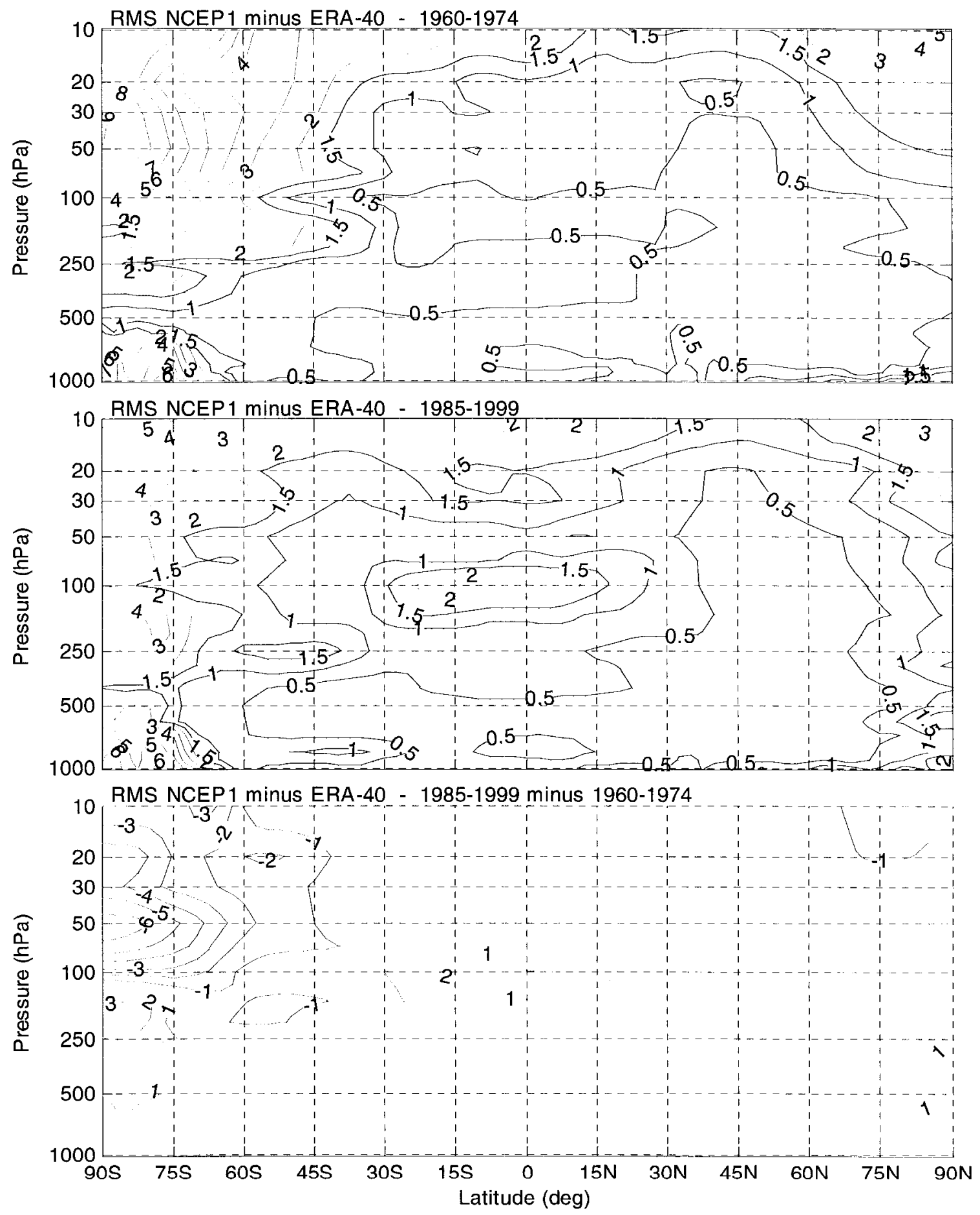

Fig. 2-2. Rms differences of NCEP1 minus ERA-40 monthly-and zonally-averaged temperatures for years 1960-74 (upper) and years 1985-99 (middle). NCEP1 minus ERA-40 differences for years 1958-99 minus differences for years 1960-74 (lower). Rms temperature differences in $K$. 
The areas of large rms difference in the southern polar lower troposphere in the upper two subplots of Fig. 2-2 result in part from errors in model heights of nine Antarctic stations located at an average latitude of $71^{\circ} \mathrm{S}$. The model heights of these stations are lower than the actual station heights by 381 meters average, 565 meters maximum (NCEP1) and 252 meters average, 744 meters maximum (ERA-40). The resulting average observed surface temperature biases are $-4.9 \mathrm{~K}(\mathrm{NCEP} 1)$ and $-3.1 \mathrm{~K}$ (ERA-40) (Bromwich and Fogt 2004). While not directly relevant to the stratospheric focus of this thesis, this case, where errors in only nine static constants, not corrected in a timely fashion, produce such glaring anomalies, illustrates the difficulty in accurately assembling the immense databases required for a multi-decade global reanalysis. Other cases of "known problems" are contained in the descriptions of the individual reanalyses in Sections 2.2.1 through 2.2.5. Some of the areas of large rms difference in Fig. 2-2 are below local topographical surface level. Some of the difference shown in the upper two subplots are an artifact of the model extrapolation from sigma surfaces to pressure surfaces below these local topographic surfaces.

Figure 2-3 compares zonal wind speed in exactly the same manner as Fig. 2-2 compares temperature. In addition to the Southern Hemisphere differences in the upper two subplots, large differences are now apparent in the Tropics from 100 to $10 \mathrm{hPa}$. These are the result of differing characterizations of the QBO by the two reanalyses, differences that are analyzed and discussed in Section 5.5. Because the tropical reanalyses were primarily forced by radiosonde data, there is minimal difference in the lower subplot. The effect of the lack of constraining observations in the Southern 
Hemisphere stratosphere discussed above in the context of temperature is also present in zonal wind speed differences in the lower subplot. Finally, although the 3DVAR assimilation of the ERA-40 reanalysis would be expected to carry the temperature variations at 200 and $30 \mathrm{hPa}$ adjacent to the South Pole to zonal winds, this was clearly not the case, as confirmed by Figs. 5-72 and 5-73 of Section 5.5. 

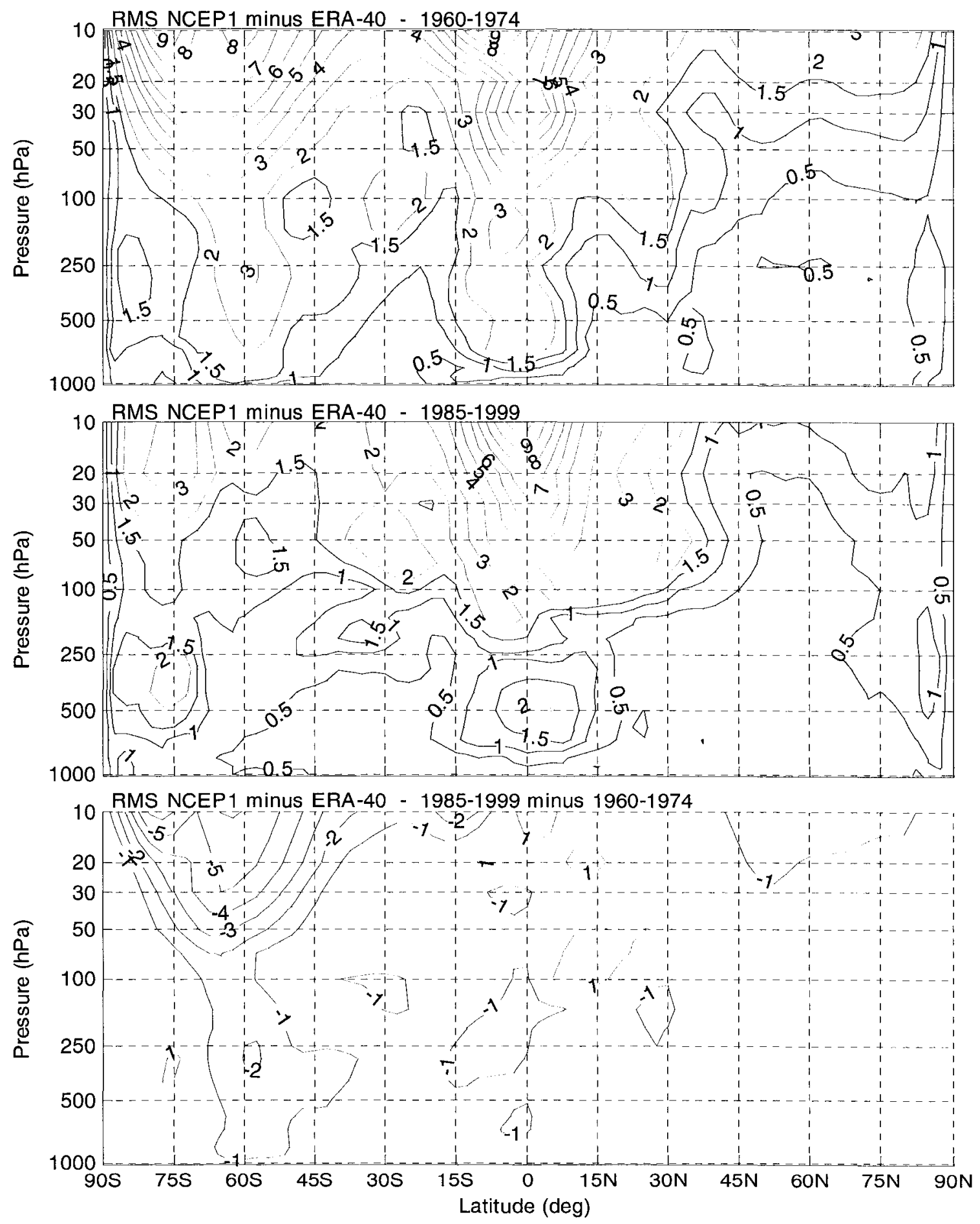

Fig. 2-3. Rms differences of NCEP1 minus ERA-40 monthly-and zonally-averaged zonal wind speeds for years 1960-74 (upper) and years 1985-99 (lower). NCEPI minus ERA-40 differences for years 1985-99 minus differences for years 1960-74 (lower). Rms wind speed differences in $\mathrm{m} \mathrm{s}^{-1}$. 
A particularly subtle limitation on reanalysis projects arises from their very charter. They assimilate "long time series of observations with an unvarying assimilation system" (Rood et al. 2006). Their preparation and testing requires major investments in staff and computing resources, even for large national centers. Their operational phases monopolize computer resources for long periods of time. Understandably, they are not easily repeated. Only one multi-decade global reanalysis, ERA-15, has been replaced by a then current state-of-the-art project, ERA-40.

Reanalysis systems evolve from the state of the art that operational forecast systems have achieved at or near the beginning of the reanalysis project. Because of computational and financial constraints, these operational forecast systems are incentivized to model the stratosphere only to the extent that such modeling can be demonstrated to improve their raison d'être, accurate tropospheric forecasts. Thus the stratospheric height of reanalysis systems and their operational experience and performance assessment is limited to that of their parent operational forecast system.

Reanalysis projects select the largest and fastest affordable computing systems available prior to the beginning of production. "Re-analysis projects must proceed at sufficient speed for them not to be continually overtaken by developments in dataassimilation technique and large-scale computing" (Simmons and Gibson 2006). These computing resources and the project timeline define the forecast model resolution and parameterizations, and also assimilation techniques and quality control. While the forecast-assimilation system is frozen in its operational role (1) computer capabilities (capacity and speed) continue to increase and (2) meteorological research and operational 
experience create and validate new forecast model and assimilation techniques. But the reanalysis system is frozen and the project must continue with its original technology.

Therefore, reanalysis projects will lag the meteorological and computational state of the art soon after the beginning of production; this gap will continue to widen with time.

\subsection{Reanalysis projects}

This section introduces the five major global reanalyses listed above and briefly discusses each with respect to its: (1) motivation and history, (2) design, (3) known problems, and (4) evaluation of its stratospheric performance in the research literature.

Papers written by project staffs often do not completely describe their design and are not forthcoming about the implications of design details or the deficiencies of their results. Developmental and production timelines are also often not clearly stated. Often the papers of others employing or analyzing the results of these projects provide more thorough descriptions of technical details of the projects and the problems with the reanalysis output. Conference and workshop proceedings and preprints have also been found to contain revealing information not found in peer-reviewed papers written by the same authors. A number of individuals in leadership positions of the various reanalysis projects and of other data sources (identified in the "Acknowledgements" section) have responded graciously and expeditiously to e-mail queries from the author of this thesis with complete and thorough answers. Their contributions are appreciated and are cited as "personal communication" where used. 


\subsubsection{NCEP-NCAR Reanalysis (NCEP1)}

\section{NCEP1 Motivation and history}

The United States National Meteorological Center (NMC), in cooperation with the National Center for Atmospheric Research (NCAR), began the NCEP-NCAR Reanalysis project in 1991 . (NMC would become the Environmental Modeling Center (EMC) of the National Centers for Environmental Prediction (NCEP) in the fall of 1995 (McPherson 1994) but today EMC is universally referenced by its parent's name, "NCEP"). Broadly, NCAR would be responsible for acquisition and preparation of data; NMC would develop the reanalysis system, which contained components of their existing operational forecast system, and execute the reanalysis. This project was initially intended to produce a Climate Data Assimilation System (CDAS) that would remain unchanged over time as a successor to the operational Global Data Assimilation System (GDAS). The CDAS Advisory Panel, constituted in 1989, suggested that CDAS would be much more valuable if combined with a state-of-the-art atmospheric model in order to perform a long-term analysis.

At an April 1991 NMC/NCAR Reanalysis Workshop, a "pocket" reanalysis for the period $1957-58$ to 1993 was proposed. This project would utilize a lower resolution but otherwise state-of-the-art system, start in 1993, and complete in 1995 or 1996. This approach would result in expeditious results for at least one research group (Kalnay and Jenne 1991). Although not discussed in the published meeting review summary, the pocket reanalysis would provide invaluable experience in the preparation for and execution of a "full" global reanalysis. Yet another unstated motivation may have been 
transatlantic rivalry. If Bengtsson and Shukla (1988) was a "message of intent," ECMWF was certainly planning for a reanalysis, and in 1991 would have been as well prepared as NMC. Although endorsed at the meeting, no other reference to this "pocket reanalysis" was discovered in the literature. NMC's resources appear to have been focused on the "full" reanalysis, which had the same horizontal resolution as that proposed for the pocket reanalysis, T62, and began production about six months before ECMWF's own "pocket" analysis, ERA-15 (Section 2.2.3) (Kalnay et al. 1996; Gibson et al. 2006).

The production phase of the NCEP-NCAR reanalysis project started in April 1994 and the first 40 years of reanalysis, 1957-96, was completed in October 1997 (Kalnay et al. 1997). Interestingly, the forecast model employed in the reanalysis system "is identical to the NGEP global operational model implemented on 10 January 1995." Except for decreased resolution, this was the NMC Medium Range Forecast Model (MRF) (Mo et al. 1995). Thus the model began service in the reanalysis system approximately nine months before its operational "debut," a dramatic leap of faith. During the production of the reanalysis, eight-day forecasts were produced every five days for "predictability studies and for monitoring the quality of the observing systems" (Kalnay et al. 1996).

Early in the project it was decided to use all available data to produce the most accurate reanalysis possible over the longest time, rather than selecting a subset of observations that remained stable over the originally-planned forty-year (1957-96) 
reanalysis period, which would result in the most apparently stable climate. It was optimistically asserted that

the analysis cycle, with the use of the 6-h forecast as a first guess, is able to transport information from data-rich to data-poor regions, so that even in relatively data-void areas the reanalysis can estimate the evolution of the atmosphere over both synoptic and climatological timescales (Kalnay et al. 1996).

NCEP1's 3DVAR assimilation has shown this assertion to be limited in the data-poor regions of the atmosphere south of $50^{\circ} \mathrm{S}$ latitude and above the tropopause because some information is transported. This is demonstrated in Fig. 2-2 and in the intercomparison sections of this thesis. Kanamitsu (2006) argues that 4DVAR will better propagate observational information into data-poor areas.

\section{NCEP1 Design}

Table 2-1 summarizes the primary characteristics of the NCEP1 reanalysis system. It incorporates information from Kalnay et al. (1996), Kistler et al. (2001), Pawson and Fiorino (1998a), and Mo et al. (1995). 
Table 2-1. NCEP1 characteristics.

\begin{tabular}{|c|c|}
\hline Reanalysis period & Jan 1948 to present \\
\hline \multicolumn{2}{|l|}{ Horizontal computational - } \\
\hline Triangular truncation & T62 \\
\hline Grid-point resolution & $210 \mathrm{~km}$ \\
\hline Horizontal available resolution & Uniform $2.5^{\circ} \times 2.5^{\circ}$ grid \\
\hline $\begin{array}{l}\text { Vertical levels: computational / available } \\
\text { (See Fig. 2-6) }\end{array}$ & $28 / 17$ \\
\hline Vertical levels $\leq 100 \mathrm{hPa}$ : computational / available & $8 / 6$ \\
\hline Model minimum pressure / maximum height & $2.73 \mathrm{hPa} / 40 \mathrm{~km}$ \\
\hline Available minimum pressure / maximum height & $10 \mathrm{hPa} / 31 \mathrm{~km}$ \\
\hline Vertical coordinate system & $\sigma$ from surface to top \\
\hline Upper boundary condition & "Artificial rigid lid" \\
\hline Cycle time & Six hours \\
\hline Clouds & Diagnosed \\
\hline Satellite temperature data & $\begin{array}{l}\text { NESDIS temperature retrievals } \\
\text { from cloud-cleared radiance data } \\
\text { used directly }\end{array}$ \\
\hline Variational Analysis & $\begin{array}{l}\text { Spectral statistical interpolation } \\
\text { (SSI) - } \\
\text { A 3DVAR analysis scheme }\end{array}$ \\
\hline
\end{tabular}

The reanalysis was performed on a Cray YMP ( 8 processors), previously used for operational forecasting, and a Cray EL2.

The model employed in the NCEP1 reanalysis is identical to the NMC operational global forecast model implemented on 10 January 1995 with the exception of the T126 
$(105 \mathrm{~km})$ horizontal resolution of the operational forecast model (Kalnay et al. 1996). This modification no doubt was driven by processing time considerations.

To illustrate the breadth and depth of the observational data employed in the NCEP1 reanalysis, Table 2-2 lists the archived sources (Woolen and Zhu 1997;

Kistler et al. 2001). Except for the last four items, all data (from many countries and sources) had been archived at NCAR. 
Table 2-2. NCEP1 observation archive.

NMC daily operational decode, 1962-97

US Control raobs/pibals (radiosonde balloons and pilot balloons), 1957-67

Tape data recordings of surface observations, 1957-67

Tape data recordings of raobs/pibals, 1957-67

Tape data recordings of aircraft observations, 1957-59

US Air Force recording of surface observations, 1967-76, aircraft reports 1976-78

Raobs and aircraft from GARP (Global Atmospheric Research Program), 1974

Aircraft reports from GASP (Global Atmospheric Sampling Program), 1975-79

Raob-like reports from constant-level balloons, 1974-75

Tropical aircraft reports, 1960-73

New Zealand national aircraft reports, 1978-88

Surface marine reports from COADS (Comprehensive Ocean Atmosphere Data Set), 1957-94

Arctic ice buoy surface reports, 1980-93

Thirty sets (from at least 18 countries) of raob/pibal reports gathered at NCAR, 1957-93

Observations from First GARP Global Experiment (FGGE), Dec. 1978 - Nov 1979 (from ECMWF)

ECMWF decode of raobs/pibals (data missing from NMC daily operational decode), 1989-93

Japanese Meteorological Agency archive of raob/pibal, aircraft, and satob data from Asia and the Asian Pacific, 1978-94

Australian PAOBs (PAid (meaning "fitted") OBservations) - estimates of sea level pressure produced by Australian analysts from satellite data, conventional data, and time continuity for the Southern Ocean, especially south of $40^{\circ}$ S (Seaman and Hart 2003) 
Climatological concentrations of aerosols, $\mathrm{CO}_{2}$ and $\mathrm{O}_{3}$, were specified in the model. No interannual variability of these concentrations was included (Pawson and Fiorino 1998a). No other description of these fields was discovered in the literature by the author of this thesis.

\section{NCEP1 Known problems}

The SPARC Intercomparison of Middle-Atmosphere Climatologies

(Randel et al. 2002, 2004) for the period 1992-99 identifies the two "largest apparent biases" in the NCEP1 reanalysis:

- "Warm tropical tropopause"

- "Satellite data discontinuity across 1978-1979"

These defects may be observed in Figs. 2-1 and 2-2. Randel et al. (2002, 2004) point out that the tropical tropopause presents analysis problems because the large vertical temperature gradients in that region are not well resolved by satellite measurement and by forecast models with vertical resolution of two kilometers or less.

Kistler et al. (2001) listed three human errors that were discovered in the reanalysis too late for correction. Their stratospheric impact should be minimal but they are listed for completeness. All were corrected in NCEP2.

- The 1973 snow cover was used for years 1974-94.

- Australian analytical estimates of sea level pressure were entered with a longitude error of $180^{\circ}$ from $1980-92$.

- The horizontal moisture diffusion formulation of the forecast model caused "unreasonable snowfall over high-latitude valleys in the winter." 
In their discussion of the NCEP2 reanalysis (Section 2.2.2),

Kanamitsu et al. (2002) identified three additional NCEP1 deficiencies, again hopefully

having minimal impact on stratospheric results, that existed over the entire reanalysis:

- The ocean albedo was too large.

A programming error resulted in an average NCEP1 ocean albedo of 0.15 at the Equator rather than a more realistic 0.06 to 0.07 .

- The relative humidity-cloudiness table was discontinuous at $0^{\circ}$ and $180^{\circ}$ longitude.

- An error in the snowmelt term overestimated the conversion of snow to water by a factor of 1,000 .

\section{NCEP1 Performance evaluation}

The primary problems (or shortcomings) for the stratospheric fidelity of the

NCEP1 reanalysis are:

- The upper model limit of $\sim 3 \mathrm{hPa}$

- An artificial rigid lid at the model top

the presence of the artificial rigid lid in atmospheric models results in a spurious "upside-down" intense baroclinic instability near the baroclinic stratospheric winter jet (Mo et al. 1995).

- A vertical sigma coordinate system from surface to model top. A two-delta sigma vertical wave in the divergence of the wind field above steep topography (e.g., the Andes) is caused by NCEP1's vertical sigma coordinate system from surface to top of model, and lack of a diffusive upper boundary condition. This behavior can corrupt E-P flux calculations above $50 \mathrm{hPa}$ (Trenberth and Stepaniak 2002). Figure 4-43 (mean difference in NCEP1 and ERA-40 $10 \mathrm{hPa}$ temperature fields) illustrates this condition.

- Pawson and Fiorino (1998a) demonstrate that the NCEP1 "meridional [cross equatorial] velocity shows a clear mass transport from the Southern to Northern Hemisphere throughout the year in the entire stratosphere" rather than the expected "annual oscillation and, generally, opposing flow in the lower and upper stratosphere with only a very weak annual mean flow at any level." This finding is confirmed throughout Section 6 . 
- The tropical climatology (QBO, etc.) suffers from use of only satellite data (Pawson and Fiorino 1998a). The referenced study

suggests that the use of the NESDIS temperature retrievals in the NCEP system may have degraded the temperature analyses near $100 \mathrm{hPa}$ in the Tropics. This is most likely a consequence of the problems associated with inverting radiance measurements to reveal the pronounced temperature minimum at the tropical tropopause.

- The Pawson and Fiorino (1998a) study also shows that the ERA-15 method, which gives priority to radiosondes and the forecast model over tropical oceans, is superior.

In their comparison of analyses and reanalyses of the 2002 Antarctic winter,

Manney et al. (2005) state that NCEP1 and NCEP2, while acceptable in the large, are not as useful for "detailed studies" (of detailed polar vortex dynamics) as observational analyses with higher resolutions.

While the overall structure and evolution of temperatures, winds, and wave diagnostics in the different analyses provide a consistent picture of the SH 2002 winter, several significant differences may affect detailed studies. [NCEP1 and NCEP2] are not recommended for detailed studies, especially those related to polar processing, because of lower-stratosphere temperature biases that result in underestimates of polar processing potential, and because their winds and wave diagnostics show increasing differences from other analyses between $\sim 30$ and $10 \mathrm{hPa}$ (their top level).

Manney et al. are incorrect in stating that $10 \mathrm{hPa}$ is the highest vertical level of the NCEP1 and NCEP2 models: their model top level is approximately $3 \mathrm{hPa}$ but $10 \mathrm{hPa}$ is the highest available vertical level. They suggest that lower horizontal model resolution and fewer model levels in the stratosphere than other data sets in the cited study, and also assimilation of satellite temperature data rather than raw radiances, may account for the behavior quoted above. 
Errors in stratospheric temperatures in NCEP1 are related to its inability to capture the cooling that results from seasonal ozone loss. This ability improved with satellite sounder data (Marshall 2002).

To assess the value of reanalyses before the satellite era, Mo et al. (1995) conducted experimental reanalyses for the month of August 1985. In the "NOSAT" case, satellite data (temperatures and cloud wind drift) were not included while the "SAT" case assimilated this data. Such disparate conclusions as

overall, these results indicate that without satellite data, the reanalyses monthly averaged temperatures are not reliable in either the stratosphere or south of $60^{\circ} \mathrm{S}$, but otherwise agree well with the SAT analyses in the troposphere;

and

these encouraging results [squared SAT-NOSAT correlation values of approximately 0.9 for heights and zonally asymmetric eddies below $100 \mathrm{hPa}$ in the $\mathrm{NH}$ and in the $\mathrm{SH}$ between $30^{\circ}$ and $60^{\circ}$ ] indicate that it is indeed worth performing a reanalysis for the years before satellite data were available, because a large component of both the daily and monthly anomalies can be captured even in the absence of the satellite data.

imply that the study was intended as a justification and would not seem to be an unbiased investigation. The study was performed about the time NCEP1 production was beginning.

Another study by Kanamitsu et al. (1997a), undertaken when the 40-year production was approaching $90 \%$ completion, reached the following conclusions:

- Satellite data have a large impact on reanalyses in data-void areas in the eastern oceanic areas of the Southern Hemisphere and in the stratosphere.

- "Satellite CMV [cloud motion vectors] in early years tend to have a low wind bias for strong winds. They also have a tendency to reduce temporal variance of winds." 
- Satellite retrieval data does not affect the horizontal temperature gradient of the reanalysis, perhaps due to the low resolution of the NCEP1 reanalysis (T62).

- Reduced temporal variance may occur with satellite data.

Ebisuzaki and Kistler (1999) conducted an examination of a data-constrained assimilation. They reanalyzed the year 1998 with aircraft and satellite data removed (but with 1998 surface observations included) to demonstrate the impact of modern observing systems. Not surprisingly, they concluded that "trend studies using NCEP/NCAR Reanalysis should be restricted to Australia, New Zealand, North America, Asia and Europe" if these studies span the 1978-79 beginning of the modern satellite era.

\subsubsection{NCEP-DOE AMIP-/l Reanalysis (R-2) (NCEP2)}

\section{NCEP2 Motivation and history}

The National Centers for Environmental Prediction-Department of Defense Atmospheric Model Intercomparison Project (NCEP-DOE AMIP-II Reanalysis (R-2)) (NCEP2) was initiated in 1998. At that time the Program for Climate Model Diagnosis and Intercomparison (PCMDI) at Lawrence Livermore National Laboratory (LLNL) provided computer resources to produce a modestly updated and corrected reanalysis, based on NCEP1 (or R-1), to provide a baseline for use in evaluation of AMIP-II simulations. "R-2 should not be considered as a next-generation reanalysis, but should be regarded as an updated and human error-fixed version of R-1" (Kanamitsu et al. 2002).

Production began in early 1998 with the reanalysis of January 1979; reanalysis of the year 2000 was completed in May 2001 (Kanamitsu et al. 2002). Production continues in near-real-time so that late in 2005 a 26-year reanalysis (through December 2004) was available. It would appear that this reanalysis will not be extended to the pre-satellite era. 
Kanamitsu et al. (2002) and its earlier draft, Kanamitsu et al. (1999) appear to be the only papers published on NCEP2.

\section{NCEP2 Design}

With the exceptions described below, the primary characteristics of the NCEP2 reanalysis system are the same as those for NCEP1 listed in Table 2-1. NCEP2 began with the "prior experience, infrastructure, and datasets from R-1." NCEP2 either "fixed" NCEP1's known problems (Section 2.2.1) or replaced original algorithms. Five of the seven model physics improvements addressed surface or boundary-layer issues, but replacing the computation of radiation on a linear grid by computation on the full Gaussian grid and decreasing the radiation computation interval from three hours to one hour (enhancements possible with increased computing speed) have the potential to improve stratospheric performance. A new ozone climatology was used and the fixed $\mathrm{CO}_{2}$ field was set to $350 \mathrm{ppmv}$ (Kanamitsu et al. 2002). Special Sensor Microwave Imager (SSM/I) data is not used in NCEP2.

\section{NCEP2 Known problems}

The SPARC Intercomparison of Middle-Atmosphere Climatologies (Randel et al. 2002, 2004) for the period 1992-99 identifies the two "largest apparent biases" in the NCEP1 reanalysis as (1) "warm tropical tropopause" and (2) "satellite data discontinuity across 1978-79." The NCEP2 reanalysis begins in 1979 and thus avoids (2) but the "warm tropical tropopause" would be expected to remain. 
Four human errors, discovered too late in the production cycle to be removed by reruns, remain in NCEP2. Three involve surface-layer physics and computations and should not be of particular interest here. The fourth is of potential significance in the region addressed in this thesis. The zonally-averaged seasonal climatological ozone data used in the radiation computation was reversed in latitude. "Limited experiments revealed only a minor impact, but problems in the radiation fluxes in the stratosphere may be expected" (Kanamitsu et al. 2002).

Thus while NCEP2 would appear to have improved the NCEP1 reanalysis at the surface and lower troposphere, it seems to have compromised stratospheric performance.

There are other minor but notable differences. For example, some differences exist in the upper-air height and temperature analyses over Northern Hemisphere oceans, where most of the observations are from satellites. ... There are differences in the equatorial divergent wind and stratospheric temperature in the Southern Hemisphere, where in R-2 the upper branch of the Hadley circulation is lower and the Southern Hemisphere jet is located slightly farther north (Kanamitsu et al. 2002).

\section{NCEP2 Performance evaluation}

Manney et al. (2005), quoted and discussed in Section 2.2.1, is the only independent stratospheric evaluation of NCEP2 performance found by the author of this thesis.

\subsubsection{ECMWF ERA-15 Reanalysis (ERA-15)}

\section{ERA-15 Motivation and history}

The ERA-15 project produced "a new, validated 15-year data set of assimilated data for the period 1979 to 1993." The project began in February 1993 with production beginning in 1994 and concluding in September 1996 (Gibson et al. 2006). 
Kanamitsu (1989) credits ECMWF with demonstrating in the "late 1970s" the value of

a state-of-the-art assimilation system, combining a sophisticated optimum interpolation objective analysis and a high-resolution global model with comprehensive physics and nonlinear normal mode initialization, all of which used the most advanced techniques and ideas available at the time.

Thus ECMWF was uniquely prepared with operationally-acquired experience and an operationally-proven system to begin a reanalysis. In fact, the ERA-15 assimilation system was the ECMWF operational system employed from April 1995 to February 1996 (Gibson et al. 2006). As was the case with NCEP1, the reanalysis system seems to have led the operational system into production. By beginning the reanalysis in 1979, coincident with the beginning of the modern satellite era, ECMWF avoided the observational discontinuity encountered in NCEP1 and ERA-40 (Section 2.2.4).

ERA-15 was, at least in hindsight, the ECMWF's pilot or "pocket" project. The experience would be similar to that gained in the construction and operation of a chemical "pilot plant" to validate research concepts before building a full scale production plant. Brooks (1975) urged software development projects to "plan to throw one away" for exactly the same purpose.

The "ECMWF Re-Analysis Project Report Series: 1. ERA-15 Description (Version 2 - January 1999)" (Gibson et al. 2006) is a superb description of the ERA-15 project, containing thorough discussions of the sources and processing of input data, the numerous experiments that led to the final configuration, the data assimilation and model employed, production, and the analysis output. The "electronic title" of this document is dated "May 1999" although the title page states "January 1999." 


\section{ERA-15 Design}

Table 2-3 summarizes the primary characteristics of the ERA-15 reanalysis system. It incorporates information from Gibson et al. (2006), Pawson and Fiorino (1998a), and Trenberth and Stepaniak (2002). Table 2-4 contains logic for satellite temperature radiance data use. Table 7 of Gibson et al. (2006) diagrams this logic.

Table 2-3. ERA-15 characteristics.

Reanalysis period

1979 through 1993

Horizontal computational -

Triangular truncation

T106

Grid-point resolution

$125 \mathrm{~km}$

Horizontal available resolution

Uniform $2.5^{\circ} \times 2.5^{\circ}$ grid

Vertical levels: computational / available

$31 / 17$

(See Fig. 2-6)

Vertical levels $\leq 100 \mathrm{hPa}$ : computational/available $\quad 5 / 5$

Model minimum pressure / maximum height

$10 \mathrm{hPa} / 31 \mathrm{~km}$

Available minimum pressure / maximum height

$10 \mathrm{hPa} / 31 \mathrm{~km}$

Vertical coordinate system

Hybrid:

$\sigma$ from surface to $100 \mathrm{hPa}$, pressure coordinates above

Upper boundary condition

Highly diffusive damping

Cycle time

Six hours

Clouds

Predicted

Satellite temperature data

See Table 2-4

Variational Analysis

Optimal interpolation 
Table 2-4. Use of satellite temperature data in ERA-15.

\section{Below $100 \mathrm{hPa}$}

1DVAR assimilation of TOVS cloud-cleared radiances into temperature fields over sea and ice areas only.

Only cloud-free TOVS data used equatorward of latitude $30^{\circ}$.

$100 \mathrm{hPa}$ and above

NESDIS temperature retrievals from cloud-cleared radiance data used directly poleward of latitude $30^{\circ}$.

Cloud-free TOVS data used between latitudes $30^{\circ}$ and $20^{\circ}$.

No satellite temperature data used equatorward of latitude $20^{\circ}$.

A Cray Research Inc. C90 computer was the central processor for the ERA-15 project.

The beginning of the ERA-15 reanalysis period, 1979, coincides with the beginning of ECMWF operational activities. Thus the ECMWF operational archive from the World Meteorological Organization (WMO) Global Telecommunications System (GTS) was available in-house. Table 2-5 provides an overview of additional data sources employed in ERA-15 (Gibson et al. 2006). 
Table 2-5. ERA-15 data sources.

TOVS Television InfraRed Observation Satellite (TIROS) Operational Vertical Sounder Cloud track winds (METEOSAT, GOES, GMS)

Observations from First GARP Global Experiment (FGGE), Dec. 1978 - Nov 1979 Surface marine reports from COADS (Comprehensive Ocean Atmosphere Data Set) Japanese Meteorological Agency archive of raob/pibal, aircraft, and satob data from Asia and the Asian Pacific

Australian PAOBs (PAid (meaning "fitted") OBservations) - estimates of sea level pressure produced by Australian analysts from satellite data, conventional data, and time continuity for the Southern Ocean, especially south of $40^{\circ} \mathrm{S}$ (Seaman and Hart 2003)

ERA-15 employed the "ECMWF operational radiation parametization with prescribed concentrations of aerosols, $\mathrm{CO}_{2}$ and $\mathrm{O}_{3}$, with $\mathrm{O}_{3}$ varying geographically, vertically, and seasonally; the aerosols varying geographically and vertically; and $\mathrm{CO}_{2}$ held constant at 353 ppmv" (Gibson et al. 2006). No interannual variability was included (Pawson and Fiorino 1998a).

\section{ERA-15 Known problems}

The SPARC Intercomparison of Middle-Atmosphere Climatologies (Randel et al. 2002, 2004) for the period 1992-99 identifies the "largest apparent bias" in the ERA-15 reanalysis as "Global cold biases $(\sim 3 \mathrm{~K})$ at 30 and $10 \mathrm{hPa}$." This same intercomparison shows the ERA-15 equivalent QBO amplitude in zonal wind speed to be $14 \%$ less than that of the Freie Universität Berlin (FUB) Singapore data at $30 \mathrm{hPa}$ and $60 \%$ less at $10 \mathrm{hPa}$ (the upper limit of the ERA-15 reanalysis). 
The fifteen-year span of ERA-15 and its model upper vertical limit of $10 \mathrm{hPa}$ severely limit its value as a long-term stratospheric climatology. However, for six years (until the advent of ERA-40) it was the only reanalysis climatology against which that of NCEP1 could be compared.

\section{ERA-15 Performance evaluation}

Pawson and Fiorino found ERA-15 annual cycle and tropical tropopause temperatures consistent with radiosonde observations. They attribute this consistency to the one-dimensional assimilation of satellite radiance measurements and the exclusive use of radiosondes in the tropical stratosphere $\left(\leq 100 \mathrm{hPa}, \leq\left|20^{\circ}\right|\right.$ latitude). They also attribute the positive temperature anomalies following the two major volcanic eruptions (E1 Chichon in April 1982 and Mt. Pinatubo in June 1990) to data assimilation because the model does not include longwave radiation absorption by sulfate aerosol. When examining the correlation of the QBO meridional circulation with the zonal wind speed at $1.25^{\circ} \mathrm{N}, 50 \mathrm{hPa}$, they generally found expected correlations over the region of interest $\left(100-10 \mathrm{hPa}, 30^{\circ} \mathrm{S}-30^{\circ} \mathrm{N}\right)$. However, in the region $100-70 \mathrm{hPa}, 15^{\circ} \mathrm{S}-15^{\circ} \mathrm{N}$, there were two cells of "intense" correlation and opposite polarity "on the vertical scale of the model levels" (Pawson and Fiorino 1998b).

Additionally, ERA-15 experienced "unrealistic spectral ripples in the model orography that are most obvious over level regions close to major mountain ranges." ERA-40's (Section 2.2.4) increased spectral resolution, T159, vs. ERA-15's T106, but with the same grid point resolution, dramatically reduced the amplitude of the spectral ripples (Simmons 2006a). 


\subsubsection{ECMWF ERA-40 Reanalysis (ERA-40)}

\section{ERA-40 Motivation and history}

The ERA-40 project began with the most complete experience base of any of the major global reanalyses. At the time of its first External Advisory Group meeting in January 1998 (Simmons and Gibson 2006), the NCEP1 reanalysis was complete to the current date, preparation for NCEP2 was complete, and most critically, ERA-15 had completed one year before and the experience from it was available in-house. Numerous independent comparisons and analyses of NCEP1 and ERA-15 had been published or were underway. Evolved from ERA-15, ERA-40 is truly a "second generation reanalysis." As the ERA-40 Project Plan points out, "ERA-40 will provide an important second source of re-analysis data for the period $1957-78$ previously covered only by the NCEP/NCAR re-analysis" (Simmons and Gibson 2006).

Each reanalysis has available the experience of its predecessors; by the time of ERA-40, a critical mass of experience had been reached. More important, the veterans of the ERA-15 project formed the core of the ERA-40 team. This team, having "built one to throw away" and open to the positive consequences of the "second system effect" (Brooks 1975), significantly advanced the reanalysis state of the art as outlined in the next section. In addition to reanalysis-experienced staff, the project benefited from the assignment of experienced individuals from China, Japan, and the United States (Simmons and Gibson 2006a).

The ERA-40 reanalysis production began in the spring of 2001 (A. J. Simmons 2006, personal communication) and concluded in April 2003 (Uppala et al. 2005). The 
assimilation system contained changes incorporated into the ECMWF operational system in January and April 2002 (Simmons 2006a). As was the case with NCEP1 and ERA-15, the reanalysis system led operational features into production. Two six-hour forecasts and two three-day forecasts were produced each reanalysis day.

ERA-40 Design

Table 2-6 summarizes the primary characteristics of the ERA-40 reanalysis system. It incorporates information from Kållberg et al. (2006) and Simmons (2006a). 
Table 2-6. ERA-40 characteristics.

Reanalysis period

Horizontal computational -

Triangular truncation

Grid-point resolution

Horizontal available resolution

Vertical levels: computational / available

(See Fig. 2-6)

Vertical levels $\leq 100 \mathrm{hPa}$ : computational/ available

Model minimum pressure / maximum height

Available minimum pressure / maximum height

Vertical coordinate system

Upper boundary condition

Cycle time

Clouds

Satellite temperature data

Variational Analysis
Sep 1957 through Aug 2002

T159

$125 \mathrm{~km}$

Uniform $2.5^{\circ} \times 2.5^{\circ}$ grid

$60 / 23$

$23 / 11$

$0.1 \mathrm{hPa} / 65 \mathrm{~km}$

$1 \mathrm{hPa} / 48 \mathrm{~km}$

Hybrid:

$\sigma$ from surface to $100 \mathrm{hPa}$, Pressure coordinates above

Highly diffusive damping

Six hours

Predicted and advected

Raw radiance data

3DVAR with FGAT (See below)

Fujitsu Ltd. provided "substantial computing support" for the ERA-40 project (Simmons and Gibsons 2006). ECMWF was free to select computing hardware of their choice, unlike United States government centers that are required to use "Made in USA" products. Much more significant was that during the six years since the beginning of NCEP1 processing, computing speed, throughput, and storage capacities had dramatically 
evolved, permitting increased horizontal resolution and vertical levels, improved assimilation algorithms, more accurate parameterizations, etc., given the same processing rate (months of reanalysis completed per hour of computing time).

Uppala et al. (2005), Simmons and Gibson (2006), Dethof and Hólm (2006), and Kållberg et al. (2006) identify major state-of-the-art reanalysis advancements introduced in ERA-40:

- Direct variational assimilation of raw radiance data from satellite sensors used globally

- Higher triangular truncation / grid-point resolution:

- ERA-40 T159/125 km

- ERA-15 T106/125 km

- NCEP1 T62/210 km

- A two-time-level semi-Lagrangian scheme

- A "linear grid" option that markedly reduced unrealistic orographic spectral ripples in level regions adjacent to major mountain ranges

- 3DVAR data assimilation (replacing ERA-15's Optimal Interpolation)

- FGAT (First Guess at Appropriate Time) implemented in the 3DVAR assimilation system: background and observed values are compared at observation time (rather than analysis time) and differences applied at analysis time

- A dramatically increased number of model pressure levels and the upper model pressure level: 60 and $0.1 \mathrm{hPa}$, respectively (from 28 and $\sim 3 \mathrm{hPa}$ in NCEP1 and 31 and $10 \mathrm{hPa}$ in ERA-15) and the introduction of cubic finite element discretization in the vertical

- Ozone added as a prognostic model variable, uncoupled from the radiation parameterization, and validated by ozonesonde data

- Atmospheric model coupled with ocean-wave model 
- Improved parameterizations of deep convection, radiation, clouds, orography, and numerous surface and boundary layer features

As the fourth major global reanalysis, ERA-40 benefited from the design, implementation, production, and archiving experiences of NCEP1, NCEP2, and ERA-15. They also had access to data not in ECMWF archives and the experience in preparing and assimilating it from United States sources (NCAR, NCEP, Navy, NASA, NOAA, and others). Data was also supplied by the Japanese Meteorological Agency (JMA), and British, Canadian, and Australian agencies (Kållberg et al. 2006).

\section{ERA-40 Known problems}

The SPARC Intercomparison of Middle-Atmosphere Climatologies (Randel et al. 2002, 2004) for the period 1992-99 identifies the two "largest apparent biases" in the ERA-40 reanalysis:

- "Cold temperature biases $(\sim 5 \mathrm{~K})$ in the upper stratosphere $(2-5 \mathrm{hPa})$ "

- "Oscillatory vertical structure in temperature, especially large over Antarctica" The oscillatory structure is evident throughout Section 4.1 and in particular Figs. 4-19 through 4-24. The cause of the "oscillatory vertical structure in temperature" is the consequence of (1) the nature of radiance data produced by satellite sensors, (2) assumptions in the ECMWF 3DVAR analysis system, (3) lack of sufficient radiosonde data to control the reanalysis, and (4) satellite orbital mechanics. Radiance data from satellite sensors is defined over deep vertical layers and contains limited information on vertical radiance/temperature distribution. Thus it is the responsibility of the analysis system to distribute this layered data to model levels. Simmons (2006b) provides a useful overview of the situation: 
The 3D-Var analysis scheme used for ERA-40 is prone to fit the measured radiances (which are sensitive to distributions of temperature over relatively deep stratospheric layers) by introducing oscillatory temperature structures (peaking close to $3 \mathrm{hPa}$ ) with vertical scales finer than those to which the measured radiances are most sensitive. The problem ... is most marked in polar regions, particularly the Antarctic, and particularly later in the period when both SSU [Stratospheric Sounding Unit] and AMSU-A [Advanced Microwave Sounding Unit] data are assimilated.

McNally (2006) supplies the final clue:

in the ECMWF analysis system temperature errors are assumed to be anticorrelated over very short vertical scales (essentially encouraging oscillatory solutions).

Finally, although not mentioned in the literature, converging spacecraft polar orbits result in a greater density of radiance data at the poles (A. J. Simmons 2006, personal communication). Compounding the problem, during winter and spring in the Southern Hemisphere, when the oscillatory vertical behavior is strongest, radiosondes often do not reach their maximum altitudes because their balloons burst in the extreme cold. This reduced availability of observational data to control the reanalysis contributes to the presence of the oscillatory vertical behavior in the vicinity of the poles and particularly the South Pole. Comparison of NCEP1 and ERA-40 temperature standard deviation is presented in Figs. 4-19 through 4-24 of Section 4.1. The temperature oscillations in the vertical are not present in the current "interim" ECMWF reanalysis (discussed in Section 7), which includes additional data from Global Positioning System (GPS) satellite radio occultation (A. J. Simmons 2006, personal communication).

Simmons (2006b) assigns responsibility for the ERA-40 cold temperature biases in the upper stratosphere in the 1992-99 time period to model biases also again to 
3DVAR analysis problems of fitting measured radiances distributed over deep stratospheric layers to model levels.

\section{ERA-40 Performance evaluation}

Baldwin and Gray (2005) compared ERA-40 tropical stratospheric zonal wind speeds to rawinsonde data, which is assimilated in the ERA-40 reanalysis, and to rocketsonde data, which is not. They concluded, "the ERA-40 data provide a good representation of tropical wind speeds up to 2-3 hPa" and "zonal-mean ERA-40 wind speeds could be used, for most purposes, in place of rawinsonde station observations." This was also the conclusion of Randel et al. $(2002,2004)$ in the SPARC intercomparison, which included NCEP1 and ERA-40 in its set of climatologies.

The quasi-biennial oscillation (QBO) dominates interannual variability for zonal winds in the Tropics, but there are significant differences in magnitude derived from various datasets. ... ERA-40 appears best at capturing the strength of the westerly phase at $10 \mathrm{hPa}$.

With reference to their Fig. 14,

for altitudes - 24-37 km ... ERA-40 exhibits the largest QBO amplitude (in best agreement with the radiosonde climatology and Singapore data). ... The ERA-40, ERA-15, METO, and NCEP datasets show approximately similar amplitudes between 70 and $30 \mathrm{hPa}$, whereas above $30 \mathrm{hPa}$ there are larger differences. Only ERA-40 approaches the Singapore and rocketsonde amplitudes over 20-10 hPa.

Manney et al. (2005) in their comparison of analyses and reanalyses during the 2002 Antarctic winter state that ERA-40, while acceptable in the large, is not as useful for "detailed studies" (of detailed polar vortex dynamics) as observational analyses with higher resolutions. 
While the overall structure and evolution of temperatures, winds, and wave diagnostics in the different analyses provide a consistent picture of the SH 2002 winter, several significant differences may affect detailed studies. ... Southern Hemisphere polar stratospheric temperatures in the ECMWF 40-Yr Re-analysis (ERA-40) show unrealistic vertical structure, so this long-term reanalysis is also unsuited for quantitative studies.

\subsubsection{Japanese Re-Analysis 25 Years (JRA-25)}

\section{JRA-25 Motivation and history}

The "Japanese Re-Analysis 25 Years" project, JRA-25, is chronologically the final project of the five considered in this section. Its target period is 1979 through 2004. It is a cooperative research project of the Japan Meteorological Agency (JMA) and the Central Research Institute of Electric Power Industry (CRIEPI), "in combination with other research institutes or universities."

It was initiated because

in Japan recent urgent needs for more accurate environmental and climate diagnostic information boosted the plan to produce another reanalysis dataset by ... [JMA and CRIEPI]. ... Data assimilation techniques are rapidly developing now, so that available reanalysis products do not necessarily agree with each other and their qualities depend on generating systems. Another version of reanalysis by Japan provides another reference for the historical global atmosphere and can potentially contribute to reducing its error-bar. From this point of view, the target of JRA-25 is to offer the reanalysis dataset and consistent real-time objective analysis, which is comparable to NCEP/NCAR Reanalysis-1 (CDAS) in quality. Other intensive targets in JRA-25 are (1) to depict positions of tropical disturbances correctly and (2) to describe the Asian climate accurately (JRA-25 Working Group 2006, with minor modifications supplied by author of this thesis).

In April 2001 JMA and CRIEPI signed a cooperative research contract. CRIEPI

was to be responsible for the computation: their supercomputer, a Fujitsu

VPP5000/32PE, would host the 25-year reanalysis, with follow-on processing to be 
performed at JMA computational facilities. In addition, CRIEPI was to be responsible for the porting and optimization of the JMA forecast model and assimilation system, development of automated processing, operation, and archiving the reanalysis. JMA was to be responsible for the meteorology: (1) acquisition and modification of the observation database; (2) data conversion and quality control; and (3) preparation, support, and evaluation of the forecast model and assimilation system. The model was frozen and production began in April 2004 (M. Sakamoto 2006, personal communication). At the completion of the JMA-CRIEPI contract on 31 March 2006, the JRA-25 Evaluation Group Secretariat announced that "all calculations including recalculations have been completed" (K. Takahashi 2006, personal communication). However, as of that date, erroneous output after 1994 had been discovered and recalculations were planned. On 14 July 2006 the Secretary of JRA-25 announced that "we have completed JRA-25 calculations" (Secretary of JRA-25 2006, personal communication). As of August 2006 the reanalysis was continuing from January 2005 with its output soon to be available (M. Sakamoto 2006, personal communication).

JRA-25 descriptive material is found only at the Japanese 25-year Reanalysis Project web site: http://www.jreap.org. Only two papers and one abstract are available: the 2001 "Japanese 25-year Reanalysis Plan" (JRA-25 Working Group 2006), an undated possible preprint (Sakamoto et al. 2006a) primarily concerned with assimilation of TOVS data, and a conference poster session abstract with a project overview (Sakamoto et al. 2006b). A comprehensive journal article describing JRA-25, similar to Kalnay et al. (1996), Kistler et al. (2001), and Uppala et al. (2005), is planned for 
publication in the Journal of the Meteorological Society of Japan (M. Sakamoto 2006, personal communication). In the absence of such a paper, numerous details included in this thesis have been supplied by frequent e-mails from the JRA-25 Project (Secretary of JRA-25 2006, personal communication) and personal communication with Masami Sakamoto of the Japanese Meteorological Agency.

\section{JRA-25 Design}

Table 2-7 summarizes the primary characteristics of the JRA-25 reanalysis system. It incorporates information from JRA-25 (2006), Sakamoto et al. (2006a; 2006b), and M. Sakamoto (2006, personal communication). 
Table 2-7. JRA-25 characteristics.

Reanalysis period

Horizontal computational -

Triangular truncation

Grid-point resolution

Horizontal available resolution

Vertical levels: computational / available

(Fig. 2-6)

Vertical levels $\leq 100 \mathrm{hPa}$ : computational/ available

Model minimum pressure / maximum height

Available minimum pressure / maximum height

Vertical coordinate system

Upper boundary condition

Cycle time

Clouds

Satellite temperature data

Variational Analysis
1979 to present

$\mathrm{T} 106$

$110 \mathrm{~km}$

Uniform $2.5^{\circ} \times 2.5^{\circ}$ grid

$40 / 23$

$13 / 12$

$0.4 \mathrm{hPa} / 55 \mathrm{~km}$

$0.4 \mathrm{hPa} / 55 \mathrm{~km}$

Hybrid

Rigid

Six hours

Unknown

TOVS and ATOVS radiance data assimilated directly

3DVAR 
Table 2-8 provides an overview of the data sources employed in JRA-25. It incorporates information from Sakamoto et al. (2006a).

Table 2-8. JRA-25 data sources.

JMA observational data from 1989

"Conventional observational data" used in ERA-40 1957-2002 from ECMWF

TOVS and ATOVS radiance data used in ERA-40 1978-2003 from ECMWF

$\mathrm{SSM} / \mathrm{I}$ data from NCDC and NOAA

JMA AMV (atmospheric motion vector) data

Chinese snow depth data from Chinese Meteorological Administration

COBE sea surface data

Three-dimensional ozone concentration derived from Total Ozone Mapping Spectrometer (TOMS) data

Snow coverage distribution from SSM/I with interpolated NCDC data used when SSM/I data is missing

\section{JRA-25 Known problems}

Sakamoto et al. (2006a), in their list of "unfavorable points," state "Relatively low reliability of the performance in the upper stratosphere. ... Taking substantial disagreement between JRA-25 and ERA-40 into account, our forecast and assimilation are not going so well regarding those layers."

\section{JRA-25 Performance evaluation}

Understandably, since the reanalysis is still underway, no independent evaluations of JRA-25 were discovered by the author of this thesis. Sakamoto et al. (2006a) claim 
that JRA-25 is the only reanalysis without "considerable distortion around volcanic eruptions." They appear to have restricted observational data to avoid "contamination to a certain extent." They are also satisfied with their precipitation results: "Precipitation distribution particularly around the Tropics of JRA-25 shows more reasonable agreement with CMAP [CPC Merged Analysis of Precipitation], even when it is compared with ERA-40's. ... So far, our system reproduces more relevant distribution of precipitation than other major reanalyses" (Sakamoto et al. 2006b).

\subsubsection{Reanalysis project and coverage timelines}

Figures 2-4 and 2-5 graph the project and coverage timelines, respectively, of the five reanalysis projects discussed in this section. Figure 2-4 incorporates information from NCEP1: Kalnay and Jenne (1991), Kalnay et al. (1996) and Kistler et al. (2001); NCEP2: Kanamitsu et al. (2002); ERA-15: Gibson et al. (2006); ERA-40: A. J. Simmons (2006, personal communication) and Uppala et al. (2005); JRA-25: M. Sakamoto (2006, personal communication). Figure 2-5 incorporates information from NCEP1:

Kistler et al. (2001); NCEP2: Kanamitsu et al. (2002); ERA-15: Gibson et al. (2006); ERA-40: Uppala et al. (2005); JRA-25: M. Sakamoto (2006, personal communication). 


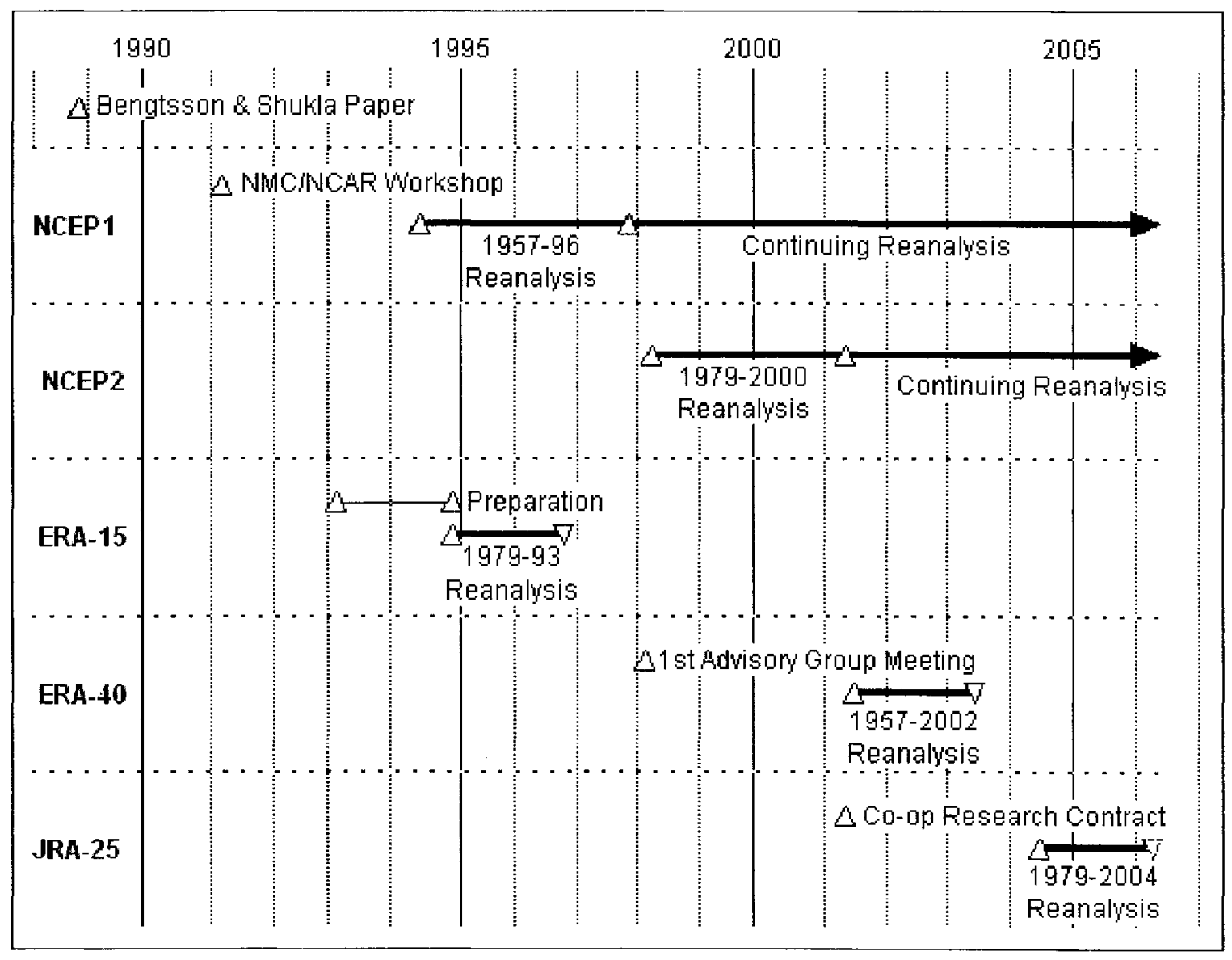

Fig. 2-4. Reanalysis project timelines. 


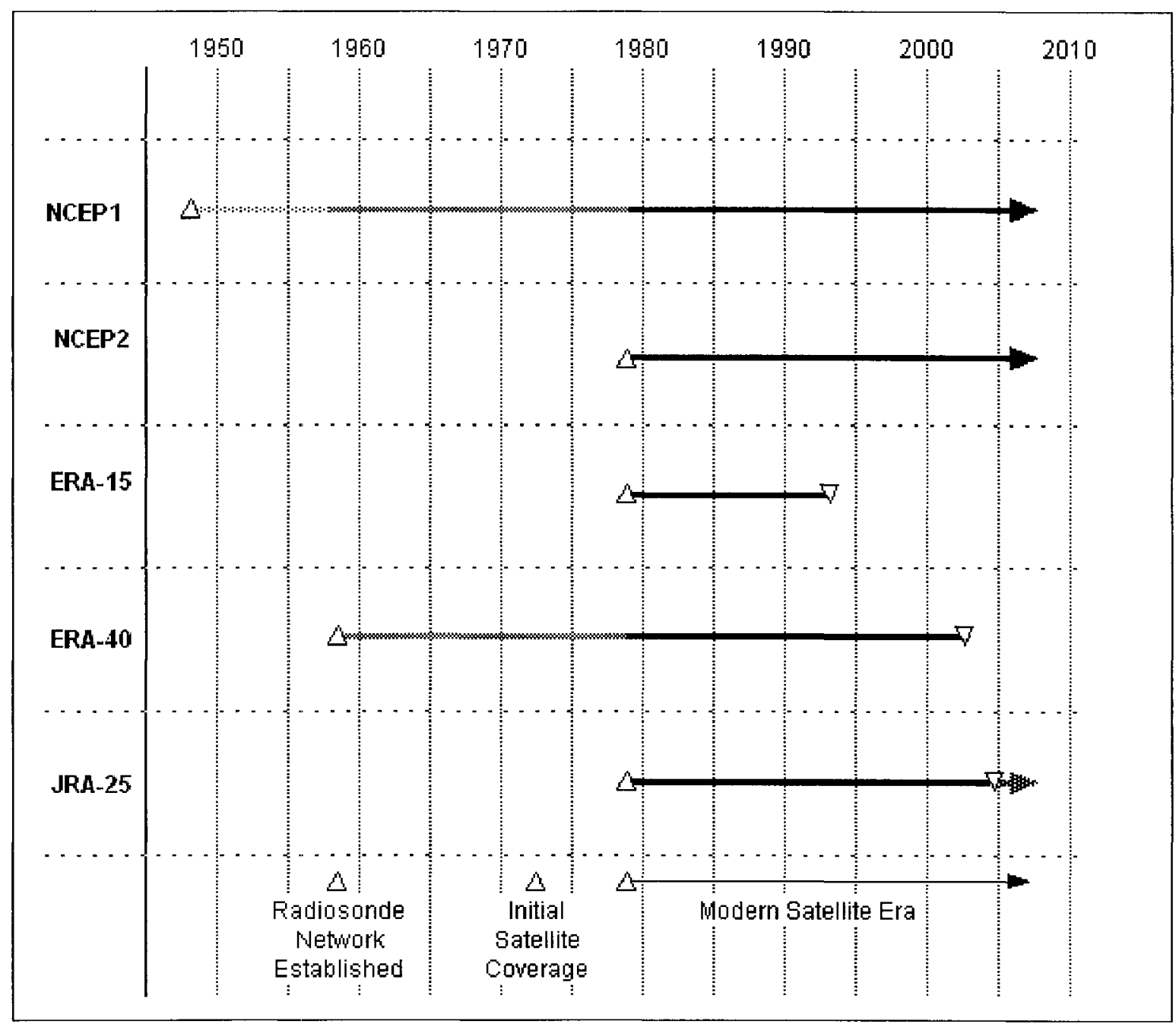

Fig. 2-5. Reanalysis coverage timelines. 


\subsubsection{Reanalysis and supporting data pressure levels}

Figure 2-6 graphs the pressure levels of the five reanalyses (Sections 2.2.1

through 2.2.5) and of the four sets of comparison data to be discussed in Section 2.3. The major reanalyses are positioned from left to right in chronological order of finalization of forecast and assimilation systems and beginning of production. The dramatic increase in number and maximum height of model levels is an indication of the increase in computational power from 1994 to 2004 . The four comparison data sets are:

- "METO" - UKMO Stratospheric Analysis. The United Kingdom Meteorology Office (UKMO) daily stratospheric analyses, produced since 1991.

- "FUB" - Freie Universität Berlin. The Institut für Meteorologie of the Freie Universität Berlin produced Northern Hemisphere subjective analyses from 1964 to 2001.

- "RATPAC" - NOAA Radiosonde Atmospheric Temperature Products for Assessing Climate. Expertly analyzed and highly refined radiosonde data from 1958 through 2005.

- "TLS" - Satellite Temperature Lower Stratosphere. Remote Sensing Systems analyses and electronically publishes integrated Microwave Sounding Unit (MSU) Channel 4 and Advanced Microwave Sounding Unit (AMSU) Channel 9 brightness temperature observations from NOAA polar-orbiting spacecraft data from 1979 to the present.

Figure 2-6 incorporates information from NCEP1: Kalnay et al. (1996); NCEP2:

Kalnay et al. (1996); ERA-15: Gibson et al. (2006); ERA-40: Kållberg et al. (2006);

JRA-25: JRA-25 (2006), M. Sakamoto (2006, personal communication); METO: netCDF control files and R. Swinbank (2006, personal communication); FUB:

Labitzke et al. (2006); RATPAC: Seidel et al. (2004); TLS: Remote Sensing Systems (2006). In the "TLS" segment of Fig. 2-6, the curve to the right of vertical illustrates the relative contribution of radiance at each pressure level to the observed radiance signal. 
To adequately simulate TLS observations, reanalysis data at pressure levels of 150,200, and $250 \mathrm{hPa}$ must be included with the six stratospheric levels as discussed in Section 4.3 . 


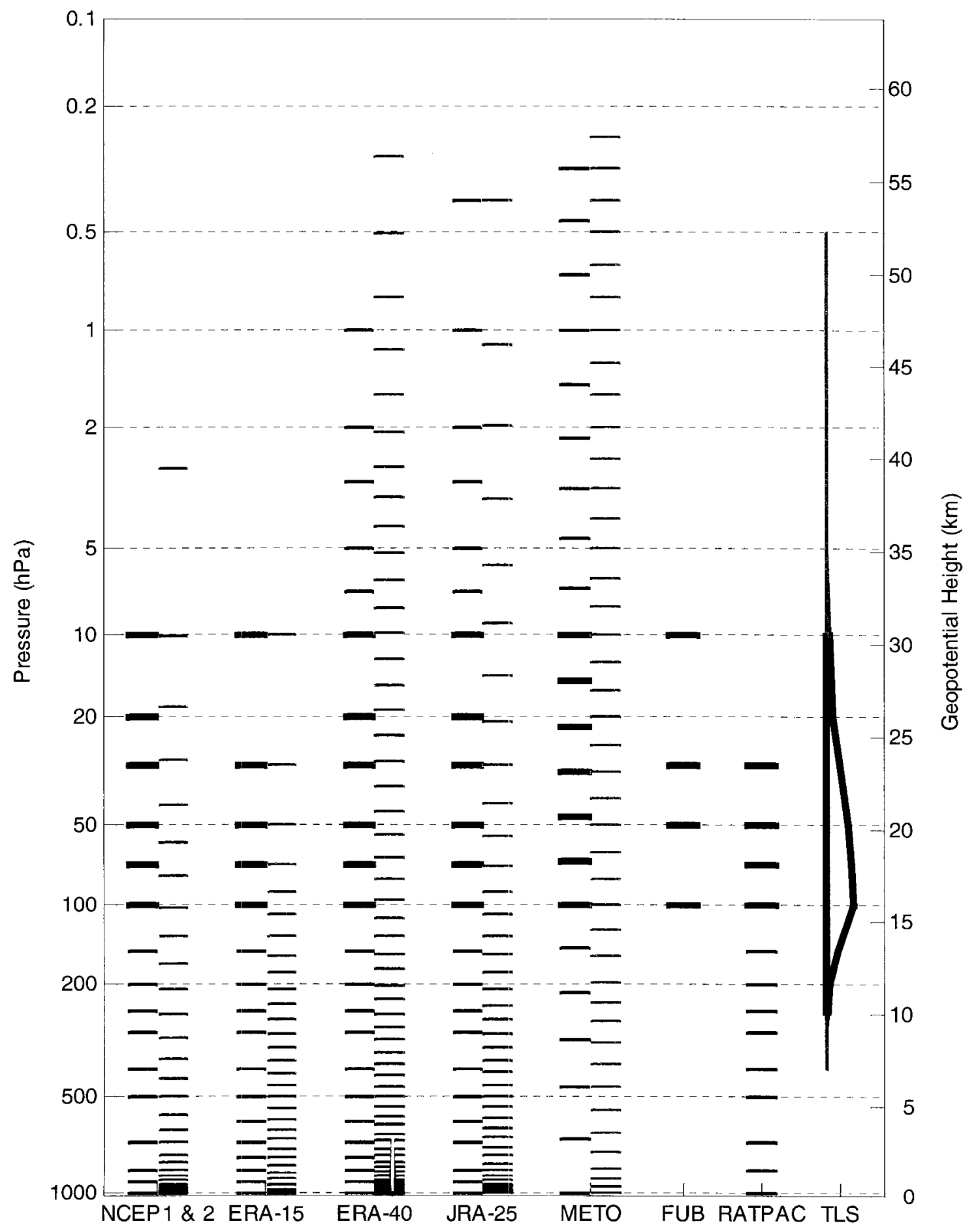

Fig. 2-6. Available data pressure levels (left/black) and model pressure levels (right/red) in the reanalyses and comparison data sets. Available pressure levels between 100 and $10 \mathrm{hPa}$ are bolded. See text for discussion of "TLS" diagram 


\subsubsection{Other reanalyses}

A number of reanalyses are not candidates for global stratospheric climatologies because (1) their results are of limited spatial or temporal coverage, (2) their data is unavailable, or (3) their results contain no additional independent data. They are briefly described below to demonstrate the breadth of the field of reanalysis.

\section{North American Regional Reanalysis (NARR)}

The goal of the NCEP North American Regional Reanalysis (NARR) is the creation of a "long-term set of consistent climate data on a regional scale for the North American domain." It is advertised as superior to the NCEP1 in both resolution and accuracy because of the "use of a regional model (Eta) and advances in modeling and data assimilation since 1995 , especially (1) precipitation assimilation, (2) direct assimilation of radiances, and (3) land-surface model updates." The reanalysis period is from 1979 to near-real time. Fedor Mesinger is the author and aggressive proponent of the Eta model. The summary of NARR in this section is excerpted from the briefing charts of Mesinger et al. (2006).

The Eta model is operated in a fully cycled three-hour EDAS (Eta Data Assimilation System) with horizontal boundary conditions from NCEP2. The North American domain includes most of North America and large parts of the adjacent oceans. Resolution is $32 \mathrm{~km}$ in the horizontal with 45 vertical layers to $25 \mathrm{hPa}$. Observed improvements include:

- "Near-surface temperatures and winds closer to observations" than NCEP2 
- "Winds in the upper troposphere, in addition to land-surface hydrology of the ConUS, maybe the strongest areas of improvement"

- "Improvements greater in winter than in summer"

Observed weaknesses include:

- "Excessive strength of summertime Gulf of California low-level jet"

- "Precipitation over Canada: the number of gauge observations available may have been insufficient to do better than the model might have done"

- "Precipitation over northern Atlantic not simulated well"

- "Atlantic hurricanes apparently not an NARR feature"

The disappointing consequences of several long-anticipated state-of-the-art improvements that were expected to have significant impacts on performance must have been extremely discouraging to the development team for the following reasons:

- The three-hour assimilation cycle time, versus the almost universal 6-hours, increased time resolution but not the accuracy of analyzed variables. The threehour first guesses do not appear be closer to observations than six-hour ones would have been.

- Assimilation of 2-meter land surface station air temperatures produced poorer first guesses throughout the troposphere.

- There was no benefit from direct assimilation of radiance data, possibly because the top of the Eta model at $25 \mathrm{hPa}(25 \mathrm{~km})$ is too low.

\section{Modern Era Retrospective-analysis for Research and Applications (MERRA)}

This is a NASA Goddard GMAO (Global Modeling and Assimilation Office)

project with "special focus" on the hydrological cycle. The project will apparently

assimilate remotely-sensed rain rates to improve "precipitation predictions" and outgoing longwave radiation estimates. The reanalysis period will extend from 1979 to the present, "the modern era of remotely sensed data." No schedule for this project was 
stated. The single page, cited in Section 9 (References) as "MERRA (2006)" contains neither technical content nor links to additional information. Without further information it is assumed that the term "retrospective-analysis" is simply a "reanalysis."

\section{NASA Data Assimilation Office (DAO)}

A sixteen-year reanalysis from 1980 was produced by the Data Assimilation Office (DAO) of the National Aeronautics and Space Administration (NASA) (Schubert et al. 1995; Schubert 1997). The GEOS Goddard Earth Observing System Data Assimilation System consisted of the GEOS-1 GCM and an assimilation component. The GEOS-1 GCM had a horizontal resolution of $2.0^{\circ}$ in latitude and $2.5^{\circ}$ in longitude, and 20 sigma levels (four above $100 \mathrm{hPa}$, highest $19.9 \mathrm{hPa}$ ). The assimilation was carried out using optimal interpolation (OI) at 14 pressure levels with five stratospheric pressure levels $(20,30,50,70$, and $100 \mathrm{hPa})$. This project was a first-generation reanalysis on which later reanalyses would be based (Rood et al. 2006). However, these later reanalyses are not documented in the literature. Uppala et al. (2005) cite Schubert et al. (1995) and Schoeberl et al. (2003) as the definitive description of this reanalysis. This reanalysis was not considered further because of its limited duration and difficulties in locating substantive documentation, data, and third-party performance evaluations. 


\section{U.S. Navy}

The only reference to this activity is "other plans presented in the October 1993 review [of the NCEP/NCAR project] were those of ... the U. S. Navy (1985 to the present)" (Kalnay et al. 1996).

\subsubsection{Selection of reanalyses for intercomparison}

Sections 2.2.1 through 2.2.5 discuss the five major global reanalyses. The following outline summarizes the salient stratospheric characteristics of the five for the purpose of selecting the reanalyses to be intercompared in this thesis:

1. NCEP-NCAR Reanalysis (NCEP1)

Long, 59-year reanalysis beginning 31 years before the modern satellite era and continuing to the present

Low, $3 \mathrm{hPa}$, artificial rigid model top, $\sigma$ coordinates from surface to top

Only eight model vertical layers above $100 \mathrm{hPa}$

2. NCEP-DOE AMIP Reanalysis (R-2) (NCEP2)

A modestly updated and corrected reanalysis, not a next-generation reanalysis

The zonally-averaged seasonal climatological ozone data used in the radiation computation reversed in latitude, apparently compromising stratospheric performance

\section{ECMWF ERA-15 (ERA-15)}

Short, 15 -year reanalysis

$10 \mathrm{hPa}$ model upper vertical limit

Superseded by ERA-40 


\section{ECMWF ERA-40 (ERA-40)}

Long, 44.7-year reanalysis beginning 21 years before the modern satellite era and ending in August 2002

Highly diffusive model top at $0.1 \mathrm{hPa}, \sigma$ coordinates to $100 \mathrm{hPa}$, pressure coordinates above

Twenty-five model vertical layers above $100 \mathrm{hPa}$

Direct assimilation of satellite radiance data

Ozone analyzed

Additional and improved observational data

"Assimilated" the experience of three prior reanalysis projects

Advantage of six years evolution in computing speed, throughput, and storage capacities

5. Japanese Reanalysis Project (JRA-25)

Completion of production announced on 14 July 2006; too late for this thesis

Project team not satisfied with stratospheric performance

NCEP2 is a modestly updated and corrected NCEP1 with questionable behavior in the stratosphere. ERA-15 is of short duration and has been superseded by ERA-40. JRA-25 is also of short duration, containing questionable stratospheric characterizations, and was not completed in time for use in this thesis. Thus only the two long running reanalyses, NCEP1 and ERA-40, remain.

The above outline and summary argue that only two reanalyses are candidates for detailed intercomparison: NCEP1 and ERA-40. In this pairing we have the chronologically first reanalysis, with its limited domain, resolution, and technology, 
especially in the stratosphere, and the next to last, with its advantages of advanced modeling and assimilation technology, mature observational data sets, faster and larger computing resources, and the experiences of previous projects.

Table 2-9 compares salient characteristics of the NCEP1 and ERA-40 reanalyses. It summarizes data from Tables 2-1 and 2-6. 
Table 2-9. Comparison of NCEP1 and ERA-40 characteristics.

\begin{tabular}{|c|c|c|}
\hline & $\underline{\mathrm{NCEP} 1}$ & ERA-40 \\
\hline Reanalysis period & Jan 1948 to present & $\begin{array}{l}\text { Sep } 1957 \text { through } \\
\text { Aug } 2002\end{array}$ \\
\hline Horizontal computational - & & \\
\hline $\begin{array}{l}\text { Triangular truncation } \\
\text { Grid-point resolution }\end{array}$ & $\begin{array}{l}\mathrm{T}-62 \\
210 \mathrm{~km}\end{array}$ & $\begin{array}{l}\mathrm{T}-159 \\
125 \mathrm{~km}\end{array}$ \\
\hline $\begin{array}{l}\text { Available horizontal } \\
\text { resolution }\end{array}$ & $2.5^{\circ} \times 2.5^{\circ}$ grid & $2.5^{\circ} \times 2.5^{\circ}$ grid \\
\hline $\begin{array}{l}\text { Vertical levels: } \\
\text { computational / available }\end{array}$ & $28 / 17$ & $60 / 23$ \\
\hline $\begin{array}{l}\text { Vertical levels } \leq 100 \mathrm{hPa}: \\
\text { computational / available }\end{array}$ & $8 / 6$ & $25 / 11$ \\
\hline $\begin{array}{l}\text { Model minimum pressure / } \\
\text { maximum height }\end{array}$ & $2.73 \mathrm{hPa} / 40 \mathrm{~km}$ & $0.1 \mathrm{hPa} / 65 \mathrm{~km}$ \\
\hline $\begin{array}{l}\text { Available minimum } \\
\text { pressure / maximum height }\end{array}$ & $10 \mathrm{hPa} / 31 \mathrm{~km}$ & $1 \mathrm{hPa} / 48 \mathrm{~km}$ \\
\hline Vertical coordinate system & $\sigma$ from surface to top & $\begin{array}{l}\text { Hybrid: } \\
\sigma \text { from surface to } 100 \mathrm{hPa} \text {, } \\
\text { pressure coordinates above }\end{array}$ \\
\hline Upper boundary condition & "Artificial rigid lid" & Highly diffusive damping \\
\hline Cycle time & Six hours & Six hours \\
\hline Clouds & Diagnosed & Predicted \\
\hline Satellite temperature data & $\begin{array}{l}\text { NESDIS temperature } \\
\text { retrievals from cloud- } \\
\text { cleared radiance data used } \\
\text { directly }\end{array}$ & Raw radiance data \\
\hline Variational Analysis & $\begin{array}{l}\text { Spectral statistical } \\
\text { interpolation (SSI) - a } \\
\text { 3DVAR analysis scheme }\end{array}$ & 3DVAR with FGAT \\
\hline
\end{tabular}




\subsection{Comparison data sets}

In an intercomparison of only two reanalyses, independent comparison data assumes an especially significant role. While the source observational data on which the comparison data sets described below are based may not be completely independent of that assimilated in the reanalyses, its processing is. Reanalysis and comparison data are commensurable; with little or no manipulation, they can be plotted on the same axes and/or statistically analyzed. Seidel et al. (2004) propose an $11^{\text {th }}$ climate-monitoring principle that "calls for monitoring key climate variables with multiple, independent observing systems for measuring the variable, and multiple, independent groups analyzing the data." The first ten climate monitoring principles were proposed by Karl et al. (1995) and recommended by the National Research Council (1999).

\subsubsection{UKMO Stratospheric Analysis (METO)}

The United Kingdom Meteorology Office (UKMO) has produced daily stratospheric analyses since 1991. This is not a reanalysis project; the forecast model and assimilation system have varied over time, thus invalidating trend results but nonetheless providing valuable sets of comparison data. The stratosphere-troposphere system used "analysis correction" assimilation until November 2000 when replaced by a 3DVAR system. These analyses were originally produced to support the Upper Atmosphere Research Satellite (UARS) project; since October 1995 they have been generated as a part of the UKMO operational forecasting suite. The output of this analysis consists of temperature, wind components, and geopotential height with horizontal resolutions of 
$3.75^{\circ}$ longitude and $2.5^{\circ}$ latitude. There are six equally spaced levels per decade of pressure (Randel et al. 2002, 2004).

The forecast model was modified to use a new semi-Lagrangian dynamical core in October 2003. The number of vertical levels was increased from 42 to 50 with pressures from 1000 through $0.1 \mathrm{hPa}$. "The model configuration has been designed to help to demonstrate the benefit of additional stratospheric levels on the processing of satellite temperature soundings" (UK Met Office 2006).

The SPARC Intercomparison of Middle-Atmosphere Climatologies

(Randel et al. 2002, 2004) for the period 1992-99 identifies the two "largest apparent biases" in the METO analysis:

- Cold temperature biases ( $~ 5 \mathrm{~K})$ near the stratopause (globally)

- Warm tropical temperature (1-2 K)

The acronym "METO" is that used in the SPARC Intercomparison of MiddleAtmosphere Climatologies (Randel et al. 2002, 2004). In that work it distinguishes the UKMO Stratospheric Analysis from the UKMO operational forecasts.

\subsubsection{Freie Universität Berlin (FUB)}

The Institut für Meteorologie of the Freie Universität Berlin has produced, for the Northern Hemisphere only, gridded temperature and geopotential height analyses at 100, 50,30 , and $10 \mathrm{hPa}$. The methodology employed is described by Randel et al. (2002, 2004). 
The analyses are performed by hand (subjective analysis) by experienced personnel using station observations of geopotential height, wind, and temperature; thermal wind balance is used as a constraint on the analysis, so that even though the wind field is not analyzed, it plays an important part in the analysis procedure. Hydrostatic balance and the thermal wind are used as the analyses are built up from the 100 -hPa tie-on level ... to build accurate analyses from the station data at the stratospheric levels.

Table 2-10. Time spans of FUB data.

\begin{tabular}{|cl} 
Temperature & $5^{\circ} \times 5^{\circ}$ \\
$100 \mathrm{hPa}$ & July 1964 through December 2001 \\
$50 \mathrm{hPa}$ & July 1974 through June 2001 \\
$30 \mathrm{hPa}$ & July 1974 through June 2001 \\
$10 \mathrm{hPa}$ & October 1971 through December 1997 \\
& September through February: 1972-83 \\
& September through March: 1984-91 \\
& 12 months: July 1992 through February 1997 \\
& Other random months included
\end{tabular}

Geopotential Height $5^{\circ} \times 5^{\circ}$

$100 \mathrm{hPa} \quad$ July 1964 through December 2001

$50 \mathrm{hPa} \quad$ July 1974 through June 2001

$30 \mathrm{hPa} \quad$ July 1974 through June 2001

$10 \mathrm{hPa} \quad$ November 1971 through December 1997

September through February

12 months in some years

Because FUB data does not include 70 and $20 \mathrm{hPa}$ and at $10 \mathrm{hPa}$ is intermittent and ends in 1997, thorough comparison with reanalysis and other comparison data is not possible. Its most significant shortcoming is the absence of data in the Southern Hemisphere, where confirming information is most needed. For these reasons, FUB Northern Hemisphere data is not used in this thesis. However, the FUB equatorial zonal wind speed data is the reference against which reanalysis characterizations of the QBO are compared in Section 5.5. 


\subsubsection{Radiosonde Atmospheric Temperature Products for Assessing Climate (RATPAC)}

The RATPAC-A data set is a

large-scale regional mean time series based on LKS [Lanzante Klein Seidel data set (Lanzante et al. 2003a, 2003b)], to extend those series to the present, and to allow for future updates without the LKS adjustment procedure (which is labor-intensive and requires a panel of experts) while minimizing inhomogeneities that would interfere with trend assessment" (Free et al. 2005).

Figure 2-7 illustrates the evolutionary steps that led to and will continue to produce the RATPAC dataset. All RATPAC data derives from the Comprehensive Aerological Reference Data Set (CARDS), a database of individual station soundings and histories from around the world, created by the United States National Climatic Data Center (NCDC) (Eskridge et al. 1995).

The development of RATPAC data from 1958 through 1995 differs from that of the data after 1995. Pre-1996 RATPAC data began as the monthly mean temperatures from 87 carefully-selected, near-globally distributed stations. These means were carefully adjusted using a "multifactor expert analysis by a team of three climate scientists without use of satellite data and with minimal use of neighbor station comparisons. ... The Lanzante Klein Seidel (LKS) data consist of monthly temperatures for 16 atmospheric levels from the surface to 10 mbar, from 1948 to 1997 " (Free et al. 2005). The LKS process, conducted by John Lanzante, Steven Klein, and Dian Seidel, is described in Lanzante et al. (2003a, 2003b). Data from Bombay and Calcutta were removed from the LKS data in the RATPAC process because of accuracy problems as was $1000 \mathrm{hPa}$ data. Surface data was retained. Because of the scarcity of 
data at 10 and $20 \mathrm{hPa}$, those levels were not included. Data from 1996-97 was also eliminated because of the small amount of data remaining in those years after LKS adjustments.

Post-1995 RATPAC data began with the creation of the Integrated Global Radiosonde Archive (IGRA), which eliminated inhomogeneities and other problems from the CARDS database (Durre et al. 2004). To further reduce inhomogeneities in the IGRA data, the RATPAC team applied a first difference procedure:

1. Six months of data are removed before and after each potential inhomogeneity in each station time series.

2. A first difference time series (FD series) is created for each station as the difference in temperature from one year to the next for each calendar month.

3. Within geographical regions of the RATPAC analysis, station time series with no potential inhornogeneities are combined using biweight means (Lanzante 1996).

4. First differencing is applied to this mean of uncut time series from step 3.

5. Station FD time series from step 2 are combined with the FD of the mean of the uncut series from step 4 (weighted by the number of uncut stations).

6. The combined regional FD series from step 5 is appended to the LKS series by cumulatively summing from 1995 to the present to form the RATPAC-A temperature anomaly time series.

A thorough discussion of the procedure summarized above is contained in Appendix A of Free et al. (2005). This reference also documents the development of the RATPAC-B data set, which is recommended for "individual station data, monthly data, or regional means on smaller scales."

Free et al. (2005) argue that "LKS [data] is likely to be more reliable than a data set derived by applying the FD method to the IGRA data before 1995." However, first difference processing of IGRA data is believed by the RATPAC project participants to be 
an acceptable method of continuing the LKS data set. Free et al. (2005) also warn that RATPAC data before 1965 "should be viewed with caution."

Randel and Wu (2006) compared the temperature time series of 66 RATPAC stations between $70^{\circ} \mathrm{S}$ to $70^{\circ} \mathrm{N}$ with at least $70 \%$ of monthly data during $1979-2004$ to $50 \mathrm{hPa}$ with co-located TLS data. Despite the careful analysis of LKS, Randel and Wu found numerous discontinuities in the RATPAC minus TLS time series, which they attribute to changes in radiosonde model types because the changes do not occur simultaneously and are often supported by metadata. The temperature jumps are generally negative, behavior usually associated with improvements in radiosonde instrumentation. Linear trends of zonal mean vertically integrated radiosonde minus TLS time series from 1997-2004 reveal that only 11 stations (17\%) exhibit a positive temperature trend; 9 of these 11 are north of $45^{\circ} \mathrm{N}$. Randel and Wu conclude "that cooling in the tropical lower stratosphere is substantially overestimated in these radiosonde data." 


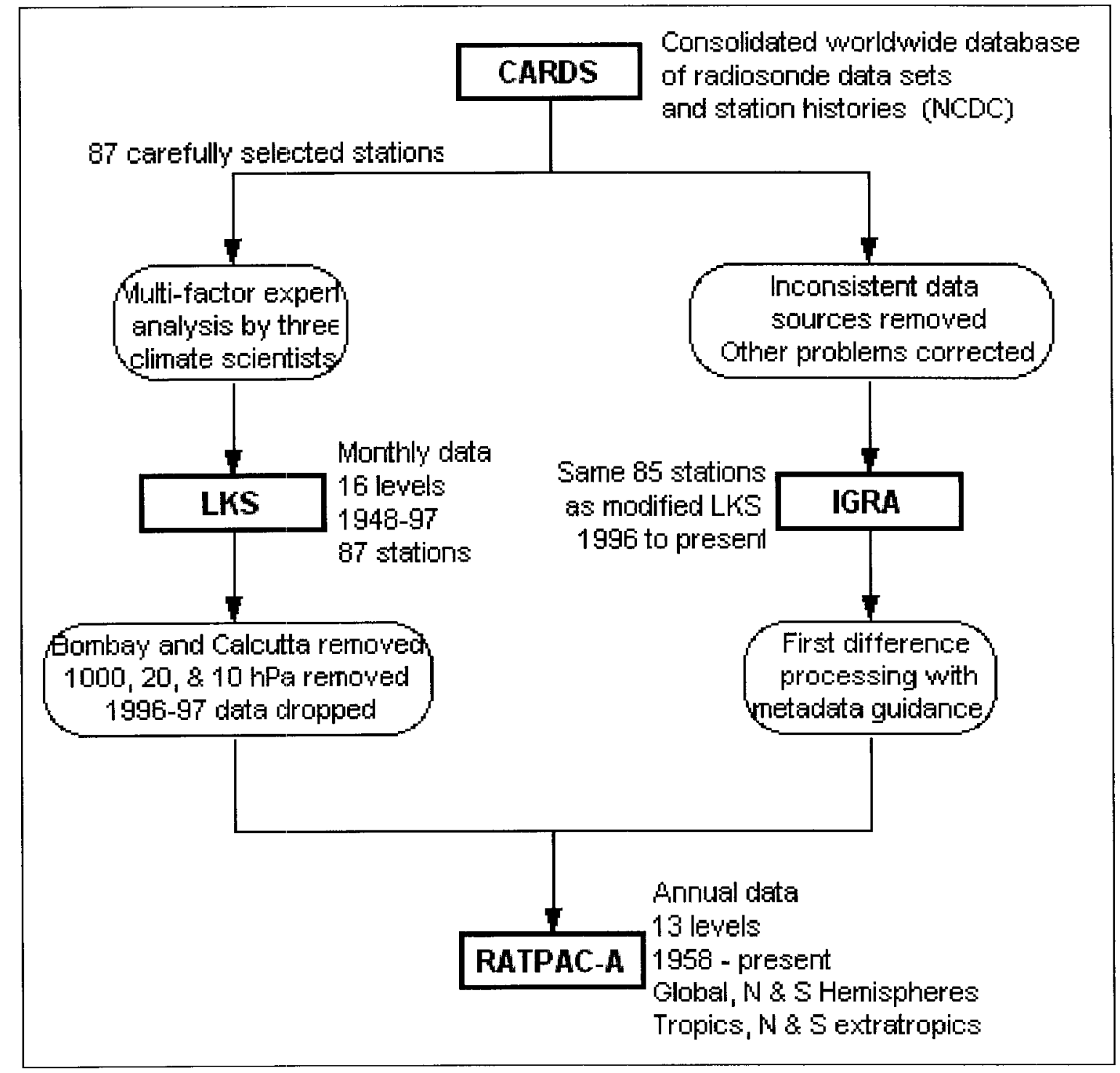

Fig. 2-7. Steps in the development of the RATPAC-A data set.

\subsubsection{Satellite Temperature Lower Stratosphere (TLS)}

Stratospheric temperature data compared in this thesis is that of Remote Sensing Systems (RSS) of Santa Rosa, CA. Their stratospheric data set includes Microwave Sounding Unit (MSU) Channel 4 brightness temperature observations from the TIROS-N, NOAA-6 through NOAA-12, and NOAA-14 polar-orbiting spacecraft and from the Advanced Microwave Sounding Unit (AMSU) Channel 9 brightness temperature 
observations from $N O A A-15$ and $N O A A-16$ polar-orbiting spacecraft. RSS has adopted the channel designation TLS (Temperature Lower Stratosphere) for their merged MSUAMSU product (Remote Sensing Systems, 2006). Seidel et al. (2004) show that RSS correlations with LKS data (see Section 2.3.3) are 0.90 for annual anomalies and 0.96 for detrended monthly anomalies.

The RSS TLS dataset is provided in netCDF format (see Appendix B for source). Temperatures are given for a single deep vertical layer beginning at $434 \mathrm{hPa}$ and extending to $0.5 \mathrm{hPa}$ with the maxima at approximately $85 \mathrm{hPa}$. The use of RSS-provided vertical weighting data to appropriately weight reanalysis data for comparison with TLS data is discussed in Section 4.3. Temperatures data is provided at the centers of $2.5^{\circ} \times 2.5^{\circ}$ grid and run from November 1978 through the present. When the temperatures are interpolated to the $2.5^{\circ} \times 2.5^{\circ}$ grid points used in NCEP 1 and ERA-40 reanalyses, the latitudinal data range is $80^{\circ} \mathrm{S}$ to $80^{\circ} \mathrm{N}$. This absence of polar data, which excludes $1.5 \%$ of the globe, is a consequence of satellite orbital mechanics; sun-synchronous polar orbits are inclined $98.8^{\circ}$ and thus do not pass over the poles (Kidder and Vonder Haar, 1995). 


\subsection{STRATOSPHERIC OVERVIEW}

This section briefly discusses general stratospheric climatology and the three major dynamic characteristics of the stratosphere:

- Polar vortices

- The quasi-biennial oscillation (QBO)

- Stratospheric sudden warmings (SSW)

These characteristics are discussed in turn in the following sections.

Earth's troposphere contains approximately $85 \%$ of the total mass of the atmosphere and essentially all atmospheric water. In this lowest 10 to $16 \mathrm{~km}$ of the atmosphere exist the weather and climate phenomena experienced by life on Earth. The Earth's stratosphere extends from the tropopause at an altitude of 10 to $16 \mathrm{~km}$ to the stratopause at 47 to $60 \mathrm{~km}$. Because of absorption of solar ultraviolet radiation by ozone, the stratosphere is characterized by an increase in mean temperature with height and is named for the resulting high static stability. Infrared radiative cooling in general balances this heating (Holton 2004). A midlatitude mean temperature profile for the stratosphere, as well as the troposphere and the mesosphere, is shown in Fig. 3-1. Heavy dashed lines indicate the region of the stratosphere considered in this thesis: from 100 to $10 \mathrm{hPa}$. The rationale for these limits is discussed in following paragraphs. 


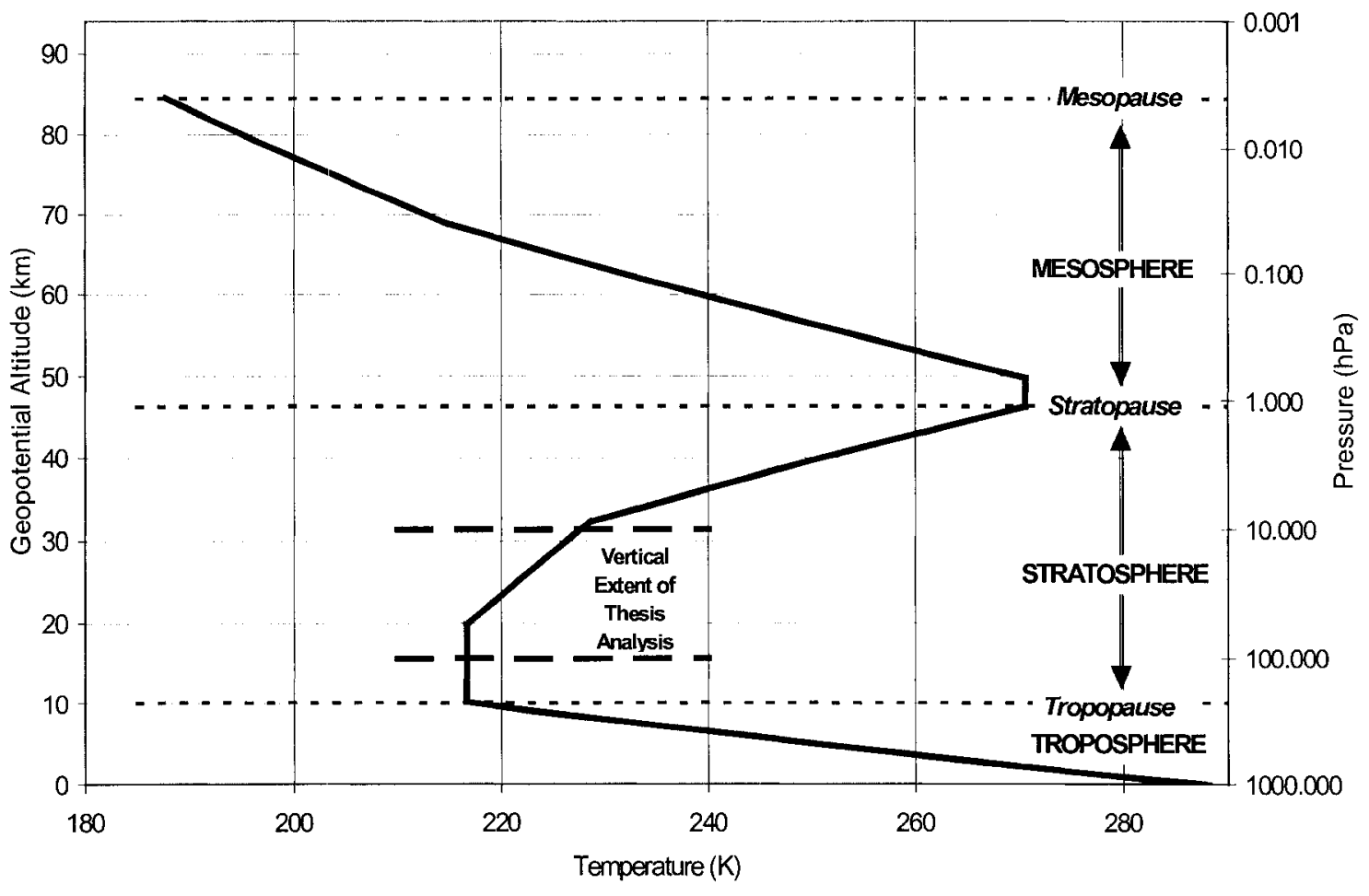

Fig. 3-1. Midlatitude mean temperature profile based on U.S. Standard Atmosphere (1976).

The climatological tropopause varies between $100 \mathrm{hPa}(16.2 \mathrm{~km})$ in equatorial latitudes and $300 \mathrm{hPa}(9.2 \mathrm{~km})$ in polar latitudes. The climatological stratopause varies between $1 \mathrm{hPa}(48 \mathrm{~km})$ in polar winter and $0.3 \mathrm{hPa}(57 \mathrm{~km})$ in polar summer (Randel et al. 2002, 2004).

This thesis intercompares the stratospheric performance of two major global reanalyses in order to identify a stratospheric climatology that can be used in the evaluation of global climate models (GCMs), such as those submitted in support of the Fourth Assessment Report of the Intergovernmental Panel on Climate Change, models employed in the WMO ozone assessments, and other stratospheric analyses. The minimum pressure level of the GCM simulation data available in IPCC models is $10 \mathrm{hPa}$. 
This is also the minimum pressure level of the NCEP1 reanalysis. In the standard atmosphere, $10 \mathrm{hPa}$ is found at $31.1 \mathrm{~km}$. The maximum pressure level of stratospheric climatological analyses is typically $100 \mathrm{hPa}$, (Randel et al. 2002, 2004; Pawson and Fiorino 1998a, 1998b). Because stratospheric behavior of interest in general occurs above $100 \mathrm{hPa}$, and $10 \mathrm{hPa}$ is the minimum common pressure level, this thesis considers the stratosphere from 100 to $10 \mathrm{hPa}$, a vertical distance of $14.9 \mathrm{~km}$. These data-driven constraints limit the analyses of this thesis to approximately $37 \%$ of the stratosphere in height and $62 \%$ in mass.

The existence of the stratosphere was discovered essentially simultaneously about 1900 by Teisserenc de Bort, at Trappe, near Paris, and by Richard Assmann near Berlin. The two scientists had been conducting independent research projects with selfregistering instruments carried aloft by unmanned balloons for more than five years. Both had resorted to night launches to avoid the effects of solar radiation on temperature readings, but both had detected a temperature inversion at heights of 8 to $13 \mathrm{~km}$ (de Bort) and 10 to $15 \mathrm{~km}$ (Assmann). During the summer of 1901 Assmann telephoned de Bort with his successful results. On 1 May 1902, they simultaneously published their findings in prestigious scientific journals in their respective countries.

The two long-time friends, who had stayed in contact about their research through correspondence, thus simultaneously revealed the discovery of the stratosphere to the world. They had arrived at the same conclusions from their observations which each had obtained by different methods and equipment, but which they had held back until they were certain that the data were correct (P. Dubois (1993) as quoted in Labitzke and van Loon 1999). 
Teisserenc de Bort must also be recognized for his contribution to a fundamental meteorological classification. It was he who "divided" the atmosphere as known at that time, as a result of the work of Assmann and himself, into two layers that he named troposphere and stratosphere. The discussion of the discovery of the stratosphere in the preceding paragraphs is summarized from Labitzke and van Loon (1999).

\subsection{Polar vortices}

In addition to the zonal structure illustrated in Fig. 3-1, the stratosphere contains meridional temperature structure and as a consequence, significant circulations. It is these meridional and vertical components of the eddy circulation that drive the middle atmosphere (stratosphere and mesosphere) away from a purely radiatively-determined state, especially in the winter stratosphere (Holton 2004).

The meridional structure of the stratosphere is illustrated in the pressure level vs. latitude contour plots of zonal wind speed, horizontal temperature gradient, and temperature in Figs. 3-2 through 3-7. These plots were generated with data from the United Kingdom Meteorological Office stratospheric analysis (METO). Section 2.3.1 describes this analysis and Appendix B identifies its data source. While the METO "climatology" consists of only eight years of data, Figs. 4-5, 4-6, 5-5, 5-6 and Randel et al. $(2002,2004)$ demonstrate that it is quite consistent with reanalyses and other climatologies and, as is demonstrated in Fig. 2-6, it is the source of the highest level of available data, data that extends to the stratopause. 
This thesis deals with atmospheric data fields (temperature and wind speed) and their derivatives. These fields exist in "four space": longitude and latitude in the horizontal, pressure levels in the vertical, and time. To succinctly identify the processing that has reduced these four-dimensional fields to two-dimensional paper, in this thesis a bracketed description of the processing that has been applied to each dimension is provided with each applicable figure. For example: [lon: mean of $0^{\circ}-356.25^{\circ}$, lat: $90^{\circ} \mathrm{S}-90^{\circ} \mathrm{N}$, press: $1000-0.316 \mathrm{hPa}$, time: monthly mean of Jan 1992-Dec 1999]. In these descriptions, the symbol " _. " means that the set of data averaged or displayed includes the end points of the set. Pressure levels in reanalysis comparisons are 100, 70, $50,30,20$, and $10 \mathrm{hPa}$. The relatively coarse spacing of these levels in the lower stratosphere at times produces sharp corners in pressure level vs. latitude contour plots. Unless otherwise explicitly noted, all heights in this thesis are geopotential heights. Geopotential heights of pressure levels are the midlatitude values of the U. S. Standard Atmosphere (1976).

Horizontal temperature gradient is included in Figs. 3-2 through 3-5 since it is the link between the temperature (lower) and zonal wind speed (upper) subplots. The thermal wind speed is the vertically integrated horizontal gradient (Eqs. 3.31 and 3.32 in Holton 2004):

$$
\vec{V}_{T} \equiv \vec{V}_{g}\left(p_{1}\right)-\vec{V}_{g}\left(p_{0}\right)=-\frac{R}{f} \int_{p_{0}}^{p_{1}}\left(\hat{k} \times \nabla_{p} T\right) d \ln p
$$

and in component form, 


$$
u_{T}=-\frac{R}{f}\left(\frac{\partial\langle T\rangle}{\partial y}\right)_{p} \ln \left(\frac{p_{0}}{p_{1}}\right) ; \quad v_{T}=\frac{R}{f}\left(\frac{\partial\langle T\rangle}{\partial x}\right)_{p} \ln \left(\frac{p_{0}}{p_{1}}\right)
$$

Thus, in Figs. 3-2 through 3-5, physical consequences flow from the temperature field (bottom subplot), to the horizontal temperature gradient field (center subplot), and finally to the zonal wind speed field (upper subplot).

Temperature in the Earth's lower stratosphere is strongly influenced by the upper troposphere and is characterized by colder regions above the Equator and the winter pole, and warmer regions above the summer pole and in the winter midlatitudes. Equatorial and winter polar latitudes of the lower stratosphere are the coldest portions of the Earth's atmosphere below the middle mesosphere. The thermal gradient from midlatitude to pole in the winter hemisphere produces a westerly circumpolar jet, the "polar night jet" (PNJ). These jets form the cyclonic vortices that dominate the stratosphere and mesosphere. Maximum zonal wind speeds in these PNJs occur in the high latitudes of the upper stratosphere or lower mesosphere. The Southern Hemisphere winter jet is significantly stronger and longer lived than that of the Northern Hemisphere (Holton 2004; Waugh and Randel 1999). The strength of these jets is modulated by the long-wave climatology of large-scale planetary waves. An easterly jet forms in the summer hemisphere. The characterization of polar vortices by the NCEP1 and ERA-40 reanalyses is presented and discussed in Section 5.4 .

The behavior described in the previous paragraph is illustrated in Fig. 3-2 for the months of December, January, and February (DJF) and Fig. 3-4 for the months of June, July, and August (JJA). The horizontal temperature gradient maxima of approximately 
$\pm 0.8 \mathrm{~K} \mathrm{deg}^{-1}$ at $30^{\circ}$ to $45^{\circ}$ latitude in both hemispheres at $100 \mathrm{hPa}$ are permanent features of the tropopause equatorial "cold pool." The gradient maxima at the tropopause in the winter hemisphere moves equatorward as the polar region cools, establishing a gradient of reverse polarity poleward and above the $100 \mathrm{hPa}$ maxima. It is these regions of reversed polarity that produce the PNJ. During JJA, the local horizontal temperature gradient maxima at $60-65^{\circ} \mathrm{S}, 40-20 \mathrm{hPa}$ are $50 \%$ greater than the permanent gradients that are otherwise the largest thermal gradients below the stratopause. Above and poleward of these regions of reversed polarity, the horizontal temperature gradient reverses once again, tilting the lower polar vortex equatorward with height. In the summer hemisphere, horizontal temperature gradients other than the permanent ones are relatively light and everywhere of the same polarity as gradient maxima at the tropopause in that hemisphere, resulting in easterly jets.

Figure 3-3 for the months of March, April, and May (MAM) and Fig. 3-5 for the months of September, October, and November (SON) show westerly jets in both hemispheres during spring and fall seasons. Fall hemisphere cooling produces horizontal temperature gradient regions of reversed polarity poleward and above the $100 \mathrm{hPa}$ maxima, and the jets begin to build toward their maximum westerly winter velocities. In the Southern Hemisphere spring, the warming polar tropopause region creates a significant horizontal temperature gradient maxima at $65-70^{\circ} \mathrm{S}, 100 \mathrm{hPa}$ while poleward and above the $100 \mathrm{hPa}$ maxima, this warming does not occur and the gradient is of the same polarity as the Southern Hemisphere tropospheric permanent maximum. This gradient pattern produces a westerly jet with its maximum zonal wind speed in the 
midstratosphere, much lower in height than the PNJ as the PNJ decays. A region of reversed horizontal temperature gradient polarity above the $100 \mathrm{hPa}$ maxima also exists in the Northern Hemisphere spring; however there is no significant temperature gradient maxima at the tropopause poleward of the permanent gradient. This gradient pattern results in a light westerly jet in the upper stratosphere.

Only in the spring hemispheres of Fig. 3-3 (MAM) and Fig. 3-5 (SON) is the core of a polar vortex found in the stratosphere. Section 5.4 demonstrates that monthly averaged polar vortex cores are found below $10 \mathrm{hPa}$ in spring hemispheres in both NCEP1 and ERA-40 reanalyses. 


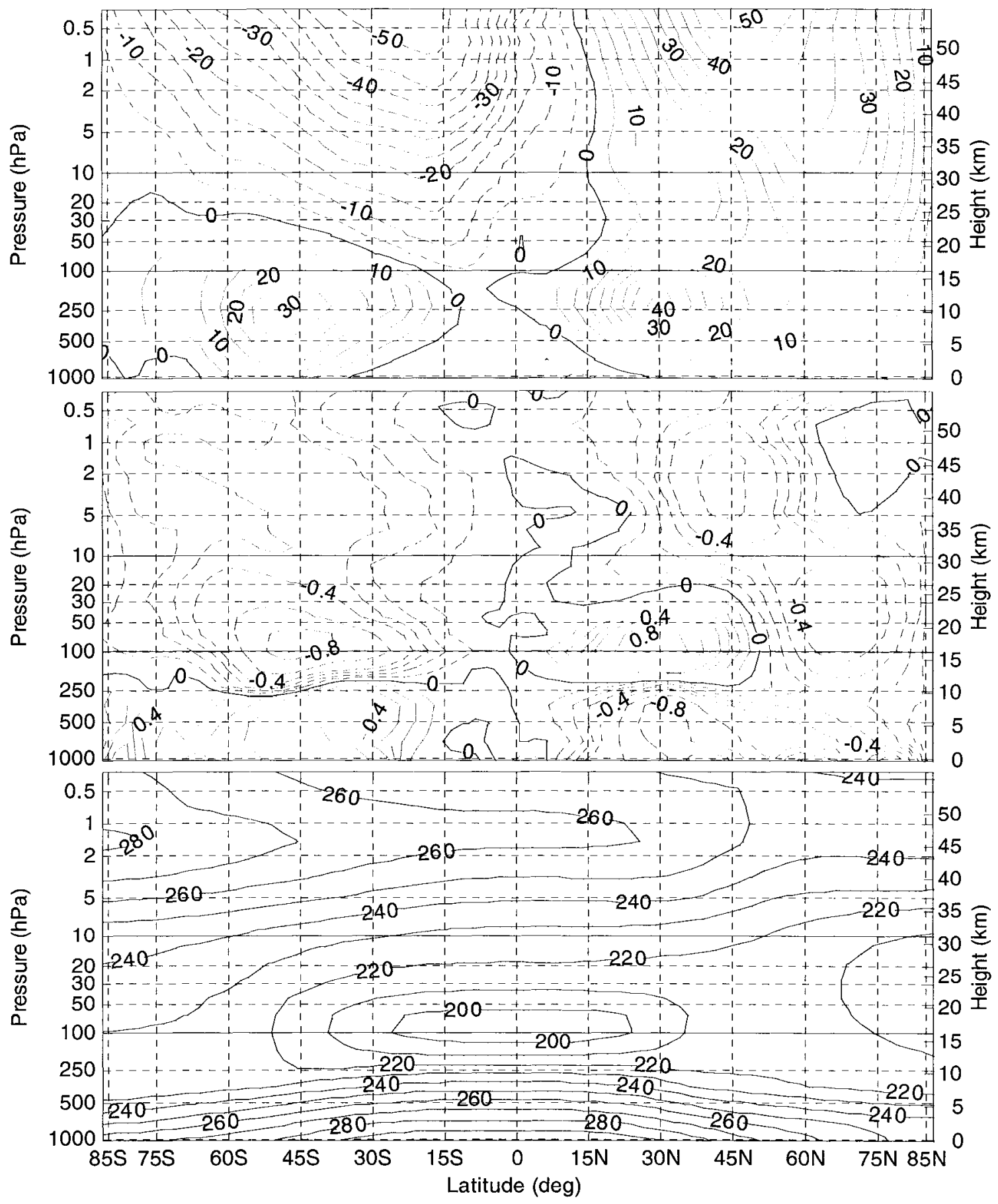

Fig. 3-2. Meridional structure of zonal climatology for December, January, and February (DJF): zonal wind speeds ( $m s^{-1}$ ) (upper), horizontal temperature gradients $\left(\mathrm{K} \mathrm{deg}^{-1}\right)$ (middle), temperatures (K) (lower) [lon: mean of $0^{\circ}-356.25^{\circ}$, lat: $86.25^{\circ} \mathrm{S}$ $86.25^{\circ} \mathrm{N}$, press: $1000-0.316 \mathrm{hPa}$, time: monthly mean of Jan 1992-Dec 1999]. 


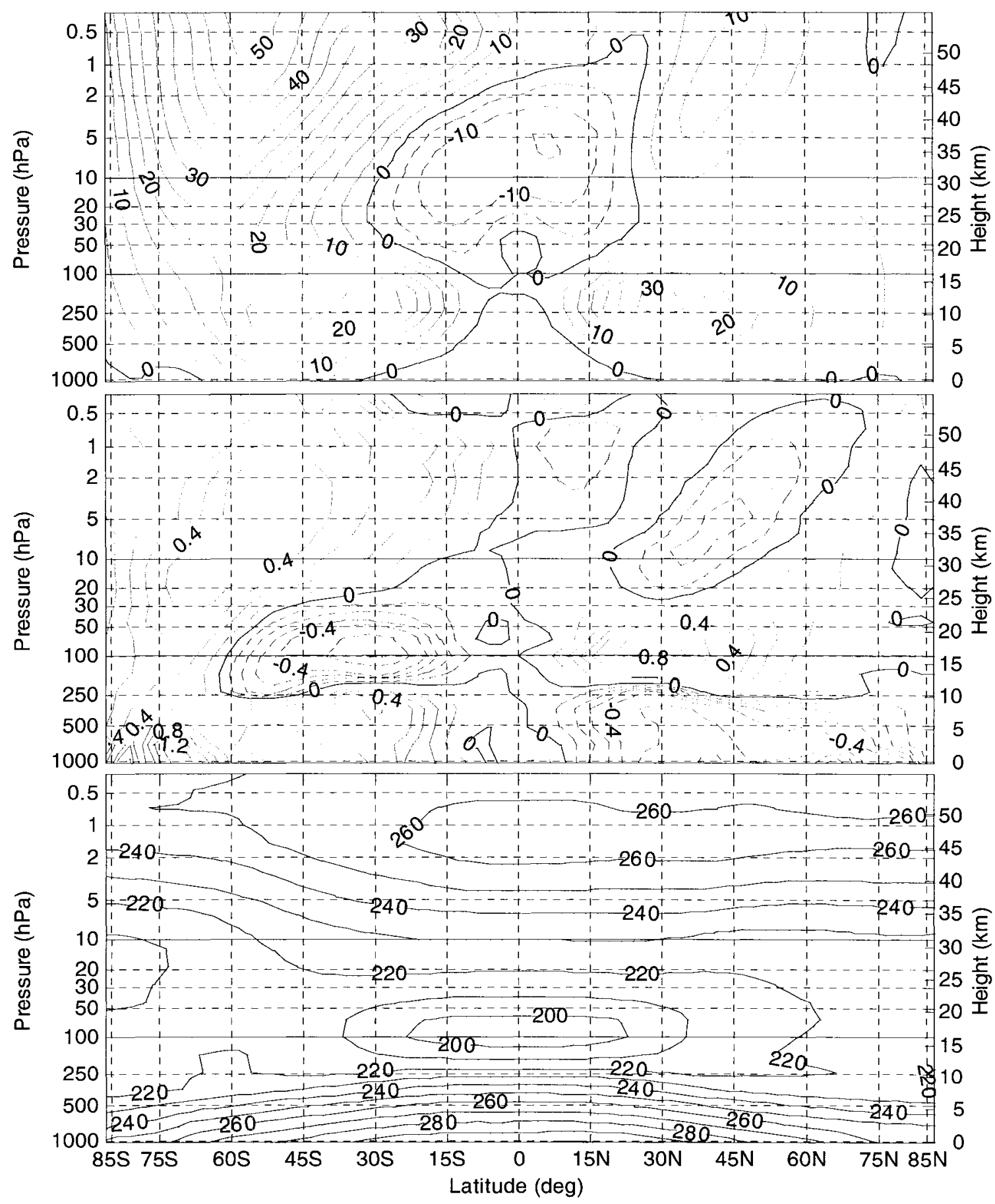

Fig. 3-3. Same as Fig. 3-2 except climatology for March, April, and May (MAM). 


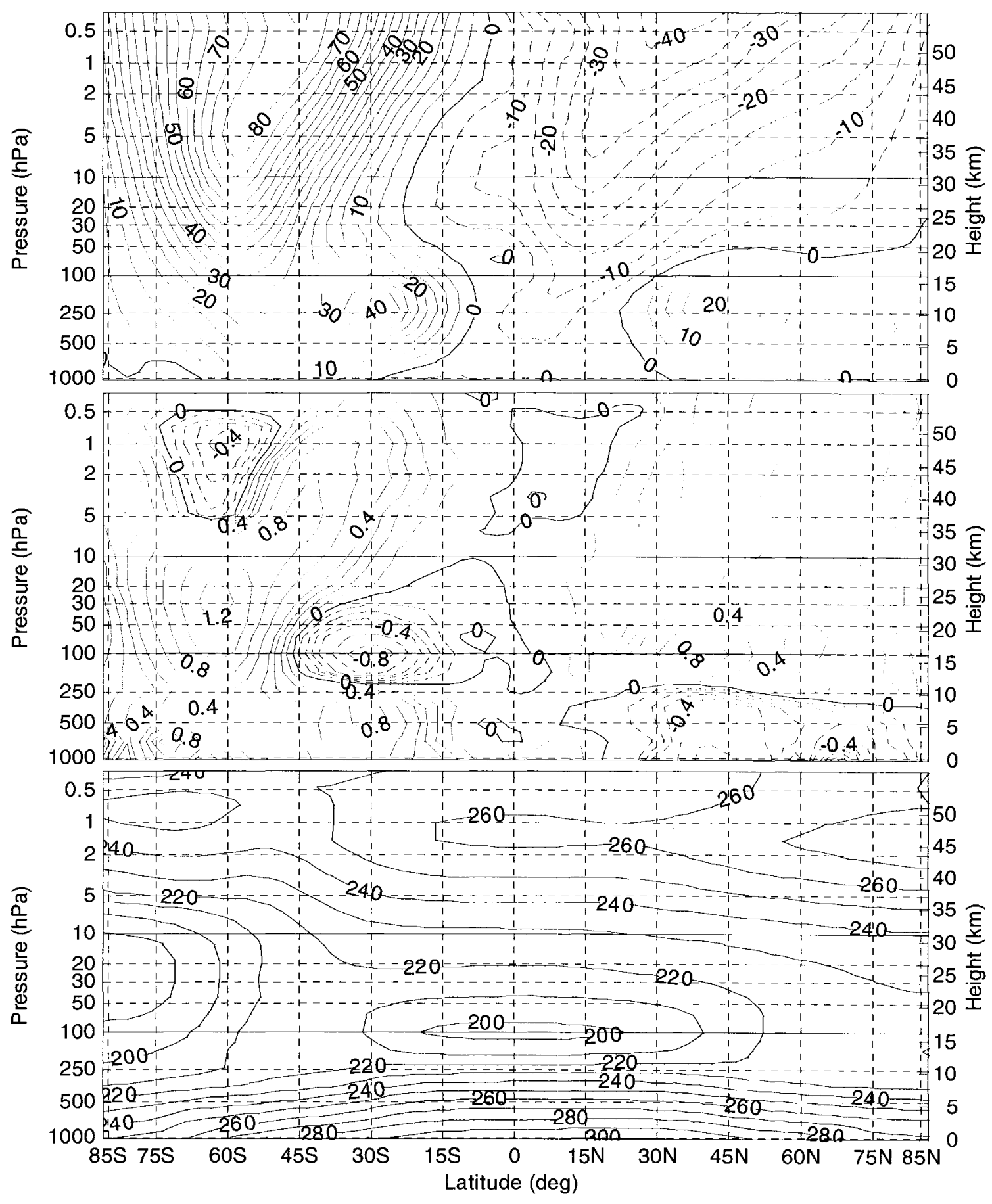

Fig. 3-4. Same as Fig. 3-2 except climatology for June, July, and August (JJA). 


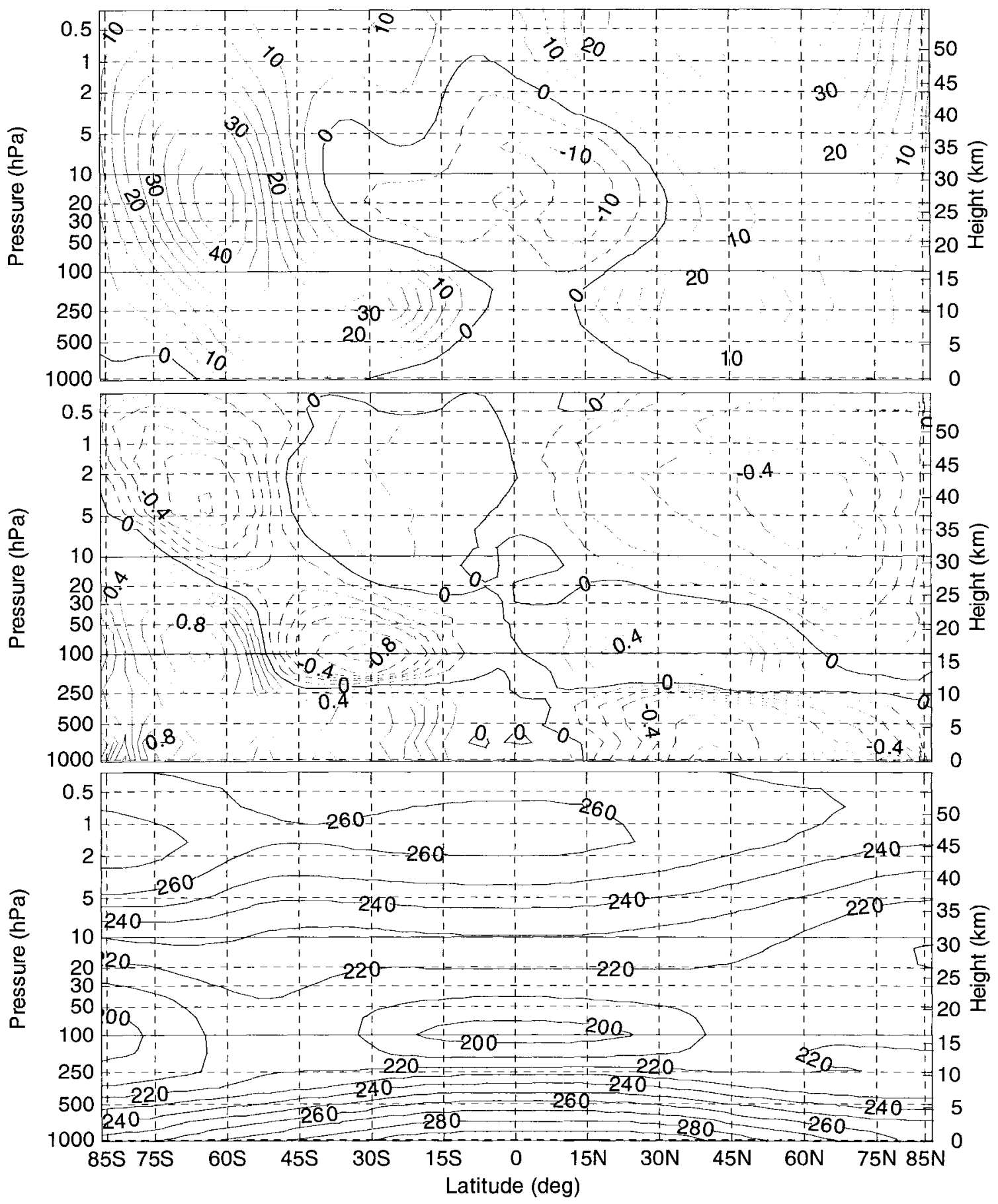

Fig. 3-5. Same as Fig. 3-2 except climatology for September, October, and November (SON). 
Plots (not shown) for the middle month of each season of Figs. 3-2 through 3-5 are almost indistinguishable from the corresponding seasonal plots. This implies that the climatology of the middle month dominates the season.

Figure 3-6 demonstrates that the mean flow from surface to stratopause is westerly from midlatitudes to the poles; the magnitude of the summer easterly jet is no match for that of the westerlies of the remaining three quarters of the year but does attenuate maximum values of the seasonal jets. Maximum standard deviations of zonal wind speed are equatorward of the stratospheric polar jets. The tropical mean flow, especially above $50 \mathrm{hPa}$, is easterly; this is the signature of the quasi-biennial oscillation (QBO), introduced in the next section. The QBO's easterly phase zonal wind speeds are stronger and more sustained than its westerlies. Figures 5-72 and 5-73 plot the reanalyzed seasonal standard deviation of zonal wind speed with annual variance removed.

The mean tropopause is shown in Fig. 3-7 at $100 \mathrm{hPa}$ and the mean stratopause at approximately $0.5 \mathrm{hPa}$. However, strong thermal variance extends well below the mean tropopause to a level of $300 \mathrm{hPa}$ in the polar regions. Holton (2004) derives a QBO temperature perturbation of approximately three $\mathrm{K}$ at the Equator so the lack of a strong thermal signature in the temperature fields is to be expected. Figures 4-19 and 4-20 plot the reanalyzed seasonal standard deviation of temperature with annual variance removed. 


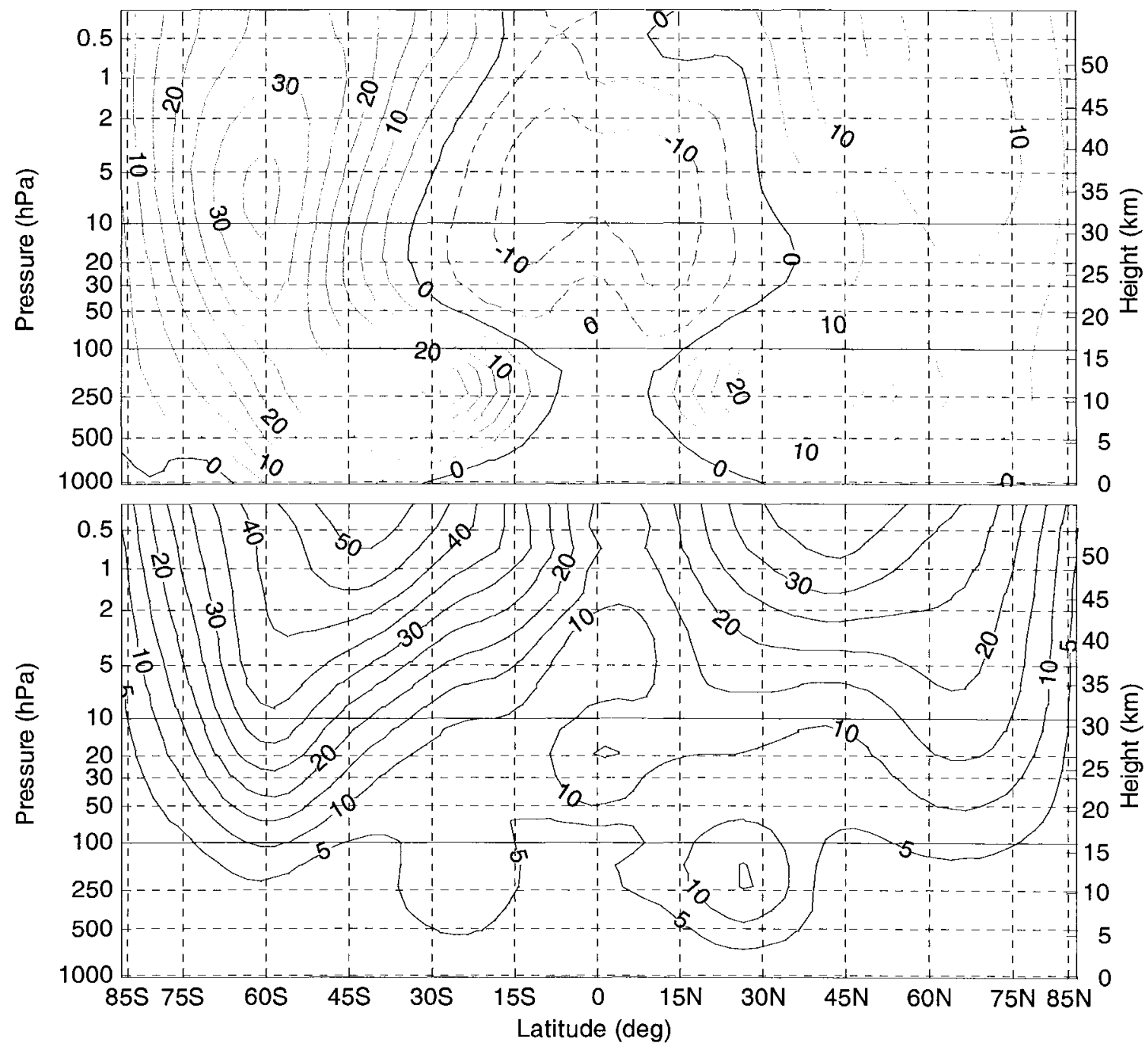

Fig. 3-6. Eight-year pressure level (height) vs. latitude structure of zonal climatology: mean zonal wind speeds $\left(\mathrm{m} \mathrm{s}^{-1}\right)$ (upper), standard deviation of zonal wind speeds $\left(\mathrm{m} \mathrm{s}^{-1}\right)$ (lower) [lon: mean of $0^{\circ}-356.25^{\circ}$, lat: $86.25^{\circ} \mathrm{S}-86.25^{\circ} \mathrm{N}$, press: $1000-0.316 \mathrm{hPa}$, time: mean of Jan 1992-Dec 1999]. 


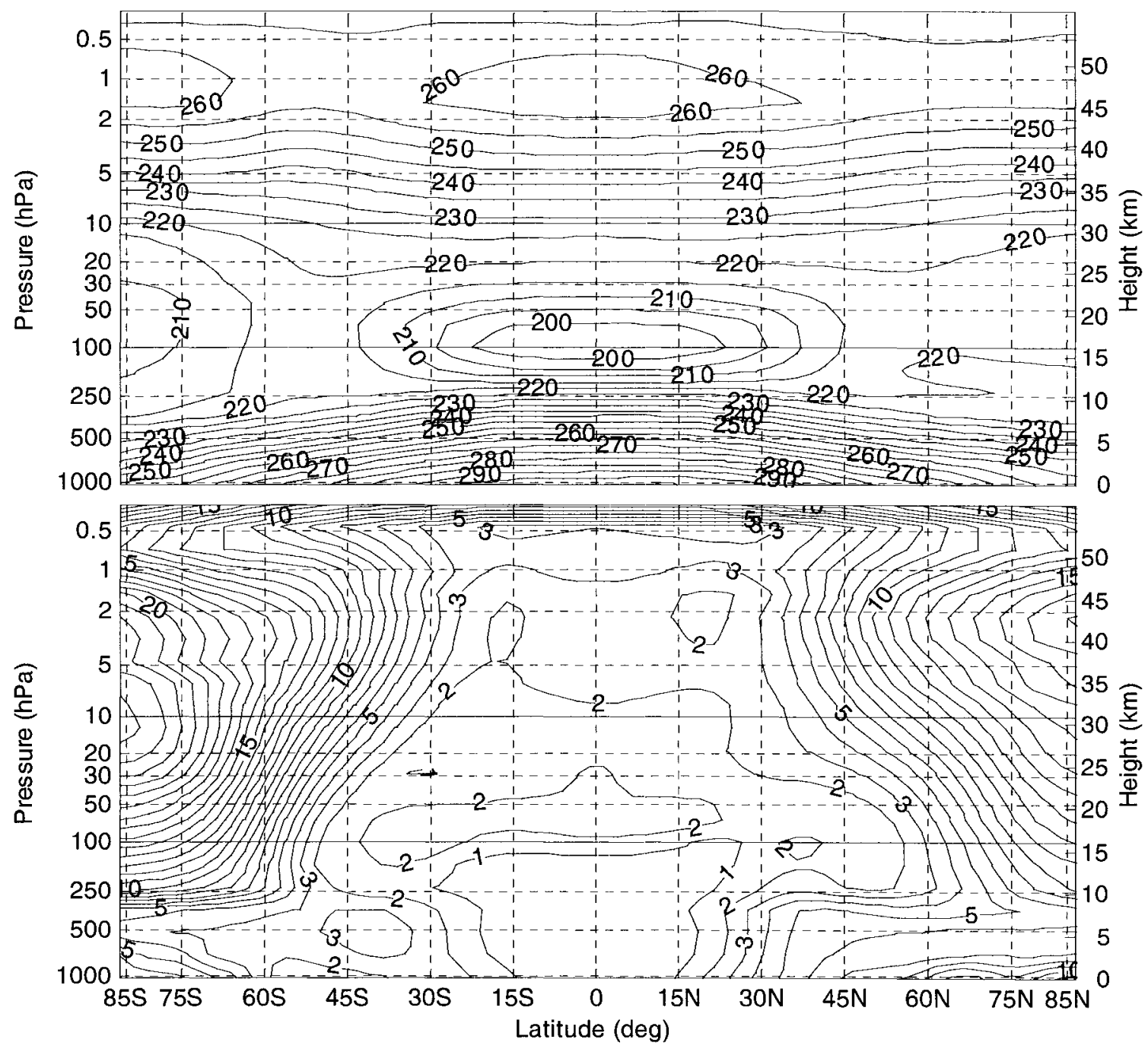

Fig. 3-7. Eight-year pressure level (height) vs. latitude structure of zonal climatology: mean temperatures $(K)$ (upper), standard deviation of temperatures ( $K)$ (lower) [lon: mean of $0^{\circ}-356.25^{\circ}$, lat: $86.25 \mathrm{~S}-86.25^{\circ} \mathrm{N}$, press: $1000-0.316 \mathrm{hPa}$, time: monthly mean of Jan 1992-Dec 1999].

Table 3-1 summarizes the general features of the polar vortices. It is based on the work of Waugh and Randel (1999), who analyzed elliptical diagnostics (Waugh 1997) of potential vorticity on isentropic surfaces using NCEP stratospheric analyses (not reanalyses) from October 1978 through April 1998. This table, the earlier discussion of this section, and Section 5.4 confirm and reinforce one another. 
Table 3-1. General nature of polar vortices.

\begin{tabular}{|c|c|c|}
\hline & ARCTIC VORTEX & ANTARCTIC VORTEX \\
\hline Onset & $\begin{array}{l}\text { Sep }(3 \mathrm{hPa})-\mathrm{Nov}(10 \mathrm{hPa}) \\
\text { typically Oct } 1\end{array}$ & $\begin{array}{l}\text { Mar }(3 \mathrm{hPa})-\text { May }(10 \mathrm{hPa}) \\
\text { typically Apr } 1 \\
\text { Onset is more gradual than decay }\end{array}$ \\
\hline $\begin{array}{l}\text { Maximum } \\
\text { areal extent }\end{array}$ & Mid-Dec - mid Jan & Late Jun - late Aug \\
\hline $\begin{array}{l}\text { Extent - } \\
\text { equivalent } \\
\text { latitude }\end{array}$ & $\begin{array}{l}\sim 57^{\circ}(3 \mathrm{hPa}) \\
\sim 64^{\circ}(10 \mathrm{hPa}) \\
\sim 69^{\circ}(55 \mathrm{hPa}) \\
\text { Midwinter radii of vortices increa } \\
\text { but decreases until no difference }\end{array}$ & $\begin{array}{l}\sim 47^{\circ}(3 \mathrm{hPa}) \\
\sim 57^{\circ}(10 \mathrm{hPa}) \\
\sim 63^{\circ}(55 \mathrm{hPa}) \\
\text { ses with height in both hemispheres } \\
\text { in spring }\end{array}$ \\
\hline Decay & $\begin{array}{l}\text { Mar }(3 \mathrm{hPa})-\text { May }(10 \mathrm{hPa}) \\
\text { typically Apr } 15\end{array}$ & $\begin{array}{l}\text { Oct }(3 \mathrm{hPa})-\mathrm{Dec}(10 \mathrm{hPa}) \\
\text { typically Nov } 10\end{array}$ \\
\hline Duration & $\begin{array}{l}\text { Arctic vortex has a life span } \\
\text { more than a month shorter } \\
\text { Trend toward longer-lasting vort }\end{array}$ & ces in both hemispheres \\
\hline \multirow{2}{*}{$\begin{array}{l}\text { Distortion } \\
\text { from zonal } \\
\text { symmetry }\end{array}$} & $\begin{array}{l}\text { Latitude of center: } \sim 14^{\circ} \\
\text { Aspect ratio: } \sim 1.7\end{array}$ & $\begin{array}{l}\text { Latitude of center: } \sim 44^{\circ} \\
\text { Aspect ratio: } \sim 1.2\end{array}$ \\
\hline & $\begin{array}{l}\text { Increases during life-cycle } \\
\text { Lack of zonal symmetry } \\
\text { associated with warming events } \\
\text { Little correlation of elongation or } \\
\text { in both hemispheres }\end{array}$ & vortex with movement off pole \\
\hline $\begin{array}{l}\text { Interannual } \\
\text { variability }\end{array}$ & $\begin{array}{l}\text { Highly variable during life } \\
\text { cycle, especially late winter }\end{array}$ & Small except during breakdown \\
\hline $\begin{array}{l}\text { Zonal shifts of } \\
\text { vortex } \\
\text { climatological } \\
\text { latitude }\end{array}$ & $\begin{array}{l}\text { Early winter - two preferred } \\
\text { locations: } \\
\text { Large shift in Nov - Dec } \\
\text { Small shift in late Jan - early } \\
\text { Feb }\end{array}$ & Large changes in late winter thru spring \\
\hline
\end{tabular}




\subsection{Quasi-biennial oscillation (QBO)}

The QBO is the second major circulation feature of the middle atmosphere. Its characterization by the NCEP1 and ERA-40 reanalyses is presented and discussed in Section 5.5. Essentially longitudinally-symmetric zonal wind speeds in the equatorial stratosphere alternate at a given height between easterly and westerly with periods varying from 24 to 30 months. These wind regimes, which are also symmetric about the Equator, appear above $30 \mathrm{~km}$ and propagate downward at a rate of approximately one $\mathrm{km}$ per month to a lower level of approximately $23 \mathrm{~km}$ (Holton 2004). Their maximum zonal wind speed amplitude is approximately +20 to $-40 \mathrm{~m} \mathrm{~s}^{-1}$ at $10 \mathrm{hPa}$ and +15 to $-20 \mathrm{~m} \mathrm{~s}^{-1}$ at $50 \mathrm{hPa}$. Figure 3-8 is a height vs. time contour plot of the QBO from the ERA-40 reanalysis.

Easterlies from 100 to $1 \mathrm{hPa}$ and extending to $30^{\circ}$ latitude in Fig. 3-6 are the signature of the QBO (Pascoe et al. 2005; Baldwin et al. 2001). Characterization of the QBO by the NCEP1 and ERA-40 reanalyses is evaluated in Section 5.5. 


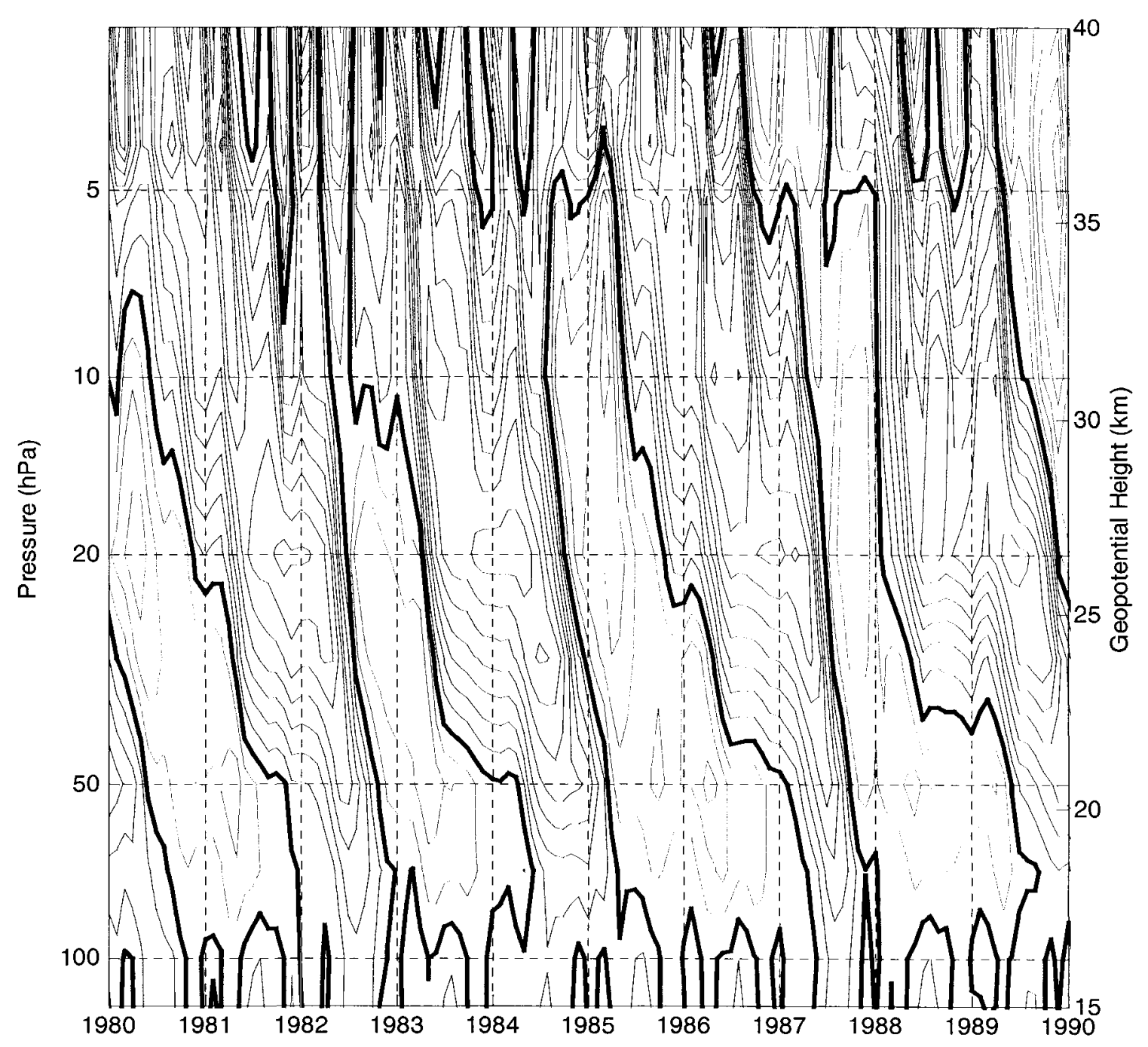

Fig. 3-8. Height vs. time contour of $Q B O$, westerly winds (red), easterly winds (blue), contour interval $5 \mathrm{~m} \mathrm{~s}^{-1}$ ERA-40 [lon: mean of $0^{\circ}-356.25^{\circ}$, lat: $0^{\circ}$, press: 120-2.8 hPa, time: 1980-1989].

\subsection{Stratospheric sudden warming}

Stratospheric sudden warmings (SSW) occur when the Northern Hemisphere winter circumpolar vortex is disrupted to the extent that the polar stratosphere warms sufficiently in temperature and space to reverse the meridional thermal gradient and/or reverse the circumpolar flow. They represent the strongest dynamical coupling between 
the stratosphere and troposphere (Charlton and Polvani, 2007, hereinafter "C\&P"). The onset of these events occurs in a few days; the effects may last for weeks. Over the 45 years from 1958 through 2002, they occurred at an average rate of approximately 0.8 per year during the months of November through April (Labitzke et al. 2006). Characterization of stratospheric sudden warmings by the NCEP1 and ERA-40 reanalyses is presented and discussed in Section 4.5.

The following discussion incorporates material from The Berlin Stratospheric Data Series (BSDS) prepared at the Freie Universität Berlin (FUB) (Labitzke et al. 2006) and also Labitzke and Naujokat (2006). There are numerous definitions of the four categories of stratospheric sudden warmings as outlined below:

1. Major midwinter warmings

- The World Meteorological Organization standard definition of a stratospheric sudden warming is "at $10 \mathrm{hPa}$, easterly winds at $60^{\circ} \mathrm{N}$ and warmer temperatures at the pole than at $60^{\circ} \mathrm{N}$." These conditions were used by Labitzke et al. (2006) to identify the events in the BSDS (Baldwin and Dunkerton 1999). To complete this definition, daily-mean, zonally-averaged, zonal winds and zonal temperatures at $10 \mathrm{hPa}$ and $60^{\circ} \mathrm{N}$ are assumed.

- Labitzke and Naujokat (2006) define the "breakdown of the polar vortex" as a reversal of the $10 \mathrm{hPa}, 60^{\circ} \mathrm{N}$ zonal winds and a displacement of the vortex center south of $60-65^{\circ} \mathrm{N}$, but they also include the possibility that the vortex is split in two.

- The first and only recorded major midwinter warming in the Southern Hemisphere occurred in September 2002, splitting the vortex and separating the Antarctic ozone hole into two parts (Baldwin et al. 2006).

- The absence of major midwinter warmings from March 1991 through November 1998 is noted by Labitzke et al. (2006) without suggestions of probable cause.

- Section 4.5 discusses a second climatology of major midwinter warmings. C\&P developed this climatology by applying an SSW identification and classification algorithm to NCEP1 and ERA-40 reanalysis data. 
2. Minor midwinter warmings

- Warming polar temperatures and at times reversal of the temperature gradient characterize minor warmings. They do not reverse the $10 \mathrm{hPa}, 60^{\circ} \mathrm{N}$ winds. Minor warmings also occur in the Southern Hemisphere (Labitzke and Naujokat 2006).

3. Canadian warmings

- Canadian warmings result from the simultaneous intensification and poleward movement of the Aleutian stratospheric high, which displaces or disrupts the polar vortex (Baldwin and Dunkerton 1999).

- These warmings, which occurred at a rate of 0.74 per year from 1958 through 1984 were observed only four times from 1989 through 2001, or 0.31 per year. They have always been observed in November and December. This decrease is attributed to climatological changes in the troposphere, since the Aleutian stratospheric high is coupled with the tropospheric Aleutian low (Labitzke et al. 2006).

4. Final warmings

- The transition to the warm summer polar high is defined as the final warming of the cold winter vortex. These events signal the breakdown of winter polar vortices without subsequent recovery. They vary in intensity and time of occurrence; there are major and minor as well as early and late final warmings (Labitzke and Naujokat 2006).

- The BSDS SSW climatology includes 12 final warmings, 1 in February, 9 in March, and 2 in April, and only 1 minor final warming in February. No final warmings have occurred since February 1989. The cause of this variability is unknown (Labitzke et al. 2006).

The multi-step development of stratospheric sudden warmings summarized from

Holton $(2004$, Sec. 12.4$)$ is outlined below.

1. Amplitudes of quasi-stationary planetary waves of wavenumbers one and two, produced by Northern Hemisphere orographic forcing, amplify rapidly in the troposphere.

2. The polar vortex provides wave guides for the vertical propagation of these waves into the stratosphere. 
3. The interaction between these waves and the mean flow results in wave growth and increased dissipation. This produces a convergent Eliassen-Palm (EP) flux, which in turn decelerates the westerly polar vortex.

4. EP flux convergence weakens the polar vortex, allowing even more waves to propagate into the stratosphere until the mean zonal wind reverses direction and a critical layer forms.

5. Strong EP flux convergence and faster easterly acceleration occur below the critical layer, which limits the vertical propagation of the waves.

6. Deceleration of the polar vortex leads to a warming of the polar stratosphere.

7. Further increases of the easterly flow inhibit vertical propagation which in turn initiates a decrease in the easterly flow.

8. Radiative cooling slowly reestablishes the normal Northern Hemisphere polar meridional temperature gradient and thermal-wind balance returns the westerly circumpolar vortex.

Table 3-2 lists the stratospheric sudden warming events compiled by

Labitzke et al. (2006). $C$ indicates months with "Canadian warmings"; $M$, months with "major midwinter warmings"; and $F$, months with "final warmings." 
Table 3-2. Stratospheric sudden warming events.

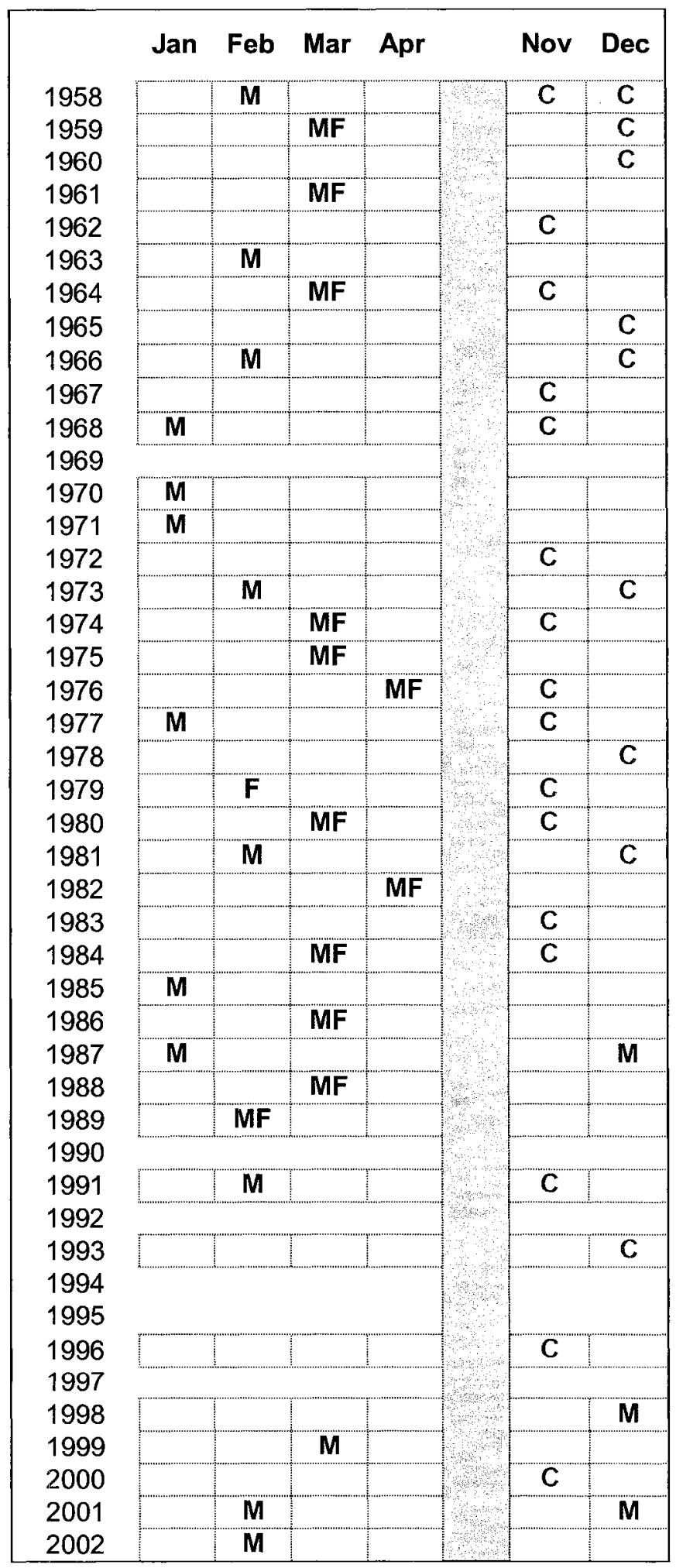


Figure 3-9 is a polar stereographic plot of daily-averaged $10 \mathrm{hPa}$ geopotential heights on 22 February 1979 , when the vortex was clearly split by a stratospheric sudden warming. This is the date identified by C\&P as the central date, i.e., the first day of a SSW that the daily-mean, zonally-averaged, zonal wind at $10 \mathrm{hPa}$ and $60^{\circ} \mathrm{N}$ becomes easterly. Labitzke et al. (2006) identify this event as a minor final warming; the C\&P classification algorithm identifies it as a major midwinter warming and definitely not a final warming. Palmer (1981) describes this event as an "intense wavenumber-2 stratospheric warming"; the maximum and minima annotated in the Fig. 3-9 are consistent with values for 0000 UTC on 22 February 1979 in Palmer's Fig. 2. Holton (2004) plots the previous day at 1200 UTC from ERA-40 as part of his Fig. 12.10. 


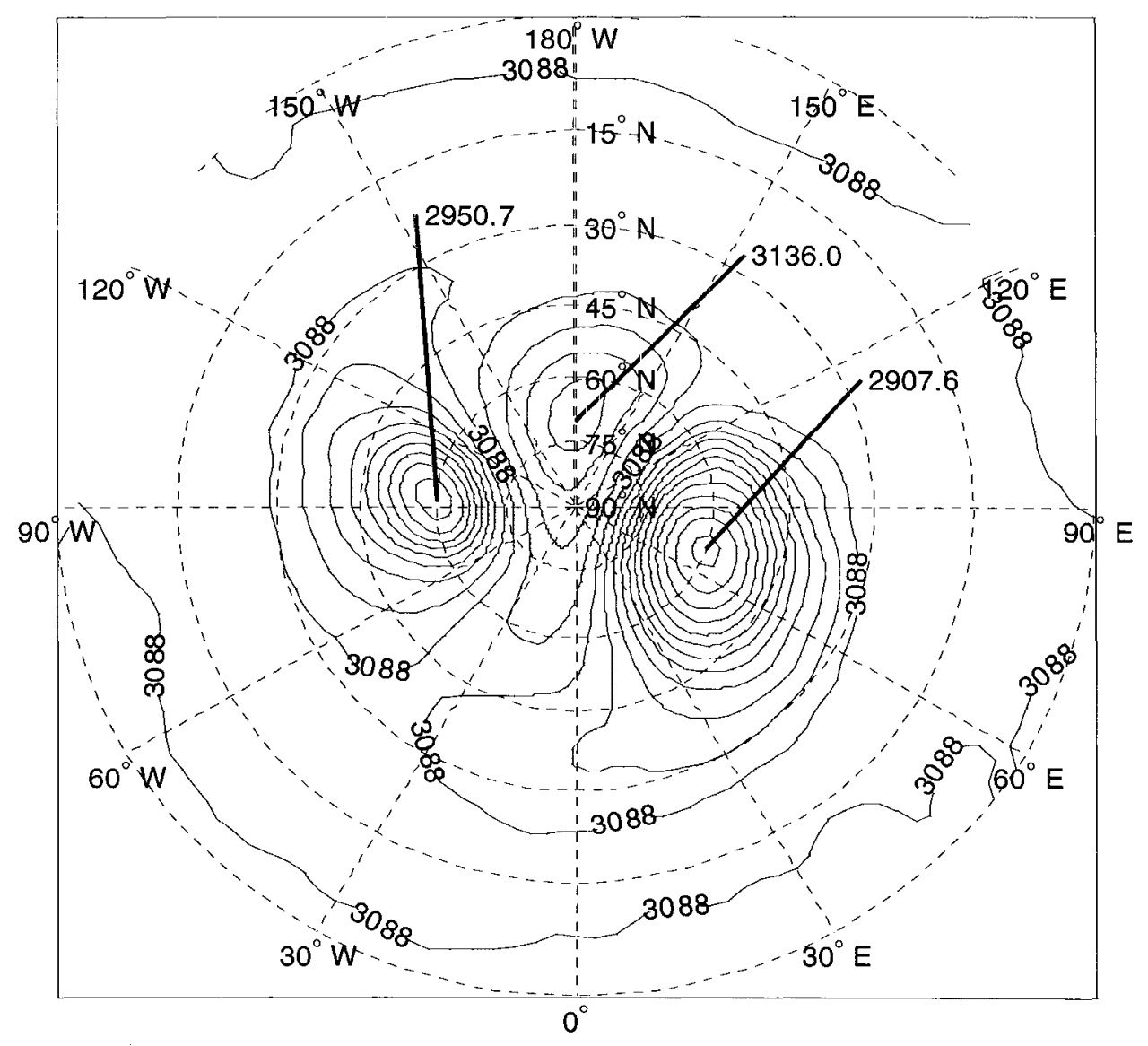

Fig. 3-9. Geopotential height reanalysis of major midwinter warming event of 22 February 1979, NCEP2: Heights in decameters, contour interval: 16 decameters [lon: $0^{\circ}-357.5^{\circ}$, lat: $0^{\circ}-90^{\circ} \mathrm{N}$, press: $10 \mathrm{hPa}$, time: daily mean 22 February 1979]. 


\subsection{TEMPERATURE COMPARISON}

This section compares NCEP1, ERA-40, METO, RATPAC, and TLS stratospheric temperature data. Comparisons of reanalyses and comparison data are performed in three domains:

- Pressure level (height) vs. latitude

- Time series at six stratospheric pressure levels of 100, 70, 50, 30, 20, and $10 \mathrm{hPa}$

- Pressure surface (latitude vs. longitude at the six stratospheric pressure levels) Temperature trends of the reanalyses are compared in polar regions in conjunction with zonal wind speed trends in these regions in Section 5.5. Finally, the presence of stratospheric sudden warming signals in monthly reanalysis data is evaluated.

\section{Introduction to comparisons}

In this section, as in Sections 5 (zonal winds) and 6 (meridional winds), conventional statistical measures, e.g., mean, standard deviation, and rms are utilized, rather than "resistant, robust, and nonparametric techniques" such as median and interquartile range/pseudo-standard deviation (Lanzante 1996). The latter approaches tend to focus on the removal of outlying data from statistical analyses. However, in this thesis, differences, both average and extreme, are of primary interest; thus the increased weighting of large differences by these conventional measures is not a liability. An exception to the use of conventional statistics is the computation of the maximum difference of data at the same coordinates in two data sets (1) over time or (2) over space and time. These differences are evaluated and the maximum value, either positive or negative, displayed with appropriate polarity. 
Pressure level (height) vs. latitude plots generally contain four subplots, each providing a unique perspective of the difference data:

- Mean subplots illustrate the average values of the differences over the indicated time span at each point in pressure level vs. latitude space.

- Standard deviation subplots show quantitatively the contours of the variance of the difference at each point.

- Rms (root-mean-square) subplots demonstrate the combined effect of the mean and variance at each point. The combination of these two quantities is not linear. The rms value of a sinusoidal waveform is $2^{-1 / 2}$ of its peak value.

- Maxima subplots are discussed in the preceding paragraph.

Mercator-like projections (latitude abscissa and longitude ordinate) are plotted with a linear latitude scale. While cartographically not a true Mercator projection, this format allows clearer viewing of the polar regions, which are of particular interest in the stratosphere.

The maximum time interval of direct NCEP1 and ERA-40 comparison is limited by the duration of ERA-40 data, which begins with September 1957 and ends with August 2002. For computational and analytic simplicity, this period is truncated to the full calendar years from January 1958 through December 2001. This constraint applies in all three comparison sections: Section 4 (temperature), Sections 5 (zonal winds), and 6 (meridional winds). 


\subsection{Height - latitude structure of monthly temperature differences}

This section evaluates temperature differences between the NCEP1 and ERA-40 reanalyses from five perspectives:

- Presents differences of conventional zonally-averaged time series

- Introduces the concept of a "longitude-time series" to retain the variance lost in zonal averaging

- Quantifies the variance lost by zonal averaging

- Demonstrates the impact of the annual cycle on longitude-time series differences

- Explores the nature of standard deviations of temperature as a function of pressure level, especially at the poles

Zonally-averaged time series (ZTS)

Figures 4-1 through 4-6 each display the mean, standard deviation, rms, and maxima of the difference of the monthly-and zonally-averaged temperatures of the two reanalyses. The temperature difference maxima are the maximum "single event" differences, either positive or negative, of each zonally-averaged time series. In this section these statistical measures are applied to zonally-averaged time series at each of 438 points (73 latitudes from $90^{\circ} \mathrm{S}$ thru $90^{\circ} \mathrm{N}$ and six pressure levels from 100 thru $10 \mathrm{hPa}$ ) as illustrated in Appendix D. The figures listed below display the four statistical measures for the six cases that cross-compare NCEP1, ERA-40, and METO temperature data:

Fig. 4-1. NCEP1 minus ERA-40 January 1958 through December 2001 (the early radiosonde era through mature satellite sensor systems)

Fig. 4-2. NCEP1 minus ERA-40 January 1958 through December 1978 (the early radiosonde era to the beginning of the modern satellite era) 
Fig. 4-3. NCEP1 minus ERA-40 January 1979 through December 2001 (the modern satellite era)

Fig. 4-4. NCEP1 minus ERA-40 January 1992 through December 1999 (to provide a comparison for Figs. 4-5 and 4-6)

Fig. 4-5. NCEP1 minus METO January 1992 through December 1999

Fig. 4-6. ERA-40 minus METO January 1992 through December 1999 Discussion of Figs. 4-1 through 4-6

Figure 4-1 displays the mean, standard deviation, rms, and maxima of the difference in the zonally-averaged time series of NCEP1 minus ERA-40 monthly temperatures over the 44-year period from January 1958 through December 2001. During this period, upper-air meteorological observational technology evolved from the early radiosonde era through mature satellite sensor systems. Northward of $75^{\circ} \mathrm{S}$, the mean of the monthly differences between the two reanalyses is no greater than $1.64 \mathrm{~K}$; that difference is the result of the NCEP1 "warm tropical tropopause (2-3 K)." South of this latitude, ERA-40's “oscillatory vertical structure in temperature, especially large over Antarctica" becomes evident (Randel et al. 2002, 2004).

Figure 4-1 also shows that the standard deviation and rms of temperature differences increase approximately twice as rapidly from $30^{\circ} \mathrm{S}$ to the South Pole as they do from the same latitude toward the North Pole. The 55 to $20 \mathrm{hPa}$ ERA-40 oscillatory bulge dominates this region as the NCEP1 warm tropical tropopause does the $100 \mathrm{hPa}$ equatorial region. The small downward protuberance in standard deviation at the Equator between 30 and $20 \mathrm{hPa}$ may be associated at least in part with ERA-40's stronger and more accurate characterization of the QBO as shown in Figs. 5-70 through 5-75. 
Randel et al. (2002, 2004) show that at $30 \mathrm{hPa}$, QBO amplitude is negligible poleward of latitude $20^{\circ}$.

The temperature difference maxima are the maximum values, either positive or negative, of each zonally-averaged time series. There is considerable congruency between positive and negative regions in the maxima and mean subplots, with steep gradients between positive and negative regions. The Southern Hemisphere maxima structures are again evidence of the ERA-40 "oscillatory vertical structure in temperature, especially large over Antarctica" (Randel et al. 2002, 2004). The congruency of mean and maxima suggest the possibility that maxima are unduly influencing mean values. Temperature difference plots (not shown) of the median of zonally-averaged time series demonstrate that this is not the case: congruency of median and maxima of the differences also exists.

Figures 4-2 and 4-3 again display the four statistical measures of the differences in the zonally-averaged time series of monthly temperatures of NCEP1 minus ERA-40 as in Fig. 4-1 but over shorter periods: January 1958 through December 1978 (21 years from the early radiosonde era to the beginning of the modern satellite era) and January 1979 through December 2001 (23 years from the beginning of the modern satellite era through mature satellite sensor systems). As expected, mean, standard deviation, and rms differences in the Southern Hemisphere polar regions are generally larger in Fig. 4-2 (before 1979) and smaller in Fig. 4-3 (after 1979) than in Fig. 4-1 (1958-2001), demonstrating that the addition of satellite data to the assimilation process reduced the variance of the difference between the individual forecast models. These results are 
similar to those of Fig. 2-3. Mean differences southward of $60^{\circ} \mathrm{S}$ in Fig. 4-2 are the only instance of differences greater than $1.7 \mathrm{~K}$, again illustrating ERA-40's oscillatory structure and demonstrating that it is more pronounced after 1979 than before. This condition is discussed more thoroughly in "Standard deviations of seasonal temperatures" later in this section. However, in Fig. 4-3 (1979-2001) the mean difference at the tropical tropopause is more pronounced than in Figs. 4-1 (1958-2001) and 4-2 (1958-78) because of the " $4 \mathrm{~K}$ discontinuity" that occurred in 1979 at the beginning of assimilation of satellite temperature data and continued until 2000. Maxima for 1958-78 are essentially the same as for 1958-2001; thus these maxima occurred before 1979. The 1979-2001 maxima are generally 50 to $75 \%$ smaller than those for $1958-78$.

Figure 4-4 displays the mean, standard deviation, rms, and maxima of the difference in the zonally-averaged time series of monthly temperatures of NCEP1 minus ERA-40, as in Figs. 4-1 through 4-3, but over the shorter eight-year period of 1992 through 1999. This permits METO data, available only for this shorter period, to be accurately compared with the zonally-averaged time series of NCEP1 (Fig. 4-5) and ERA-40 (Fig. 4-6). The mean tropical tropopause temperature difference is more pronounced in Fig. 4-4 (1992-99) than in Fig. 4-1 (1958-2001). Randel et al. (2002, 2004) show that ERA-40 closely matches radiosonde data in this region. The ERA-40 southern polar temperature "oscillation" is still present, and both the variance and rms of the differences increase with increasing height in the polar regions. In general the rms differences are less in Fig. 4-4 (1992-99) than in Fig. 4-3 (1979-2001). 
The excellent agreement of NCEP1 and METO climatologies is demonstrated in Fig. 4-5. The largest maxima over the range of latitudes and pressure levels during the 96-month period is $5.5 \mathrm{~K}$. Unfortunately, this agreement is in part the result of similar errors; both climatologies are too warm with a year-round bias of approximately $2-3 \mathrm{~K}$ at $100 \mathrm{hPa}$ in the Tropics and the QBO amplitudes of both are too small (Randel et al. 2002, 2004).

ERA-40 minus METO (Fig. 4-6) is similar to NCEP1 minus ERA-40 (Fig. 4-4) for the same time period, except for the oscillatory vertical temperature structure above the South Pole; increasing variance, rms, and maxima of the differences with increasing height in the polar regions; and significant mean and rms differences in the tropical tropopause. These characteristics are as large in magnitude as in the case of NCEP1 minus ERA-40 for the 1992-99 period.

The four statistical measures of the difference between NCEP1 and ERA-40 temperature data decrease in magnitude after 1978. Generally, as may be observed in Figs. 4-1 through 4-4, NCEP1 is cooler than ERA-40 in the Tropics above $70-50 \mathrm{hPa}$ and below $20 \mathrm{hPa}$, and warmer elsewhere. In these seven cases the mean difference is less than three $\mathrm{K}$ except southward of $60^{\circ} \mathrm{S}$ from 1958-78. Almost every case illustrates three of the "largest apparent biases" identified by Randel et al. $(2002,2004)$ : "warm tropical tropopause" and the "satellite data discontinuity across 1978-79" (NCEP1) and "oscillatory vertical structure in temperature, especially large over Antarctica" (ERA-40 for the period 1992-99). NCEP1 minus METO agreement is closer in all measures than 
ERA-40 minus METO but both reanalyses exhibit a too warm tropical tropopause (Randel et al. 2002, 2004).

There is considerable congruency between positive and negative regions in maxima and mean plots in Figs. 4-1 through 4-6, with steep gradients between positive and negative regions. Maxima occur in the Southern Hemisphere with the exception of NCEP1 minus ERA-40 (1992-99). Maxima in both hemispheres occur not at $10 \mathrm{hPa}$ at the poles as do the standard deviation and rms values in more than $80 \%$ of the cases.

Temperature maxima for the first nine cases of this section are summarized in Table 4-1. In every longitude-time series vs. zonal averaging comparison, the longitudetime series maxima and minima are larger in absolute magnitude than those from zonal averaging. Differences in a majority of the comparisons are quite small. NCEP1 minus ERA-40 1958--78 maxima and minima are equal to those of NCEP1 minus ERA-40 1958-2001, demonstrating that the maximum and minimum values occurred before the beginning of the modern satellite era. In only 4 cases of the 20 listed do maxima or minima occur at a pole; the remainder, with the exception of one at the Equator, range from $82.5^{\circ}$ to $67.5^{\circ}$ latitude. 

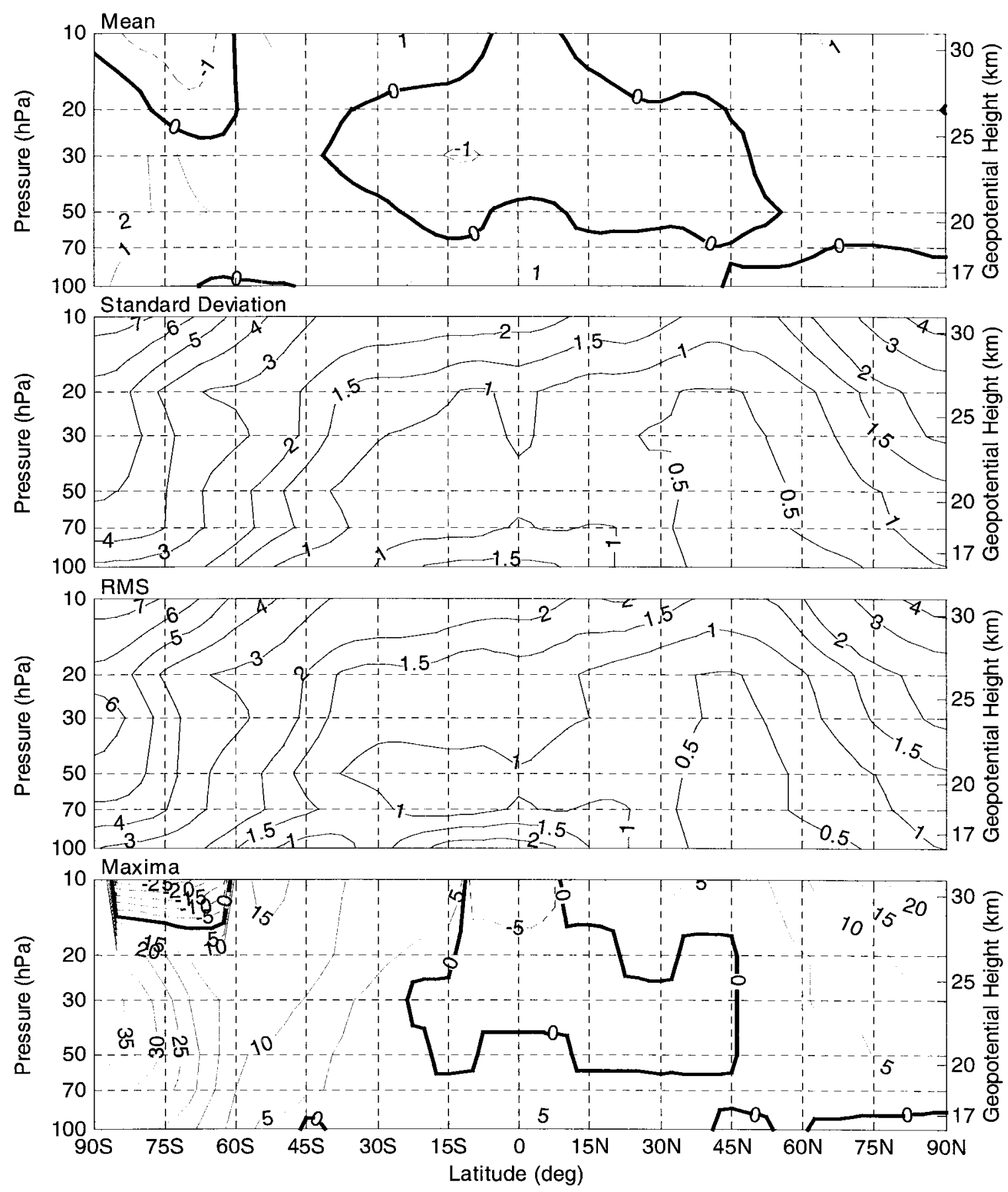

Fig. 4-1. Mean, standard deviation, rms, and maxima of time series of monthly-and zonally-averaged temperature differences $(K)$ :

NCEPI minus ERA-40 [lon: mean of $0^{\circ}-357.5^{\circ}$, lat: $90^{\circ} \mathrm{S}-90^{\circ} \mathrm{N}$, press: $100-10 \mathrm{hPa}$, time: Jan 1958-Dec 2001]. 

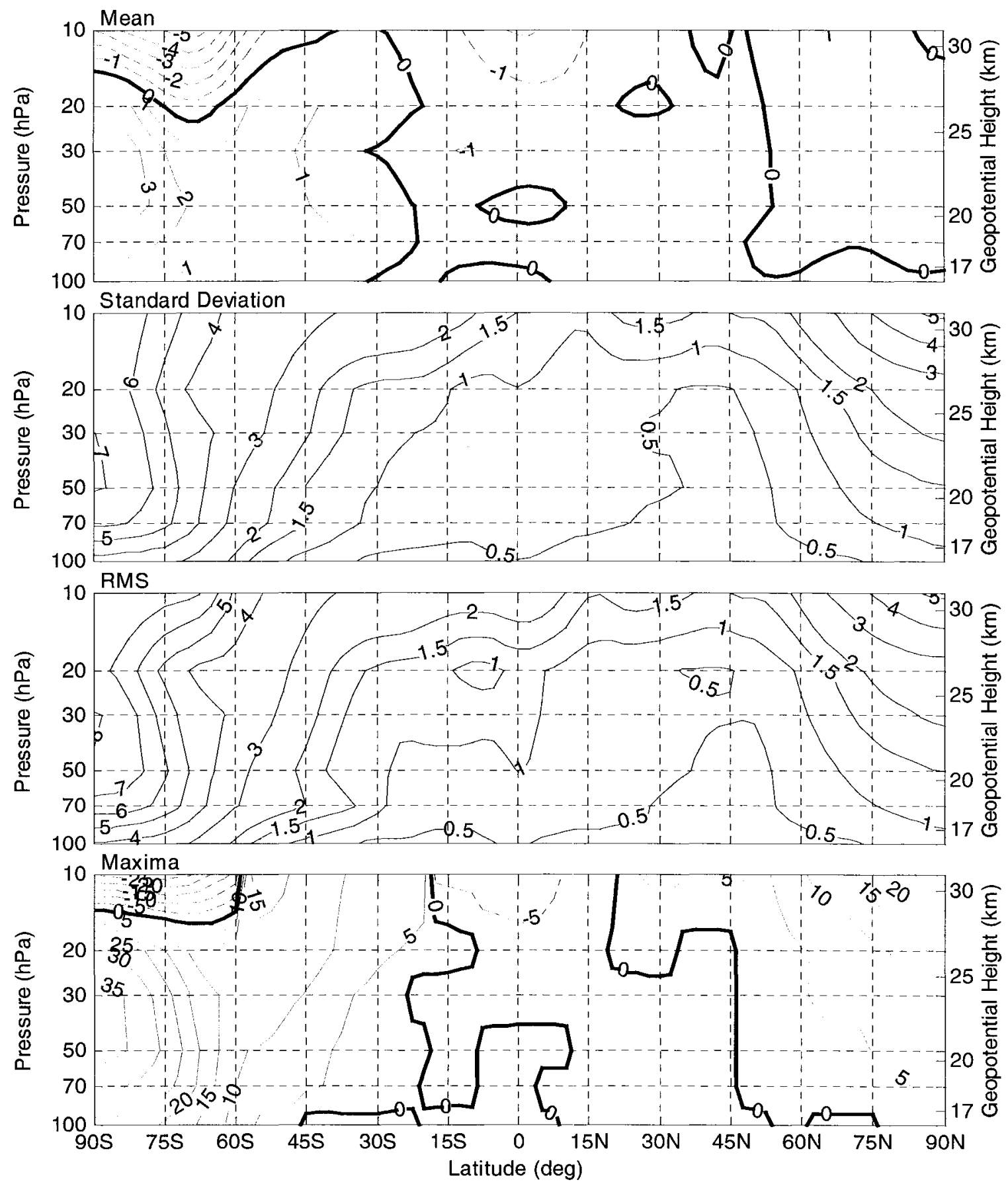

Fig. 4-2. Same as Fig. 4-1 except time: Jan 1958-Dec 1978. 

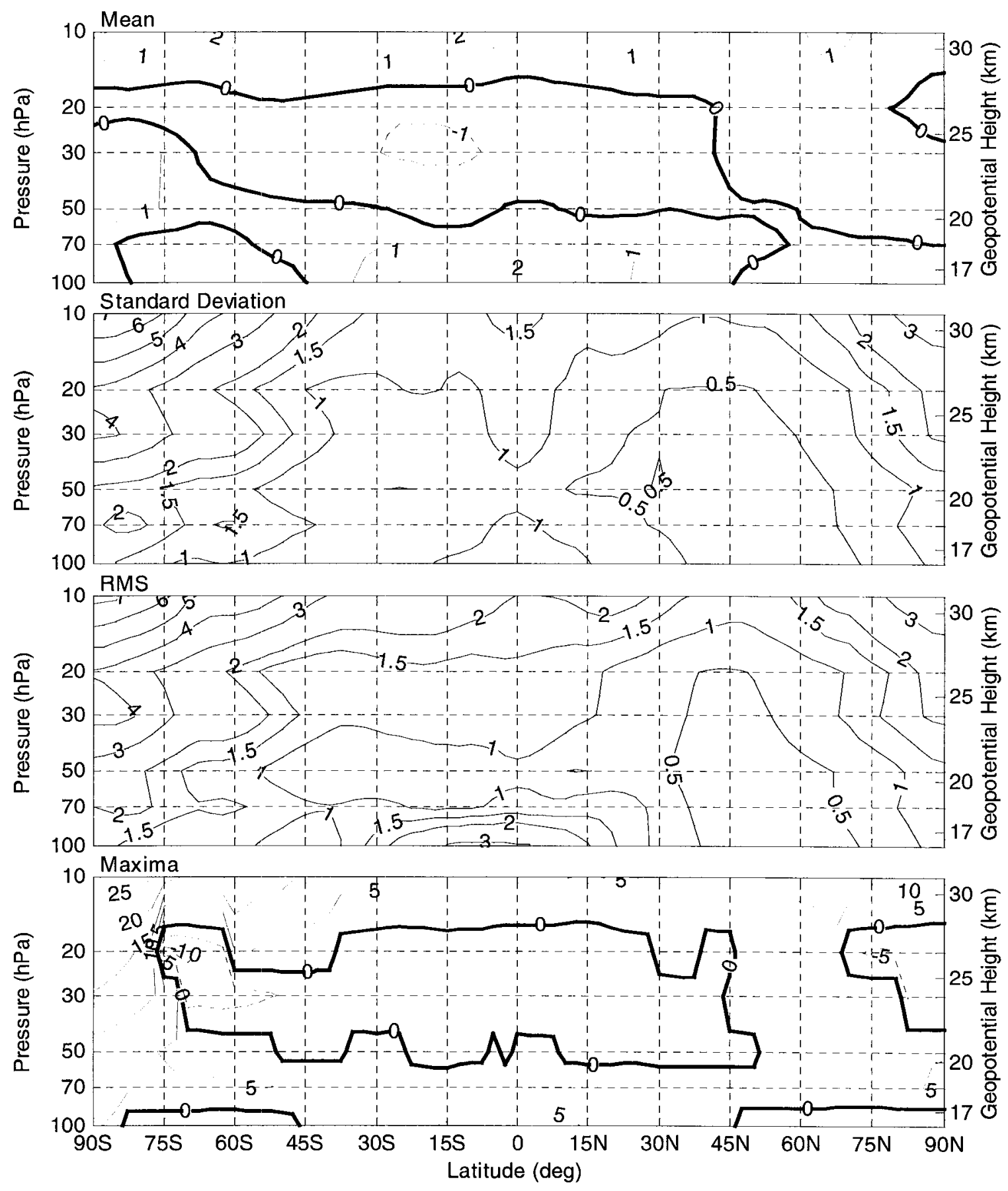

Fig. 4-3. Same as Fig. 4-1 except time: Jan 1979-Dec 2001. 

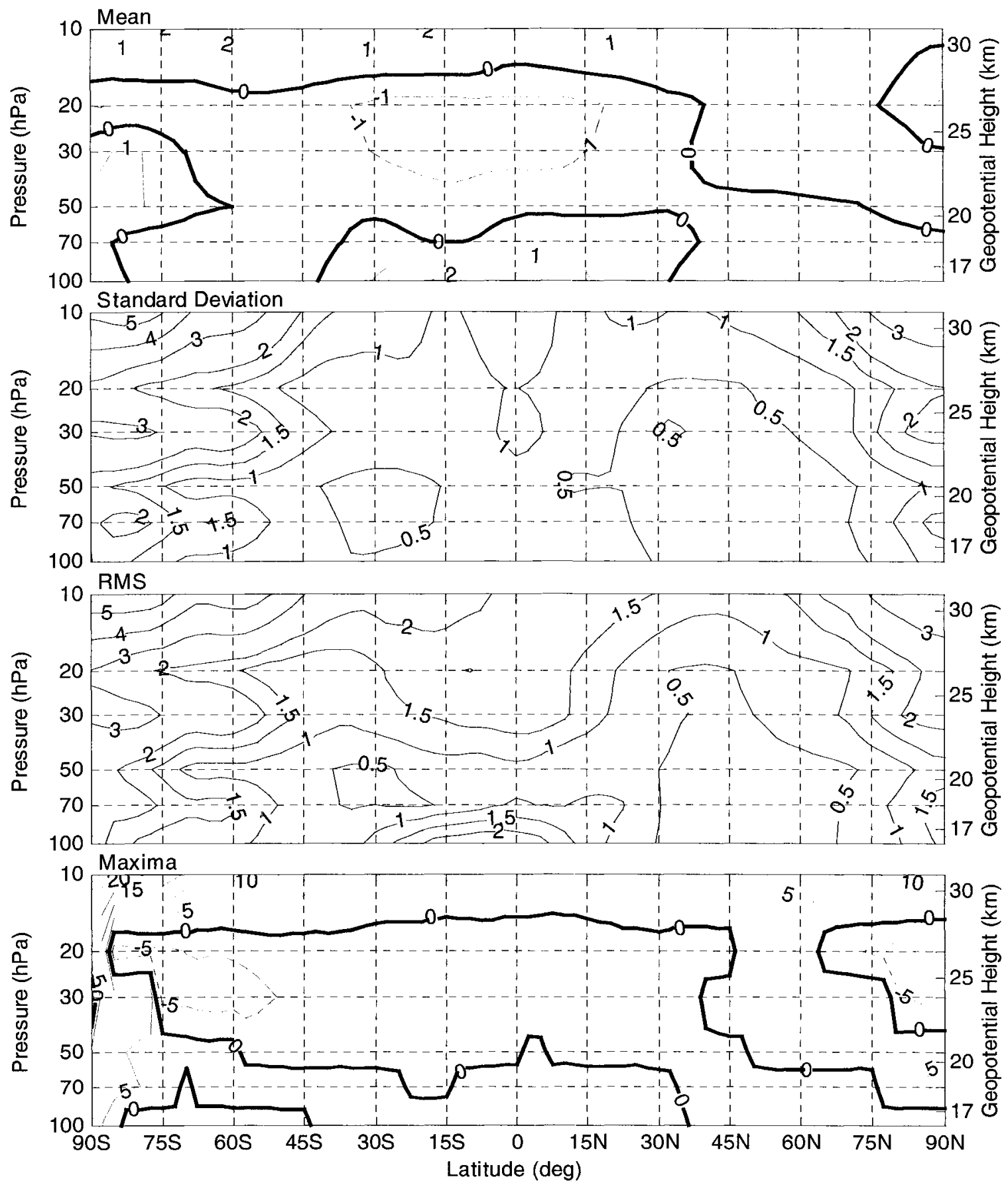

Fig. 4-4. Same as Fig. 4-1 except time: Jan 1992-Dec 1999. 

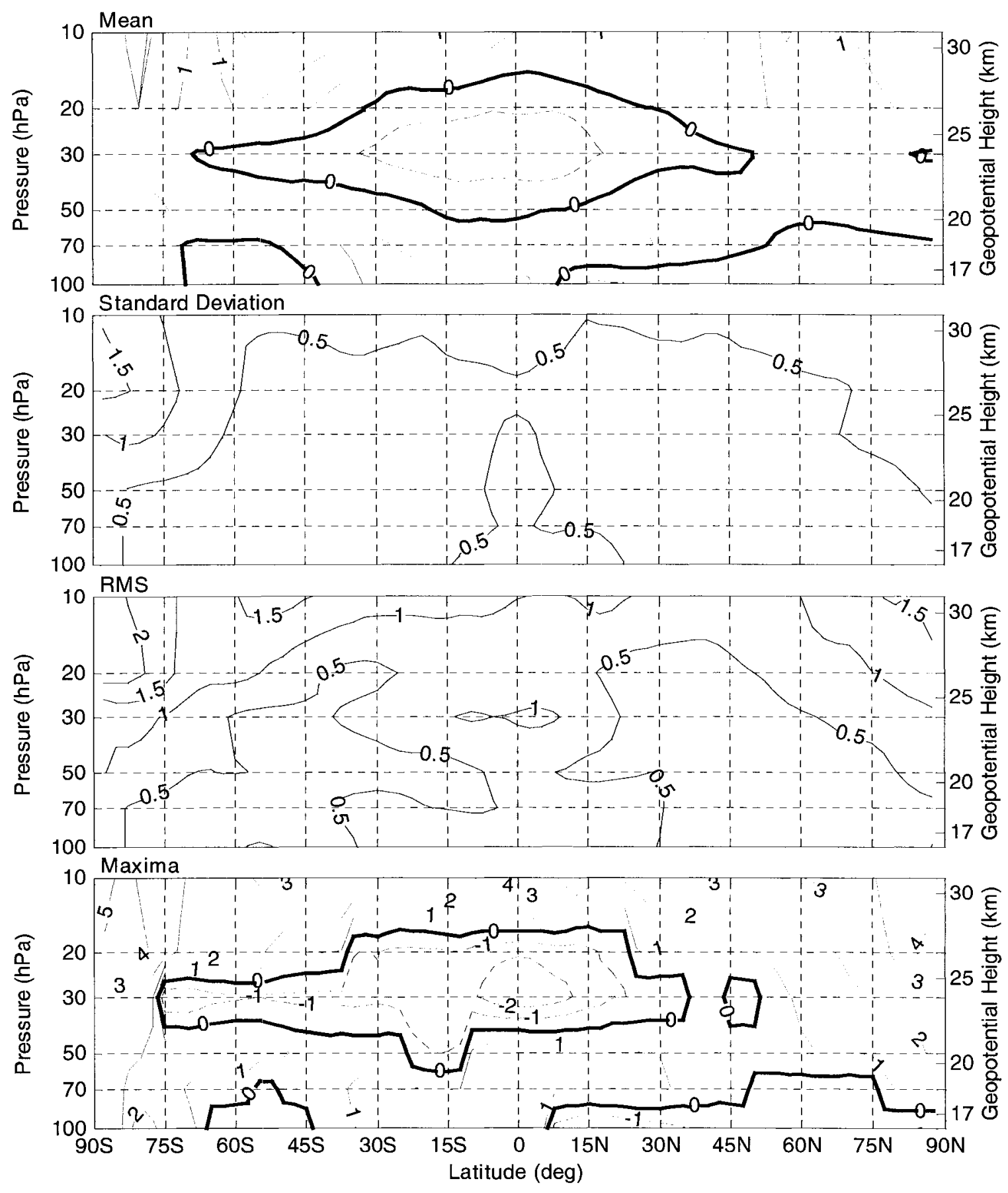

Fig. 4-5. Same as Fig. 4-1 except NCEPI minus METO, lat: $87.5^{\circ} \mathrm{S}-87.5^{\circ} \mathrm{N}$, and time: Jan 1992-Dec 1999. 

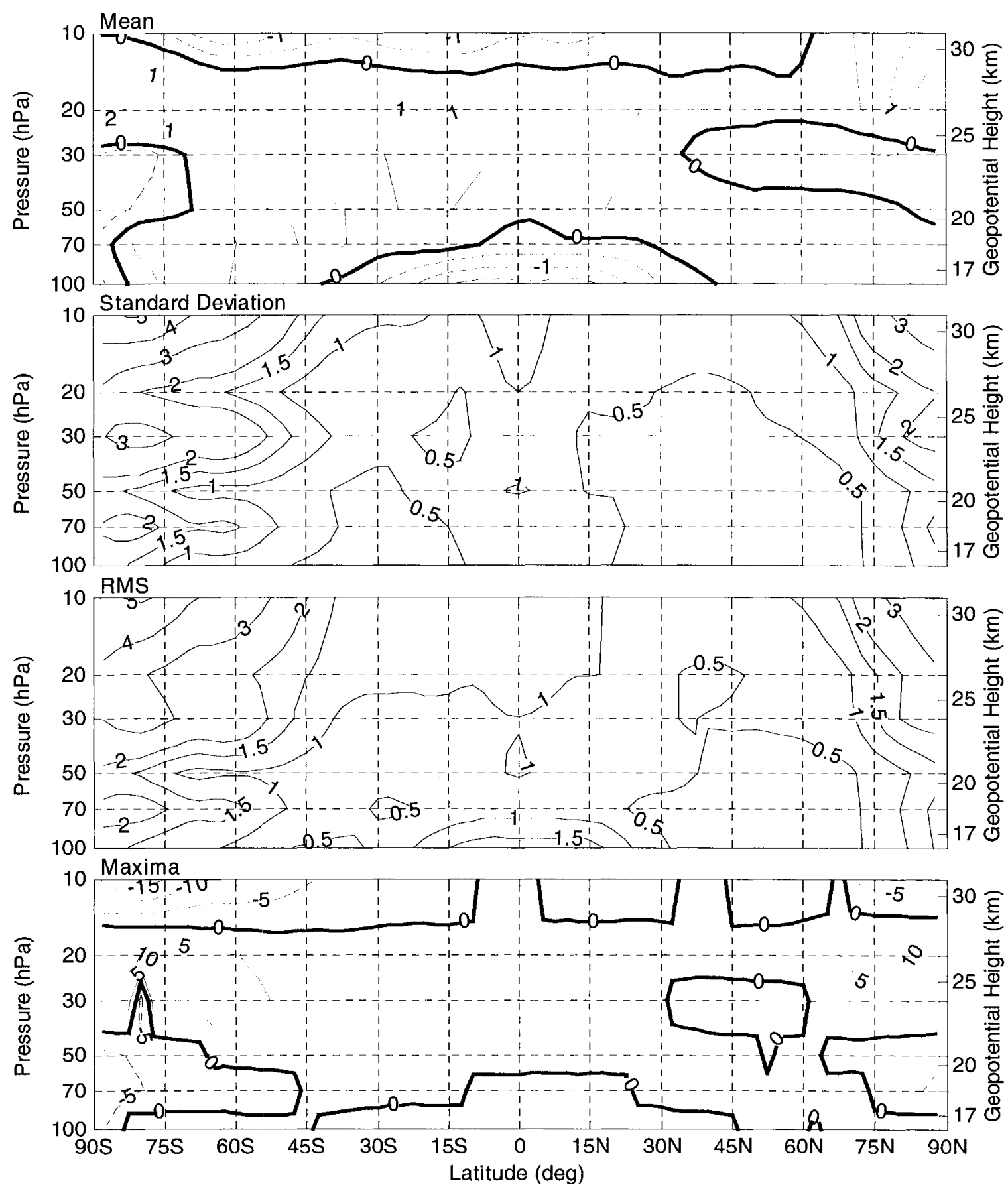

Fig. 4-6. Same as Fig. 4-5 except ERA-40 minus METO. 
Longitude-time series (LTS)

Figures 4-7 through 4-9 display the mean, standard deviation, root-mean-square (rms), and maxima of the difference in the monthly temperatures of the two reanalysis data sets. The last three measures, standard deviation, rms, and maxima, are evaluated over all longitudes and times at each of 438 points ( 73 latitudes from $90^{\circ} \mathrm{S}$ through $90^{\circ} \mathrm{N}$ and six pressure levels from 100 through $10 \mathrm{hPa}$ ). Computationally, temperature data for each time series and each longitude associated with a pressure-level vs. latitude coordinate is assembled into a "longitude x time" array. This array is then "reshaped" into a linear vector and treated, without loss of generality in this application since time order is not relevant, as in a simple zonally-averaged time series, referred to hereinafter as a "longitude-time series." This concept is further discussed and illustrated in Appendix D.

The figures listed below display the four statistical measures for three representative longitude-time series cases:

Fig. 4-7. NCEP1 minus ERA-40 January 1958 through December 2001 (the early radiosonde era through mature satellite sensor systems)

Fig. 4-8. NCEP1 minus ERA-40 January 1958 through December 1978 (the early radiosonde era to the modern satellite era)

Fig. 4-9. NCEP1 minus ERA-40 January 1979 through December 2001 (the modern satellite era) 


\section{Longitude-time series minus zonally-averaged time series}

Figures 4-10, 4-11, and 4-12 display the standard deviation, rms, and maxima of the differences between the respective statistics generated from longitude-time series (LTS) minus those generated from the zonally-averaged time series (ZTS) illustrated in Figs. 4-1 through 4-3. These figures graphically quantify the loss of variance that results when the computationally simpler zonal averaging technique is employed.

Fig. 4-10. NCEP1 minus ERA-40 January 1958 through December 2001 (Fig. 4-7 (LTS) minus Fig. 4-1 (ZTS))

Fig. 4-11. NCEP1 minus ERA-40 January 1958 through December 1978 (Fig. 4-8 (LTS) minus Fig. 4-2 (ZTS))

Fig. 4-12. NCEP1 minus ERA-40 January 1979 through December 2001 (Fig. 4-9 (LTS) minus Fig. 4-3 (ZTS))

\section{Discussion of Figs. 4-7 through 4-12}

The statistics of the difference between longitude-time series and zonallyaveraged time series provide indications of the efficacy of zonal averaging and of the relative magnitude of longitudinal variations. The mean difference between results of these two techniques is zero at all points in pressure level vs. latitude space, which serves as at least partial confirmation of the computational process. Figures 4-10 through 4-12 demonstrate that significant variance may be ignored if statistics other than the mean are applied to zonally-averaged data. The reason for the increase in standard deviation and rms occurring at approximately $\pm 60^{\circ}$ latitude and $10 \mathrm{hPa}$ in each hemisphere is explained in Section 4.3. Maxima subplots demonstrate that in large regions, especially at the 
borders of the meridional domains, there are maximum differences that are hidden by zonal averaging. 

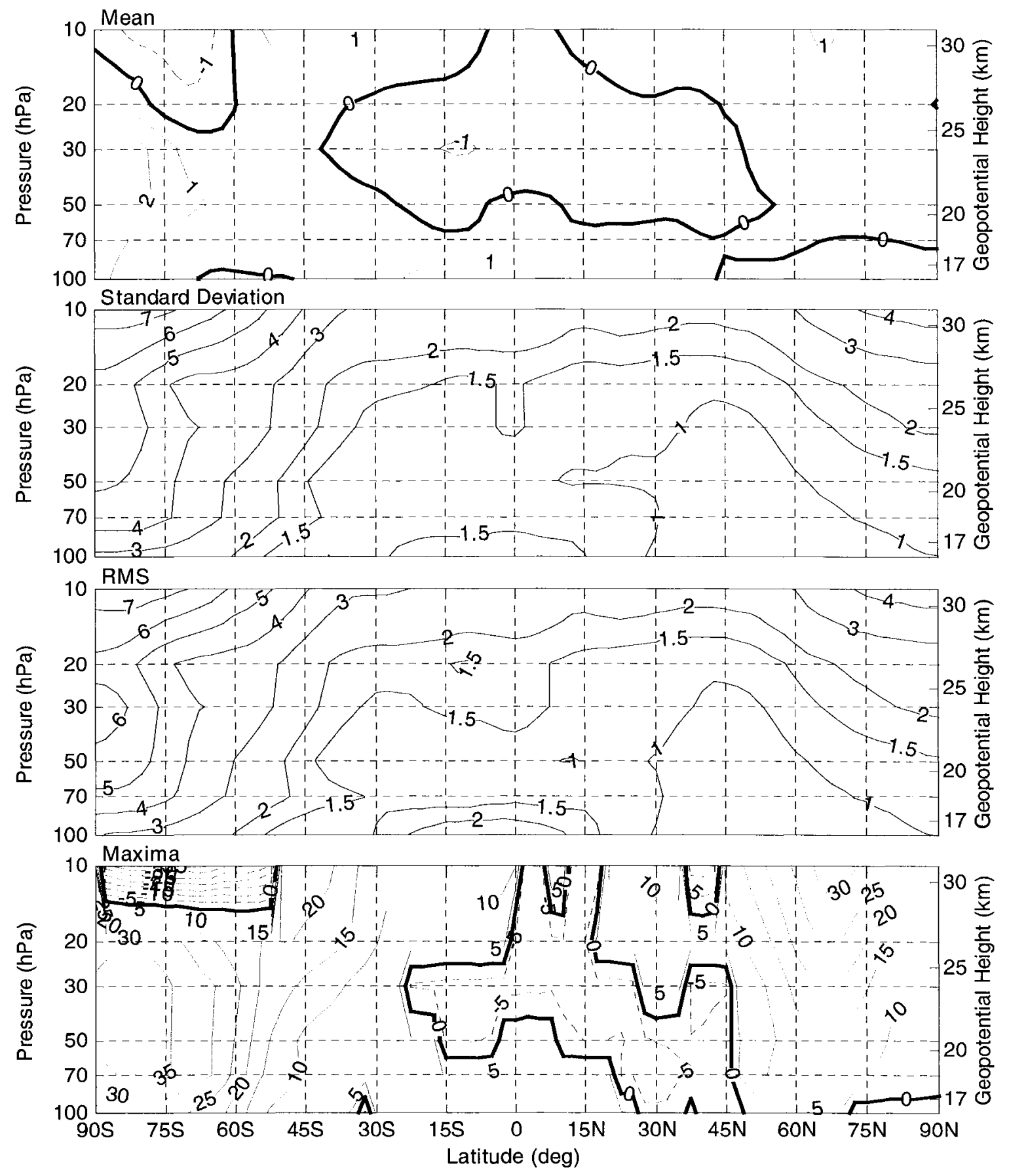

Fig. 4-7. Mean, standard deviation, rms, and maxima of longitude-time series of monthly-averaged temperature differences $(K)$ :

NCEPI minus ERA-40 [lon: mean of $0^{\circ}-357.5^{\circ}$, lat: $90^{\circ} \mathrm{S}-90^{\circ} \mathrm{N}$, press: 100-10 hPa, time: Jan 1958-Dec 2001]. 

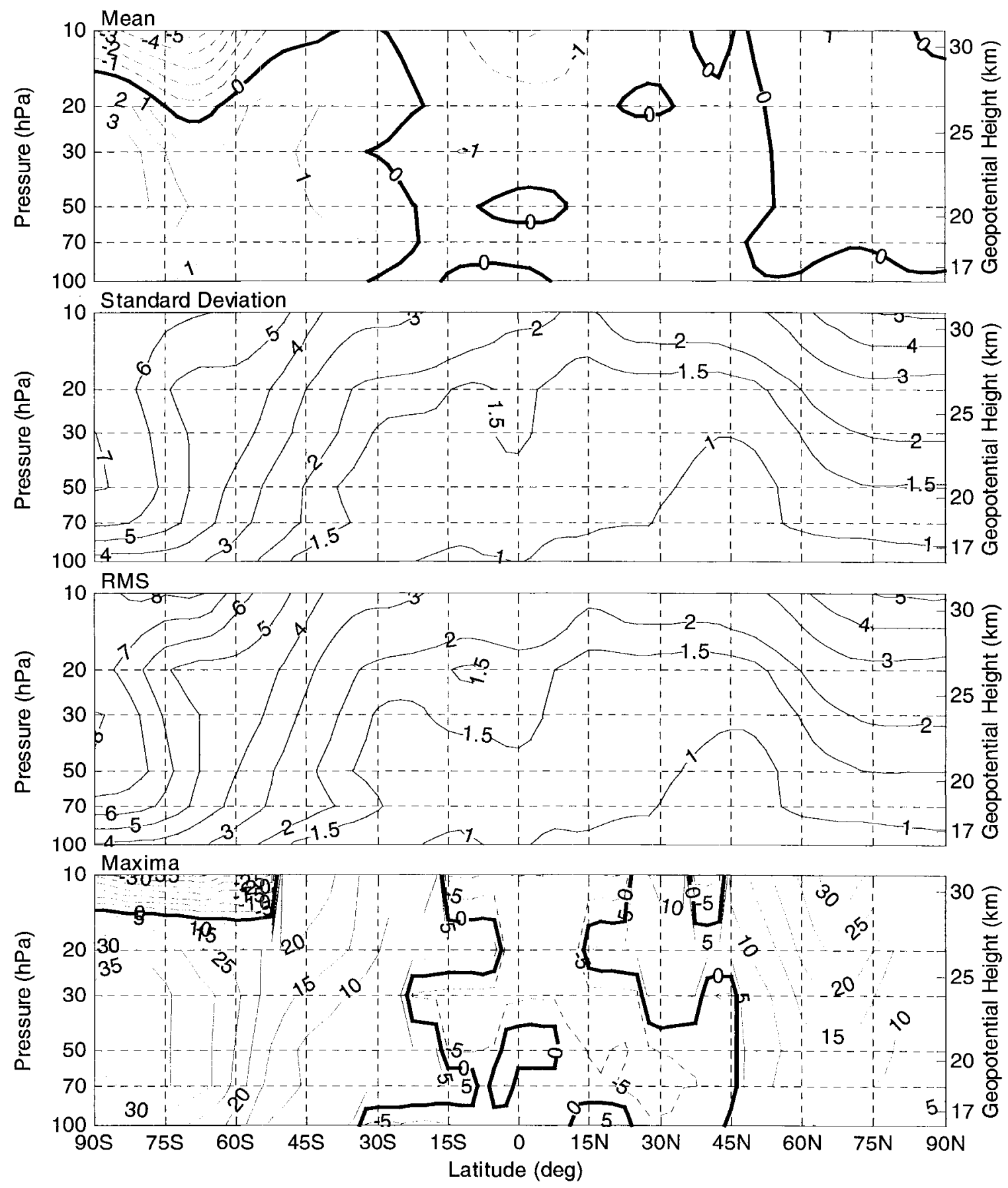

Fig. 4-8. Same as Fig. 4-7 except time: Jan 1958-Dec 1978. 

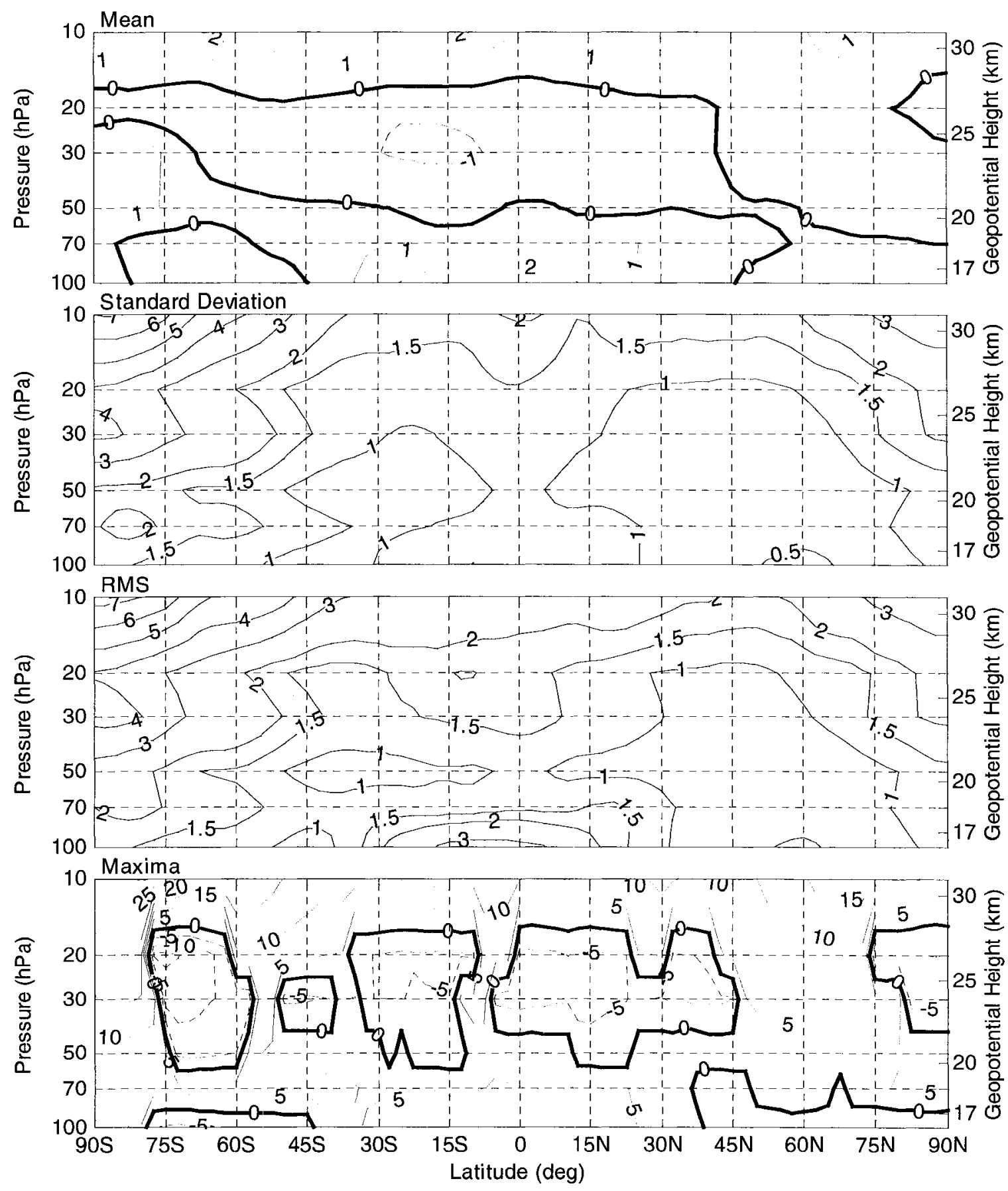

Fig. 4-9. Same as Fig. 4-7 except time: Jan 1979-Dec 2001. 

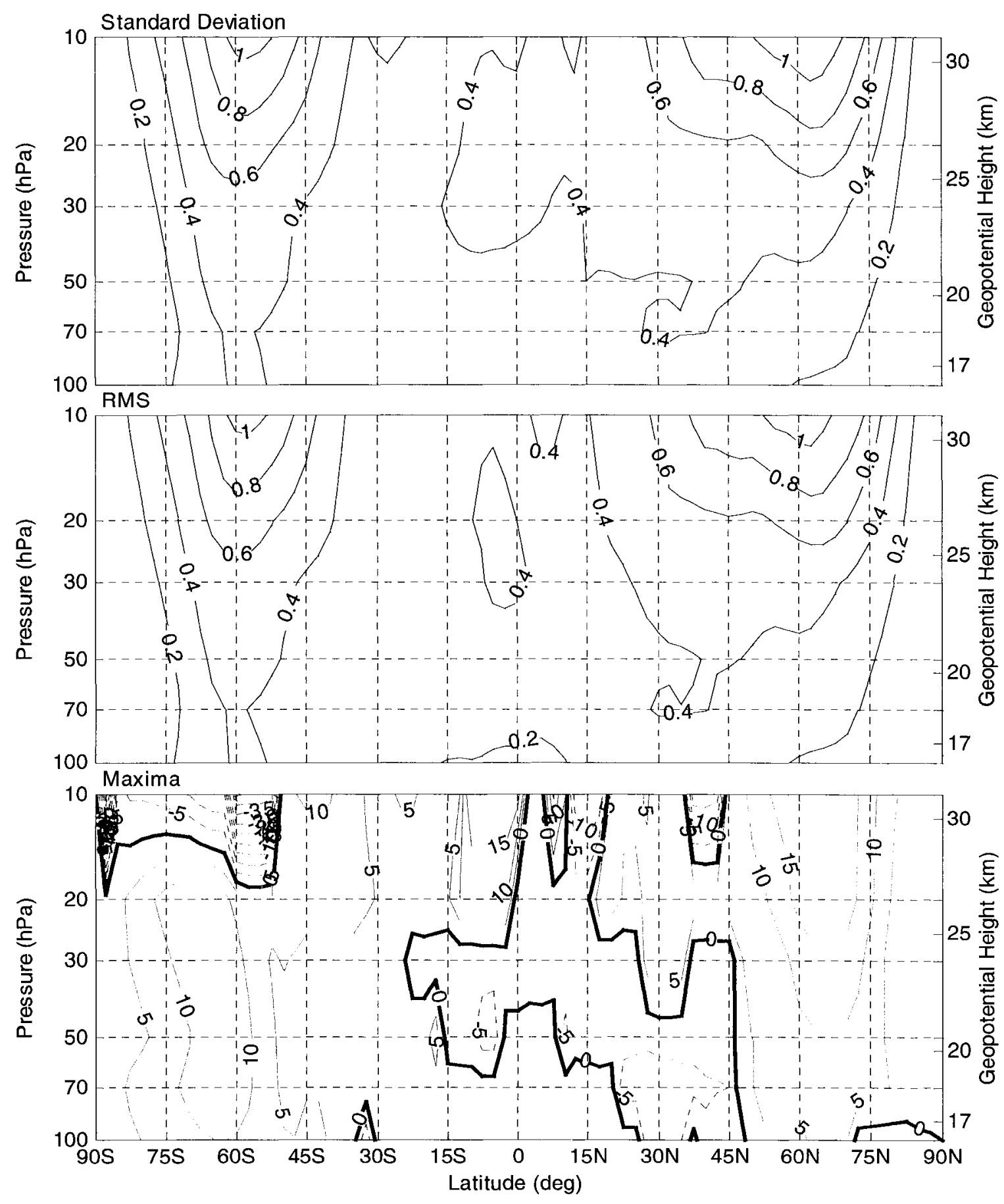

Fig. 4-10. Standard deviation, rms, and maxima of longitude-time series (Fig. 4-7) minus time series of monthly-and zonally-averaged (Fig. 4-1) temperature differences (K): NCEP1 minus ERA-40 [lon: mean of $0^{\circ}-357.5^{\circ}$, lat: $90^{\circ} \mathrm{S}-90^{\circ} \mathrm{N}$, press: $100-10 \mathrm{hPa}$, time: Jan 1958-Dec 2001]. 

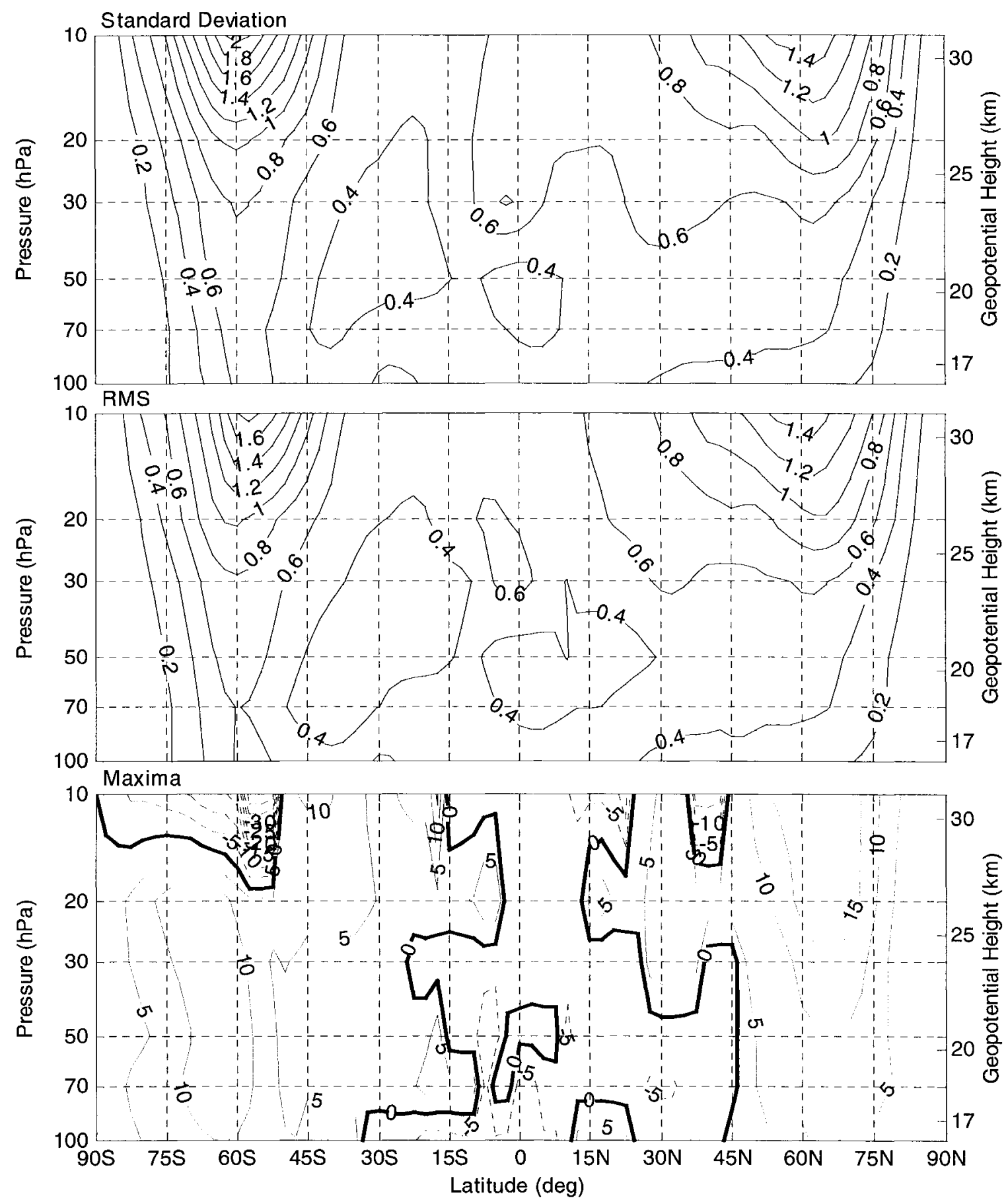

Fig. 4-11. Same as Fig. 4-10 except longitude-time series is Fig. 4-8 and zonally-averaged time series is Fig. 4-2, and time: Jan 1958-Dec 1978. 

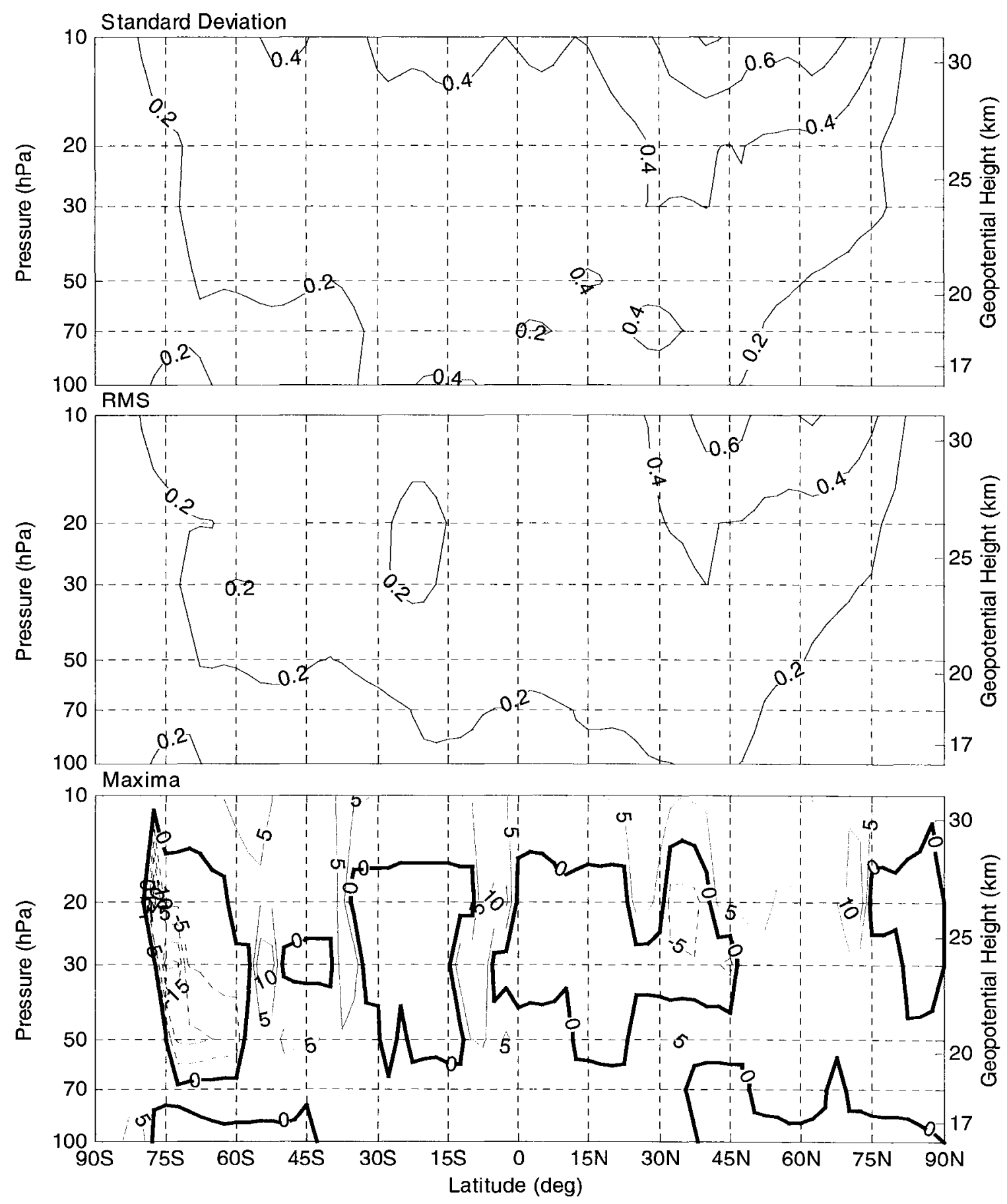

Fig. 4-12. Same as Fig. 4-10 except longitude-time series is Fig. 4-9 and zonally-averaged time series is Fig. 4-3, and time: Jan 1979-Dec 2001. 
Table 4-1. Maximum temperature differences $(\mathrm{K})$ and their locations for cases presented in Figs. 4-1 through 4-6 and Figs. 4-7 through 4-9.

\begin{tabular}{|c|c|c|c|c|c|c|c|c|c|}
\hline \multirow{3}{*}{$\begin{array}{c}\text { NCEP1 minus } \\
\text { ERA-40 } \\
(1958-2001)\end{array}$} & \multirow{3}{*}{$\begin{array}{l}\text { LTS } \\
\text { ZTS }\end{array}$} & \multicolumn{3}{|c|}{ Max Positive } & \multicolumn{3}{|c|}{ Max Negative } & \multirow{2}{*}{$\begin{array}{l}90^{\circ} \mathrm{S} \\
10 \mathrm{hPa} \\
27.5\end{array}$} & \multirow{2}{*}{$\begin{array}{l}90^{\circ} \mathrm{N} \\
10 \mathrm{hPa} \\
27.0\end{array}$} \\
\hline & & 39.4 & $82.5^{\circ} \mathrm{S}$ & $30 \mathrm{hPa}$ & -36.7 & $67.5^{\circ} \mathrm{S}$ & $10 \mathrm{hPa}$ & & \\
\hline & & 37.1 & $90^{\circ} \mathrm{S}$ & $30 \mathrm{hPa}$ & -29.2 & $80^{\circ} \mathrm{S}$ & $10 \mathrm{hPa}$ & 27.5 & 27.0 \\
\hline $\begin{array}{l}\text { NCEP1 minus } \\
\text { ERA }-40\end{array}$ & LTS & 39.4 & $82.5^{\circ} \mathrm{S}$ & $30 \mathrm{hPa}$ & -36.7 & $67.5^{\circ} \mathrm{S}$ & $10 \mathrm{hPa}$ & -27.1 & 27.0 \\
\hline$(1958-1978)$ & ZTS & 37.1 & $90^{\circ} \mathrm{S}$ & $30 \mathrm{hPa}$ & -29.2 & $80^{\circ} \mathrm{S}$ & $10 \mathrm{hPa}$ & -27.1 & 27.0 \\
\hline $\begin{array}{l}\text { NCEP1 minus } \\
\text { ERA-40 }\end{array}$ & LTS & 30.4 & $80^{\circ} \mathrm{S}$ & $10 \mathrm{hPa}$ & -16.2 & $77.5^{\circ} \mathrm{S}$ & $20 \mathrm{hPa}$ & 27.5 & 13.9 \\
\hline$(1979-2001)$ & ZTS & 27.5 & $87.5^{\circ} \mathrm{S}$ & $10 \mathrm{hPa}$ & -12.1 & $75^{\circ} \mathrm{S}$ & $20 \mathrm{hPa}$ & 27.5 & 13.9 \\
\hline $\begin{array}{l}\text { NCEP1 minus } \\
\text { FRA } 40\end{array}$ & LTS & 23.9 & $80^{\circ} \mathrm{S}$ & $10 \mathrm{hPa}$ & -10.1 & $87.5^{\circ} \mathrm{N}$ & $20 \mathrm{hPa}$ & 22.7 & 12.8 \\
\hline (1992-1999) & ZTS & 22.7 & $90^{\circ} \mathrm{S}$ & $10 \mathrm{hPa}$ & -9.4 & $90^{\circ} \mathrm{N}$ & $20 \mathrm{hPa}$ & 22.7 & 12.8 \\
\hline $\begin{array}{l}\text { NCEP1 minus } \\
\text { METO } \\
(1992-1999)\end{array}$ & ZTS & 5.5 & $87.5^{\circ} \mathrm{S}$ & $10 \mathrm{hPa}$ & -2.9 & $\mathrm{EQ}$ & $30 \mathrm{hPa}$ & 5.5 & 3.6 \\
\hline $\begin{array}{c}\text { ERA-40 } \\
\text { minus METO } \\
(1992-1999)\end{array}$ & ZTS & 12.5 & $87.5^{\circ} \mathrm{S}$ & $20 \mathrm{hPa}$ & -19.3 & $87.5^{\circ} \mathrm{S}$ & $10 \mathrm{hPa}$ & -19.3 & 10.6 \\
\hline
\end{tabular}

LTS: Longitude-time series

ZTS: Zonally-averaged time series 


\section{Effect of annual cycle on time series differences}

Figures 4-1 through 4-12 present differences based on complete monthly- and zonally-averaged reanalysis temperature data and thus include differences between NCEP1 and ERA-40 characterizations of the annual cycle and its harmonics. Figures 4-13 through 4-15 illustrate the effect of the annual cycle and its harmonics on temperature differences by removing them with frequency-domain filtering, which eliminates all variance with period less than 13 months. This filtering technique is discussed in Appendix E. Hereinafter in this thesis, all references to "annual cycle" include its harmonics. The figures listed below display the four statistical measures for three cases with all variance less than 13 months removed; these three figures correspond to the unfiltered longitude-time series of Figs. 4-7 through 4-9.

Fig. 4-13. NCEP1 minus ERA-40 January 1958 through December 2001

Fig. 4-14. NCEP1 minus ERA-40 January 1958 through December 1978

Fig. 4-15. NCEP1 minus ERA-40 January 1979 through December 2001

The figures listed below illustrate the effect of frequency-domain filtering by displaying longitude-time series, filtered (plotted in Figs. 4-13 through 4-15) minus unfiltered (plotted in Fig. 4-7 through 4-9).

Fig. 4-16. NCEP1 minus ERA-40 January 1958 through December 2001

Fig. 4-17. NCEP1 minus ERA-40 January 1958 through December 1978

Fig. 4-18. NCEP1 minus ERA-40 January 1979 through December 2001 


\section{Discussion of Figs. 4-13 through 4-18}

Figures 4-13 through 4-15 plot the mean, standard deviation, rms, and maxima of longitude-time series of monthly averaged temperature differences with the annual cycle removed before differencing. Frequency-domain filtering is employed to remove all variance with period less than 13 months from each reanalysis longitude-time series, as discussed in Appendix E, and the filtered data differenced. The two three-figure sets referenced in this paragraph cover the periods 1958-2001, 1958-1978, and 1979-2001. Comparison of Figs. 4-13 through 4-15 with Fig. 4-1 through 4-3 (which include annual cycle), respectively, reveals strong congruency in the corresponding contours, but maxima less so. As expected, magnitudes of the four statistics of the reanalysis temperature differences are smaller when the annual cycle is removed. Figures 4-16 through 4-18 display the effect of this removal. Maximum and mean rms differences decrease by $2.0 \mathrm{~K}$ and $0.5 \mathrm{~K}, 2.1 \mathrm{~K}$ and $0.6 \mathrm{~K}$, and $1.4 \mathrm{~K}$ and $0.3 \mathrm{~K}$, respectively, for the three time periods. Area weighting of the mean values minimizes the contribution of maximum values that generally exist at higher latitudes. Removal of the annual cycle and its harmonics with notch filtering in the frequency-domain (Appendix E) was also evaluated but results not plotted. Because of the additional variance retained with this technique, the reduction in difference statistics is less than with low-pass filtering: $1.1 \mathrm{~K}$ and $0.2 \mathrm{~K}, 1.2 \mathrm{~K}$ and $0.3 \mathrm{~K}$, and $1.3 \mathrm{~K}$ and $0.2 \mathrm{~K}$, respectively, for the three time periods. Table 4-2 quantitatively summarizes the results of the three methods of processing NCEP1 and ERA-40 temperature differences. The pressure level vs. latitude data is area weighted as a function of latitude to form mean values of each statistic, 
minimizing the contribution of higher latitudes, where maximum values are generally found. 

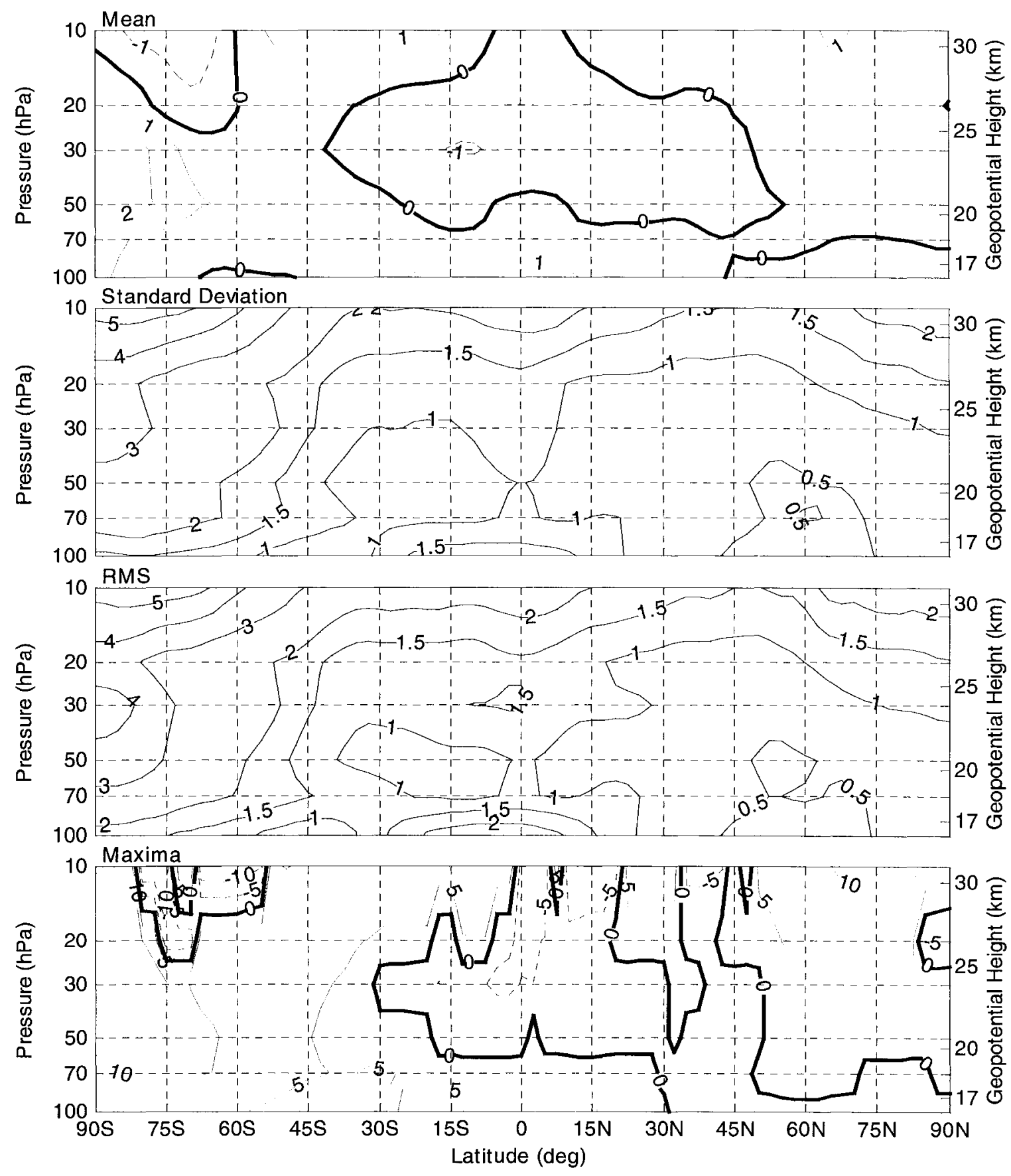

Fig. 4-13. Mean, standard deviation, rms, and maxima of longitude-time series of monthly-averaged temperature differences with all variance with period less than 13 months removed before differencing (K): NCEP1 minus ERA-40

[lon: mean of $0^{\circ}-357.5^{\circ}$, lat: $90^{\circ} \mathrm{S}-90^{\circ} \mathrm{N}$, press: $100-10 \mathrm{hPa}$,

time: Jan 1958-Dec 2001]. 

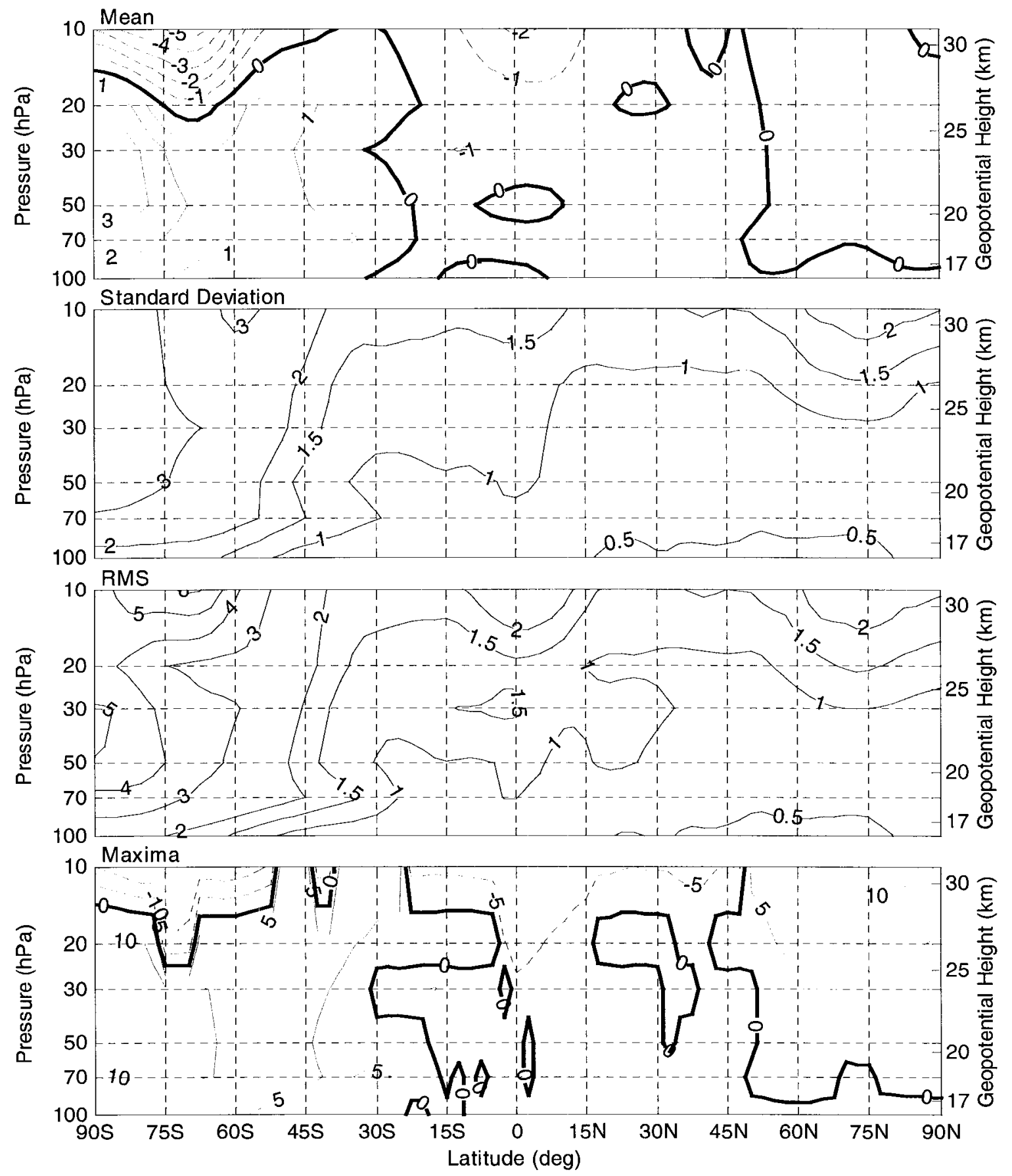

Fig. 4-14. Same as Fig. 4-13 except time: Jan 1958-Dec 1978. 

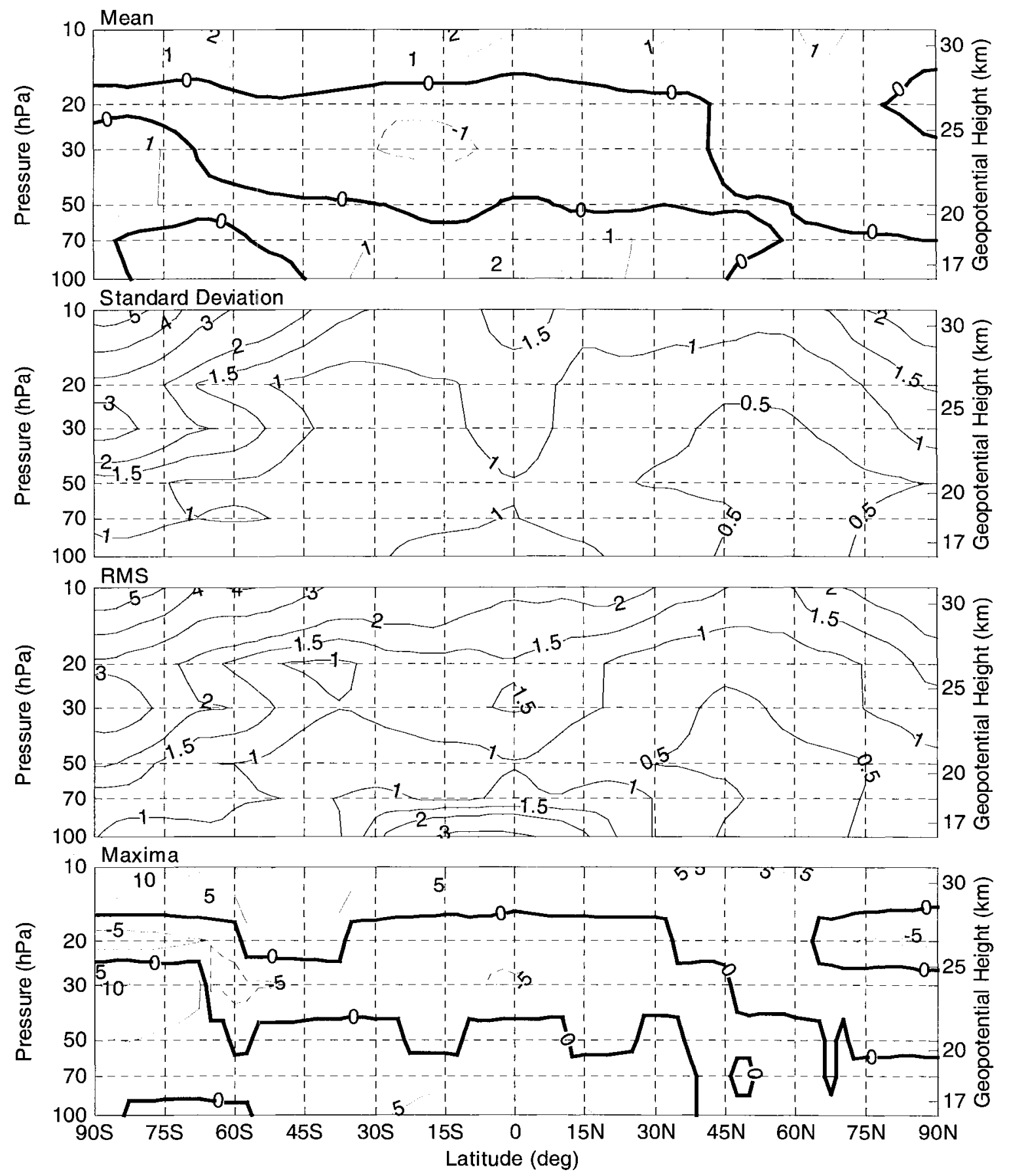

Fig. 4-15. Same as Fig. 4-13 except time: Jan 1979-Dec 2001. 

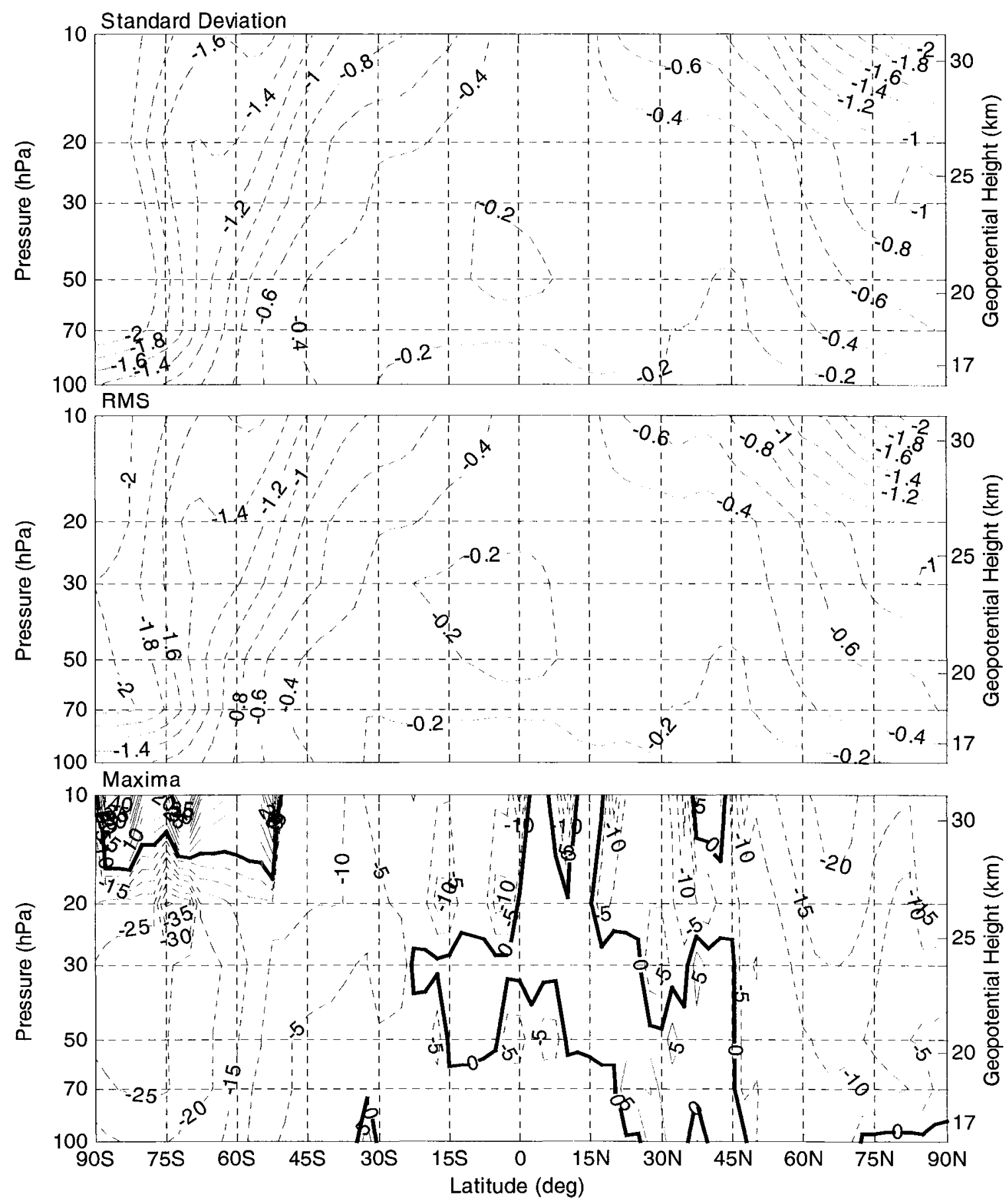

Fig. 4-16. Standard deviation, rms, and maxima of filtered longitude-time series (Fig. 4- 13) minus unfiltered longitude-time series (Fig. 4-7) temperature differences (K): NCEPI minus ERA-40 [lon: mean of $0^{\circ}-357.5^{\circ}$, lat: $90^{\circ} \mathrm{S}-90^{\circ} \mathrm{N}$, press: $100-10 \mathrm{hPa}$, time: Jan 1958-Dec 2001]. 


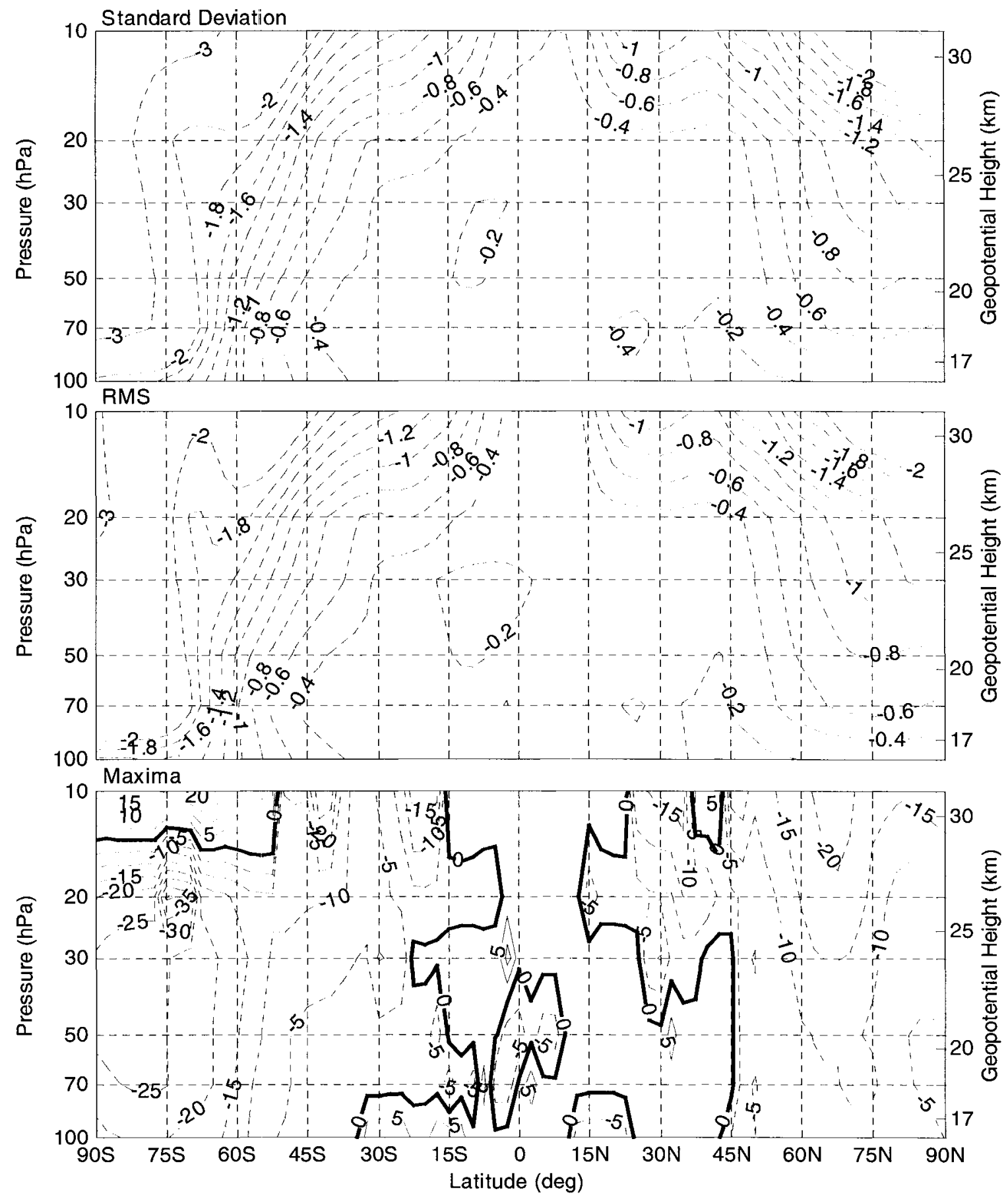

Fig. 4-17. Same as Fig. 4-16 except filtered longitude-time series is Fig. 4-14, unfiltered longitude-time series is Fig. 4-8, and time: Jan 1958-Dec 1978. 


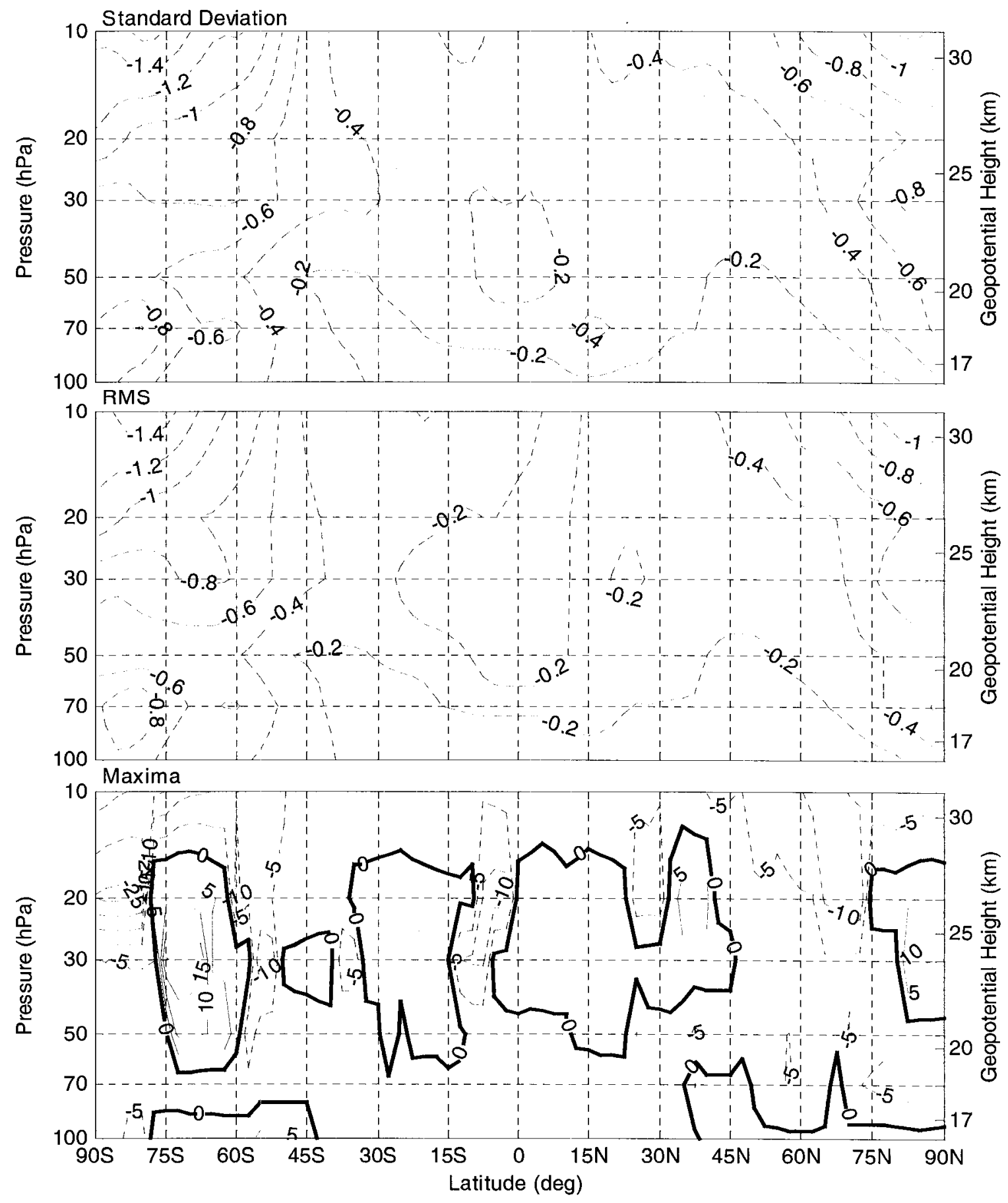

Fig. 4-18. Same as Fig. 4-16 except filtered longitude-time series is Fig. 4-15, unfiltered longitude-time series is Fig. 4-9, and time: Jan 1979-Dec 2001. 
Table 4-2. Comparison of Section 4.1 reanalysis temperature difference processing (K).

\begin{tabular}{|c|c|c|c|c|c|c|}
\hline \multirow[t]{2}{*}{$1958-2001$} & \multicolumn{2}{|c|}{$\begin{array}{l}\text { Zonally- } \\
\text { Averaged }\end{array}$} & \multicolumn{2}{|c|}{$\begin{array}{c}\text { Longitude-Time } \\
\text { Series }\end{array}$} & \multicolumn{2}{|c|}{$\begin{array}{c}\text { Annual Cycle } \\
\text { Removed }\end{array}$} \\
\hline & $\max$ & mean & $\max$ & mean & $\max$ & mean \\
\hline mean & 2.66 & 0.18 & 2.66 & 0.18 & 2.66 & 0.18 \\
\hline SD & 7.48 & 1.34 & 7.56 & 1.75 & 5.51 & 1.27 \\
\hline RMS & 7.50 & 1.47 & 7.66 & 1.85 & 5.68 & 1.40 \\
\hline \multirow[t]{2}{*}{ MAX } & $\begin{array}{l}+37 \\
-29\end{array}$ & 3.48 & $\begin{array}{l}+40 \\
-37\end{array}$ & 6.31 & $\begin{array}{l}+15 \\
-15\end{array}$ & 2.05 \\
\hline & \multicolumn{2}{|c|}{ Fig. 4-1 } & \multicolumn{2}{|c|}{ Fig. 4-7 } & \multicolumn{2}{|c|}{ Fig. 4-13 } \\
\hline \multirow[t]{2}{*}{$1958-1978$} & \multicolumn{2}{|c|}{$\begin{array}{l}\text { Zonally- } \\
\text { Averaged }\end{array}$} & \multicolumn{2}{|c|}{$\begin{array}{l}\text { Longitude-Time } \\
\text { Series }\end{array}$} & \multicolumn{2}{|c|}{$\begin{array}{c}\text { Annual Cycle } \\
\text { Removed }\end{array}$} \\
\hline & $\max$ & mean & $\max$ & mean & $\max$ & mean \\
\hline mean & -5.50 & -0.06 & -5.50 & -0.06 & -5.50 & -0.06 \\
\hline SD & 7.07 & 1.30 & 7.07 & 1.87 & 3.40 & 1.19 \\
\hline RMS & 8.07 & 1.46 & 8.26 & 1.99 & 6.17 & 1.36 \\
\hline \multirow[t]{2}{*}{ MAX } & $\begin{array}{l}+37 \\
-29\end{array}$ & 2.42 & $\begin{array}{r}+39 \\
-37\end{array}$ & 4.39 & $\begin{array}{l}+12 \\
-15\end{array}$ & 0.27 \\
\hline & \multicolumn{2}{|c|}{ Fig. 4-2 } & \multicolumn{2}{|c|}{ Fig. 4-8 } & \multicolumn{2}{|c|}{ Fig. 4-14 } \\
\hline \multirow[t]{2}{*}{$1979-2001$} & \multicolumn{2}{|c|}{$\begin{array}{l}\text { Zonally- } \\
\text { Averaged }\end{array}$} & \multicolumn{2}{|c|}{$\begin{array}{l}\text { Longitude-Time } \\
\text { Series }\end{array}$} & \multicolumn{2}{|c|}{$\begin{array}{l}\text { Annual Cycle } \\
\text { Removed }\end{array}$} \\
\hline & $\max$ & mean & $\max$ & mean & $\max$ & mean \\
\hline mean & 2.99 & 0.39 & 2.99 & 0.39 & 2.99 & 0.39 \\
\hline SD & 7.05 & 1.00 & 7.08 & 1.31 & 5.64 & 0.97 \\
\hline RMS & 7.38 & 1.38 & 7.38 & 1.63 & 6.02 & 1.34 \\
\hline \multirow[t]{2}{*}{ MAX } & $\begin{array}{l}+28 \\
-12\end{array}$ & 1.55 & $\begin{array}{l}+30 \\
-16\end{array}$ & 3.09 & $\begin{array}{c}+15 \\
-9\end{array}$ & 1.60 \\
\hline & \multicolumn{2}{|c|}{ Fig. 4-3 } & \multicolumn{2}{|c|}{ Fig. 4-9 } & \multicolumn{2}{|c|}{ Fig. 4-15 } \\
\hline
\end{tabular}

- Zonally-Averaged: Mean, standard deviation, rms, and maxima of monthly- and zonally-averaged temperature differences: NCEP1 minus ERA-40

- Longitude-Time Series: Same as "Zonally-Averaged" except longitude-time series of monthly-averaged temperature differences formed from individual monthlyaveraged temperature time series

- Annual Cycle Removed: Same as "Longitude-Time-Series" except all variance with period less than 13 months removed from individual monthly- and zonally-averaged temperature time series before differencing and longitude-time series formed 
- Figure numbers below each case indicate the figures containing the numerical values shown above

Standard deviations of seasonal temperatures

Surprisingly large standard deviations of ERA-40 $10 \mathrm{hPa}$ JJA temperatures as compared with similar NCEP1 data have been observed in related but independent studies, e.g., Randel et al. (2002, 2004). The manifestation of the "oscillatory vertical structure in temperature, especially large over Antarctica" in the standard deviation of temperature has been illustrated in NCEP1 minus ERA-40 temperature difference plots throughout Section 4.1 and discussed therein. Figures 4-19 through 4-21 reconfirm this finding and demonstrate that ERA-40 standard deviations of temperature, especially at 10,3 , and $1 \mathrm{hPa}$ near the South Pole and at 3 and $1 \mathrm{hPa}$ near the North Pole, are anomalously large throughout the year. These figures consider ERA-40 reanalysis data to their maximum available height of one $\mathrm{hPa}$, rather than the value of $10 \mathrm{hPa}$ used for comparison with NCEP1 reanalysis data (whose maximum available height is $10 \mathrm{hPa}$ ). 
Figure 4-21 plots the standard deviation as a function of pressure level, demonstrating that the ERA-40 oscillatory behavior at the geographic poles continues above $10 \mathrm{hPa}$ in the Southern Hemisphere and, to a lesser extent, is present primarily at $3 \mathrm{hPa}$ in the Northern Hemisphere. NCEP1 standard deviation shows no oscillatory behavior and is generally consistent in magnitude with ERA-40 without its oscillations. It is the absence of NCEP1 oscillatory behavior that accentuates the standard deviation of NCEP1 minus ERA-40 temperature differences throughout Section 4.1. Because areaweighted analyses omits data at a geographic pole, Fig. 4-21 includes the results of areaweighted standard deviations for the following latitude bands:

- $90^{\circ}$ (bold, largest magnitude)

- $90^{\circ}$ through $75^{\circ}$ (fine line, $2^{\text {nd }}$ largest magnitude)

- $90^{\circ}$ through $60^{\circ}$ (fine line, $3^{\text {rd }}$ largest magnitude)

- $90^{\circ}$ through $45^{\circ}$ (fine line, $4^{\text {th }}$ largest magnitude)

- $90^{\circ}$ through $30^{\circ}$ (bold, smallest magnitude)

in both hemispheres for both NCEP1 and ERA-40 reanalyses. These figures illustrate the decreasing effect of the polar anomalies as the latitude bands are broadened to include increasingly larger areas.

Figures 4-22 and 4-23 display moving three-year standard deviations at $10 \mathrm{hPa}$. In the Southern Hemisphere the $10 \mathrm{hPa}$ variance during JJA and SON is dominated by an ERA-40 transient occurring in 1984-85, while transients during the late 1990's are significant during DJF and MAM. A temperature spike from 1978 through 1980 dominates the NCEP1 $10 \mathrm{hPa}$ variance in the JJA and SON seasons and is still significant during the remainder of the year. To again demonstrate the mitigating effects of area 
averaging over extratropics from $90^{\circ} \mathrm{S}$ through $30^{\circ} \mathrm{S}$ (Fig. 4-22) and $30^{\circ} \mathrm{N}$ through $90^{\circ} \mathrm{N}$ (Fig. 4-23), area-averaged curves are also included in these figures. The time series of Fig. 4-24 illustrates the timing and magnitude of the transients of the previous two figures. Extratropical time series corresponding to the conditions of Fig. 4-24 are plotted in Fig. 4-30. 

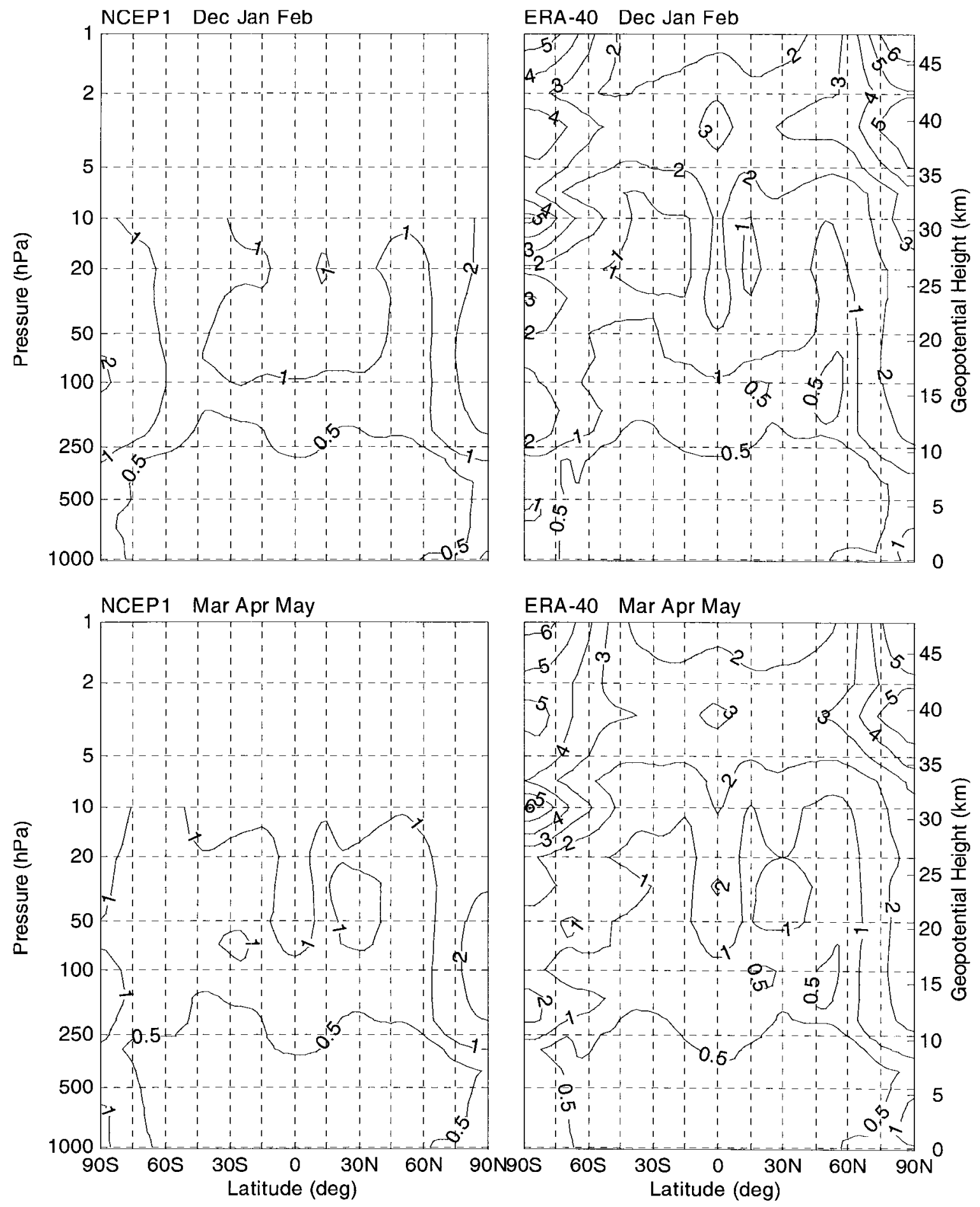

Fig. 4-19. Standard deviation of monthly-and zonally-averaged temperatures for DJF and MAM seasons $(K)$, all variance less than 13 months removed: [lon: mean of $0^{\circ}-357.5^{\circ}$, lat: $90^{\circ} \mathrm{S}-90^{\circ} \mathrm{N}$, press: $100-1 \mathrm{hPa}$, time: Jan 1979-Dec 1999]. 

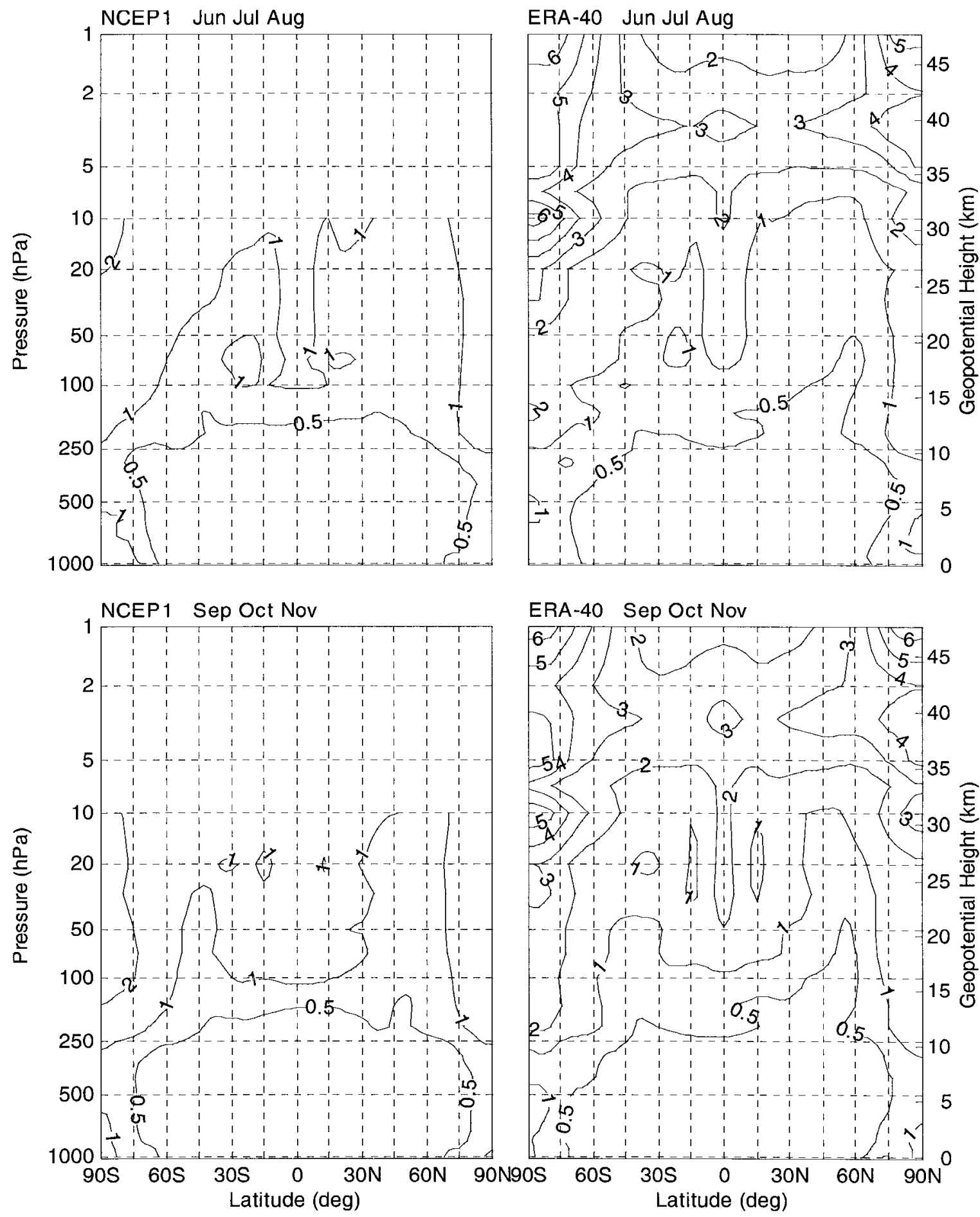

Fig. 4-20. Same as Fig. 4-19 except JJA and SON seasons. 

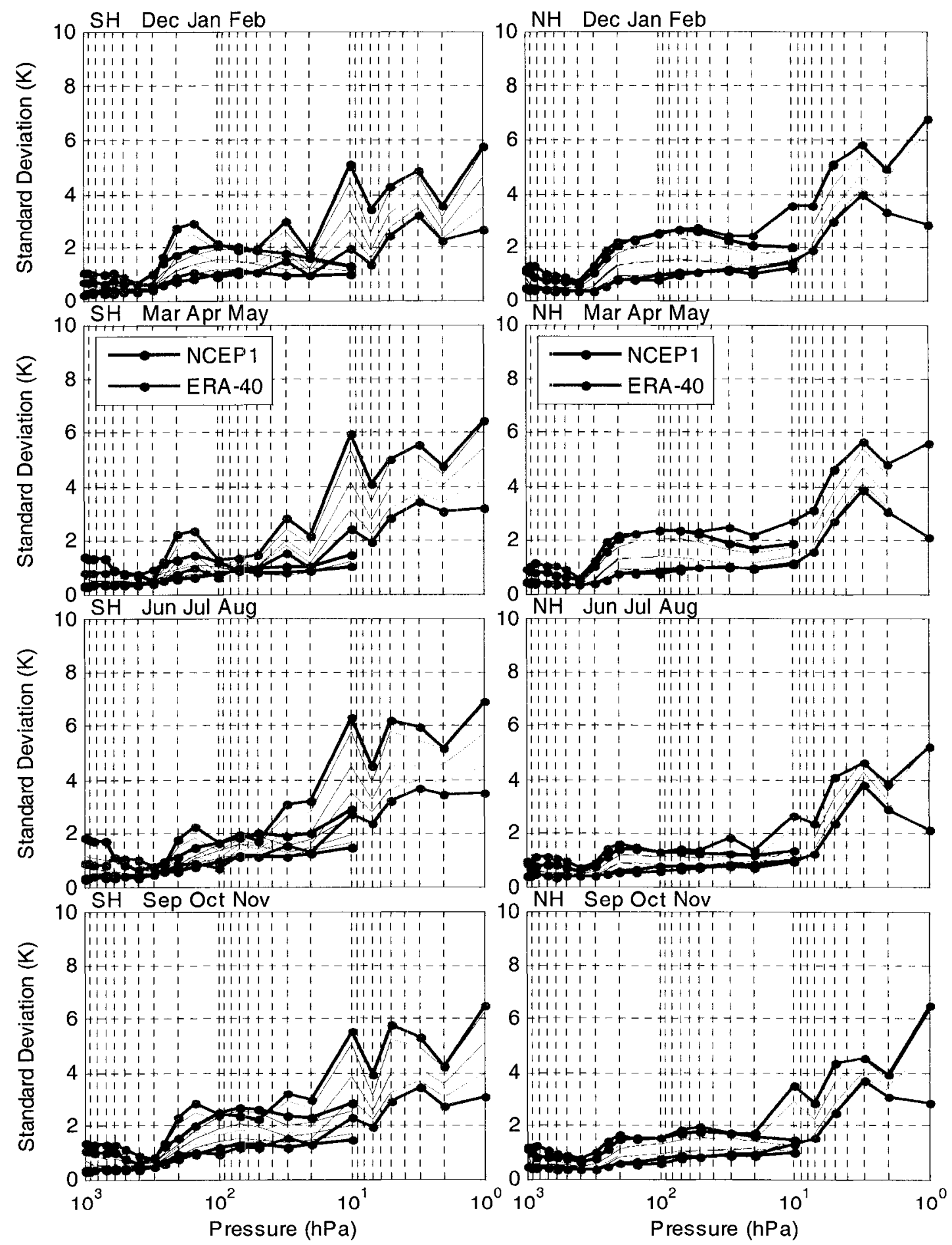

Fig. 4-21. Standard deviation of monthly-and zonally-averaged seasonal temperatures as a function of pressure level (K), all variance less than 13 months removed: [lon: mean of $0^{\circ}-357.5^{\circ}$, lat: see text, press: 100-1 hPa, time: Jan 1979-Dec 2001]. Significance of individual traces discussed in text. 

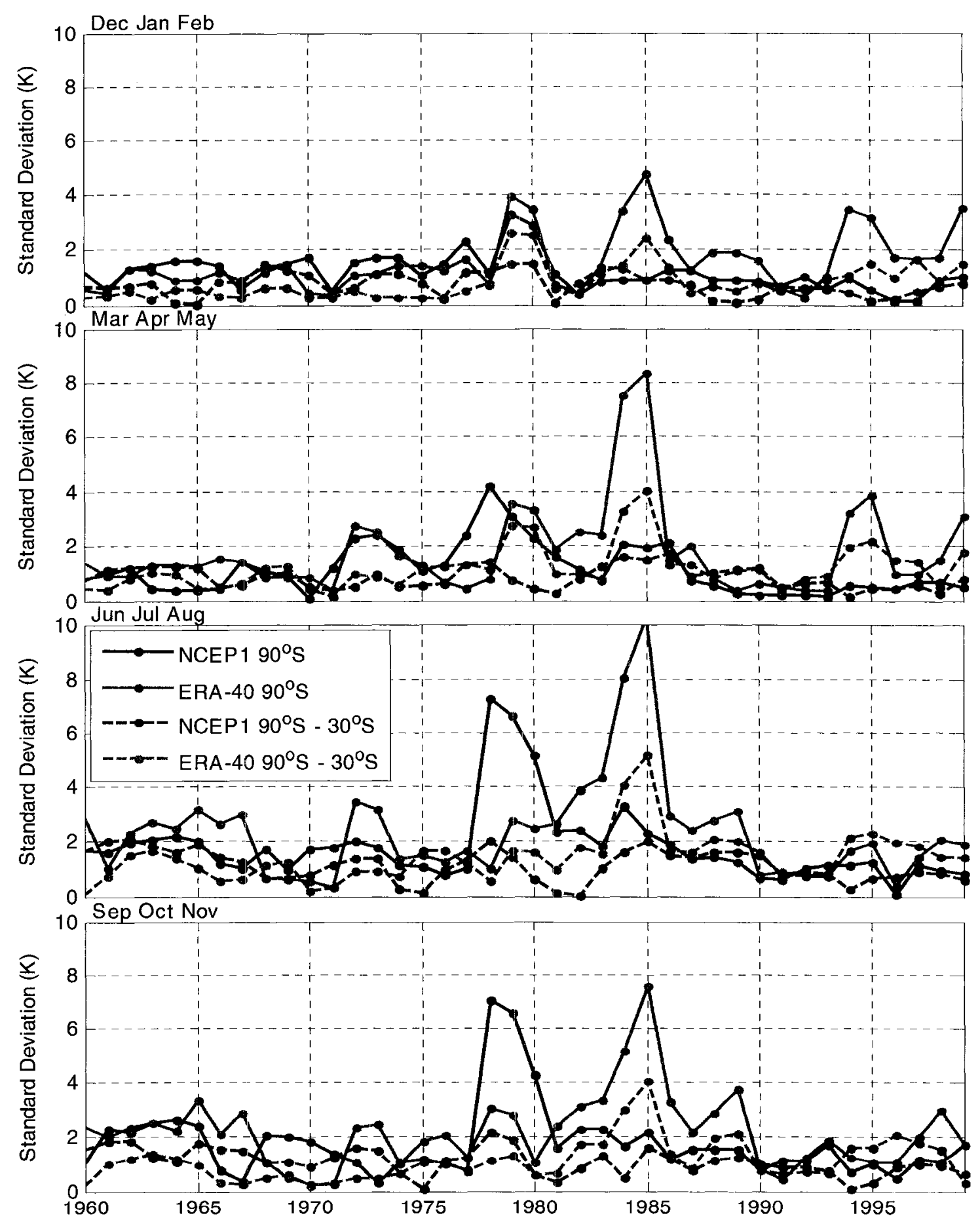

Fig. 4-22. Three-year moving standard deviation of South Pole $\left(90^{\circ} S\right.$ - bold) and Southern Hemisphere $\left(90^{\circ} \mathrm{S}-30^{\circ} \mathrm{S}\right.$ - dashed) temperature (K) (all variance with period less than 13 months removed by frequency-domain filtering): [lon: mean of $0^{\circ}-357.5^{\circ}$, lat: as stated, press: $10 \mathrm{hPa}$, time: Jan 1958-Dec 2001]. 

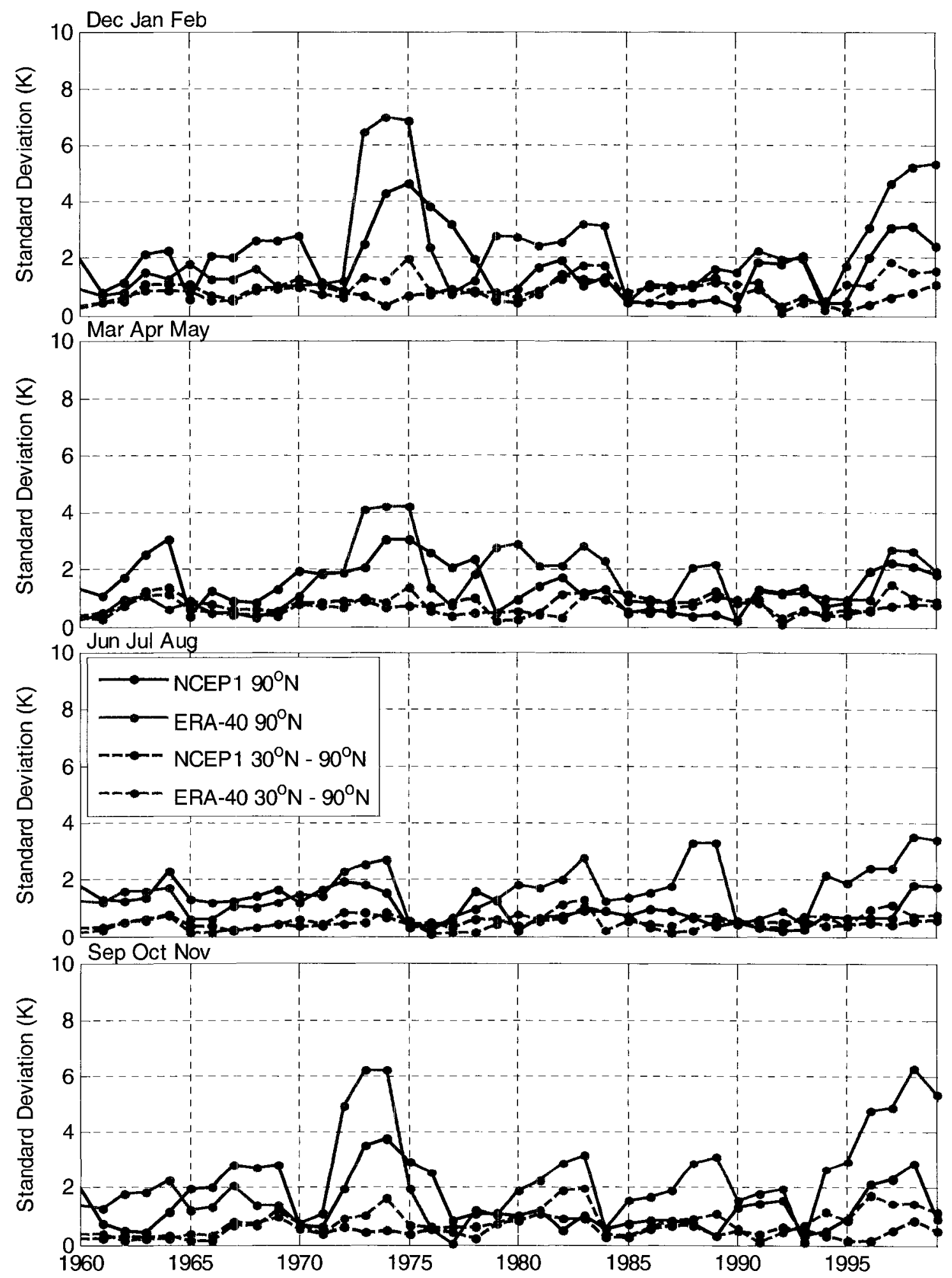

Fig. 4-23. Same as Fig. 4-22 except North Pole $\left(90^{\circ} \mathrm{N}\right.$-bold) and Northern Hemisphere $\left(30^{\circ} \mathrm{N}-90^{\circ} \mathrm{N}\right.$-dashed). 

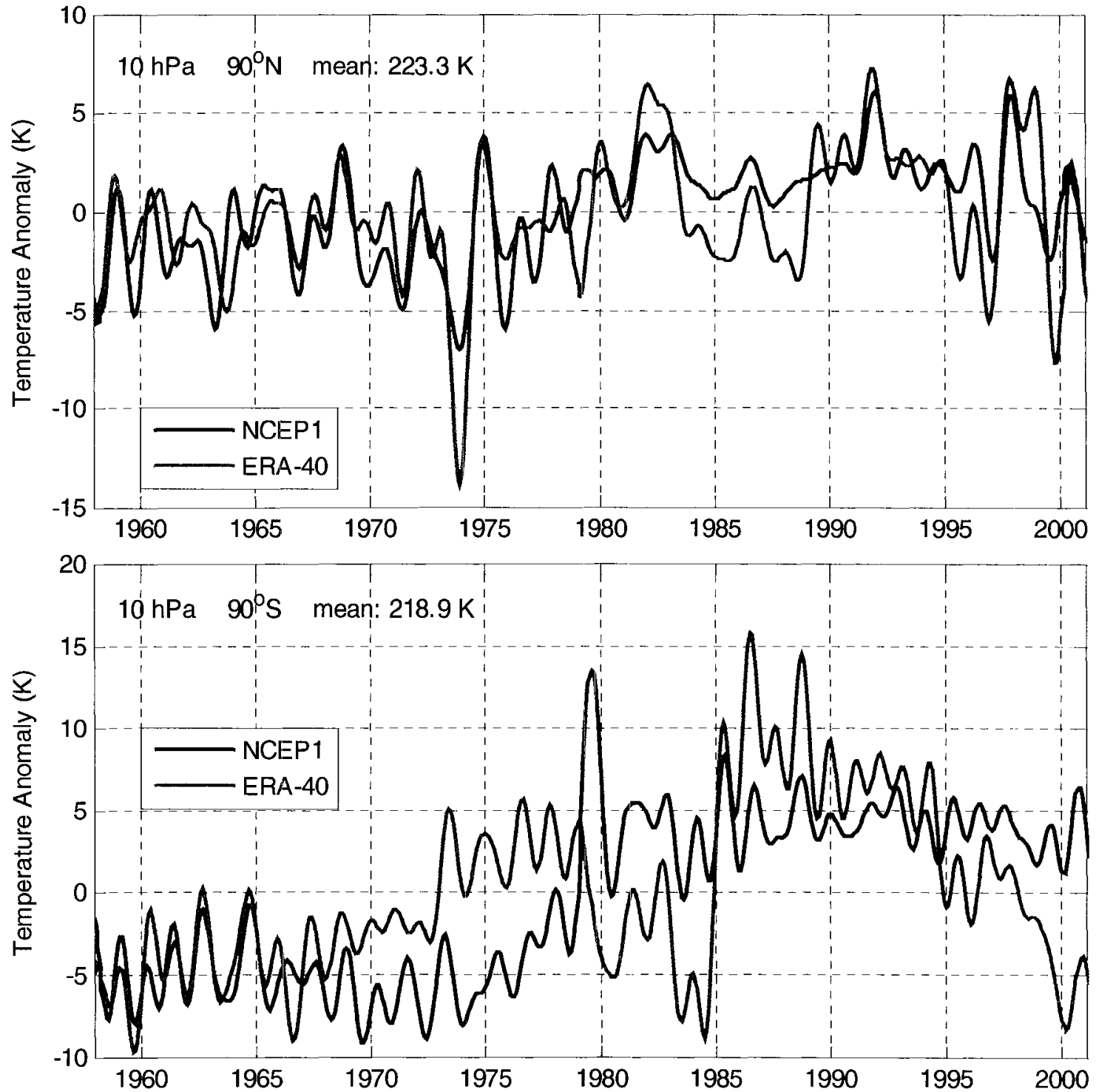

Fig. 4-24. Anomaly time series of zonally-averaged monthly-averaged temperatures $(K)$ (all variance with period less than 13 months removed by frequency-domain filtering): [lon: mean of $0^{\circ}-357.5^{\circ}$; lat: as stated; press: $10 \mathrm{hPa}$; time: Jan 1958-Dec 2001]. 


\subsection{Temperature anomaly time series}

Figures 4-25 through 4-30 display time series temperatures for:

- NCEP1 (January 1958 through December 2005: 48 years, 576 months, monthly- and zonally-averaged)

- ERA-40 (January 1958 through December 2001: 44 years, 528 months, monthly- and zonally-averaged)

- METO (January 1992 through December 1999: 8 years, 96 months, monthly- and zonally-averaged)

- RATPAC (January 1958 through December 2005: 48 years, annually- and latitudinally-averaged, $100 \mathrm{hPa}$ through $30 \mathrm{hPa}$ only)

The latitude ranges $90^{\circ} \mathrm{S}$ through $30^{\circ} \mathrm{S}$ (Southern Hemisphere extratropical), $30^{\circ} \mathrm{S}$ through $30^{\circ} \mathrm{N}$ (tropical), $30^{\circ} \mathrm{N}$ through $90^{\circ} \mathrm{N}$ (Northern Hemisphere extratropical), and Global correspond to the ranges available in the RATPAC data set. These ranges contain $25 \%, 50 \%, 25 \%$, and $100 \%$ of the global surface area, respectively. The four ranges represent the same percentages of the global temperature at the six stratospheric pressure levels.

Temperatures in each latitude range are weighted by the cosine of their latitude and averaged using trapezoidal integration. To remove the annual temperature cycle, which can exceed the underlying data by more than an order of magnitude, these areaweighted temperatures are then filtered in the frequency domain to remove all variance of period less than 13 months. This filtering technique is described and illustrated in detail in Appendix E. As Fig. E-4 demonstrates, filtered time series plots contain much more information than does the calendar-year average. Although filtered data contain higher frequency components than RATPAC annual averages, Fig. E-4 also confirms that low- 
pass filtered data passes through (or extremely close to) data points annually averaged from the same original source. Thus annually averaged data may be considered the "core" of a filtered plot.

For clarity and figure-to-figure consistency, the time series are presented as temperature anomalies. Removed mean values, shown in each subplot, are the average of the means of the area-weighted, unfiltered NCEP1 and ERA-40 means over their full duration. Preparation of RATPAC data results (Section 2.2.3) in annualized anomalies; therefore mean values of RATPAC data do not exist. Thus, it is reassuring that the RATPAC anomalies generally closely match the three monthly time series.

In Fig. 4-25 the $100 \mathrm{hPa}$ Northern Hemisphere extratropical time series are highly correlated with one another. Southern Hemisphere correlation is intermittent, improving after 1987. The tropical time series are highly correlated with one another until 1979, the beginning of the modern satellite era. This is the infamous four-degree temperature discontinuity (Huesmann and Hitchman 2003). RATPAC agrees more closely with ERA-40 after 1985. By 2000 NCEP1 converges to RATPAC values.

At $70 \mathrm{hPa}$, Fig. 4-26, the Northern Hemisphere extratropical and tropical time series are again highly correlated. The tropical time series displays considerably more variance, primarily because of the three major volcanic eruptions during the period: Agung in March 1963, El Chichon in April 1982, and Mt. Pinatubo in June 1990. The reanalyses demonstrate similar responses in each case, although NCEPI's El Chichon transient is larger than ERA-40's. Transients in RATPAC's annual data are generally less than those of the reanalyses. During the first 13 years of the Southern Hemisphere 
extratropical time series, except for 1963, ERA-40 is approximately $2 \mathrm{~K}$ less than NCEP1 and RATPAC but by 1971 has converged with NCEP1 and RATPAC. Another brief departure of ERA-40 from NCEP1 and RATPAC occurs from 1980 to 1982. The variations discussed above in this paragraph are minimized when globally averaged.

The relative behaviors of the time series at 50, 30, and $20 \mathrm{hPa}$ in Figs. 4-27 through 4-29 are surprisingly similar to those at $70 \mathrm{hPa}$ in Fig. 4-26, with slowly increasing variance with height. The most notable exception is the $1979-85$ positive excursion of ERA-40 at $30 \mathrm{hPa}$ in the Southern Hemisphere extratropics, the only extended interval when ERA-40 is significantly larger than RATPAC.

The $10 \mathrm{hPa}$ time series in Fig. 4-30 exhibits large month-to-month variance and differences not seen at any lower heights. It is indeed unfortunate that RATPAC data is not available at this level to provide an independent data set for comparison. As will be demonstrated quantitatively in Figs. 4-31 and Table 4-3, the largest temperature differences are approximately seven K during 1979-85 in the Southern Hemisphere extratropics, when simultaneously NCEP1 moves positively and ERA-40 negatively, and in 1995-97 when ERA-40 again moves negatively. The 1979 excursion coincides with the beginning of the modern satellite era; the reason for the other difference is not known.

Throughout these six pressure levels NCEP1 is almost always highly correlated with METO. This may result from the similar treatment of satellite data in NCEP1 and METO, which convert radiance data to temperature, while ERA-40 directly assimilates "raw" radiance data. 

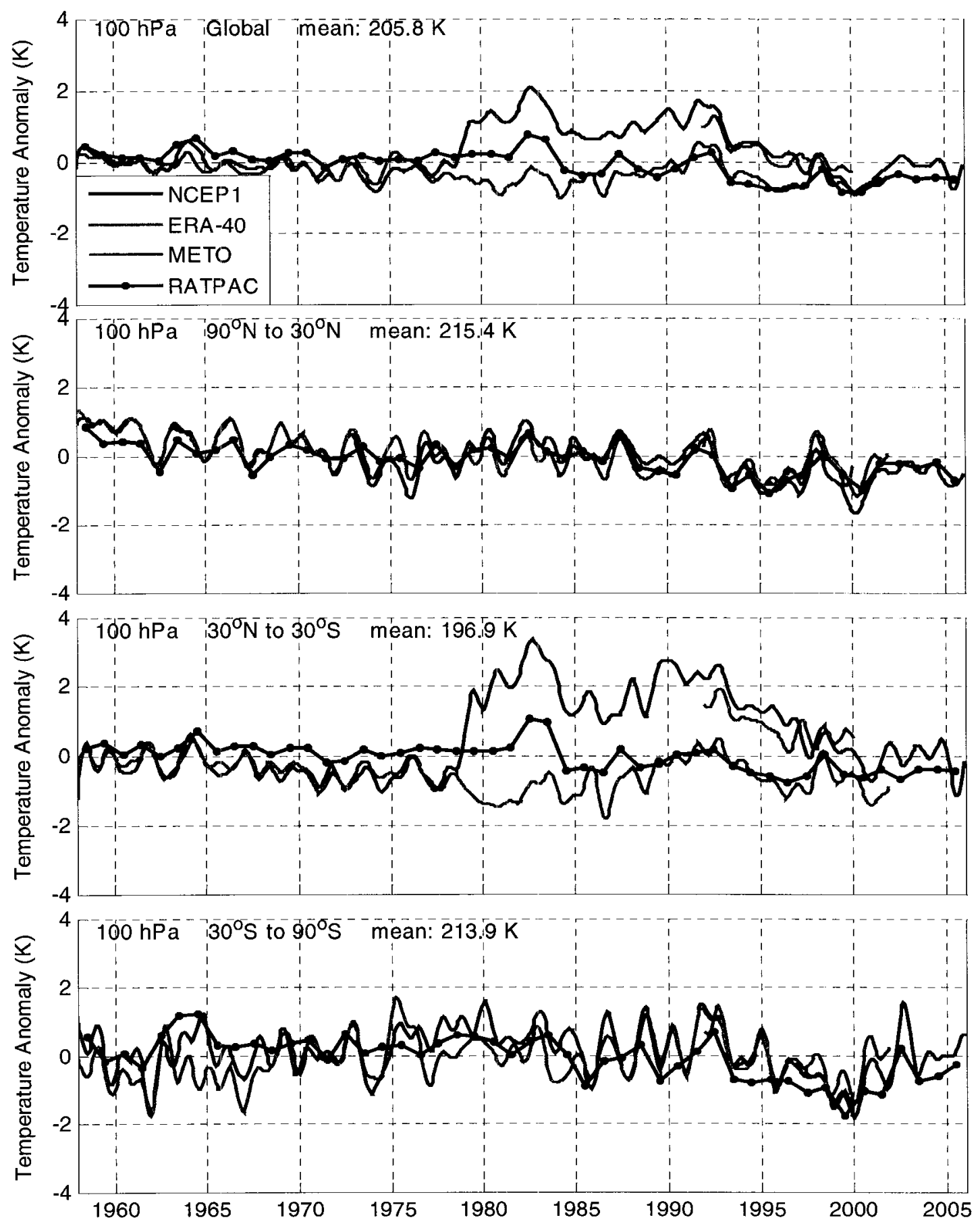

Fig. 4-25. Anomaly time series of monthly-and zonally-averaged temperatures (all variance with period less than 13 months removed by frequency-domain filtering):

[lon: mean of $0^{\circ}-357.5^{\circ}$; lat: as stated; press: $100 \mathrm{hPa}$; time: Jan 1958-Dec 2005 (NCEPI), Jan 1958-Dec 2001 (ERA-40), Jan 1992-Dec 1999 (METO), Jan 1958-Dec 2005 (RATPAC)]. 

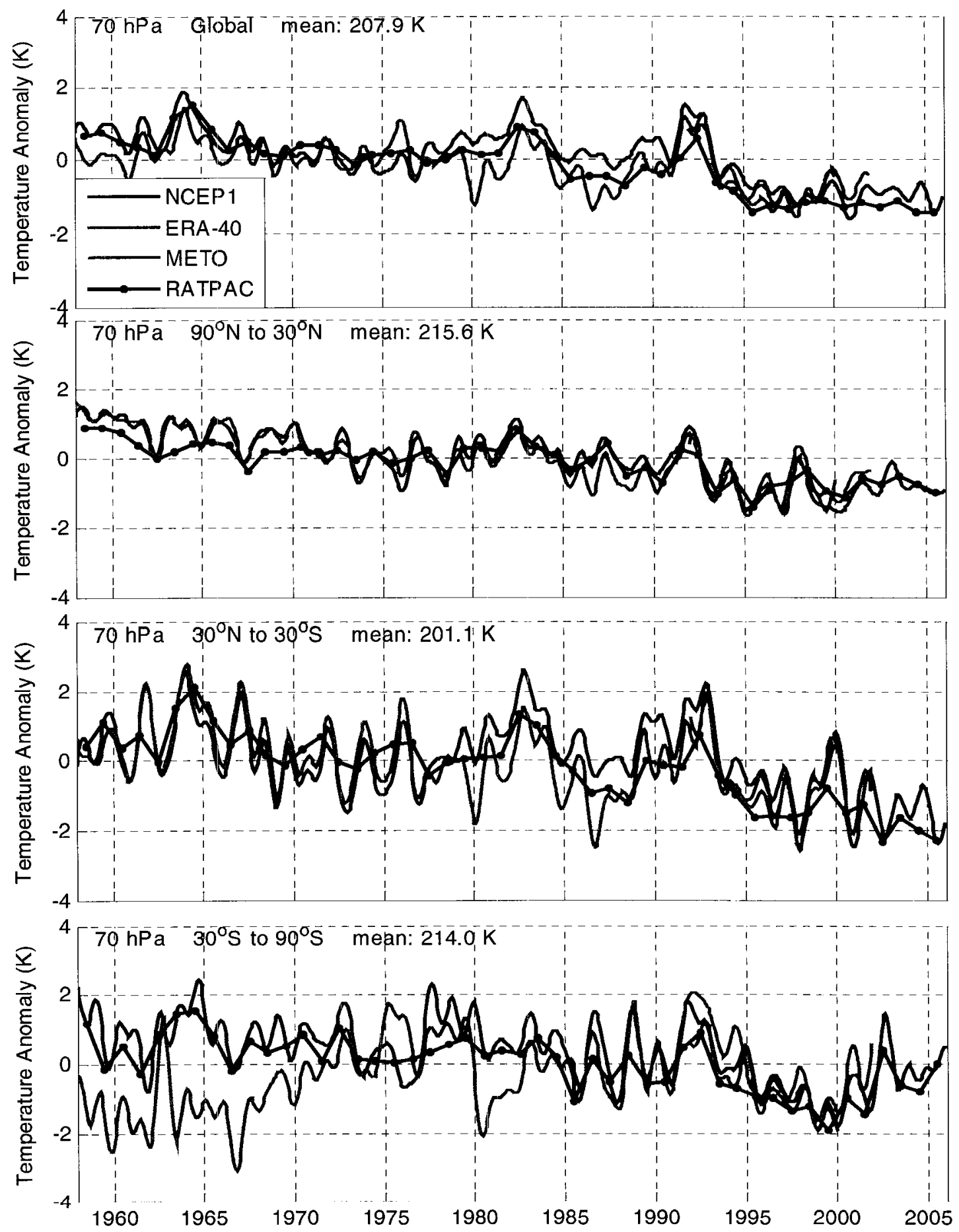

Fig. 4-26. Same as Fig. 4-25 except press: $70 \mathrm{hPa}$. 

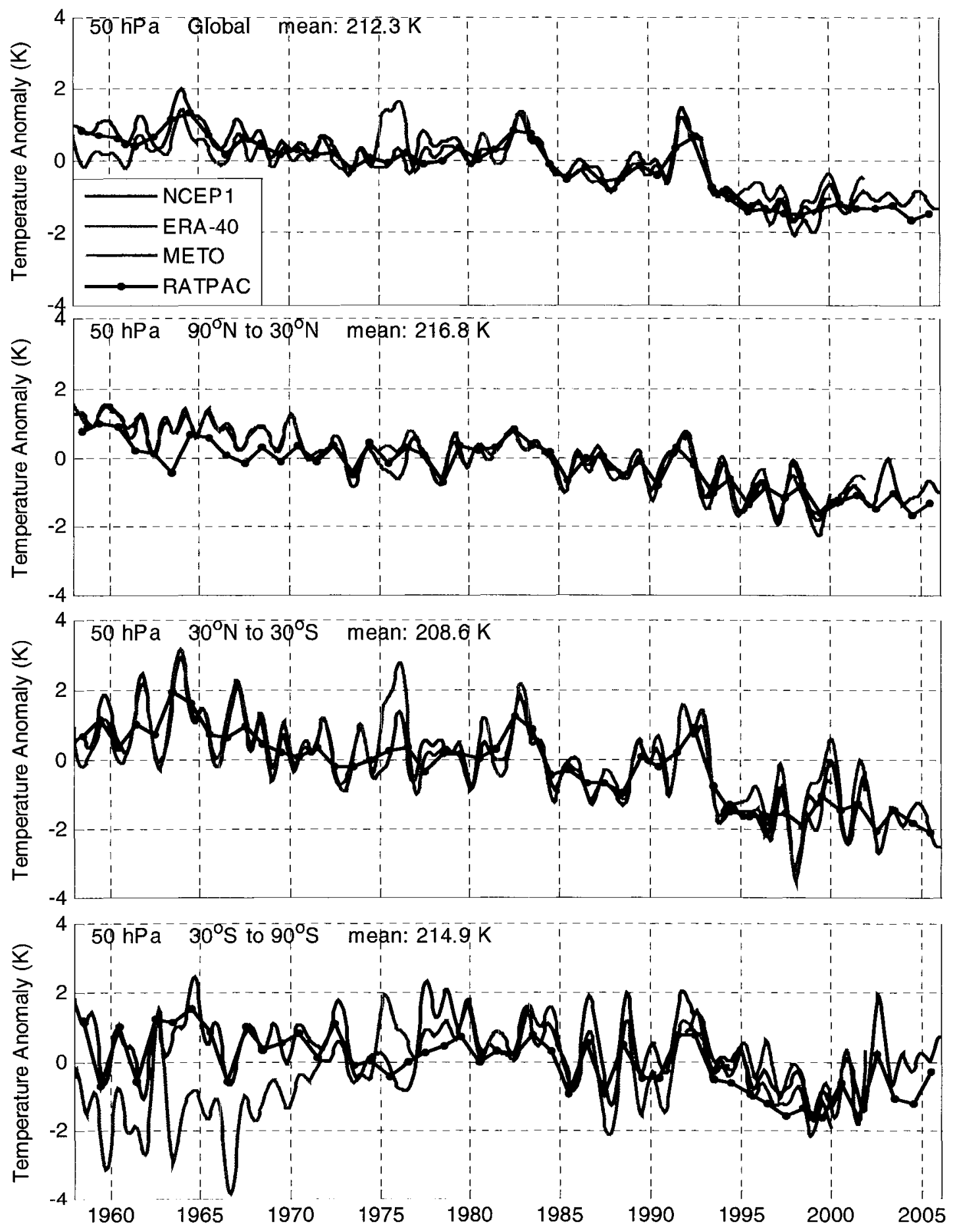

Fig. 4-27. Same as Fig. 4-25 except press: $50 \mathrm{hPa}$. 

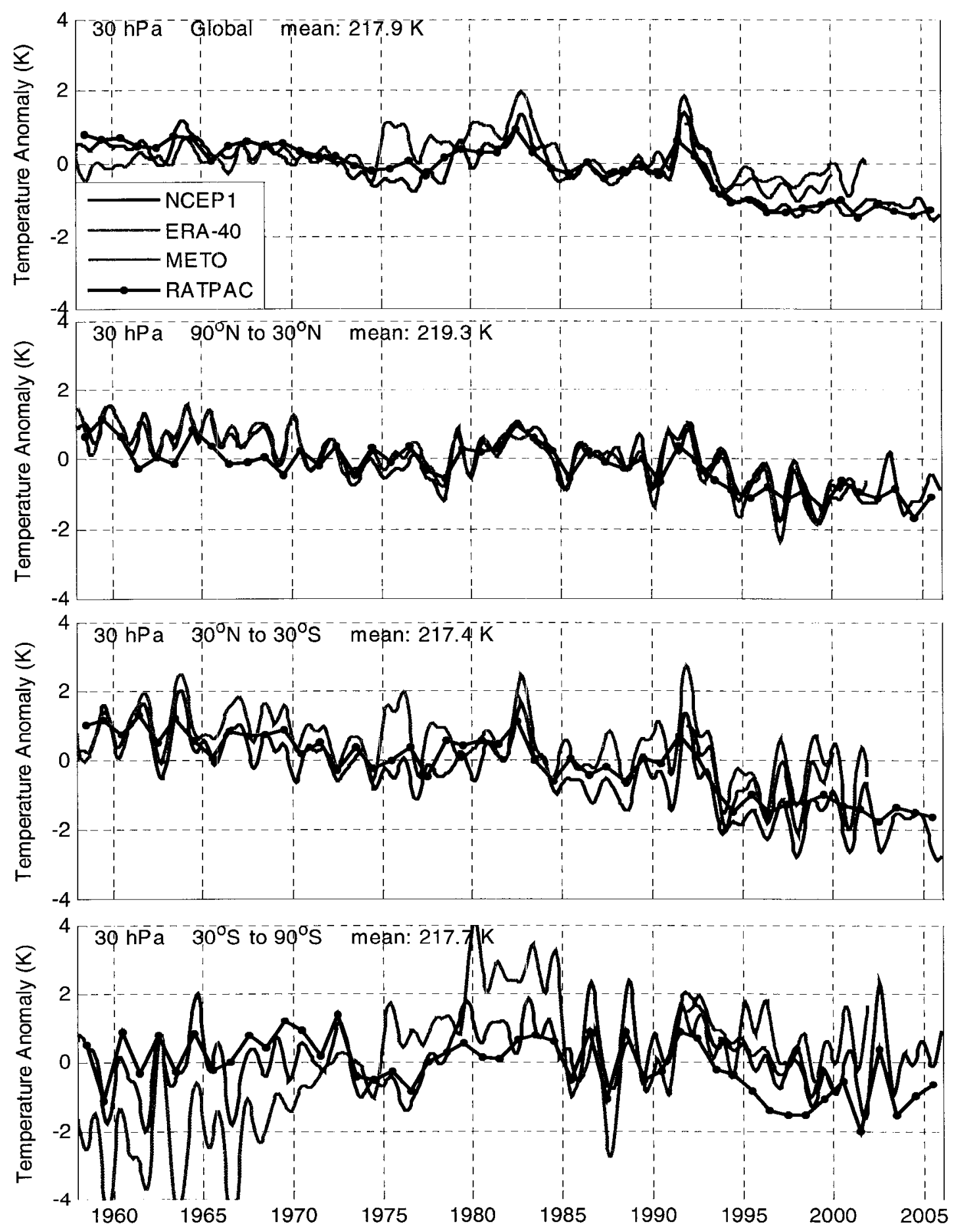

Fig. 4-28. Same as Fig. 4-25 except press: $30 \mathrm{hPa}$. 

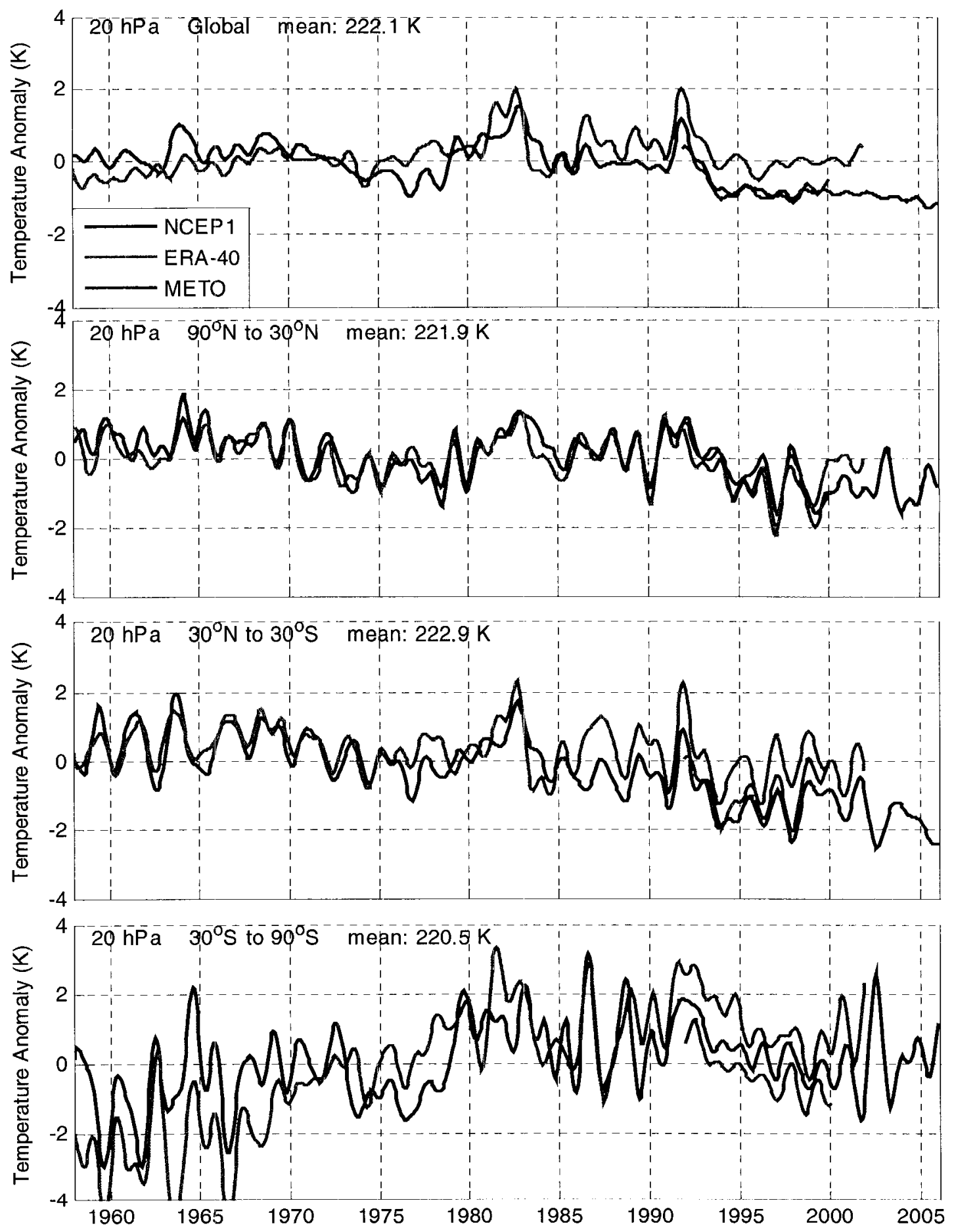

Fig. 4-29. Same as Fig. 4-25 except press: $20 \mathrm{hPa}$ and RATPAC data not available. 


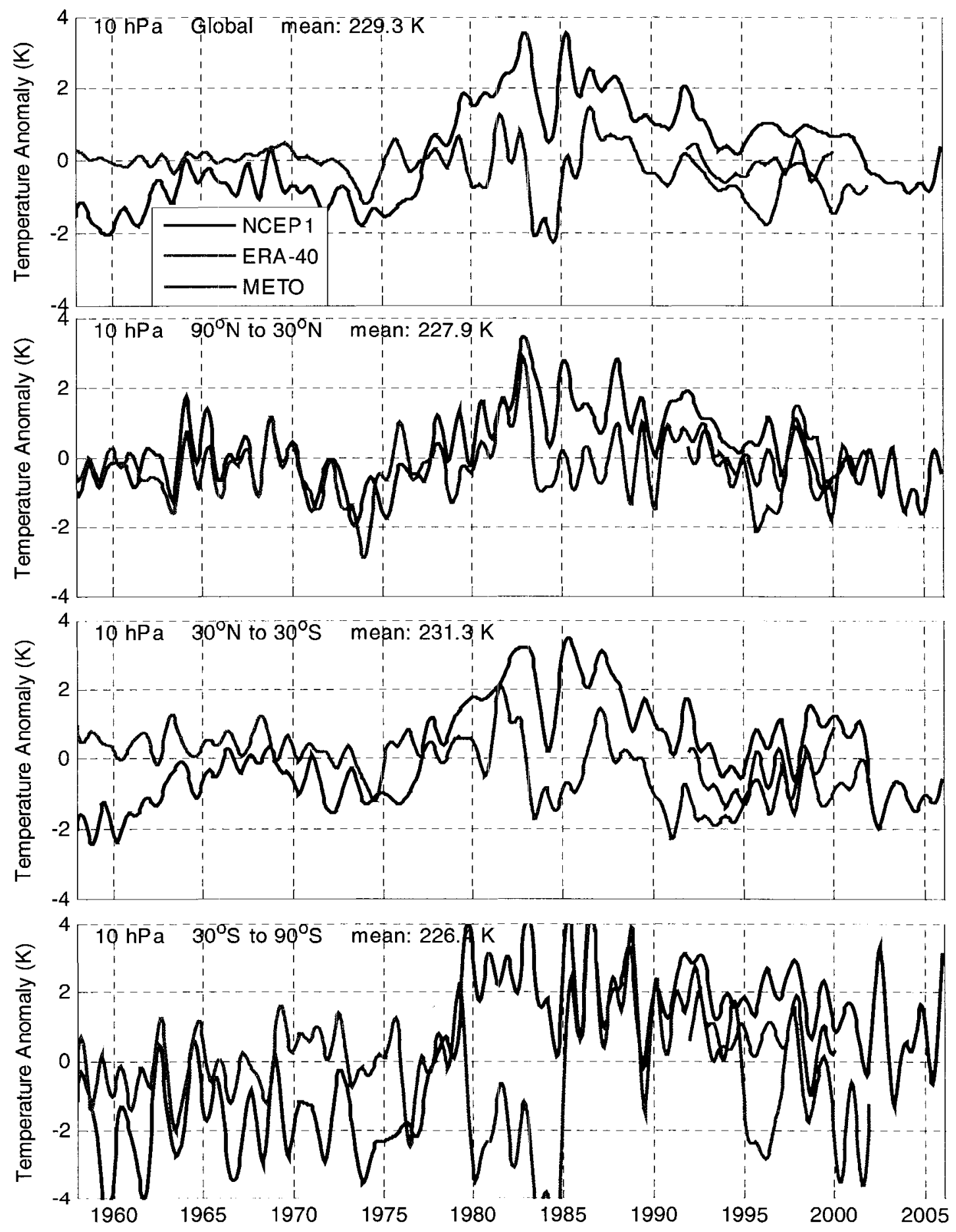

Fig. 4-30. Same as Fig. 4-25 except press: $10 \mathrm{hPa}$ and RATPAC data not available. 
Figures 4-31 and 4-32 display the differences in the monthly- and zonallyaveraged temperatures of NCEP1 minus ERA-40 for latitude ranges of $90^{\circ} \mathrm{S}$ through $30^{\circ} \mathrm{S}, 30^{\circ} \mathrm{S}$ through $30^{\circ} \mathrm{N}$, and $30^{\circ} \mathrm{N}$ through $90^{\circ} \mathrm{N}$ (the three subglobal regions of Figs. 4-25 through 4-30) at pressure levels of 100, 70, and 50 (Fig. 4-31), and 30, 20, and $10 \mathrm{hPa}$ (Fig. 4-32) for the period January 1958 through December 2001. Green lines are the differences of the latitude-weighted time series for the indicated case. Black lines are the latitude-weighted time series with all variance less than 13 months removed by frequency-domain filtering and then differenced. The unfiltered maximum and mean difference values listed in each subplot and the filtered maximum and unfiltered standard deviation of each time series are tabulated in Table 4-3 for easier comparison. Table 4-3 also lists global values. Lower panels of Figs. 4-31 and 4-32 contain the 12-month moving average rms of the latitude-weighted differences and also display their maximum and mean values.

Table 4-3 summarizes the graphical data of Figs. 4-31 and 4-32 and identifies the differences between NCEP1 and ERA-40 implied by the time series plots of Figs. 4-25 through 4-30. The absolute values of the maximum differences form an almost monotonic surface from the Southern Hemisphere extratropics at $10 \mathrm{hPa}$ to the Northern Hemisphere at $100 \mathrm{hPa}$, the most significant outliers from this surface being the Tropics at 100 and $70 \mathrm{hPa}$. The same monotonic behavior with tropical outliers is found in the standard deviation of the time series at $100 \mathrm{hPa}$ in the Tropics. These results are consistent with the pressure level vs. latitude plot of Fig. 4-1, which displays contours of the zonal time series at each pressure level and latitude. 

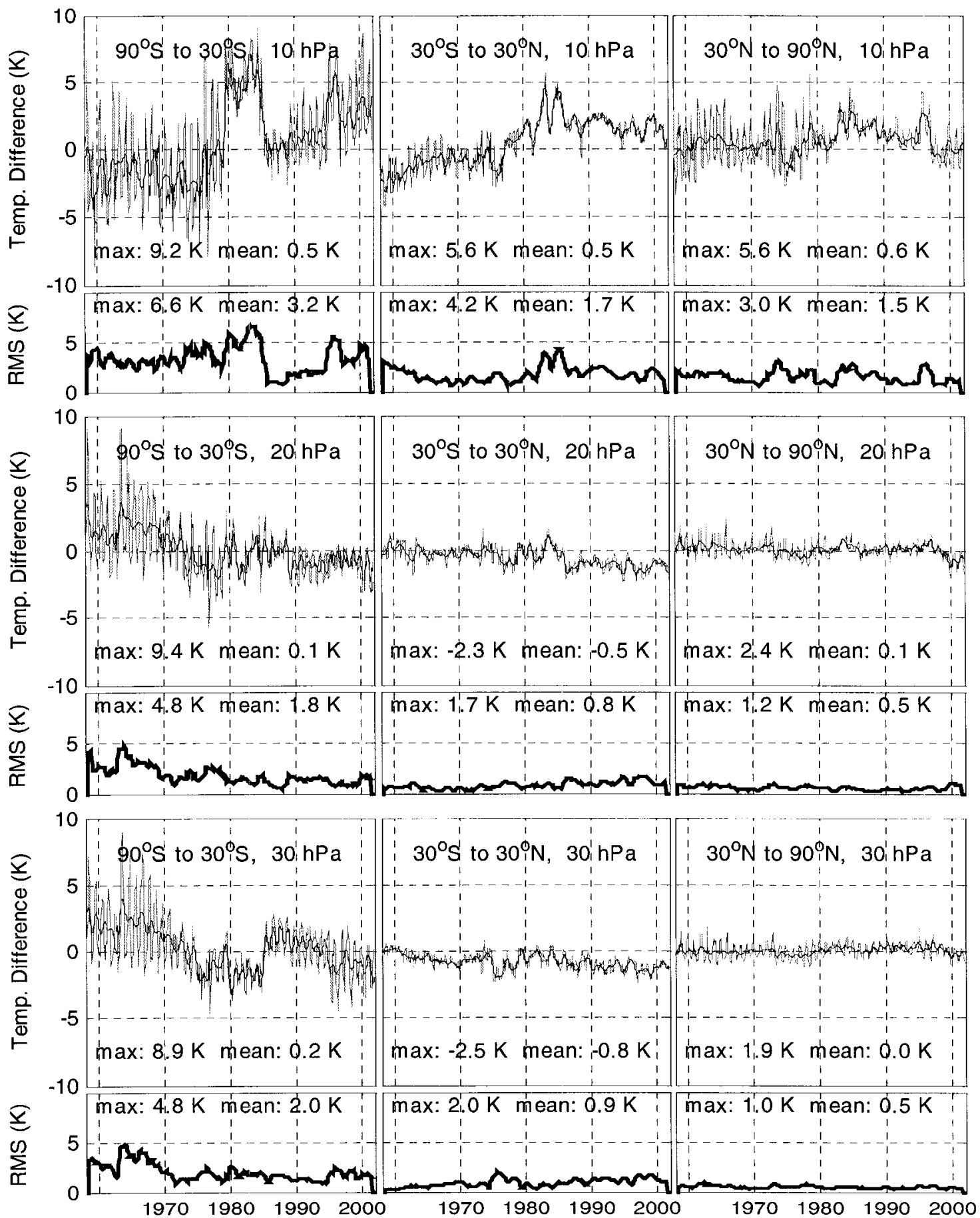

Fig. 4-31. Time series of monthly-and zonally-averaged temperature differences (see text) and 12-month moving rms: NCEPI minus ERA-40: [lon: mean of $0^{\circ}-357.5^{\circ}$; lat: as stated; press: as stated; time: Jan 1958-Dec 2001]. 

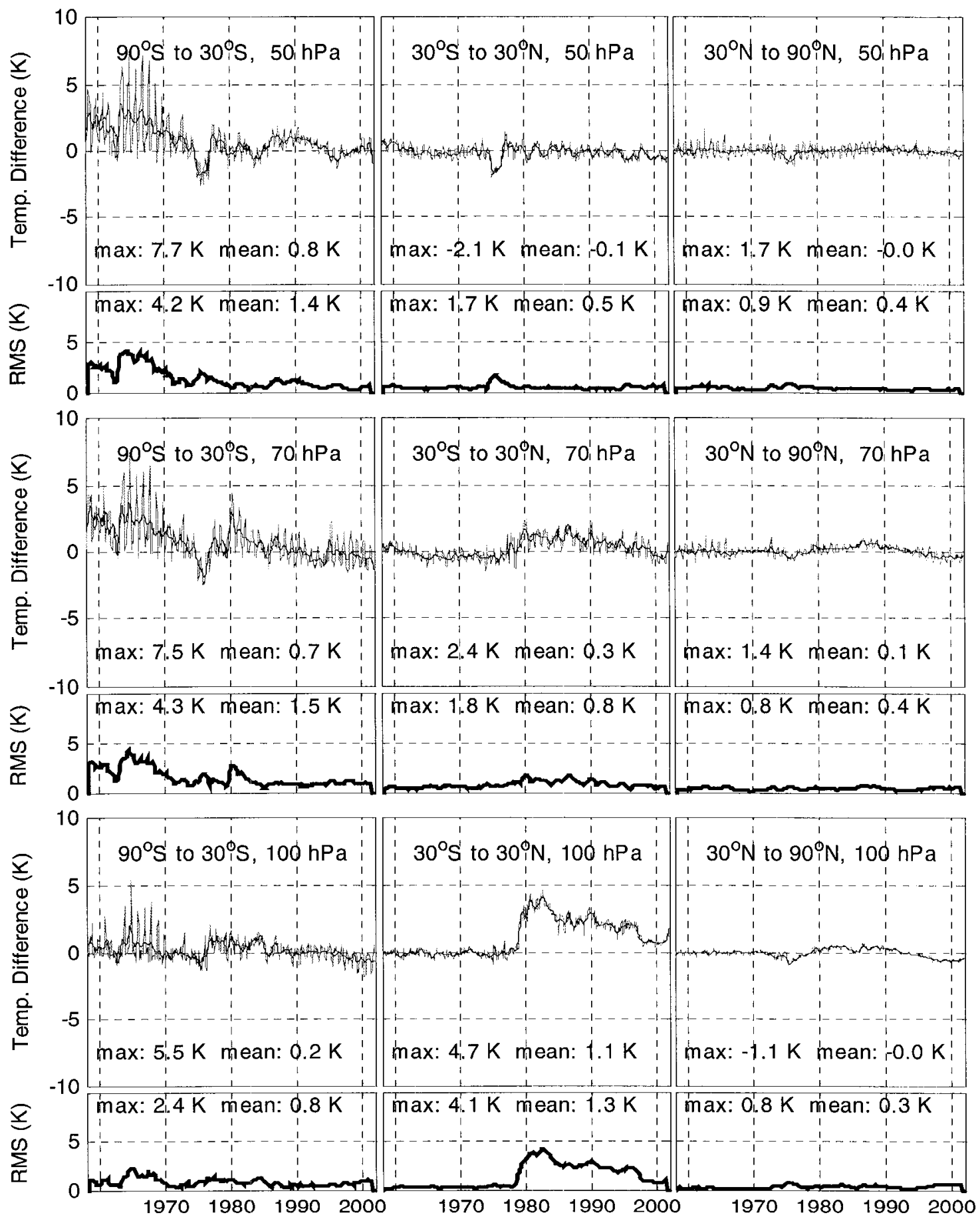

Fig. 4-32. Same as Fig. 4-31 except pressure levels. 
Table 4-3. Unfiltered maximum, filtered maximum, mean, and unfiltered standard deviation of temperature difference time series (K) for subplots of Figs. 4-31 and 4-32.

\begin{tabular}{|c|c|c|c|c|}
\hline \multicolumn{5}{|c|}{ MAXIMUM (Unfiltered) } \\
\hline & $90 S-30 S$ & $30 \mathrm{~S}-30 \mathrm{~N}$ & $30 \mathrm{~N}-90 \mathrm{~N}$ & Global \\
\hline $10 \mathrm{hPa}$ & 9.2 & 5.6 & 5.6 & 5.7 \\
\hline $20 \mathrm{hPa}$ & 9.4 & -2.3 & 2.4 & 2.8 \\
\hline $30 \mathrm{hPa}$ & 8.9 & -2.5 & 1.9 & -1.9 \\
\hline $50 \mathrm{hPa}$ & 7.7 & -2.1 & 1.7 & 2.0 \\
\hline $70 \mathrm{hPa}$ & 7.5 & 2.4 & 1.4 & 2.2 \\
\hline $100 \mathrm{hPa}$ & 5.5 & 4.7 & -1.1 & 2.7 \\
\hline \multicolumn{5}{|c|}{ MAXIMUM (Filtered) } \\
\hline & $90 S-30 S$ & $30 \mathrm{~S}-30 \mathrm{~N}$ & $30 \mathrm{~N}-90 \mathrm{~N}$ & Global \\
\hline $10 \mathrm{hPa}$ & 7.1 & 4.5 & 2.9 & 4.6 \\
\hline $20 \mathrm{hPa}$ & 3.7 & -1.8 & -1.2 & -1.6 \\
\hline $30 \mathrm{hPa}$ & 4.0 & -1.9 & 0.8 & -1.6 \\
\hline $50 \mathrm{hPa}$ & 3.6 & -1.8 & -0.9 & -1.6 \\
\hline $70 \mathrm{hPa}$ & 3.8 & 2.0 & 0.9 & 1.7 \\
\hline $100 \mathrm{hPa}$ & 2.0 & 4.1 & -0.9 & 2.3 \\
\hline \multicolumn{5}{|c|}{ MEAN (Unfiltered = Filtered) } \\
\hline & $90 S-30 S$ & $30 \mathrm{~S}-30 \mathrm{~N}$ & $30 N-90 N$ & Global \\
\hline $10 \mathrm{hPa}$ & 0.5 & 0.5 & 0.6 & 0.5 \\
\hline $20 \mathrm{hPa}$ & 0.1 & -0.5 & 0.1 & -0.2 \\
\hline $30 \mathrm{hPa}$ & 0.2 & -0.8 & 0.0 & -0.3 \\
\hline $50 \mathrm{hPa}$ & 0.8 & -0.1 & 0.0 & 0.1 \\
\hline $70 \mathrm{hPa}$ & 0.7 & 0.3 & 0.1 & 0.3 \\
\hline $100 \mathrm{hPa}$ & 0.2 & 1.1 & 0.0 & 0.6 \\
\hline \multicolumn{5}{|c|}{ STANDARD DEVIATION (Unfiltered) } \\
\hline & $90 S-30 S$ & $30 \mathrm{~S}-30 \mathrm{~N}$ & $30 N-90 N$ & Global \\
\hline $10 \mathrm{hPa}$ & 3.4 & 1.8 & 1.5 & 1.6 \\
\hline $20 \mathrm{hPa}$ & 2.0 & 0.7 & 0.6 & 0.7 \\
\hline $30 \mathrm{hPa}$ & 2.1 & 0.6 & 0.6 & 0.7 \\
\hline $50 \mathrm{hPa}$ & 1.5 & 0.5 & 0.4 & 0.6 \\
\hline $70 \mathrm{hPa}$ & 1.6 & 0.8 & 0.4 & 0.6 \\
\hline $100 \mathrm{hPa}$ & 0.8 & 1.3 & 0.3 & 0.8 \\
\hline
\end{tabular}




\subsection{Pressure surface temperature anomalies and differences}

Sections 4.1 and 4.2 compare NCEP1, ERA-40, and METO temperature data in pressure level (height) vs. latitude projections and in time series at the six stratospheric pressure levels. In this section the latitude vs. longitude structure of temperature is explored. As discussed at the beginning of Section 4.0, because of their linear latitude scale, the latitude vs. longitude plots of this section are not cartographically-correct Mercator plots.

The pressure level (height) vs. latitude analyses of Figs. 4-1, 4-2, and 4-3 in Section 4.1 and the time series at the six stratospheric pressure levels of Section 4.2 employ zonally-averaged data. To reduce the four-dimensional reanalysis data set to two dimensions for analysis and display, both of these sections assume that the variation of a given variable (temperature, zonal wind, etc.) from its longitudinal average is small with respect to its variation in latitude, pressure, and especially time. That this may not be a valid assumption is demonstrated by Figs. 4-10 through 4-12, which compare the difference between longitude-time series and zonally-averaged time series in the pressure level (height) vs. latitude domain, and by the following discussion.

\section{Temperature anomaly with respect to longitudinal mean}

To determine the temperature anomaly from the zonal average at each latitude, the following steps are performed with NCEP1 reanalysis data at each pressure level:

1. The mean temperature on each latitude circle is computed for each month of a 48-year period (January 1958 through December 2005). 
2. The corresponding monthly-averaged temperature for each latitude and longitude is obtained.

3. For each month and each latitude, the longitudinally-averaged temperature of step 1 is subtracted from the temperature of step 2 at each longitude to produce a temperature anomaly with respect to the longitudinal mean for each month.

The results of step 3 are plotted in Figs. 4-33 (Northern Hemisphere) and 4-34 (Southern Hemisphere) as the longitude vs. latitude view of the 48-year mean temperature anomalies for the month with the largest Northern Hemisphere rms temperature difference at each pressure level. Figure 4-33 reveals a planetary wavenumber one anomaly in temperature that migrates eastward with decreasing height from a maximum at $\sim 140^{\circ} \mathrm{E}$ and minimum at $\sim 25^{\circ} \mathrm{W}$ at $10 \mathrm{hPa}$, to a maximum at $\sim 160^{\circ} \mathrm{W}$ and minimum at $\sim 65^{\circ} \mathrm{E}$ at $100 \mathrm{hPa}$. The latitudinal center of the wave varies from $57.5^{\circ} \mathrm{N}(10 \mathrm{hPa})$ to $67.5^{\circ} \mathrm{N}(100 \mathrm{hPa})$ and its half width from $33^{\circ}$ to $40^{\circ}$. The peakto-peak amplitude of the wave also decreases with height. The wave is essentially a winter phenomenon, strongest from October or November through March or April but present, although weakly, through the remainder of the year. Labitzke and van Loon (1999) identify the source of the Aleutian high as the divergence of the upper tropospheric jet east of Japan and associated sinking to the north of the jet. This planetary wave pattern also may be observed in the FUB data shown in Fig. 2.7 of that reference. It is this longitudinal temperature anomaly that produces the increase in standard deviation and rms occurring at approximately $\pm 60^{\circ}$ latitude when zonallyaveraged time series are contrasted with longitude-time series in Figs. 4-10 through 4-12. The wavenumber one temperature anomaly is limited to the northern extratropics 
poleward of $30^{\circ} \mathrm{N}$. There is no appreciable mean temperature anomaly from the longitudinal mean in the Northern Hemisphere Tropics.

Generally similar behavior is observed in the Northern Hemisphere ERA-40 temperature anomaly from longitudinal mean plots (not shown). Latitude and longitude positions of the maxima and minima are effectively the same at corresponding pressure levels. Maxima have lower peak magnitudes at higher levels than NCEP1 and the same peak magnitudes at lower ones. Minima exhibit slightly lower magnitude at $10 \mathrm{hPa}$ and essentially the same magnitude at lower heights. The small standard deviation of differences in values of longitude-time series and zonally-averaged time series, $1.0 \mathrm{~K}$ (1958-2001), 1.4 K (1958-78), and 0.7 K (1979-2001), in Figs. 4-10 through 4-12, respectively, demonstrate that temperature anomalies from the longitudinal mean are similarly represented by the two reanalyses. These standard deviations are the largest values in their respective figures. 


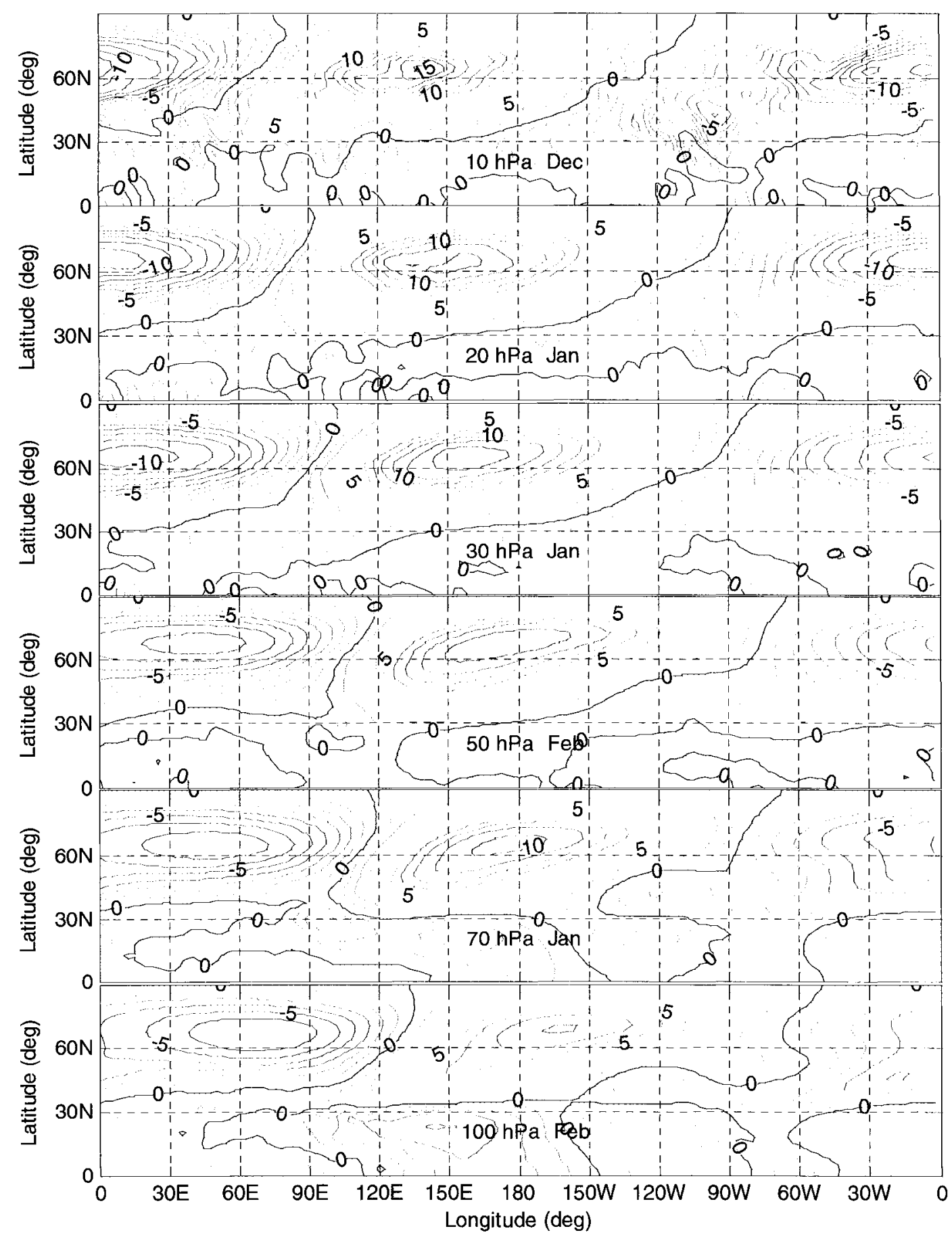

Fig. 4-33. Mean Northern Hemisphere temperature anomaly from longitudinal mean (K): NCEP1 [lon: $0^{\circ}-357.5^{\circ}$, lat: $0^{\circ}-90^{\circ} \mathrm{N}$, press: as stated, time: monthly means from Jan 1958-Dec 2005]. 
A similar temperature anomaly from the longitudinal mean exists in the Southern Hemisphere extratropics as illustrated in Fig. 4-34. Again the wavenumber one shifts eastward with decreasing height from a maximum at $\sim 90^{\circ} \mathrm{E}$ and minimum at $\sim 90^{\circ} \mathrm{W}$ at $10 \mathrm{hPa}$ to a maximum at $\sim 170^{\circ} \mathrm{E}$ and minimum at $\sim 20^{\circ} \mathrm{W}$ at $100 \mathrm{hPa}$. The latitudinal center of the wave varies from $60^{\circ} \mathrm{S}(10 \mathrm{hPa})$ to $70^{\circ} \mathrm{S}(100 \mathrm{hPa})$ and its half width from $25^{\circ}$ to $40^{\circ}$. The peak-to-peak amplitude of the wave also decreases with height. Latitude vs. longitude views of the temperature for months with maximum Southern Hemisphere rms temperature anomalies over the 48-year reanalysis are plotted at each pressure level. The wave is essentially a spring phenomena: strongest from August or October through November but present, although weakly, through the remainder of the year.

Charlton et al. (2005) discuss and illustrate the presence, typically in October, of a middle-stratosphere quasi-stationary anticyclone in the quadrant $90^{\circ} \mathrm{E}-180^{\circ}$. This is the region of the temperature maxima of Fig. 4-34.

The wavenumber one temperature anomaly is limited to the southern extratropics poleward of $30^{\circ} \mathrm{S}$. There is no appreciable mean temperature anomaly from the longitudinal mean in the Southern Hemisphere Tropics.

Similar behavior is observed in the Southern Hemisphere ERA-40 temperature anomaly from longitudinal mean plots (not shown). Latitudinal and longitudinal placement of maxima and minima are the same as NCEP1. Maxima have a lower peak magnitude at $10 \mathrm{hPa}$, higher at $50 \mathrm{hPa}$, and are approximately the same otherwise. Minima exhibit lower magnitude at $10 \mathrm{hPa}$, higher at $20 \mathrm{hPa}$ and $50 \mathrm{hPa}$, and are approximately equal otherwise. 


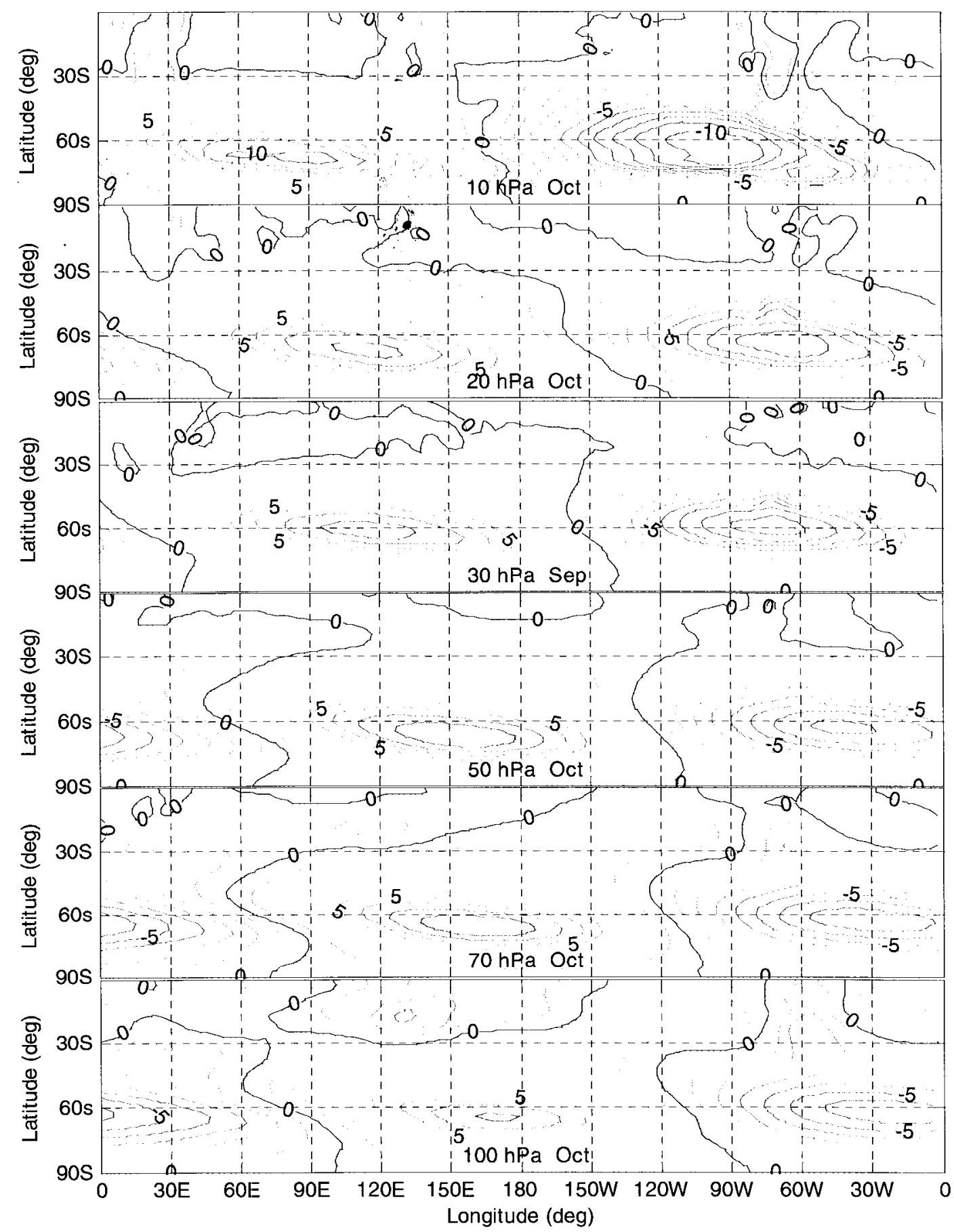

Fig. 4-34. Mean Southern Hemisphere temperature anomaly from longitudinal mean (K): NCEPl [lon: $0^{\circ}-357.5^{\circ}$, lat: $90^{\circ} \mathrm{S}-0^{\circ}$, press: as stated, time: monthly means from Jan 1958-Dec 2005]. 
The maximum and minimum temperature anomalies for December of each year and their corresponding longitude and latitude are plotted for $10 \mathrm{hPa}$ in the Northern Hemisphere in Fig. 4-35. This figure shows 48 years of NCEP1 data (1958-2005) and 44 years of ERA-40 data (1958-2001). Figure 4-36 contains comparable data for the Southern Hemisphere.

Figure 4-35 shows that, with the exception of 1979, the longitudes of the maxima and latitudes of both maxima and minima are quite constant with time. The minima move within a band from $0^{\circ}$ to $90^{\circ} \mathrm{W}$. In 1979 the NCEP1 maxima longitude moved westerly $120^{\circ}$ and the latitudes of both NCEP1 and ERA-40 maxima move to the Tropics with an accompanying decrease in maxima amplitude. Since this dramatic behavior occurred only once and in both NCEP1 and ERA-40 data, it is more likely a result of the beginning of modern satellite data assimilation than a true atmospheric phenomena. Tables 3-2 (Section 3.3) and 4-7 (Section 4.5) contain no stratospheric sudden warming events for December 1979. The major midwinter warmings in December 1987, 1998, and 2001 do not appear in any of the variables of Fig. 4-35. Summarizing the data of Fig. 4-35, anomalies are $20 \mathrm{~K}$ to $40 \mathrm{~K}$ above and below the longitudinal mean, respectively, for a wavenumber one amplitude of $10 \mathrm{~K}$ to $20 \mathrm{~K}$ (wave amplitude is onehalf of the difference). This significant stationary wave is eliminated by zonal averaging.

Figure 4-36 repeats the information of Fig. 4-35 for the month of October at $10 \mathrm{hPa}$ in the Southern Hemisphere. The locations of the anomalies over time are reasonably consistent, with the exception of several transients. The 2002 departure from the longitude mean may be a consequence of the first Southern Hemisphere stratospheric 
sudden warming in late September 2002. However, the behavior of the anomaly magnitudes changes dramatically after 1979 from large magnitudes and large variance to very consistent levels. Their values after 1979 are $5 \mathrm{~K}$ to $10 \mathrm{~K}$, approximately half of the Northern Hemisphere value, but still significant. 

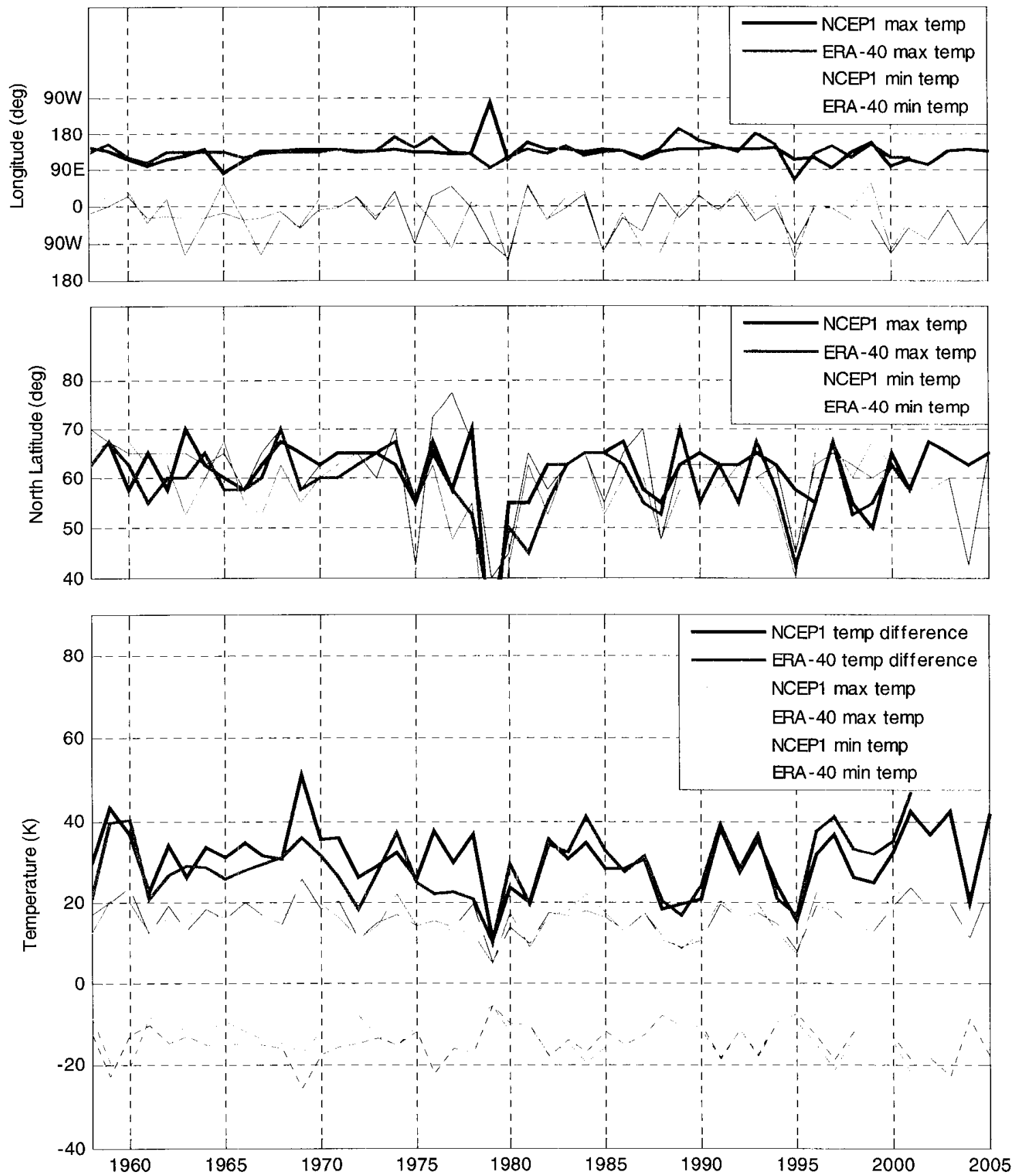

Fig. 4-35. December Northern Hemisphere monthly-averaged temperature anomaly from longitudinal mean $(K)$. Longitude (upper) and latitude (middle) location of maximum and minimum anomalies and magnitudes of anomalies (lower).

[lon: $0^{\circ}-357.5^{\circ}$, lat: $0^{\circ}-90^{\circ} \mathrm{N}$, press: $10 \mathrm{hPa}$,

time: Dec monthly average 1958-2005 (NCEPI), 1958-2001 (ERA-40)]. 

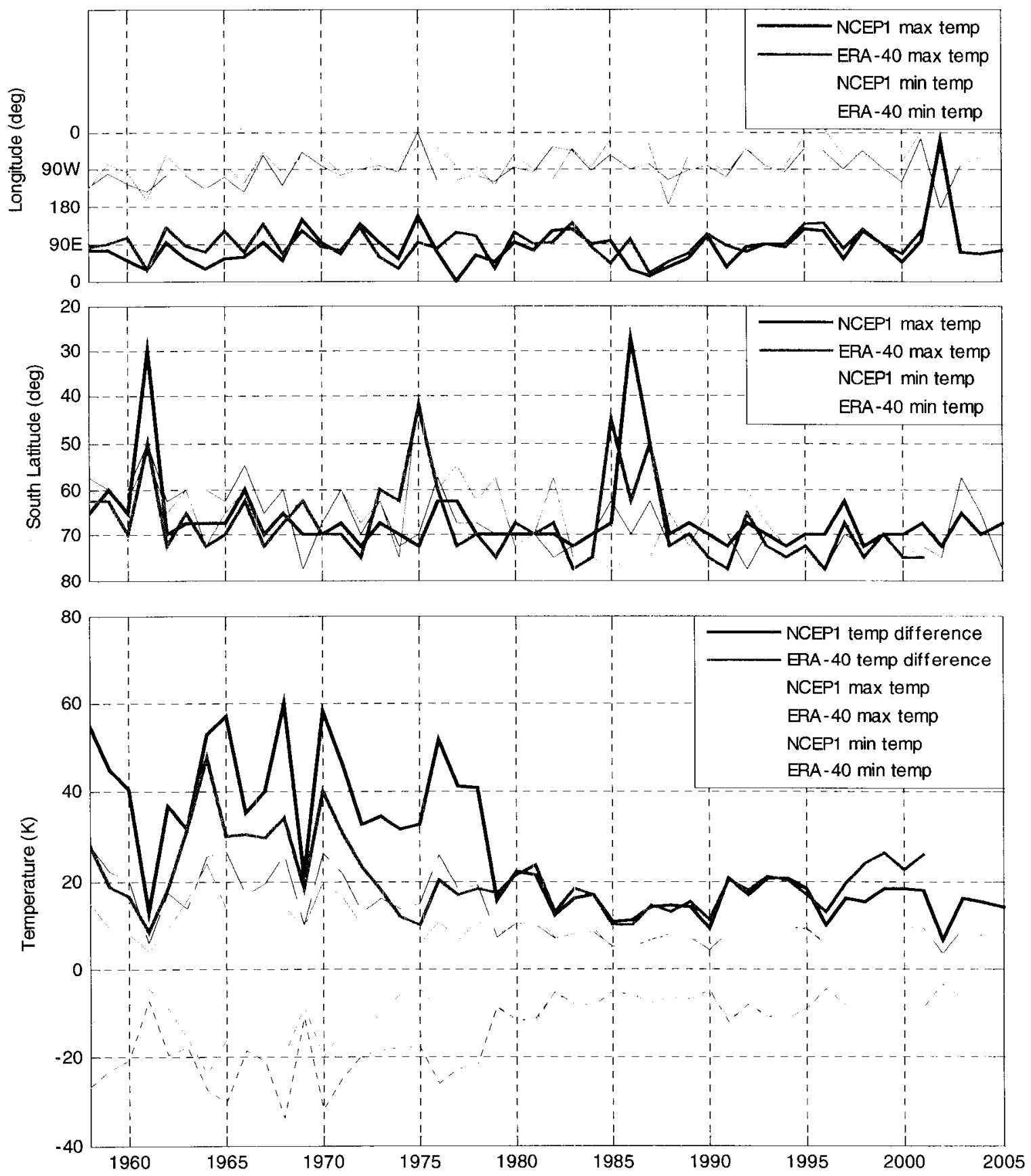

Fig. 4-36. October Southern Hemisphere monthly-averaged temperature anomaly from longitudinal mean $(K)$. Longitude (upper) and latitude (middle) location of maximum and minimum anomalies and magnitudes of anomalies (lower).

[lon: $0^{\circ}-357.5^{\circ}$, lat: $90^{\circ} \mathrm{S}-0^{\circ}$, press: $10 \mathrm{hPa}$,

time: Oct monthly average 1958-2005 (NCEP1), 1958-2001 (ERA-40)]. 
Table 4-4 summarizes the mean and standard deviation of temperature anomaly maxima and minima, and their differences for the month with largest rms temperature anomaly difference at each pressure level. In the Northern Hemisphere all mean anomalies and their differences decrease with height while in the Southern Hemisphere maximum means are found at $50 \mathrm{hPa}$. Again the amplitude of the wavenumber one at the latitude of these anomalies is one-half the magnitude of the tabulated difference.

Anomaly latitude and longitude standard deviations for heights below $10 \mathrm{hPa}$ are similar to those at $10 \mathrm{hPa}$. They generally decrease (NCEP1) with decreasing heights but increase then decrease (ERA-40) slightly with decreasing heights. Figure 4-37, which plots the standard deviation of the temperature anomaly longitude-time series for both NCEP1 and ERA-40, illustrates these trends in a full pressure level vs. latitude domain. The figure results from the following procedure where, for each pressure level:

1. The zonally-averaged temperature for each latitude and month is obtained for the 264 months from January 1980 through December 2001.

2. The temperature for each longitude, latitude, and month is obtained.

3. The results of step 1 are subtracted from those of step 2, creating an array of longitude, temperature, and time.

4. A longitude-time series (Appendix D) is formed for each latitude.

5. The standard deviation of the longitude-time series is computed for each latitude. The dominant variance in the figure is centered at $\pm 60^{\circ}$ latitude, the latitudes of the anomalies discussed throughout this section. The magnitude of this variance, computed over all months of the year for a full 22 years, is diminished from the 7 to $14 \mathrm{~K}$ (Northern Hemisphere) and 3.5 to $7 \mathrm{~K}$ (Southern Hemisphere) observed above during months of peak wavenumber one amplitude. Figure 4-37 graphically confirms that 
essentially no temperature anomalies from longitudinal mean exist equatorward of $\pm 30^{\circ}$ latitude in both hemispheres. 
Table 4-4. Mean (and standard deviation) of maxima and minima temperature anomalies from longitudinal mean and their differences (1980-2001) (K).

\begin{tabular}{|c|c|c|c|c|c|c|c|}
\hline \multirow[b]{2}{*}{$\begin{array}{c}\text { Pressure } \\
\text { level } \\
\text { (hPa) }\end{array}$} & \multirow[b]{2}{*}{ Month } & \multicolumn{2}{|c|}{ MAXIMA } & \multicolumn{2}{|c|}{ MINIMA } & \multicolumn{2}{|c|}{ DIFFERENCE } \\
\hline & & NCEP1 & ERA-40 & NCEP1 & ERA-40 & NCEP1 & ERA-40 \\
\hline \multicolumn{8}{|c|}{ NORTHERN HEMISPHERE } \\
\hline 10 & Dec & $\begin{array}{l}15.2 \\
(3.9)\end{array}$ & $\begin{array}{l}16.6 \\
(4.8)\end{array}$ & $\begin{array}{l}-13.0 \\
(3.6)\end{array}$ & $\begin{array}{l}-14.1 \\
(4.0)\end{array}$ & $\begin{array}{l}28.3 \\
(7.1)\end{array}$ & $\begin{array}{l}30.6 \\
(8.4)\end{array}$ \\
\hline 20 & Jan & $\begin{array}{l}14.9 \\
(3.8)\end{array}$ & $\begin{array}{l}15.7 \\
(4.2)\end{array}$ & $\begin{array}{l}-13.9 \\
(4.6)\end{array}$ & $\begin{array}{l}-14.2 \\
(4.8)\end{array}$ & $\begin{array}{l}28.8 \\
(7.7)\end{array}$ & $\begin{array}{l}29.9 \\
(8.2)\end{array}$ \\
\hline 30 & Jan & $\begin{array}{l}14.6 \\
(4.0)\end{array}$ & $\begin{array}{l}15.3 \\
(4.1)\end{array}$ & $\begin{array}{l}-12.7 \\
(4.4)\end{array}$ & $\begin{array}{l}-13.0 \\
(4.5)\end{array}$ & $\begin{array}{l}27.3 \\
(7.7)\end{array}$ & $\begin{array}{l}28.3 \\
(8.1)\end{array}$ \\
\hline 50 & Feb & $\begin{array}{r}12.0 \\
(3.7)\end{array}$ & $\begin{array}{l}12.7 \\
(4.0)\end{array}$ & $\begin{array}{l}-11.4 \\
(3.7)\end{array}$ & $\begin{array}{l}-11.7 \\
(3.7)\end{array}$ & $\begin{array}{l}23.4 \\
(7.1)\end{array}$ & $\begin{array}{l}24.5 \\
(7.3)\end{array}$ \\
\hline 70 & Jan & $\begin{array}{r}11.9 \\
(3.0)\end{array}$ & $\begin{array}{l}12.1 \\
(3.0)\end{array}$ & $\begin{array}{c}-9.4 \\
(3.0)\end{array}$ & $\begin{array}{c}-9.5 \\
(3.1)\end{array}$ & $\begin{array}{l}21.3 \\
(5.7)\end{array}$ & $\begin{array}{l}21.6 \\
(5.8)\end{array}$ \\
\hline 100 & Feb & $\begin{array}{c}9.9 \\
(2.7)\end{array}$ & $\begin{array}{l}10.2 \\
(2.8)\end{array}$ & $\begin{array}{l}-8.9 \\
(2.4)\end{array}$ & $\begin{array}{c}-8.9 \\
(2.3)\end{array}$ & $\begin{array}{r}18.8 \\
(4.7)\end{array}$ & $\begin{array}{l}19.0 \\
(4.8)\end{array}$ \\
\hline \multicolumn{8}{|c|}{ SOUTHERN HEMISPHERE } \\
\hline 10 & Oct & $\begin{array}{c}8.0 \\
(1.7)\end{array}$ & $\begin{array}{c}8.9 \\
(2.3)\end{array}$ & $\begin{array}{c}-8.2 \\
(2.3)\end{array}$ & $\begin{array}{c}-9.1 \\
(2.7)\end{array}$ & $\begin{array}{l}16.2 \\
(3.8)\end{array}$ & $\begin{array}{l}17.9 \\
(4.9)\end{array}$ \\
\hline 20 & Oct & $\begin{array}{c}9.0 \\
(2.4)\end{array}$ & $\begin{array}{l}10.8 \\
(2.4)\end{array}$ & $\begin{array}{l}-10.2 \\
(2.5)\end{array}$ & $\begin{array}{l}-12.4 \\
(3.0)\end{array}$ & $\begin{array}{l}19.1 \\
(4.7)\end{array}$ & $\begin{array}{l}23.3 \\
(5.2)\end{array}$ \\
\hline 30 & Sep & $\begin{array}{l}10.6 \\
(3.9)\end{array}$ & $\begin{array}{l}12.7 \\
(4.9)\end{array}$ & $\begin{array}{l}-10.8 \\
(3.9)\end{array}$ & $\begin{array}{l}-12.5 \\
(4.4)\end{array}$ & $\begin{array}{l}21.4 \\
(7.5)\end{array}$ & $\begin{array}{l}25.2 \\
(9.0)\end{array}$ \\
\hline 50 & Oct & $\begin{array}{l}11.9 \\
(3.5)\end{array}$ & $\begin{array}{l}13.1 \\
(3.8)\end{array}$ & $\begin{array}{l}-12.1 \\
(3.8)\end{array}$ & $\begin{array}{l}-13.2 \\
(4.2)\end{array}$ & $\begin{array}{l}23.9 \\
(7.0)\end{array}$ & $\begin{array}{l}26.2 \\
(7.7)\end{array}$ \\
\hline 70 & Oct & $\begin{array}{l}11.7 \\
(3.8)\end{array}$ & $\begin{array}{l}12.9 \\
(3.9)\end{array}$ & $\begin{array}{c}-11.6 \\
(4.0)\end{array}$ & $\begin{array}{l}-12.5 \\
(4.1)\end{array}$ & $\begin{array}{l}23.4 \\
(7.4)\end{array}$ & $\begin{array}{l}25.4 \\
(7.7)\end{array}$ \\
\hline 100 & Oct & $\begin{array}{l}10.5 \\
(3.7)\end{array}$ & $\begin{array}{l}11.4 \\
(3.9)\end{array}$ & $\begin{array}{l}-10.2 \\
(3.5)\end{array}$ & $\begin{array}{c}-10.8 \\
(3.5)\end{array}$ & $\begin{array}{l}20.7 \\
(6.8)\end{array}$ & $\begin{array}{l}22.2 \\
(7.1)\end{array}$ \\
\hline
\end{tabular}



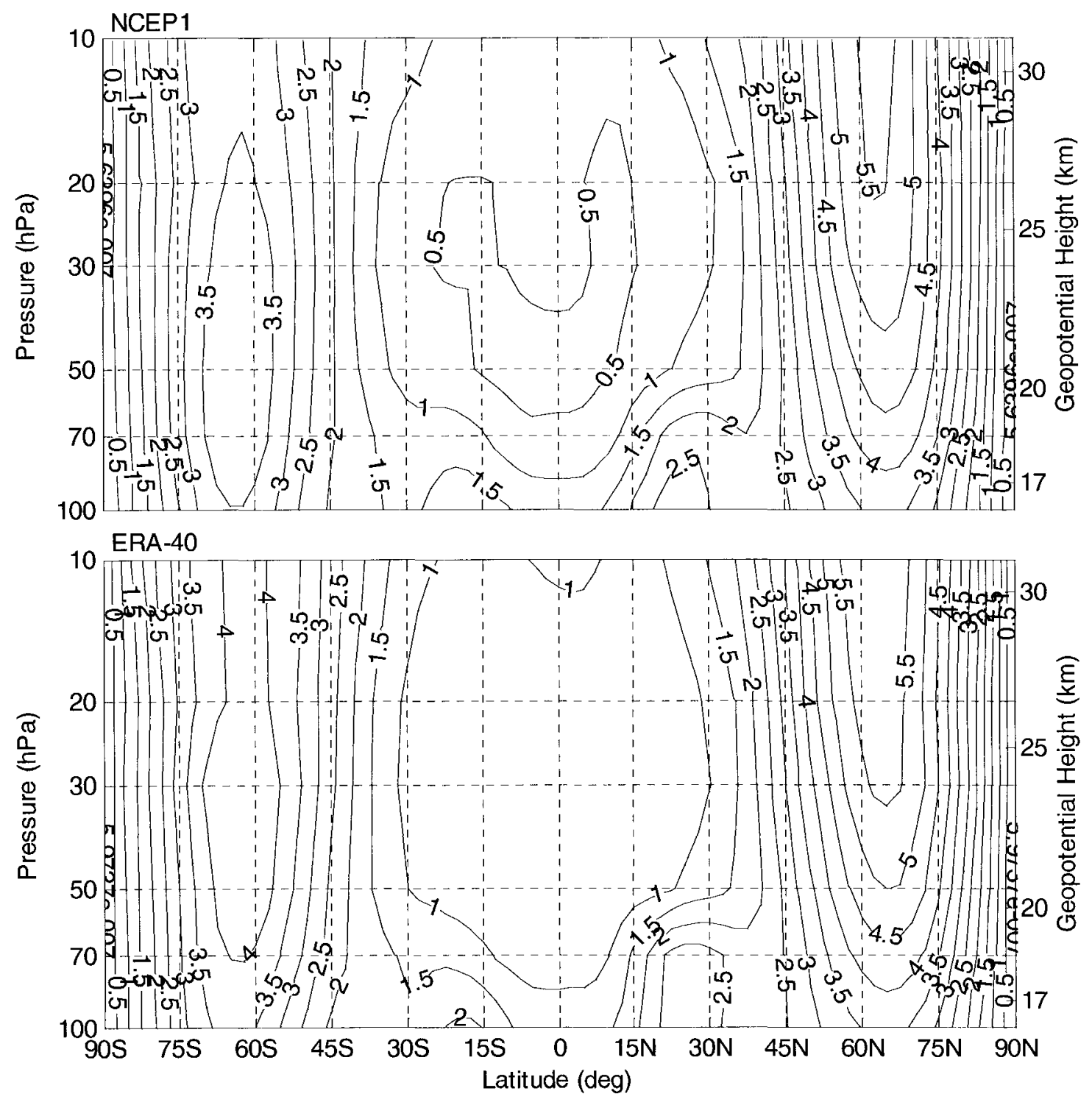

Fig. 4-37. Standard deviation of temperature anomaly longitude-time series (K). [lon: $0^{\circ}-357.5^{\circ}, \quad$ lat: $90^{\circ} \mathrm{S}-90^{\circ} \mathrm{N}$, press: $100-10 \mathrm{hPa}$, time: Jan 1980 through Dec 2001].

\section{Latitude vs. longitude view of temperature differences}

Figures 4-38 through 4-43 plot the mean, standard deviation, and maxima of monthly-averaged NCEP1 minus ERA-40 temperature differences from 1958 through 2001 for the six stratospheric pressure levels from $100 \mathrm{hPa}$ through $10 \mathrm{hPa}$, respectively. 
The figures represent level-by-level latitude vs. longitude views of the temperature differences projected on to the pressure level vs. latitude plots of the temperature longitude-time series of Fig. 4-7. Additional insight into the three-dimensional structure of the NCEP1 minus ERA-40 temperature differences may be obtained by comparison of Fig. 4-1 with Figs. 4-38 through 4-43.

The infamous four K step in equatorial temperature occurring in 1979 appears in Fig. 4-38 as the two K contours that are the centroids of the discontinuity. These regions, one spanning the Indian Ocean and the other west of Ecuador in the eastern Pacific Ocean, are displaced 180 degrees from one another. As in Fig. 4-1, there is no extratropical mean temperature difference at $100 \mathrm{hPa}$ greater than one $\mathrm{K}$. Maximum standard deviation and maximum temperature difference occur above coastal Antarctica from the Weddell Sea westward to Marie Byrd Land. North of $50^{\circ} \mathrm{S}$, there are no maximum temperature differences greater than five K. Over a large portion of the Earth, especially between latitude $30^{\circ} \mathrm{S}$ and $30^{\circ} \mathrm{N}, \mathrm{NCEP} 1$ is warmer, in both mean and maxima, than ERA-40; this is consistent with the "warm tropical tropopause" identified in Randel et al. $(2002,2004)$ for the period 1992-99.

Above the tropopause, the $70 \mathrm{hPa}$ pressure surface displayed in Fig. 4-39 contains mean differences of approximately one $\mathrm{K}$, again with NCEP1 warmer than ERA-40. The standard deviation of the temperature differences is quite zonal with the maximum magnitude at the South Pole slightly larger than at $100 \mathrm{hPa}$. Again, the maximum monthly difference is located over Antarctica and is greater than at $100 \mathrm{hPa}$ as would be expected by the bulge centered at approximately $40 \mathrm{hPa}$ and the South Pole in Fig. 4-1 
maxima. This is the lowest altitude at which Southern Hemisphere differences begin to become significant.

Figure 4-40 shows that at $50 \mathrm{hPa}$ the mean temperature difference is greater than one $\mathrm{K}$ only in the southern polar regions, with NCEP1 warmer than ERA-40 except in several isolated areas. As at $70 \mathrm{hPa}$, the standard deviation of the temperature difference is zonal and on the order of one $\mathrm{K}$ except again in the southern subpolar and polar regions where it continues to increase over that of 100 and $70 \mathrm{hPa}$. The maxima subplot is very similar to that at $70 \mathrm{hPa}$ except that the southern subpolar and polar temperature differences are slightly more zonal.

Figure 4-41 shows the that mean temperature difference in the Tropics at $30 \mathrm{hPa}$ is generally negative (ERA-40 is warmer) while in the southern subpolar region the difference is positive (NCEP1 is warmer) at about the same value as at $50 \mathrm{hPa}$. The tropical standard deviation at $30 \mathrm{hPa}$ is similar to that at $50 \mathrm{hPa}$ but larger in magnitude in the northern subpolar regions. The maxima subplot is very similar to that at $50 \mathrm{hPa}$ except for larger values in the northern subpolar and polar regions; it is also less zonal in structure.

Mean temperature differences in Fig. 4-42 at $20 \mathrm{hPa}$ are essentially negative with only small positive subpolar and polar areas. The $20-\mathrm{hPa}$ standard deviation of the temperature differences is essentially identical to that at $30 \mathrm{hPa}$. Maxima, as is the case at 70,50 , and $30 \mathrm{hPa}$, are negative in the Tropics and subtropics. At $20 \mathrm{hPa}$ maximum differences are slightly less in the southern subpolar regions than at $30 \mathrm{hPa}$ and greater in the northern subpolar regions. 
The mean temperature difference at $10 \mathrm{hPa}$ in Fig. 4-43 illustrates "a pathological problem" (Trenberth and Stepaniak 2002) resulting from NCEP1's use of a sigma coordinate system to the top of the assimilating model and a rigid top (Section 2.2.1). Although initially detected as a "two-delta vertical wave in the divergence of the wind field above steep topography," the consequences of this problem are seen here in the temperature difference field. From east to west along $30^{\circ} \mathrm{S}$, significantly rippled temperature gradients are evident at or near South Africa, east and west Australia, and especially the Andes, where the "problem" was first observed. In the Northern Hemisphere from west to east, the Himalayas, the west coast of North America, and Greenland are also sites of significant temperature gradients. The gradients centered at $180^{\circ}$ over Antarctica may be caused by the mountain range at that longitude. At $10 \mathrm{hPa}$, the average temperature difference is again positive, i.e., NCEP1 is warmer. The structure of the standard deviation field is similar to those at lower heights but with higher values throughout. The maximum temperature difference field at $10 \mathrm{hPa}$ is unique among all levels because of its large region of large negative differences in the Southern Hemisphere.

Simmons (2006a) discusses the ERA-40 solution to unrealistic spectral ripples in level regions adjacent to major mountain ranges. The use of a finer spectral resolution with unchanged grid-point resolution markedly reduced these ripples in their model orography in these regions.

In the mean subplots of Figs. 4-38 through 4-43, which are averaged over 528 months, the variations from longitudinal means seen in Figs. 4-33 and 4-34 are not 
apparent. In addition, the differences between zonal and longitude-time series of Figs. 4-11 through 4-14 are not seen in Figs. 4-38 through 4-43 because the former reflect the larger variance and maxima of month-by-month differences of the two methods (zonally-averaged time series and longitude-time series) while the latter figures show the month-by-month differences of the two reanalyses.

Latitude vs. longitude plots for the time period 1979-2001 (not shown) differ only slightly from those of Figs. 4-38 through 4-43. During this later interval, slightly larger mean temperature differences exist at $100 \mathrm{hPa}$ and the Equator. Standard deviation and rms values of differences from 100 to $20 \mathrm{hPa}$ southward of $45^{\circ} \mathrm{S}$ are effectively half the corresponding values of those for 1958-2001; in all other regions of pressure level vs. latitude space there is minimal difference. Maxima of temperature differences poleward of $\pm 45^{\circ}$ latitude for 1979-2001 are again approximately half the corresponding values for 1958-2001 but otherwise only small differences exist. Comparison of Fig. 4-7 (pressure level vs. latitude plots for 1958-2001) with Fig. 4-9 (similar plots for 1979-2001) will illustrate the differences discussed above. 

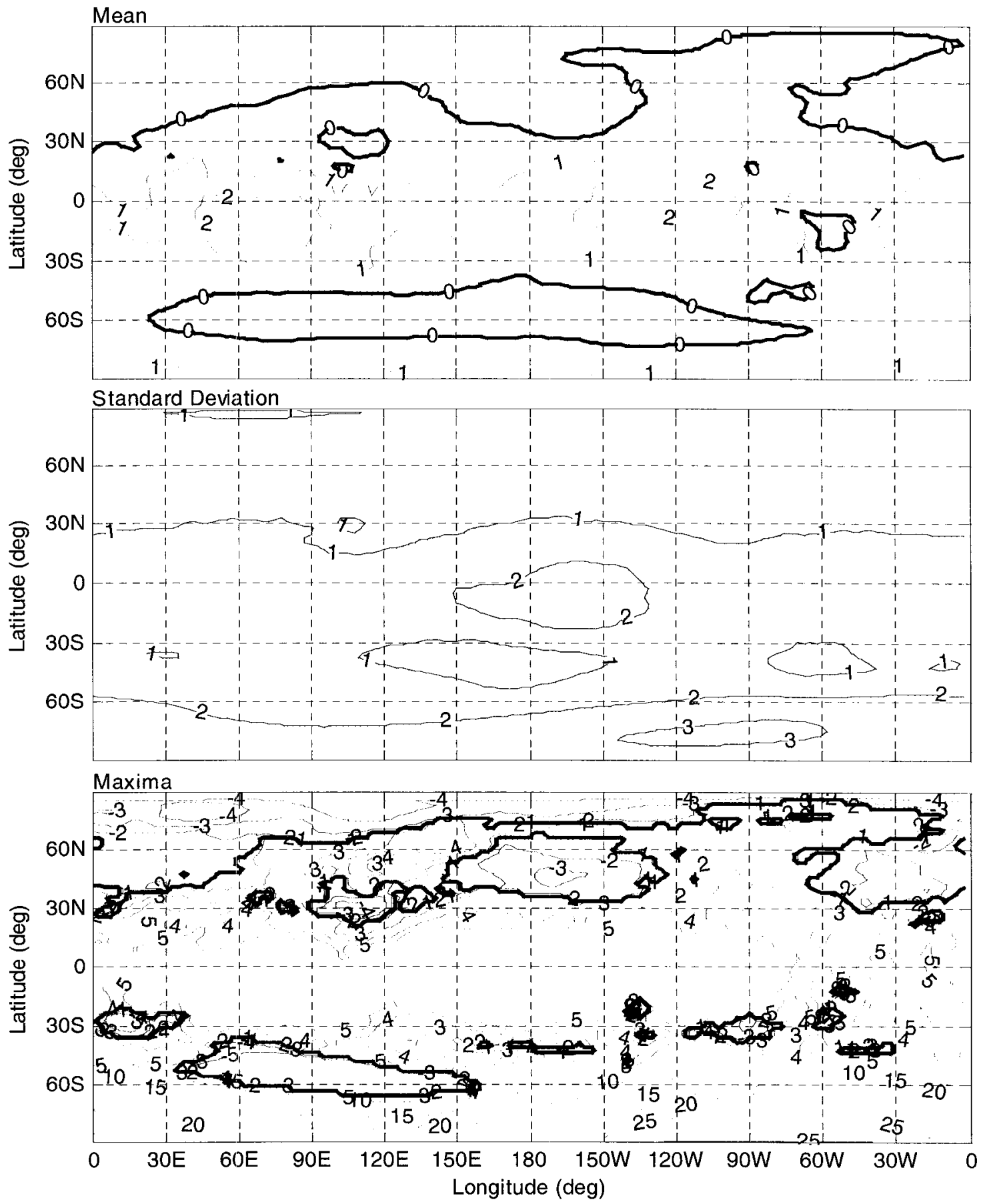

Fig. 4-38. Mean, standard deviation, and maxima of time series of monthly-averaged temperature differences $(K):$ NCEPI minus ERA-40

[lon: $0^{\circ}-357.5^{\circ}$, lat: $90^{\circ} \mathrm{S}-90^{\circ} \mathrm{N}$, press: $100 \mathrm{hPa}$, time: Jan 1958-Dec 2001]. 

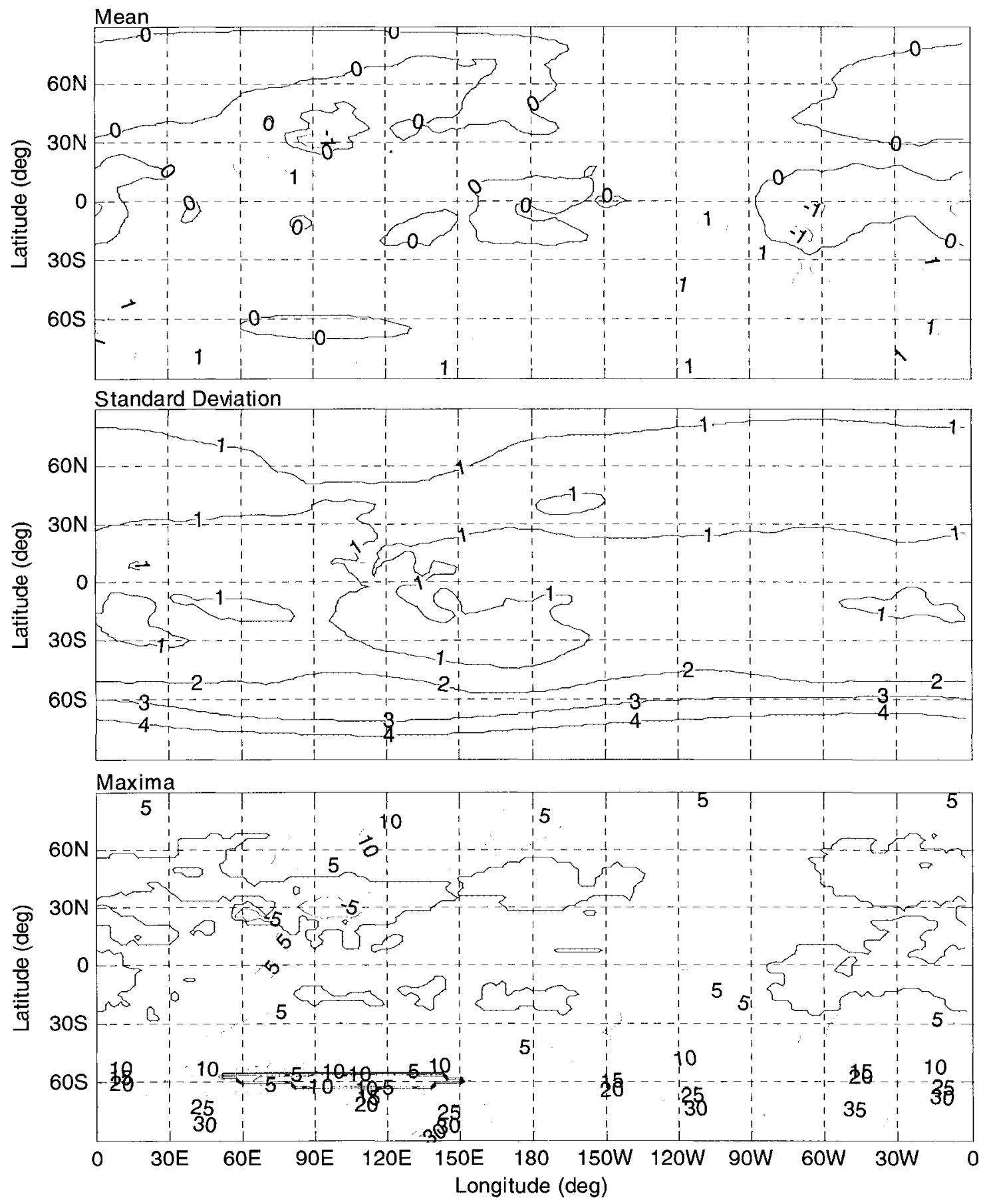

Fig. 4-39. Same as Fig. 4-38 except press: $70 \mathrm{hPa}$. 

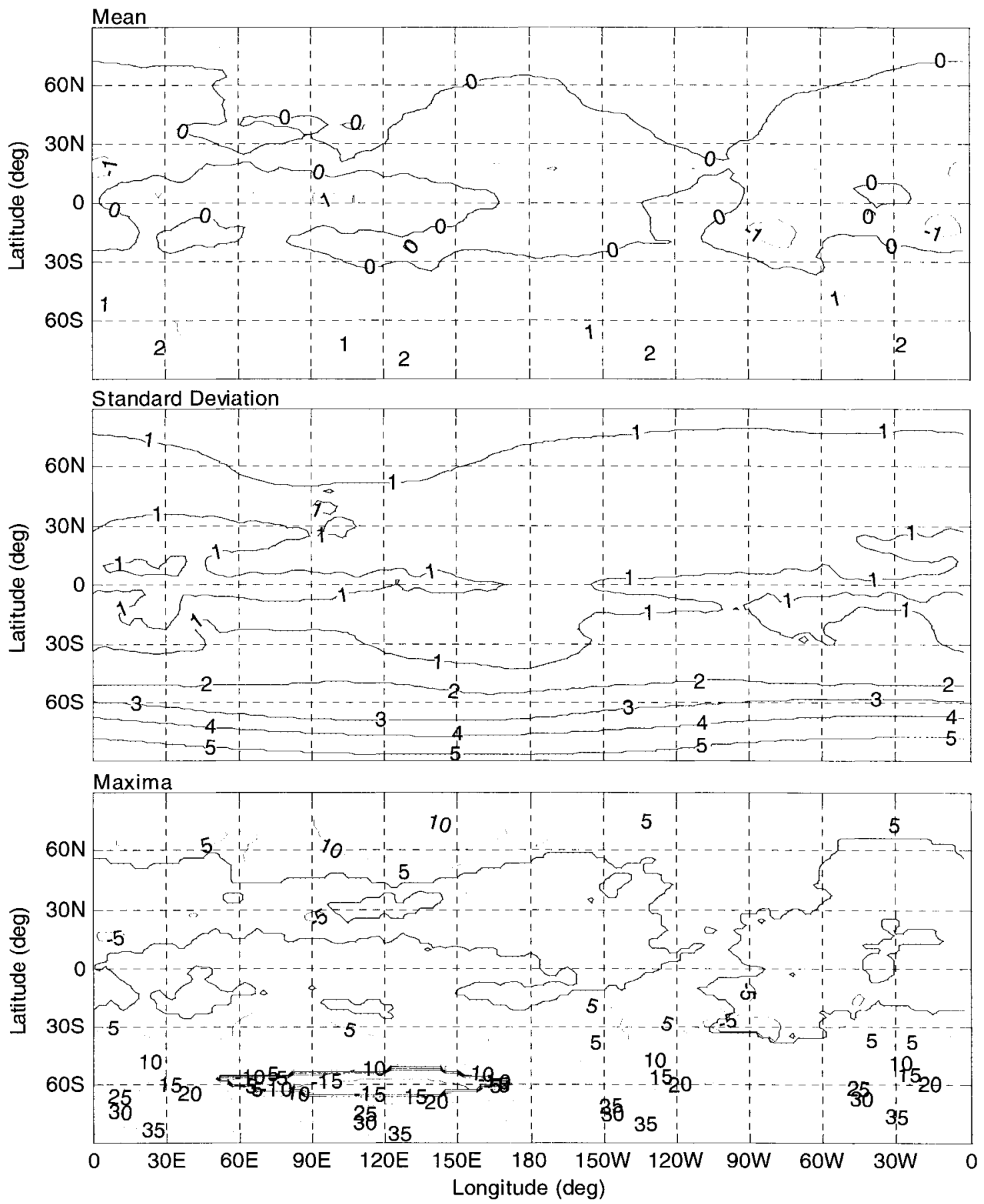

Fig. 4-40. Same as Fig. 4-38 except press: $50 \mathrm{hPa}$. 

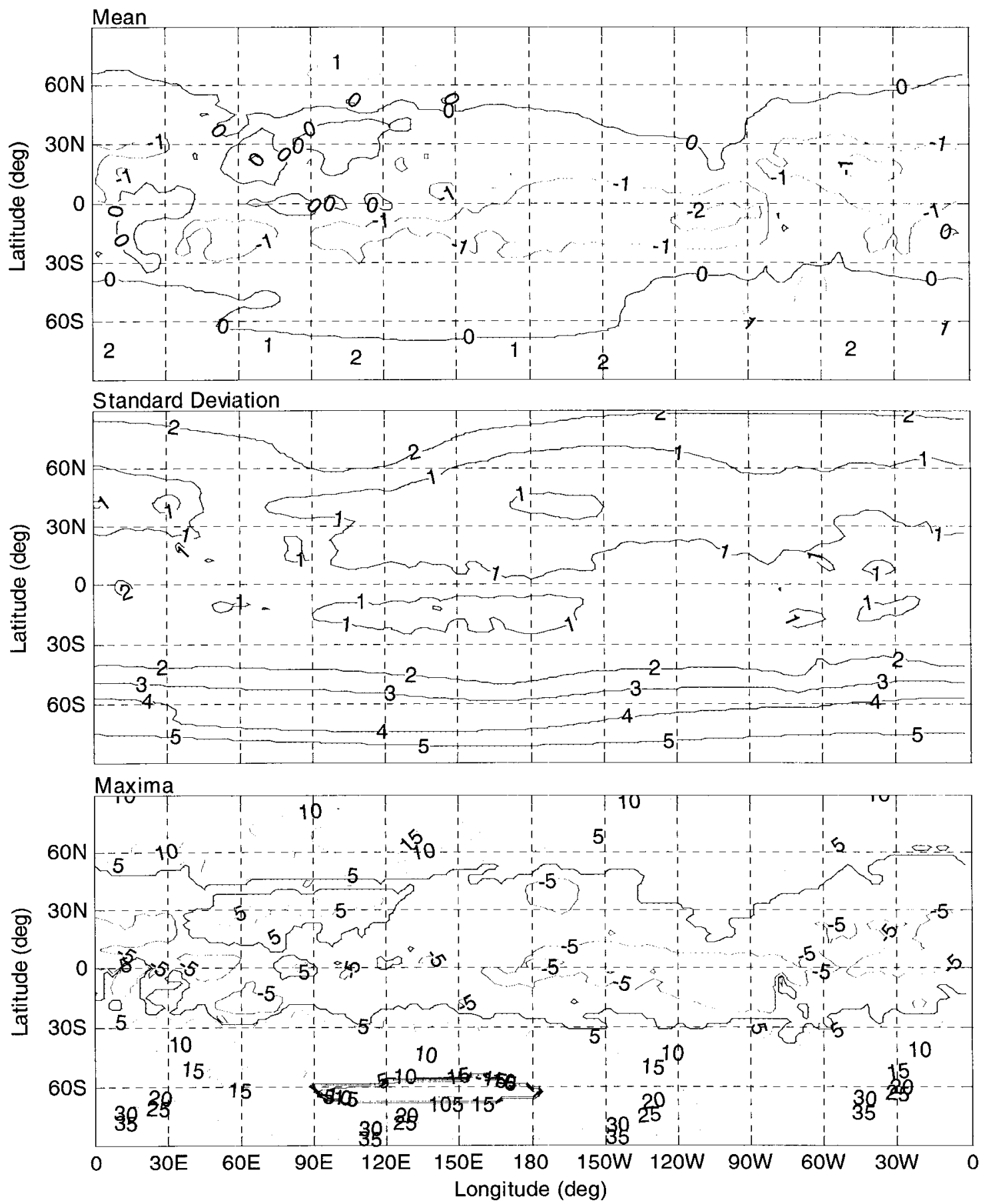

Fig. 4-41. Same as Fig. 4-38 except press: $30 \mathrm{hPa}$. 

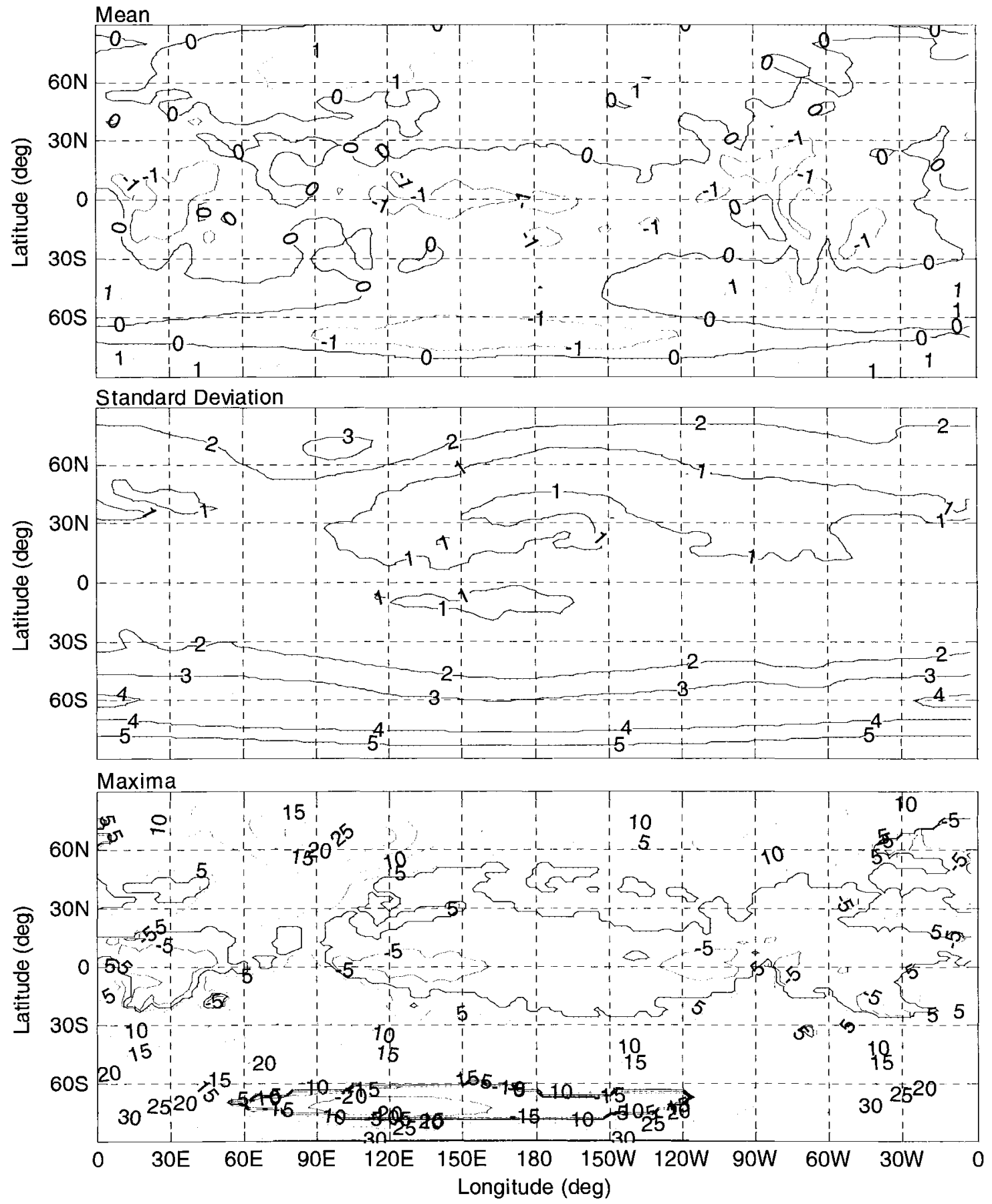

Fig. 4-42. Same as Fig. 4-38 except press: $20 \mathrm{hPa}$. 

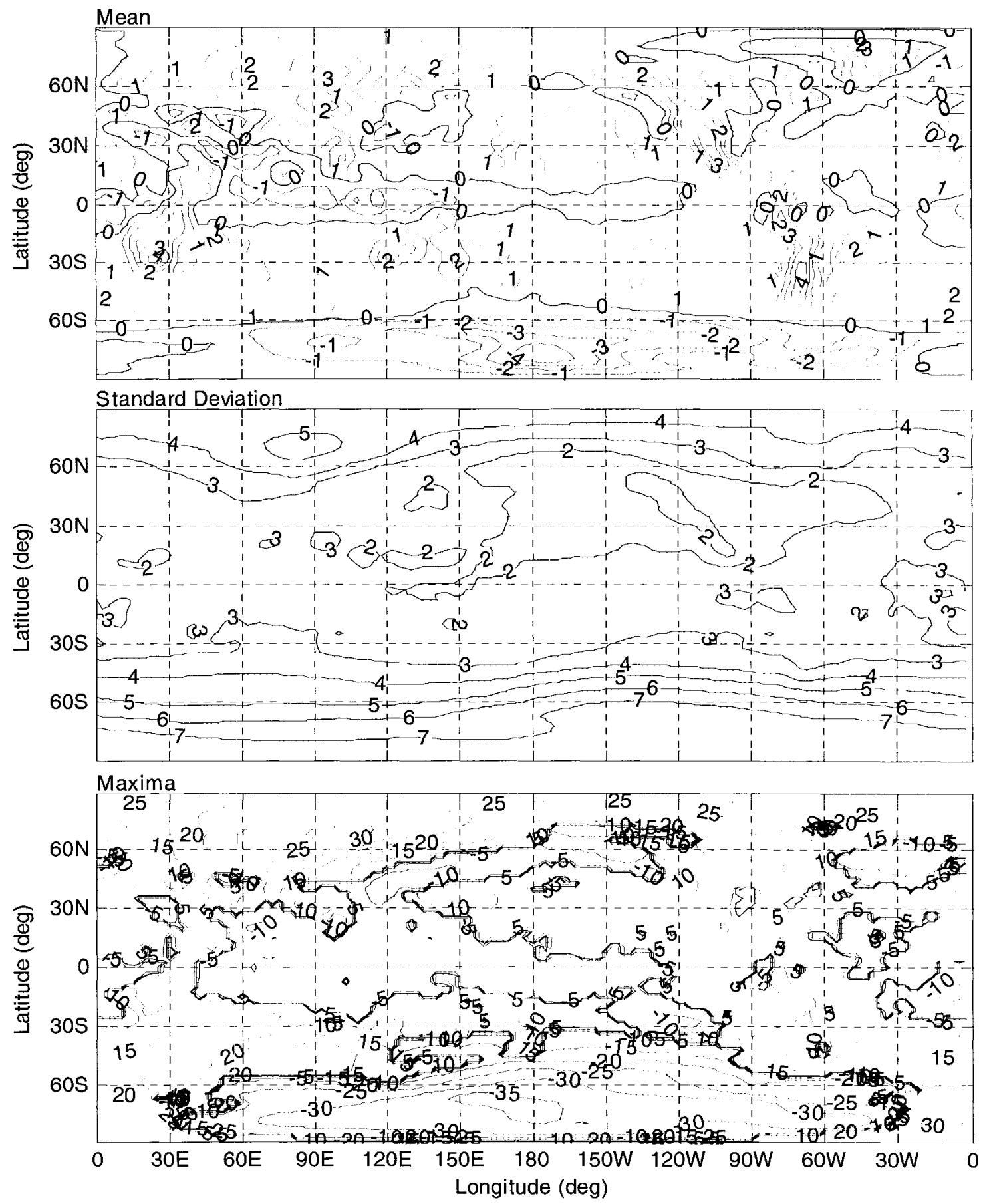

Fig. 4-43. Same as Fig. 4-38 except press: $10 \mathrm{hPa}$. 


\section{TLS comparison}

Throughout this thesis, the period from 1979 to the present has been identified as the modern satellite era and the incorporation and influence of satellite meteorological data on the various reanalysis efforts described. This section directly compares reanalysis and satellite temperature data.

Radiance data from satellite sensors are defined over deep vertical layers and contain limited information on vertical radiance/temperature distribution. Figure 2-6 illustrates that the range of lower stratospheric radiance sensed by MSU4 and AMSU9 includes radiance from 453 to $0.45 \mathrm{hPa}$. Significant contributions ( $1 \%$ of peak) are present from 290 to $5.5 \mathrm{hPa}$.

To compare reanalysis and satellite temperature data, weighting factors must be applied to reanalysis temperatures at their available pressure levels, corresponding to those sensed by the satellite-borne sensors, to simulate the temperature reported by the TLS system. Figure 4-44 plots the weighting factors for MSU4 as graphically extracted from Fig. 1 of Seidel et al. (2004). Hereinafter, this set of weighting factors from Seidel et $\underline{\text { al. }}$ as well as graphical and tabular results derived from them are identified as 'SEA' in this section. A second set of weighting factors was computed from weighting functions downloaded from Remote Sensing Systems (RSS) (Section 2.3.4 and Appendix B). RSS weighting functions were interpolated to mid-points between the pressure levels shown in Fig. 4-44 and trapezoidally integrated to form the second set of weighting factors, which are also plotted in Fig. 4-44. Differences between the two sets 
of weighting factors at every level are small but, as will be demonstrated, the resulting temperature differences are not negligible.

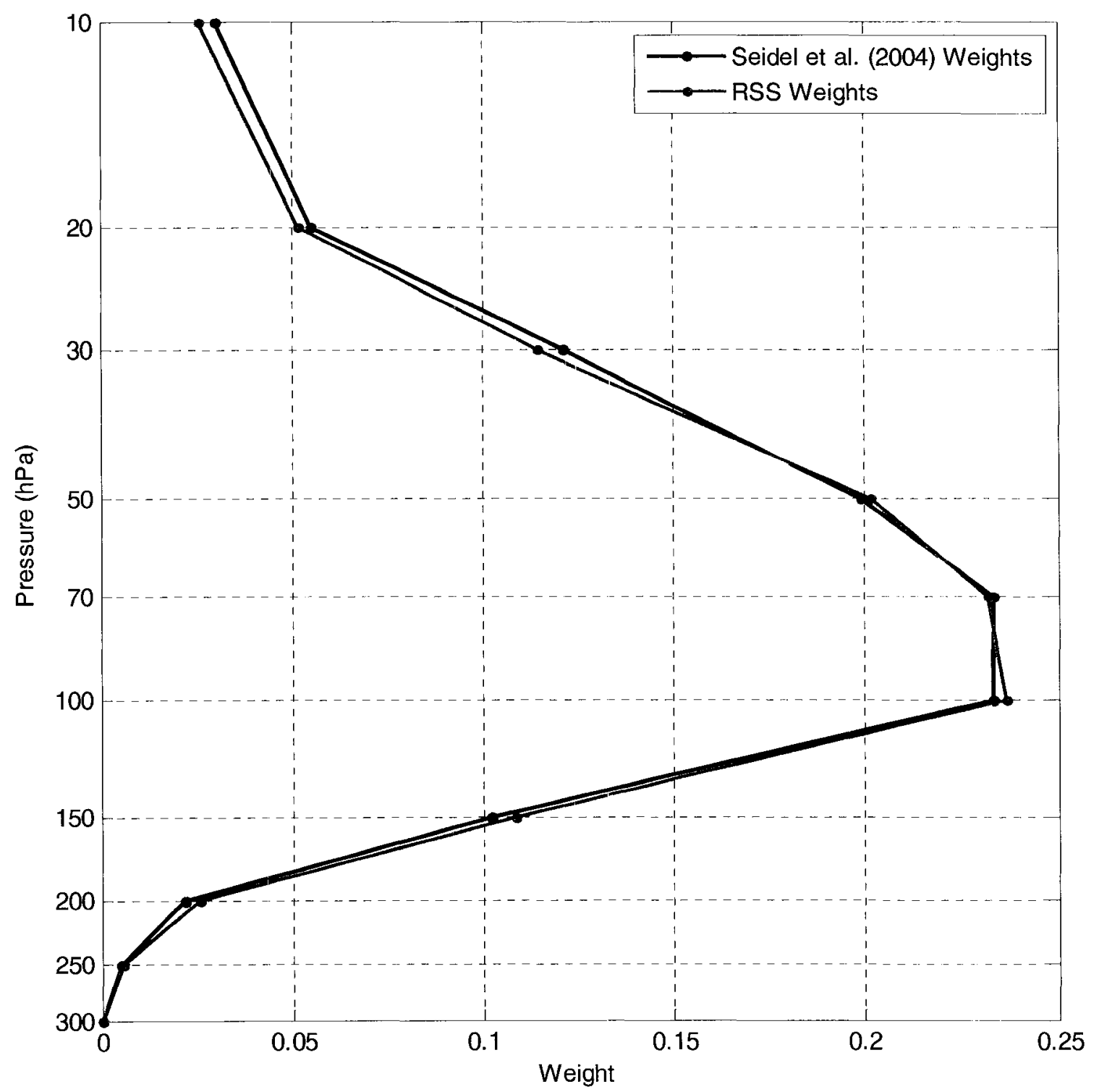

Fig. 4-44. Seidel et al. (2004) and RSS weighting factors vs. pressure level.

Figures 4-45 and 4-46 display the mean, standard deviation, and maxima of temperature differences of NCEP1 and ERA-40 reanalyses with RSS weighting, respectively, minus TLS data. Significant bias exists in these results, with both 
reanalyses everywhere warmer than TLS by approximately $1.5 \mathrm{~K}$ globally. RSS

arbitrarily chose $N O A A-10$ as its temperature reference, applying offsets to the data of all other satellites. The intent and implications of this strategy are summarized by Carl Mears (2006, personal communication).

If we had chosen a different satellite, the mean temperature of our dataset might be different by a value up to around $1 \mathrm{~K}$. So absolute comparisons are not so meaningful. Our datasets are developed to study changes in temperature more than absolute values. 

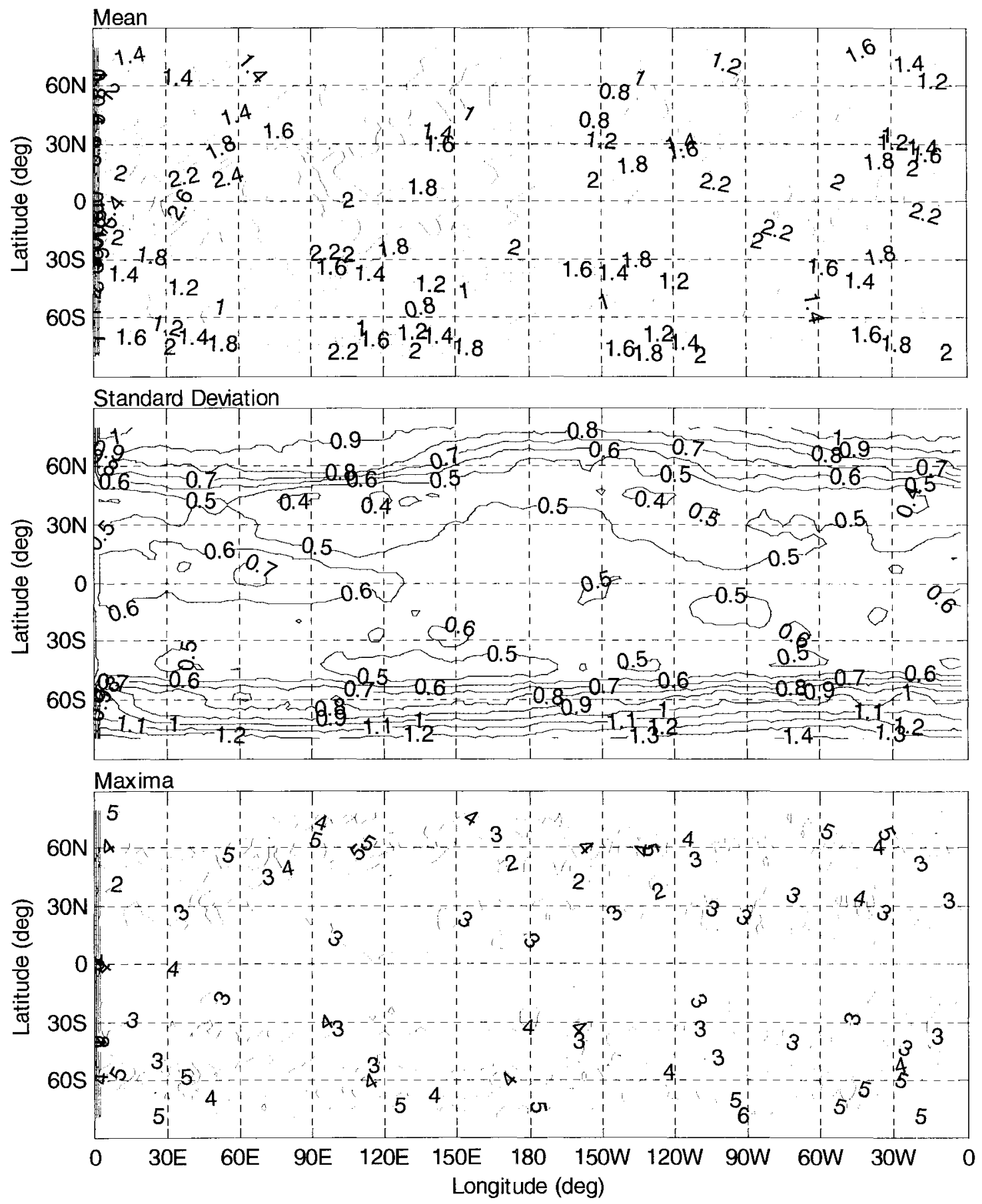

Fig. 4-45 Mean, standard deviation, and maxima of time series of monthly-averaged temperature differences (K): NCEPl with RSS weighting minus TLS. [lon: $2.5^{\circ}-357.5^{\circ}$, lat: $80^{\circ} \mathrm{S}-80^{\circ} \mathrm{N}$, press: $250-10 \mathrm{hPa}$, time: Jan 1979-Dec 2001]. 

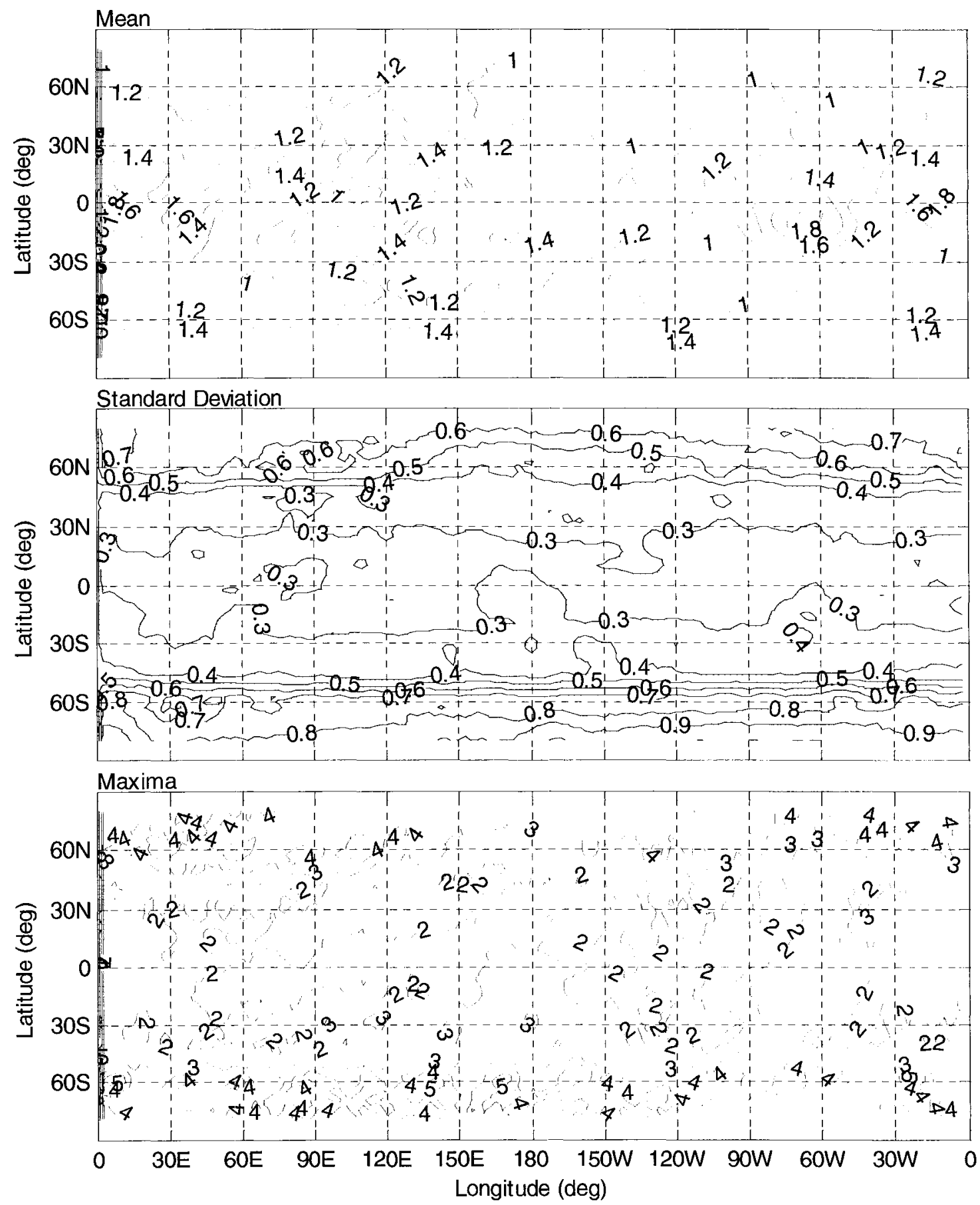

Fig. 4-46. Same as Fig. 4-45 except ERA-40. 
The figures that follow consider the four cases of NCEP1 and ERA-40 reanalysis temperatures from 250 to $10 \mathrm{hPa}$, each weighted by RSS and SEA factors. Figure 4-47 plots the zonally-averaged mean temperature differences for these four cases. The NCEP1 "warm tropical tropopause" (Randel et al. 2002, 2004) is the dominant feature of this figure. The mean differences increase rapidly poleward of $\pm 45^{\circ}$ latitude, dramatically in the Southern Hemisphere. Reanalysis temperatures with SEA weightings are also everywhere slightly greater than those with RSS weightings, especially in the Tropics, most likely because of the larger SEA weightings at 30 to $10 \mathrm{hPa}$, levels with the warmest temperatures in the vertical region considered in the TLS analysis. Figure 4-48 plots the zonally-averaged standard deviation of temperature differences for these four cases. Again, as with means, variances increase rapidly poleward of $\pm 45^{\circ}$ latitude, more so in the Southern Hemisphere. Standard deviation of longitude-time series differ almost imperceptibly from zonally-averaged values, confirming the minimal longitudinal variation in differences seen in Figs. 4-45 and 4-46. Figure 4-49 shows the monthly- and zonally-averaged temperature difference time series for the four cases above. The extratropical series are quite consistent. The tropical subplot again illustrates the warm tropical tropopause that slowly dissipates by 2000 . Similar behavior is also present in the $100 \mathrm{hPa}$ tropical temperature anomaly time series plot of Fig. 4-25. 


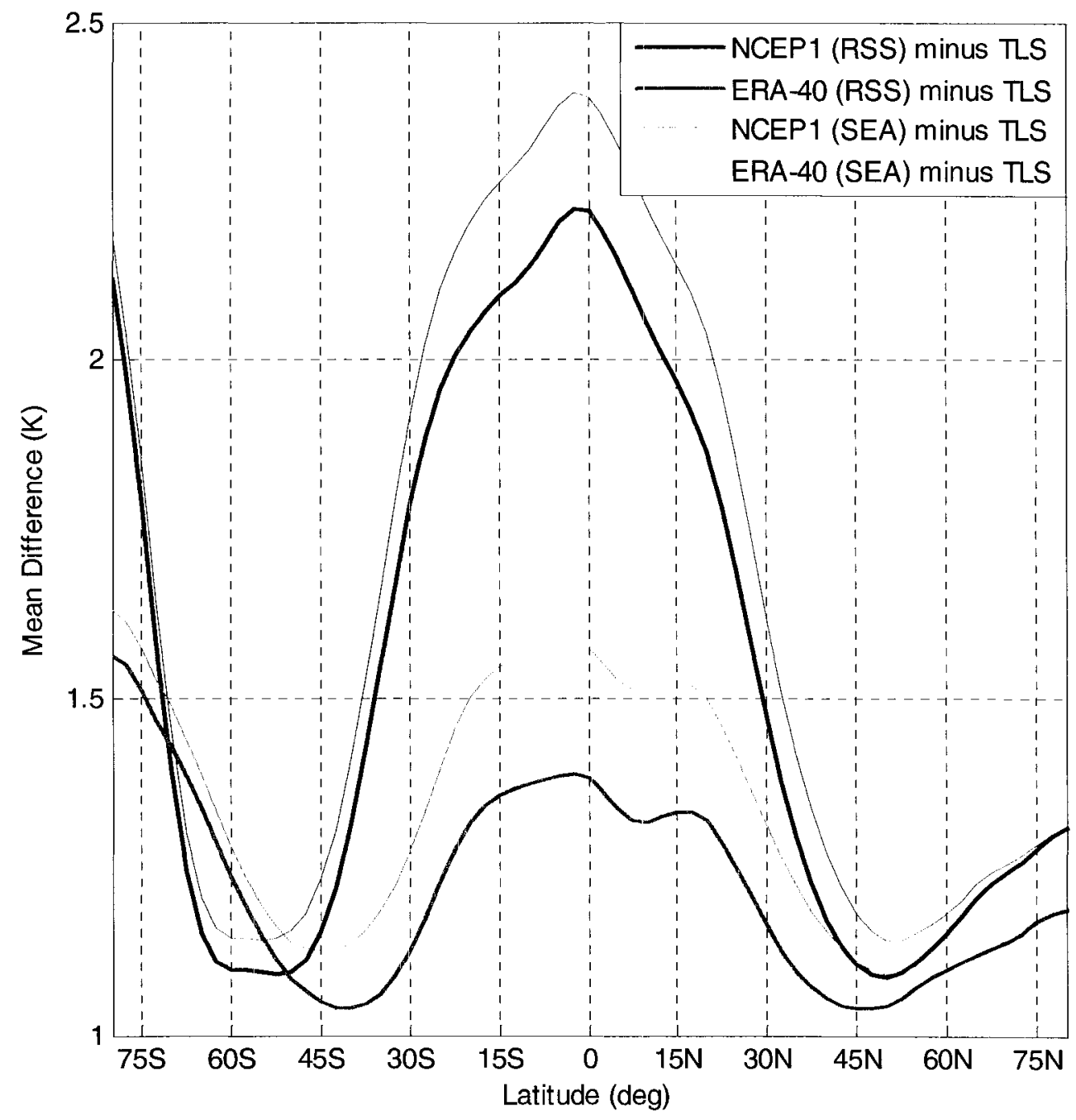

Fig. 4-47. Mean monthly-and zonally-averaged temperature differences $(K)$.

[lon: $2.5^{\circ}-357.5^{\circ}$, lat: $80^{\circ} \mathrm{S}-80^{\circ} \mathrm{N}$, press: $250-10 \mathrm{hPa}$, time: Jan 1979-Dec 2001]. 


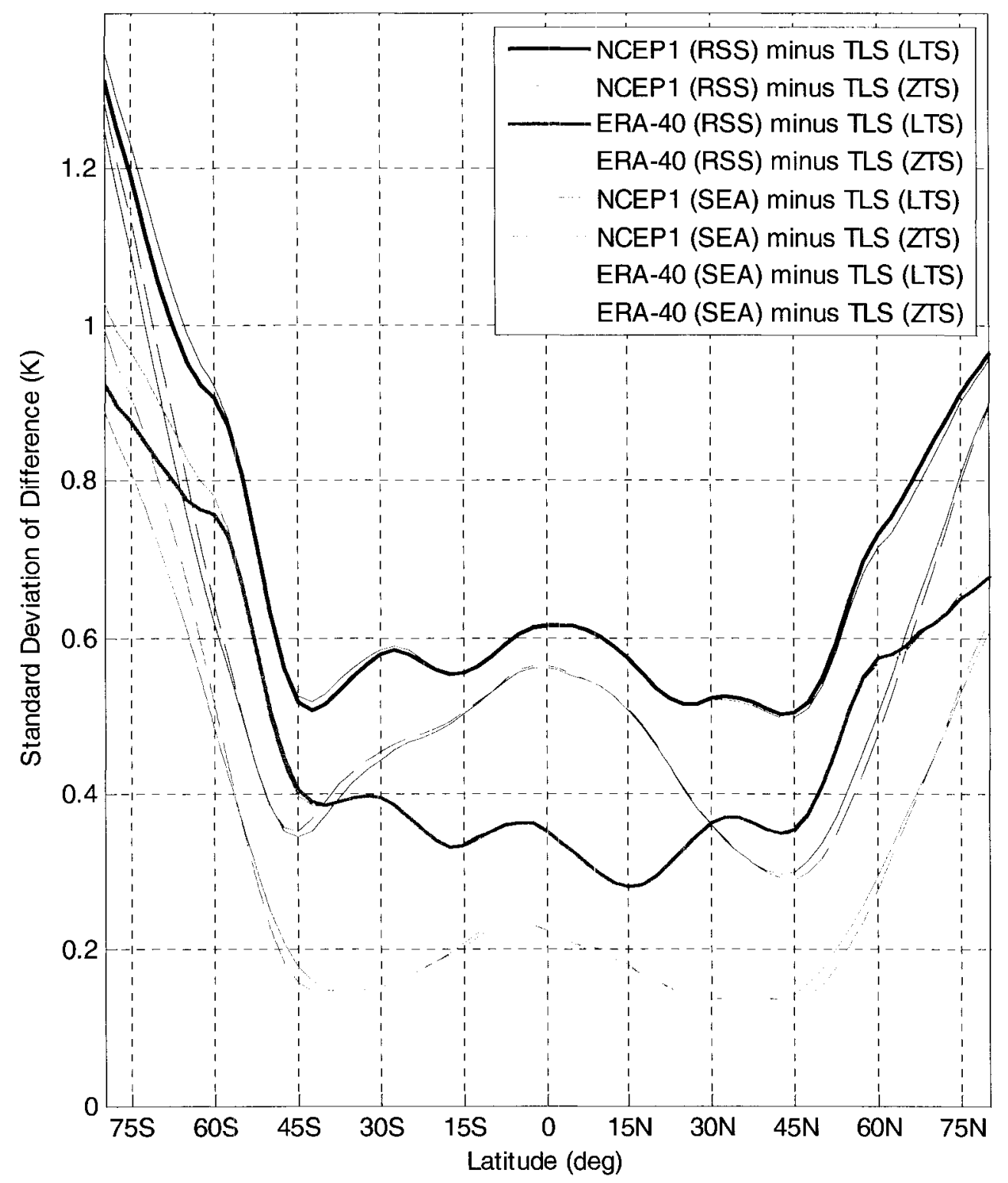

Fig. 4-48. Same as Fig. 4-47 except standard deviation (LTS: Longitude-time series ZTS: Zonally-averaged time series). 

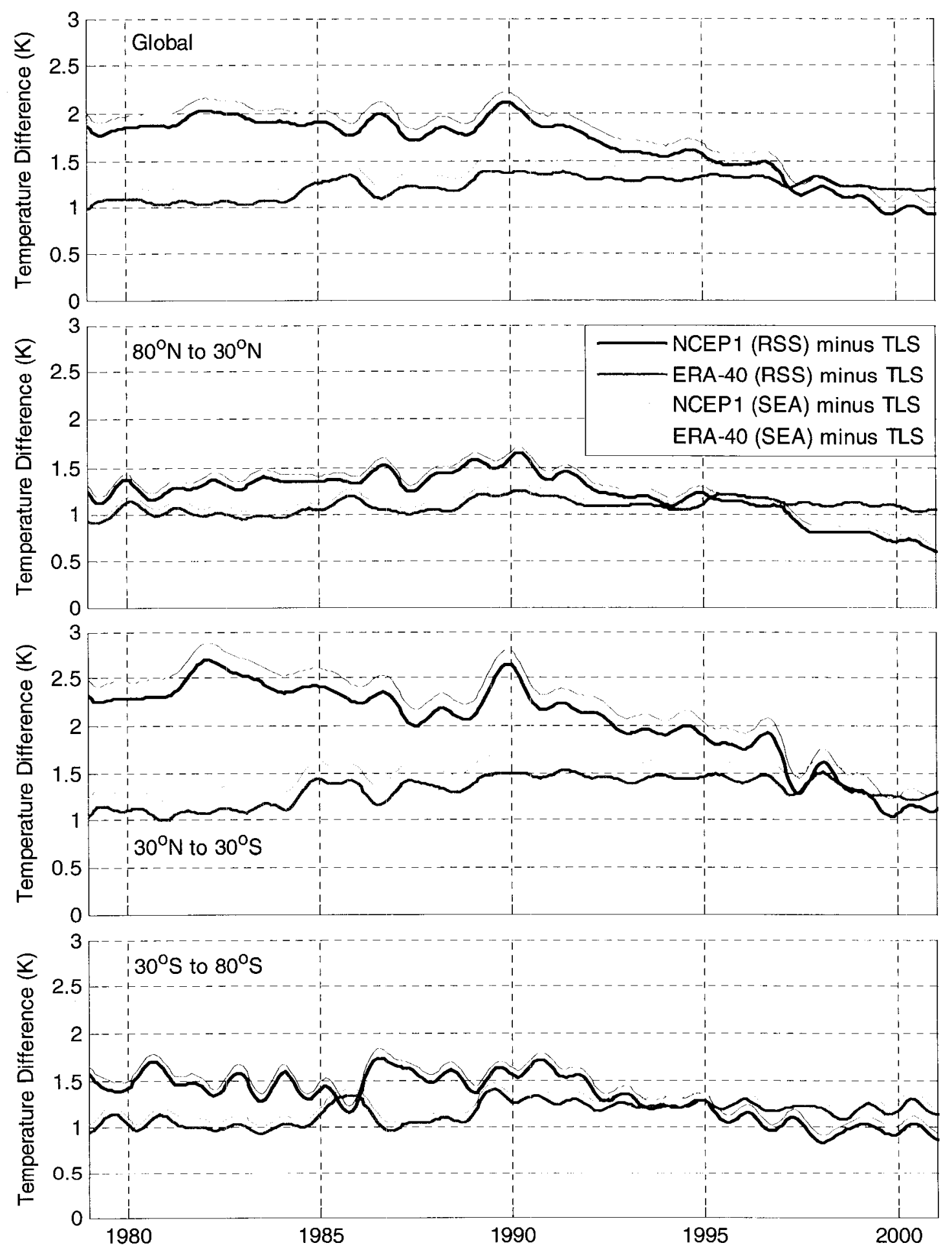

Fig. 4-49. Time series of NCEPI and ERA-40 with RSS and SEA weighting minus TLS time series in latitude bands (all variance with period greater than 13 months removed). [lon: 2.5'-357.5\%, lat: as stated, press: 250-10 hPa, time: Jan 1979-Dec 2001]. 
Table 4-5 quantitatively summarizes the differences illustrated in the preceding five figures.

Table 4-5. Temperature differences between NCEP1 and ERA-40 reanalyses and TLS $(\mathrm{K})$.

NCEP1 weighted by RSS minus TLS

\section{NCEP1}

weighted by SEA minus

TLS
ERA-40 weighted by RSS minus TLS
ERA-40 weighted by SEA minus TLS

23-year global mean differences

\begin{tabular}{|c|c|c|c|c|c|c|c|}
\hline 1.55 & $\begin{array}{l}+1.25 \\
-0.84\end{array}$ & 1.65 & $\begin{array}{l}+1.32 \\
-0.89\end{array}$ & 1.22 & $\begin{array}{l}+0.79 \\
-0.40\end{array}$ & 1.32 & $\begin{array}{l}+0.88 \\
-0.44\end{array}$ \\
\hline \multicolumn{8}{|c|}{ 23-year global standard deviation of differences } \\
\hline 0.66 & $\begin{array}{l}+0.76 \\
-0.27\end{array}$ & 0.66 & $\begin{array}{l}+0.79 \\
-0.28\end{array}$ & 0.45 & $\begin{array}{l}+0.57 \\
-0.26\end{array}$ & 0.46 & $\begin{array}{l}+0.67 \\
-0.27\end{array}$ \\
\hline \multicolumn{8}{|c|}{ 23-year global maximum differences } \\
\hline 3.52 & $\begin{array}{l}+2.73 \\
-1.93\end{array}$ & 3.65 & $\begin{array}{l}+3.06 \\
-1.99\end{array}$ & 2.75 & $\begin{array}{l}+2.90 \\
-1.25\end{array}$ & 2.89 & $\begin{array}{l}+2.92 \\
-1.16\end{array}$ \\
\hline
\end{tabular}

\subsection{Temperature trends}

Temperatures in polar regions are closely related to and correlated with vortex zonal wind speeds. For this reason, analyses of polar temperature and their trends are combined with the analyses of polar vortex zonal wind speeds and their trends in Section 5.4. However, a brief summary of temperature trend results is included in Section 4.6.

\subsection{Stratospheric sudden warmings}

This section explores the efficacy of employing the monthly-averaged reanalysis data used in this thesis to detect occurrences of stratospheric sudden warmings (SSWs), 
phenomena whose existence is often measured in days. To this end, a set of historical SSW events is used to evaluate three candidate detection algorithms applied to NCEP1 and ERA-40 reanalysis data, and successful detection and false-positive results produced by these algorithms tabulated.

The World Meteorological Organization standard definition of a stratospheric sudden warming is "at $10 \mathrm{hPa}$, easterly winds at $60^{\circ} \mathrm{N}$ and warmer temperatures at the pole than at $60^{\circ} \mathrm{N}$." These conditions were used by Labitzke et al. (2006) to identify the events in the Berlin Stratospheric Data Series (BSDS) (Baldwin and Dunkerton 1999). Charlton and Polvani (2007), in defining an acceptance criteria only for major midwinter warmings, add the constraints of daily-mean, zonally-averaged, zonal wind speeds at $10 \mathrm{hPa}$ and $60^{\circ} \mathrm{N}$. They do not include temperature gradient as a criteria. As in Section 3.3, the Charlton and Polvani paper is referenced as "C\&P." In Table 4-8 "CP" indicates the same paper.

Table 4-7, in the columns headed $F$ (indicating FUB), includes the 56 SSW events from January 1958 through February 2002 identified by Labitzke et al. (2006): 25 Canadian warmings, $C$; 18 major midwinter warmings, $M$; and 13 final warmings, $F$. Months in Table 4-7 with both major midwinter and final warmings, $M F$, are considered final warmings. C\&P developed "a new objective identification algorithm ... [which] identifies sudden warmings based on the zonally-averaged mean zonal wind speed at $60^{\circ} \mathrm{N}$ and $10 \mathrm{hPa}$, and classifies them into events which do and do not split the stratospheric polar vortex." This algorithm, applied to NCEP1 and ERA-40 reanalysis data by C\&P, reclassified five FUB Canadian warmings and five major midwinter and 
final warmings as major midwinter warmings. Seventeen of eighteen FUB major midwinter warmings were similarly classified by C\&P, leaving only one FUB major midwinter warming, in February 1991, undetected by their algorithm. The C\&P algorithm also detected four major midwinter warmings not listed in Labitzke et al. (2006), all in the month of March. Thus the C\&P climatology, and that employed in this section, contains 20 Canadian warmings, 31 major midwinter warmings (including the February 1991 major midwinter warming), and 8 final warmings. As expected, 12 of the $15 \mathrm{C} \& \mathrm{P}$ detections of major midwinter warmings at times of FUB Canadian warmings are displacements of the vortex. The results of C\&P are also listed in Table 4-7 in the columns $N$ and $E$ (events detected in NCEP1 and/or ERA-40 reanalyses, respectively). $S(s)$ or $D(d)$ identify events in which the polar vortex was found to split or displace, respectively, and '--' indicates events detected by one reanalysis but not detected by the other. Capital letters indicate extreme stratospheric events (ESEs), where the value of the Northern Annular Mode (NAM) index at $10 \mathrm{hPa}$ is greater than +1.5 (Baldwin and Dunkerton 2001). Where FUB and C\&P dates for apparently the same event occur in adjacent months, doubtless because of different definitions of starting dates (known as central dates), their letter codes are grouped by a graphical box. Table 4-6 summarizes the characters and symbols used to identify sources, warmings, and detections in Tables 4-7 and 4-8.

The most valuable contribution of the C\&P paper to this thesis is the set of SSW events whose signatures clearly exist in NCEP1 and/or ERA-40 reanalysis data. Thus 
algorithms applied to monthly-averaged data in this thesis are seeking phenomena known to exist in daily reanalysis data.

Table 4-6. Character and symbol definitions in Table 4-7.

Column Headings:

F: $\quad$ FUB (Freie Universität Berlin) climatology

N: NCEP1 detection (C\&P)

E: $\quad$ ERA-40 detection (C\&P)

Warmings:

C: Canadian

M: Major midwinter

F: Final warmings

MF: Major midwinter warming that is also a final warmings

C\&P Detections:

S (s) or D (d): Polar vortex detected as split or displaced, respectively

Upper case: Extreme stratospheric events (ESEs) (definition in text)

-- Warming not detected in indicated reanalysis 
Table 4-7. Stratospheric sudden warming events.

(See Table 4-6 for definitions of characters and symbols.)

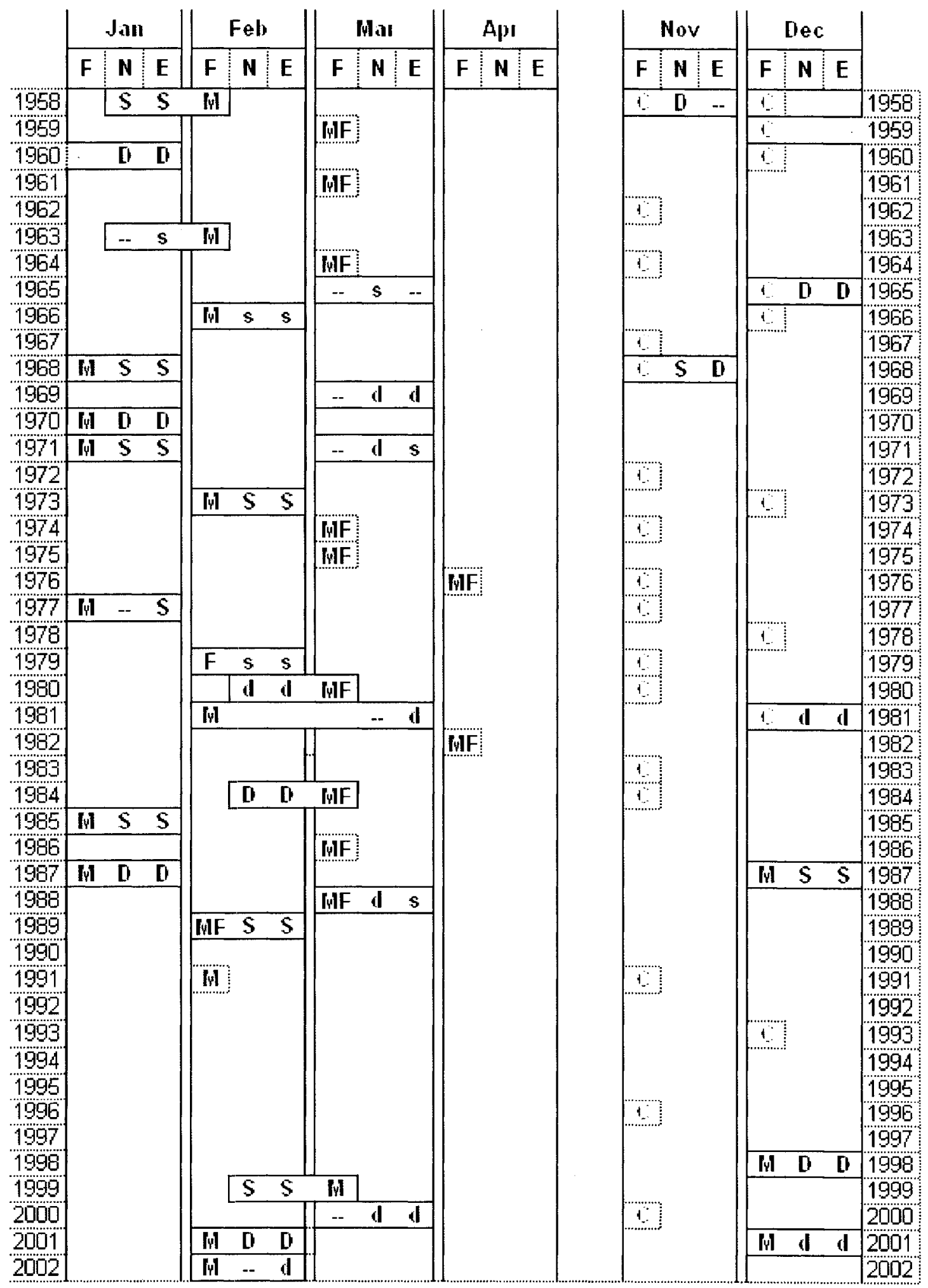


Table 4-8 compares the performance of the NCEP1 and ERA-40 reanalyses over the four classifications of stratospheric sudden warmings. The results show that the skill of the two reanalyses is essentially equal; ERA-40 leads in total events by an insignificant 29 to 27. However, ERA-40 does contain more of the non-ESE major midwinter warmings, both split and displaced, than NCEP1, indicating greater skill in characterizing these weaker warmings. Canadian warmings are rarely detected by the C\&P algorithm because it tests only for reversals of zonally-averaged zonal wind speeds. Canadian warmings are the consequence of polar vortex displacement and thus are less likely to reverse the zonally-averaged flow than a major midwinter warming.

Table 4-8. Characterization of SSW by NCEP1 and ERA-40 reanalyses.

\begin{tabular}{|c|c|c|c|c|c|c|}
\hline & & FUB & & NCEP1 & ERA-40 & \\
\hline \multirow{2}{*}{$\begin{array}{l}\text { Canadian } \\
\text { Warmings }\end{array}$} & \multirow{2}{*}{$\begin{array}{r}\mathrm{ESE} \\
\text { non-ESE }\end{array}$} & \multirow{2}{*}{5} & & 4 & 3 & \multirow{2}{*}{$\begin{array}{l}\text { 20 Canadian Warmings } \\
\text { not detected by CP }\end{array}$} \\
\hline & & & & 1 & 1 & \\
\hline \multirow{6}{*}{$\begin{array}{c}\text { Major } \\
\text { Midwinter } \\
\text { Warmings }\end{array}$} & \multirow{2}{*}{ ESE } & \multirow{2}{*}{12} & $\mathrm{~S}$ & 7 & 8 & \multirow{6}{*}{$\begin{array}{l}\text { One MMWW } \\
\text { not detected by CP }\end{array}$} \\
\hline & & & $\mathrm{D}$ & 4 & 4 & \\
\hline & \multirow{2}{*}{ non-ESE } & \multirow{2}{*}{5} & $\mathrm{~s}$ & 1 & 2 & \\
\hline & & & $\mathrm{d}$ & 1 & 3 & \\
\hline & \multirow{2}{*}{$\begin{array}{r}\text { Not in FUB } \\
\text { non-ESE }\end{array}$} & \multirow{2}{*}{4} & $\mathrm{~s}$ & 1 & 1 & \\
\hline & & & $\mathrm{d}$ & 3 & 2 & \\
\hline \multirow{4}{*}{$\begin{array}{c}\text { Major } \\
\text { Midwinter } \\
\text { and Final } \\
\text { Warmings }\end{array}$} & \multirow{2}{*}{$\mathrm{ESE}$} & \multirow{2}{*}{2} & $\mathrm{~S}$ & 1 & 1 & \multirow{4}{*}{$\begin{array}{l}\text { Eight MMWW that are also } \\
\text { final } \\
\text { not detected by CP }\end{array}$} \\
\hline & & & $\mathrm{D}$ & 1 & 1 & \\
\hline & \multirow{2}{*}{ non_ESE } & \multirow{2}{*}{2} & $\mathrm{~s}$ & 0 & 1 & \\
\hline & & & $\mathrm{d}$ & 2 & 1 & \\
\hline $\begin{array}{c}\text { Final } \\
\text { Warming }\end{array}$ & non-ESE & 1 & $\mathrm{~s}$ & 1 & 1 & \\
\hline
\end{tabular}


The three candidate detection algorithms applied to NCEP1 and ERA-40

reanalysis data in this thesis are:

1. The WMO definition: Easterly monthly- and zonally-averaged zonal wind speeds at $10 \mathrm{hPa}$ and $60^{\circ} \mathrm{N}$ and warmer monthly- and zonally-averaged temperatures at the pole than at $60^{\circ} \mathrm{N}$

2. Temperature reversal: Monthly- and zonally-averaged temperatures warmer during a given month than the preceding and following month

3. Wind reversal: Monthly- and zonally-averaged zonal wind speeds weaker during a given month than the preceding and following month

Trade-off analyses discussed below demonstrate that the reversal algorithms are most effective for temperatures and zonal wind speeds area-averaged at latitudes $85^{\circ} \mathrm{N}$ through $90^{\circ} \mathrm{N}$ at a pressure level of $10 \mathrm{hPa}$. These algorithms are applied to both NCEP1 and ERA-40 data for the months of November through April, and successful detection and false-positive results are tabulated in Table 4-9.

Figure 4-50 is a graphical example of the behavior of the temperature reversal algorithm for the conditions stated in its captions. Event labels are those defined in Table 4-6. Six Canadian warmings are not detected but one is; none are in the C\&P climatology. Ten major midwinter warmings are detected, again one was not; two of the detected events are not in the C\&P climatology and the one not detected is in the C\&P climatology. Only one final warming was not detected but two are; none are in the C\&P climatology. Three false positives, March 1978, February 1983, and January 1986, are obvious in the figure. 


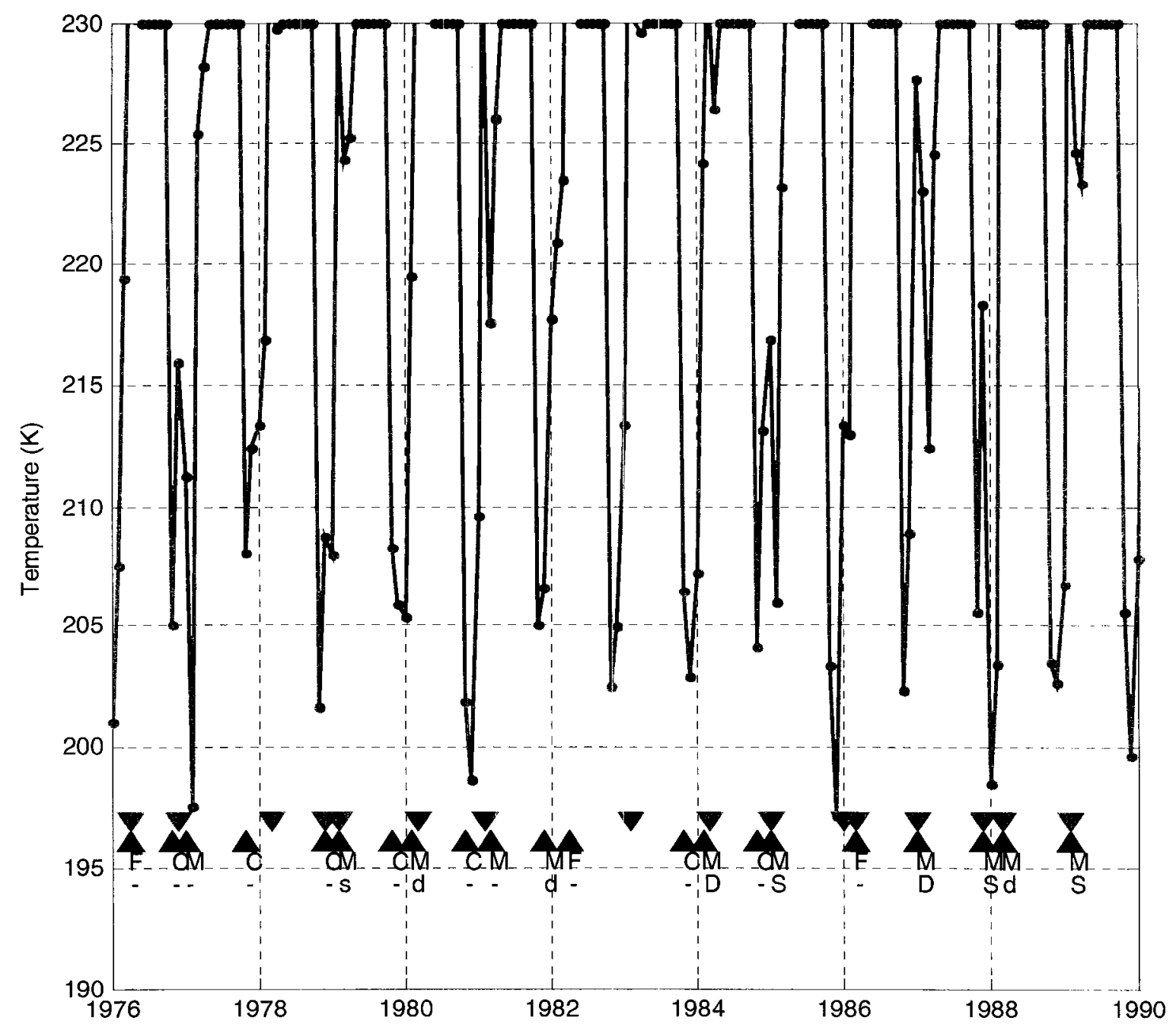

Fig. 4-50. Application of temperature detection algorithm to NCEPI monthly-and zonally-averaged temperatures. Downward red triangles represent detections, upward black triangles and their labels indicate SSW events from the climatology of Table 4-7. [lon: mean of $0^{\circ}-357.5^{\circ}$, lat: $85^{\circ} \mathrm{N}-90^{\circ} \mathrm{N}$, press: $10 \mathrm{hPa}$, time: Jan 1976-Dec 1989]. See text and Table 4-6 for definition of characters and symbols.

Table 4-9 summarizes the results of the application of the three algorithms.

Performance is measured by the percent of each warming event type detected by the algorithm and by the percent of false-positive detections. 
The WMO definition algorithm was used to test the NCEP1 and ERA-40 monthly- and zonally-averaged zonal wind speed at $10 \mathrm{hPa}$ and $60^{\circ} \mathrm{N}$ for easterly flow and the monthly- and zonally-averaged temperatures for a negative gradient between $60^{\circ} \mathrm{N}$ and $90^{\circ} \mathrm{N}$. The results of the application of this algorithm are shown in the leftmost data column of Table 4-9. The implementation of the WMO definition with monthly-averaged data does poorly, detecting at most $13 \%$ of major midwinter warmings with a false detection rate greater than $60 \%$. The temperature and wind components of the WMO definition applied alone detect $44-53 \%$ and $16-19 \%$ of major midwinter warmings, respectively, but again with greater than $60 \%$ false detection rates.

The lower right quarter of Table 4-9 presents the performance of the temperature and wind reversal algorithms. The zonal wind speed reversal algorithm very effectively detects high percentages of all three event types but produces as many false-positive detections as correct ones. This is clearly not acceptable performance.

Comparison of temperature reversal algorithm performance applied to NCEP1 and ERA-40 reanalyses over the range of pressure levels illustrates a tradeoff between detection efficiency and false-positive detections. However, the detection rates for both reanalysis data sets generally increase slightly faster with height than do the false-positive rates. Thus the temperature reversal algorithm applied to the temperature mean from $85^{\circ} \mathrm{N}$ through $90^{\circ} \mathrm{N}$ at $10 \mathrm{hPa}$ appears to give the best indication of stratospheric sudden warmings in monthly- and zonally-averaged data. Other latitude ranges for the temperature and wind reversal algorithms result in performance inferior to that of $85^{\circ} \mathrm{N}$ through $90^{\circ} \mathrm{N}$ (not shown). 
Canadian warmings are weakly detected by the temperature reversal algorithm because it tests only for reversals of monthly- and zonally-averaged temperatures.

Canadian warmings are the consequence of polar vortex displacement and thus are less likely to cause a temperature reversal in a monthly average than a major midwinter warming. 
Table 4-9. Percentages of warmings of known SSW events detected with WMO definition and with temperature or wind speed reversals and percentages of false detections.

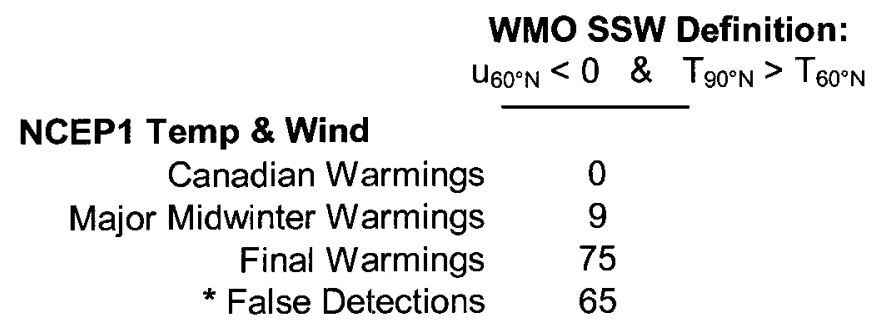

ERA-40 Temp and Wind

Canadian Warmings 0

Major Midwinter Warmings 13

Final Warmings 75

False Detections $\quad 64$

\section{NCEP1 Temperature}

Canadian Warmings

0

Major Midwinter Warmings

Final Warmings

44

False Detections

100

66

\begin{tabular}{cccc}
\multicolumn{5}{c}{ Temperature or Wind Reversal } \\
\hline $50 \mathrm{hPa}$ & $30 \mathrm{hPa}$ & $20 \mathrm{hPa}$ & $10 \mathrm{hPa}$ \\
$85^{\circ} \mathrm{N}-$ & $85^{\circ} \mathrm{N}-$ & $85^{\circ} \mathrm{N}-$ & $85^{\circ} \mathrm{N}-$ \\
$90^{\circ} \mathrm{N}$ & $90^{\circ} \mathrm{N}$ & $90^{\circ} \mathrm{N}$ & $90^{\circ} \mathrm{N}$ \\
& & & \\
15 & 25 & 10 & 30 \\
69 & 72 & 69 & 81 \\
38 & 63 & 88 & 100 \\
22 & 23 & 28 & 30
\end{tabular}

NCEP1 Zonal Wind Speed

Canadian Warmings

Major Midwinter Warmings

Final Warmings

$\begin{array}{cc}0 & 65 \\ 16 & 94 \\ 100 & 88 \\ 63 & 55\end{array}$

$\begin{array}{llcl}65 & 75 & 80 & 85 \\ 94 & 97 & 100 & 94 \\ 88 & 75 & 75 & 88 \\ 55 & 56 & 52 & 55\end{array}$

\section{ERA-40 Temperature}

Major Midwinter Warmings

Canadian Warmings

\section{0}

53

100

Final Warmings

False Detections

64

15
66
25
13

20

20

25

13

75

72

75

38

100

14

50

26

\section{ERA-40 Zonal Wind Speed}

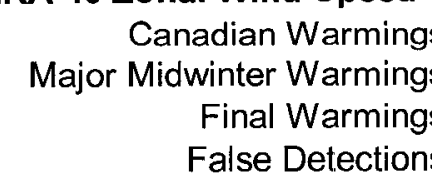

0
19
88
61

85
94
88
50

70
94
88
51

90

65

91

100

61

50

88

50

* Percentage of false detections: Number of false-positive detections divided by total number of detections 


\subsection{Summary}

Section 4 compares stratospheric temperature data from the NCEP1 and ERA-40 reanalyses with the aid of comparison data in three domains: pressure level vs. latitude, time series at the six stratospheric pressure levels considered in this thesis, and pressure surfaces (latitude vs. longitude) at those six stratospheric pressure levels.

NCEP1 minus ERA-40 multiyear pressure level vs. latitude temperature differences are analyzed with three processing methods: (1) monthly- and zonallyaveraged data, (2) longitude-time series of monthly-averaged data, and (3) longitude-time series of monthly-averaged data with the annual cycle removed. Figures 4-1 through 4-6 (1), 4-7 through 4-9 (2), and 4-13 through 4-15 (3) illustrate the results of these three methods. These figures show that multiyear mean differences between NCEP1 and ERA-40 are three $\mathrm{K}$ or less north of $60^{\circ} \mathrm{S}$, and in many regions the mean difference is less than one $\mathrm{K}$. Only south of $60^{\circ} \mathrm{S}$ do mean values exceed six $\mathrm{K}$ and only during 1958-78. Similarly, multiyear rms differences of four $\mathrm{K}$ or less (three $\mathrm{K}$ when the annual cycle is removed) are found north of $60^{\circ} \mathrm{S}$, with maximum values of seven to eight $\mathrm{K}$ south of $60^{\circ} \mathrm{S}$ during $1958-78$. The multiyear maximum and mean values of mean, standard deviation, rms, and maxima over the entire pressure level vs. latitude space for the 1958-2001, 1958-78, and 1978-2001 time periods are contained in Table 4-2. In addition to the statistics above, this table show that the longitude-time series increased mean rms values by 20 to $45 \%$ and mean maxima by 81 to $99 \%$, numerically representing the variance removed by zonal averaging. Removal of the annual cycle reduced the mean rms values by 3 to $9 \%$ and, because the majority of variance is 
removed from polar regions, as illustrated in Figs. 4-16 through 4-18, peak rms values decreased by 23 to $36 \%$.

Cross comparison of NCEP1 and ERA-40 monthly- and zonally-averaged data with that of METO for the period of 1992-99 shown in Figs. 4-5 and 4-6 discloses much larger mean and rms temperature differences between ERA-40 and METO than between NCEP1 and METO, primarily south of $60^{\circ} \mathrm{S}$ and north of $75^{\circ} \mathrm{N}$. The ERA-40 minus METO Southern Hemisphere differences are approximately twice as large as those in the Northern Hemisphere.

Figures 4-19 through 4-21 show significantly greater variance in ERA-40 temperatures than in NCEP1 temperatures at $10 \mathrm{hPa}$ at the South Pole. This variance is evidence of the "oscillatory vertical structure in temperature, especially large over Antarctica" in ERA-40 (Randel et al. 2002, 2004). This "large apparent bias" in the pressure level vs. latitude domain is illustrated by the anomalous transients in the time series of Figs. 4-22 through 4-24 during 1973, 1979-80, and 1994-2000 (South Pole) and 1974, 1983, 1988, and 1995-2000 (North Pole). The causes of this oscillatory vertical structure are discussed in Section 2.2.4.

Time series for the latitude bands of $90^{\circ} \mathrm{S}-30^{\circ} \mathrm{S}, 30^{\circ} \mathrm{S}-30^{\circ} \mathrm{N}, 30^{\circ} \mathrm{N}-90^{\circ} \mathrm{N}$, and global at the six stratospheric pressure levels of this thesis are presented in Figs. 4-25 through 4-30. They benefit from commensurate comparison data from RATPAC during 1958-2005 from 100-30 hPa (but not available where most needed: 20 and $10 \mathrm{hPa}$ ) and from METO from 1992-99 at all pressure levels. In general, the NCEP1, ERA-40, RATPAC, and METO time series agree within fractions of a degree K. Not only are 
multi-year trends simillar but most short-term transients (volcanic eruptions) match in time if not in absolute magnitude. There are several departures from this consistency:

$\begin{array}{lclll}\text { NCEP1 } & 1980-2000 & 100 \mathrm{hPa} & \text { Tropics } & \text { As much as three K warmer } \\ \text { ERA-40 } & 1958-73 & 70-20 \mathrm{hPa} & \text { SH extratropics } & \text { As much as four K colder } \\ \text { ERA-40 } & 1980-83 & 70 \mathrm{hPa} & \text { Tropics } & \text { One K colder }\end{array}$

Also at $10 \mathrm{hPa}$ a six $\mathrm{K}$ difference exists between NCEP1 (warmer) and ERA-40 (colder) from 1980-85 in the Southern Hemisphere extratropics. Thus, although in many regions of pressure level vs. latitude space, the temperatures of either reanalysis appear accurate, there are regions and time periods where the data of one or the other should be avoided.

A Northern Hemisphere planetary wavenumber one temperature anomaly is present primarily during winter months with maximum at $58^{\circ} \mathrm{N}, 140^{\circ} \mathrm{E}(10 \mathrm{hPa})$ to $68^{\circ} \mathrm{N}$, $160^{\circ} \mathrm{W}(100 \mathrm{hPa})$ and a year-to-year amplitude with respect to longitudinal means of 10 to $20 \mathrm{~K}$. A similar wave exists during the Southern Hemisphere winter with maximum at $60^{\circ} \mathrm{S}, 90^{\circ} \mathrm{E}(10 \mathrm{hPa})$ to $70^{\circ} \mathrm{S}, 170^{\circ} \mathrm{E}(100 \mathrm{hPa})$ and a year-to-year amplitude of 5 to $10 \mathrm{~K}$ after 1979 (in earlier years wave amplitudes and their variance are much larger). These departures from longitudinal means are removed by zonal and time averaging. Figures 4-33 through 4-37 and Table 4-4 show the characteristics and magnitudes of these waves. Latitude vs. longitude temperature difference plots at the six stratospheric pressure levels of this thesis are consistent with and confirm the pressure level vs. latitude and time series analyses summarized above. Of particular interest, the 100-hPa plot in Fig. 4-38 locates the two centroids of the four K "satellite data discontinuity across 1978-1979" (Randel et al. 2002, 2004). One spans the Indian Ocean and the other is west 
of Ecuador in the eastern Pacific Ocean, displaced 180 degrees from one another. The 10-hPa plot illustrates "a pathological problem" (Trenberth and Stepaniak 2002) resulting from NCEP1's use of a sigma coordinate system to the top of the assimilating model and a rigid top (Section 2.2.1). Rippled temperature gradients in the vicinity of major mountain range systems in Fig. 4-43 are evidence of this problem in the temperature domain.

\section{TLS Comparison}

Over time and space, simulated deep vertical layer temperatures created using weighted reanalysis data are warmer than TLS temperatures; the 23-year global average differences are $1.60 \mathrm{~K}$ (NCEP1 minus TLS) and 1.25 (ERA-40 minus TLS). Maximum differences occur in the tropics where NCEP1 minus TLS is $2.3 \mathrm{~K}$ and ERA-40 minus TLS is $1.3 \mathrm{~K}$. NCEP1 is warmer than ERA-40 from 1979 to 1997 in the tropics and 1979 to 1993 in the extratropics; after these intervals, temperatures of the two reanalyses with respect to TLS are essentially equal. Mean, standard deviation, and maxima differences are larger in the Southern Hemisphere polar regions than in the Northern Hemisphere and are consistent in magnitude in the two reanalyses.

\section{Northern Hemisphere temperature trends}

Temperature trend analyses in both hemispheres consider only NCEP1 reanalysis data. Comparison of NCEP1 and ERA-40 temperature trends in Figs. 5-44, 5-46, 5-48, 5-55, 5-57, 5-59, and 5-61 reveals dramatic differences in structure and magnitude of these trends. These differences, in light of the oscillatory character of ERA-40 polar 
temperatures with height, lead to the assumption that ERA-40 temperature trends are incorrect. For this reason, ERA-40 temperature trends are not considered further. Because of the small (23-year) sample size and the large variance in reanalysis data, 1979-2001 temperature trends are generally statistically insignificant.

In the Northern Hemisphere, October (Fig. 5-52), November (Fig. 5-44), February (Fig. 5-50), and March (Fig. 5-54) exhibit negative temperature trends over the entire region displayed $\left(100-10 \mathrm{hPa}, 45^{\circ} \mathrm{N}-90^{\circ} \mathrm{N}\right)$. The December trend (Fig. 5-46) is positive over most of the displayed region, and January's trend (Fig. 5-48) is negative at heights above $30 \mathrm{hPa}$ and equatorward of $60^{\circ} \mathrm{N}$. Most of the displayed region is statistically significant in October; none is statistically significant in December; varying minor portions of the displayed region are statistically significant in the remaining months. Generally, statistical significance in temperature trends is less likely to be found in vortex regions and polar regions at upper heights. Northern Hemisphere temperature trends in the analyzed region are generally negative, exhibiting a cooling trend, but not in all months and not with consistent statistical significance.

\section{Southern Hemisphere temperature trends}

In the Southern Hemisphere, May (Fig. 5-63), June (Fig. 5-55), and July (Fig. 5- 57) all exhibit negative temperature trends over the entire region displayed (100-10 hPa, $90^{\circ} \mathrm{S}-45^{\circ} \mathrm{S}$ ). August (Fig. 5-59), September (Fig. 5-61), and October (Fig. 5-65) contain mixed trends. Statistical significance is present in the trends of each month; it is found in larger areas of the first three months, May, June, and July and in lesser amounts in the last three. As in the Northern Hemisphere, statistical significance is 
less likely to be found in vortex regions and polar regions at upper heights. Southern Hemisphere temperature trends are stronger in magnitude and more consistently negative than those of the Northern Hemisphere but again without consistent statistical significance.

\section{Stratospheric Sudden Warmings}

C\&P detected essentially equal numbers of Stratospheric Sudden Warmings of all classifications in the NCEP1 and ERA-40 reanalyses. The most valuable contribution of the C\&P paper to this thesis is the compilation of a set of SSW events whose signatures clearly exist in NCEP1 and/or ERA-40 daily reanalysis data. Of the three simple algorithms applied to the monthly- and zonally-averaged data employed in this thesis, "temperature reversal" proved the most effective. Here temperatures, averaged over $85^{\circ} \mathrm{N}$ through $90^{\circ} \mathrm{N}$ at $10 \mathrm{hPa}$, that are warmer during a given winter month than the preceding and following months are interpreted as a warming. The percentages of warmings and false detections by the algorithm applied to the NCEP1 (ERA-40) reanalyses are as follows: Canadian: 30 (25), major midwinter warmings: 81 (75), final warmings: 100 (100), false detections: 30 (26).

This simple temperature reversal algorithm demonstrates that SSWs can be detected in the monthly output of a GCM. Daily zonal wind speed and temperature data must be analyzed to determine if the WMO criteria of Section 3.3 are met; even more conclusive would be the application of the C\&P algorithm. 


\subsection{ZONAL WIND SPEED COMPARISON}

This section compares NCEP1, ERA-40, and METO stratospheric zonal wind speed data. Comparisons of reanalyses and comparison data are performed in three domains:

- Pressure level (height) vs. latitude

- Time series at six stratospheric pressure levels of $100,70,50,30,20$, and $10 \mathrm{hPa}$

- Pressure surface (latitude vs. longitude at the six stratospheric pressure levels) The location, speed, and time trends of zonal wind speeds of the two reanalyses are compared in polar regions in conjunction with temperature trends in these regions. Finally, the characterizations of the quasi-biennial oscillation (QBO) by the two reanalyses are compared with each other and with comparison data.

\subsection{Height - latitude structure of monthly zonal wind speed differences}

This section evaluates zonal wind speed differences between the NCEP1 and ERA-40 reanalyses from four perspectives:

- Presents differences of conventional zonally-averaged time series

- Applies "longitude-time series" to retain the variance lost in zonal averaging

- Quantifies the variance lost by zonal averaging

- Demonstrates the impact of the annual cycle on longitude-time series differences 


\section{Zonally-averaged time series (ZTS)}

Figures 5-1 through 5-6 each display the mean, standard deviation, rms, and maxima of the difference of the monthly- and zonally-averaged zonal wind speeds of two data sets. The zonal wind speed difference maxima are the maximum "single event" differences, either positive or negative, of each zonally-averaged time series. In this section these statistical measures are applied to zonally-averaged time series at each of 438 points (73 latitudes from $90^{\circ} \mathrm{S}$ to $90^{\circ} \mathrm{N}$ and six pressure levels from 100 to $10 \mathrm{hPa}$ ) as illustrated in Appendix D. The figures listed below display the four statistical measures for the six cases that cross-compare NCEP1, ERA-40, and METO zonal wind speed data:

Fig. 5-1. NCEP1 minus ERA-40 January 1958 through December 2001 (the early radiosonde era through mature satellite sensor systems)

Fig. 5-2. NCEP1 minus ERA-40 January 1958 through December 1978 (the early radiosonde era to the beginning of the modern satellite era)

Fig. 5-3. NCEP1 minus ERA-40 January 1979 through December 2001 (the modern satellite era)

Fig. 5-4. NCEP1 minus ERA-40 January 1992 through December 1999 (to provide a comparison for Figs. 5-5 and 5-6)

Fig. 5-5. NCEP1 minus METO January 1992 through December 1999

Fig. 5-6. ERA-40 minus METO January 1992 through December 1999 Discussion of Figs. 5-1 through 5-6

In Figs. 5-1 through 5-6, mean, standard deviation, and rms differences in the northern extratropics are less than those at corresponding heights and latitudes in the southern extratropics. These hemispheric differences are most pronounced in Figs. 5-1 (1958-2001) and 5-2 (1958-79), reflecting the lack of observations in the pre-modern satellite era. A core of high variance on the Equator projects downward with a maximum 
at $10 \mathrm{hPa}$ and minimum at $100 \mathrm{hPa}$. These differences are in part a result of ERA-40's stronger characterization of the QBO (presented in Section 5.5). Randel et al. (2002, 2004) show that at $30 \mathrm{hPa}, \mathrm{QBO}$ amplitude is negligible poleward of $\pm 20^{\circ}$ latitude. In

Figs. 5-1 through 5-3, reanalysis differences in the tropical QBO region are roughly equivalent to those in the southern extratropics at $10 \mathrm{hPa}$ for all three statistical measures. In Figs. 5-1 through 5-5, NCEP1 winds are stronger than ERA-40 in the Tropics except in the vicinity of $10 \mathrm{hPa}$, where they are weaker, and ERA-40 winds are stronger northward of $45^{\circ} \mathrm{N}$ to $60^{\circ} \mathrm{N}$ and southward of $60^{\circ} \mathrm{S}$.

Figures 5-2 (1958-78) and 5-3 (1979-2001) illustrate significantly differing mean, standard deviation, and rms behavior before and during the modern satellite era, respectively. Reanalysis differences in these three statistical measures in the northern extratropics are generally a factor of two less in the later interval while in the southern extratropics, differences are factors of three to four less. Maximum differences also decrease by similar factors in the southern extratropics while effectively disappearing in the northern extratropics and remaining unchanged in the Tropics.

Figures 5-4 (NCEP1 minus ERA-40), 5-5 (NCEP1 minus METO), and 5-6 (ERA-40 minus METO) provide a three-way comparison of zonal wind speed over the brief 1992-99 interval. As in the case of the comparison of temperature data from the same sources for the same time period in Figs. 4-4 through 4-6, the NCEP1 minus METO differences are significantly smaller than those of ERA-40 minus METO. Also, as with temperature, the small NCEP1 minus METO differences in the Tropics are a result of deficiencies in the METO characterization of the QBO. In the extratropics, all four 
statistical measures of the three sets of zonal wind speed differences exhibit similar contours.

There is considerable congruency between positive and negative regions in maxima and mean plots in Figs. 5-1 through 5-6, with steep gradients between positive and negative regions. Maxima occur in the Southern Hemisphere with the exception of NCEP1 minus ERA-40 (1979-2001).

Zonal wind speed maxima for the first nine cases of this section are summarized in Table 5-1. In every longitude-time series vs. zonal averaging comparison, the longitude-time series maxima and minima are larger in absolute magnitude than those from zonal averaging. Percentage differences in a majority of the comparisons are much larger than those found for temperatures in Table 4-1. NCEP1 minus ERA-40 1958-78 maxima and minima are equal to those of NCEP1 minus ERA-40 1958-2001, demonstrating that the 44-year maximum and minimum values occurred before the beginning of the modern satellite era. In only 4 cases of the 20 listed do maxima or minima occur at a pole; the remainder, with the exception of one at the Equator, range from $82.5^{\circ}$ to $67.5^{\circ}$ latitude.

None of the figures comparing the ERA-40 zonal wind speeds with others contains any hint of the "oscillatory vertical structure in temperature, especially large over Antarctica" (Randel et al. 2002, 2004). Figures 5-72 and 5-73, seasonal plots of the standard deviation of zonally- and monthly-averaged zonal wind speeds, clearly show no vertical oscillation. 

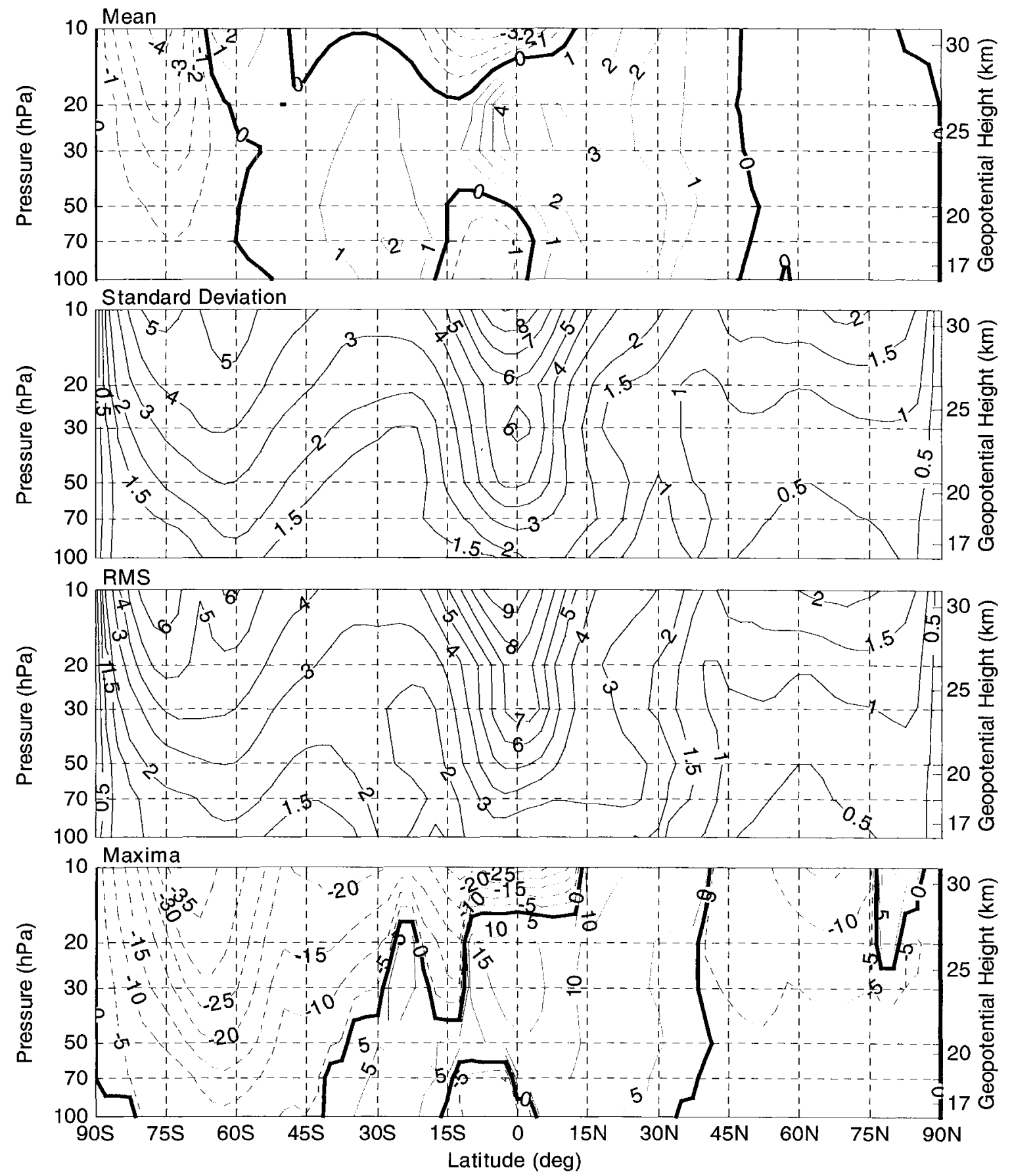

Fig. 5-1. Mean, standard deviation, rms, and maxima of time series of monthly-and zonally-averaged zonal wind speed differences $\left(\mathrm{m} \mathrm{s}^{-1}\right)$ :

NCEPI minus ERA-40 [lon: mean of $0^{\circ}-357.5^{\circ}$, lat: $90^{\circ} \mathrm{S}-90^{\circ} \mathrm{N}$, press: $100-10 \mathrm{hPa}$, time: Jan 1958-Dec 2001]. 

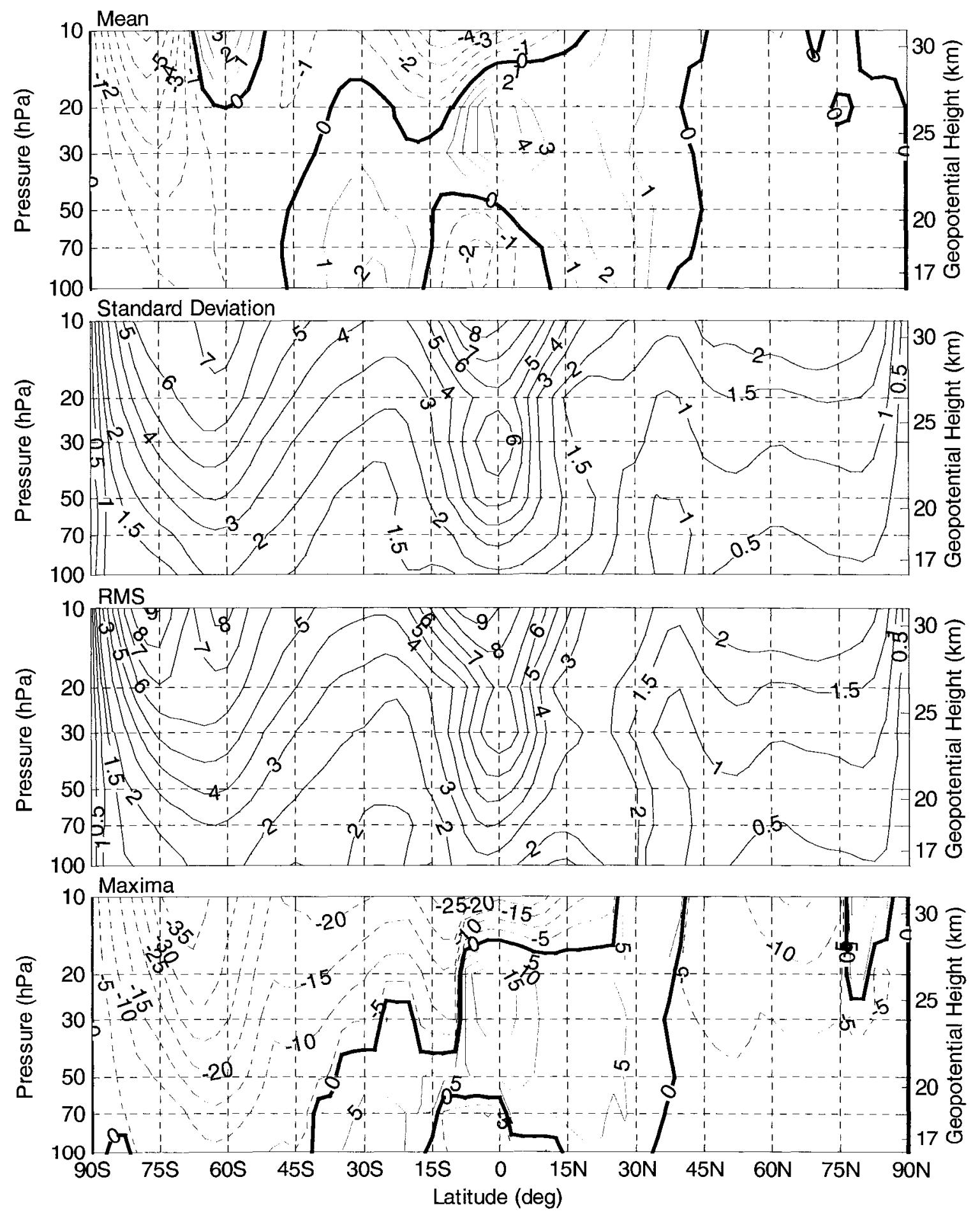

Fig. 5-2. Same as Fig. 5-1 except time: Jan 1958-Dec 1978. 

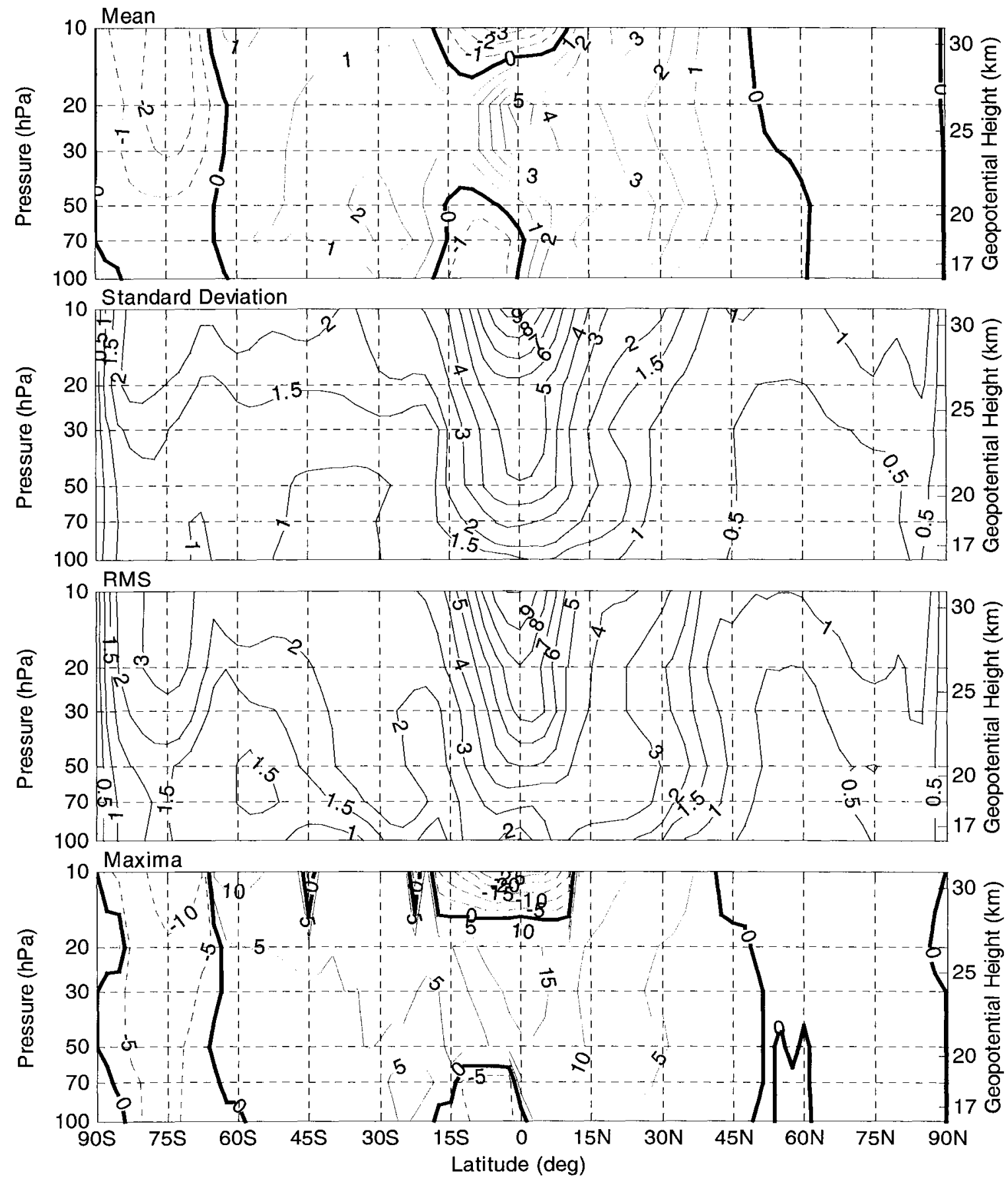

Fig. 5-3. Same as Fig. 5-1 except time: Jan 1979-Dec 2001. 

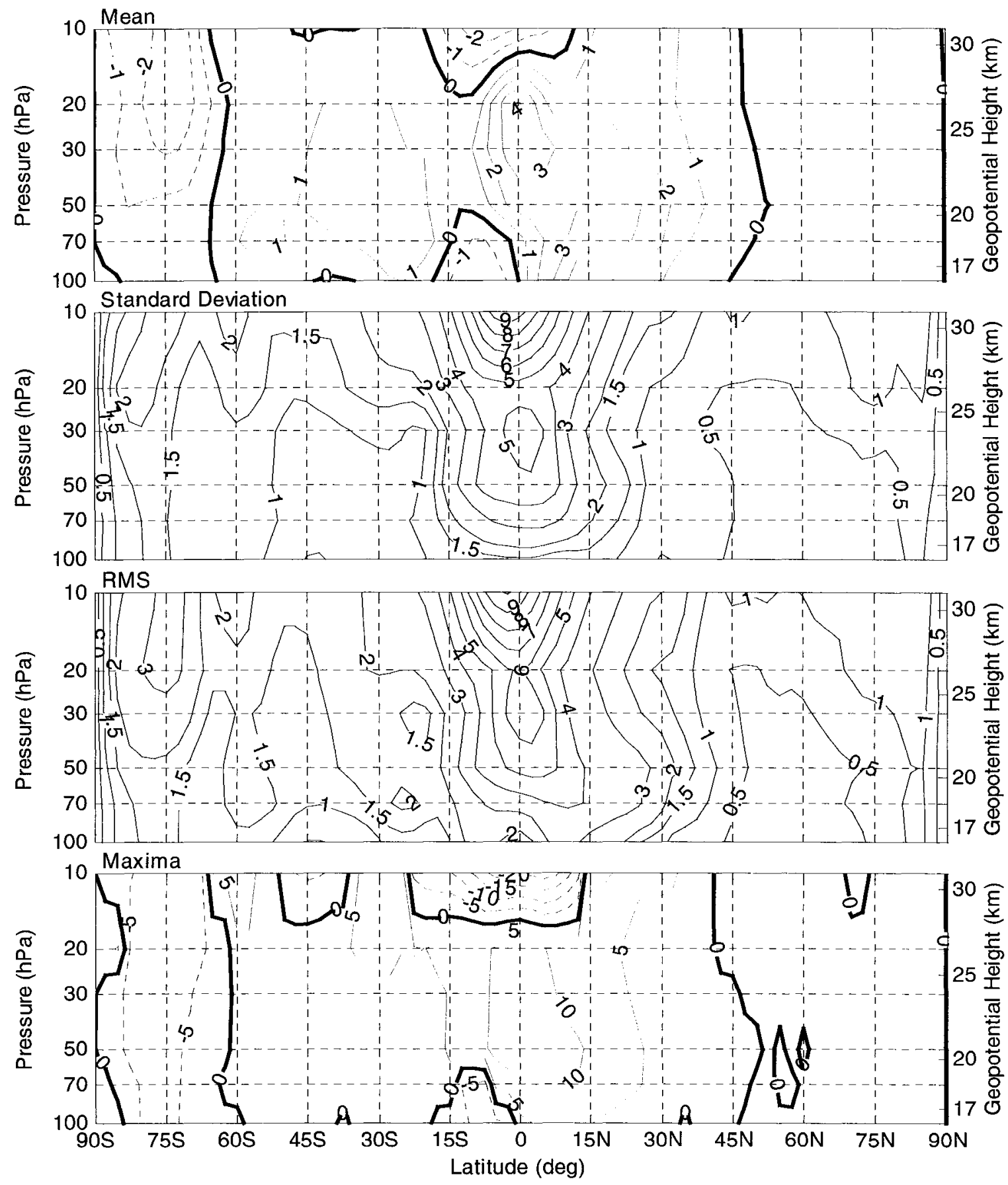

Fig. 5-4. Same as Fig. 5-1 except time: Jan 1992-Dec 1999. 

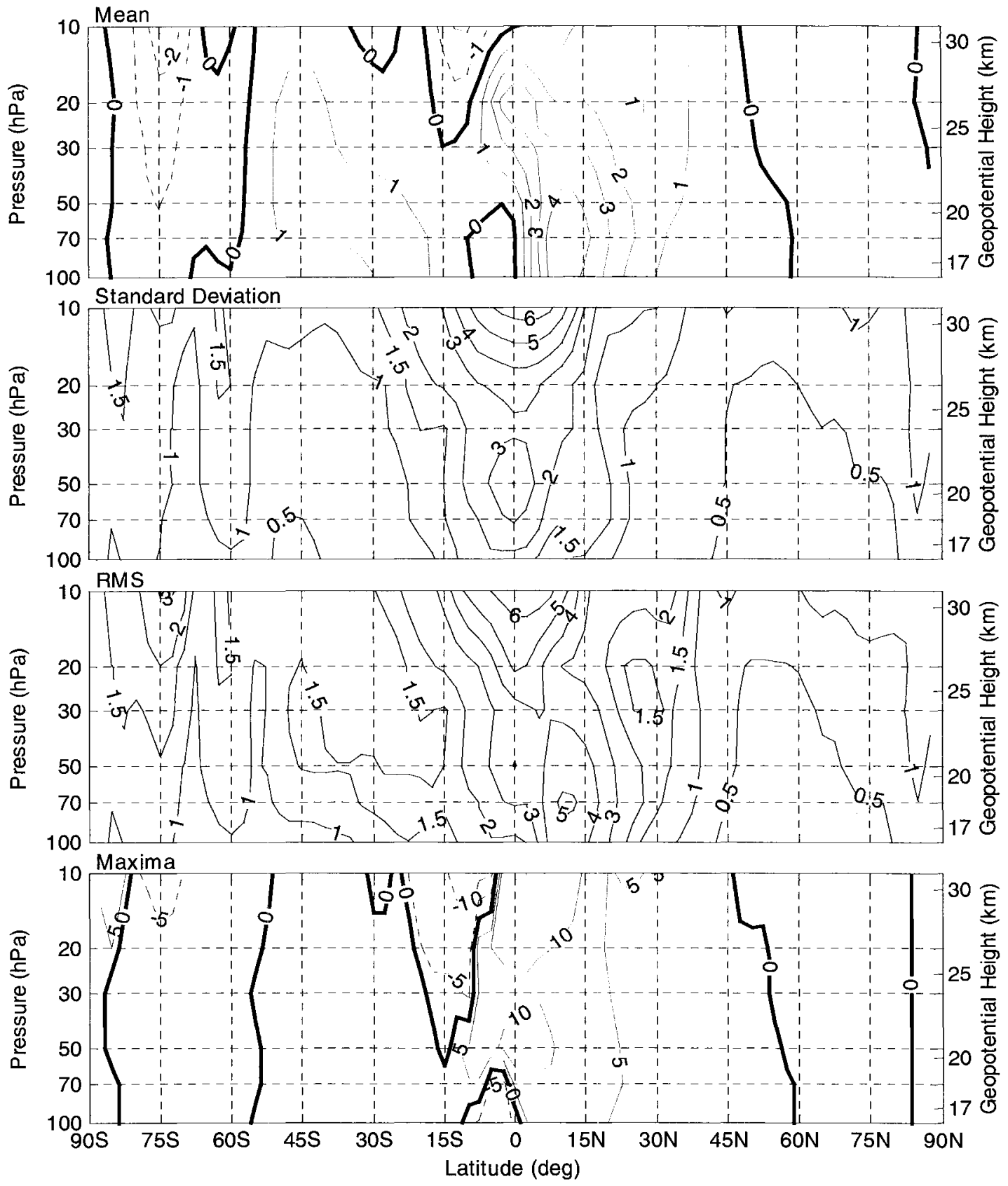

Fig. 5-5. Same as Fig. 5-1 except NCEPI minus METO, [lat: 87.5 S-87.5 $\mathrm{N}$, and time: Jan 1992-Dec 1999]. 

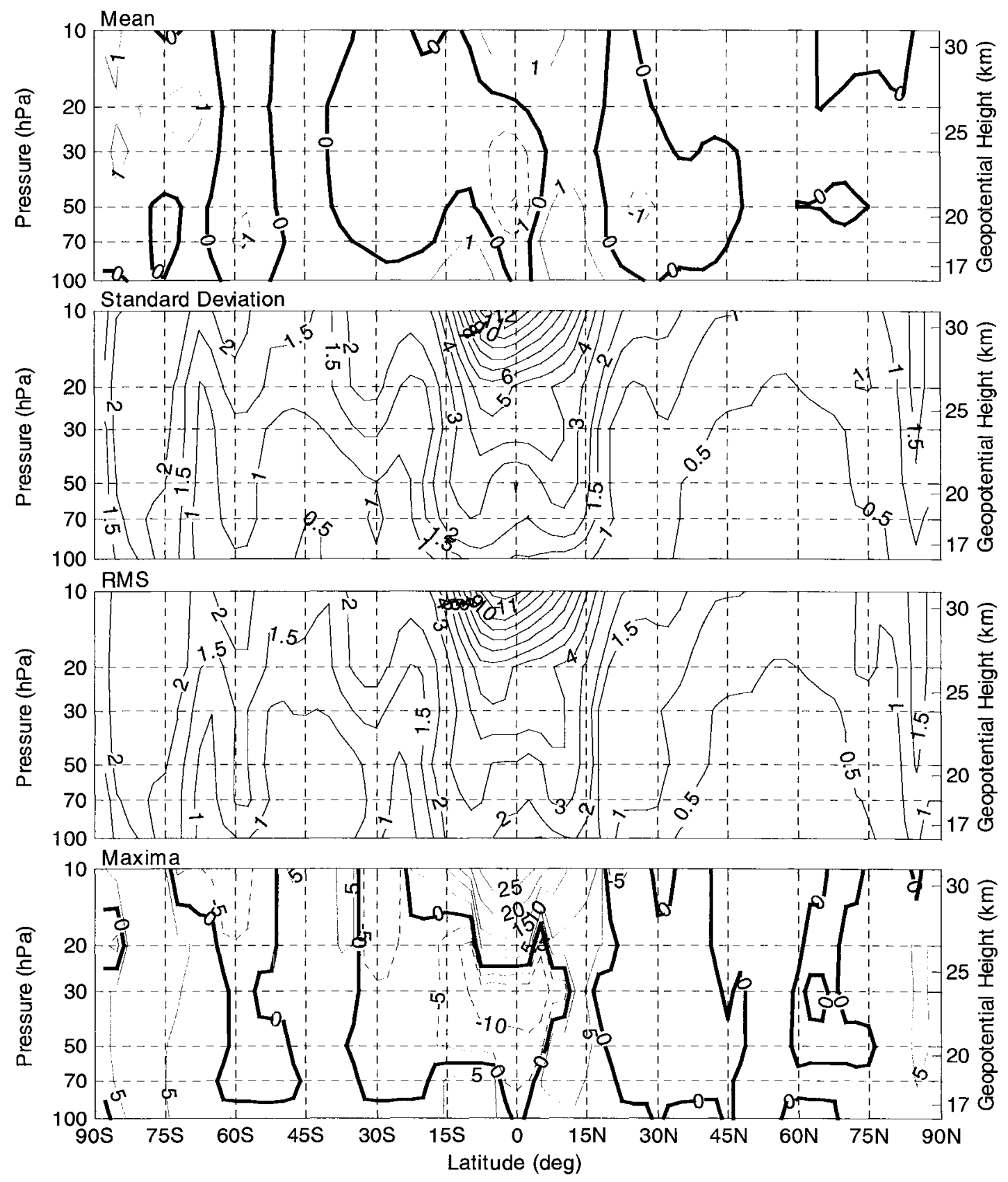

Fig. 5-6. Same as Fig. 5-5 except ERA-40 minus METO. 


\section{Longitude-time series (LTS)}

Figures 5-7 through 5-9 display the mean, standard deviation, root-mean-square (rms), and maxima of the difference in the monthly zonal wind speeds of the two reanalysis data sets. The last three measures: standard deviation, rms, and maxima, are evaluated over all longitudes and times at each of 438 points ( 73 latitudes from $90^{\circ} \mathrm{S}$ through $90^{\circ} \mathrm{N}$ and six pressure levels from 100 through $10 \mathrm{hPa}$ ). The concept of "longitude-time series" is introduced in Section 4.1 and further discussed and illustrated in Appendix D.

The figures listed below display the four statistical measures for the three longitude-time series cases:

Fig. 5-7. NCEP1 minus ERA-40 January 1958 through December 2001 (the early radiosonde era through mature satellite sensor systems)

Fig. 5-8. NCEP1 minus ERA-40 January 1958 through December 1978 (the early radiosonde era to the beginning of the modern satellite era)

Fig. 5-9. NCEP1 minus ERA-40 January 1979 through December 2001 (the modern satellite era)

\section{Longitude-time series minus zonally-averaged time series}

Figures 5-10 through 5-12 display the standard deviation, rms, and maxima of the differences between the respective statistics generated from longitude-time series (LTS) minus those generated from zonally averaged time series (ZTS). These figures graphically quantify the loss of variance that results when the computationally simpler zonal averaging technique is employed. 
Fig. 5-10. NCEP1 minus ERA-40 January 1958 through December 2001 (Fig. 5-7 (LTS) minus Fig. 5-1 (ZTS))

Fig. 5-11. NCEP1 minus ERA-40 January 1958 through December 1978 (Fig. 5-8 (LTS) minus Fig. 5-2 (ZTS))

Fig. 5-12. NCEP1 minus ERA-40 January 1979 through December 2001 (Fig. 5-9 (LTS) minus Fig. 5-3 (ZTS))

\section{Discussion of Figs. 5-7 through 5-12}

Northward of $75^{\circ} \mathrm{S}$, the means of the monthly differences between the two reanalyses in Figs. 5-7 through 5-9 are greater than five $\mathrm{m} \mathrm{s}^{-1}$ in only one small region, and only at $80^{\circ} \mathrm{S}$ in Fig. 5-8 (1958-78) are the difference greater than six m s${ }^{-1}$. Northward of $30^{\circ} \mathrm{N}$, mean differences are very rarely greater than two $\mathrm{m} \mathrm{s}^{-1}$, and in large regions, especially northward of $45^{\circ} \mathrm{N}$, generally less than one $\mathrm{m} \mathrm{s}^{-1}$. The mean differences are generally positive; i.e., ERA-40's wind speeds are greater than NCEP1's only southward of $60^{\circ} \mathrm{S}$ and at $10 \mathrm{hPa}$ at the Equator.

The nature of the standard deviation and rms pressure level (height) vs. latitude differences are similar in character to those of the means just discussed. Standard deviation and rms southward of $30^{\circ} \mathrm{S}$ are approximately twice those northward of $30^{\circ} \mathrm{N}$. In Fig. 5-9, covering the years 1979-2001, these extratropical variances in each hemisphere are one-half to one-third those of the first two figures, again demonstrating that the addition of satellite data to the assimilation process reduced the variance of the individual forecast models. However, the tropical standard deviation and rms, driven by the differences in QBO reanalyses, are not appreciably reduced in the modern satellite era. 
Extremely large maximum differences of NCEP1 minus ERA-40 zonal wind speeds are found in Figs. 5-7 (1958-2001) and 5-8 (1958-78), decreasing from more than $55 \mathrm{~m} \mathrm{~s}^{-1}$ in the Southern Hemisphere midlatitudes and $35 \mathrm{~m} \mathrm{~s}^{-1}$ in the Tropics and Northern Hemisphere midlatitudes at $10 \mathrm{hPa}$ to approximately $15 \mathrm{~m} \mathrm{~s}^{-1}$ at $100 \mathrm{hPa}$. In Fig. 5-9 (1979-2001), these extratropical maxima in each hemisphere are significantly less than those of the first two figures but the tropical maxima are unchanged.

Figures 5-10 through 5-12 quantify the variance lost by zonally averaging zonal wind speed. At $100 \mathrm{hPa}$ at the Equator, the three figures all display regions of standard deviation and rms with maxima of $1.8 \mathrm{~m} \mathrm{~s}^{-1}$. Additionally, at $10 \mathrm{hPa}$ there are regions of increased standard deviation and rms that vary from approximately $1.8 \mathrm{~m} \mathrm{~s}^{-1}$ (Fig. 5-10, 1958-2001), greater than $2 \mathrm{~m} \mathrm{~s}^{-1}$ (Fig. 5-11, 1958--78), and $1.4 \mathrm{~m} \mathrm{~s}^{-1}$ (Fig. 5-12, 1979-2001). These regions are the result of zonal wind speed anomalies with respect to zonal means that are illustrated and discussed in Section 5.3. Finally, at both poles, standard deviation and rms of greater than three $\mathrm{m} \mathrm{s}^{-1}$ (Fig. 5-10, 1958-2001) and greater than four $\mathrm{m} \mathrm{s}^{-1}$ (Fig. 5-11, 1958-78) decrease to approximately two $\mathrm{m} \mathrm{s}^{-1}$ (Fig. 5-12, 1979-2001) with satellite input to the assimilation process. The longitude-time series minus zonal time series differences enumerated in this paragraph are small with respect to maximum polar vortex wind speeds but are significant when compared with the multiyear mean zonal wind speed time series shown in Section 5.2. 

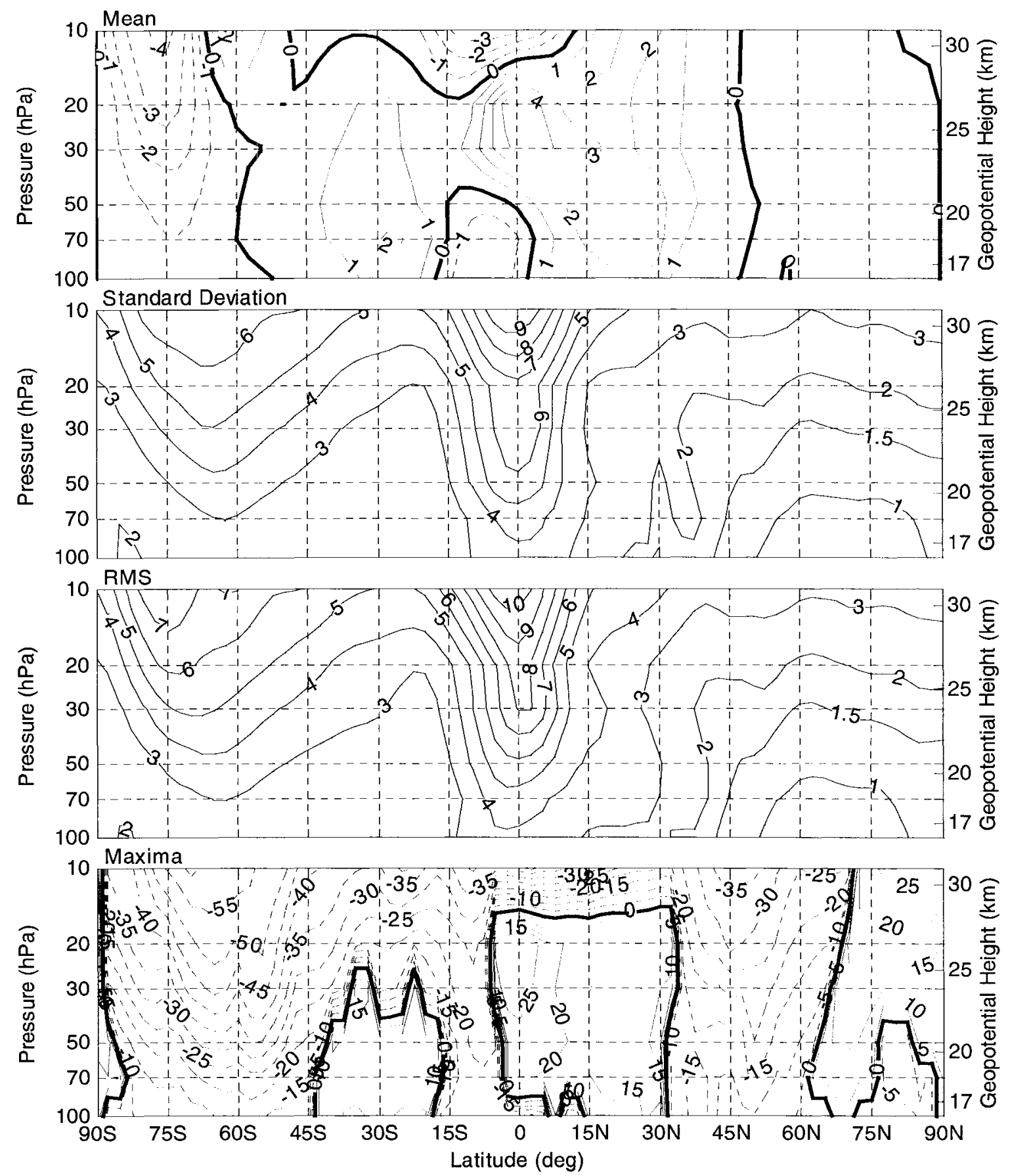

Fig. 5-7. Mean, standard deviation, rms, and maxima of longitude-time series of differences in monthly averaged zonal wind speed $\left(\mathrm{m} \mathrm{s}^{-1}\right)$ :

NCEP1 minus ERA-40 [lon: mean of $0^{\circ}-357.5^{\circ}$, lat: $90^{\circ} \mathrm{S}-90^{\circ} \mathrm{N}$, press: 100-10 hPa, time: Jan 1958-Dec 2001]. 

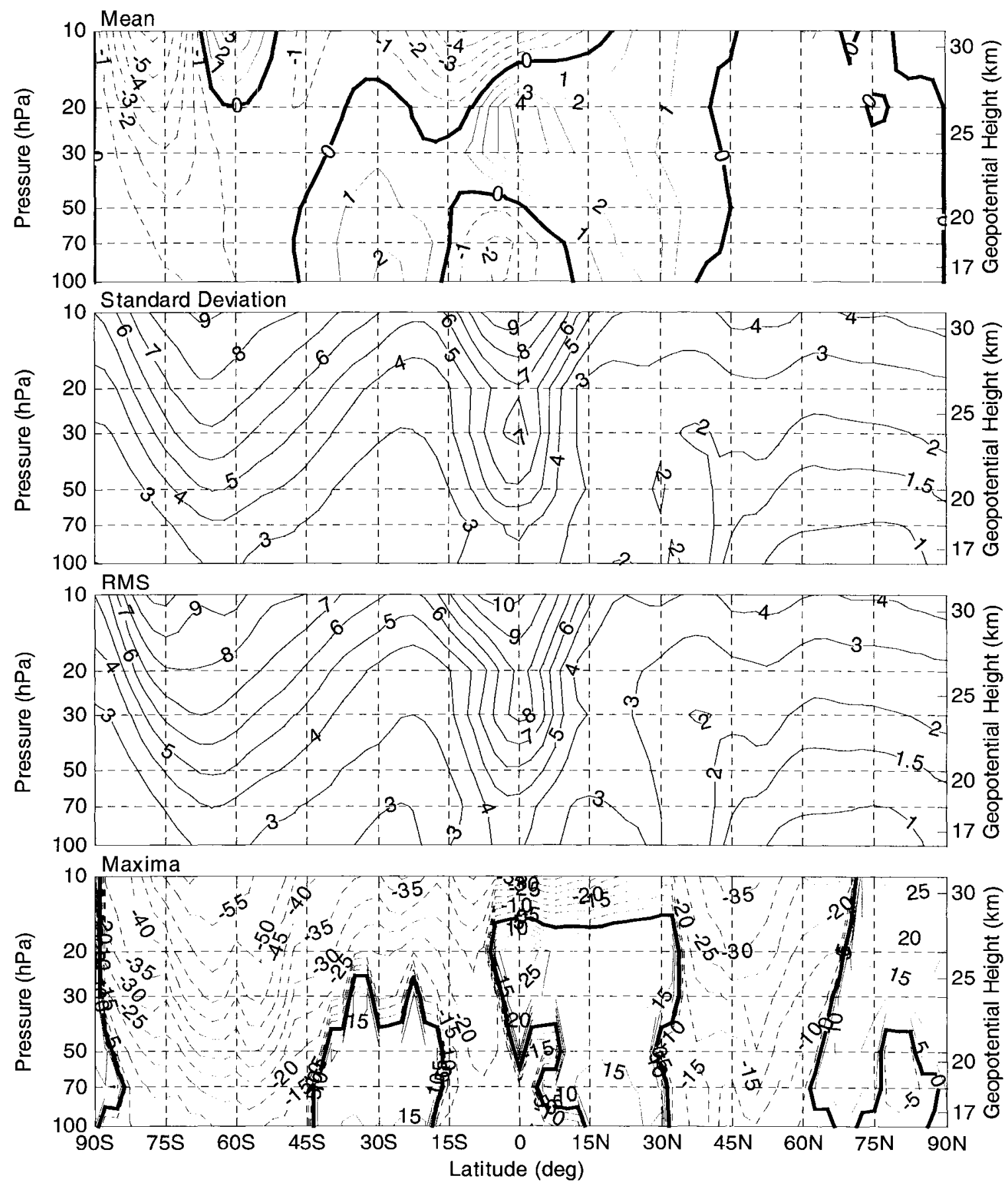

Fig. 5-8. Same as Fig. 5-7 except time: Jan 1958-Dec 1978. 

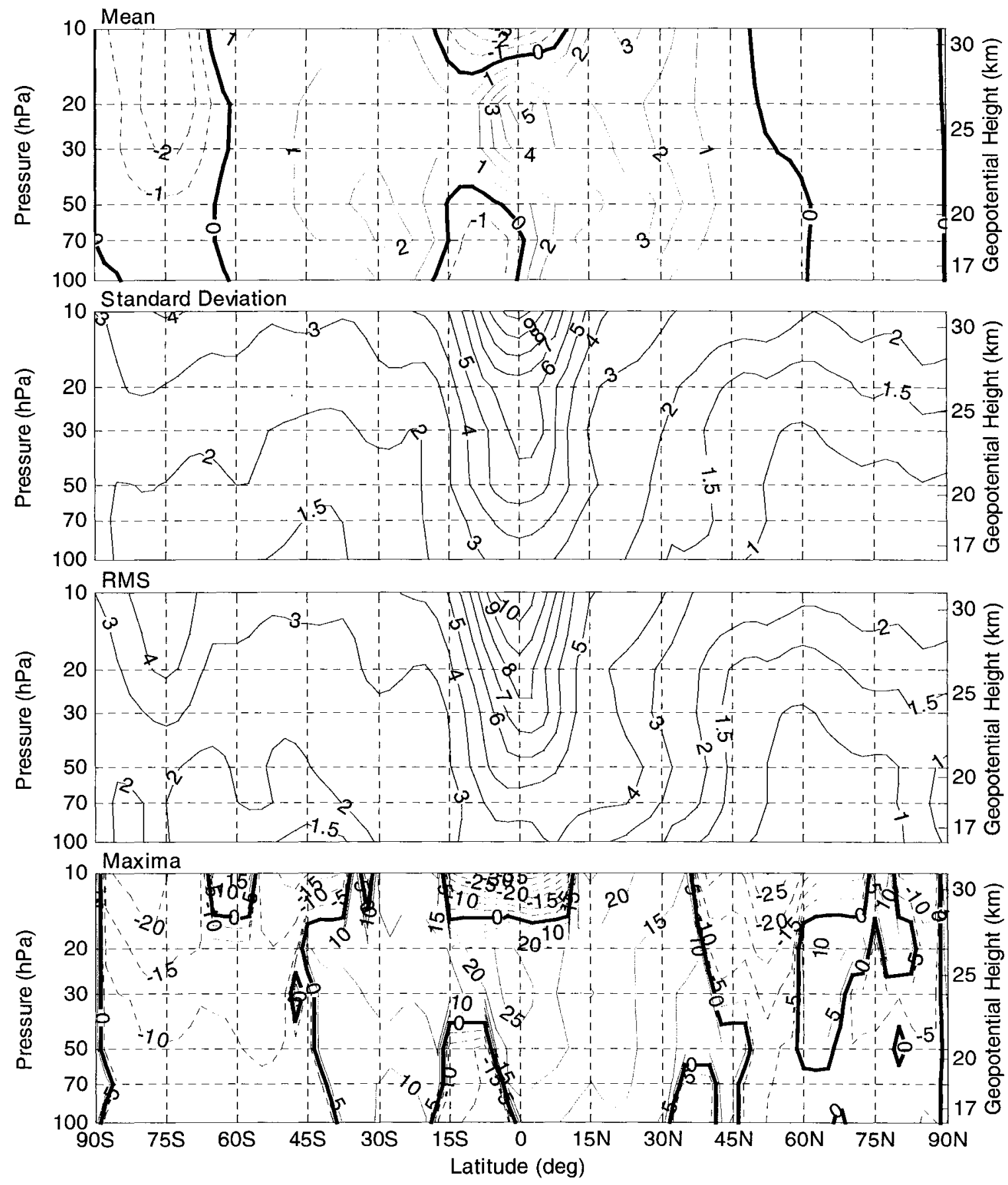

Fig. 5-9. Same as Fig. 5-7 except time: Jan 1979-Dec 2001. 

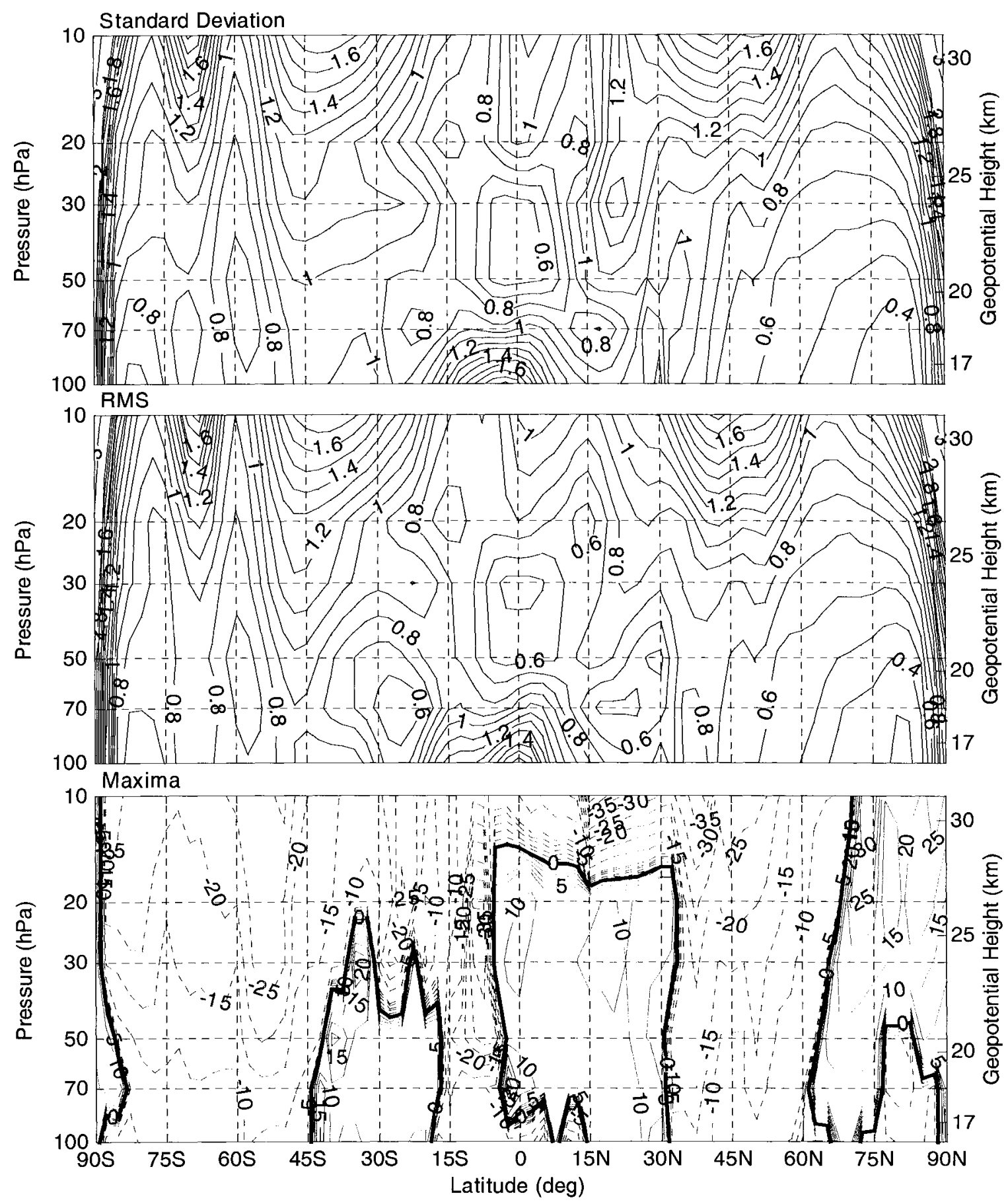

Fig. 5-10. Standard deviation, rms, and maxima of longitude-time series (Fig. 5-7) minus time series of differences in monthly-and zonally-averaged (Fig. 5-1) zonal wind speed $\left(\mathrm{m} \mathrm{s}^{-1}\right)$ : NCEP1 minus ERA-40 [lon: mean of $0^{\circ}-357.5^{\circ}$, lat: $90^{\circ} \mathrm{S}-90^{\circ} \mathrm{N}$, press: 100-10 hPa, time: Jan 1958-Dec 2001]. 

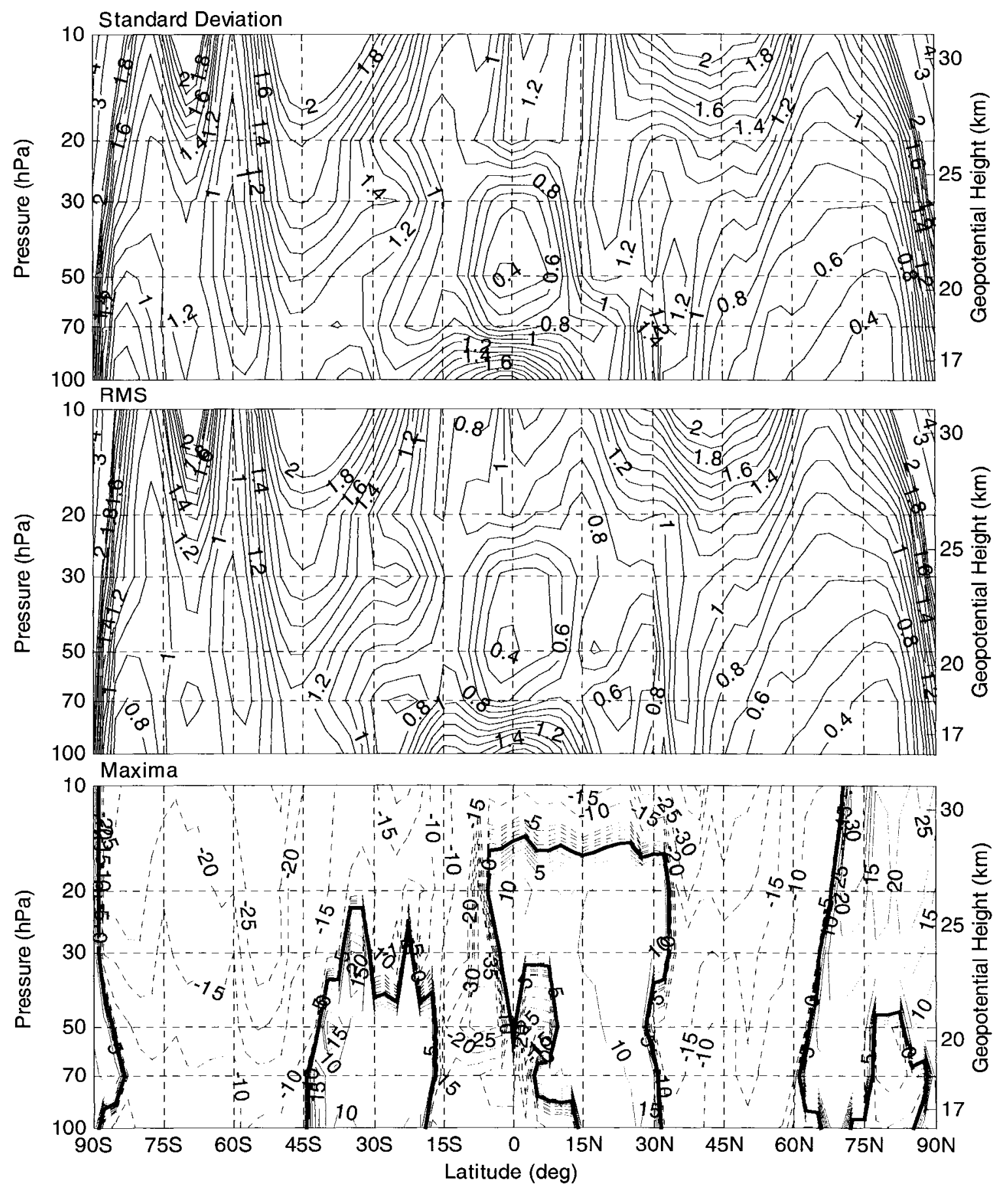

Fig. 5-11. Same as Fig. 5-10 except longitude-time series is Fig. 5-8 and zonallyaveraged time series is Fig. 5-2, and time: Jan 1958-Dec 1978. 

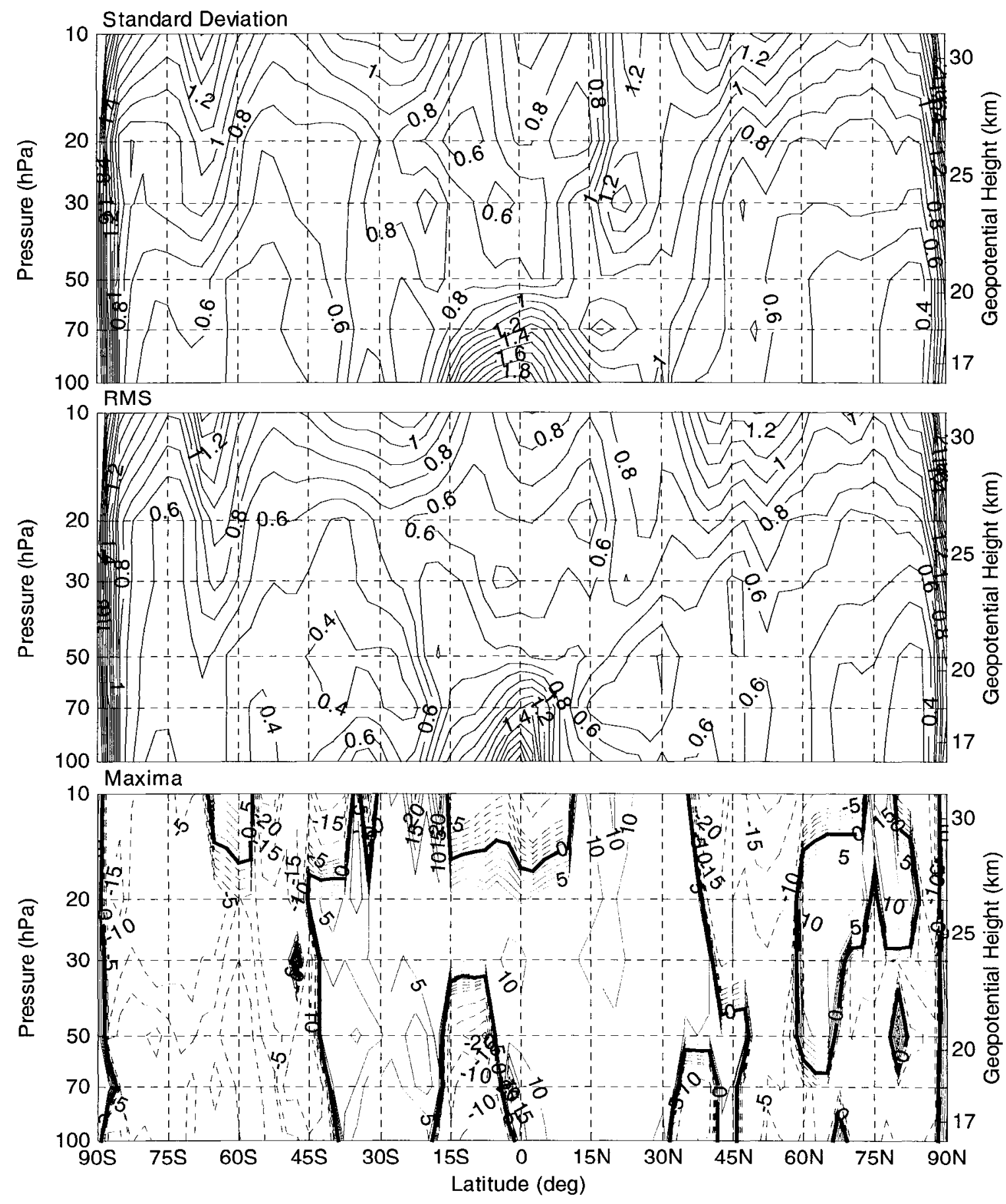

Fig. 5-12. Same as Fig. 5-10 except longitude-time series is Fig. 5-9 and zonallyaveraged time series is Fig. 5-3, and time: 1979-2001. 
Table 5-1. Maximum zonal wind speed differences $\left(\mathrm{m} \mathrm{s}^{-1}\right)$ and their locations for cases presented in Figs. 5-1 through 5-9.

\begin{tabular}{|c|c|c|c|c|c|c|c|c|c|}
\hline \multirow[b]{2}{*}{$\begin{array}{c}\text { NCEP1 minus } \\
\text { ERA-40 } \\
(1958-2001)\end{array}$} & \multirow[b]{2}{*}{ LTS } & \multicolumn{3}{|c|}{ Max Positive } & \multicolumn{3}{|c|}{ Max Negative } & \multirow{2}{*}{$\begin{array}{c}90^{\circ} \mathrm{S} \\
10 \mathrm{hPa} \\
32 \\
0.0\end{array}$} & \multirow{2}{*}{$\begin{array}{c}90^{\circ} \mathrm{N} \\
10 \mathrm{hPa} \\
24.7 \\
0.0\end{array}$} \\
\hline & & $\begin{array}{l}32.0 \\
20.1\end{array}$ & $\begin{array}{l}90^{\circ} \mathrm{S} \\
2.5^{\circ} \mathrm{S}\end{array}$ & $\begin{array}{l}10 \mathrm{hPa} \\
20 \mathrm{hPa}\end{array}$ & $\begin{array}{l}-61.6 \\
-38.7\end{array}$ & $\begin{array}{l}70^{\circ} \mathrm{S} \\
70^{\circ} \mathrm{S}\end{array}$ & $\begin{array}{l}10 \mathrm{hPa} \\
10 \mathrm{hPa}\end{array}$ & & \\
\hline $\begin{array}{c}\text { NCEP1 minus } \\
\text { ERA-40 } \\
(1958-1978)\end{array}$ & $\begin{array}{l}\text { LTS } \\
\text { ZTS }\end{array}$ & $\begin{array}{l}32.0 \\
18.4\end{array}$ & $\begin{array}{l}90^{\circ} \mathrm{S} \\
2.5^{\circ} \mathrm{S}\end{array}$ & $\begin{array}{l}10 \mathrm{hPa} \\
20 \mathrm{hPa}\end{array}$ & $\begin{array}{l}-61.6 \\
-38.7\end{array}$ & $\begin{array}{l}70^{\circ} \mathrm{S} \\
70^{\circ} \mathrm{S}\end{array}$ & $\begin{array}{l}10 \mathrm{hPa} \\
10 \mathrm{hPa}\end{array}$ & $\begin{array}{l}32 \\
0.0\end{array}$ & $\begin{array}{c}24.7 \\
0.0\end{array}$ \\
\hline $\begin{array}{c}\text { NCEP1 minus } \\
\text { ERA-40 } \\
(1979-2001)\end{array}$ & $\begin{array}{l}\text { LTS } \\
\text { ZTS }\end{array}$ & $\begin{array}{l}29.0 \\
20.1\end{array}$ & $\begin{array}{c}\mathrm{EQ} \\
2.5^{\circ} \mathrm{S}\end{array}$ & $\begin{array}{l}30 \mathrm{hPa} \\
20 \mathrm{hPa}\end{array}$ & $\begin{array}{l}-41.7 \\
-31.4\end{array}$ & $\begin{array}{l}2.5^{\circ} \mathrm{N} \\
2.5^{\circ} \mathrm{S}\end{array}$ & $\begin{array}{l}10 \mathrm{hPa} \\
10 \mathrm{hPa}\end{array}$ & $\begin{array}{l}17.7 \\
0.0\end{array}$ & $\begin{array}{l}12.7 \\
0.0\end{array}$ \\
\hline $\begin{array}{c}\text { NCEP1 minus } \\
\text { ERA-40 } \\
(1992-1999)\end{array}$ & $\begin{array}{l}\text { LTS } \\
\text { ZTS }\end{array}$ & $\begin{array}{l}23.9 \\
14.5\end{array}$ & $\begin{array}{l}5^{\circ} \mathrm{N} \\
2.5^{\circ} \mathrm{N}\end{array}$ & $\begin{array}{l}50 \mathrm{hPa} \\
50 \mathrm{hPa}\end{array}$ & $\begin{array}{l}-41.7 \\
-23.4\end{array}$ & $\begin{array}{l}2.5^{\circ} \mathrm{N} \\
5^{\circ} \mathrm{N}\end{array}$ & $\begin{array}{l}10 \mathrm{hPa} \\
10 \mathrm{hPa}\end{array}$ & $\begin{array}{l}7.5 \\
0.0\end{array}$ & $\begin{array}{l}8.6 \\
0.0\end{array}$ \\
\hline $\begin{array}{l}\text { NCEP1 minus } \\
\text { METO } \\
(1992-1999)\end{array}$ & ZTS & 15.4 & $5^{\circ} \mathrm{N}$ & $10 \mathrm{hPa}$ & -12.8 & $10^{\circ} \mathrm{S}$ & $10 \mathrm{hPa}$ & 5.8 & 3.7 \\
\hline $\begin{array}{c}\text { ERA-40 } \\
\text { minus METO } \\
(1992-1999)\end{array}$ & ZTS & 30.7 & $2.5^{\circ} \mathrm{S}$ & $10 \mathrm{hPa}$ & -14.2 & $5^{\circ} \mathrm{S}$ & $30 \mathrm{hPa}$ & 5.1 & 4.1 \\
\hline
\end{tabular}

LTS: Longitude-time series

ZTS: Zonally-averaged time series 


\section{Effect of annual cycle on time series differences}

Figures 5-1 through 5-12 present differences based on complete monthly- and zonally-averaged reanalysis zonal wind speed data and thus include differences between NCEP1 and ERA-40 characterizations of the annual cycle and its harmonics. Figures 5-15 through 5-17 illustrate the effect of the annual cycle and its harmonics on zonal wind speed differences by removing them with frequency-domain filtering, which eliminates all variance with period less than 13 months. The figures listed below display the four statistical measures for three cases with all variance less than 13 months removed; these three figures correspond to the unfiltered longitude-time series of Figs. 5-7 through 5-9.

Fig. 5-13. NCEP1 minus ERA-40 January 1958 through December 2001

Fig. 5-14. NCEP1 minus ERA-40 January 1958 through December 1978

Fig. 5-15. NCEP1 minus ERA-40 January 1979 through December 2001 The figures listed below illustrate the effect of the frequency-domain filtering by displaying longitude-time series filtered (Figs. 5-13 through 5-15) minus longitude-time series unfiltered (Fig. 5-7 through 5-9).

Fig. 5-16. NCEP1 minus ERA-40 January 1958 through December 2001

Fig. 5-17. NCEP1 minus ERA-40 January 1958 through December 1978

Fig. 5-18. NCEP1 minus ERA-40 January 1979 through December 2001

\section{Discussion of Figs. 5-13 through 5-18}

Figures 5-13 through 5-15 plot the mean, standard deviation, rms, and maxima of longitude-time series of monthly averaged zonal wind speed differences with the annual 
cycle removed before differencing. Frequency-domain filtering is employed to remove all variance with period less than 13 months from each reanalysis longitude-time series, as discussed in Appendix E, and the filtered data differenced. The two three-figure sets referenced in this paragraph cover the periods 1958-2001, 1958-1978, and 1979-2001. Comparison of Figs. 5-13 through 5-15 with Fig. 5-1 through 5-3 (which include annual cycle data), respectively, reveals strong congruency in the corresponding contours, maxima less so than the first three subplots. As expected, magnitudes of the four statistics of the reanalysis differences are smaller when the annual cycle is removed. Figures 5-16 through 5-18 display the effect of this removal. Maximum and mean rms differences decrease by $1.4 \mathrm{~m} \mathrm{~s}^{-1}$ and $0.9 \mathrm{~m} \mathrm{~s}^{-1}, 1.5 \mathrm{~m} \mathrm{~s}^{-1}$ and $1.1 \mathrm{~m} \mathrm{~s}^{-1}$, and $1.4 \mathrm{~m} \mathrm{~s}^{-1}$ and $0.6 \mathrm{~m} \mathrm{~s}^{-1}$ for the three time periods. Area weighting of the mean values minimizes the contribution of maximum values that generally exist at higher latitudes. Removal of the annual cycle and its harmonics with notch filtering in the frequency-domain (Appendix E) was also evaluated but results not plotted. Because of the additional variance retained with this technique, the reduction in difference statistics is less than with low-pass filtering: $0.5 \mathrm{~m} \mathrm{~s}^{-1}$ and $0.4 \mathrm{~m} \mathrm{~s}^{-1}, 0.7 \mathrm{~m} \mathrm{~s}^{-1}$ and $0.4 \mathrm{~m} \mathrm{~s}^{-1}$, and $0.5 \mathrm{~m} \mathrm{~s}^{-1}$ and $0.3 \mathrm{~m} \mathrm{~s}^{-1}$, respectively, for the three time periods.

Table 5-2 quantitatively summarizes the results of the three methods of processing the NCEP1 and ERA-40 zonal wind speed differences. The pressure level vs. latitude data is area weighted to form mean values of each statistic, minimizing the contribution of higher latitudes, where maximum values are generally found. 

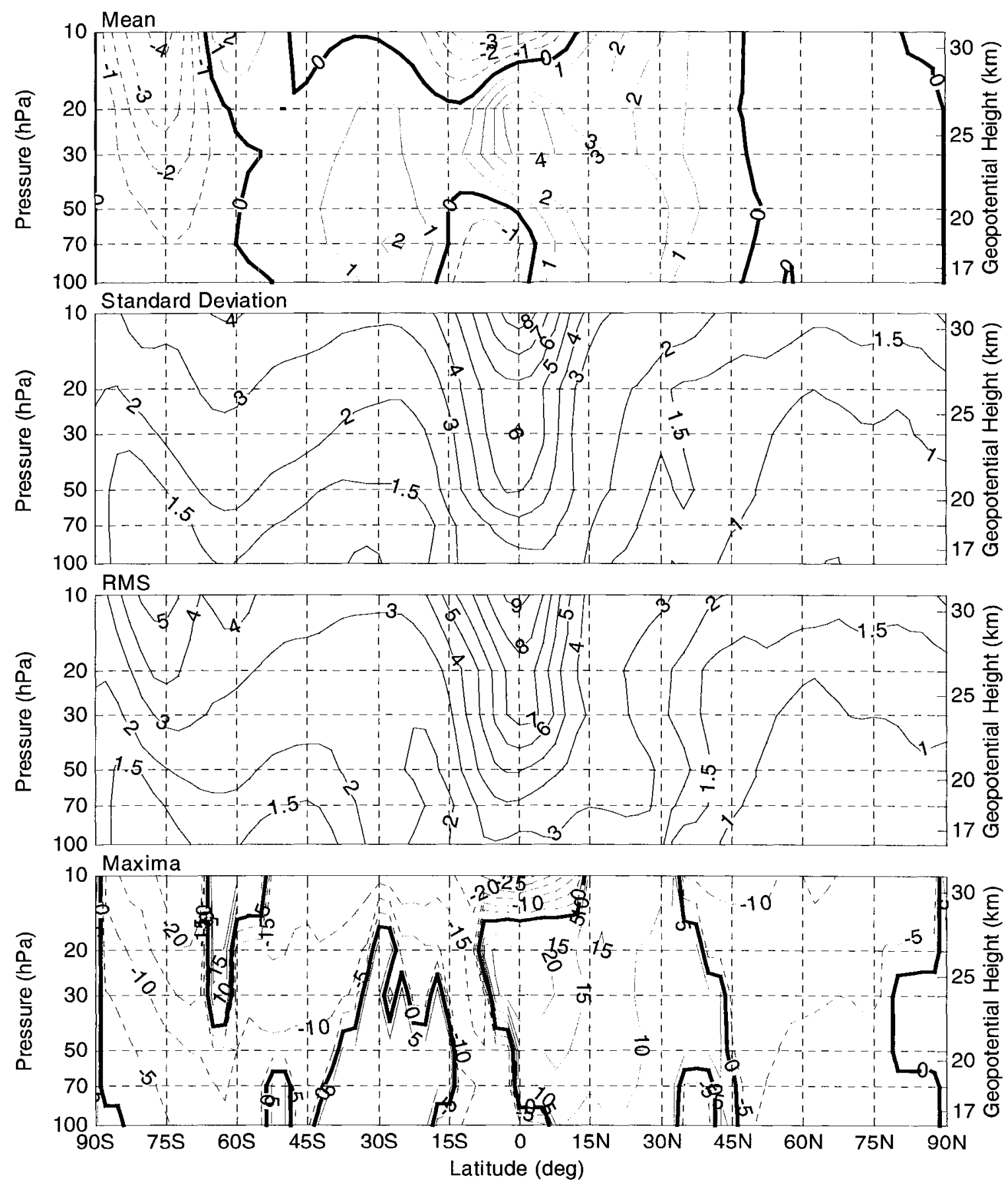

Fig. 5-13. Mean, standard deviation, rms, and maxima of longitude-time series of monthly-averaged zonal wind speed differences with all variance with period less than 13 months removed before differencing $\left(\mathrm{m} \mathrm{s}^{-1}\right)$ : NCEPI minus ERA-40 [lon: mean of $0^{\circ}-357.5^{\circ}$, lat: $90^{\circ} \mathrm{S}-90^{\circ} \mathrm{N}$, press: 100-10 hPa, time: Jan 1958-Dec 2001]. 

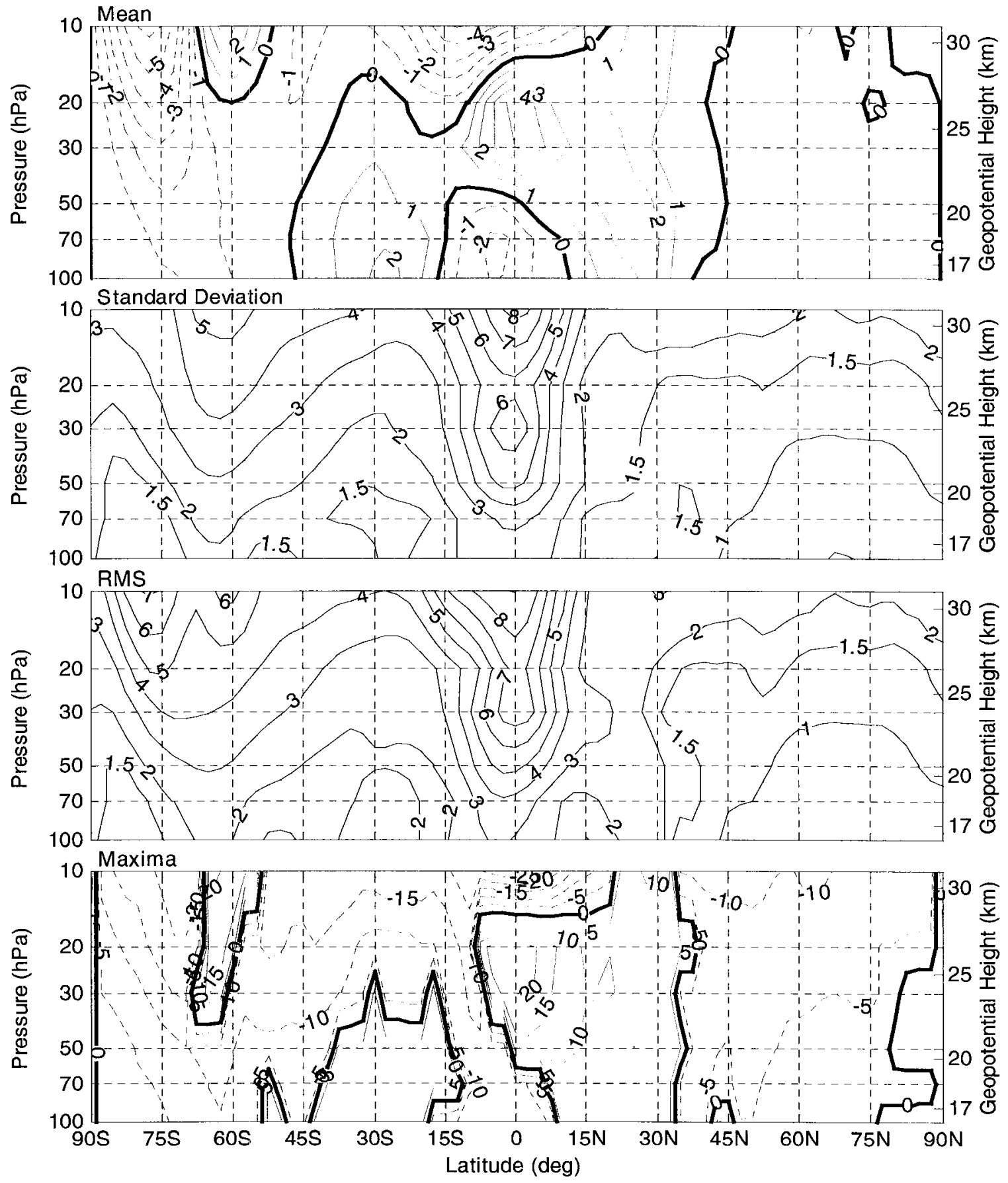

Fig. 5-14. Same as Fig. 5-13 except time: 1958-1978. 

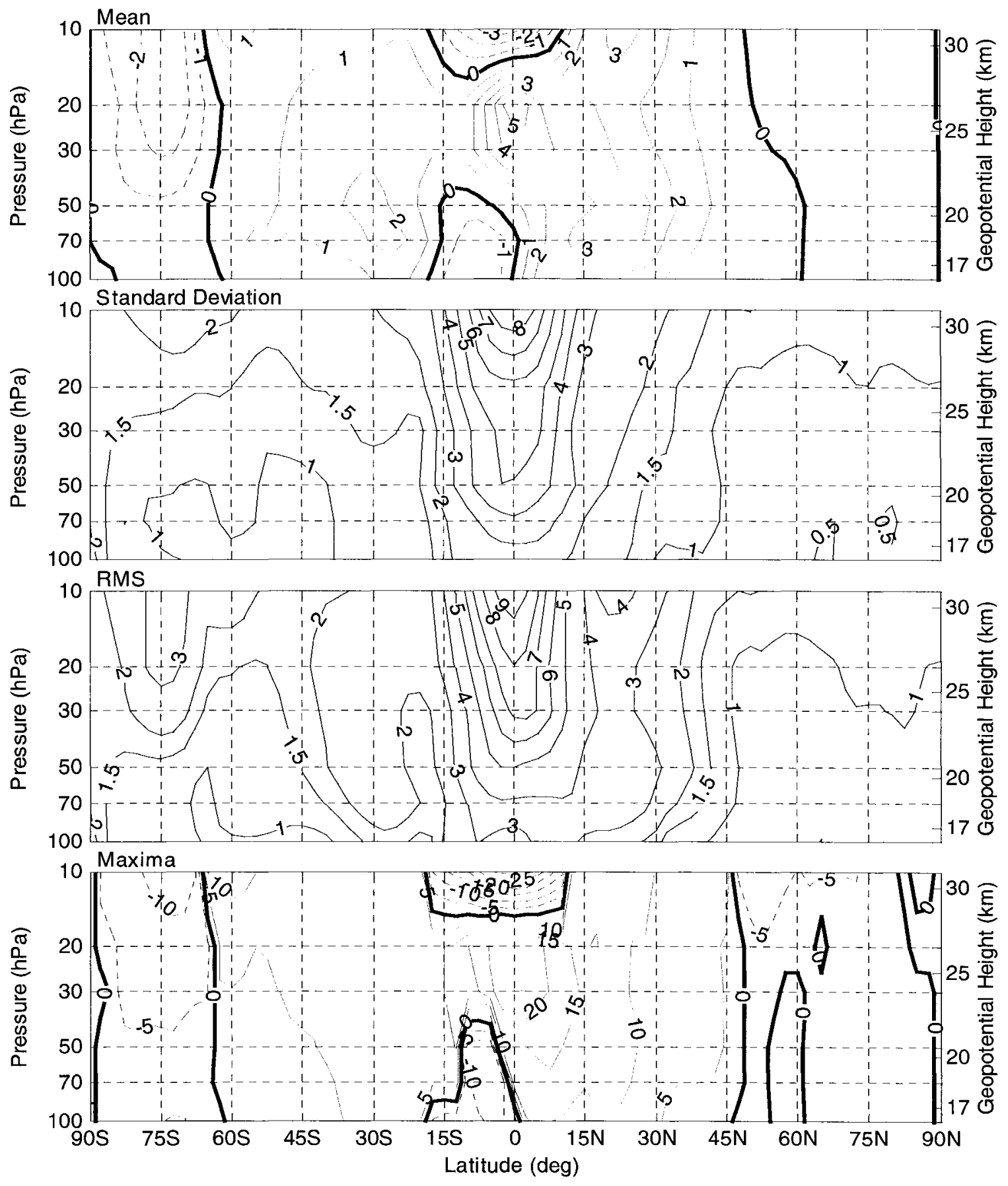

Fig. 5-15. Same as Fig. 5-13 except time: 1979-2001. 


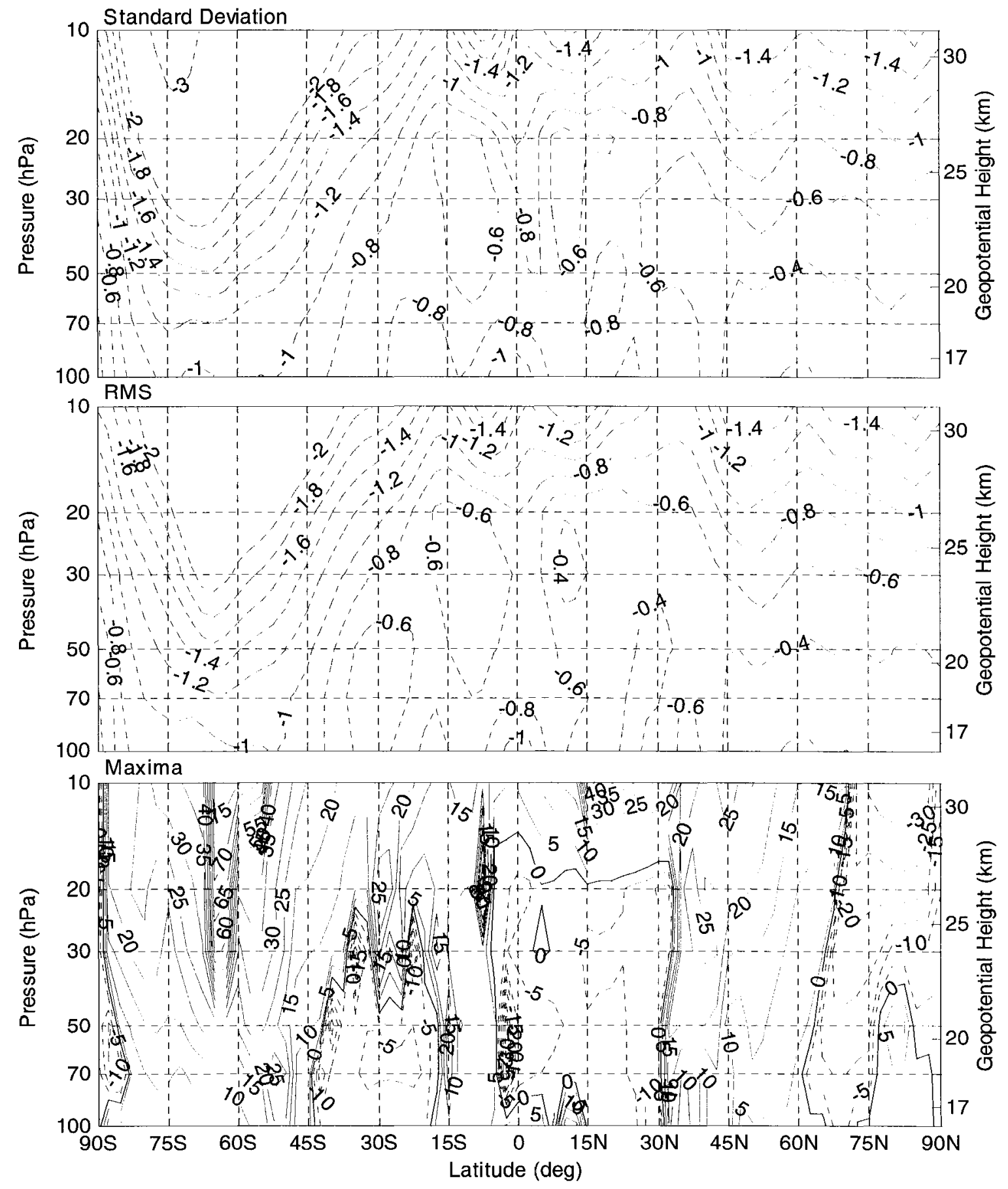

Fig. 5-16. Standard deviation, rms, and maxima of filtered longitude-time series (Fig. 5- 13) minus unfiltered longitude-time series (Fig. 5-7) zonal wind speed differences $\left(\mathrm{m} \mathrm{s}^{-1}\right)$ : NCEP1 minus ERA-40 [lon: mean of $0^{\circ}-357.5^{\circ}$, lat: $90^{\circ} \mathrm{S}-90^{\circ} \mathrm{N}$, press: 100-10 hPa, time: Jan 1958-Dec 2001]. 


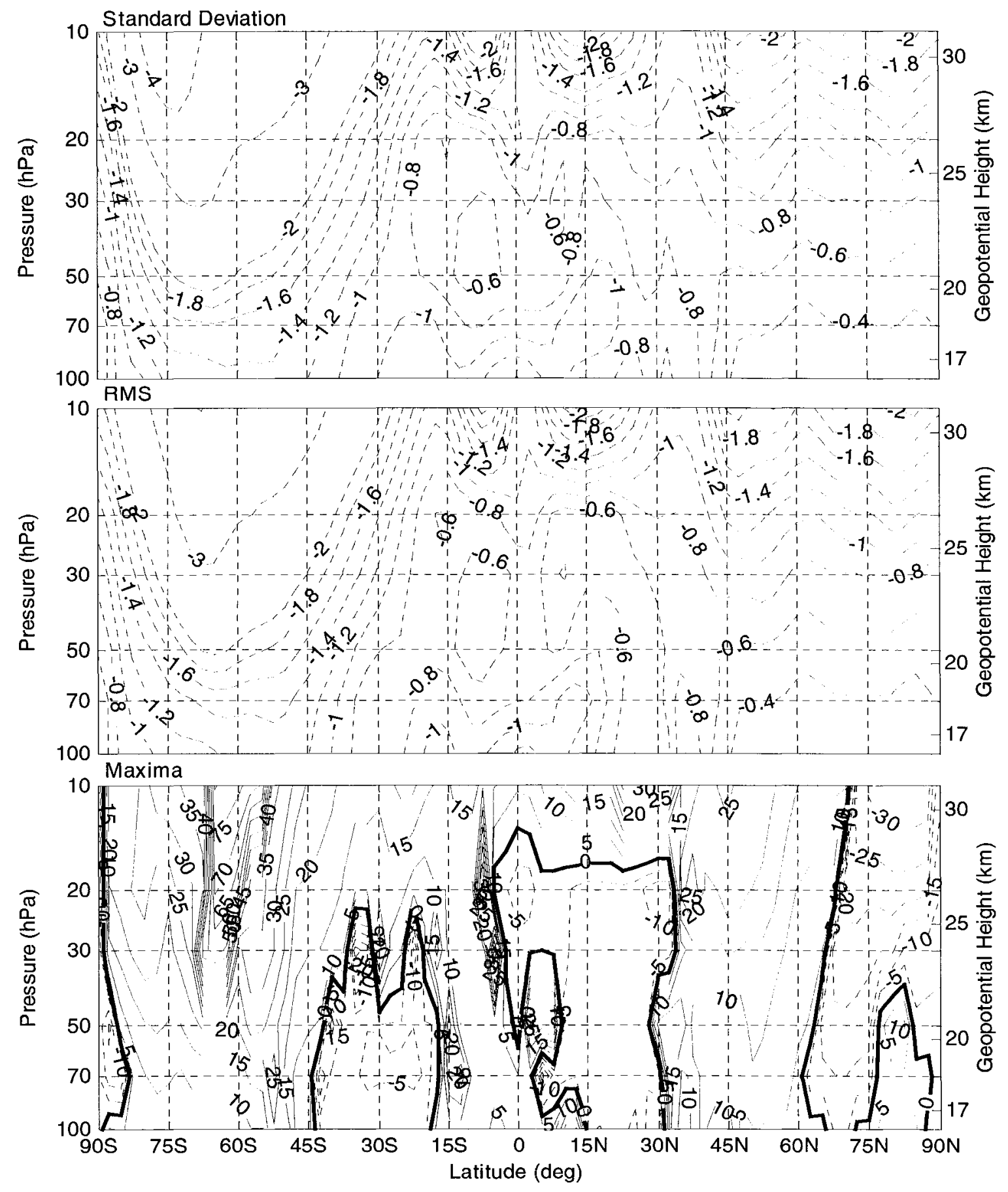

Fig. 5-17. Same as Fig. 5-16 except filtered longitude-time series is Fig. 5-14, unfiltered longitude-time series is Fig. 5-8, and time: 1958-1978. 

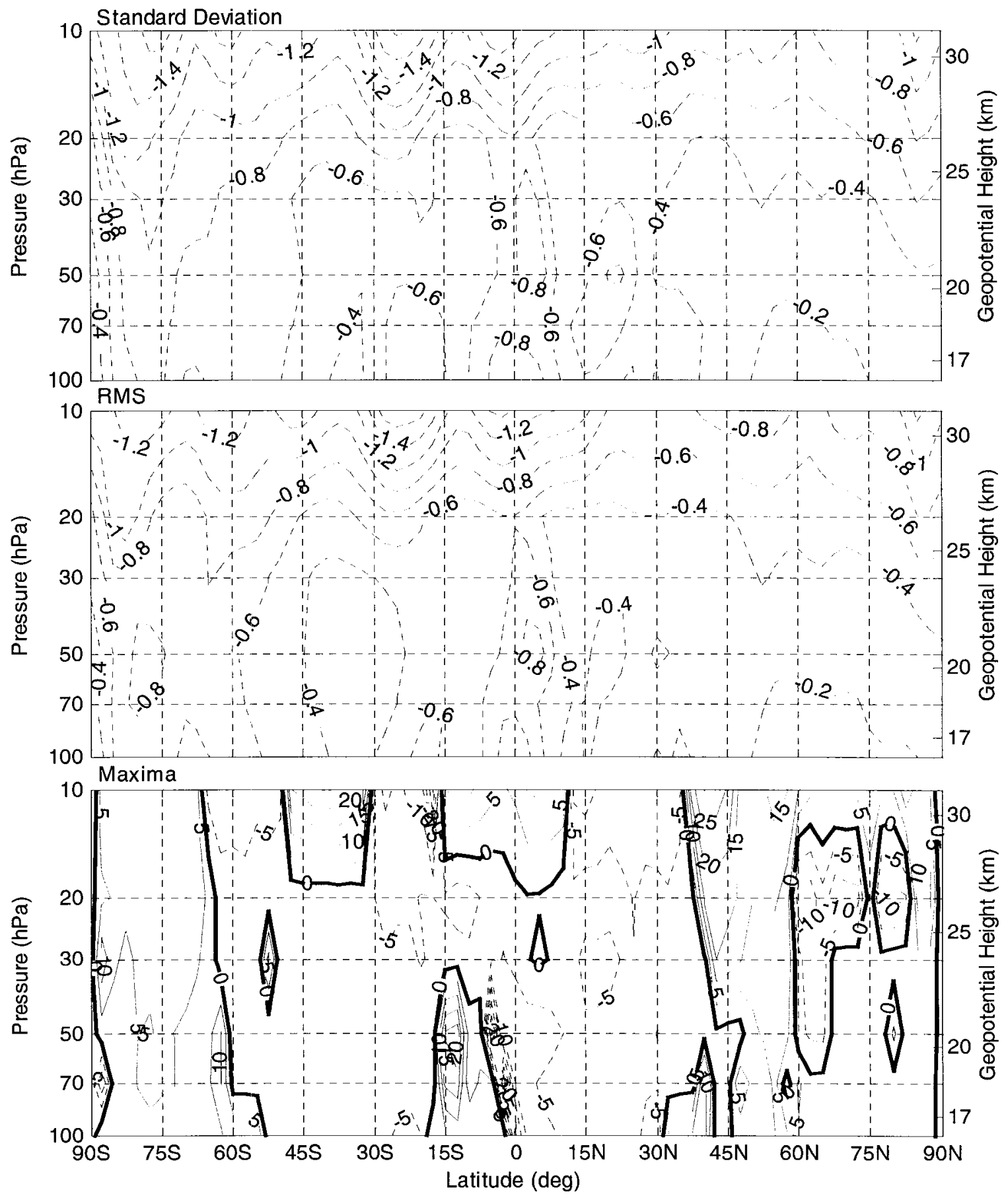

Fig. 5-18. Same as Fig. 5-16 except filtered longitude-time series is Fig. 5-15, unfiltered longitude-time series is Fig. 5-9, and time: 1979-2001. 
Table 5-2. Comparison of Section 5.1 reanalysis zonal wind speed difference processing.

\begin{tabular}{|c|c|c|c|c|c|c|}
\hline \multirow[t]{2}{*}{1958 - 2001} & \multicolumn{2}{|c|}{$\begin{array}{l}\text { Zonally- } \\
\text { Averaged }\end{array}$} & \multicolumn{2}{|c|}{$\begin{array}{c}\text { Longitude-Time } \\
\text { Series }\end{array}$} & \multicolumn{2}{|c|}{$\begin{array}{c}\text { Annual Cycle } \\
\text { Removed }\end{array}$} \\
\hline & $\max$ & mean & $\max$ & mean & $\max$ & mean \\
\hline mean & 5.04 & 0.60 & 5.04 & 0.60 & 5.04 & 0.60 \\
\hline $\mathrm{SD}$ & 9.08 & 2.38 & 10.02 & 3.36 & 8.66 & 2.39 \\
\hline RMS & 9.58 & 2.83 & 10.73 & 3.69 & 9.33 & 2.82 \\
\hline \multirow[t]{2}{*}{ MAX } & $\begin{array}{l}+20 \\
-39\end{array}$ & -2.56 & $\begin{array}{l}+32 \\
-62\end{array}$ & -8.99 & $\begin{array}{l}+25 \\
-33\end{array}$ & -0.98 \\
\hline & \multicolumn{2}{|c|}{ Fig. 5-1 } & \multicolumn{2}{|c|}{ Fig. 5-7 } & \multicolumn{2}{|c|}{ Fig. 5-13 } \\
\hline \multirow[t]{2}{*}{$1958-1978$} & \multicolumn{2}{|c|}{$\begin{array}{l}\text { Zonally- } \\
\text { Averaged }\end{array}$} & \multicolumn{2}{|c|}{$\begin{array}{c}\text { Longitude-Time } \\
\text { Series }\end{array}$} & \multicolumn{2}{|c|}{$\begin{array}{l}\text { Annual Cycle } \\
\text { Removed }\end{array}$} \\
\hline & $\max$ & mean & $\max$ & mean & $\max$ & mean \\
\hline mean & -6.34 & 0.17 & 4.49 & 0.17 & 4.49 & 0.17 \\
\hline $\mathrm{SD}$ & 8.76 & 2.56 & 9.73 & 3.72 & 8.40 & 2.48 \\
\hline RMS & 9.82 & 2.96 & 10.59 & 4.01 & 9.07 & 2.88 \\
\hline \multirow[t]{2}{*}{ MAX } & $\begin{array}{l}+18 \\
-39\end{array}$ & -3.85 & $\begin{array}{l}+32 \\
-62\end{array}$ & -10.55 & $\begin{array}{l}+23 \\
-30\end{array}$ & -2.50 \\
\hline & \multicolumn{2}{|c|}{ Fig. 5-2 } & \multicolumn{2}{|c|}{ Fig. 5-8 } & \multicolumn{2}{|c|}{ Fig. 5-14 } \\
\hline \multirow[t]{2}{*}{1979 - 2001} & \multicolumn{2}{|c|}{$\begin{array}{l}\text { Zonally- } \\
\text { Averaged }\end{array}$} & \multicolumn{2}{|c|}{$\begin{array}{c}\text { Longitude-Time } \\
\text { Series }\end{array}$} & \multicolumn{2}{|c|}{$\begin{array}{l}\text { Annual Cycle } \\
\text { Removed }\end{array}$} \\
\hline & $\max$ & mean & $\max$ & mean & $\max$ & mean \\
\hline mean & 5.55 & 1.00 & 5.55 & 1.00 & 5.55 & 1.00 \\
\hline $\mathrm{SD}$ & 9.45 & 1.92 & 10.40 & 2.77 & 8.93 & 2.10 \\
\hline RMS & 10.11 & 2.57 & 10.98 & 3.25 & 9.59 & 2.69 \\
\hline MAX & $\begin{array}{l}+20 \\
-31\end{array}$ & 3.07 & $\begin{array}{l}+29 \\
-42 \\
\end{array}$ & 4.67 & $\begin{array}{l}+25 \\
-33\end{array}$ & 4.44 \\
\hline
\end{tabular}

- Zonally-Averaged: Mean, standard deviation, rms, and maxima of monthly- and zonally-averaged zonal wind speed differences: NCEP1 minus ERA-40

- Longitude-Time Series: Same as "Zonally-Averaged" except longitude-time series of monthly averaged zonal wind speed differences formed from individual monthlyaveraged zonal wind speed time series

- Annual Cycle Removed: Same as "Longitude-Time-Series" except all variance with period less than 13 months removed from individual monthly- and zonally-averaged zonal wind speed time series before differencing and longitude-time series formed 
- Figure numbers below each case reference the figures containing the numerical values shown above

\subsection{Zonal wind speed anomaly time series}

Figures 5-19 through 5-24 display time series of the monthly- and zonallyaveraged zonal wind speeds for

- NCEP1 (January 1958 through December 2005: 48 years, 576 months)

- ERA-40 (January 1958 through December 2001: 44 years, 528 months)

- METO (January 1992 through December 1999: 8 years, 96 months)

The latitude ranges $90^{\circ} \mathrm{S}$ through $30^{\circ} \mathrm{S}$ (Southern Hemisphere extratropical), $30^{\circ} \mathrm{S}$ through $30^{\circ} \mathrm{N}$ (tropical), and $30^{\circ} \mathrm{N}$ through $90^{\circ} \mathrm{N}$ (Northern Hemisphere extratropical) are the component ranges employed for temperatures in Section 4.2. The data in these ranges represent averages of $25 \%, 50 \%$, and $25 \%$, of the global surface area, respectively. The three ranges represent the same percentages of the global zonal wind speed at the six stratospheric pressure levels.

In each latitude range, zonal wind speeds are weighted by the cosine of their latitude and averaged using trapezoidal integration. These weighted zonal wind speeds are then filtered in the frequency domain to remove all variance of period less than 13 months. Equatorial time series from 70 through $10 \mathrm{hPa}$ are filtered to remove all variance of period less than 36 months, in order to remove the QBO signal that is discussed in Section 5.5. These equatorial time series do not include METO data because an inadequate amount of the eight-year span of this data remains after the three-year filtering for an accurate filtered time series. The initial and final 36 months of the 
NCEP1 and ERA-40 equatorial time series are also removed for end-point reasons. The frequency domain filtering technique is described and illustrated in detail in Appendix E.

For clarity and figure-to-figure consistency, the time series are presented as zonal wind speed anomalies. Removed mean values, listed for each plot, are the average of the means of the area-weighted, unfiltered NCEP1 and ERA-40 means over their full duration.

Throughout this section NCEP1, ERA-40, and METO time series, with the exception of equatorial series at heights above $100 \mathrm{hPa}$, are remarkably consistent, often to the point of overlay, and even when their longer-term means diverge, their higher frequency waveforms are still highly correlated. Differences in zonal wind speed anomalies, where they exist, are small with respect to mean values. This behavior is in marked contrast to the differences in the temperature time series discussed in Section 4.2.

Northern extratropic time series display similar behavior except during 1979-90. In this latitude band, METO more closely matches ERA-40 than NCEP1. Equatorial zonal wind time series at heights above $100 \mathrm{hPa}$ illustrate the impact of the 36-month low-pass filter. They also illustrate the mean zonal wind speed difference between the NCEP1 and ERA-40 reanalyses, which reaches a maximum of four to five $\mathrm{m} \mathrm{s}^{-1}$ between 20 and $30 \mathrm{hPa}$ at the Equator (Figs. 5-1 through 5-4). Southern extratropic time series are best matched after 1987, with differences becoming larger and occurring earlier as height increases. 

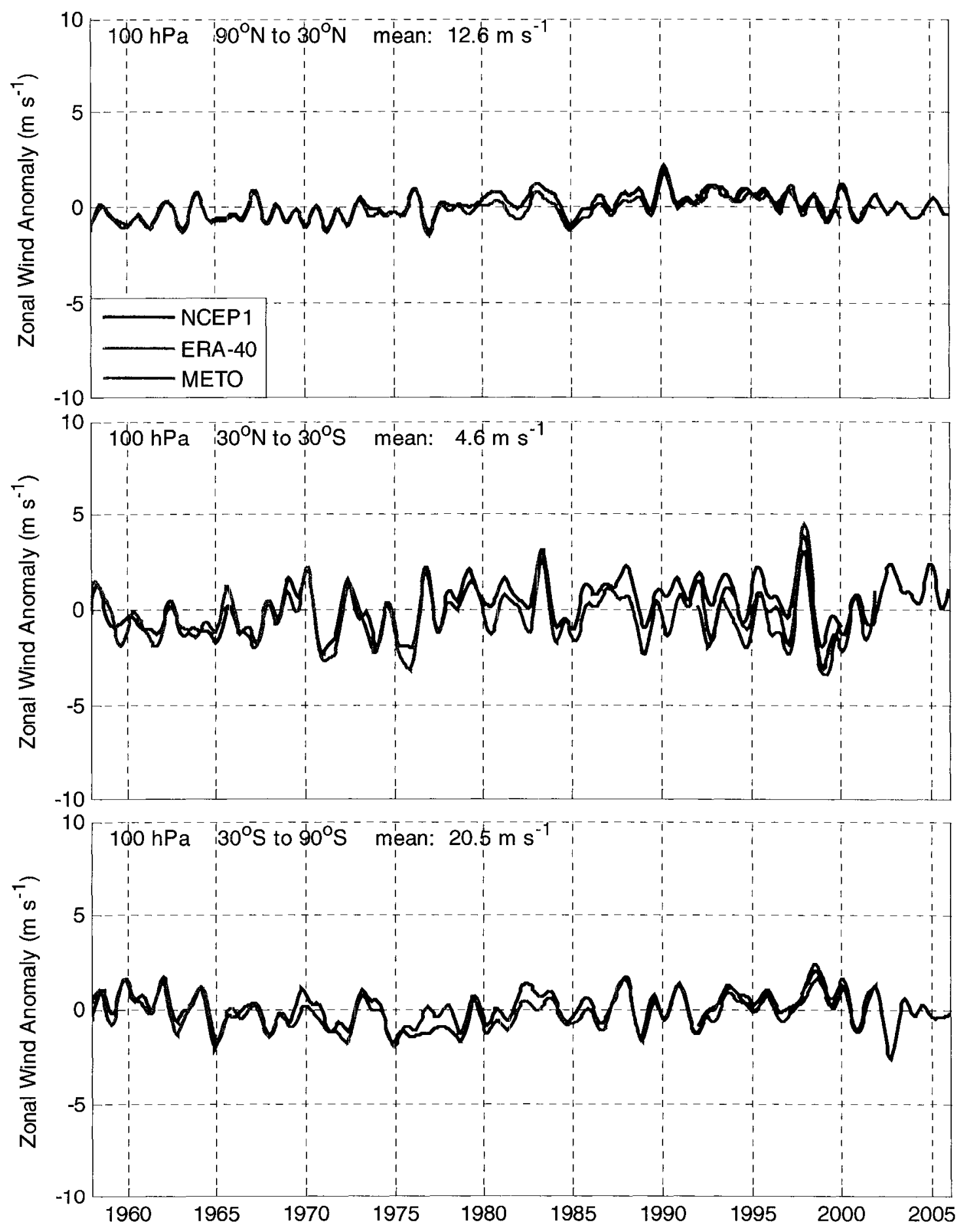

Fig. 5-19. Anomaly time series of monthly- and zonally-averaged zonal wind speed (all variance with period less than 13 months removed by frequency-domain filtering): [lon: mean of $0^{\circ}-357.5^{\circ}$; lat: as stated; press: $100 \mathrm{hPa}$; time: Jan 1958-Dec 2005 (NCEP1), Jan 1958-Dec 2001 (ERA-40), Jan 1992-Dec 1999 (METO)]. 

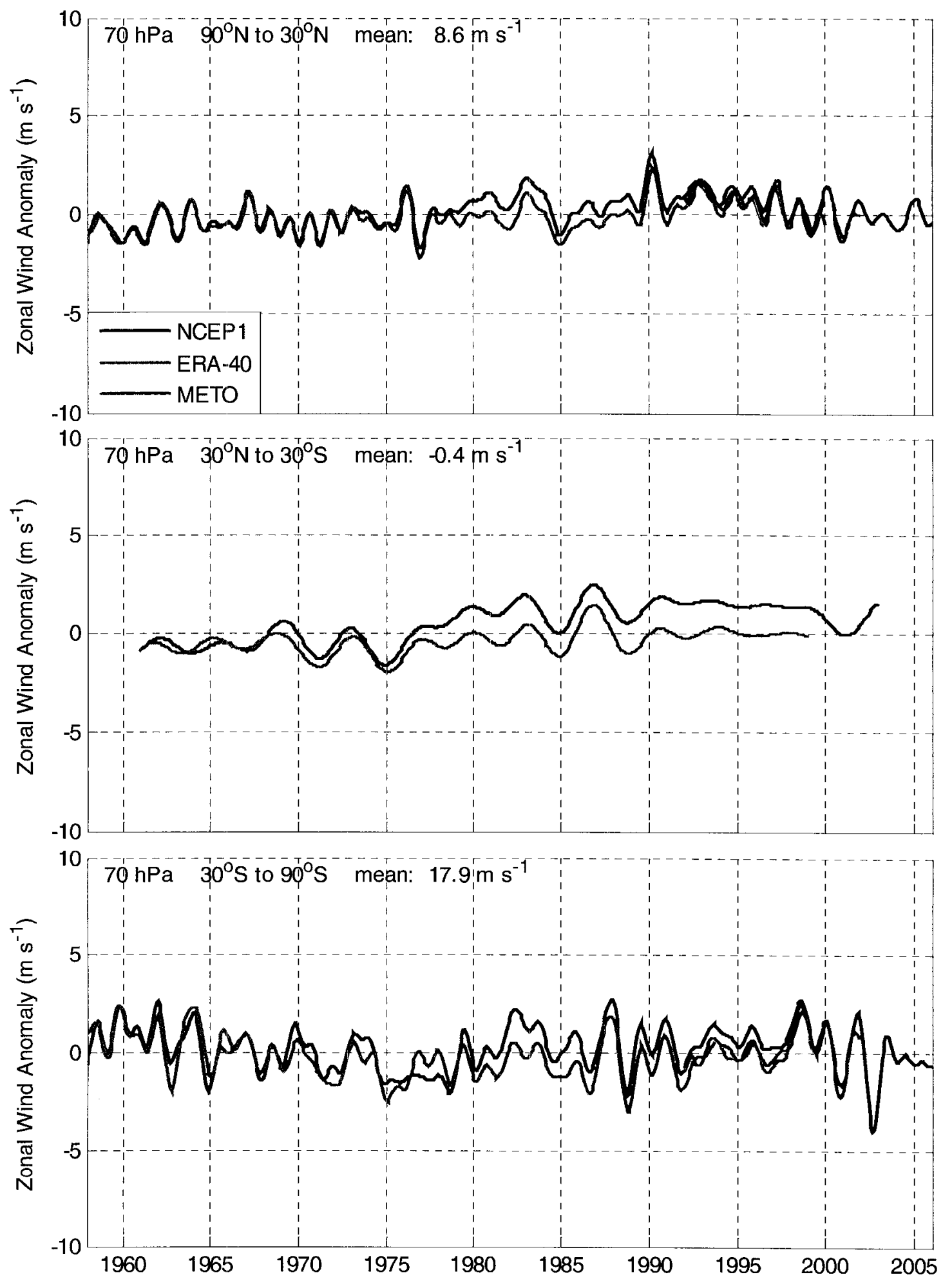

Fig. 5-20. Same as Fig. 5-19 except press: 70 hPa and for equatorial series all variance with period less than 36 months removed by frequency-domain filtering. 

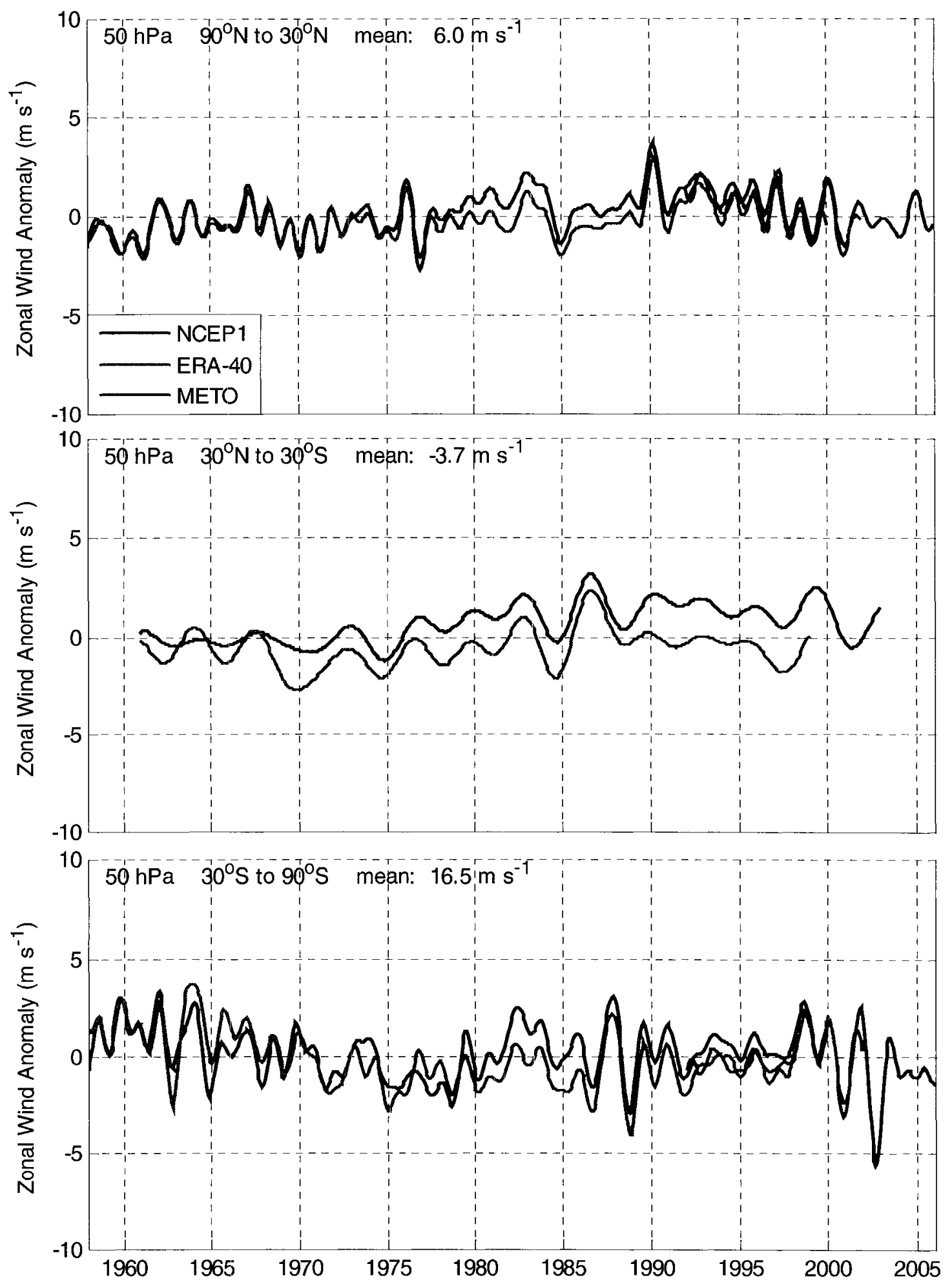

Fig. 5-21. Same as Fig. 5-19 except press: $50 \mathrm{hPa}$ and for equatorial series all variance with period less than 36 months removed by frequency-domain filtering. 

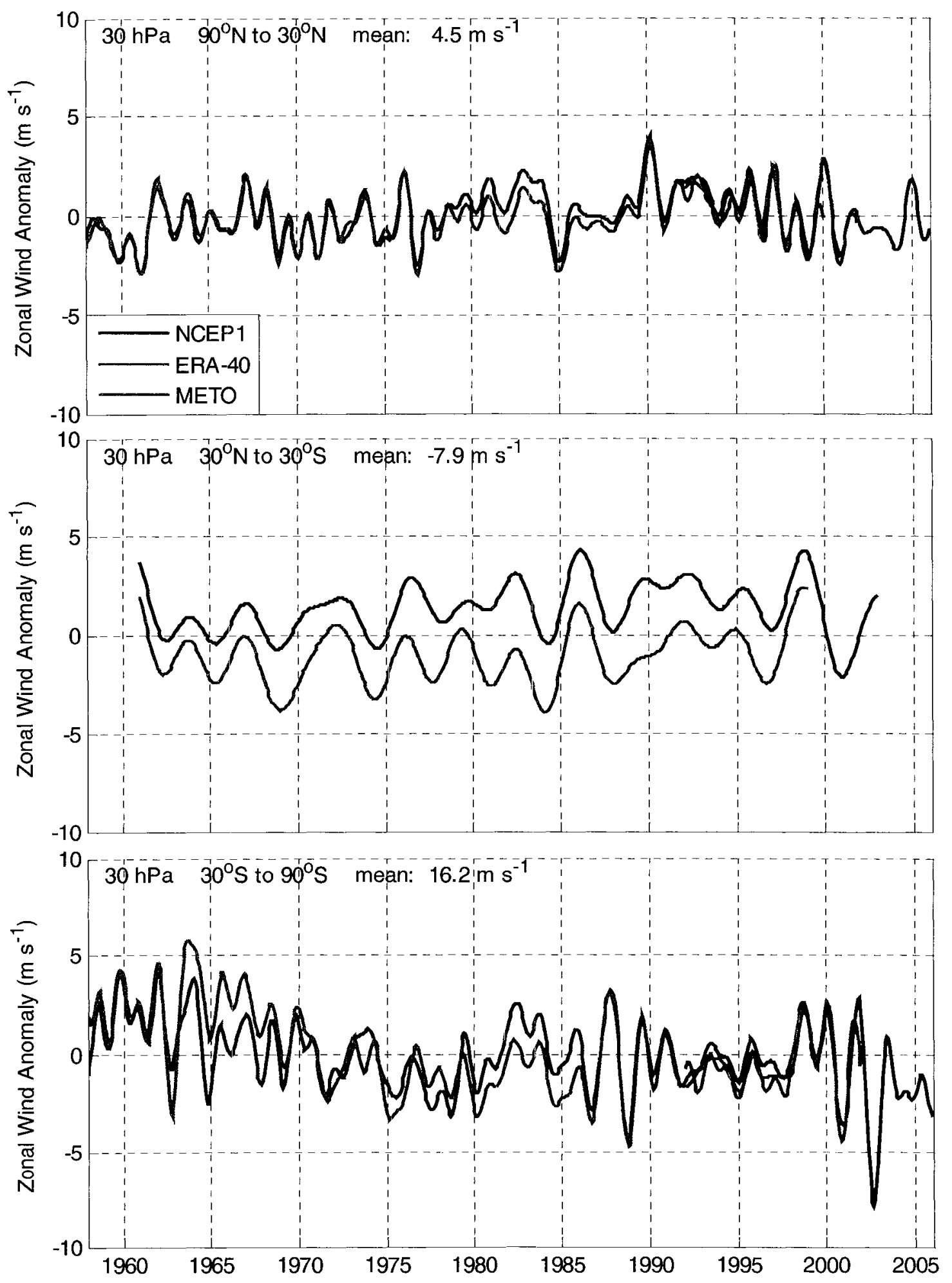

Fig. 5-22. Same as Fig. 5-19 except press: $30 \mathrm{hPa}$ and for equatorial series all variance with period less than 36 months removed by frequency-domain filtering. 

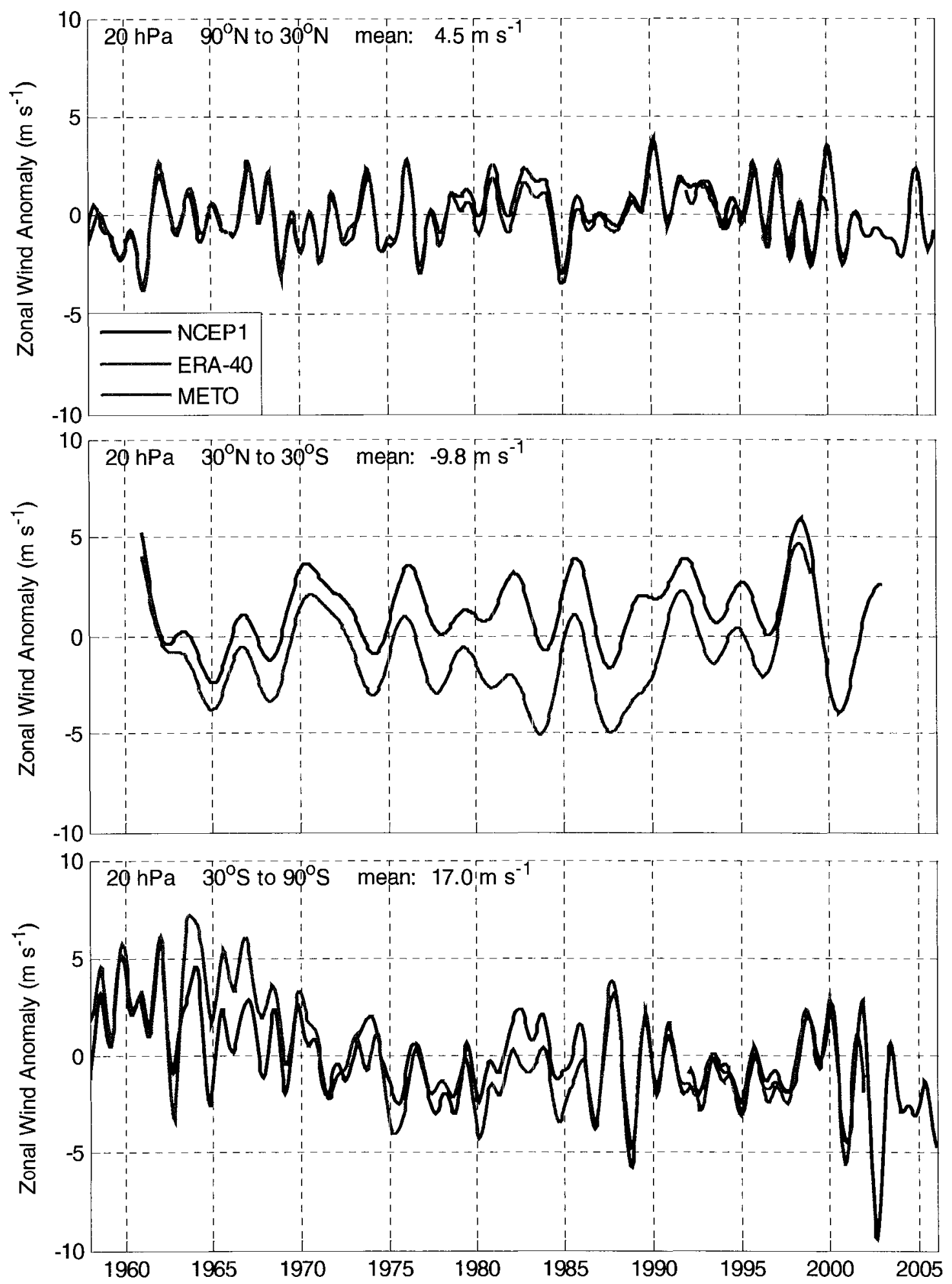

Fig. 5-23. Same as Fig. 5-19 except press: 20 hPa and for equatorial series all variance with period less than 36 months removed by frequency-domain filtering. 

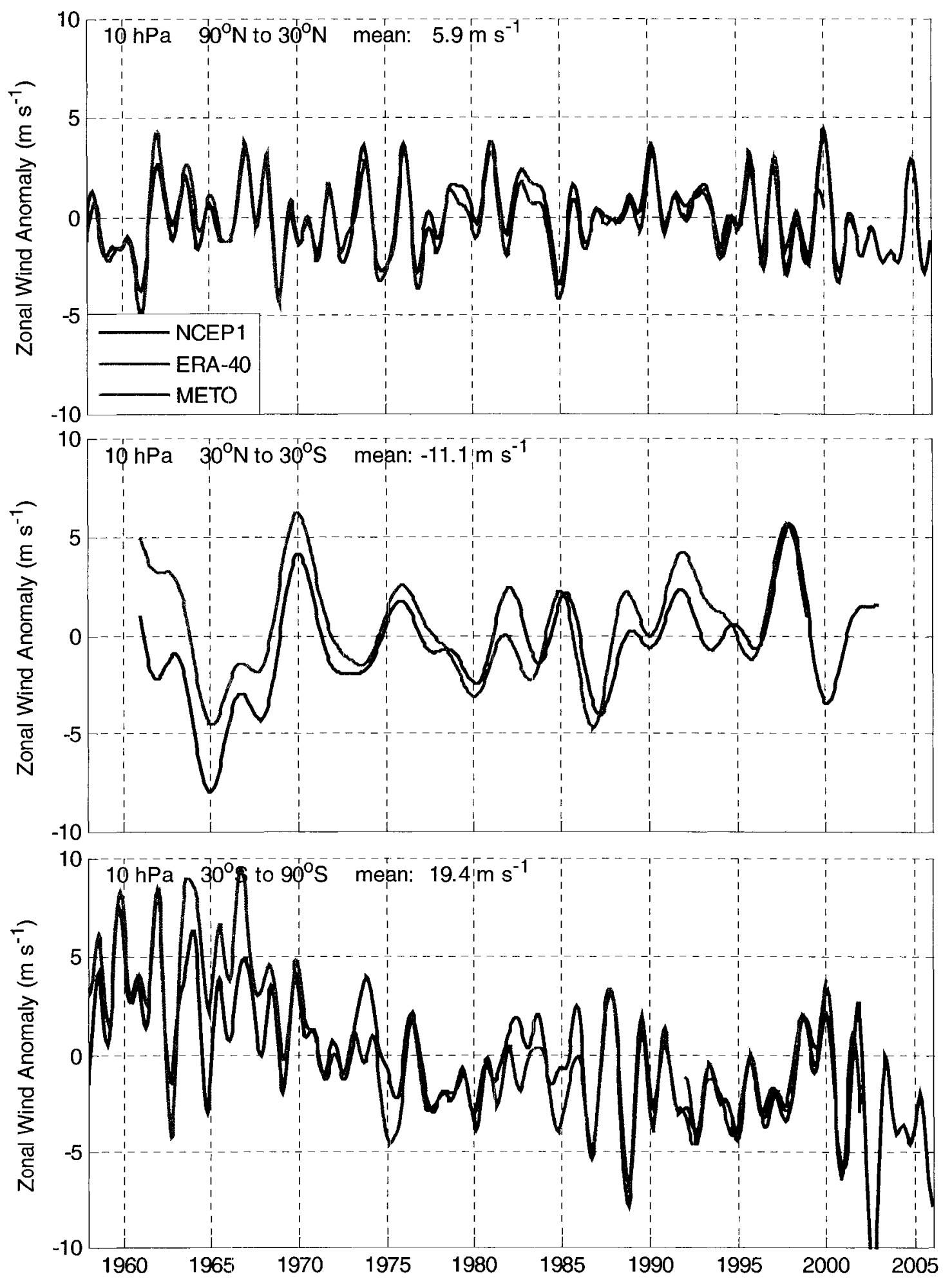

Fig. 5-24. Same as Fig. 5-19 except press: $10 \mathrm{hPa}$ and for equatorial series all variance with period less than 36 months removed by frequency-domain filtering. 
Figures 5-25 and 5-26 display the differences in the monthly- and zonallyaveraged zonal wind speeds of NCEP1 minus ERA-40 for latitude ranges of $90^{\circ} \mathrm{S}$ through $30^{\circ} \mathrm{S}, 30^{\circ} \mathrm{S}$ through $30^{\circ} \mathrm{N}$, and $30^{\circ} \mathrm{N}$ through $90^{\circ} \mathrm{N}$ (the three subglobal regions of Figs. 5-19 through 5-24) at pressure levels of 100, 70, and 50, (Fig. 5-25) and 30, 20, and $10 \mathrm{hPa}$ (Fig. 5-26) for the period January 1958 through December 2001. Green lines are the differences of the latitude-weighted time series for the indicated case. Black lines within the green are the latitude-weighted time series with all variance less than 13 months removed by frequency-domain filtering and then differenced. The unfiltered maximum and mean difference values listed on each subplot, and the filtered maximum and unfiltered standard deviation of each time series, are tabulated for easier comparison in Table 5-3, which also lists global values. Lower panels of Figs. 5-25 and 5-26 contain the 12-month moving average rms of the latitude-weighted differences and display maximum and mean values.

Table 5-3 summarizes the graphical data of Figs. 5-25 and 5-26 and illustrates the differences between NCEP1 and ERA-40 implied by the time series plots of Figs. 5-19 through 5-24. The absolute values of the maximum differences form a monotonic surface from the Southern Hemisphere extratropics at $10 \mathrm{hPa}$ to the Northern Hemisphere at $100 \mathrm{hPa}$. Mean differences are largest at 50 and $70 \mathrm{hPa}$ in the extratropics and at 20 to $30 \mathrm{hPa}$ in the Tropics, while standard deviations increase monotonically with increasing height. In both cases, tropical values are larger than extratropical. These results are consistent with the pressure level vs. latitude plot of Fig. 5-1, which displays the zonal time series at each pressure level and latitude. Again, the largest values of mean, 
standard deviation, and filtered maximum differences, $2.4,3.8$, and $5.5 \mathrm{~m} \mathrm{~s}^{-1}$, respectively, are small with respect to peak values of stratospheric zonal wind speeds. 

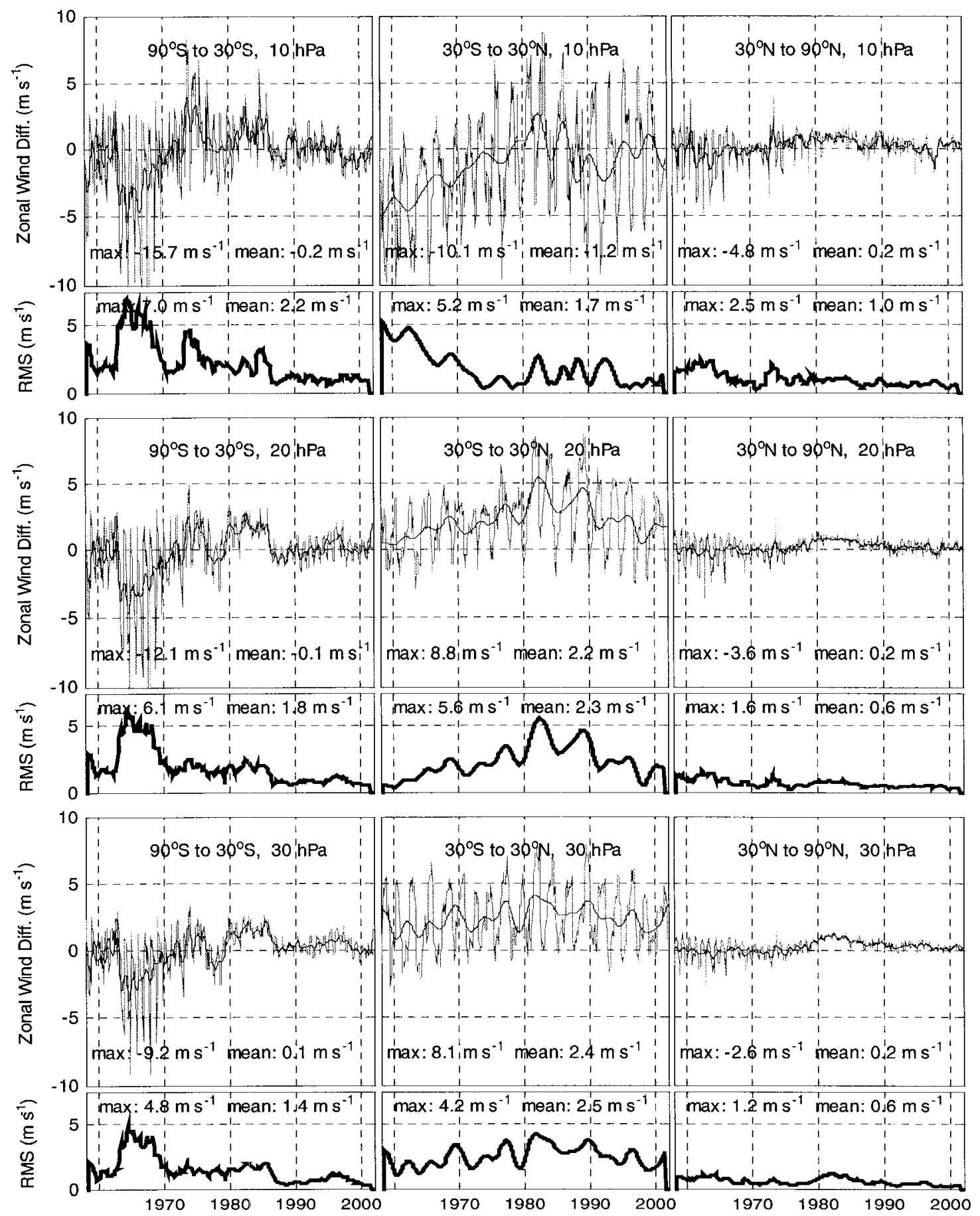

Fig. 5-25. Time series of monthly-and zonally-averaged zonal wind speed differences (see text) and 12-month moving rms: NCEP1 minus ERA-40: [lon: mean of $0^{\circ}-357.5^{\circ}$; lat: as stated; press: as stated; time: Jan 1958-Dec 2001]. 

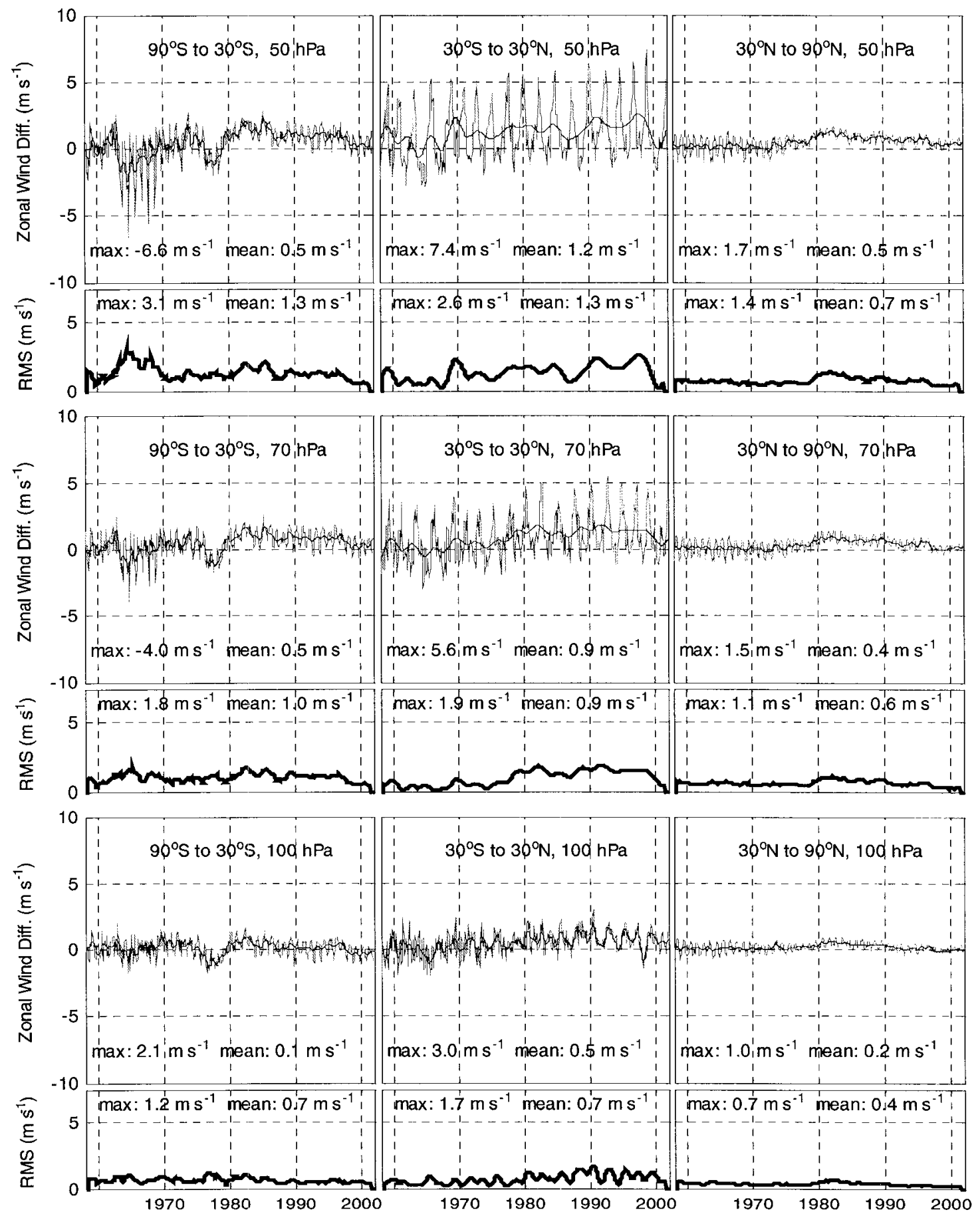

Fig. 5-26. Same as Fig. 5-25 except pressure levels. 
Table 5-3. Unfiltered maximum, filtered maximum, mean, and unfiltered standard deviation of zonal wind speed difference time series $\left(\mathrm{m} \mathrm{s}^{-1}\right)$

for subplots of Figs. 5-25 and 5-26.

\begin{tabular}{lcccc}
\multicolumn{5}{c}{ MAXIMUM (Unfiltered) } \\
& 90S - 30S & 30S - 30N & 30N - 90N & Global \\
$10 \mathrm{hPa}$ & -15.7 & -10.1 & -4.8 & -7.7 \\
$20 \mathrm{hPa}$ & -12.1 & 8.8 & -3.6 & 5.0 \\
$30 \mathrm{hPa}$ & -9.2 & 8.1 & -2.6 & 4.8 \\
$50 \mathrm{hPa}$ & -6.6 & 7.4 & 1.7 & 3.7 \\
$70 \mathrm{hPa}$ & -4.0 & 5.6 & 1.5 & 3.0 \\
$100 \mathrm{hPa}$ & 2.1 & 3.0 & 1.0 & 1.7
\end{tabular}

MAXIMUM (Filtered)

$90 \mathrm{~S}-30 \mathrm{~S} 30 \mathrm{~S}-30 \mathrm{~N} 30 \mathrm{~N}-90 \mathrm{~N}$ Global

$\begin{array}{lcccc}10 \mathrm{hPa} & -5.4 & -5.3 & -1.6 & -4.9 \\ 20 \mathrm{hPa} & -4.7 & 5.5 & 0.9 & 4.9 \\ 30 \mathrm{hPa} & -3.7 & 4.1 & 1.2 & 4.8 \\ 50 \mathrm{hPa} & -2.4 & 2.6 & 1.3 & 3.3 \\ 70 \mathrm{hPa} & 1.8 & 1.9 & 1.0 & 2.8 \\ 100 \mathrm{hPa} & -1.3 & 2.1 & 0.6 & 1.1\end{array}$

MEAN (Unfiltered $=$ Filtered)

90S - 30S 30S - 30N 30N - 90N Global

$\begin{array}{lcccc}10 \mathrm{hPa} & -0.2 & -1.2 & 0.2 & -0.6 \\ 20 \mathrm{hPa} & -0.1 & 2.2 & 0.2 & 1.1 \\ 30 \mathrm{hPa} & 0.1 & 2.4 & 0.2 & 1.3 \\ 50 \mathrm{hPa} & 0.5 & 1.2 & 0.5 & 0.8 \\ 70 \mathrm{hPa} & 0.5 & 0.9 & 0.4 & 0.6 \\ 100 \mathrm{hPa} & 0.1 & 0.5 & 0.2 & 0.3\end{array}$

STANDARD DEVIATION (Unfiltered) 90S - 30S 30S - 30N 30N - 90N Global

$\begin{array}{lllll}10 \mathrm{hPa} & 2.6 & 3.8 & 1.0 & 2.1 \\ 20 \mathrm{hPa} & 2.2 & 2.3 & 0.7 & 1.3 \\ 30 \mathrm{hPa} & 1.7 & 2.2 & 0.6 & 1.2 \\ 50 \mathrm{hPa} & 1.3 & 2.1 & 0.6 & 1.1 \\ 70 \mathrm{hPa} & 0.9 & 1.6 & 0.5 & 0.9 \\ 100 \mathrm{hPa} & 0.7 & 0.9 & 0.3 & 0.5\end{array}$




\subsection{Longitudinal distribution of zonal wind speed anomalies}

\section{Zonal wind speed anomaly with respect to longitudinal mean}

Sections 5.1 and 5.2 compare NCEP1, ERA-40, and METO zonal wind speed data in pressure level (height) vs. latitude and time series at the six stratospheric pressure level domains, respectively. In this section, the latitude vs. longitude structure of zonal wind speed is explored. As discussed at the beginning of Section 4.0, because of their linear latitude scale, the latitude vs. longitude plots of this section are not cartographically-correct Mercator plots.

To determine the zonal wind speed anomaly from the zonal average at each latitude, the following steps are performed with NCEP1 reanalysis data at each pressure level:

1. The mean zonal wind speed on each latitude circle is computed for each month of a 48-year period (January 1958 through December 2005).

2. The corresponding monthly-averaged zonal wind speed for each latitude and longitude is obtained.

3. For each month and each latitude, the longitudinally-averaged zonal wind speed of step 1 is subtracted from the zonal wind speed of step 2 at each longitude to produce a zonal wind speed anomaly with respect to the longitudinal mean for each month.

The results of step 3 are plotted in Fig. 5-27 (Northern Hemisphere) and 5-28 (Southern Hemisphere) as the longitude vs. latitude views of the 48 -year mean zonal wind speed anomalies for the month with largest rms temperature anomaly at each pressure level (from Figs. 4-33 and 4-34).

Temperature anomalies with respect to longitudinal means form planetary waves of wavenumber one at latitudes of $60^{\circ} \mathrm{N}$ to $70^{\circ} \mathrm{N}$ and $70^{\circ} \mathrm{S}$ to $60^{\circ} \mathrm{S}$ as illustrated in 
Figs. 4-33 and 4-34. However, zonal wind speed anomalies with respect to longitudinal means are characterized by two wavenumber one waves, $180^{\circ}$ out of phase; the first is poleward and equatorward of $60^{\circ} \mathrm{N}$ to $70^{\circ} \mathrm{N}$ and $70^{\circ} \mathrm{S}$ to $60^{\circ} \mathrm{S}$ latitude, and the second poleward of $30^{\circ}$ latitude. Unlike the temperature anomalies of Figs. 4-33 and 4-34, Figs. 5-27 and 5-28 show that significant variance does exist equatorward of $30^{\circ} \mathrm{N}$ and $30^{\circ} \mathrm{S}$ latitude, particularly in the regions of $15^{\circ} \mathrm{N}$ and $15^{\circ} \mathrm{S}$ latitude between 100 and $70 \mathrm{hPa}$, and $15^{\circ} \mathrm{N}$ to $30^{\circ} \mathrm{N}$ and $30^{\circ} \mathrm{S}$ to $15^{\circ} \mathrm{S}$ latitude above $70 \mathrm{hPa}$. These waves are consistent with a thermal wind balance resulting from the temperature anomalies with respect to longitudinal means. Zonal wind speed maxima and minima associated with temperature maxima are found between $180^{\circ}$ and $150^{\circ} \mathrm{W}$ at all pressure levels, while the temperature maxima move westward from $160^{\circ} \mathrm{W}$ at $100 \mathrm{hPa}$ to $140^{\circ} \mathrm{E}$ at $10 \mathrm{hPa}$. Zonal wind speed maxima and minima associated with temperature minima move westward from $90^{\circ} \mathrm{E}$ at $100 \mathrm{hPa}$ to $45^{\circ} \mathrm{E}$ at $10 \mathrm{hPa}$, while the temperature minima move westward from $65^{\circ} \mathrm{E}$ at $100 \mathrm{hPa}$ to $25^{\circ} \mathrm{W}$ at $10 \mathrm{hPa}$. 


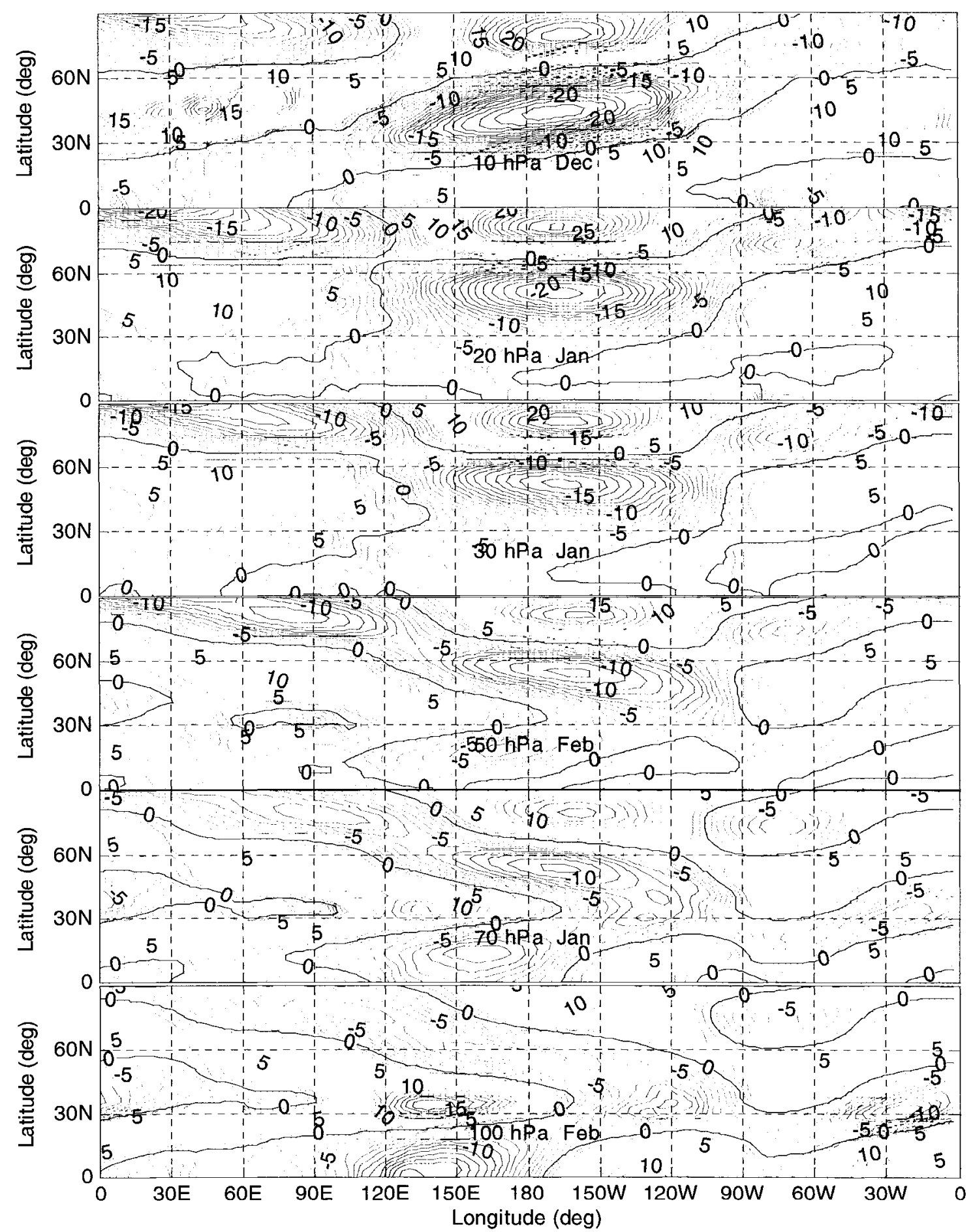

Fig. 5-27. Mean Northern Hemisphere zonal wind speed variation from longitudinal mean $\left(\mathrm{m} \mathrm{s}^{-1}\right)$ : NCEP1 [lon: mean of $0^{\circ}-357.5^{\circ}$, lat: $90^{\circ} \mathrm{S}-90^{\circ} \mathrm{N}$, press: as stated, time: variation: stated month, monthly mean within: Jan 1980-Dec 2005]. 
Similar zonal wind speed anomalies from the longitudinal mean exist in the Southern Hemisphere extratropics as illustrated in Fig. 5-28. The latitudinal extent of the waves is similar to those of the Northern Hemisphere. Again, thermal wind balance based on the temperature anomalies with respect to longitudinal means applies. Here the wavenumber one zonal wind speed maxima and minima associated with temperature maxima shift westward with decreasing height from maxima at $180^{\circ}$ at $100 \mathrm{hPa}$ to $150^{\circ} \mathrm{E}$ at $10 \mathrm{hPa}$, while the temperature maxima move westward from $170^{\circ} \mathrm{E}$ at $100 \mathrm{hPa}$ to $90^{\circ} \mathrm{E}$ at $10 \mathrm{hPa}$.

Differences in locations and magnitudes of maxima and minima between NCEP1 and ERA-40 zonal wind speed variations from longitudinal means (ERA-40 not shown) are essentially inconsequential. As is generally the case with comparisons between the two reanalyses, zonal wind speed differences are less that those of temperatures. The small standard deviation of differences between NCEP1 and ERA-40 in values of longitude-time series and monthly- and zonally-averaged time series, $1.8 \mathrm{~m} \mathrm{~s}^{-1}$ (1958-2001), $2.5 \mathrm{~m} \mathrm{~s}^{-1}$ (1958-78), and $1.5 \mathrm{~m} \mathrm{~s}^{-1}$ (1979-2001), shown in Figs. 5-10 through 5-12, respectively, demonstrate that the zonal wind speed anomalies from the longitudinal mean are similarly represented by the two reanalyses. 


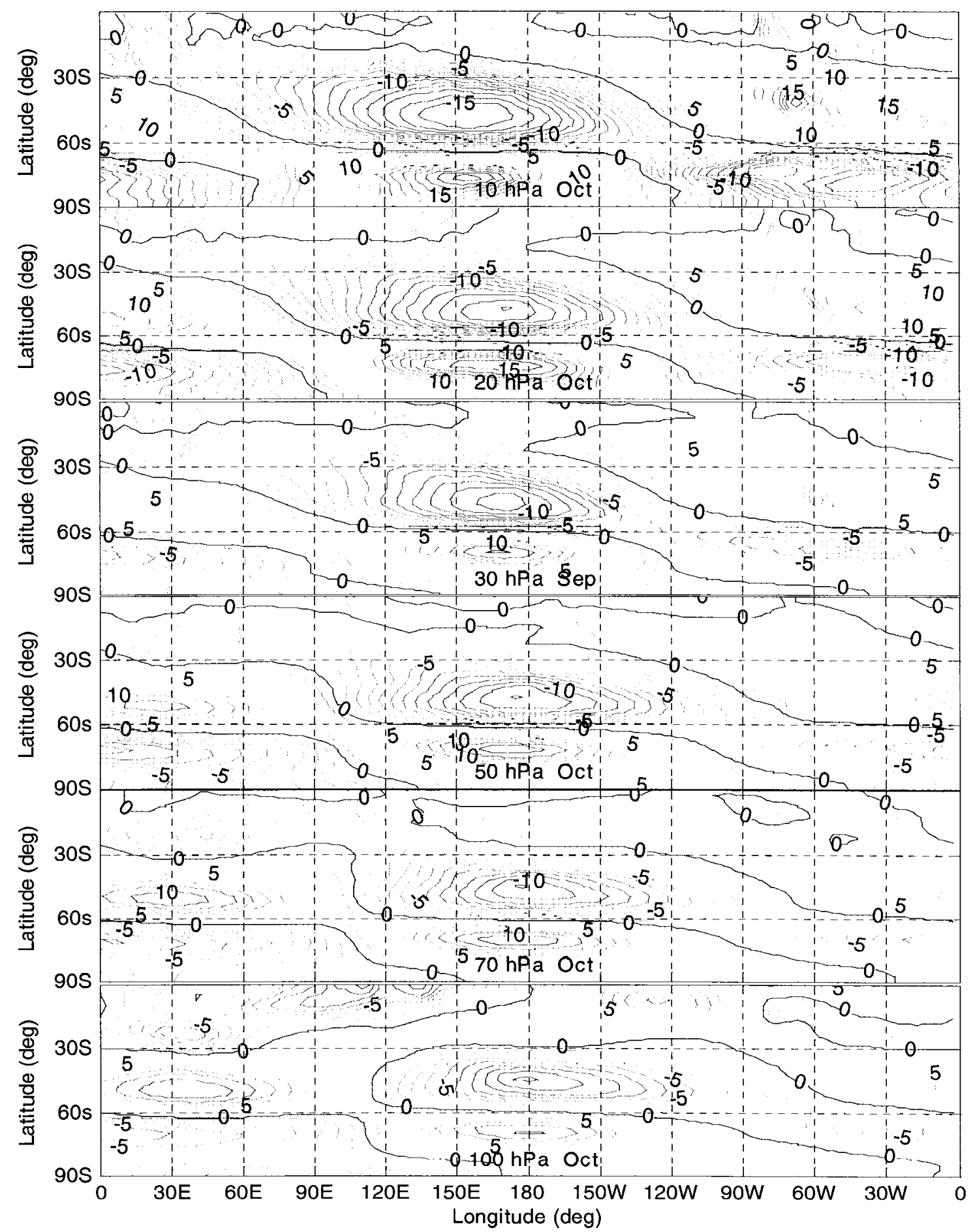

Fig. 5-28. Mean Southern Hemisphere zonal wind speed variation from longitudinal mean $\left(\mathrm{m} \mathrm{s}^{-1}\right)$ : NCEP1

[lon: mean of $0^{\circ}-357.5^{\circ}$, lat: $90^{\circ} \mathrm{S}-90^{\circ} \mathrm{N}$, press: as stated, time: variation: stated month, monthly mean within: Jan 1980-Dec 2005]. 
Figure 5-29, which plots the standard deviation of the zonal wind speed anomaly longitude-time series for both NCEP1 and ERA-40, illustrates their behavior in a full pressure level vs. latitude domain. The figure results from the following procedure where, for each pressure level:

1. The zonally-averaged zonal wind speed for each latitude and time is obtained for the 264 months from January 1980 through December 2001.

2. The zonal wind speed for each longitude, latitude, and time is obtained.

3. The results of step 1 are subtracted from those of step 2, creating an array of longitude, zonal wind speed, and time.

4. A longitude-time series (Appendix D) is formed for each latitude.

5. The standard deviation of the longitude-time series is computed for each latitude. Two dominant variance maxima exist in each hemisphere, at $50^{\circ} \mathrm{N}$ and $90^{\circ} \mathrm{N}$ in the Northern Hemisphere and at $45^{\circ} \mathrm{S}$ and $75^{\circ} \mathrm{S}$ in the Southern Hemisphere, consistent with Figs. 5-27 and 5-28. The magnitude of these variances, computed over all months of the year for a full 22 years, is diminished from that observed during months of peak wavenumber one amplitude. 

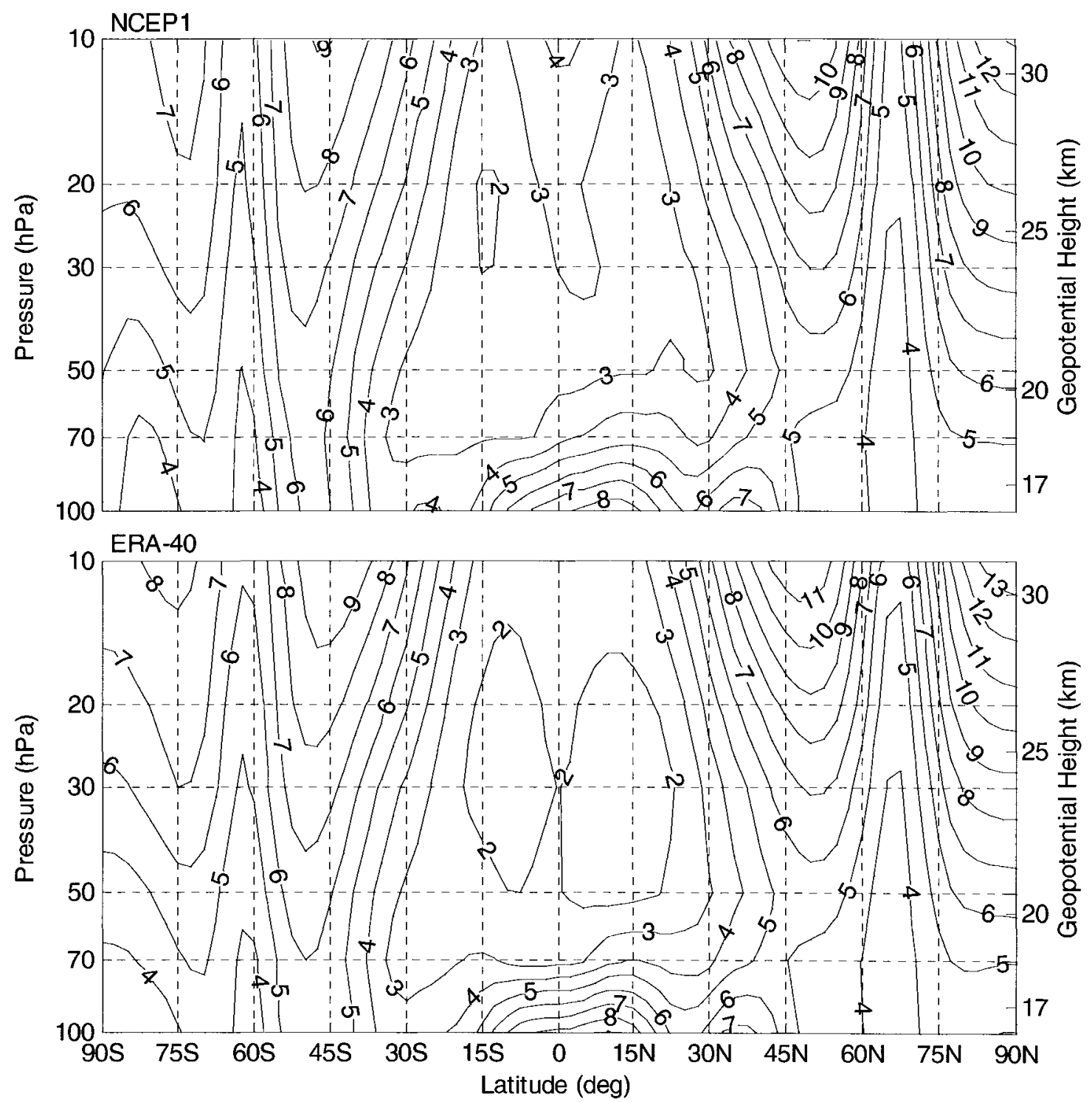

Fig. 5-29. Standard deviation of zonal wind speed anomaly longitude-time series $\left(\mathrm{m} \mathrm{s}^{-1}\right)$. [lon: $0^{\circ}-357.5^{\circ}$, lat: $90^{\circ} \mathrm{S}-90^{\circ} \mathrm{N}$, press: 100-10 hPa, time: Jan 1980-Dec 2001].

\section{Latitude vs. longitude view of zonal wind speed differences}

Figures 5-30 through 5-35 plot the mean, standard deviation, and maxima of zonal wind speed difference time series of monthly-averaged NCEP1 minus ERA-40 data from 1958 through 2001 for the six stratospheric pressure levels from $100 \mathrm{hPa}$ through $10 \mathrm{hPa}$. The figures represent level-by-level latitude vs. longitude views of the zonal wind speed 
differences projected onto the pressure level vs. latitude plots of Fig. 5-1. Additional insight into the three-dimensional structure of the NCEP1 minus ERA-40 zonal wind speed differences may be obtained by comparison of Fig. 5-1 with Figs. 5-30 through 5-35 and Figs. 5-25 and 5-26. Inspection of the pressure level vs. latitude plots of Figs. 5-1 through 5-3 and the time series of Figs. 5-19 through 5-24 shows that differences in the characterizations of the equatorial QBO and Southern Hemisphere zonal wind speeds by the NCEP1 and ERA-40 reanalyses dominate stratospheric zonal wind speed differences between the two.

Figure 5-30 shows that the largest mean differences in $100 \mathrm{hPa}$ zonal wind speed between the NCEP1 and ERA-40 reanalyses are five $\mathrm{m} \mathrm{s}^{-1}$ over the west coast of Peru, and four $\mathrm{m} \mathrm{s}^{-1}$ over the Himalayas and the coast of West Africa. From $0^{\circ}$ to $15^{\circ} \mathrm{S}, \mathrm{a}$ region with NCEP1 easterlies slightly stronger than ERA-40 easterlies exists. However, in the $30^{\circ} \mathrm{S}$ to $30^{\circ} \mathrm{N}$ equatorial band, stronger NCEP1 than ERA-40 westerlies are present and a mean westerly flow prevails. Mean extratropical zonal wind speed differences between the two reanalyses are less than two $\mathrm{m} \mathrm{s}^{-1}$.

The tropical mean flow at $70 \mathrm{hPa}$ in Fig. 5-31 is similar in direction and slightly stronger in magnitude than that at $100 \mathrm{hPa}$ with light NCEP1 westerlies and stronger ERA-40 easterlies. The West African and Himalaya maxima are still present. At the Equator both reanalyses have a mean easterly flow with NCEP1 stronger.

Equatorial and tropical mean flow differences at 50 through $20 \mathrm{hPa}$ (Figs. 5-30 through 5-34) are largely positive, and increase in extent and magnitude with height. These differences are the result of easterly flows in both reanalyses with ERA-40's 
stronger. Extratropical mean differences also increase with height through $10 \mathrm{hPa}$, more rapidly in the Southern Hemisphere than in the Northern.

At $10 \mathrm{hPa}$ (Fig. 5-35), both the equatorial and tropical mean differences are easterly as a consequence of a stronger easterly NCEP1 flow than ERA-40. At this level, extratropical mean zonal wind speed differences reach their maximum values. Mean differences at all levels exhibit structures of increased zonal wind speed in the regions of maximum zonal wind speed variation from the longitudinal mean (shown in Fig. 5-27) at $30^{\circ} \mathrm{N}, 0^{\circ}$ and $180^{\circ}$. This phenomenon is not obvious in the Southern Hemisphere.

With the exception of the Himalayas at $100 \mathrm{hPa}$, maximum values of standard deviation of reanalysis differences are confined to the Tropics (Fig. 5-30). This is the lower bound of the differences in the characterization of the QBO by the two reanalyses. Extratropical standard deviations of reanalysis differences increase with height at all latitudes, more rapidly in the Southern Hemisphere than in the Northern (Figs. 5-31 through 5-35). Differences in the QBO characterizations dominate the standard deviation, increasing rapidly with maximum values over the Indian and eastern Pacific Oceans at the Equator. Section 5.5 discusses the QBO differences.

At $100 \mathrm{hPa}$, maxima of the differences in zonal wind speed are generally widespread, zonal, confined to the Tropics and southern extratropics, and on the order of $10 \mathrm{~m} \mathrm{~s}^{-1}$ (Fig. 5-30). With increasing height, maxima increase in magnitude, again at all latitudes, but more rapidly in the Southern Hemisphere than in the Northern (Figs. 5-31 through 5-35). At lower heights, maxima of differences in the northern Tropics and 
Eastern Hemisphere to $50^{\circ} \mathrm{S}$ are positive (NCEP1 values larger than ERA-40). At $10 \mathrm{hPa}$ large portions of the plot are negative. 

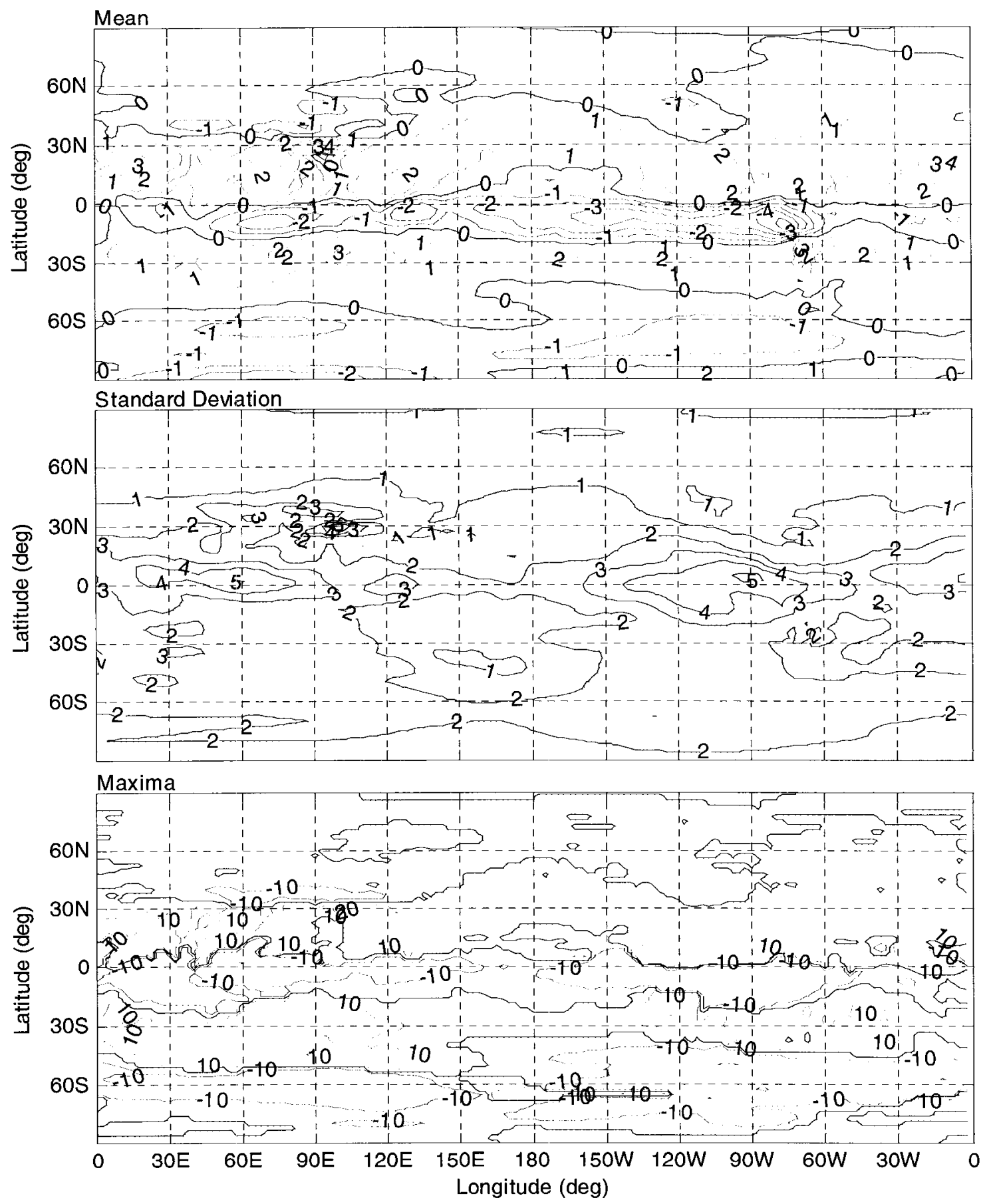

Fig. 5-30. Mean, standard deviation, and maxima of time series of monthly-averaged zonal wind speed differences $\left(\mathrm{m} \mathrm{s}^{-1}\right)$ : NCEPI minus ERA-40

[lon: $0^{\circ}-357.5^{\circ}, \quad$ lat: $90^{\circ} \mathrm{S}-90^{\circ} \mathrm{N}$, press: $100 \mathrm{hPa}$, time: Jan 1958-Dec 2001]. 

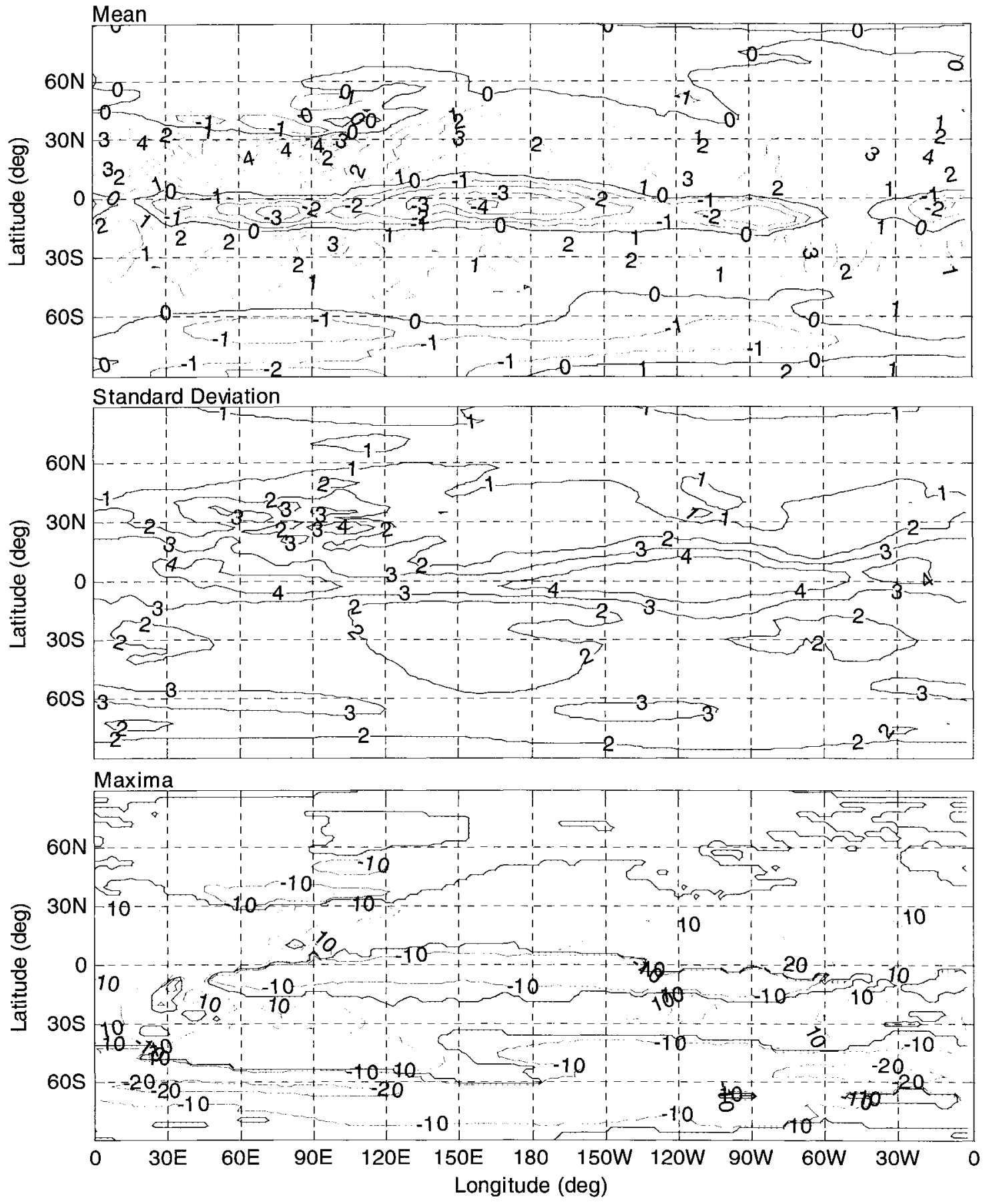

Fig. 5-31. Same as Fig. 5-30 except press: $70 \mathrm{hPa}$. 

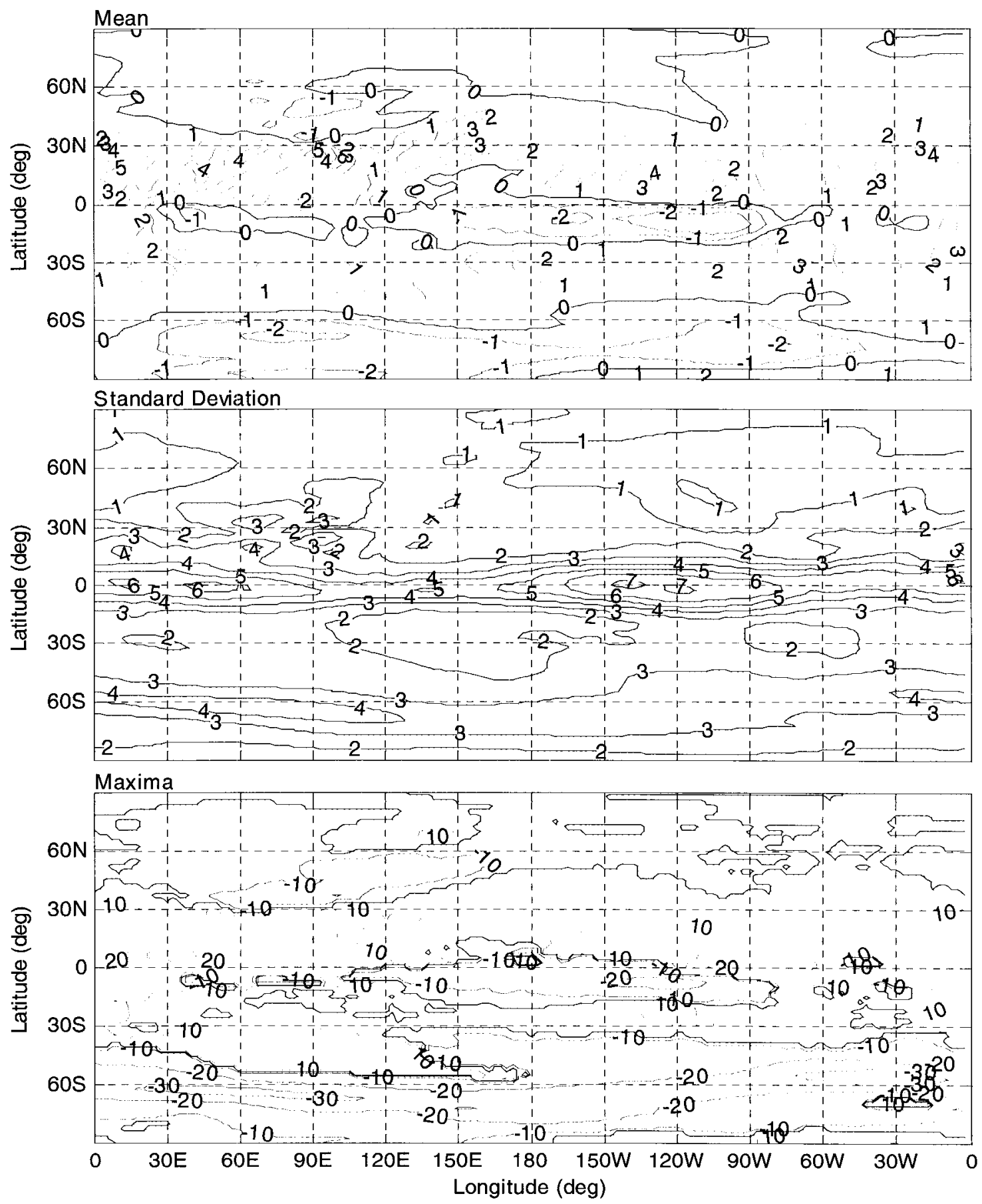

Fig. 5-32. Same as Fig. 5-30 except press: 50 hPa. 

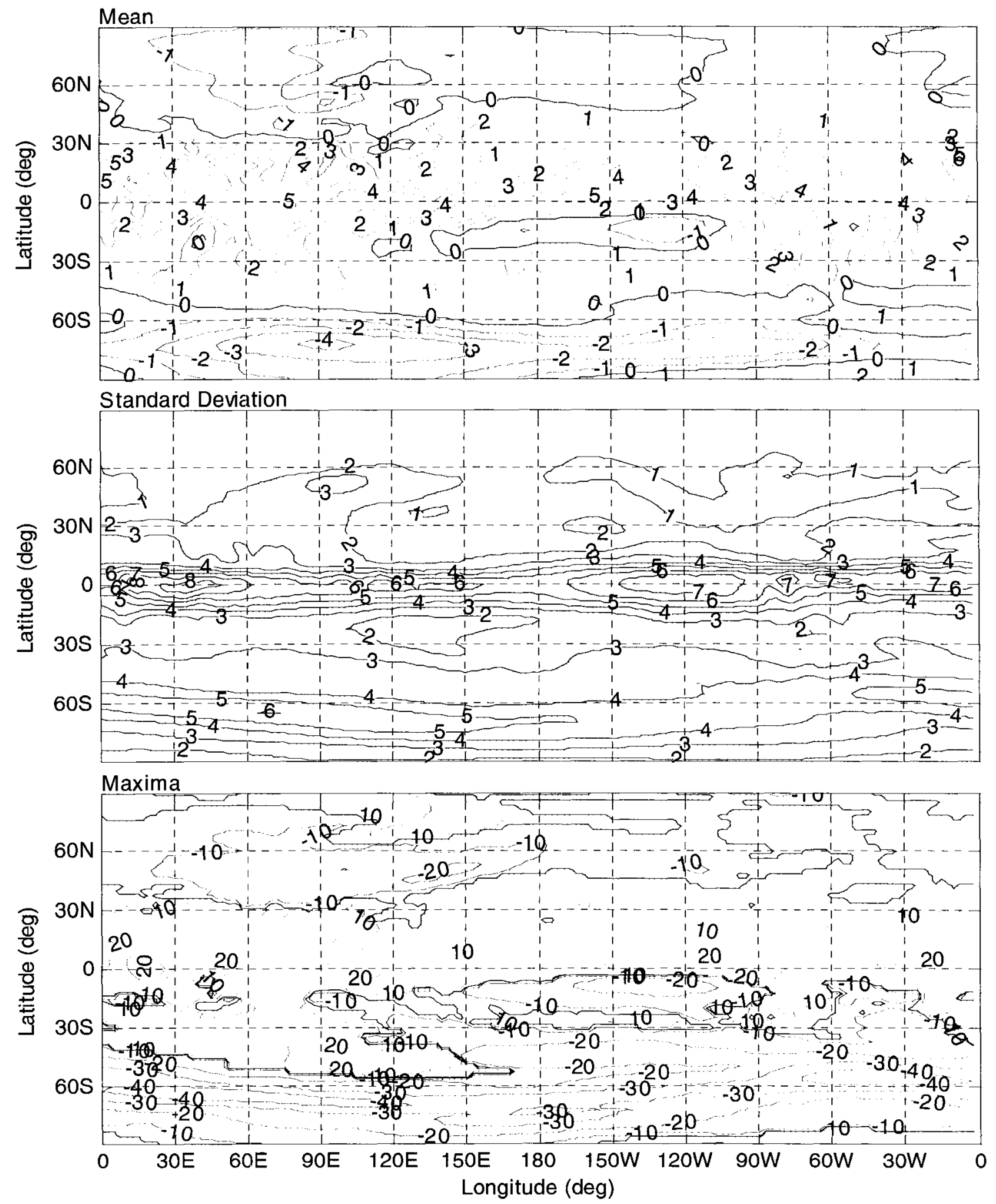

Fig. 5-33. Same as Fig. 5-30 except press: $30 \mathrm{hPa}$. 

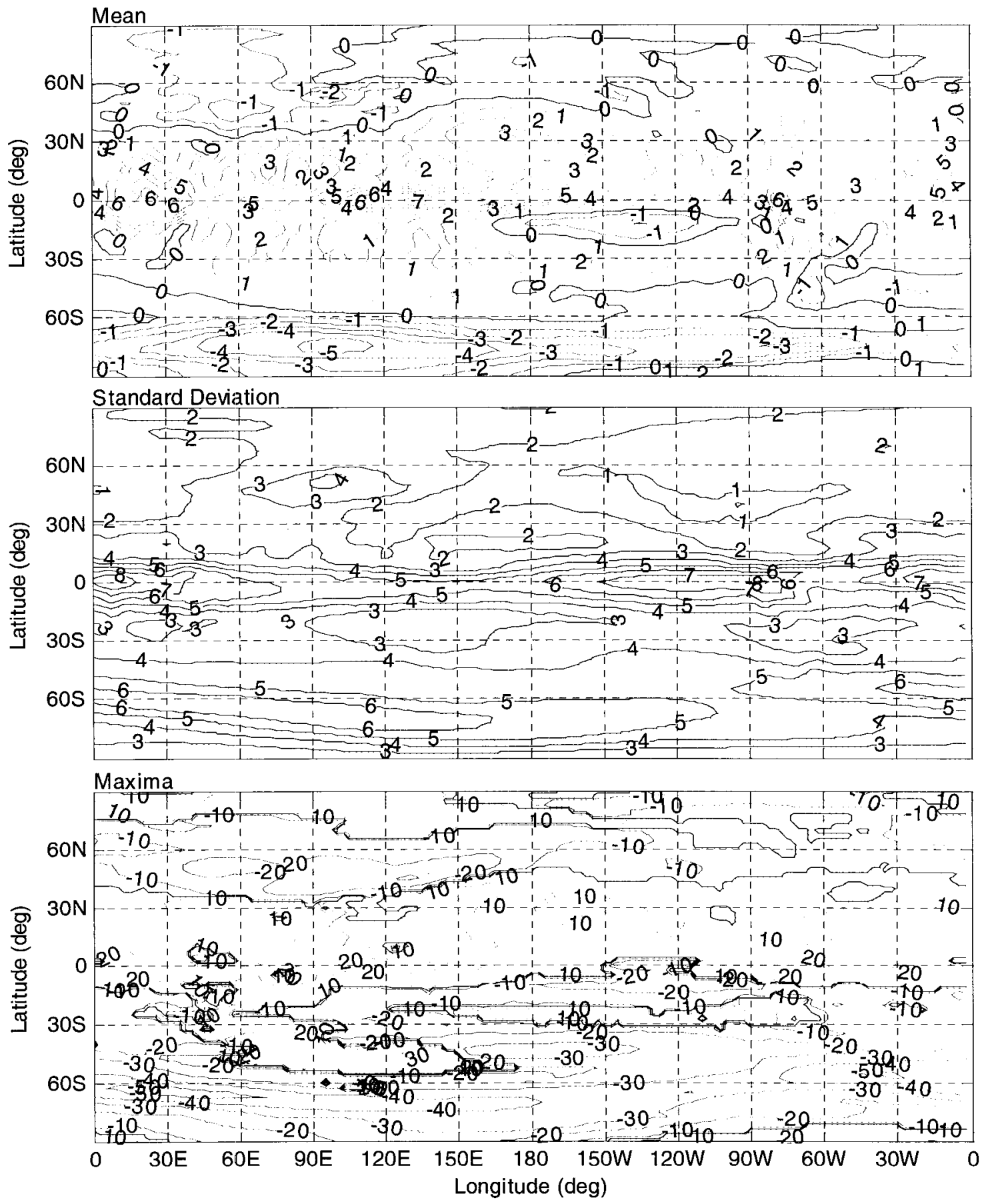

Fig. 5-34. Same as Fig. 5-30 except press: $20 \mathrm{hPa}$. 

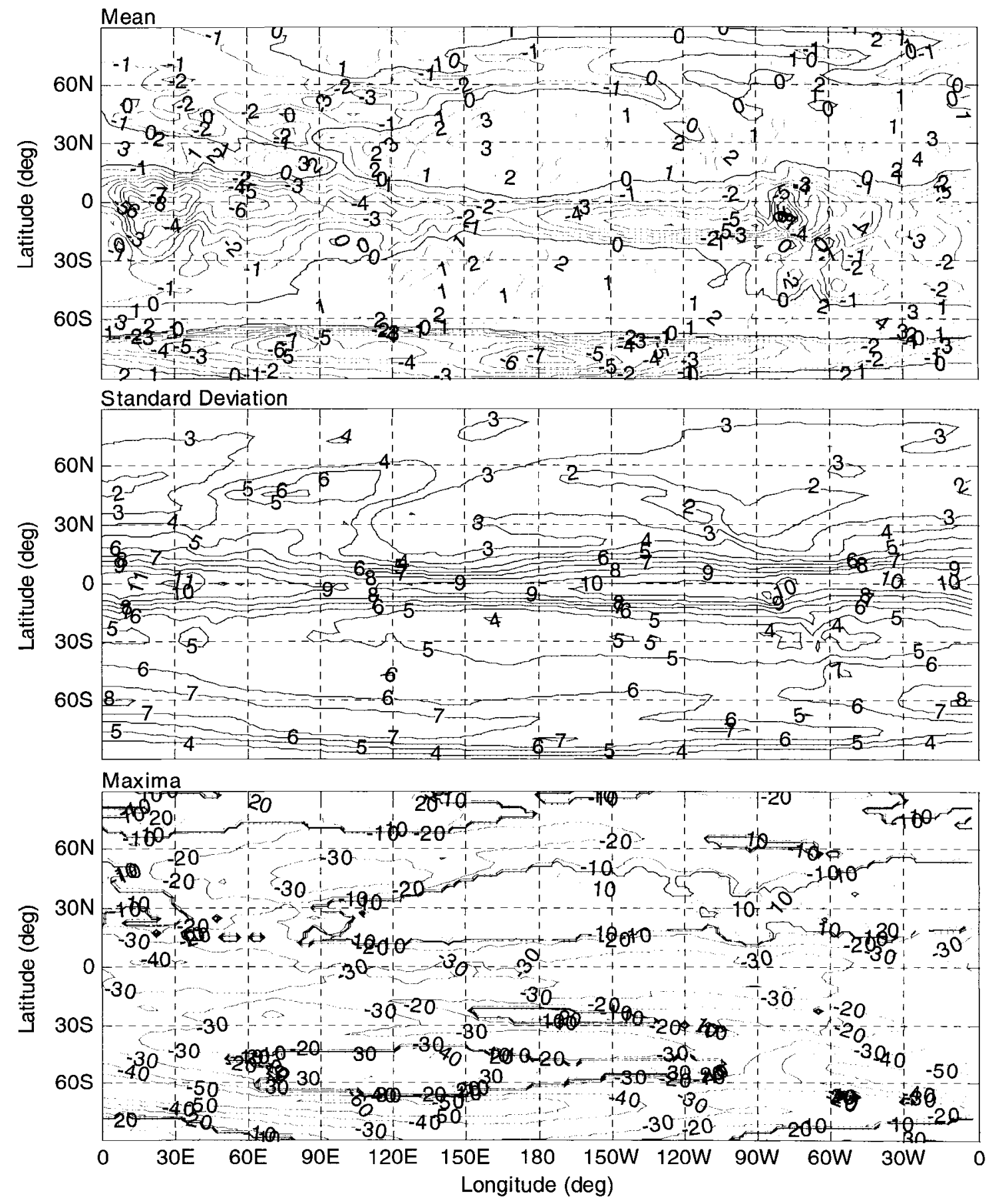

Fig. 5-35. Same as Fig. 5-30 except press: $10 \mathrm{hPa}$. 


\subsection{Location, speed, and linear trends of maximum polar wind speeds}

This section determines the locations (latitudes and pressure levels) and magnitudes of monthly- and zonally-averaged maximum northern and southern polar zonal wind speeds. It also evaluates the linear regression trends of the zonal wind speeds at the locations of their maximum wind speeds. Both NCEP1 and ERA-40 reanalyses are considered. As illustrated in Figs. 3-2 through 3-5, the cores of polar vortices are often found in the upper stratosphere and mesosphere. The $10 \mathrm{hPa}$ upper limit of this thesis prevents detection of the true core of a polar vortex and generally limits this analysis to the detection to the lower extent of a vortex.

Latitude and pressure level locations of the monthly maxima of the northern and southern polar zonal wind speeds for each month are first determined and plotted. The latitude and pressure level ranges included in these analyses are identified in the corresponding figure captions of Figs. 5-36 and 5-37. Northern and southern polar vortex zonal wind speeds at the latitudes and pressure levels of maximum wind speed are then compared with speeds at the conventional definition of the polar vortex locations: $\pm 60^{\circ}$ latitude and $10 \mathrm{hPa}$. Finally, linear regression trends and their confidence levels at the latitudes and pressure levels of maximum wind speed are computed. The polar vortex locations and zonal wind speeds are evaluated and plotted for 1958-2001 for both reanalyses; the results for this period are functionally equivalent to those of 1979-2001 (not shown).

Figures 5-36 and 5-37 plot the latitude and pressure level locations of the monthly maxima of polar zonal wind speeds for each month of the winter season for the Northern 
and Southern Hemispheres, respectively. In the Northern Hemisphere the monthly zonal wind speed maxima are widely dispersed in latitude with the highest frequencies of occurrence in both reanalyses in October at $67.5^{\circ} \mathrm{N}$. Table $5-4$ shows that mean vortex latitudes range from $57.8^{\circ} \mathrm{N}$ to $67.9^{\circ} \mathrm{N}(\mathrm{NCEP} 1)$ and from $57.8^{\circ} \mathrm{N}$ to $66.9^{\circ} \mathrm{N}(\mathrm{ERA}-40)$, with 44-year means and standard deviations of $64.9^{\circ} \mathrm{N}$ and $2.99^{\circ}(\mathrm{NCEP} 1)$ and $64.0^{\circ} \mathrm{N}$ and $2.87^{\circ}$ (ERA-40). These statistics and the contours of Fig. 5-36 clearly demonstrate that maximum Northern Hemisphere polar vortex zonal wind speeds are centered well poleward of $60^{\circ} \mathrm{N}$ at $10 \mathrm{hPa}$.

During each of the 44 years from 1958 through 2001, maximum zonal wind speed occurs at $10 \mathrm{hPa}$ during the months of September (ERA-40 only), October, and November. During these months, the height of the polar vortex core is at, or more likely above, $10 \mathrm{hPa}$. The count of polar vortices detected at $10 \mathrm{hPa}$ decreases slowly from November through March as the vortex core descends. From January through April a greater portion of detected vortex maxima are found at 20 and $30 \mathrm{hPa}$. Table 5-4 lists the monthly total of vortices detected at all levels.

The latitude location contours and pressure level plots of Fig. 5-36 and the data and statistics of Table 5-4 demonstrate that the NCEP1 and ERA-40 reanalyses are very similar in their characterization of the Northern Hemisphere polar vortex maximum zonal wind speeds and their locations. This behavior is very likely the result of a 44-year commensurate history of observations, adequate in time and space to produce quite similar results. 
In contrast to the visual and statistical consistency in Northern Hemisphere latitude locations of maximum polar vortex wind speed, Fig. 5-37 and Table 5-4 illustrate significant variation between NCEP1 and ERA-40 Southern Hemisphere locations. Although the nine-month means of latitude locations differ by only $0.1^{\circ}$, the ERA-40 standard deviation is almost twice that of NCEP1. Both contour plots in Fig. 5-37 exhibit an upwardly convex shape during the Southern Hemisphere winter but ERA-40's has a significantly greater curvature, reflected in the larger standard deviation. As in the Northern Hemisphere, the pressure level plots and statistics of the two reanalyses are quite consistent with one another; the only significant difference is a larger, by a factor of two, number of years with vortices at $20 \mathrm{hPa}$ during October in ERA-40 results.

Figures 5-38 and 5-39 plot the (1) mean, (2) mean \pm one standard deviation, and (3) maximum/minimum monthly- and zonally-averaged northern and southern, polar vortex wind speeds at $60^{\circ} \mathrm{N}$ and $60^{\circ} \mathrm{S}, 10 \mathrm{hPa}$, within the bounds of the latitudes and pressure levels shown in Figs. 5-36 and 5-37. Each figure contains both NCEP1 and ERA-40 results. As evidenced throughout Section 5, the polar vortex wind speeds from the two reanalyses are very consistent. Northern Hemisphere zonal wind speed magnitudes are slightly stronger in the "maximum speed" plots than in the $60^{\circ} \mathrm{N}$ and $60^{\circ} \mathrm{S}, 10 \mathrm{hPa}$ ones, as expected, since these speeds are the maximum for each month and, as shown by Table 5-4 and Fig. 5-36, maximum Northern Hemisphere polar vortex winds occur significantly northward of $60^{\circ} \mathrm{N}$. In the Southern Hemisphere plots of Fig. 5-39, wind speeds at $60^{\circ} \mathrm{S}, 110 \mathrm{hPa}$ are essentially equal to the "maximum speed" values 
because, as Table 5-4 shows, the mean vortex latitude for both reanalyses is approximately $60.6^{\circ} \mathrm{S}$. 

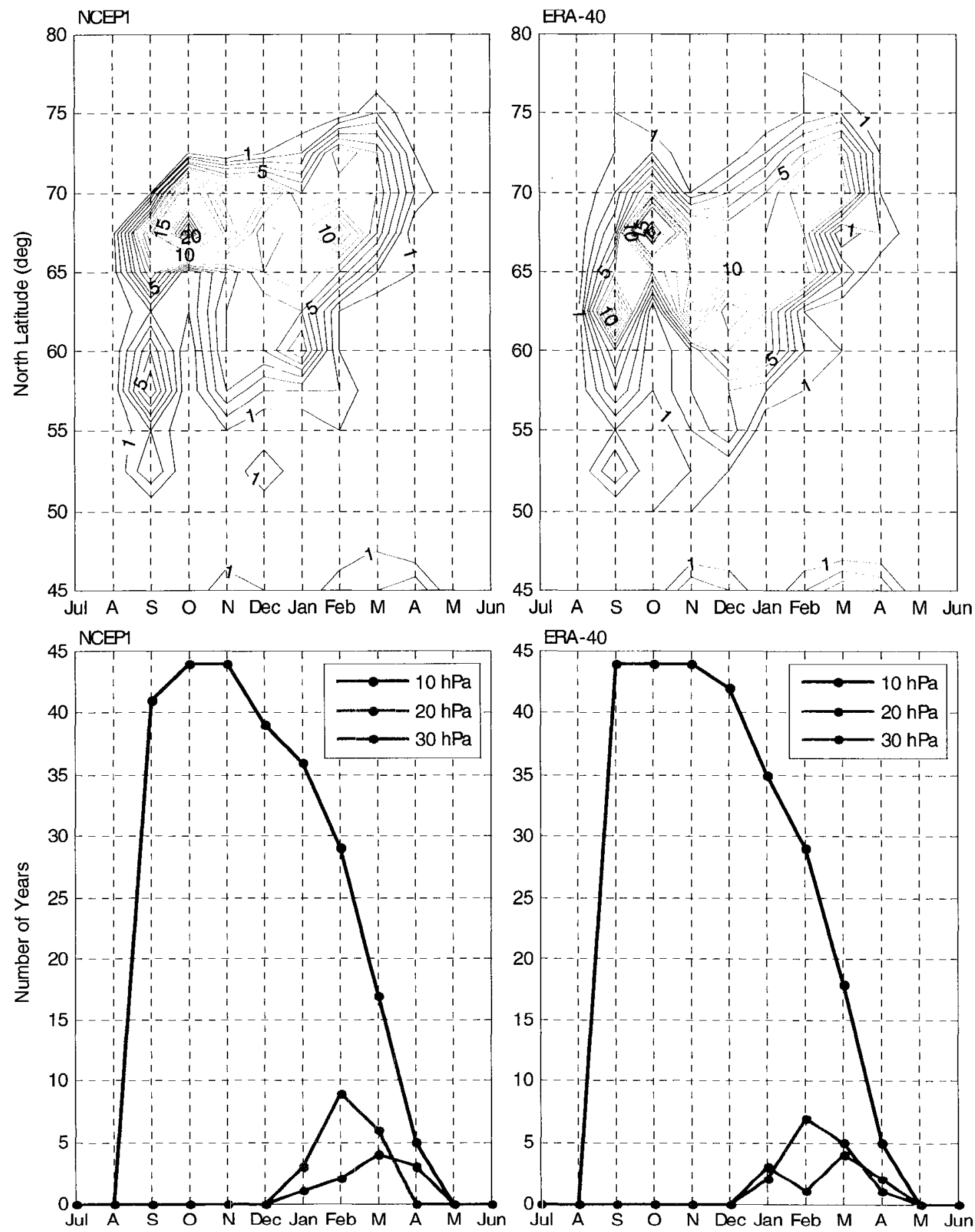

Fig. 5-36. Latitude (upper) and pressure level (lower) locations of monthly maxima of monthly-and zonally-averaged northern polar vortex wind speeds [lon: mean of $0^{\circ}-357.5^{\circ}$, lat: $45^{\circ} \mathrm{N}-80^{\circ} \mathrm{N}$, press: $30-10 \mathrm{hPa}$, time: Jan 1958-Dec 2001]. 

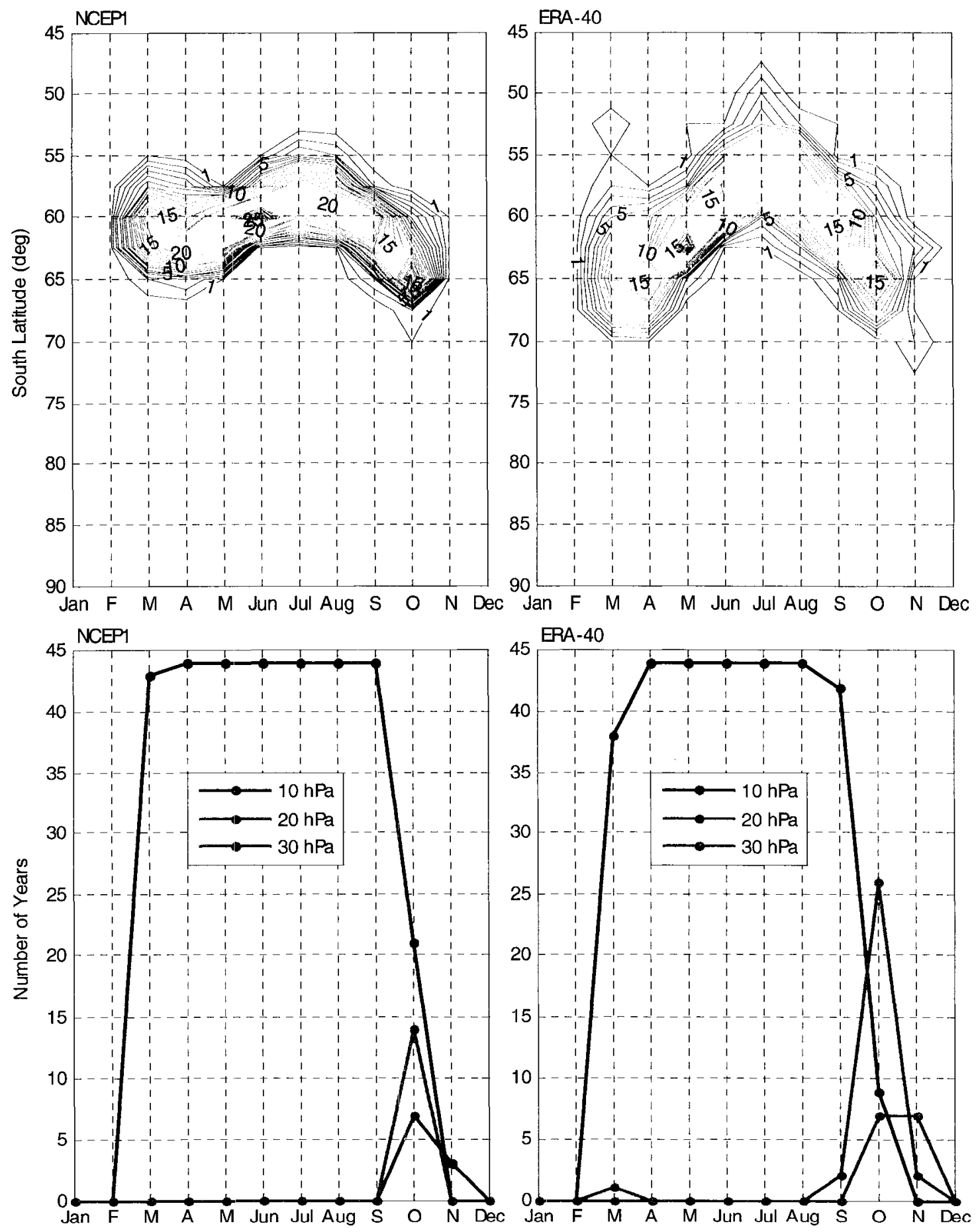

Fig. 5-37. Latitude (upper) and pressure level (lower) locations of monthly maxima of monthly-and zonally-averaged southern polar vortex wind speeds [lon: mean of $0^{\circ}-357.5^{\circ}, \quad$ lat: $45^{\circ} \mathrm{S}-80^{\circ} \mathrm{S}$, press: 30-10 hPa, time: Jan 1958-Dec 2001]. 

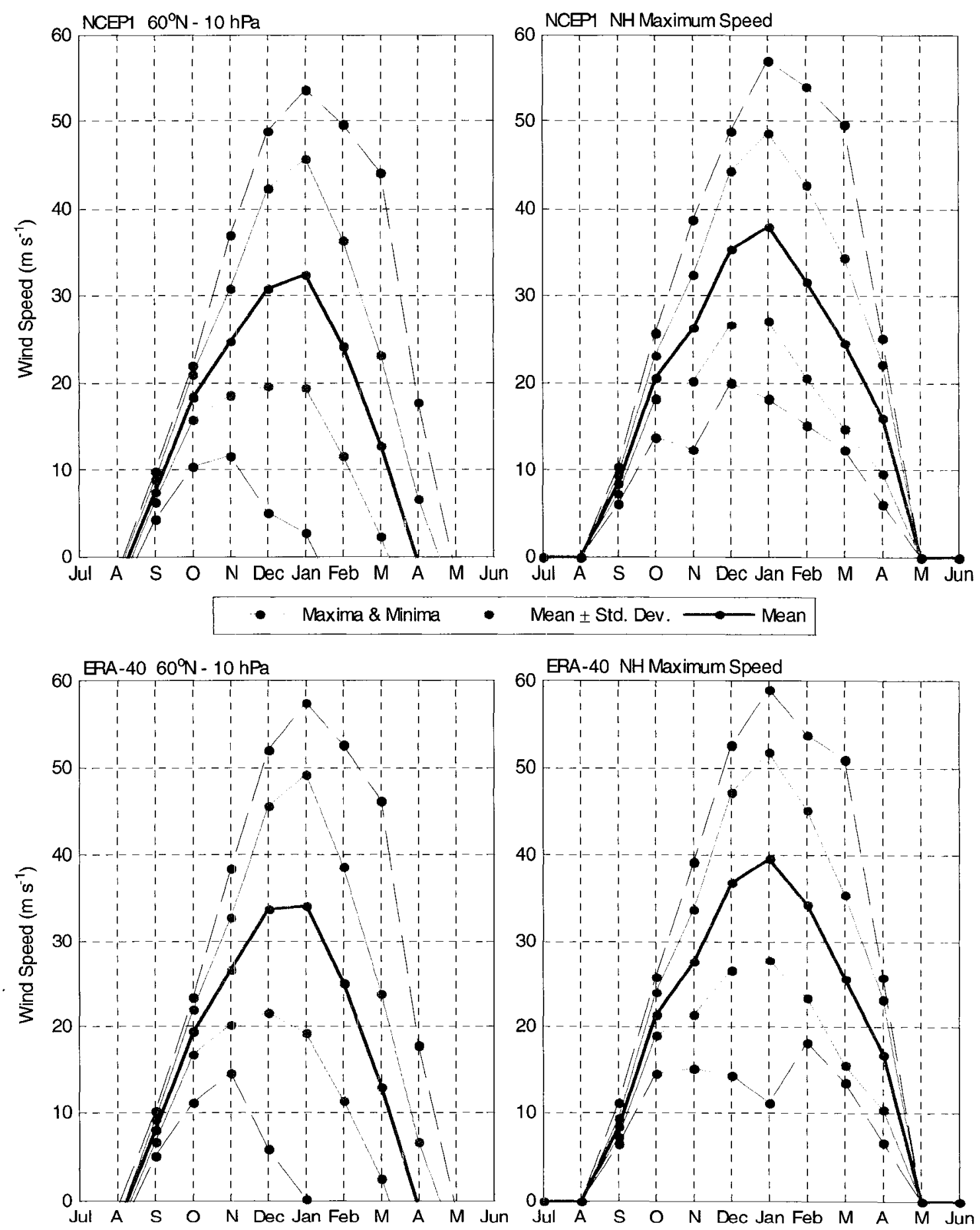

Fig. 5-38. Mean, mean \pm one standard deviation and maximum/minimum monthly-and zonally-averaged northern polar vortex wind speeds at $60^{\circ} \mathrm{N}, 10 \mathrm{hPa}$ (left) and within latitudes and pressure levels (right): [lon: mean of $0^{\circ}-357.5^{\circ}, \quad$ lat: $45^{\circ} \mathrm{N}-80^{\circ} \mathrm{N}$, press: 30-10 hPa, time: Jan 1958-Dec 2001]. 

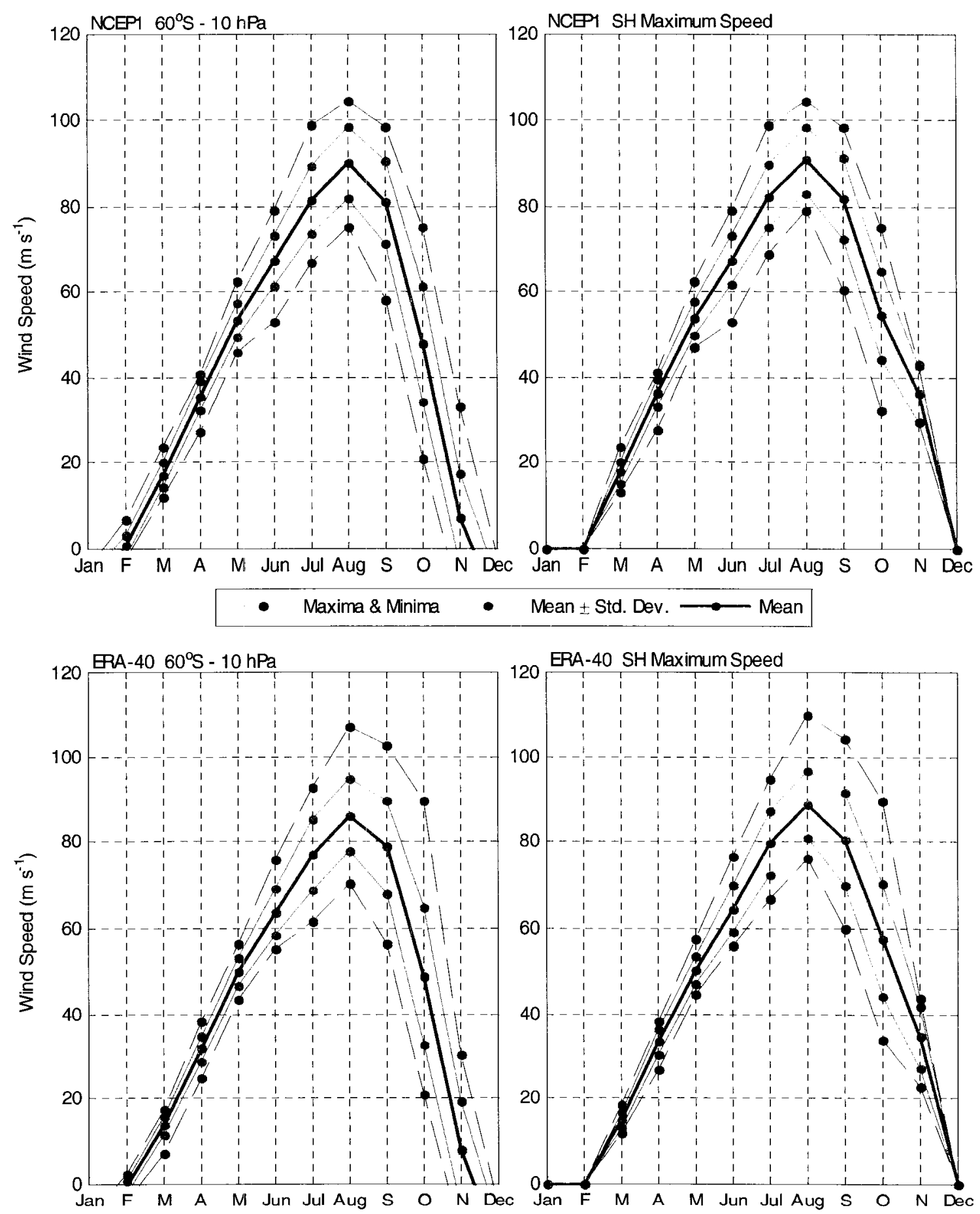

Fig. 5-39. Mean, mean \pm one standard deviation and maximum/minimum monthly-and zonally-averaged southern polar vortex wind speeds at $60^{\circ} \mathrm{N}, 10 \mathrm{hPa}$ (left) and maximum latitudes and pressure levels (right): [lon: mean of $0^{\circ}-357.5^{\circ}$, lat: $45^{\circ} \mathrm{S}-80^{\circ} \mathrm{S}$, press: $30-10 \mathrm{hPa}$, time: Jan 1958-Dec 2001]. 
Table 5-4. Total vortex counts and mean vortex latitudes for Northern and Southern Hemispheres in NCEP1 and ERA-40 reanalyses.

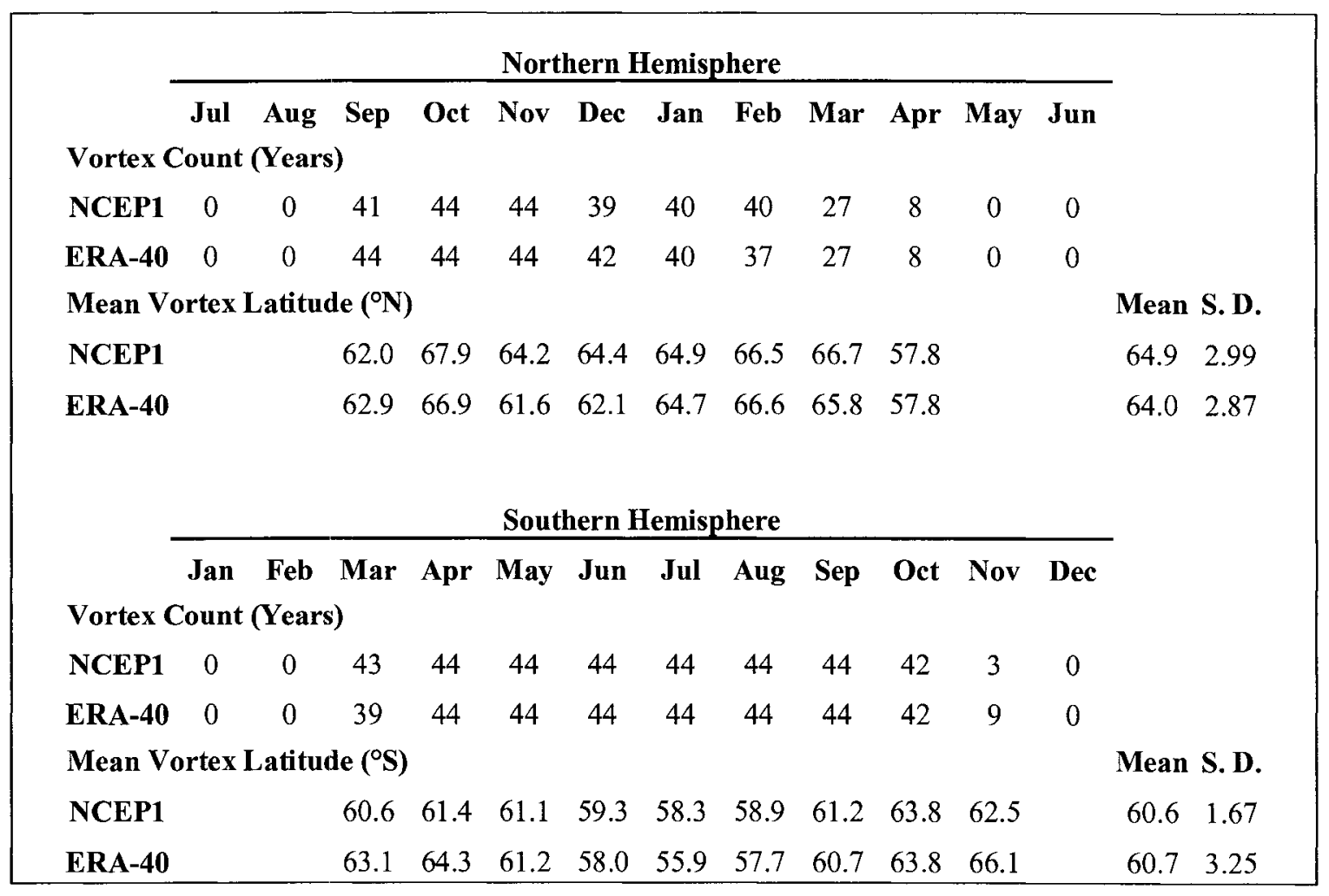

Figures 5-40 and 5-41 plot the polar vortex zonal wind speeds for the six months centered on the Northern and Southern Hemisphere winters, respectively, for both the NCEP1 and ERA-40 reanalyses. Latitudes and pressure levels are the locations of the maximum mean zonal wind speeds for each of the indicated months, i.e., averages from the data plotted in Figs. 5-36 and 5-37. Linear regression lines (blue: 1958-2001, green: 1958-78, red: 1979-2001) show that for most months, trends over these three previouslydiscussed time periods differ significantly in polarity and/or magnitude. However, the zonal wind speeds and thus the accompanying trends in any given month are quite consistent between the two reanalyses. Because of the increased confidence in the accuracy of reanalysis data in the modern satellite era (1979 to the present), only this time 
period, ending with 2001 because of the termination of ERA-40 data in August 2002, will be analyzed and discussed further.

The subplots of Figs. 5-40 and 5-41 are plotted with the same linear scale (but not the same range) to maximize the displayed slopes and emphasize the larger variance in the Northern Hemisphere, except during October. It is also apparent that the 1979-2001 trend of zonal wind speed is negative in the months of November through January and positive in February and March. In the Southern Hemisphere, although 1958-2001 trends are generally negative, 1979-2001 trends are less so.

Figure 5-42 quantifies the 1979-2001 polar vortex zonal wind speed trends and adds $5 \%$ confidence intervals. Computation of these confidence intervals includes the adjustment required by time series autocorrelation or "serial dependence" (Wilks 1995, pp. 125-127), which in the presence of positive correlation, reduces the effective number of time series samples and increases the extent of the confidence limits (Santer et al. 2000). Because of the small actual and effective sample size, a Student's $t$ statistic is employed. Of the 36 trends shown in Fig. 5.42, 23 exhibited no positive correlation; the effective number of time series samples averaged 15.4 for the remaining 13 trends. The approximately 2:1 ratio of Northern (DJF) to Southern (JJA) Hemisphere confidence intervals is confirmed by figures similar to the pressure level vs. latitude standard deviation subplots of Figs. 5-72 (DJF) and 5-73 (JJA). These figures (Figs. 5-72 and 5-73) were modified by changing the time period to 1979 to 2001 for consistency with the times of this section and by substituting notch filtering for low-pass filtering to remove the annual cycle and its harmonics but retain all other variance; the resulting plots 
are not shown. The ratio of DJF to JJA standard deviations at the latitudes and pressure levels of Figs. 5-40 through 5-42 in the modified figures (not shown) is also 2:1, confirming the confidence interval ratios of Fig. 5-42.

The Northern Hemisphere NCEP1 minus ERA-40 confidence intervals show that when the variance cormmon to both reanalyses is removed by differencing, their trends are almost equal, with high confidence. Southern Hemisphere trends, with the exception of August and September, are again almost zero but with larger confidence intervals than in the Northern Hemisphere. Trends in August and September are slightly negative with a slightly larger difference. The larger means and confidence intervals of the Southern Hemisphere differences indicates more variation between the reanalyses than in the Northern Hemisphere, a conclusion substantiated by the pressure level vs. latitude rms subplot of Fig. 5-3, which shows a value greater than two $\mathrm{m} \mathrm{s}^{-1}$ at $60^{\circ} \mathrm{S}, 10 \mathrm{hPa}$, and a value slightly greater than one $\mathrm{m} \mathrm{s}^{-1}$ at $65^{\circ} \mathrm{N}, 10 \mathrm{hPa}$. This approximately $2: 1$ ratio in Fig. 5-3 is consistent with the $\mathrm{SH}$ to $\mathrm{NH}$ means and confidence intervals of the reanalysis differences. 

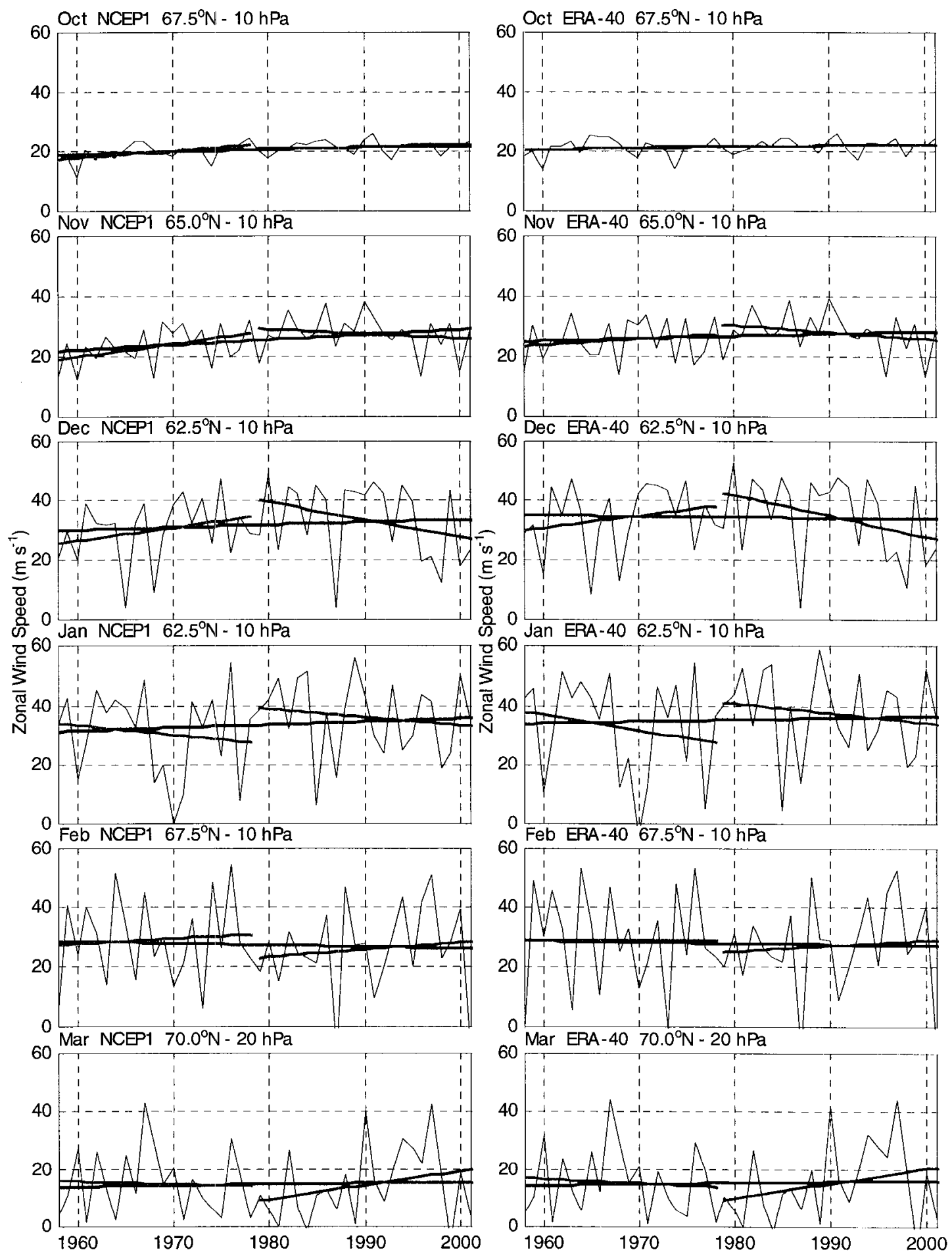

Fig. 5-40. Monthly- and zonally-averaged northern polar vortex zonal wind speeds and linear regression trends (blue: 1958-2001, green: 1958-78, red: 1979-2001). [lon: mean of $0^{\circ}-357.5^{\circ}$, lat: as stated, press: as stated, time: Jan 1958-Dec 2001]. 

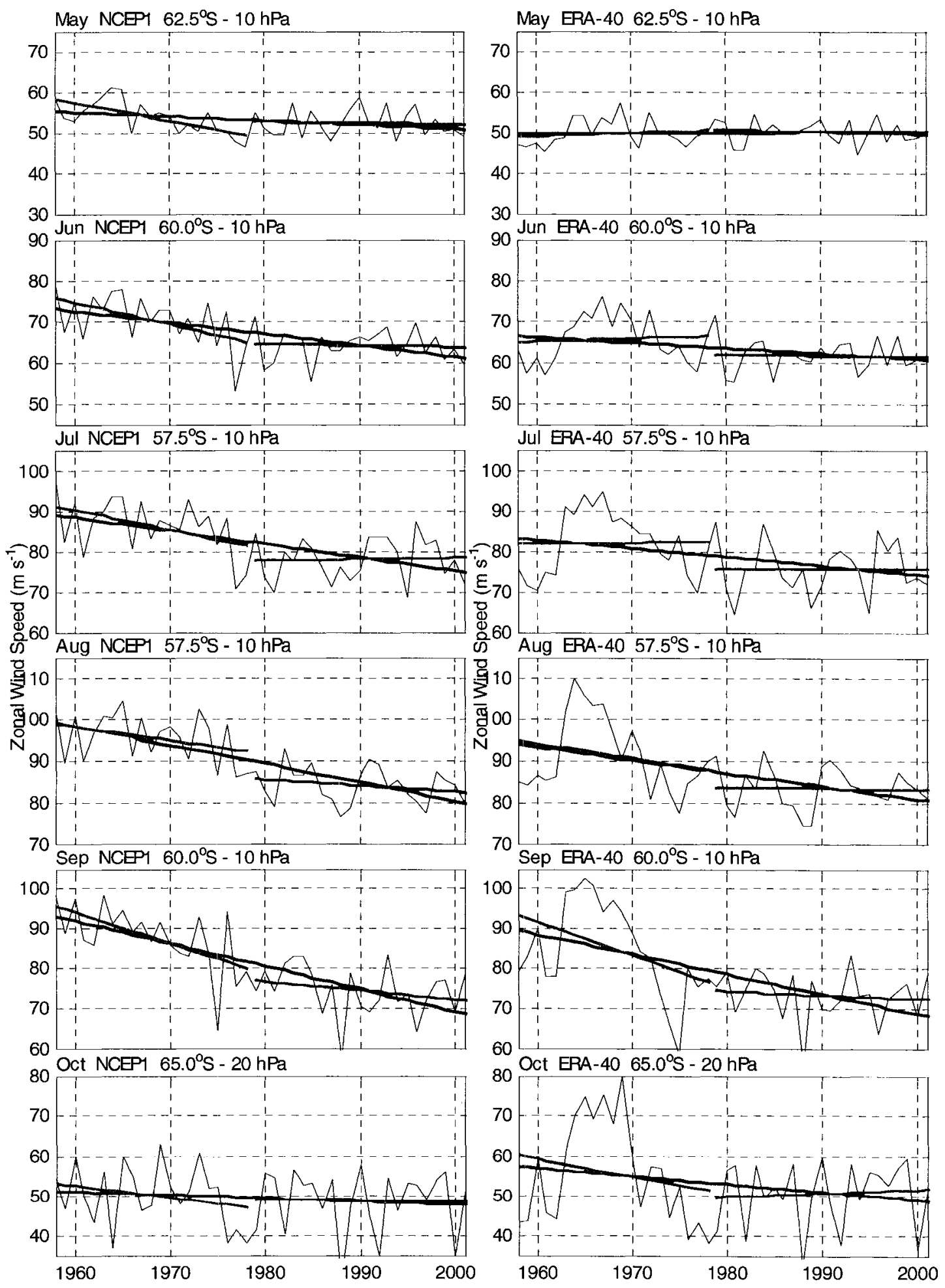

Fig. 5-41. Same as Fig. 5-40 except southern polar vortex zonal wind speeds. 

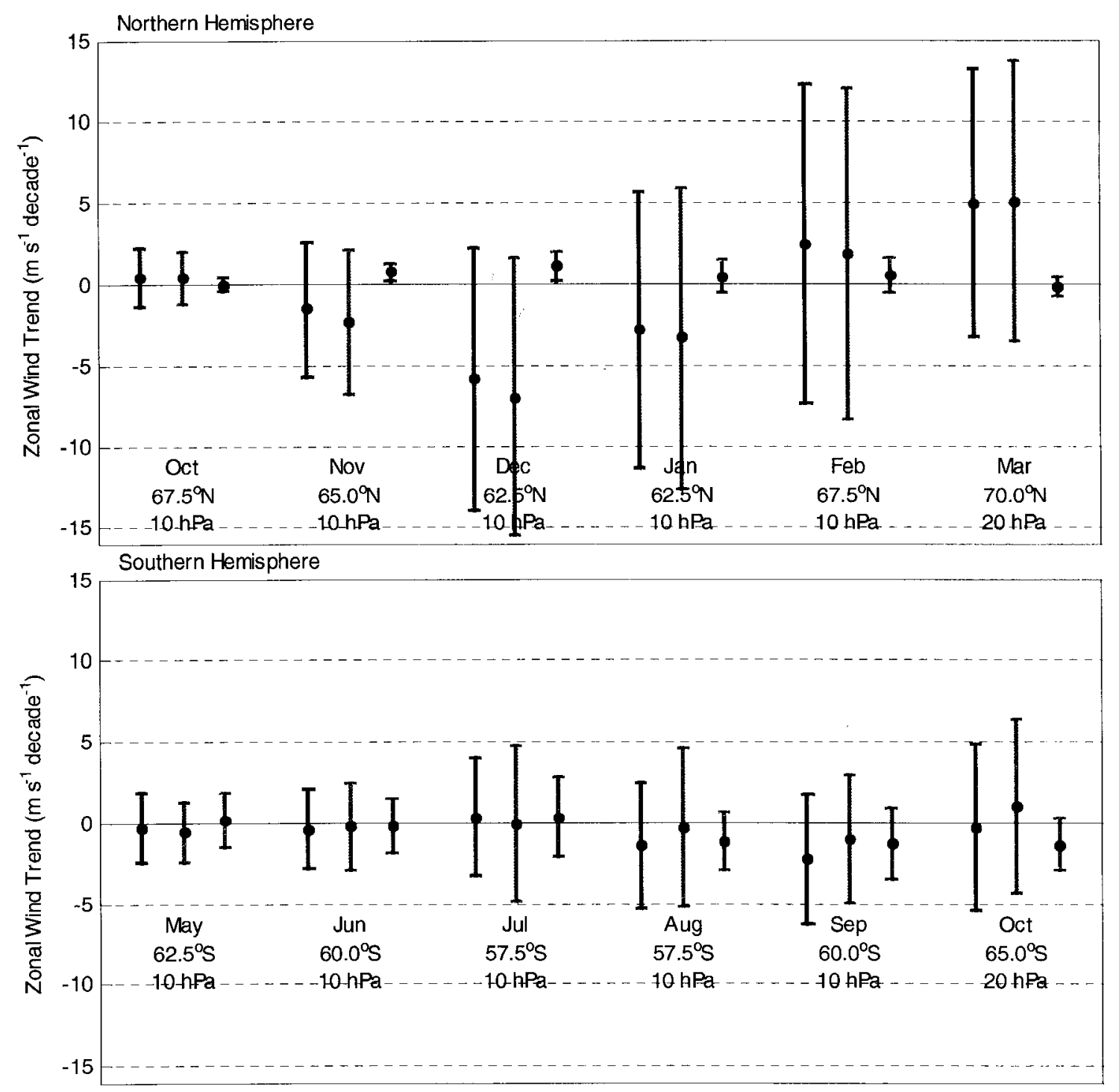

Fig. 5-42. 1979-2001 linear regression trends and 5\% confidence intervals for monthlyand zonally-averaged polar vortex zonal wind speeds - blue: NCEP1, green: ERA-40, red: NCEP1 minus ERA-40. [lon: mean of $0^{\circ}-357.5^{\circ}$, lat: as stated, press: as stated, time: 1979-2001]. 
The 22 figures that follow, Figs. 5-44 through 5-54 for the Northern Hemisphere (November through February) and Figs. 5-55 through 5-65 for the Southern Hemisphere (June through September), present polar vortex zonal wind speeds and their trends in both pressure level and latitude.

Temperatures in polar regions are closely related to vortex zonal wind speeds. For this reason, temperatures, their horizontal gradients, and the trends of both in the same polar domain are displayed in the set of figures listed above. Additionally, analyses of polar temperatures and their trends are combined with the analyses of polar vortex zonal wind speeds and their trends in this section. However, a summary of temperature trend results is included in Section 4.6.

Two figures are required to illustrate the logical sequence of physical information for each month. All subplots are contour plots. Wind data is shown over pressure levels from 30 to $10 \mathrm{hPa}$; because polar vortices (thermal winds) are a function of vertically integrated horizontal temperature gradients, temperature data is shown over pressure levels from 100 to $10 \mathrm{hPa}$. NCEP1 and ERA-40 data are arranged side-by-side for ease of comparison. The first page of each two-figure pair contains three subplots:

1. Mean Zonal Wind $\left(\mathrm{m} \mathrm{s}^{-1}\right) 30-10 \mathrm{hPa}$, $45^{\circ} \mathrm{N}-90^{\circ} \mathrm{N}$ ( $\mathrm{NH}$ figures) or $90^{\circ} \mathrm{S}-45^{\circ} \mathrm{S}$ (SH figures)

Contours illustrate location of maximum zonal wind speed

2. Mean Zonal Temperature (K) $100-10 \mathrm{hPa}$, $45^{\circ} \mathrm{N}-90^{\circ} \mathrm{N}$ (NH figures) or $90^{\circ} \mathrm{S}-45^{\circ} \mathrm{S}$ (SH figures)

Contours of stratospheric zonal temperatures

3. Trend of Temperature $\left(\mathrm{K} \mathrm{decade}^{-1}\right) 100-10 \mathrm{hPa}$, $45^{\circ} \mathrm{N}-90^{\circ} \mathrm{N}$ ( $\mathrm{NH}$ figures) or $90^{\circ} \mathrm{S}-45^{\circ} \mathrm{S}$ ( $\mathrm{SH}$ figures)

Contours of stratospheric zonal temperature trends (1979-2001) 
The second page of each pair contains four subplots:

4. Mean Horizontal Temperature Gradient $\left(\mathrm{K}\right.$ degree $\left.^{-1}\right) 100-10 \mathrm{hPa}$, $45^{\circ} \mathrm{N}-90^{\circ} \mathrm{N}$ ( $\mathrm{NH}$ figures) or $90^{\circ} \mathrm{S}-45^{\circ} \mathrm{S}$ (SH figures)

Contours of mean zonal temperature (2. above) gradient

5. Mean Zonal Wind $\left(\mathrm{m} \mathrm{s}^{-1}\right) 30-10 \mathrm{hPa}$, $45^{\circ} \mathrm{N}-90^{\circ} \mathrm{N}$ (NH figures) or $90^{\circ} \mathrm{S}-45^{\circ} \mathrm{S}$ ( $\mathrm{SH}$ figures)

Repeat of 1 . above to illustrate consequence of 4 . above and for comparison with 7. below

6. Trend of Horizontal Temperature Gradient $\left(\mathrm{K}_{\text {degree }}{ }^{-1}\right.$ decade $\left.^{-1}\right) 100-10 \mathrm{hPa}$, $45^{\circ} \mathrm{N}-90^{\circ} \mathrm{N}$ ( $\mathrm{NH}$ figures) or $90^{\circ} \mathrm{S}-45^{\circ} \mathrm{S}$ (SH figures)

Contours of stratospheric mean horizontal temperature gradient (4. above) trends (1979-2001)

7. Trend of Zonal Wind $\left(\mathrm{m} \mathrm{s}^{-1}\right.$ decade $\left.^{-1}\right) 30-10 \mathrm{hPa}$, $45^{\circ} \mathrm{N}-90^{\circ} \mathrm{N}$ ( $\mathrm{NH}$ figures) or $90^{\circ} \mathrm{S}-45^{\circ} \mathrm{S}$ (SH figures)

Contours of mean zonal wind speed (1. and 6. above) trends (1979-2001)

Six figures (Figs. 5-52 through 5-54, NH; Figs. 5-63 through 5-65, SH) plot

temperature and zonal wind speed trends as functions of latitude for three pressure levels.

NCEP1 and ERA-40 trends and their 5\% confidence intervals are superimposed in each

subplot and two months are presented on each page. These figures illustrate

consistencies and inconsistencies in magnitudes of trends and their confidence intervals

that are not readily apparent in the contour plots of Figs. 5-44 through 5-51 (NH) and

Figs. 5-55 through 5-62 (SH). For accurate comparison, the vertical scale of all

temperature trend subplots is the same: $\pm 4 \mathrm{~K} \mathrm{decade}^{-1}$; as is the vertical scale of all zonal wind speed trend subplots: $\pm 10 \mathrm{~K} \mathrm{~m} \mathrm{~s}^{-1}$ decade $^{-1}$. 
The 22-figure set is best viewed with the figures arranged as in Fig. 5-43.

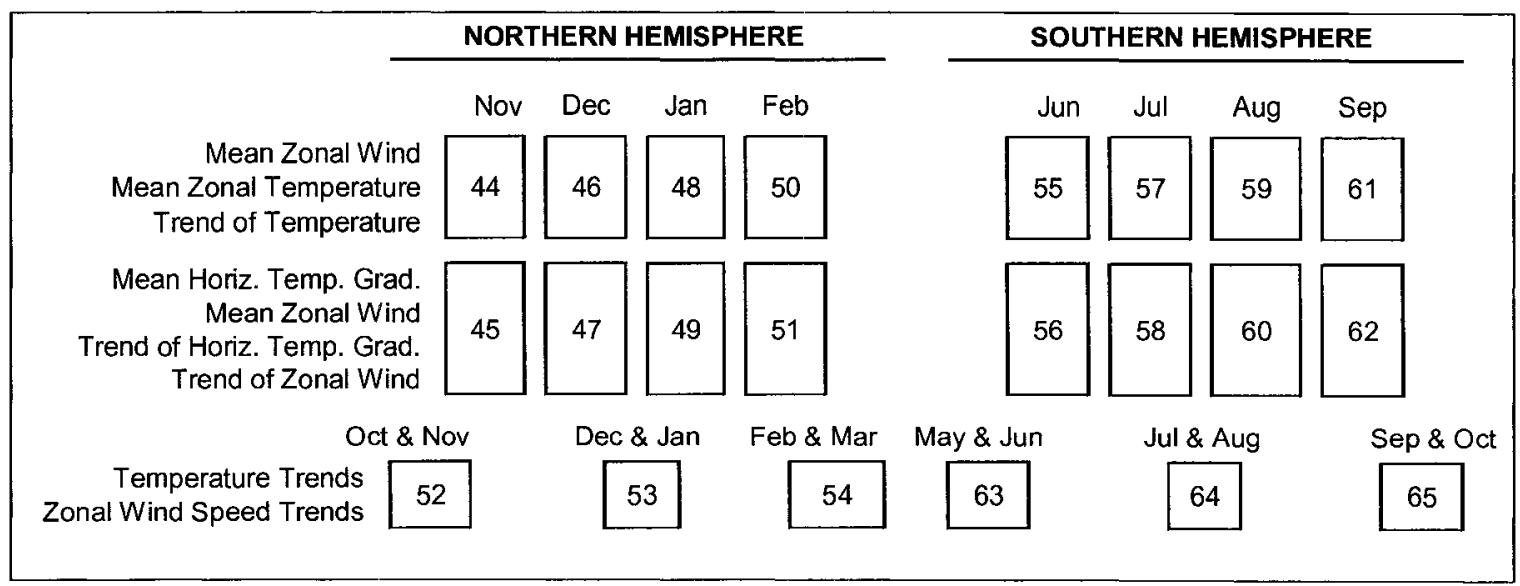

Fig. 5-43. Logical arrangement of figures.

Figures 5-44, 5-46, 5-48, and 5-50 (Northern Hemisphere) and 5-55, 5-57, 5-59, and 5-61 (Southern Hemisphere) begin with plots of zonally- and monthly averaged mean zonal wind speeds. Contours of this variable from the two reanalyses are quite similar, and as discussed above and demonstrated in Fig. 5-42, mean values of the zonal wind speed at the locations (latitudes and pressure levels to $10 \mathrm{hPa}$ ) of the maximum zonal wind speed are also consistent between reanalyses. These same figures next plot zonallyand monthly-averaged mean temperatures and again, except for latitudes near the poles, the two reanalyses have similar contours. Figure 4-3 (NCEP1 minus ERA-40 temperatures), with increasing mean differences, larger in the Southern Hemisphere and within 15 degrees latitude of the poles, confirms these results.

Unlike the first two sets of subplots discussed above, temperature trends differ dramatically between NCEP1 and ERA-40. With the exception of February (Fig. 5-50), ERA-40 temperature trend contours are significantly greater in magnitude and in internal gradients. This difference in reanalysis trends increases from $\pm 45^{\circ}$ latitude to maxima at 
the poles in both hemispheres. This behavior is confirmed by Figs. 4-3 (NCEP1 minus ERA-40) and 4-6 (ERA-40 minus METO), which contain large perturbations in temperature standard deviation at 30 and $70 \mathrm{hPa}$ within $15^{\circ}$ latitude of the poles. These anomalies in temperature standard deviation extend equatorward from the poles to $60^{\circ} \mathrm{S}-45^{\circ} \mathrm{S}$ and $75^{\circ} \mathrm{N}$. Figures $4-19$ and $4-20$ also show dramatically large values of ERA-40 Southern Hemisphere temperature standard deviation (with respect to NCEP1) at $10 \mathrm{hPa}$, and significantly larger values to $40-50 \mathrm{hPa}$. Because of this evidence of anomalous ERA-40 temperatures in stratospheric polar regions in this thesis and that documented elsewhere (Randel et. al. 2002, 2004), further discussion of temperature trends in this section will be limited to NCEP1 plots. Although not believed to be accurate, ERA-40 temperature trend subplots are included for completeness and for comparison with corresponding NCEP1 trends.

\section{Northern Hemisphere temperature trends}

In the Northern Hemisphere, October, November, February, and March exhibit negative temperature trends over the entire region displayed $\left(100-10 \mathrm{hPa}, 45^{\circ} \mathrm{N}-90^{\circ} \mathrm{N}\right)$ in the range of -0.5 to $-4 \mathrm{~K} \mathrm{decade}^{-1}$. The December trend is positive over most of the displayed region with a maximum of $2.5 \mathrm{~K} \mathrm{decade}^{-1}$. However, January's trend is negative at heights above $30 \mathrm{hPa}$ and equatorward of $60^{\circ} \mathrm{N}$ and positive elsewhere. Most of the displayed region is statistically significant in October; none is statistically significant in December; varying portions of the displayed region are statistically significant in the remaining months. Generally, statistical significance in temperature trends is less likely to be found in vortex regions and polar regions at upper heights. 
Northern Hemisphere temperature trends and their confidence intervals are also plotted as a function of latitude for pressure levels of 10, 20, and $30 \mathrm{hPa}$ in Figs. 5-52, 5-53, and 5-54.

\section{Southern Hemisphere temperature trends}

In the Southern Hemisphere, May, June, and July all exhibit negative temperature trends over the entire region displayed $\left(100-10 \mathrm{hPa}, 45^{\circ} \mathrm{N}-90^{\circ} \mathrm{N}\right)$ in the range of -0.5 to $-4 \mathrm{~K} \mathrm{decade}^{-1}$. August, September, and October contain mixed trends. Statistical significance is present in the trends of each month; it is found in larger areas of the first three months, May, June, and July, and in lesser amounts in the last three. As in the Northern Hemisphere, statistical significance is less likely to be found in vortex regions and polar regions at upper heights. Southern Hemisphere temperature trends and their confidence intervals are also plotted as a function of latitude for pressure levels of 10, 20, and $30 \mathrm{hPa}$ in Figs. 5-63, 5-64, and 5-65.

Figures 5-45, 5-47, 5-49, and 5-51 (Northern Hemisphere) and 5-56, 5-58, 5-60, and 5-62 (Southern Hemisphere) begin with plots of horizontal temperature gradients. Contours of these gradients from the two reanalyses are quite similar in their respective hemispheres and reflect seasonal variations. Again, as is the case with temperature trends, dramatic differences exist between plots of horizontal temperature gradient trends of the NCEP1 and ERA-40 reanalyses. 
Northern Hemisphere horizontal temperature gradients and trends, and zonal wind speeds and trends

In the Northern Hemisphere, negative horizontal temperature gradients dominate the area of interest during all months considered in this section. Maximum values of horizontal temperature gradient decrease in height from $10 \mathrm{hPa}$ (October) to $100 \mathrm{hPa}$ (March). Small regions of positive horizontal temperature gradient occur in the region of $100-30 \mathrm{hPa}, 45^{\circ} \mathrm{N}-50^{\circ} \mathrm{N}$. Thermal wind balance produces increasing zonal wind speed, given stronger horizontal temperature gradient maxima. Horizontal temperature gradient trend is generally positive except during October (light), February, and March. Positive horizontal temperature gradient trends in a region of mean negative horizontal temperature gradient result in negative zonal wind speed trends and vice versa. Regions where the horizontal temperature gradient trend increases with height correspond to zonal wind speed gradients that increase with height. Similarly, regions where the horizontal temperature gradient trend is constant with height correspond to zonal wind speed trends that are constant with height. Although ERA-40 horizontal temperature gradient trend has a much more complex structure than NCEP1, zonal wind speed trends of the two reanalyses and their confidence intervals are quite consistent. This consistency is evident in the lower halves of Figs. 5-52 through 5-54. Only from $45^{\circ} \mathrm{N}-60^{\circ} \mathrm{N}$ in December are zonal wind speed trends statistically significant.

Southern Hemisphere horizontal temperature gradients and trends, and zonal wind speeds and trends

In the Southern Hemisphere the horizontal temperature gradient is positive over the entire region for each of the months analyzed in this thesis. Maxima are located at 
$50-30 \mathrm{hPa}, 65^{\circ} \mathrm{S}-60^{\circ} \mathrm{S}$ except during May $(20 \mathrm{hPa})$ and October $(100-50 \mathrm{hPa})$. Stronger horizontal temperature gradient maxima lead, via the thermal wind balance, to stronger zonal wind speed.

Although the Southern Hemisphere horizontal temperature gradients are much stronger and consistent from month to month than those of the Northern Hemisphere, their trends are much less structured. Their strongest trends are positive and are found from $30-10 \mathrm{hPa}, 85^{\circ} \mathrm{S}-60^{\circ} \mathrm{S}$. Weaker negative trends occur at lower heights and equatorward of the positive ones. Positive horizontal temperature gradient trends in a region of mean positive horizontal temperature gradients result in positive zonal wind speed trends and vice versa. Months where the horizontal temperature gradient trend is positive at upper heights and negative at lower ones (June and July) exhibit a slight positive zonal wind speed trend maximum at $10 \mathrm{hPa}$ and a stronger negative trend at $30 \mathrm{hPa}$ at the same latitude. Months with negative horizontal temperature gradient trends equatorward of positive ones (August, September, and October) have positive zonal wind speed trends at all heights $(30-10 \mathrm{hPa})$ poleward of negative trends. As a consequence of mixed positive and negative horizontal temperature gradient trends, both positive and negative zonal wind speed trends exist in a given month for all months except June (completely negative zonal wind speed trend).

Again, although ERA-40 horizontal temperature gradient trends have much more complex structure than those of NCEP1, zonal wind speed trends of the two reanalyses and their confidence intervals are quite consistent as is evident in Figs. 5-63 through 5-65. Only from $15-10 \mathrm{hPa}, 90^{\circ} \mathrm{S}-75^{\circ} \mathrm{S}$ in August is there statistical significance. 
Generally, Southern Hemisphere temperature trends are stronger than those of the Northern Hemisphere. However, Northern Hemisphere zonal wind speed trends are stronger than Southern Hemisphere trends. 

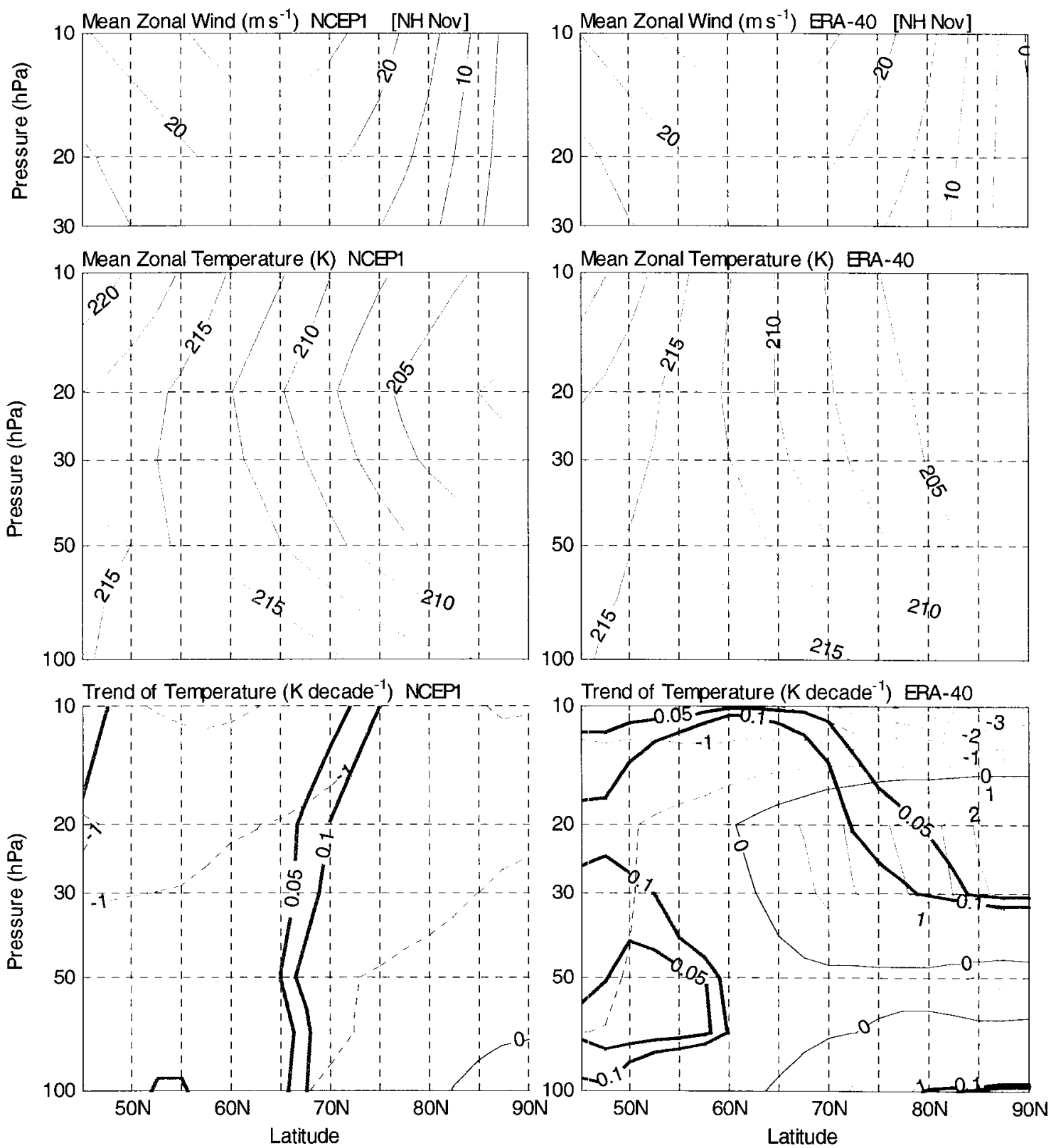

Fig. 5-44. November NH monthly-and zonally-averaged zonal wind speed and temperature and 1979-2001 temperature trend (5\% and 10\% statistical significance limits shown in green): [lon: mean of $0^{\circ}-357.5^{\circ}$, lat: $45^{\circ} \mathrm{N}-90^{\circ} \mathrm{N}$, press: $100-10 \mathrm{hPa}$, time: 1979-2001]. 

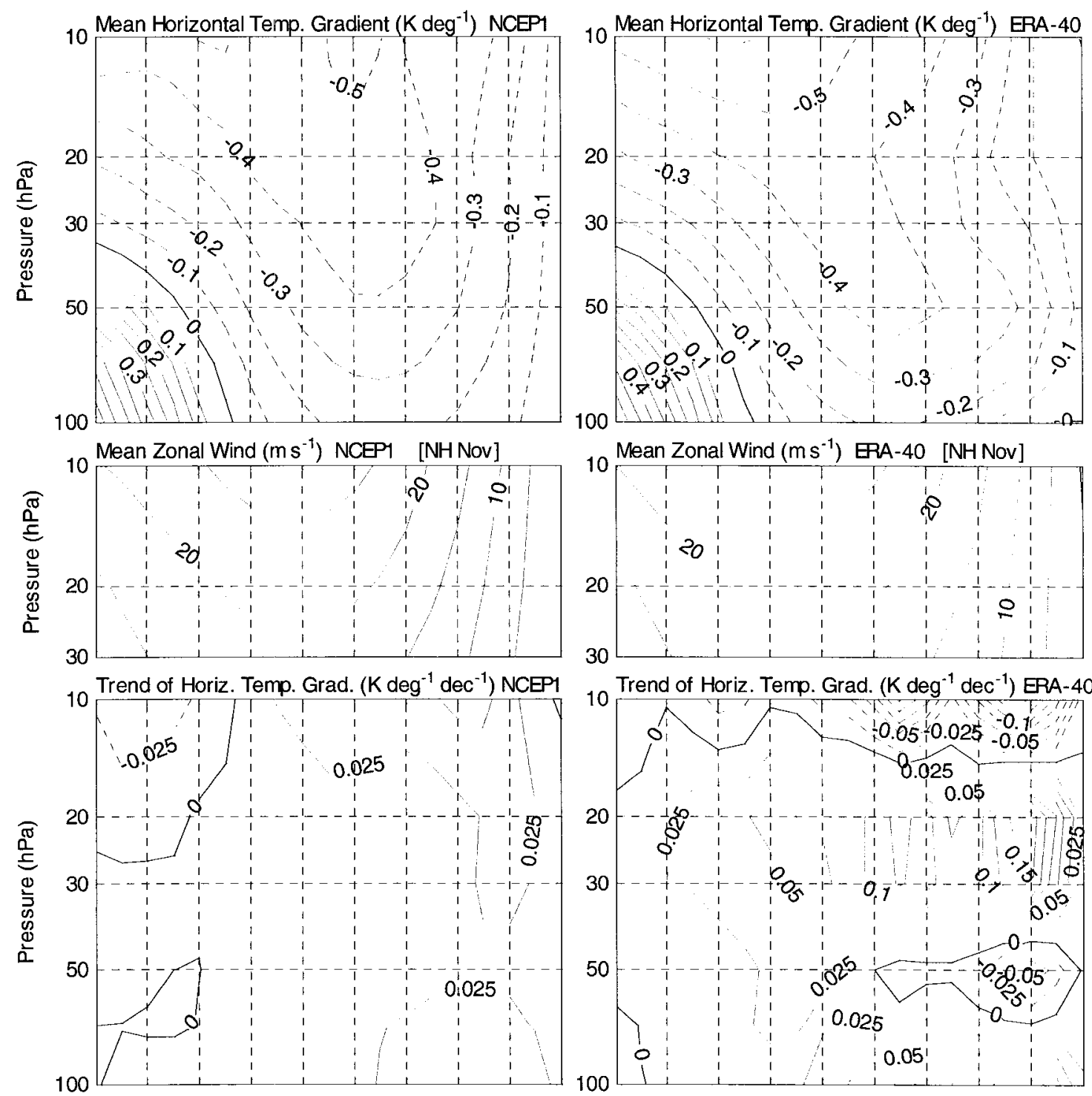

10 Trend of Horiz. Temp. Grad. $\left(\mathrm{K} \mathrm{deg}^{-1} \mathrm{dec}^{-1}\right)$ ERA-40
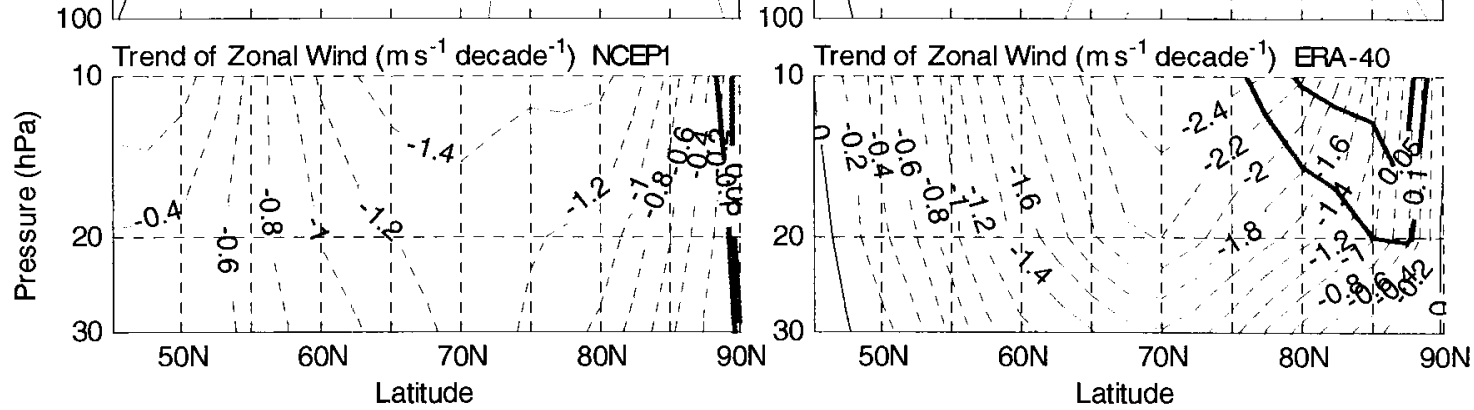

Fig. 5-45. November NH monthly-and zonally-averaged horizontal temperature gradient and its 1979-2001 trend, zonal wind speed and its 1979-2001 trend (5\% and $10 \%$ statistical significance limits shown in green):

[lon: mean of $0^{\circ}-357.5^{\circ}$, lat: $45^{\circ} \mathrm{N}-90^{\circ} \mathrm{N}$, press: $100-10 \mathrm{hPa}$, time: 1979-2001]. 

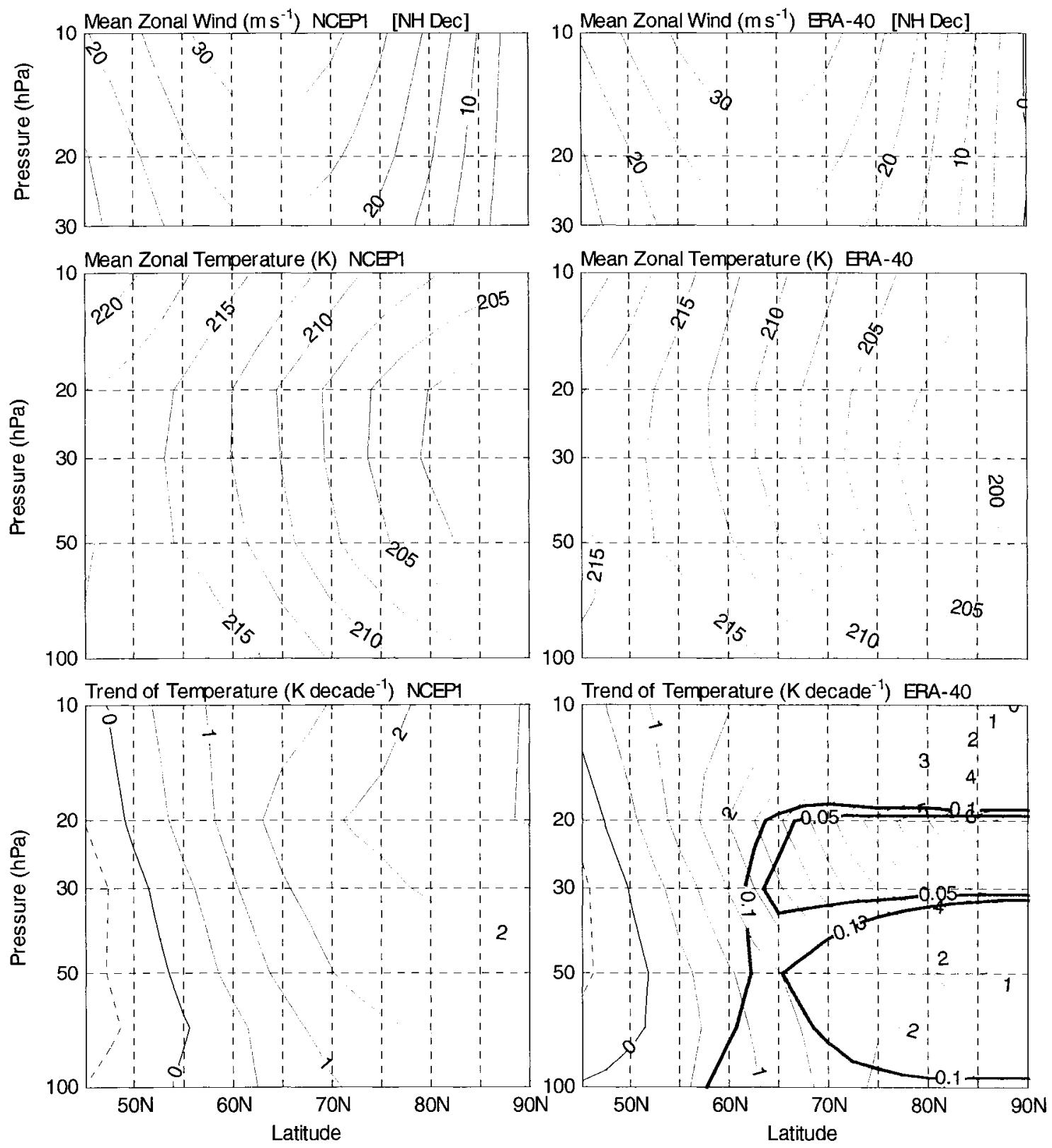

Fig. 5-46. Same as Fig. 5-44 except December. 

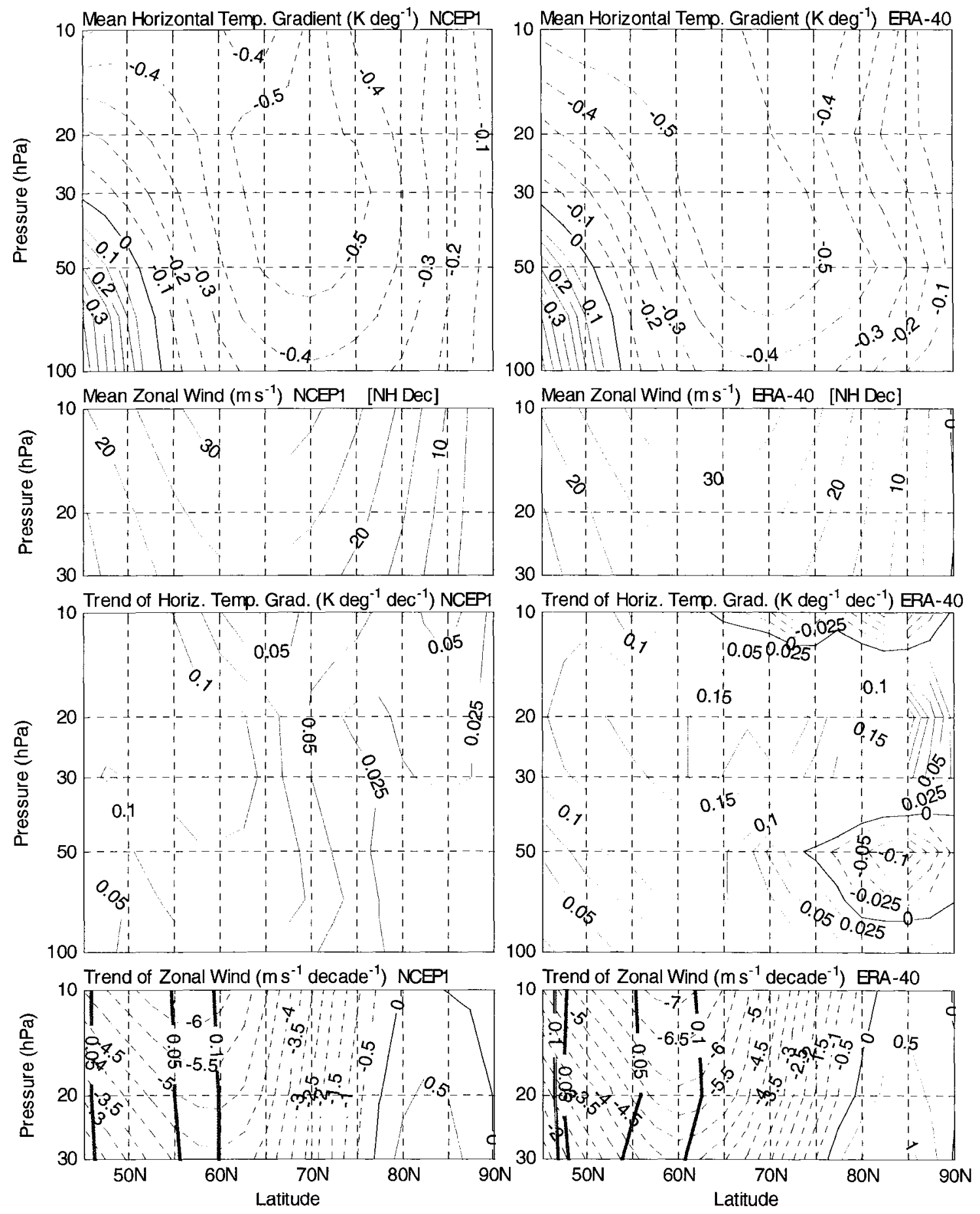

Fig. 5-47. Same as Fig. 5-45 except December. 

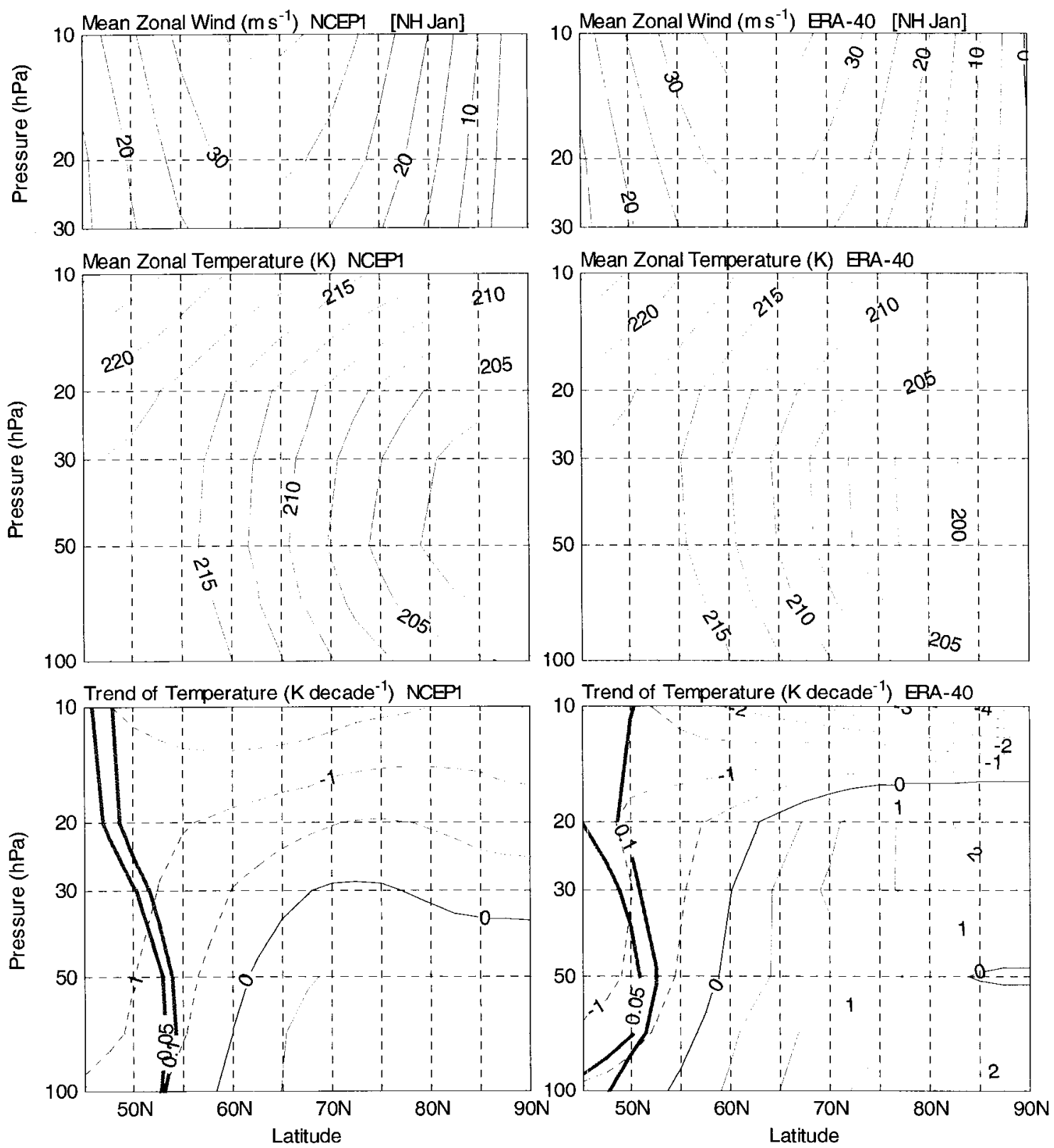

Fig. 5-48. Same as Fig. 5-44 except January. 

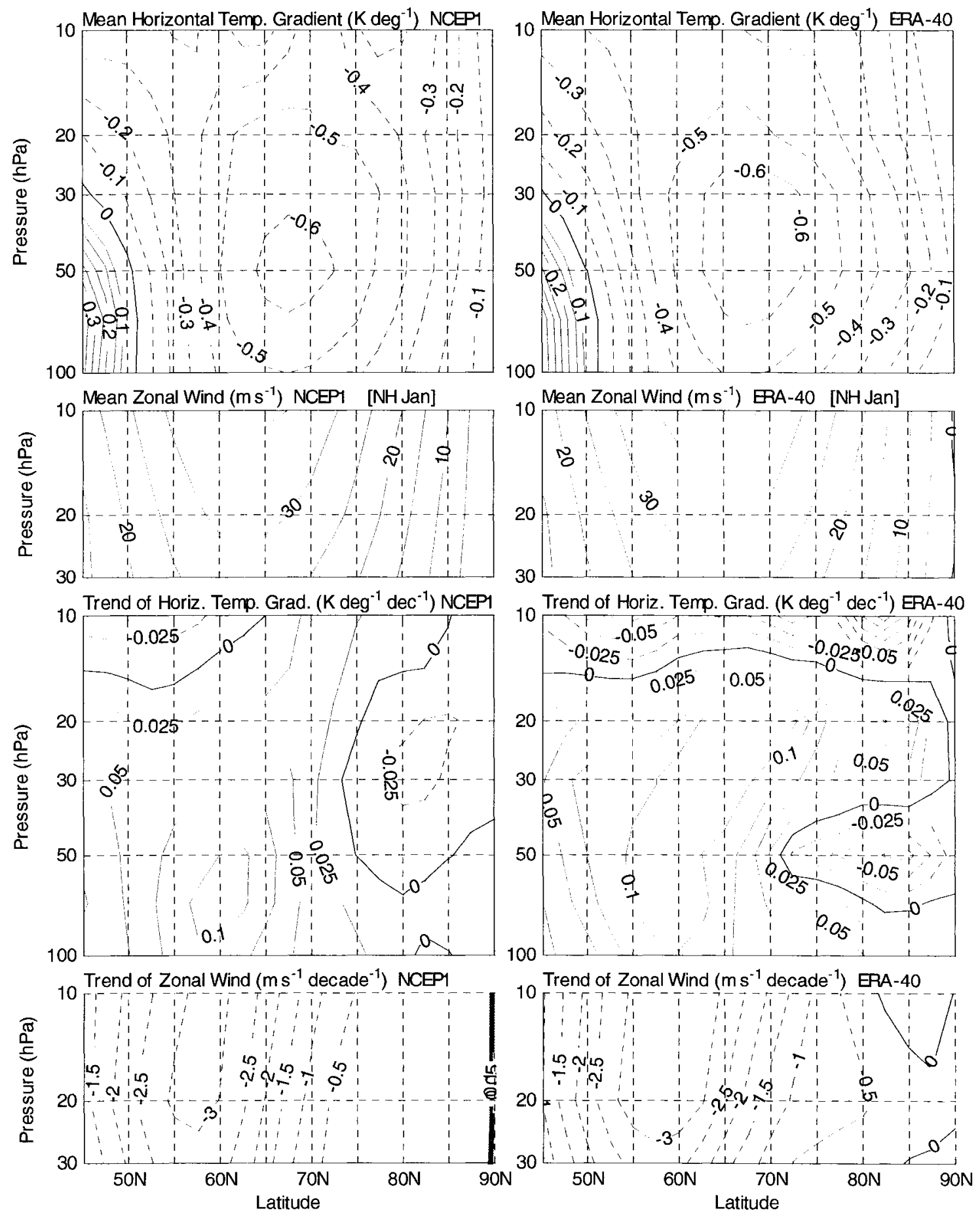

Fig. 5-49. Same as Fig. 5-45 except January. 

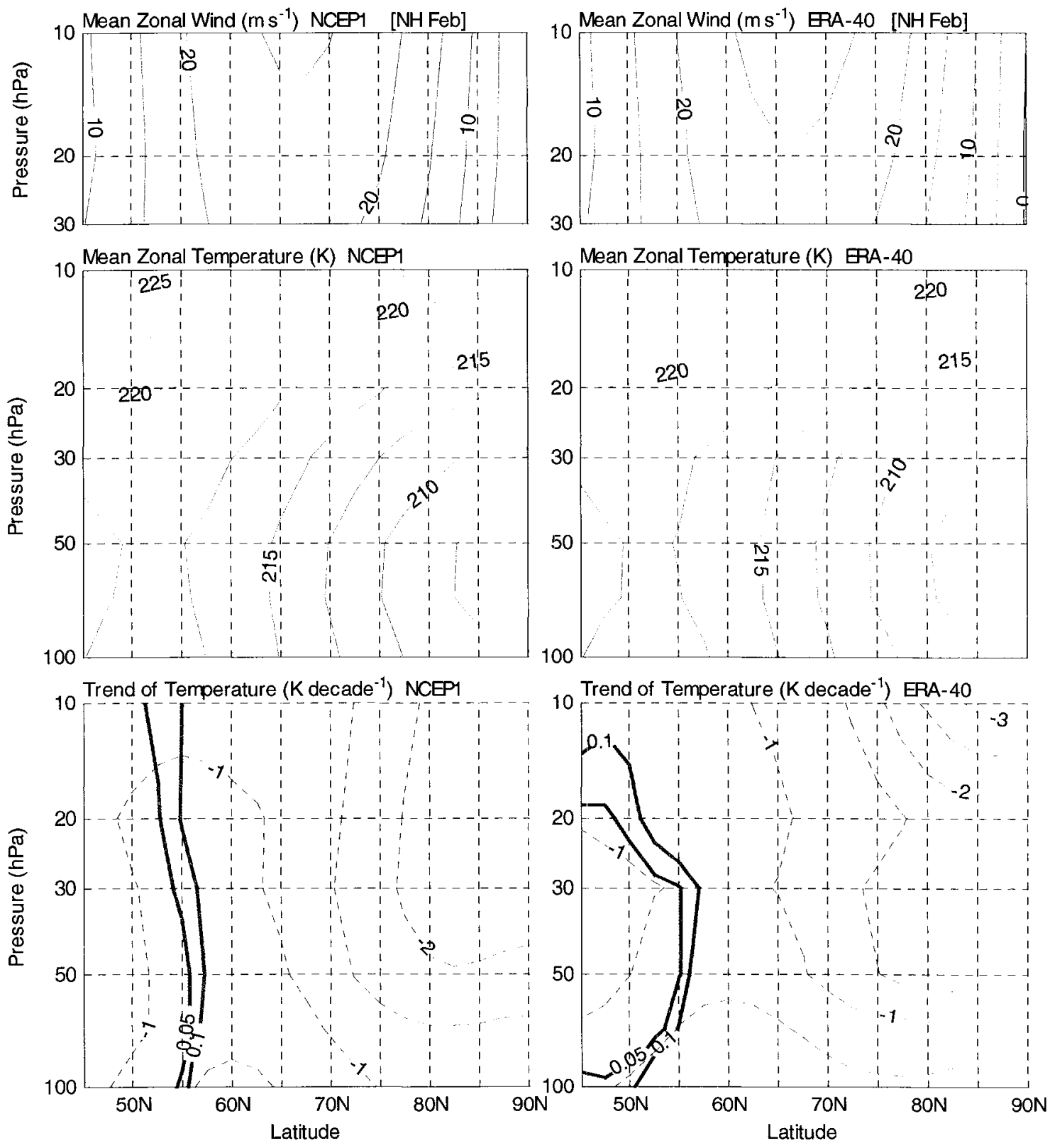

Fig. 5-50. Same as Fig. 5-44 except February. 

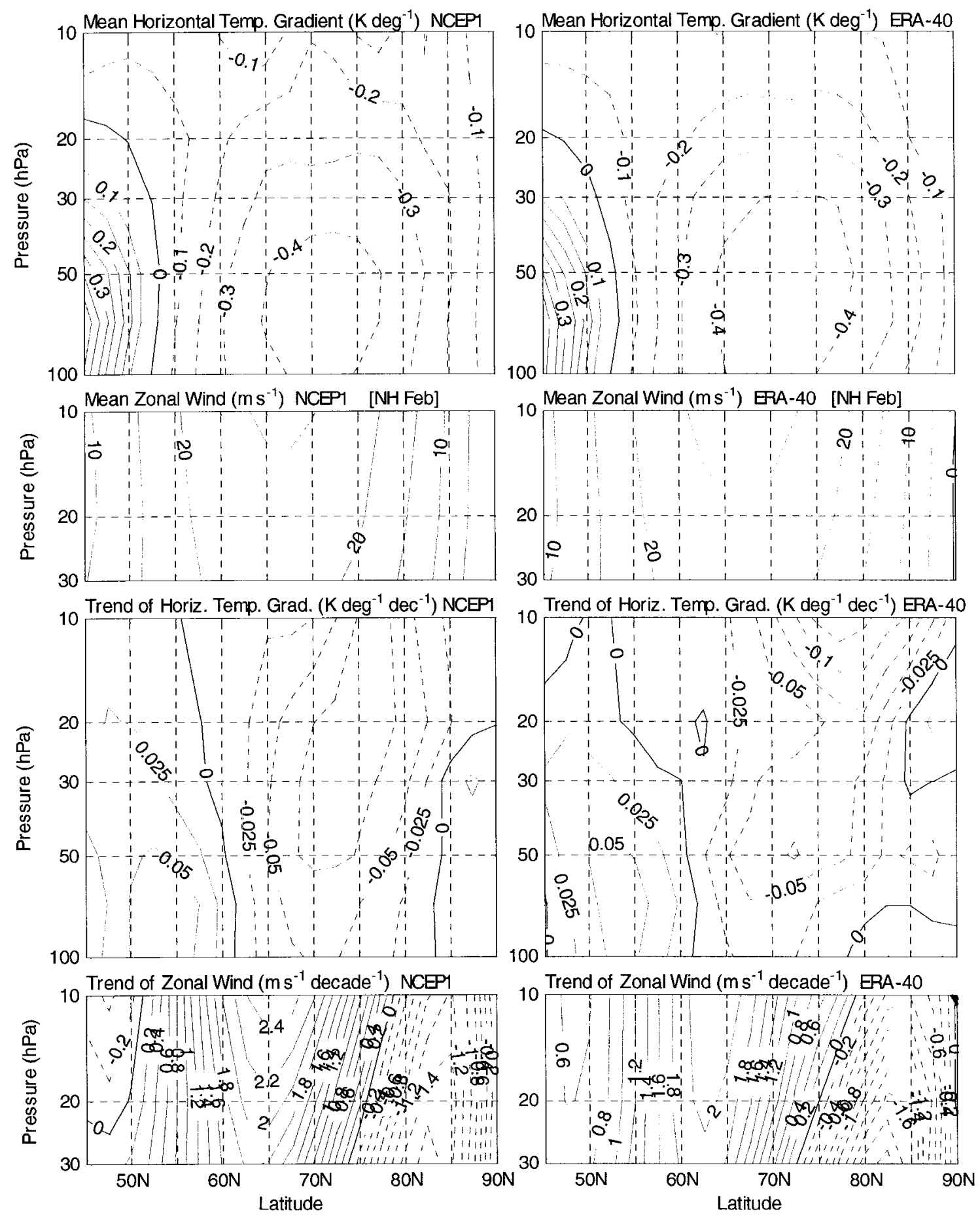

Fig. 5-51. Same as Fig. 5-45 except February. 

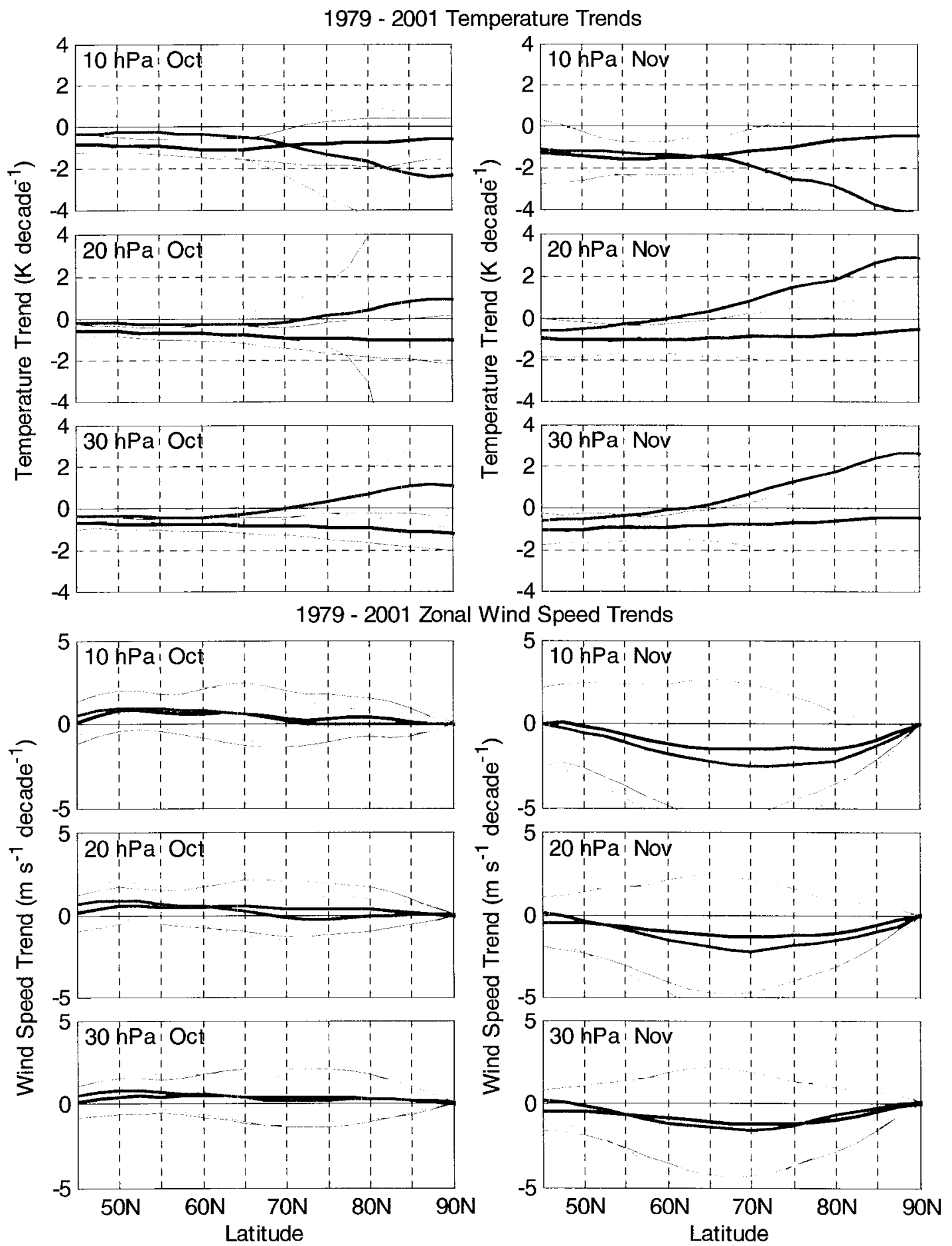

Fig. 5-52. October and November NH monthly- and zonally-averaged temperature and zonal wind speed 1979-2001 trends vs. latitude (blue: NCEP1, green: ERA-40, bold: mean trend, light lines: 5\% statistical significance): [lon: mean of $0^{\circ}-357.5^{\circ}$, lat: $45^{\circ} \mathrm{N}-90^{\circ} \mathrm{N}$, press: $100-10 \mathrm{hPa}$, time: $\left.1979-2001\right]$. 


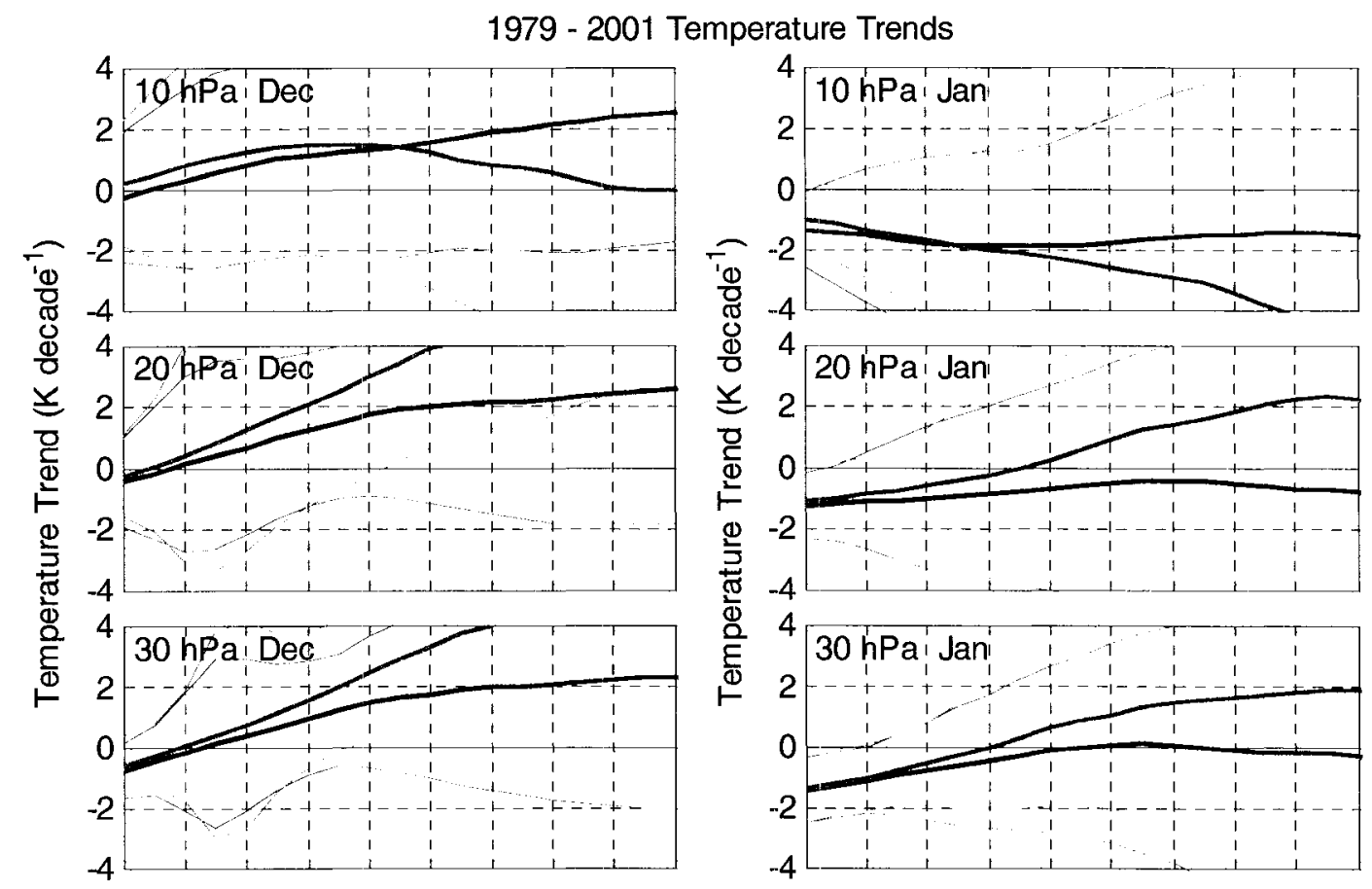

1979 - 2001 Zonal Wind Speed Trends
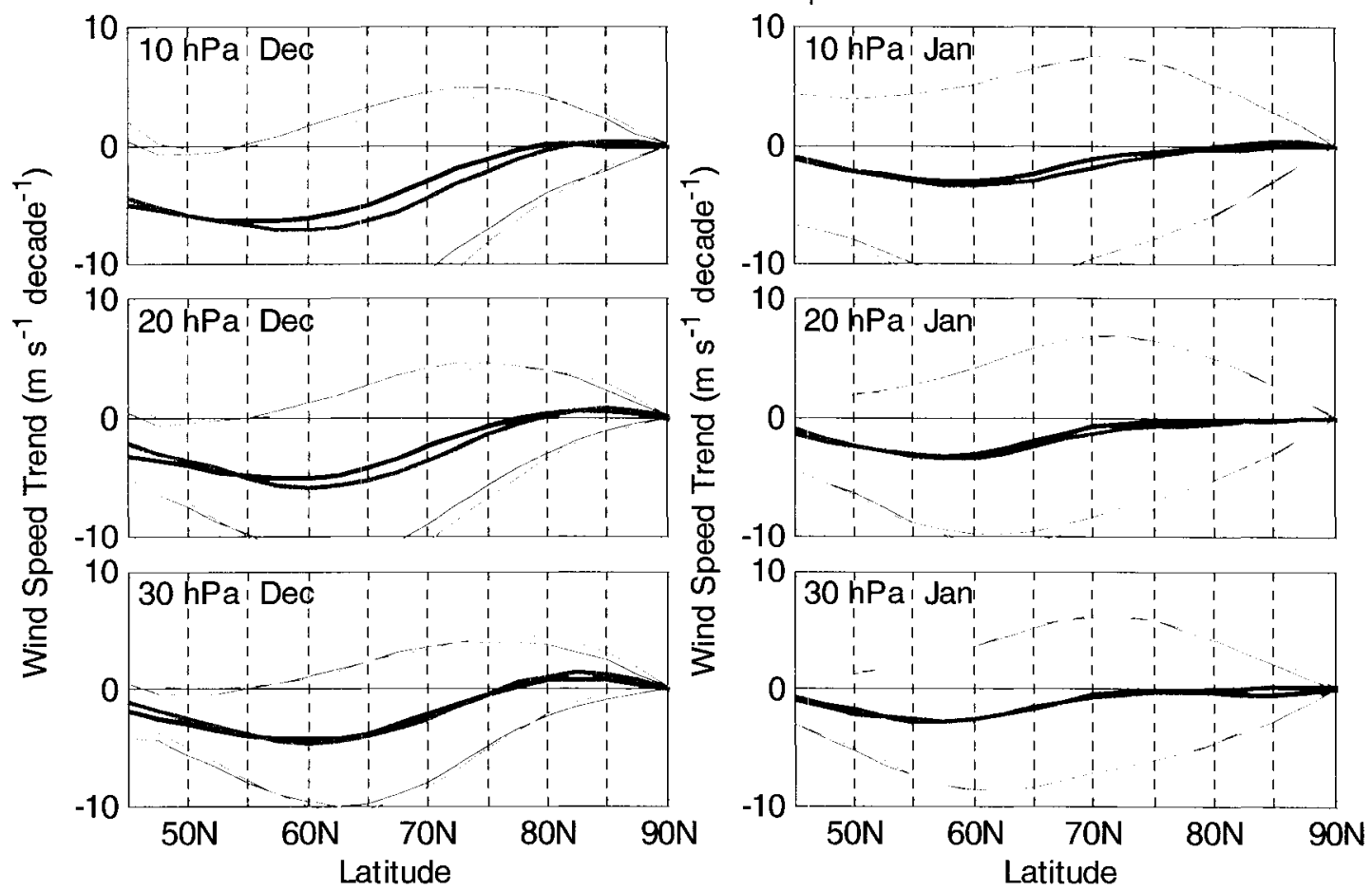

Fig. 5-53. Same as Fig. 5-52 except December and January. 
1979 - 2001 Temperature Trends

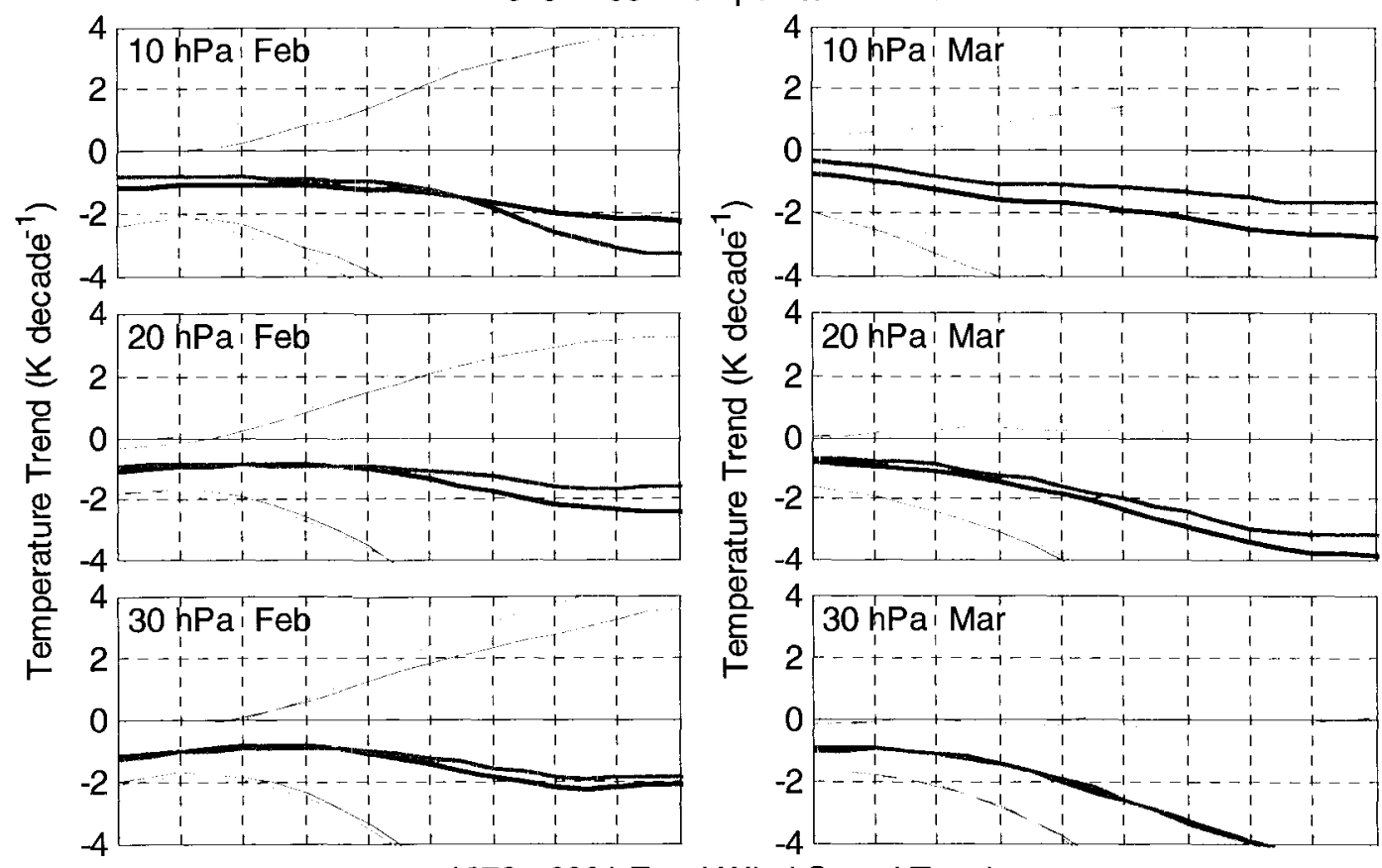

1979 - 2001 Zonal Wind Speed Trends
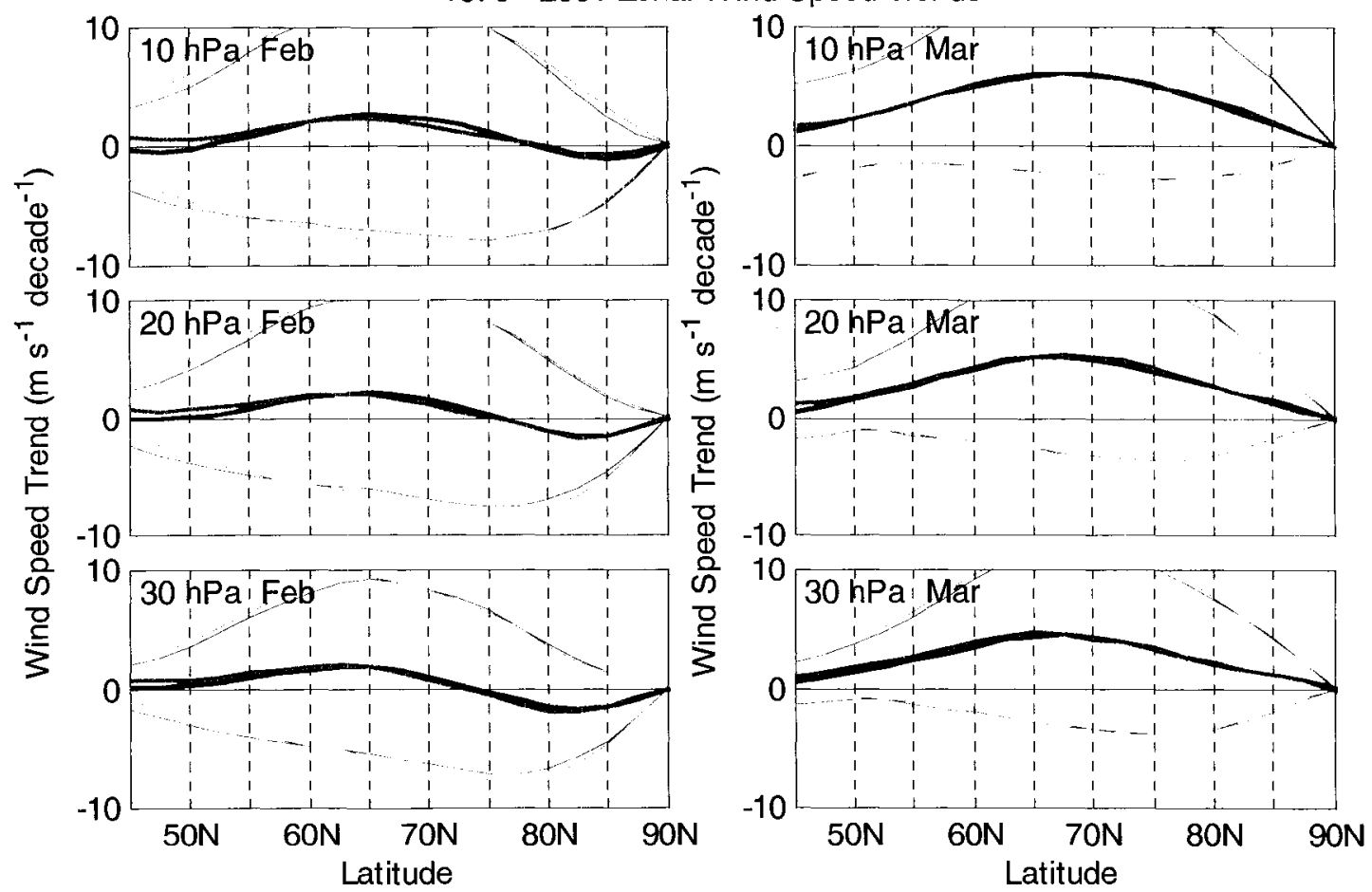

Fig. 5-54. Same as Fig. 5-52 except February and March. 

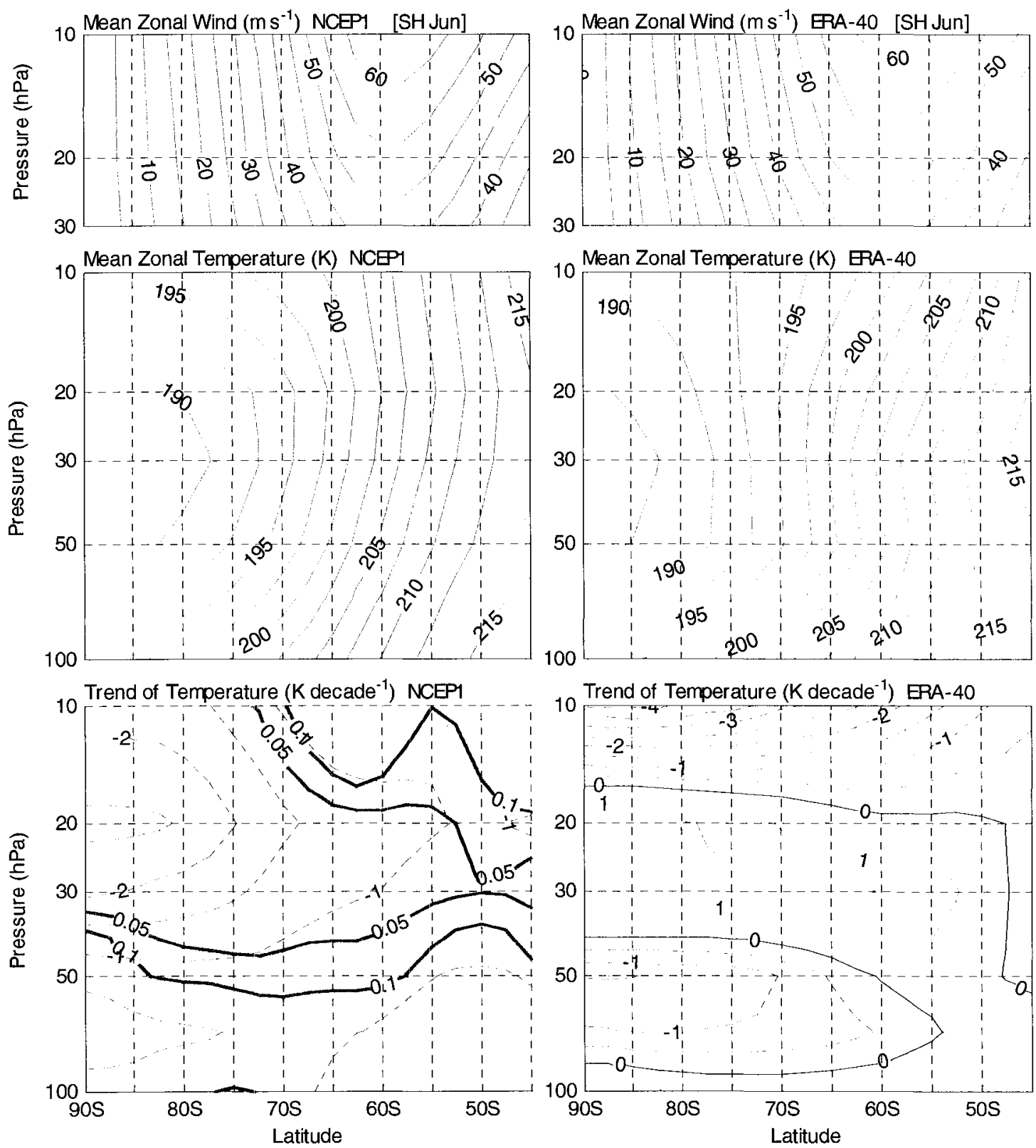

Fig. 5-55. June SH monthly- and zonally-averaged zonal wind speed and temperature and 1979-2001 temperature trend (5\% and 10\% statistical significance limits shown in green): [lon: mean of $0^{\circ}-357.5^{\circ}$, lat: $90^{\circ} \mathrm{S}-45^{\circ} \mathrm{S}$, press: $100-10 \mathrm{hPa}$,

time: 1979-2001]. 

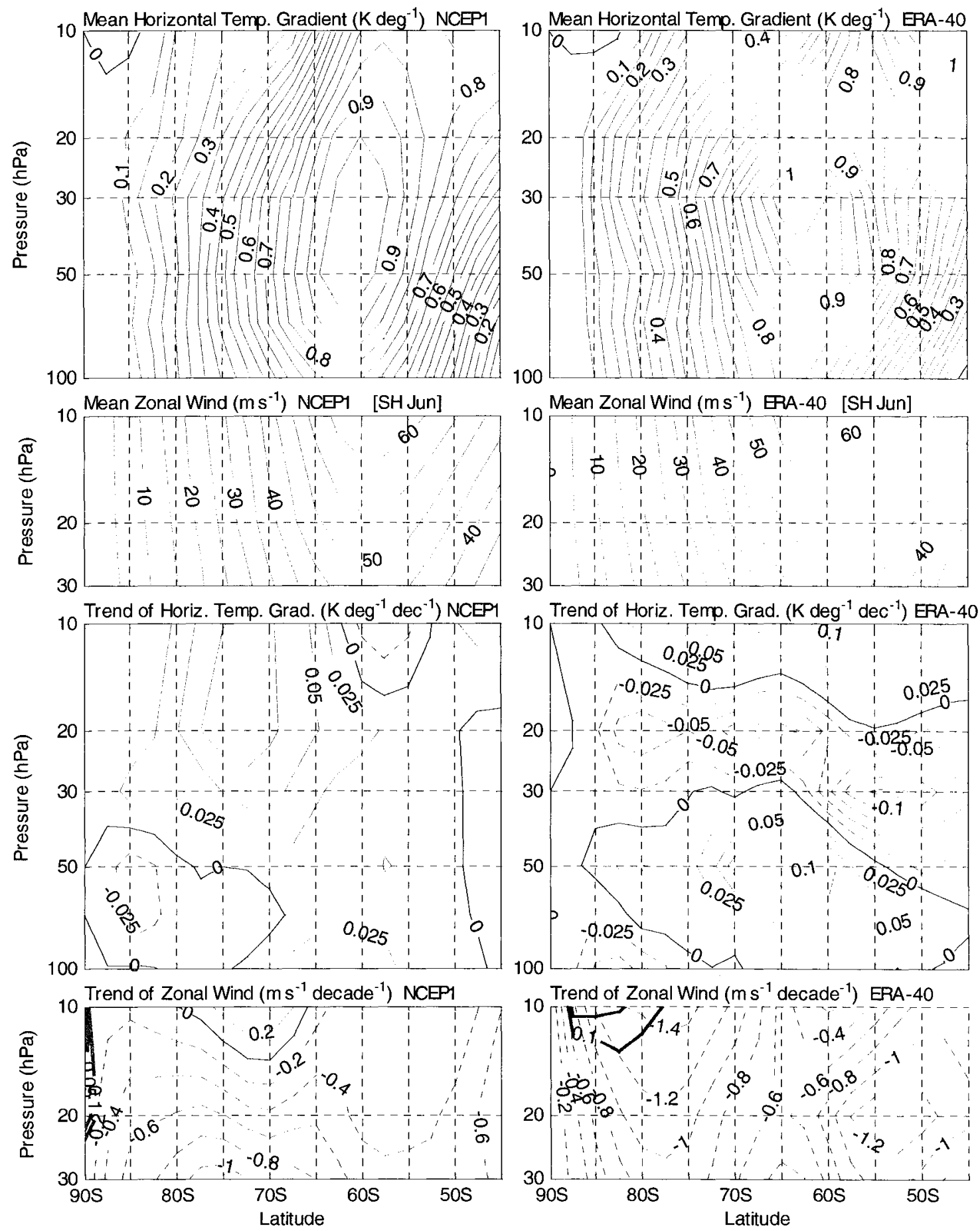

Fig. 5-56. June SH monthly- and zonally-averaged horizontal temperature gradient and its 1979-2001 trend, zonal wind speed and its 1979-2001 trend (5\% and 10\% statistical significance limits shown in green): [lon: mean of $0^{\circ}-357.5^{\circ}$, lat: $90^{\circ} \mathrm{S}-45^{\circ} \mathrm{S}$, press: 100-10 hPa, time: 1979-2001]. 

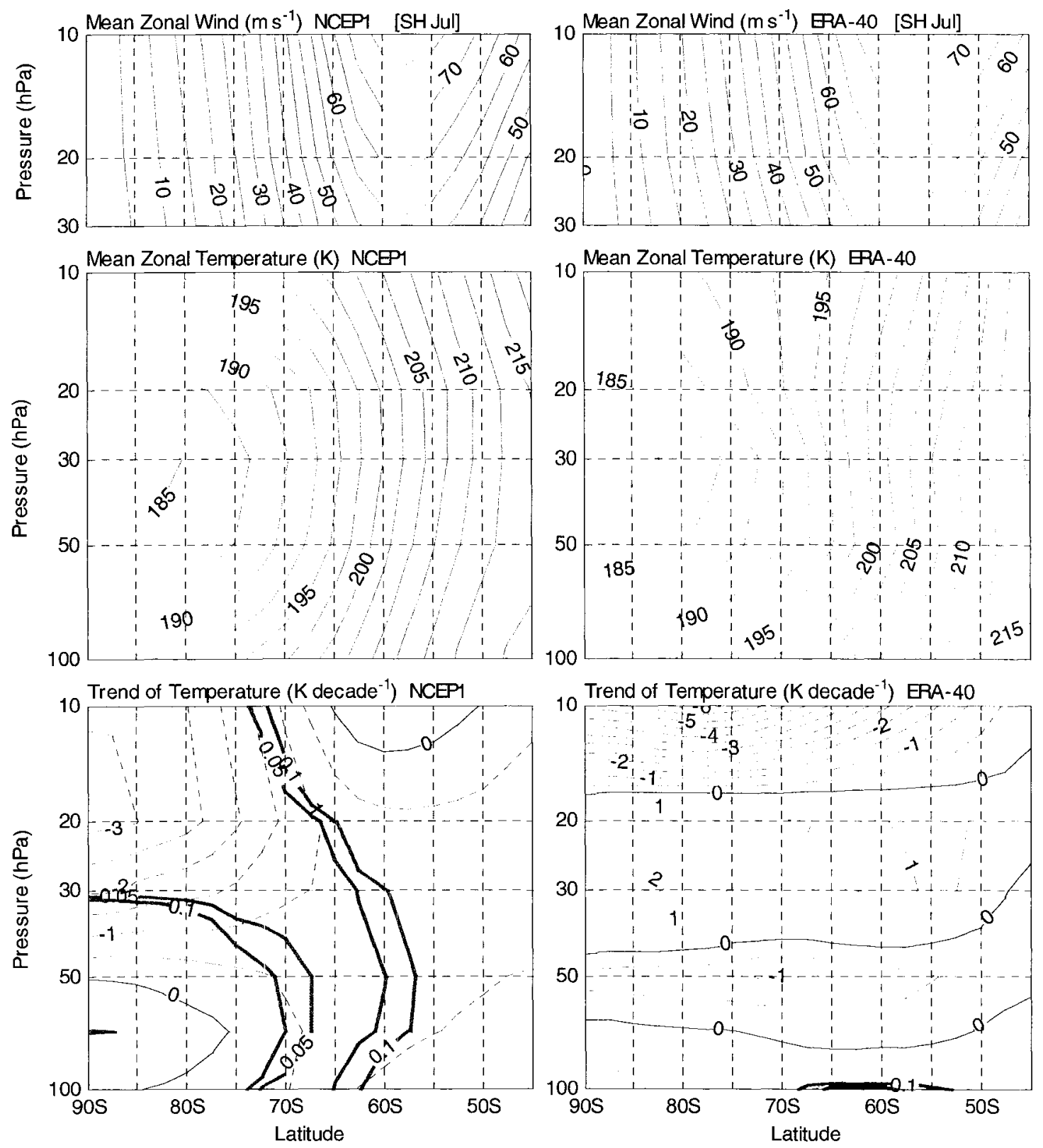

Fig. 5-57. Same as Fig. 5-55 except July. 

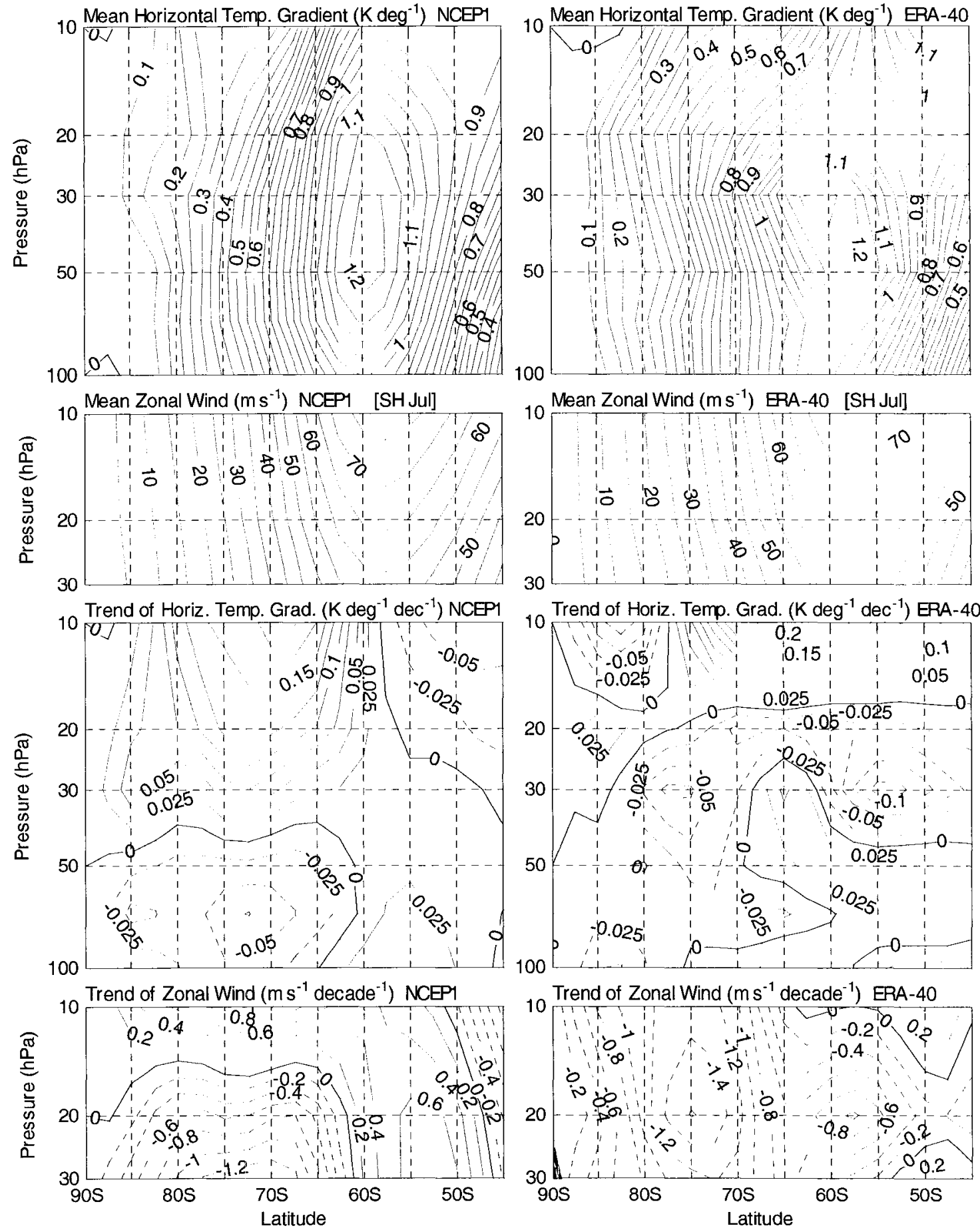

Fig. 5-58. Same as Fig. 5-56 except July. 

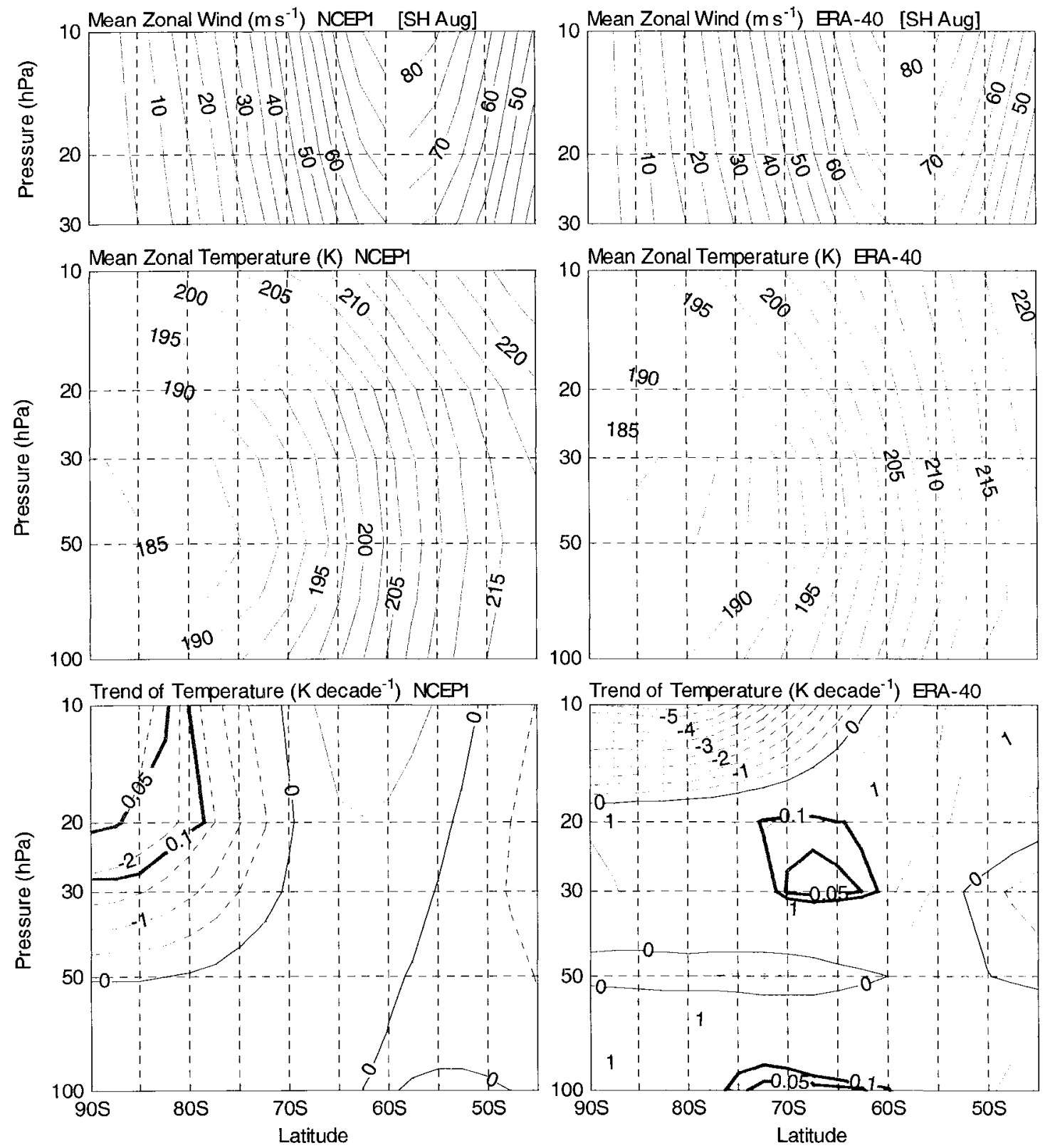

Fig. 5-59. Same as Fig. 5-55 except August. 

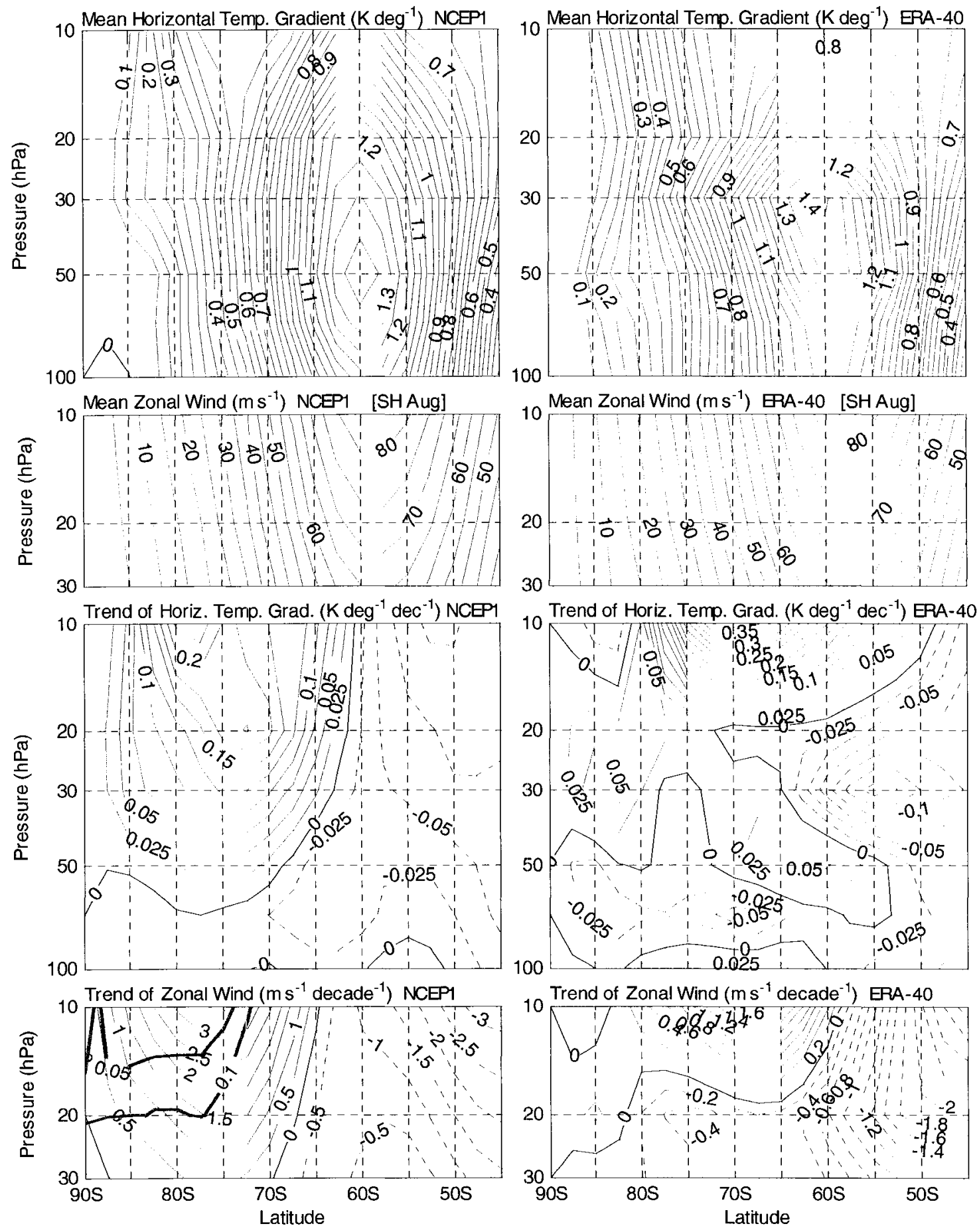

Fig. 5-60. Same as Fig. 5-56 except August. 

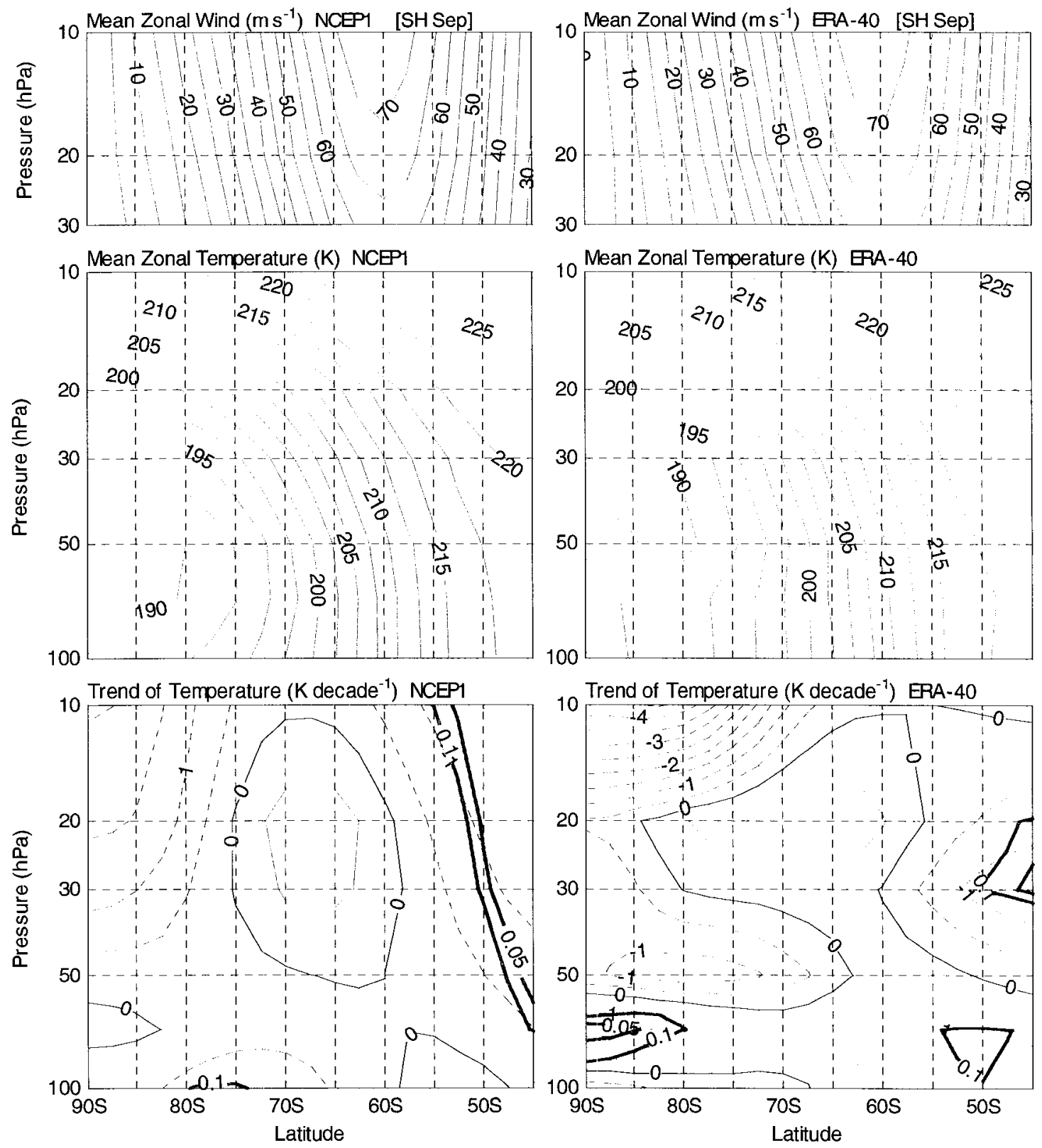

Fig. 5-61. Same as Fig. 5-55 except September. 

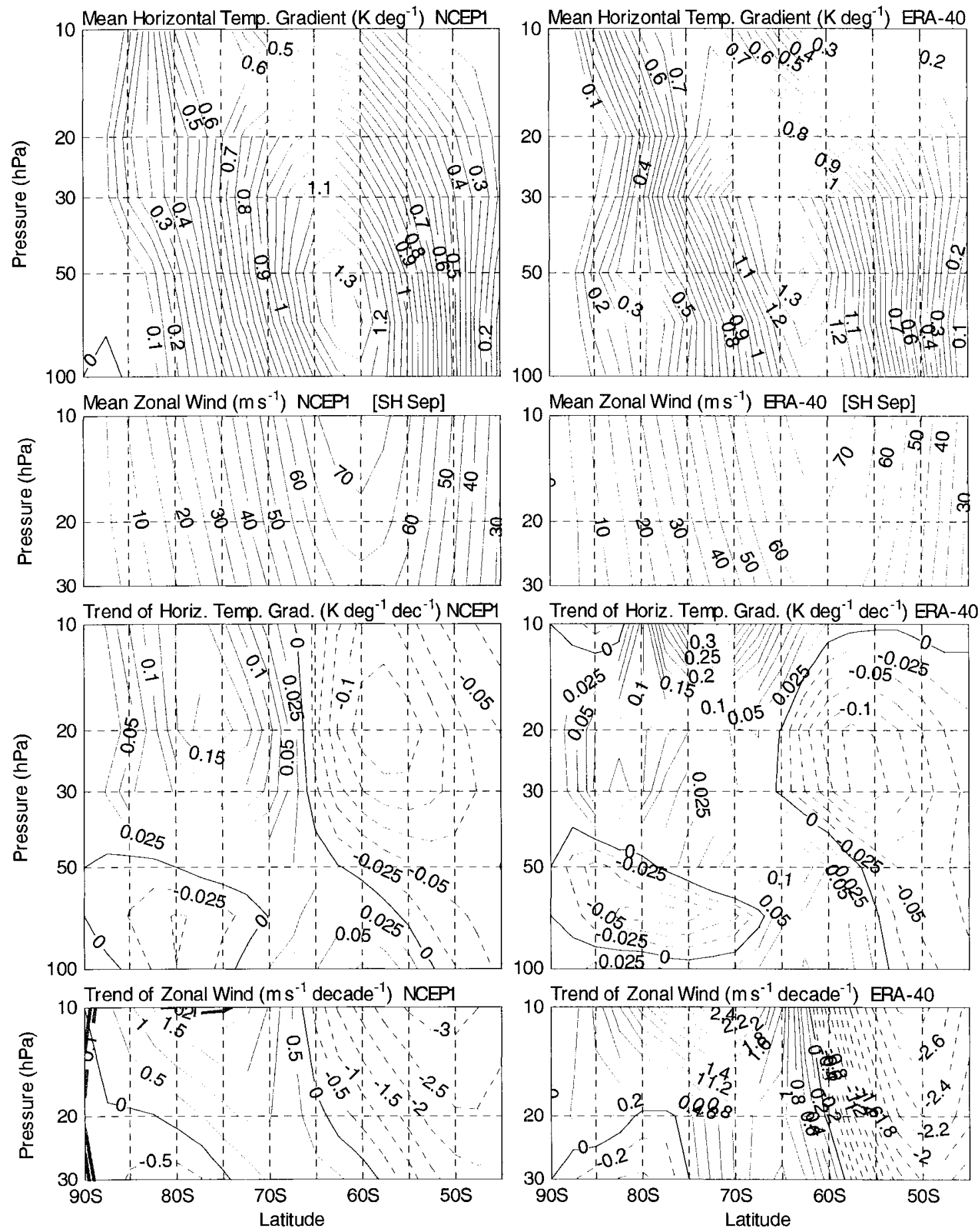

Fig. 5-62. Same as Fig. 5-56 except September. 
1979 - 2001 Zonal Temperature Trends
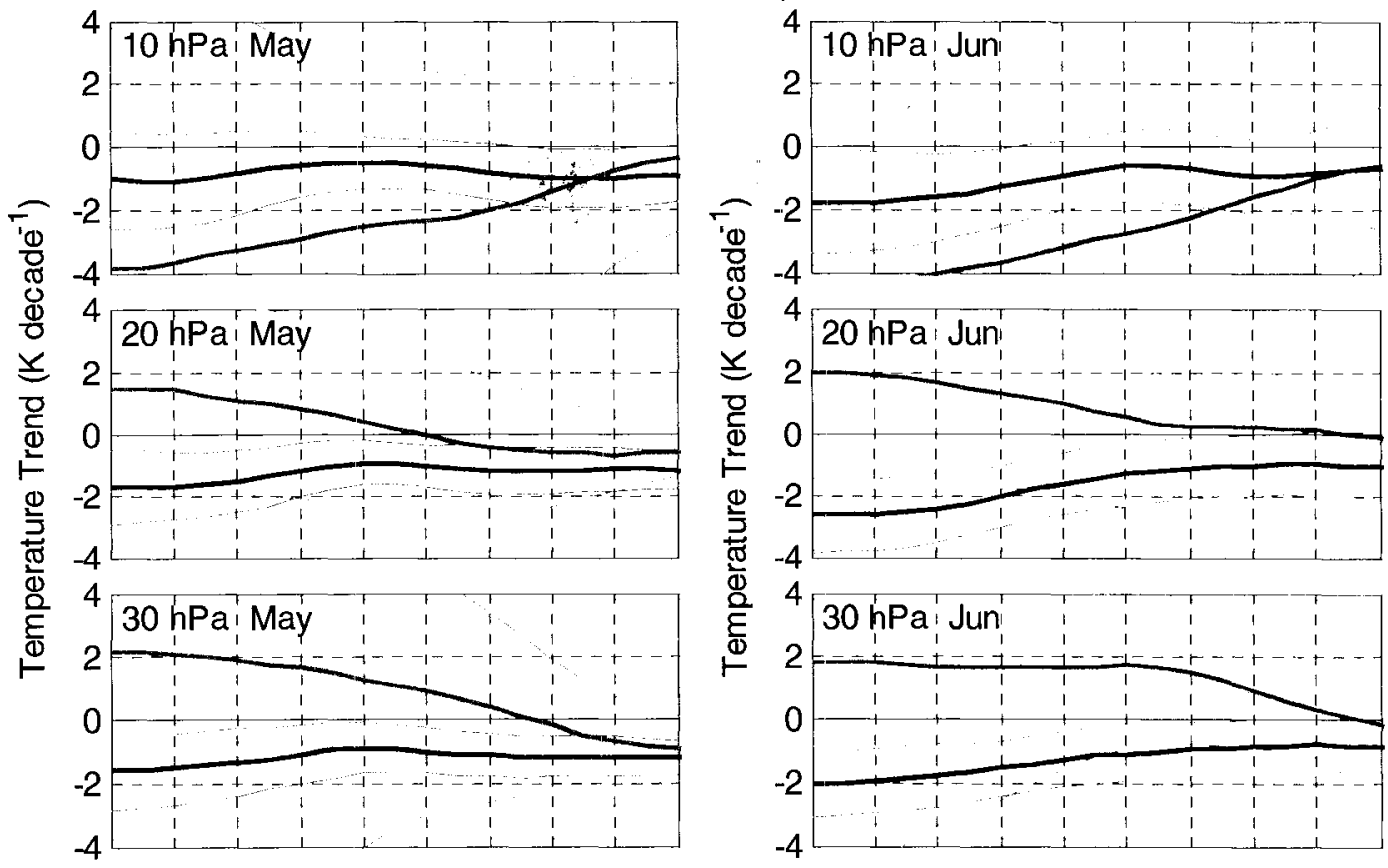

1979 - 2001 Zonal Wind Speed Trends
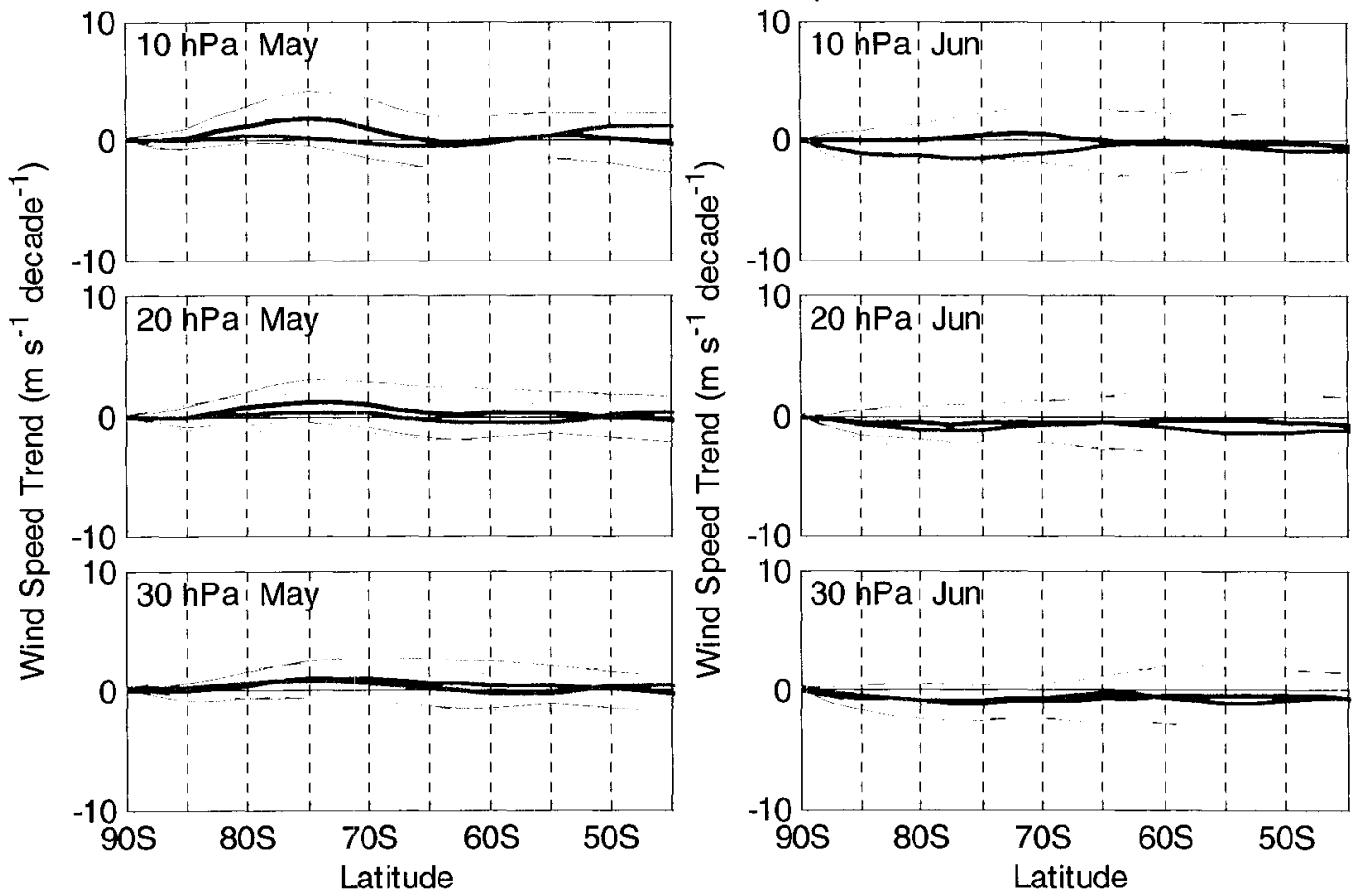

Fig. 5-63. May and June SH monthly- and zonally-averaged temperature and zonal wind speed 1979-2001 trends vs. latitude (blue: NCEP1, green: ERA-40, bold: mean trend, light lines: 5\% statistical significance): [lon: mean of $0^{\circ}-357.5^{\circ}$, lat: $90^{\circ} \mathrm{S}-45^{\circ} \mathrm{S}$, press: 100-10 hPa, time: 1979-2001]. 
1979 - 2001 Zonal Temperature Trends
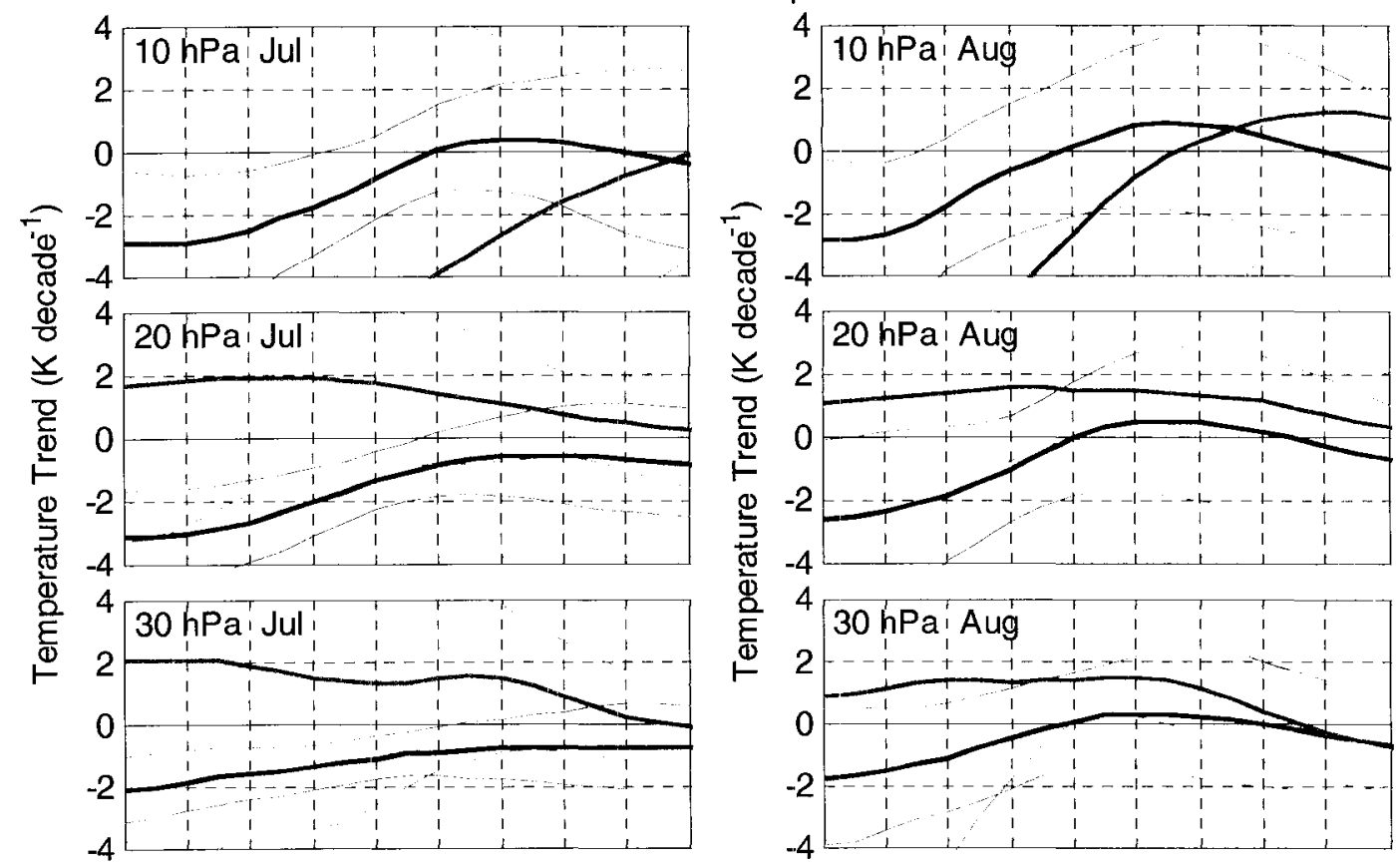

1979 - 2001 Zonal Wind Speed Trends
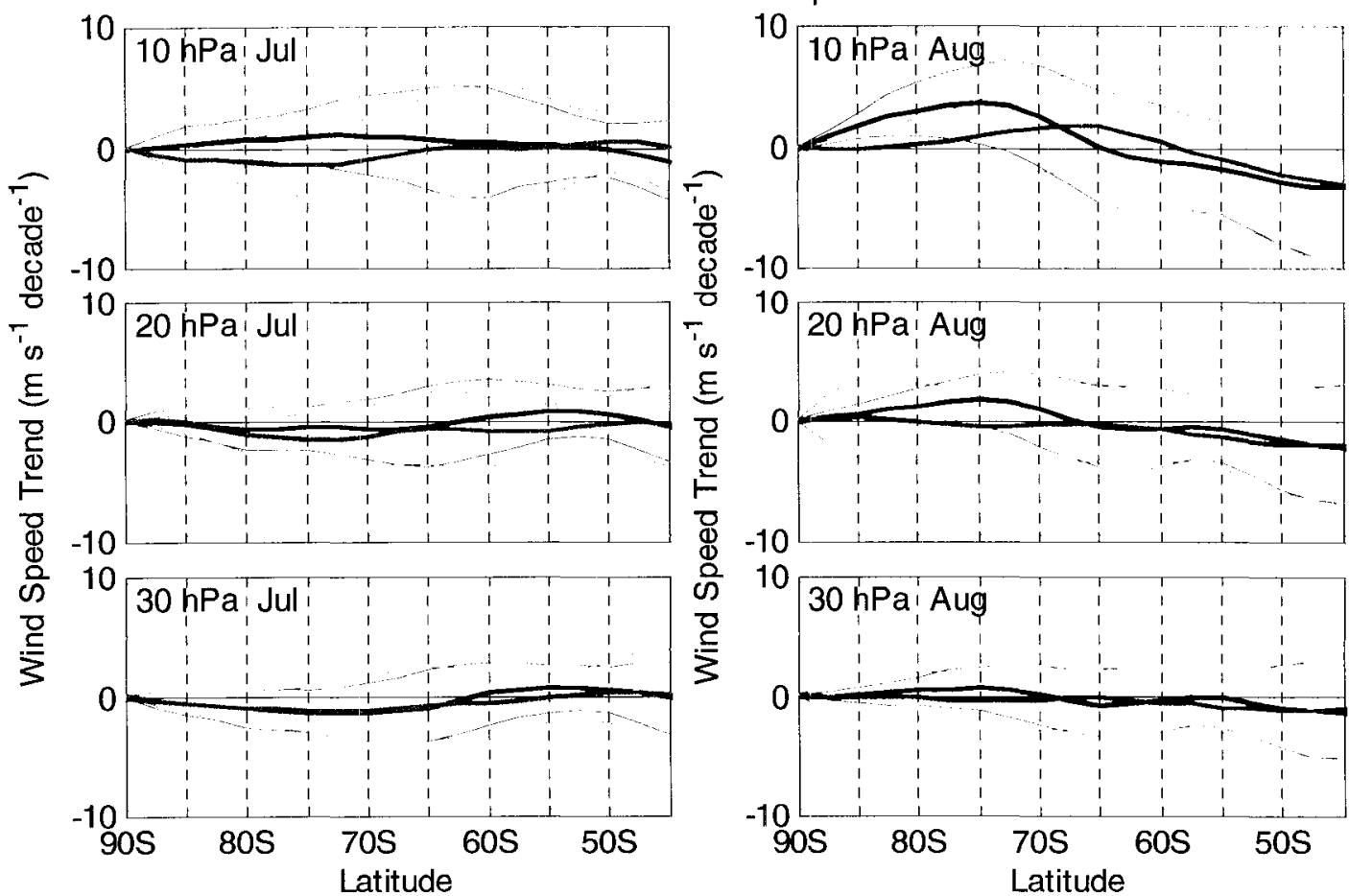

Fig. 5-64. Same as Fig. 5-63 except July and August. 


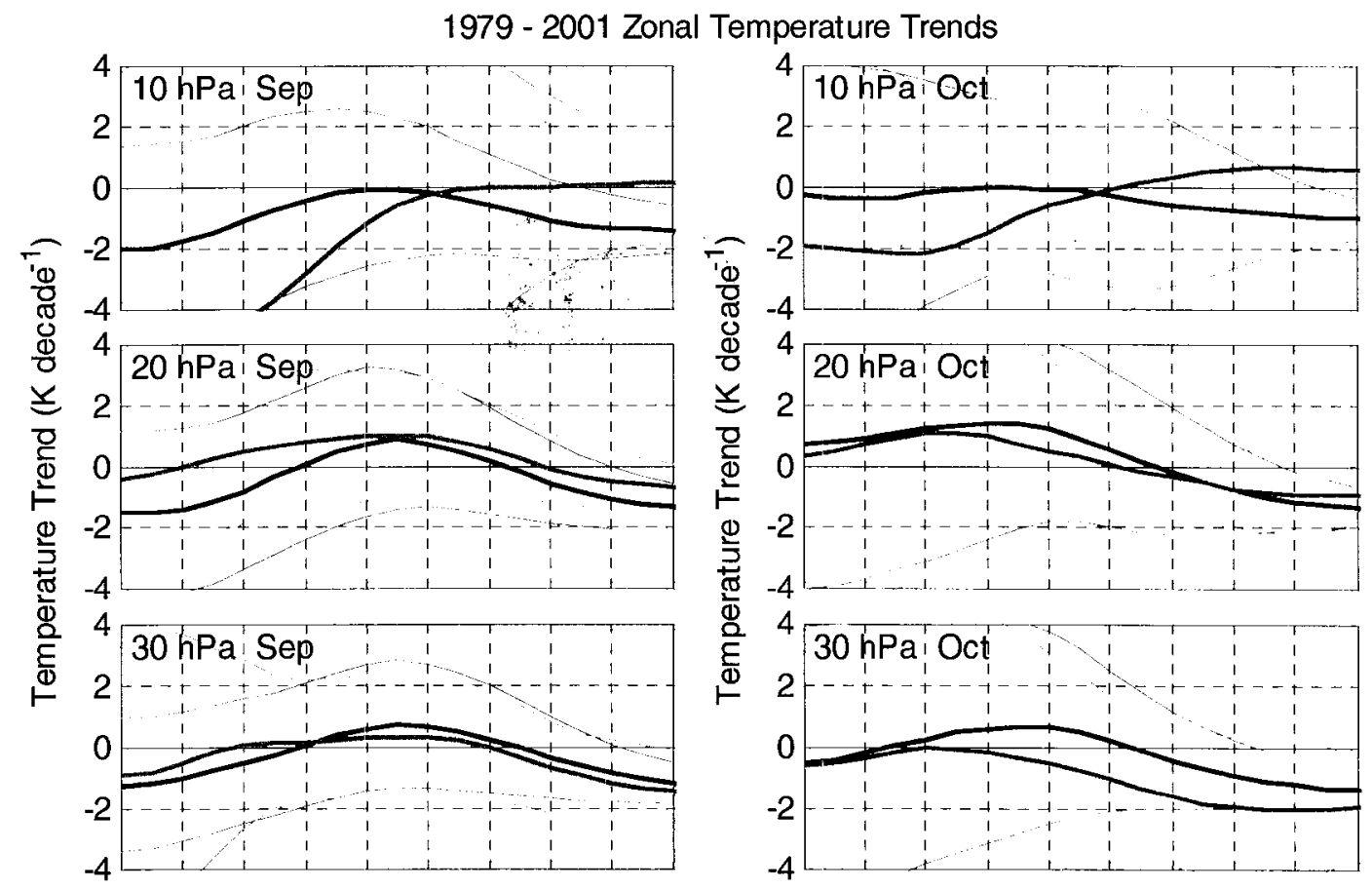

1979 - 2001 Zonal Wind Speed Trends
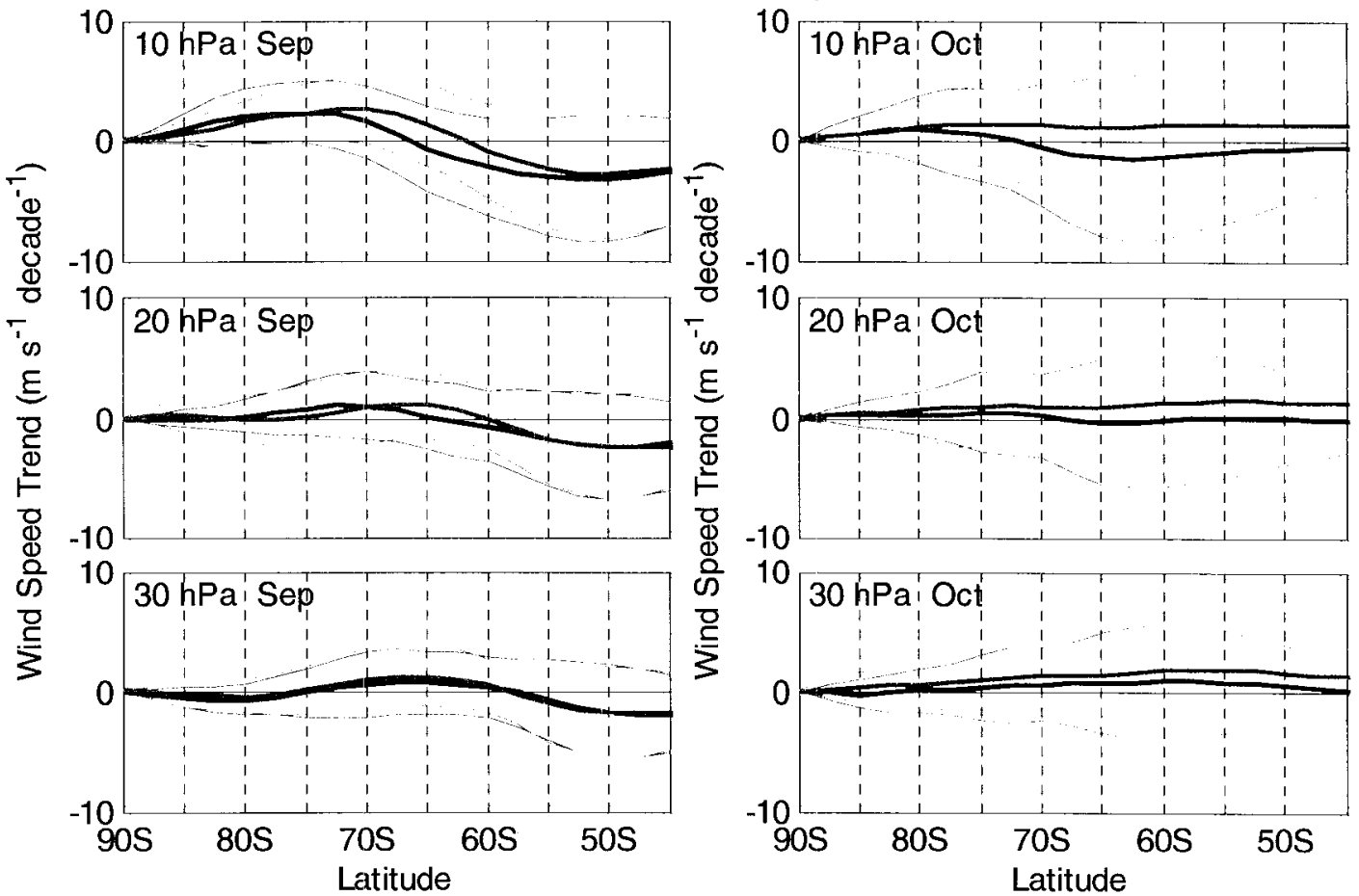

Fig. 5-65. Same as Fig. 5-63 except September and October. 
Figures 5-66 and 5-67 summarize on single pages Northern and Southern Hemisphere 1979-2001 temperature trends, respectively. Figures 5-68 and 5-69 summarize on single pages Northern and Southern Hemisphere 1979-2001 zonal wind speed trends, respectively. With the exception of October and March, (Northern Hemisphere), and May and October (Southern Hemisphere) each of the monthly subplots is repeated from corresponding subplots of Figs. 5-44 through 5-51 (Northern Hemisphere) and Figs. 5-55 through 5-62 (Southern Hemisphere). ERA-40 temperature trend subplots are included for completeness and for comparison with corresponding NCEP1 trends; they are not believed to be accurate. 

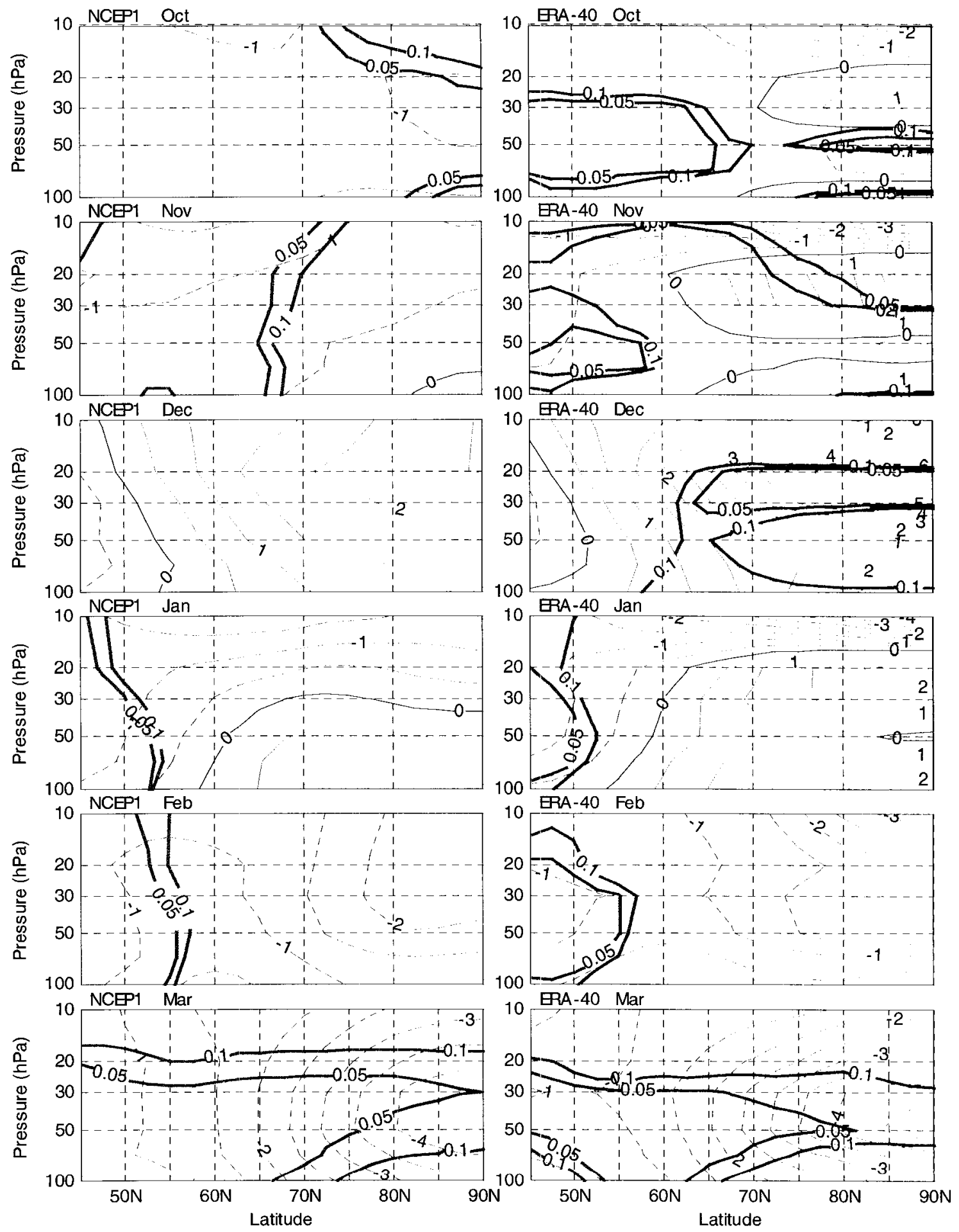

Fig. 5-66. NH monthly- and zonally-averaged 1979-2001 temperature trends $\left(\mathrm{m} \mathrm{s}^{-1}\right.$ decade $\left.{ }^{-1}\right) \quad 5 \%$ and $10 \%$ statistical significance limits shown in green: [lon: mean of $0^{\circ}-357.5^{\circ}$, lat: $45^{\circ} \mathrm{N}-90^{\circ} \mathrm{N}$, press: $100-10 \mathrm{hPa}$, time: 1979-2001]. 

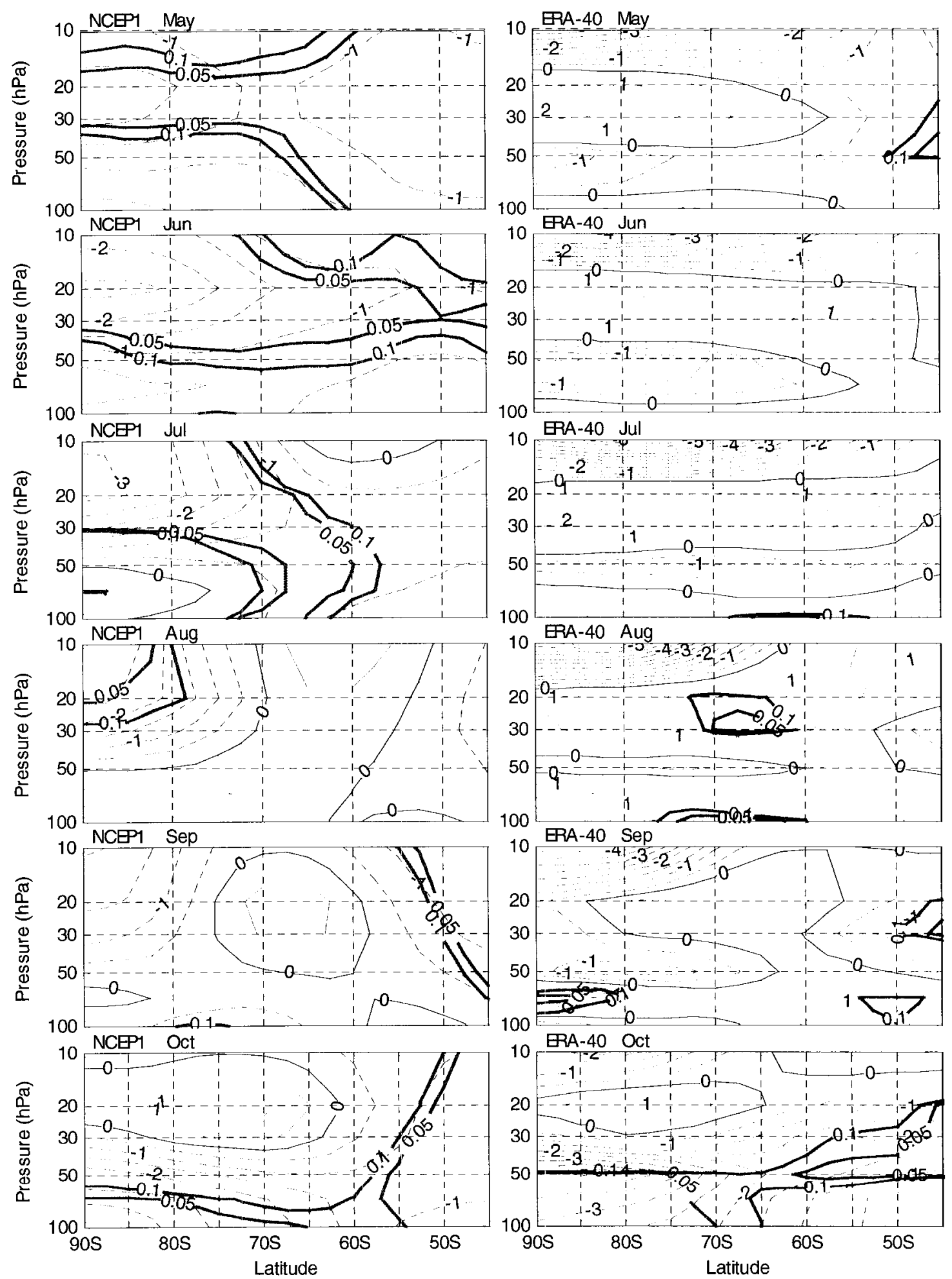

Fig. 5-67. Same as Fig. 5-66 except Southern Hemisphere with lat: $90^{\circ} \mathrm{S}-45^{\circ} \mathrm{S}$. 

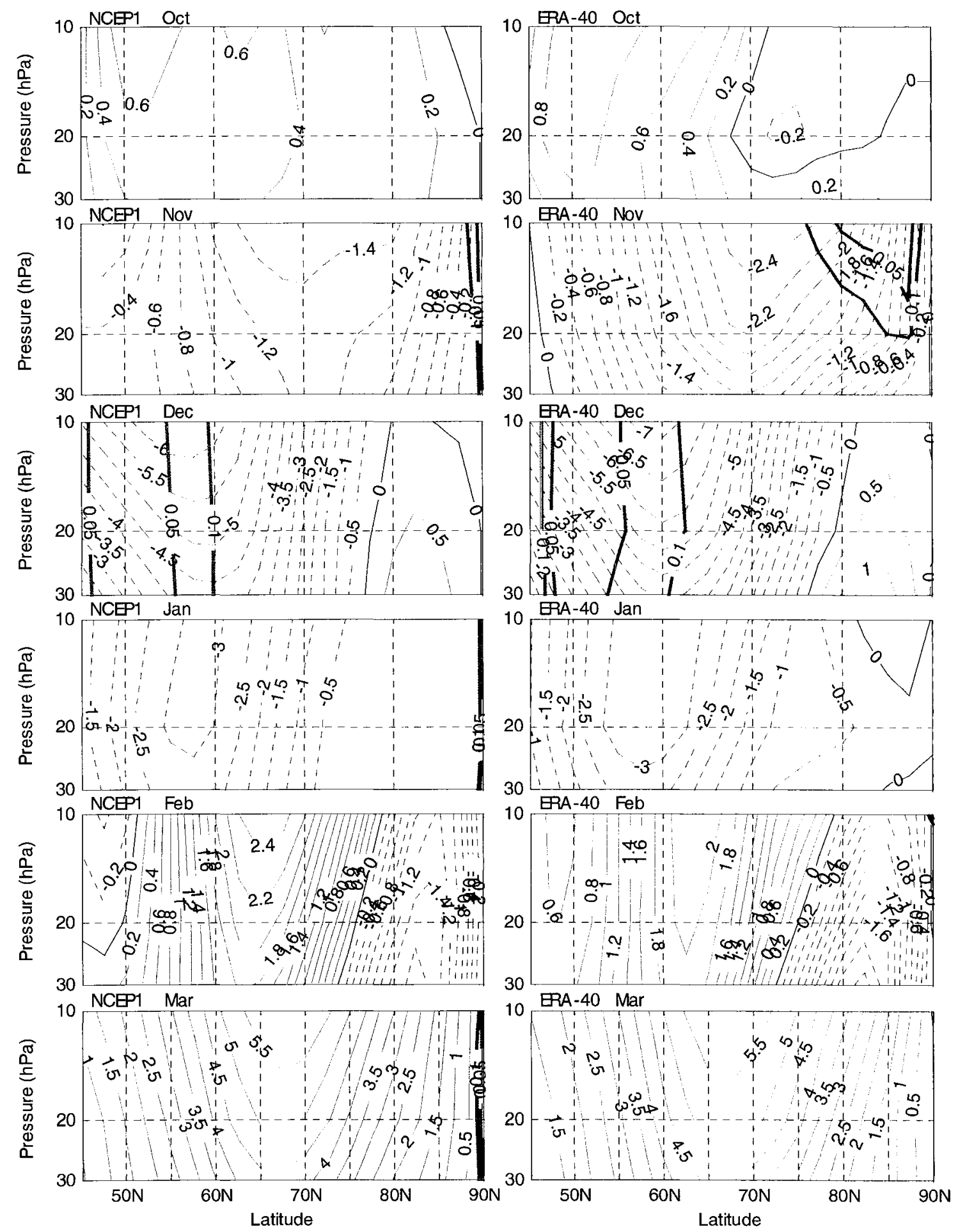

Fig. 5-68. NH monthly-and zonally-averaged 1979-2001 zonal wind speed trends ( $\mathrm{m} \mathrm{s}^{-1}$ decade $\left.{ }^{-1}\right) \quad(5 \%$ and $10 \%$ statistical significance limits shown in green): [lon: mean of $0^{\circ}-357.5^{\circ}, \quad$ lat: $45^{\circ} \mathrm{N}-90^{\circ} \mathrm{N}$, press: $30-10 \mathrm{hPa}$, time: 1979-2001]. 

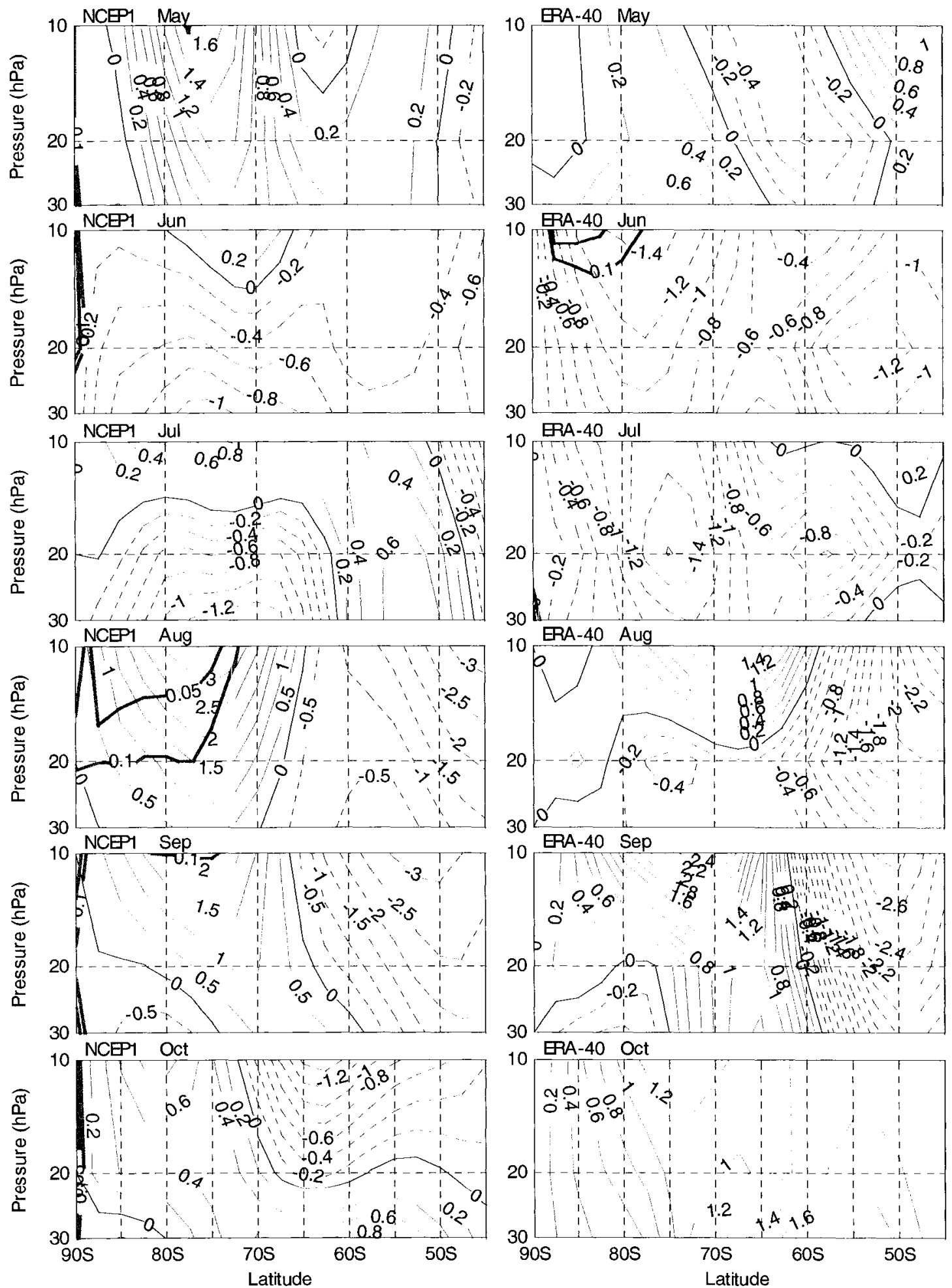

Fig. 5-69. Same as Fig. 5-68 except Southern Hemisphere with lat: $90^{\circ} \mathrm{S}-45^{\circ} \mathrm{S}$. 


\subsection{Quasi-biennial oscillation}

The $\mathrm{QBO}$ is a very active research topic. Baldwin et al. (2001) provide a comprehensive summary of this subject through 2000. Section 3.2 is an overview of this major dynamic characteristic of the stratosphere.

The Institut für Meteorologie of the Freie Universität Berlin, Germany (FUB), as one aspect of their compilation of daily stratospheric charts (Section 2.3.2), has tabulated the daily radiosonde observations of zonal wind speed at selected stations near the Equator since 1957 . Daily values have been averaged by FUB to form monthly mean zonal wind speeds at $70,50,40,30,20,15$, and $10 \mathrm{hPa}$. Observations from three radiosonde stations,

- Canton Island $2^{\circ} 46^{\prime} \mathrm{S}, \quad 171^{\circ} 43^{\prime} \mathrm{W}$ - 1953 until closed in 1967

- Gan/Maledive Islands $\quad 0^{\circ} 41^{\prime} \mathrm{S}, \quad 73^{\circ} \quad 9^{\prime} \mathrm{E} \quad-1967$ until closed in 1975

- Singapore $1^{\circ} 22^{\prime} \mathrm{N}, \quad 103^{\circ} 55^{\prime} \mathrm{E} \quad-$ since 1976

have been combined to create an equatorial data set from 1953 to the present. Naujokat (1986) is the universal reference for the original FUB data set. The online source of QBO data for this thesis is documented in Appendix B.

The single-location (latitude and longitude) radiosonde wind speed profiles of the FUB data set are appropriate comparison references for longitudinally-averaged reanalysis winds since longitudinal variations in the $\mathrm{QBO}$ wind phases are generally minimal (Hamilton et al. 2004). Figures 5-30 through 5-35 demonstrate that there are minimal longitudinal variations in the QBO zonal wind speeds in both NCEP1 and ERA-40 reanalyses. 
Figures 5-70 and 5-71 plot the equatorial winds at the upper four levels considered in this thesis. The combined rms values of the four pressure levels plotted and $70 \mathrm{hPa}$ is also graphed. From these two figures, it is apparent that ERA-40 tracks the FUB QBO much more closely than does NCEP1. 


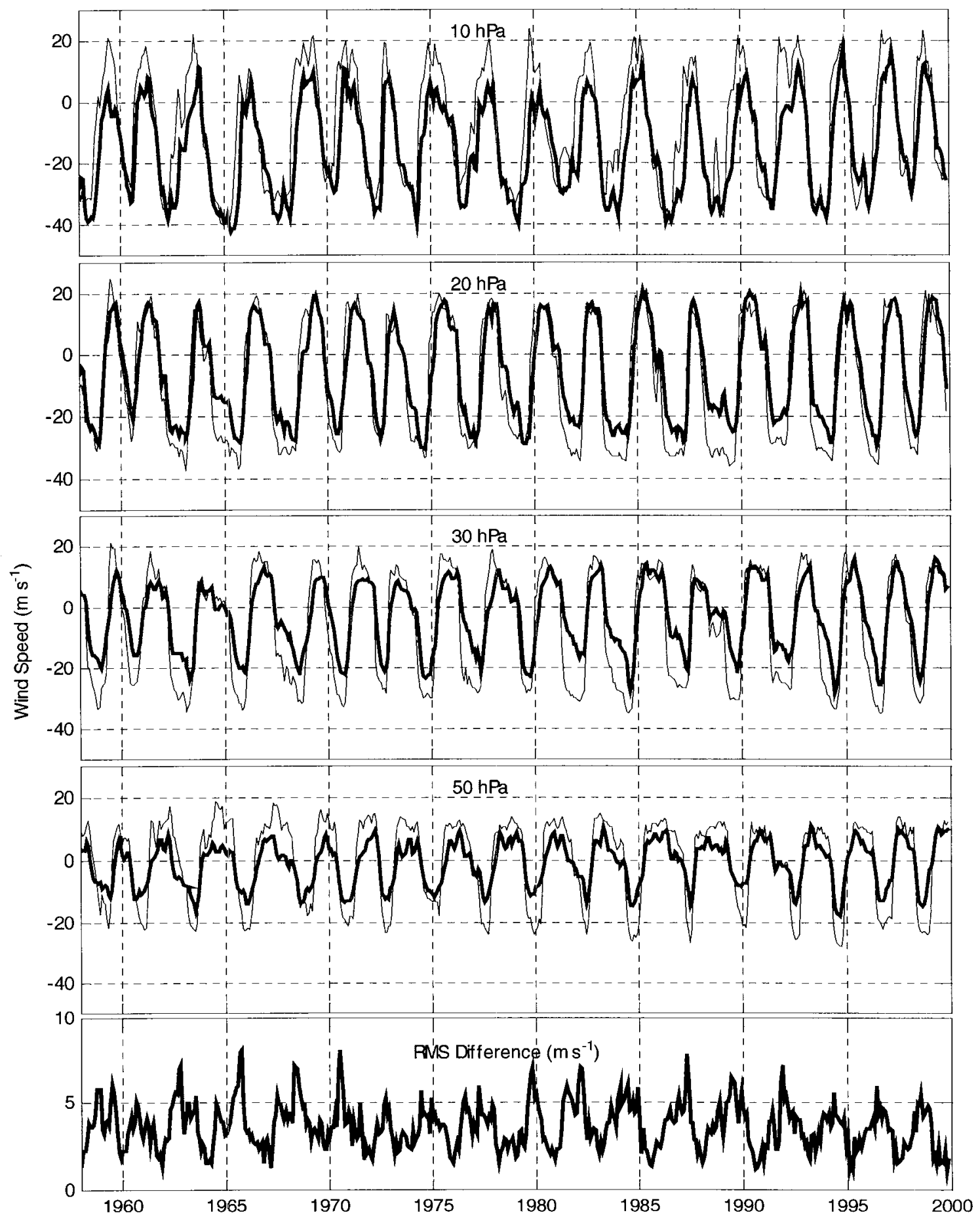

Fig. 5-70. NCEP1 monthly-and zonally-averaged equatorial zonal wind speeds (bold blue line) and FUB (light black line) equatorial data set. Rms difference computed for pressure levels shown and $70 \mathrm{hPa}$ (not shown). [NCEP1: lon: mean of $0^{\circ}-357.5^{\circ}$; lat: $0^{\circ}$, press: as noted, time: Jan 1958-Dec 1999; FUB: see text]. 


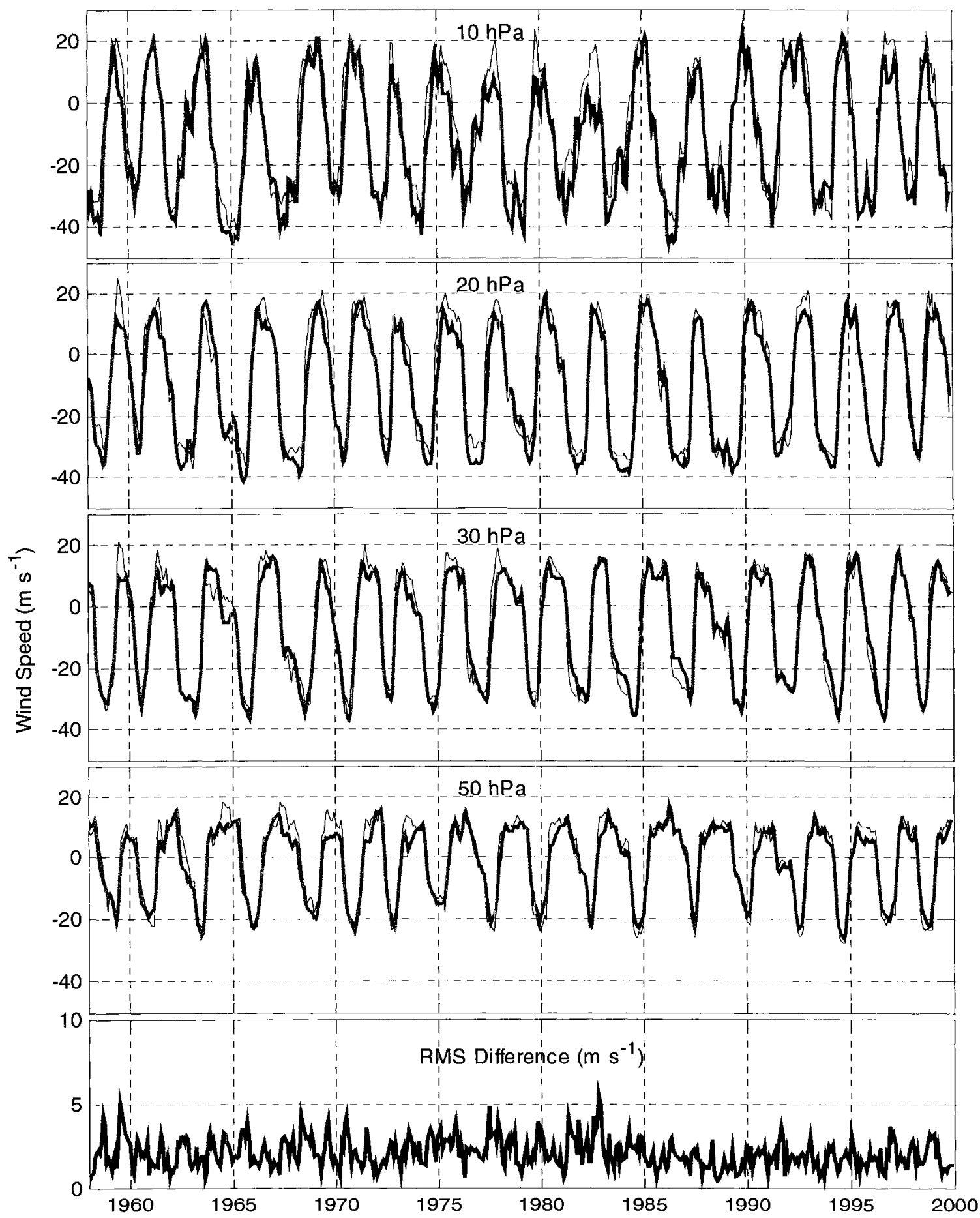

Fig. 5-71. ERA-40 monthly-and zonally-averaged equatorial zonal wind speeds (bold green line) and FUB (light black line) equatorial data set. Rms difference computed for pressure levels shown and $70 \mathrm{hPa}$ (not shown). [ERA-40: lon: mean of $0^{\circ}-357.5^{\circ}$; lat: $0^{\circ}$, press: as noted, time: Jan 1958-Dec 1999; FUB: see text]. 
The "finger print" plots of Figs. 5-72 and 5-73 confirm the SPARC estimate of QBO latitudinal width of $20^{\circ}$ about the Equator (Randel et al. 2002, 2004). Maximum standard deviations taken from these figures and summarized in Fig. 5-5 show that ERA-40 values are generally 15 to $20 \%$ larger than those of NCEP1.

Table 5-5. Ratio of ERA-40 to NCEP1 maximum standard deviations.

\begin{tabular}{cccc} 
Season & \multicolumn{2}{c}{$\begin{array}{c}\text { Maximum } \\
\text { Standard Deviation } \\
\left(\mathrm{m} \mathrm{s}^{-1}\right)\end{array}$} & Ratio \\
& NCEP1 & ERA-40 & ERA-40/ NCEP1 \\
Dec Jan Feb & 15.8 & 19.0 & 1.20 \\
Mar Apr May & 15.4 & 18.3 & 1.19 \\
Jun Jul Aug & 15.9 & 17.9 & 1.13 \\
Sep Oct Nov & 15.1 & 17.6 & 1.17
\end{tabular}



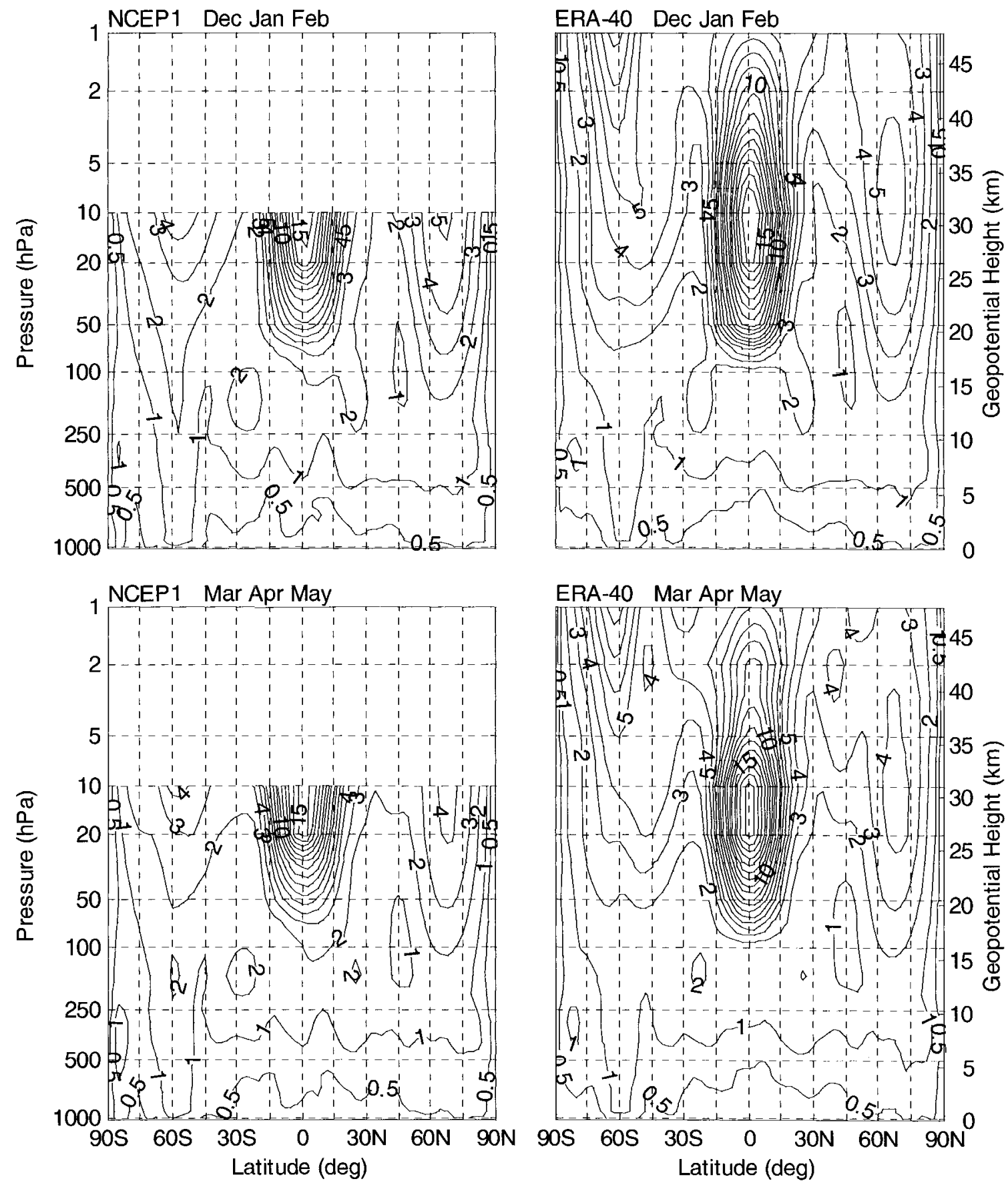

Fig. 5-72. Standard deviation of monthly-and zonally-averaged zonal wind speeds for DJF and MAM seasons $\left(\mathrm{m} \mathrm{s}^{-1}\right)$ all variance less than 13 months removed: [lon: mean of $0^{\circ}-357.5^{\circ}$, lat: $90^{\circ} \mathrm{S}-90^{\circ} \mathrm{N}$, press: 100-1 hPa, time: Jan 1958-Dec 2001]. 

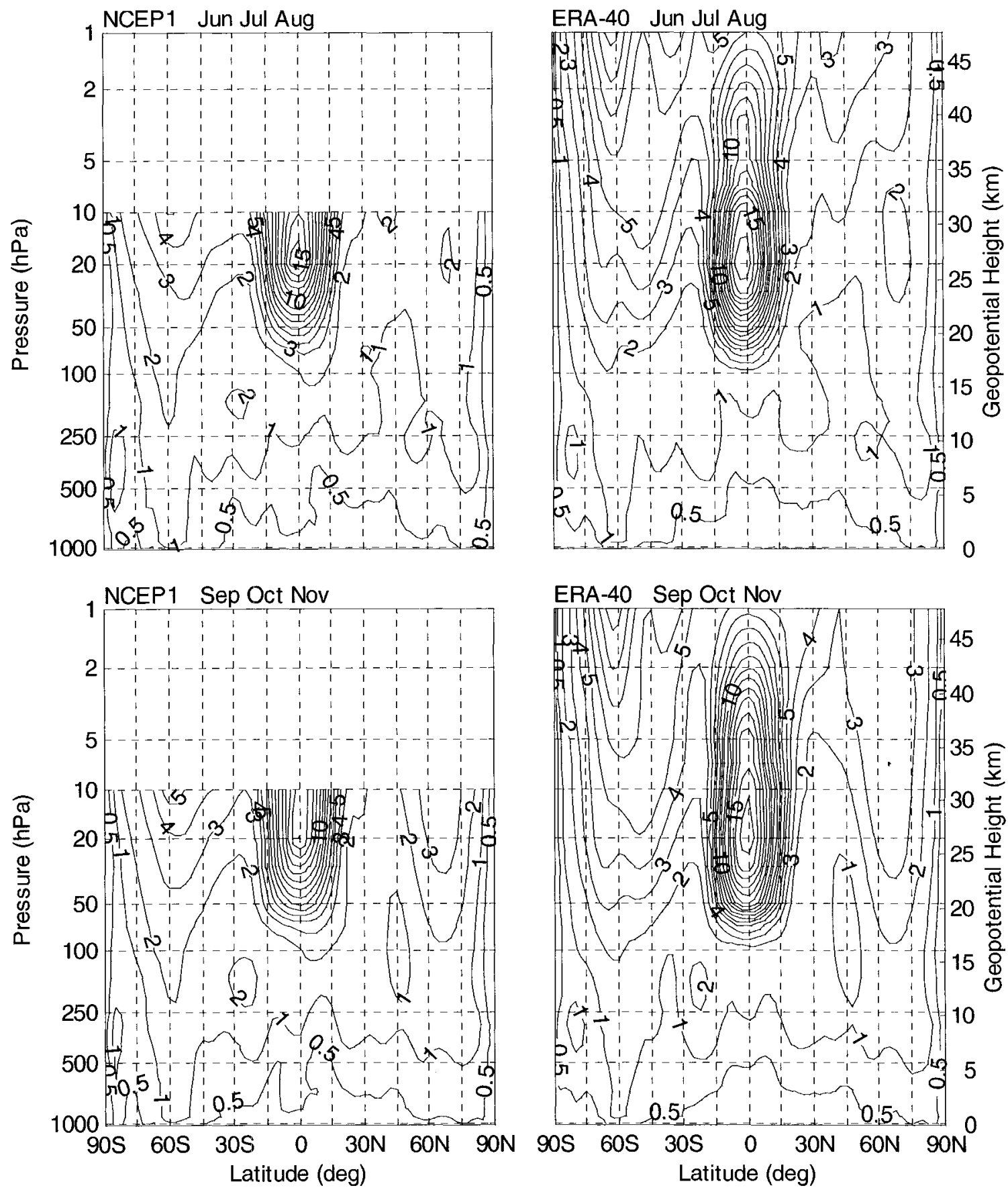

Fig. 5-73. Standard deviation of monthly-and zonally-averaged zonal wind speeds for JJA and SON seasons $\left(\mathrm{m} \mathrm{s}^{-1}\right)$ all variance less than 13 months removed: [lon: mean of $0^{\circ}-357.5^{\circ}$, lat: $90^{\circ} \mathrm{S}-90^{\circ} \mathrm{N}$, press: 100-1 hPa, time: Jan 1958-Dec 2001$]$. 
Pascoe et al. (2005) list the values of the QBO period determined by various researchers, including themselves:

- 27.7 months Naujokat (1986)

- 28.2 months Baldwin et al. (2001)

- $28.4 \pm 1.98$ months Pascoe et al. (2005)

The spectral analysis of Fig. 5-74 shows a very consistent maximum in QBO wind speed variance at 26.53 months for NCEP1, ERA-40, and FUB at 10, 20, 30, and $50 \mathrm{hPa}$, with the broadness of the peak indicative of significant variation in the QBO period from 23 to 30 months. This analysis captures the variance contained in 504 monthly wind speed samples; Pasco et al. (2005) used only the 37 zero-crossings of wind speed. The spectral peak in Fig. 5-74 is 26.53 months; due to FFT quantization its range of uncertainty is +0.74 to -0.66 months. Thus there is a 0.85 -month overlap between the lower bound of Pasco et al. (2005) and the upper bound of the spectral analysis. Although at periods less than 13 months (approximately half the QBO period), noticeable differences exist between the reanalysis magnitudes and those of the FUB data (the higher frequency differences in Figs. 5-70 and 5-71); however, the spectra above 13 months essentially differ only in magnitude. Other periods of increased variance: 11.7 months, the annual cycle, and 5.9 months, the semi-annual cycle or oscillation, differ from 12 and 6 months, respectively, due to FFT quantization. 


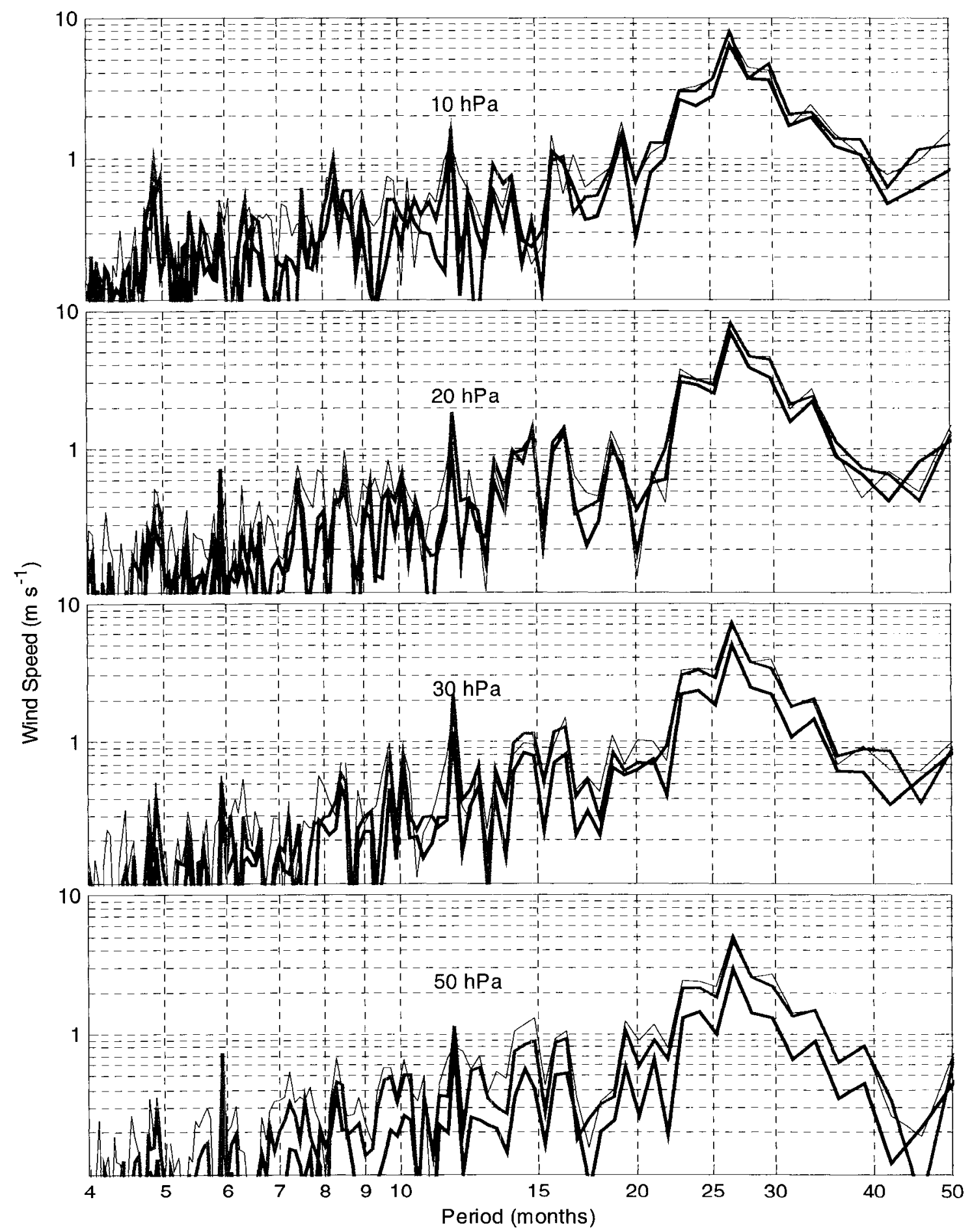

Fig. 5-74. Spectral analysis of NCEPI (bold blue) and ERA-40 (bold green) equatorial zonal wind speeds and the FUB (black) equatorial data set. [NCEP1 and ERA-40: lon: mean of $0^{\circ}-357.5^{\circ}$; $\quad$ lat: $0^{\circ}$, press: as stated, time: monthly means from Jan 1958Dec 1999; FUB: see text]. 
The phase plots of Fig. 5-75 illustrate the phase lag as well as the amplitude attenuation of the NCEP1 QBO monthly- and zonally-averaged zonal wind speed with respect to those of the FUB (reference) data set. Each plot is a trajectory of paired points at a common time, the first from a reanalysis time series and the second from the FUB time series for the indicated pressure level. Each point in a plot displays the reanalysis wind in the vertical and the FUB wind in the horizontal. If the two time series were identical, the resulting plot would be the bold black line in each plot. Since Figs. 5-70 and 5-71 demonstrate they are not, the phase plots illustrate the manner in which they differ. The quasi-elliptical trajectories in the 10, 20, and $30 \mathrm{hPa}$ NCEP1 phase plots indicate a significant phase lag of the NCEP1 QBO time series with respect to that of the FUB time series. At $50 \mathrm{hPa}$, the NCEP1 phase plot is essentially in phase but its amplitude is significantly smaller that that of FUB. The ERA-40 trajectories demonstrate minimal phase shift and attenuation at all pressure levels. 

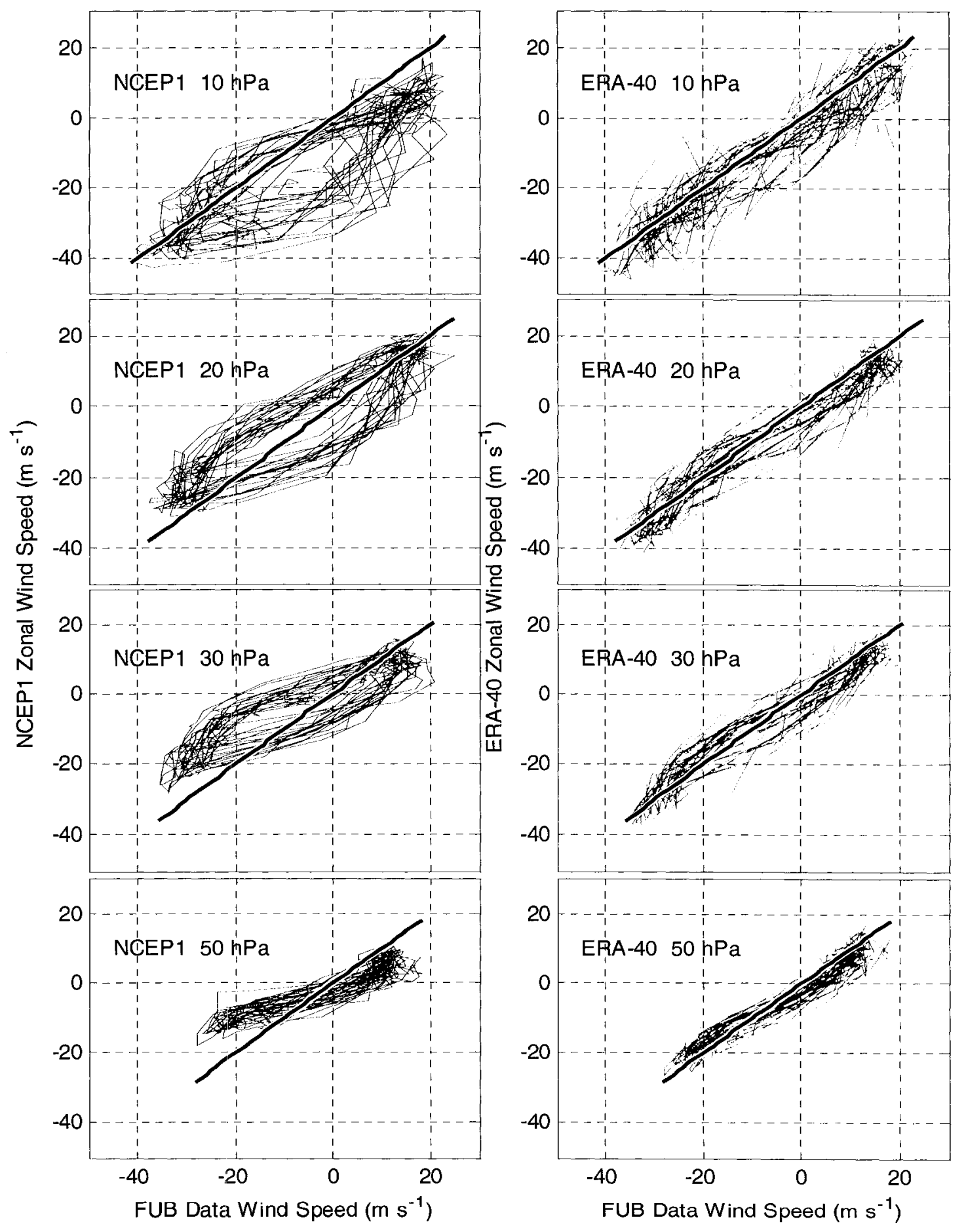

Fig. 5-75. Phase plots of NCEPI and ERA-40 equatorial zonal wind speeds plotted with respect to $F U B$ equatorial data set winds. [lon: mean of $0^{\circ}-357.5^{\circ}$; lat: $0^{\circ}$, press: as stated, time: Jan 1958-Dec 1999]. 
Figures 5-70 through 5-75 graphically illustrate from the different perspectives of the time, variance, spectral, and phase domains that the ERA-40 reanalysis represents the equatorial QBO much more accurately than does NCEP1.

This was also the conclusion of Randel et al. (2004) in the SPARC intercomparison, which included NCEP1 and ERA-40 in its set of climatologies.

The quasi-biennial oscillation (QBO) dominates interannual variability for zonal wind speeds in the Tropics, but there are significant differences in magnitude derived from various datasets. ... ERA-40 appears best at capturing the strength of the westerly phase at $10 \mathrm{hPa}$.

With reference to their Fig. 14,

for altitudes - 24-37 km ... ERA-40 exhibits the largest QBO amplitude (in best agreement with the radiosonde climatology and Singapore data).

... The ERA-40, ERA-15, METO, and NCEP datasets show approximately similar amplitudes between 70 and $30 \mathrm{hPa}$, whereas above $30 \mathrm{hPa}$ there are larger differences. Only ERA-40 approaches the Singapore and rocketsonde amplitudes over $20-10 \mathrm{hPa}$.

\subsection{Summary}

The general pattern of NCEP1 and ERA-40 stratospheric zonal wind speed differences is similar to that of the temperature differences of Section 4; differences between these two variables in the two reanalyses increase both with increasing height and with increasing southerly latitude. However, maximum zonal wind speed differences are not found at the same pressure level - latitude locations as the maximum temperature differences of Section 4. Unlike the maxima of temperature differences, which generally occur at $10 \mathrm{hPa}$ at the poles, maximum extratropical differences in zonal wind speed are usually found at $10 \mathrm{hPa}, 60^{\circ} \mathrm{S}-80^{\circ} \mathrm{S}$, the latitudinal locations of the Southern Hemisphere 
winter polar vortex. Secondary maxima are found in the vicinity of the Northern Hemisphere winter polar vortex.

However, tropical regions $\left(30^{\circ} \mathrm{S}\right.$ to $\left.30^{\circ} \mathrm{N}\right)$ of pressure level vs. latitude plots of monthly- and zonally-averaged zonal wind speed differences contain significant and often dominant structures resulting from differing characterizations of the QBO by the two reanalyses. These differences in QBO characterization appear in all the statistical measures (mean, standard deviation, rms, and maxima) used in this thesis. They are discussed and illustrated in Randel et al. $(2002,2004)$ and in Section 5.5 of this thesis, all of which consistently demonstrate that ERA-40 represents the QBO as defined by FUB Singapore data much more accurately than does NCEP1, which under-predicts the amplitude of the QBO and adds an observable and measurable phase shift. There is no evidence of ERA-40's “oscillatory vertical structure in temperature, especially large over Antarctica" (Randel et al. 2002, 2004) reflected in zonal wind speeds. Figures. 5-16 through 5-18 and 5-30 through 5-35 demonstrate that the departure of QBO winds from zonal symmetry in both NCEP1 and ERA-40 reanalyses is minimal, especially after 1979. These results validate the almost universal assumption of zonal symmetry in QBO research.

Time series of the two reanalyses are closely matched, both in absolute magnitude and in high-frequency dynamics. The only significant differences in magnitude are found in equatorial subplots from $70 \mathrm{hPa}$ to $10 \mathrm{hPa}$ (Figs. 5-20 through 5-24), where the difference in these low frequency magnitudes reflects the differing characterizations of the QBO. 
Figures 5-38 and 5-39 show that monthly- and zonally-averaged Northern and Southern Hemisphere peak polar vortex winds at $10 \mathrm{hPa}$ are approximately $40 \pm 20 \mathrm{~m} \mathrm{~s}^{-1}$ and $90 \pm 20 \mathrm{~m} \mathrm{~s}^{-1}$, respectively. These peak winds occur in the vicinity of $65^{\circ} \mathrm{N}$ and $60^{\circ} \mathrm{S}$ during the hemisphere winter. Given these magnitudes, the relatively small mean, standard deviation, and rms differences displayed in this section demonstrate remarkably similar characterizations of extratropical zonal wind speed by the two reanalyses. This analysis produced two unexpected results:

- Maximum Northern Hemisphere polar vortex wind speeds at $10 \mathrm{hPa}$ occur at 64 $65^{\circ} \mathrm{N}$ with a standard deviation of $3^{\circ}$ latitude, not the often-used $60^{\circ} \mathrm{N}$

- Maximum Southern Hemisphere polar vortex winds speeds at $10 \mathrm{hPa}$ occur in August with the three peak months July, August, and September, not the often-used June, July, and August (JJA)

At the mean locations of maximum polar vortex zonal wind speed during each Northern Hemisphere winter month, Fig. 5-64 shows that zonal wind speeds increase during the 1979-2001 period in both reanalyses in the months of October, February, and March and decrease in the months of November, December, and January. In a similar analysis (Fig. 5-65) in the Southern Hemisphere, zonal wind speeds decrease from May through October in at least one reanalysis and in July and October, the trends of the two reanalyses have opposite signs. Northern Hemisphere zonal wind speed trends are generally much larger than those in the Southern Hemisphere. Figure 5-42 plots the trends, determined by linear regression, and their confidence intervals. Because of the small (23-year) sample size and the large variance in reanalysis data, 1979-2001 trends in maximum polar vortex zonal wind speed listed above and plotted in Fig. 5-42 are generally statistically insignificant. These trends are generally consistent between 
NCEP1 and ERA-40, but their difference is statistically significant only in the Northern Hemisphere winter months of November and December.

An examination of polar vortex zonal wind speed trends in the area of $30-10 \mathrm{hPa}$ and $45^{\circ} \mathrm{N}-90^{\circ} \mathrm{N}$ and $45^{\circ} \mathrm{S}-90^{\circ} \mathrm{S}$ produced results that might be expected from those shown in Fig. 5-42. In the Northern Hemisphere the months of November (Fig. 5-45), December (Fig. 5-47), and January (Fig. 5-49) exhibited negative trends throughout the area; October (Fig. 5-52) and March (Fig. 5-54) positive trends throughout. Negative trends are present in February (Fig. 5-51) north of $75^{\circ} \mathrm{N}$, an area that includes the core of the Northern polar vortex, and positive south of this latitude. There is much more structure in the Southern Hemisphere polar vortex zonal wind speed trends. No simple catalog of the results shown in Figs. 5-56, 5-58, 5-60, and 5-62 through 5-65 is possible.

Examination of these reanalysis results leads to the conclusion that no decisive monthly polar vortex zonal wind speed trend exists in either hemisphere. The Northern Hemisphere area-wide negative trends of November, December, and January are the most consistent but even they are not statistically significant.

Finally, agreement between NCEP1 and ERA-40 zonal wind speeds is good in 1958-78 and excellent in 1979-2001 except in the Tropics (QBO). For lower stratospheric climatology, either reanalysis is acceptable. ERA-40's characterization of the QBO is much closer to FUB radiosonde data than is NCEP1, so although most GCMs do not spontaneously generate a QBO, this fidelity is of value in evaluating QBO simulations in those GCM's that do. 


\subsection{MERIDIONAL WIND SPEED COMPARISON}

In Section 2.1 meridional wind was identified as a "Type B" variable, one that is influenced by both observation and model during the reanalysis process, rather than primarily influenced by observational data as are temperature and extratropical zonal wind speed (Kistler et al. 2001). It is the influence of the "intrinsic variability" (Sterl 2004) of the NCEP1 and ERA-40 forecast models and assimilation systems that results in the differences in their characterization of the meridional wind speed as demonstrated in this section.

In this section numerous figures illustrate, and accompanying discussions note, that unusual discontinuities and transients in the results of both NCEP1 and ERA-40 reanalyses are present during 1975-79. With one exception, equatorial NCEP1 at $100 \mathrm{hPa}$, these possible anomalies are all found in ERA-40 results. The well-known NCEP1 1979 equatorial $100 \mathrm{hPa}$ temperature discontinuity is discussed by Huesmann and Hitchman (2003). VTPR system data was assimilated into the NCEP1 reanalysis during 1975-78 (Bromwich and Fogt 2004) and with extensive pre-processing, into ERA-40 (Li et al. 1999) beginning in November 1972. This pre-processing phase appears to have deleted some unusable data for ERA-40 from January through August 1976 (Li et al. 1999). Simmons (2006b) identifies ERA-40 temperature assimilation errors "stemming from erroneous bias-correction of VTPR data from NOAA-3 in 1975 and 1976."

Throughout Section 6, only total meridional wind speed is considered; no mean values are removed to form anomalies. While small in magnitude, meridional wind 
speeds are significant in mass exchange and chemical transport (Pawson and Fiorino 1998a).

\subsection{Height - latitude structure of monthly meridional wind speeds}

Figures 6-1 and 6-2 demonstrate an elementary climatology of monthly meridional wind speeds for the NCEP1 and ERA-40 reanalyses, respectively. They show mean and standard deviation contours developed from the seasonal time series of the 23-year period from January 1979 through December 2001. Figure 6-3 is a chronology of NCEP1 and ERA-40 mean meridional wind speeds for four time periods from 1958 through 2001.

Comparison of Figs. 6-1 and 6-2 illustrates the dramatic difference in the NCEP1 and ERA-40 reanalyses of meridional wind speeds. In Fig. 6-1 NCEP1 is vertically stratified with flows in one direction over a large latitudinal extent at all levels considered in this thesis. Most noticeable and surprising is that for a large portion of the year, mean meridional flow is northward at all pressure levels at latitudes from $15^{\circ} \mathrm{S}$ to $60^{\circ} \mathrm{N}$ and for the remainder of the year at latitudes from $15^{\circ} \mathrm{N}$ to the North Pole. Maximum meridional northerly flow occurs at the Equator at $100 \mathrm{hPa}$ at all times of the year, especially December - February, with a minimum during June - August. Small vertically oriented regions of southerly flow are seen to exist southward of this northerly flow region throughout the year, and northward for three of four seasons of the year. Standard deviations are significant only above $30 \mathrm{hPa}$ and at the Equator at $100 \mathrm{hPa}$; here they are of the same order of magnitude as the mean values. The year-long northerly flow implies 
a mean mass transport from the Southern Hemisphere at all levels considered, a condition that is not in "agreement with expectations" (Pawson and Fiorino 1998a).

The ERA-40 seasonal climatology of Fig. 6-2 possesses significantly more complex structure than that of NCEP1 but is also essentially vertical in nature with no evidence of horizontal stratification. In DJF there is an extensive northerly flow in the Northern Hemisphere and a southerly flow south of $15^{\circ}-30^{\circ} \mathrm{S}$. During the remainder of the year a "checkerboard" pattern of northerly and southerly flow regions exists, about $30^{\circ}$ wide in latitude and vertically separated at $30 \mathrm{hPa}$. If the meridional flow is northerly above $30 \mathrm{hPa}$ it is typically southerly below. There are permanent regions of northerly flow at $15^{\circ}-30^{\circ} \mathrm{N}$ below $30 \mathrm{hPa}$ and $10^{\circ} \mathrm{S}-10^{\circ} \mathrm{N}$ above $30 \mathrm{hPa}$. A southerly flow always exists south of the Equator at $100 \mathrm{hPa}$. Northerly flow is present north of the Equator from Northern Hemisphere fall through spring and especially strong during DJF. As in the case of the NCEP1 reanalysis, standard deviations are of the same order of magnitude as the mean flows.

The side-by-side comparison of four time periods $(10,11,11$, and 12 years) of NCEP1 and ERA-40 meridional wind speeds in Fig. 6-3 further illustrates the differences between the two reanalyses. A plot of NCEP1 meridional wind speeds from 1979-93 (not shown) duplicates Fig. 14(a) of Pawson and Fiorino (1998a).

NCEP1 is more temporally consistent than ERA-40, with alternating northerly and southerly vertically stratified regions and, with the exception of increased northerly flow at the Equator at $100 \mathrm{hPa}$ after 1979 , is seemingly unaffected by the beginning of the modern satellite era. A symmetry exists about the zero wind speed at $\sim 15^{\circ} \mathrm{N}$ with the 
primary feature the large region of northerly flow from $15^{\circ} \mathrm{S}$ to $45^{\circ} \mathrm{N}$, somewhat balanced by a southerly flow from $15^{\circ} \mathrm{S}$ to $45^{\circ} \mathrm{S}$. Also, southerly flow from $60^{\circ} \mathrm{N}$ to $75^{\circ} \mathrm{N}$ and 50 to $10 \mathrm{hPa}$ is balanced in the southern hemisphere by a similar region of northerly flow. Wind speeds at heights above $70 \mathrm{hPa}$ are never more than $0.25 \mathrm{~m} \mathrm{~s}^{-1}$ northerly. Very localized maxima of $0.75 \mathrm{~m} \mathrm{~s}^{-1}$ northerly are present at the Equator at $100 \mathrm{hPa}$ after 1979 .

There is minimal symmetry in the sequence of ERA-40 subplots in Fig. 6-3. The 10- to 12-year mean flow patterns generally are consistent with one another and with the less complex patterns of the NCEP1 sequence. There is a region of northerly flow, broader and more connected in some periods than others, north of the equator; a region of southerly flow, again more extensive in some periods than others, to the south; and upper level subpolar regions of flow with direction opposite that of latitude. As with NCEP1, strongest wind speeds at heights below $70 \mathrm{hPa}$ are at the Equator $\left(0.71 \mathrm{~m} \mathrm{~s}^{-1}\right.$ southerly $)$ but maximum speeds here are consistently at $10 \mathrm{hPa}$ in the southern extratropics (0.92 $\mathrm{m} \mathrm{s}^{-1}$ southerly). In both reanalyses, minimum variance occurs at $70 \mathrm{hPa}$, increasing with increasing height.

Figure 6-4 displays the mean, standard deviation, rms, and maxima of NCEP1 minus ERA-40 meridional wind speeds over the period from January 1958 through December 2001. Similar figures for component periods of 1958 through 1978 and 1979 through 2001 (not shown) exhibit similar characteristics. The dominant differences are the ERA-40 southerly flow in the vicinity of $30^{\circ} \mathrm{N}$ and $30^{\circ} \mathrm{S}$ between 10 and $30 \mathrm{hPa}$, which is not present in the NCEP1 reanalysis, and the southerly flow south of the Equator that again is not present in the NCEP1 reanalysis. Figure 6-4 again quantitatively 
confirms that standard deviations are of the same order of magnitude, and in some regions larger than, the mean meridional wind speed. 

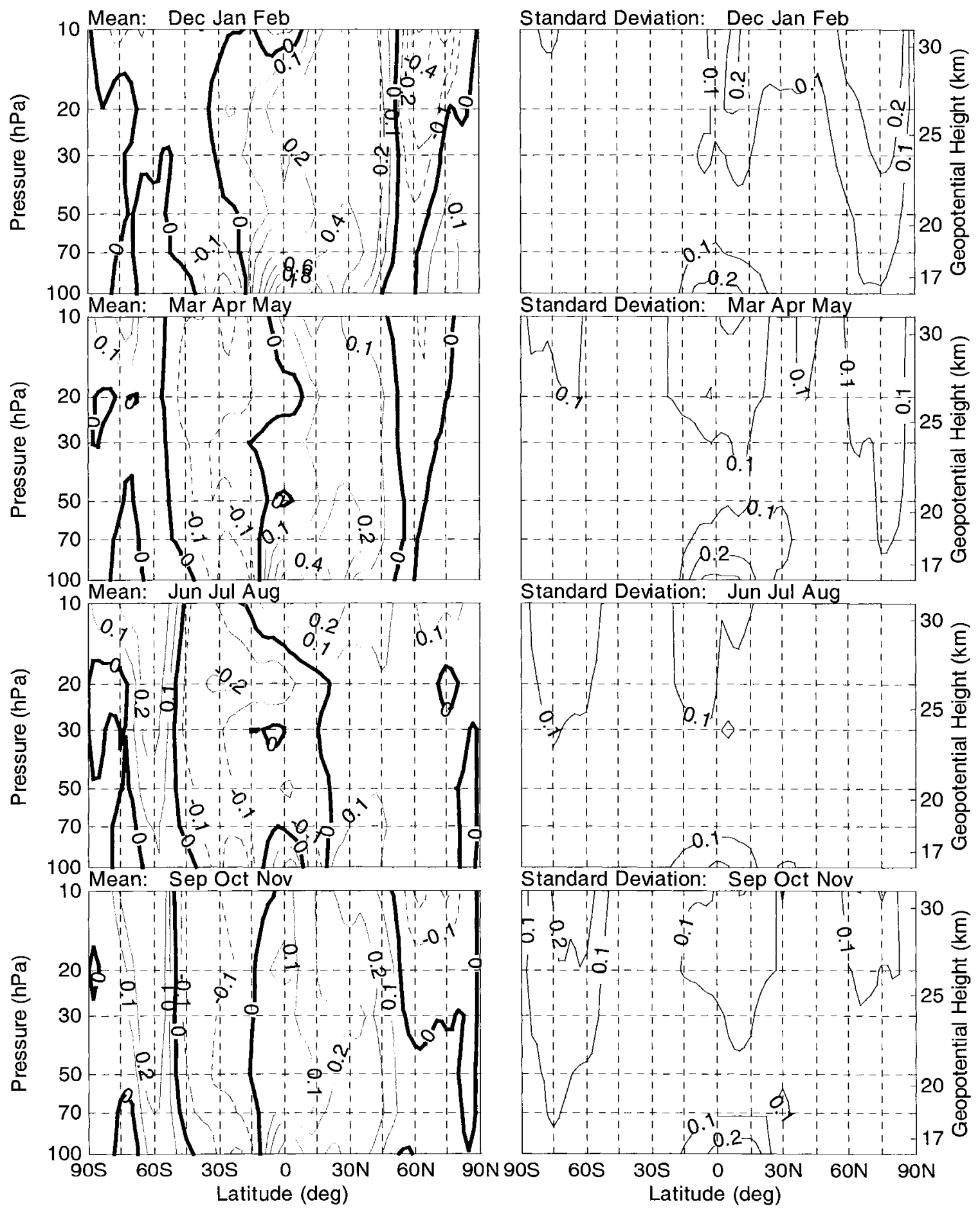

Fig. 6-1. Climatology of monthly-and zonally-averaged meridional wind speed $\left(m s^{-1}\right)$ : NCEP1: [lon: mean of $0^{\circ}-357.5^{\circ}$, lat: $90^{\circ} \mathrm{S}-90^{\circ} \mathrm{N}$, press: $100-10 \mathrm{hPa}$, time: seasonal means from Jan 1979-Dec 2001]. 

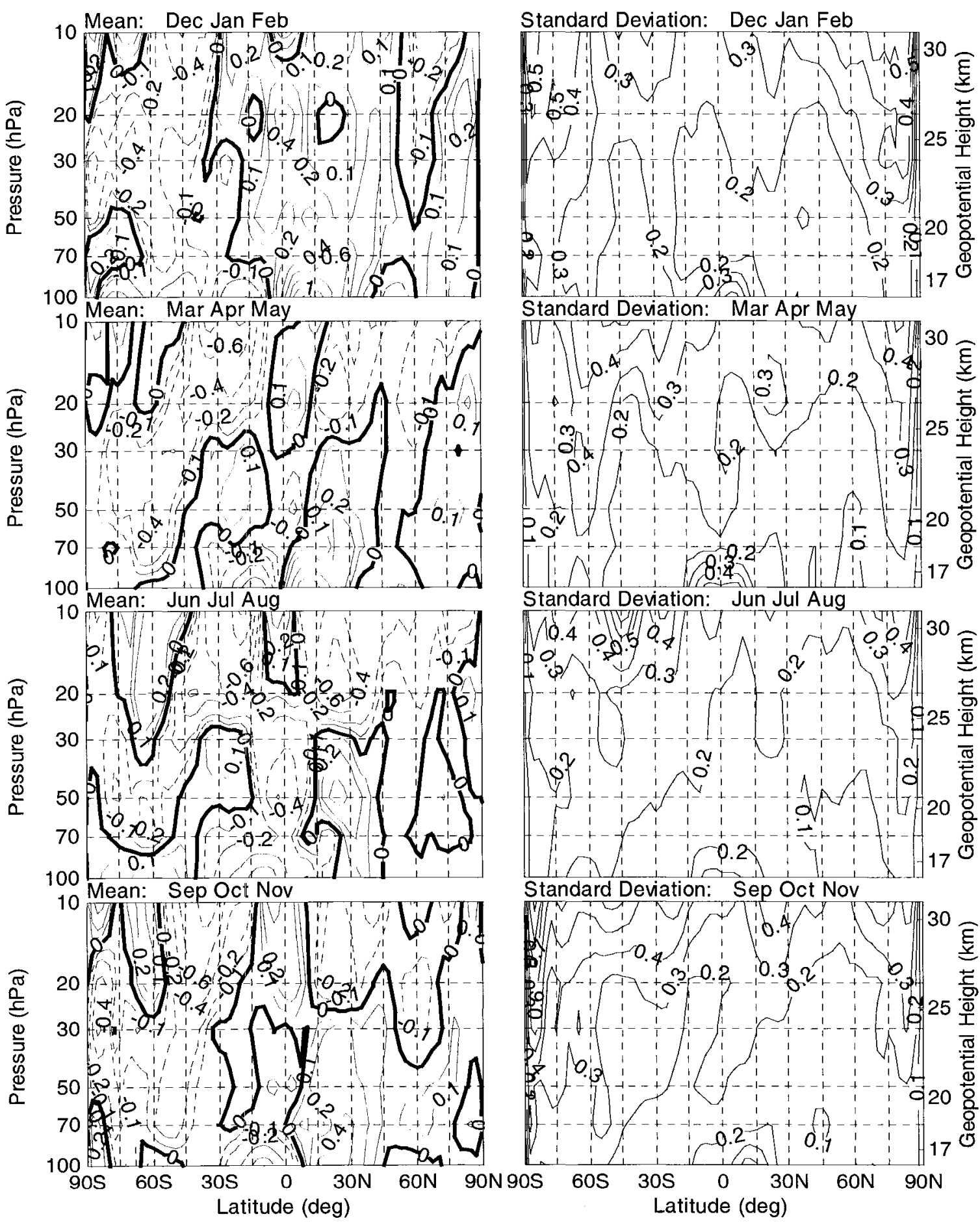

Fig. 6-2. Climatology of monthly-and zonally-averaged meridional wind speed $\left(\mathrm{m} \mathrm{s}^{-1}\right)$ : ERA-40: [lon: mean of $0^{\circ}-357.5^{\circ}$, lat: $90^{\circ} \mathrm{S}-90^{\circ} \mathrm{N}$, press: $100-10 \mathrm{hPa}$, time: seasonal means from Jan 1979-Dec 2001$].$ 

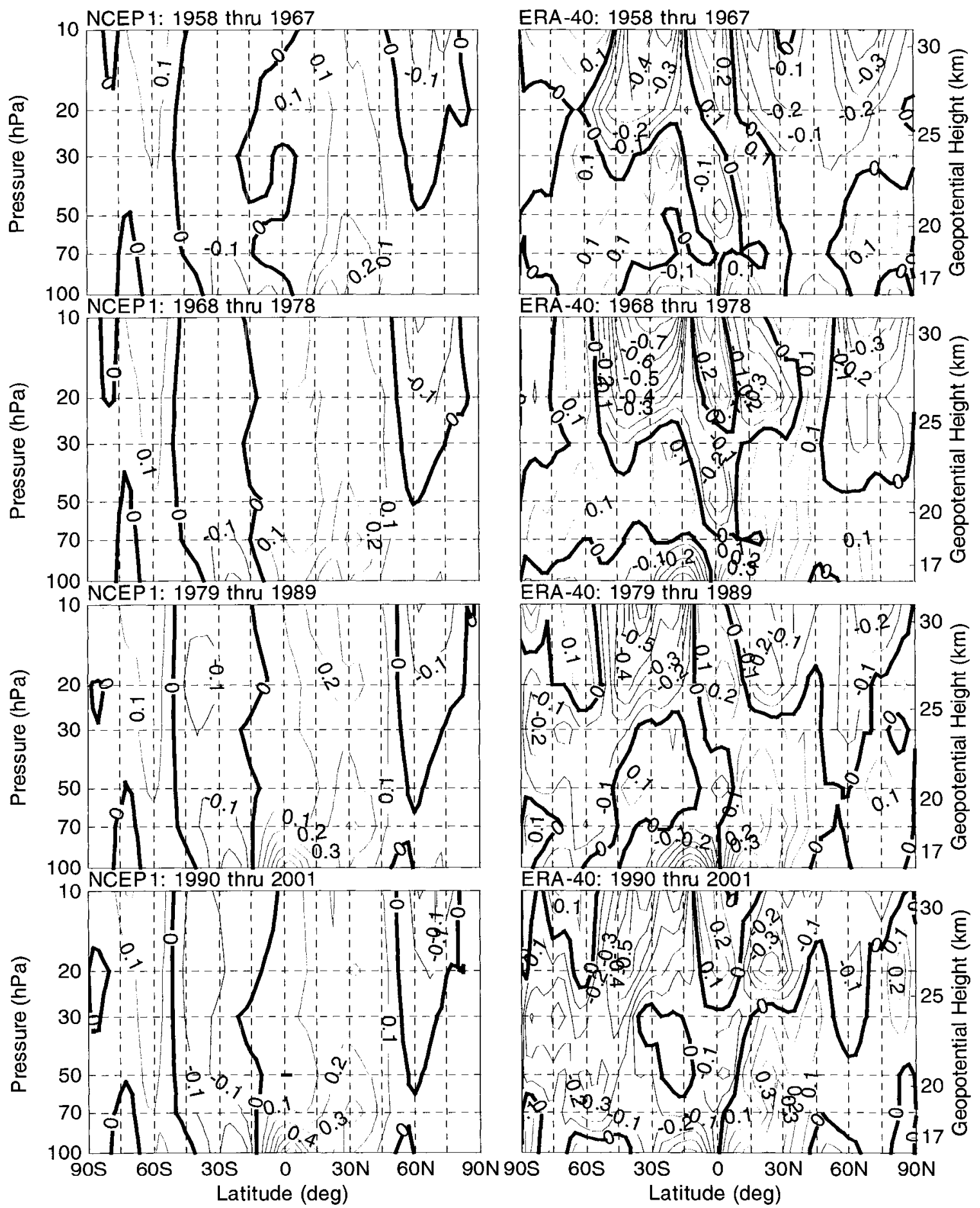

Fig. 6-3. Means of monthly- and zonally-averaged meridional wind speeds $\left(m s^{-1}\right)$ : NCEPI and ERA-40: [lon: mean of $0^{\circ}-357.5^{\circ}$, lat: $90^{\circ} \mathrm{S}-90^{\circ} \mathrm{N}$, press: $100-10 \mathrm{hPa}$, time: as stated]. 

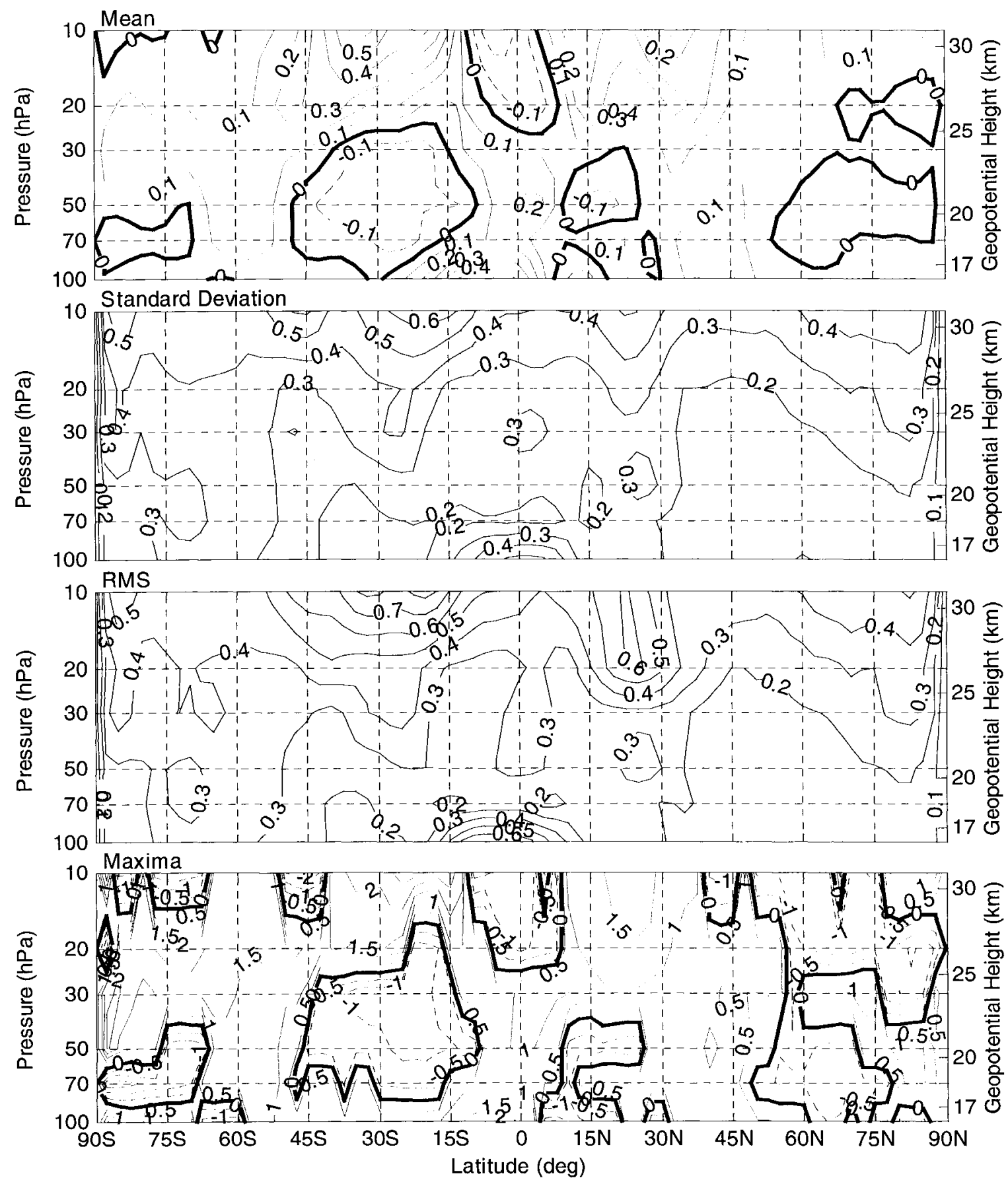

Fig. 6-4. Mean (upper), standard deviation, rms, and maximum (lower) of monthly-and zonally-averaged meridional wind speed differences $\left(\mathrm{m} \mathrm{s}^{-1}\right):$ NCEPI minus ERA-40: [lon: mean of $0^{\circ}-357.5^{\circ}$, lat: $90^{\circ} \mathrm{S}-90^{\circ} \mathrm{N}$, press: $100-10 \mathrm{hPa}$, time: Jan 1958-Dec 2001]. 


\subsection{Meridional wind speed time series}

Figures 6-5 through 6-10 compare meridional wind speed time series from NCEP1 and ERA-40 reanalyses over the 48- and 44-year periods of January 1958 through December 2005 and January 1958 through December 2001, respectively.

The figures are similar in format to those of Figs. 4-25 through 4-30 (temperature) and 5-19 through 5-24 (zonal wind speed) with the addition in this section of the meridional wind speed at the Equator (the cross-equatorial flow). In each latitude range, meridional wind speeds within the range are weighted by the cosine of their latitude and averaged using trapezoidal integration. These area-weighted meridional wind speeds are then filtered in the frequency domain to remove all variance of period less than 13 months. This filtering technique is described and illustrated in detail in Appendix E.

Table 6-1 summarizes the algebraic difference (NCEP1 minus ERA-40) of the mean, filtered meridional wind speeds at each pressure level and latitude band of Figs. 6-5 through 6-10. Differences greater in magnitude than $0.20 \mathrm{~m} \mathrm{~s}^{-1}$ are bolded for emphasis. Figure 6-2 presents the corresponding rms values of the NCEP1 minus ERA-40 differences. These differences are of the same order of magnitude as the mean values of Section 6.1. Consistent with the figures of Section 6.1, absolute values of ERA-40 means are larger than those of NCEP1 at 10, 20, and $30 \mathrm{hPa}$, except in the northern Tropics (Equator through $30^{\circ} \mathrm{N}$ ) and NCEP1 means are generally larger at lower levels (not shown). Table 6-3 lists the Pearson correlation of annual mean values of NCEP1 and ERA-40 meridional wind speeds. Correlations greater than 0.5 are bolded 
for emphasis. Mean and rms values for 1979-2001 differ by very small amounts from those of 1958-2001 with the exception of significant decreases at the Equator at $10 \mathrm{hPa}$ and the Southern Hemisphere Equator and Tropics at $100 \mathrm{hPa}$. Correlations generally decrease during the 1979-2001 period, presumably because of the reduced number of samples.

In every one of the 30 subplots with annual cycle present (not shown), the peakto-peak range of the ERA-40 annual cycle is larger than, and in many cases significantly larger than, that of NCEP1.

Table 6-1. Difference in mean meridional wind speeds $\left(\mathrm{m} \mathrm{s}^{-1}\right)$ :

NCEP1 minus ERA-40 1958-2001 and (1979-2001).

\begin{tabular}{|c|ccccc|}
\hline \multirow{5}{*}{$10 \mathrm{hPa}$} & $90^{\circ} \mathrm{S}-30^{\circ} \mathrm{S}$ & $30^{\circ} \mathrm{S}-\mathrm{EQ}$ & Equator & $\mathrm{EQ}-30^{\circ} \mathrm{N}$ & $30^{\circ} \mathrm{N}-90^{\circ} \mathrm{N}$ \\
\cline { 2 - 6 } & $\mathbf{0 . 2 5}$ & 0.18 & -0.19 & 0.18 & 0.13 \\
& $\mathbf{( 0 . 2 5 )}$ & $(0.18)$ & $(-0.05)$ & $\mathbf{( 0 . 2 0 )}$ & $(0.14)$ \\
& $\mathbf{0 . 2 0}$ & 0.07 & -0.14 & $\mathbf{0 . 2 2}$ & 0.14 \\
$30 \mathrm{hPa}$ & $(0.19)$ & $(0.04)$ & $(-0.17)$ & $\mathbf{( 0 . 2 3 )}$ & $(0.13)$ \\
& 0.07 & -0.01 & 0.11 & 0.06 & 0.07 \\
$50 \mathrm{hPa}$ & $(0.09)$ & $(0.02)$ & $(0.06)$ & $(0.08)$ & $(0.05)$ \\
& -0.01 & -0.04 & $\mathbf{0 . 2 0}$ & 0.01 & 0.03 \\
$70 \mathrm{hPa}$ & $(0.09)$ & $(0.03)$ & $(0.16)$ & $(-0.03)$ & $(0.01)$ \\
& 0.01 & 0.00 & 0.14 & 0.06 & 0.05 \\
$100 \mathrm{hPa}$ & $(0.08)$ & $(0.04)$ & $\mathbf{( 0 . 2 0 )}$ & $(0.03)$ & $(0.04)$ \\
& 0.03 & $\mathbf{0 . 3 3}$ & $\mathbf{0 . 3 8}$ & 0.04 & 0.08 \\
& $(0.00)$ & $(\mathbf{0 . 4 7 )}$ & $\mathbf{( 0 . 7 4 )}$ & $(0.12)$ & $(0.06)$ \\
\hline
\end{tabular}


Table 6-2. Rms difference in NCEP1 and ERA-40 meridional wind speeds $\left(\mathrm{m} \mathrm{s}^{-1}\right)$ 1958-2001 and (1979-2001).

\begin{tabular}{|c|ccccc|}
\hline \multirow{5}{*}{$10 \mathrm{hPa}$} & $90^{\circ} \mathrm{S}-30^{\circ} \mathrm{S}$ & $30^{\circ} \mathrm{S}-\mathrm{EQ}$ & Equator & $\mathrm{EQ}-30^{\circ} \mathrm{N}$ & $30^{\circ} \mathrm{N}-90^{\circ} \mathrm{N}$ \\
\cline { 2 - 6 } $20 \mathrm{hPa}$ & $\mathbf{0 . 3 4}$ & $\mathbf{0 . 2 6}$ & $\mathbf{0 . 3 0}$ & $\mathbf{0 . 2 2}$ & 0.18 \\
& $\mathbf{( 0 . 3 5 )}$ & $\mathbf{( 0 . 2 1 )}$ & $(0.19)$ & $\mathbf{( 0 . 2 3 )}$ & $(0.19)$ \\
& $\mathbf{0 . 2 4}$ & 0.15 & 0.19 & $\mathbf{0 . 2 5}$ & 0.16 \\
& $\mathbf{( 0 . 2 3 )}$ & $(0.11)$ & $\mathbf{( 0 . 2 1 )}$ & $\mathbf{( 0 . 2 5 )}$ & $(0.15)$ \\
& 0.15 & 0.11 & 0.19 & 0.12 & 0.10 \\
$50 \mathrm{hPa}$ & $(0.17)$ & $(0.09)$ & $(0.13)$ & $(0.12)$ & $(0.08)$ \\
& 0.15 & 0.10 & $\mathbf{0 . 2 3}$ & 0.07 & 0.07 \\
$70 \mathrm{hPa}$ & $(0.16)$ & $(0.08)$ & $(0.18)$ & $(0.07)$ & $(0.05)$ \\
& 0.13 & 0.09 & 0.18 & 0.09 & 0.07 \\
$100 \mathrm{hPa}$ & $(0.14)$ & $(0.08)$ & $\mathbf{( 0 . 2 2 )}$ & $(0.07)$ & $(0.06)$ \\
& 0.14 & $\mathbf{0 . 3 9}$ & $\mathbf{0 . 5 7}$ & 0.14 & 0.10 \\
& $(0.10)$ & $\mathbf{( 0 . 4 9 )}$ & $\mathbf{( 0 . 7 7 )}$ & $(0.16)$ & $(0.08)$ \\
\hline
\end{tabular}

Table 6-3. Correlation of NCEP1 and ERA-40 meridional wind speeds $\left(\mathrm{m} \mathrm{s}^{-1}\right)$ 1958-2001 and (1979-2001).

\begin{tabular}{|c|ccccc|}
\hline \multirow{5}{*}{$10 \mathrm{hPa}$} & $90^{\circ} \mathrm{S}-30^{\circ} \mathrm{S}$ & $30^{\circ} \mathrm{S}-\mathrm{EQ}$ & Equator & EQ-30 & $30^{\circ} \mathrm{N}-90^{\circ} \mathrm{N}$ \\
\cline { 2 - 6 } $20 \mathrm{hPa}$ & 0.17 & 0.08 & -0.21 & 0.34 & 0.11 \\
& $(0.01)$ & $(0.21)$ & $(0.01)$ & $(0.47)$ & $(0.36)$ \\
$30 \mathrm{hPa}$ & 0.14 & 0.00 & 0.25 & 0.41 & 0.26 \\
& $(0.26)$ & $(0.14)$ & $(0.26)$ & $\mathbf{( 0 . 5 2 )}$ & $(0.38)$ \\
$50 \mathrm{hPa}$ & $\mathbf{0 . 5 5}$ & 0.12 & 0.30 & 0.23 & 0.33 \\
& $\mathbf{0 . 4 3 )}$ & $(0.35)$ & $(-0.01)$ & $(0.32)$ & $(0.20)$ \\
$70 \mathrm{hPa}$ & $\mathbf{0 . 5 5}$ & 0.26 & 0.10 & 0.47 & 0.28 \\
& $(0.15)$ & $(0.36)$ & $(0.23)$ & $(0.37)$ & $(0.11)$ \\
$100 \mathrm{hPa}$ & $\mathbf{0 . 5 7}$ & 0.29 & -0.01 & $\mathbf{0 . 6 7}$ & 0.42 \\
& $(0.13)$ & $(0.42)$ & $(0.28)$ & $(0.31)$ & $(0.34)$ \\
& -0.19 & $\mathbf{- 0 . 6 4}$ & -0.18 & $\mathbf{0 . 5 5}$ & 0.21 \\
& $(-0.16)$ & $(-0.35)$ & $(0.28)$ & $(\mathbf{0 . 5 4 )}$ & $(0.18)$ \\
\hline
\end{tabular}


At $100 \mathrm{hPa}$ (Fig. 6-5) in the northern extratropics, NCEP1 latitude-averaged meridional wind speeds exhibit a steady $\sim 0.1 \mathrm{~m} \mathrm{~s}^{-1}$ northerly flow while ERA-40's vary about a zero mean. Northern tropical wind speeds are the most correlated at this level but both tend toward a more northerly flow after 1965. At the Equator the $>0.5 \mathrm{~m} \mathrm{~s}^{-1}$ NCEP1 northerly flow that appears after 1975, also noted by Pawson and Fiorino (1998a), contrasts with ERA-40 winds, which have essentially zero mean. In the southern Tropics, the NCEP1 flow has zero mean while ERA-40 shows a strong southerly flow throughout, increasing after 1970. These two time series, Equator and Equator through $30^{\circ} \mathrm{S}$, have the largest NCEP1 minus ERA-40 differences in meridional wind speed and two of the largest rms differences, 0.65 and $0.41 \mathrm{~m} \mathrm{~s}^{-1}$, respectively, in the region of interest in this thesis. In the southern extratropics, NCEP 1 winds are quiescent while ERA-40's have considerable variation about zero, most notably the possibly anomalous $0.5 \mathrm{~m} \mathrm{~s}^{-1}$ excursion from 1975-77. The correlation of these final three $100 \mathrm{hPa}$ time series is actually negative.

At the three interior pressure levels of $70 \mathrm{hPa}$ (Fig. 6-6), $50 \mathrm{hPa}$ (Fig. 6-7), and $30 \mathrm{hPa}$ (Fig. 6-8), NCEP1 and ERA-40 reanalyses are in much better agreement than at $100 \mathrm{hPa}$. The largest mean differences of the three pairs of time series occur at the Equator: $0.14,0.20$, and $0.11 \mathrm{~m} \mathrm{~s}^{-1}$, respectively. Maximum rms difference values of $0.23,0.32$, and $0.35 \mathrm{~m} \mathrm{~s}^{-1}$, respectively, are again found at the Equator. NCEP1 northern extratropical winds are steady at $0.15,0.10$, and $0.07 \mathrm{~m} \mathrm{~s}^{-1}$ and those of the southern extratropics quiescent. ERA-40 $50 \mathrm{hPa}$ southern tropical winds exhibit a seemingly anomalous $0.3 \mathrm{~m} \mathrm{~s}^{-1}$ northerly transient between 1975 and 1977 , similar to that in the 
southern extratropics at $100 \mathrm{hPa}$. At $30 \mathrm{hPa}$, a southerly $0.3 \mathrm{~m} \mathrm{~s}^{-1} 1975-77$ transient and $0.75 \mathrm{~m} \mathrm{~s}^{-1}$ peak-to-peak oscillations from 1973 to 1977 are inconsistent with the nominal behavior of the ERA-40 time series in which they occur.

In all latitude bands of the $20 \mathrm{hPa}$ time series (Fig. 6-9 and Tables 6-1 and 6-3), mean and rms NCEP1 and ERA-40 differences increase from previous levels. Rms differences range from 0.20 to $0.29 \mathrm{~m} \mathrm{~s}^{-1}$. The lower extent of the southerly ERA-40 flows seen in Fig. 6-3 may be observed in the southern extratropics and the northern Tropics, with northerly flow at the Equator. Again ERA-40's seemingly anomalous 1975-77 southerly flow transients are present in the southern Tropics, northern Tropics, and extratropics.

At $10 \mathrm{hPa}$ (Fig. 6-10), the ERA-40 southerly flow evident in Fig. 6-3 is present in all time series except at the Equator where northerly flow exists. Significant ERA-40 southerly transients occur from the South Pole to the Equator, where three of the largest mean differences and the largest rms differences may be found.

Table 6-4 identifies the latitude bands and pressure levels where spectral analysis of the meridional wind speed time series of Figs. 6-5 through 6-10 exhibits evidence of the quasi-biennial oscillation (QBO). Variance spectra of the time series were visually inspected for the presence of peaks in the range of 23 to 30 months (Fig. 5-74) after removal of all variance with period less than 13 months (Appendix E). A major QBO presence is identified in Table 6-4 if a peak in the 23 to 30 month range was the largest in the complete spectra; a minor QBO presence was noted if a significant peak, but not the dominant, one existed in the period of interest. The coupling from the equatorial zonal 
wind speed variation of the QBO to the extratropical meridional wind speed is driven by the hemispheric-scale meridional circulation in the summer hemisphere and by wavemean flow interactions in the winter season (Holton and Tan 1980). Major QBO spectral signatures are found more often in the NCEP1 reanalysis, perhaps because of its generally lighter winds.

Table 6-4. QBO variance present in meridional wind speeds 1958-2001.

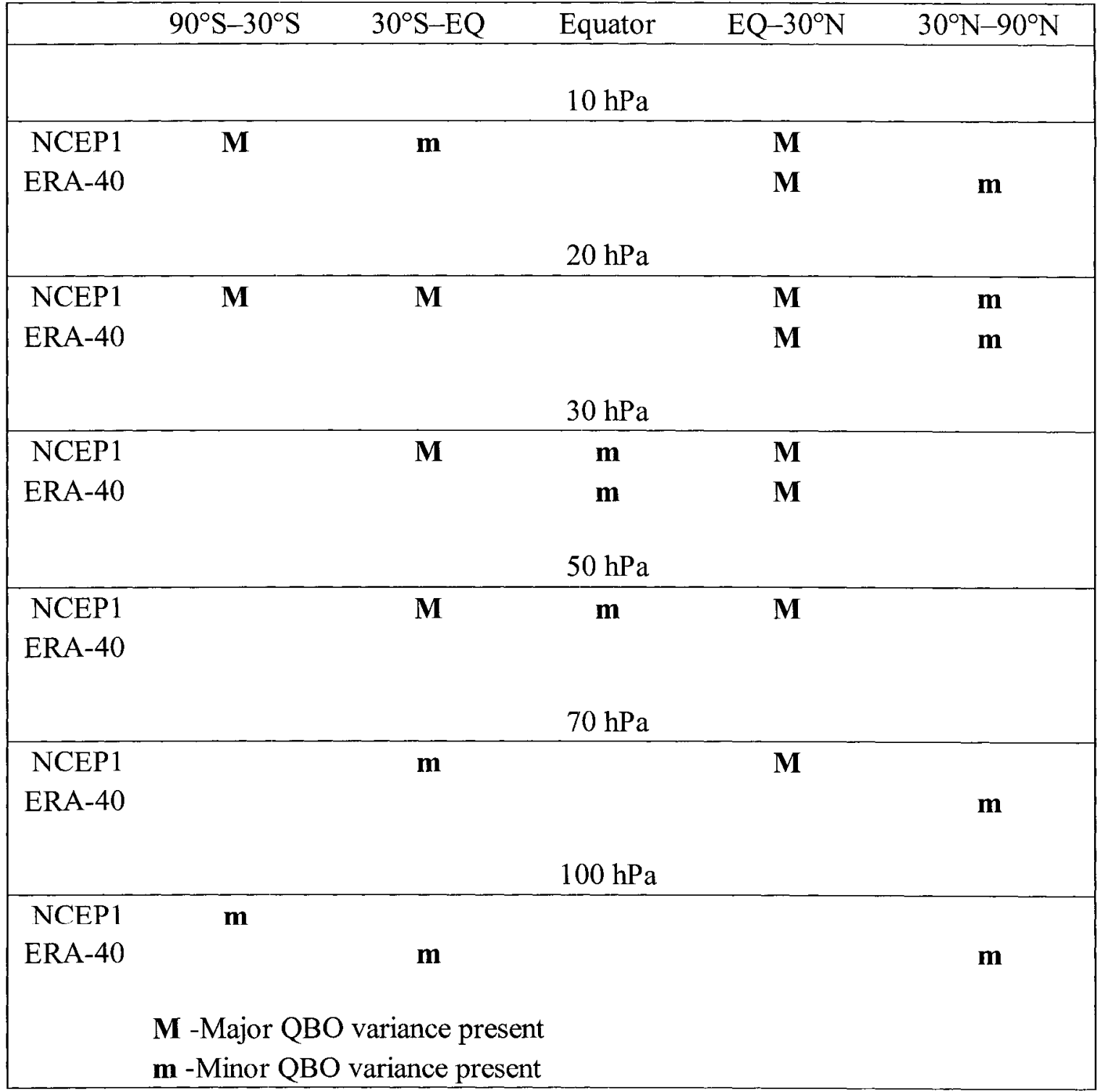



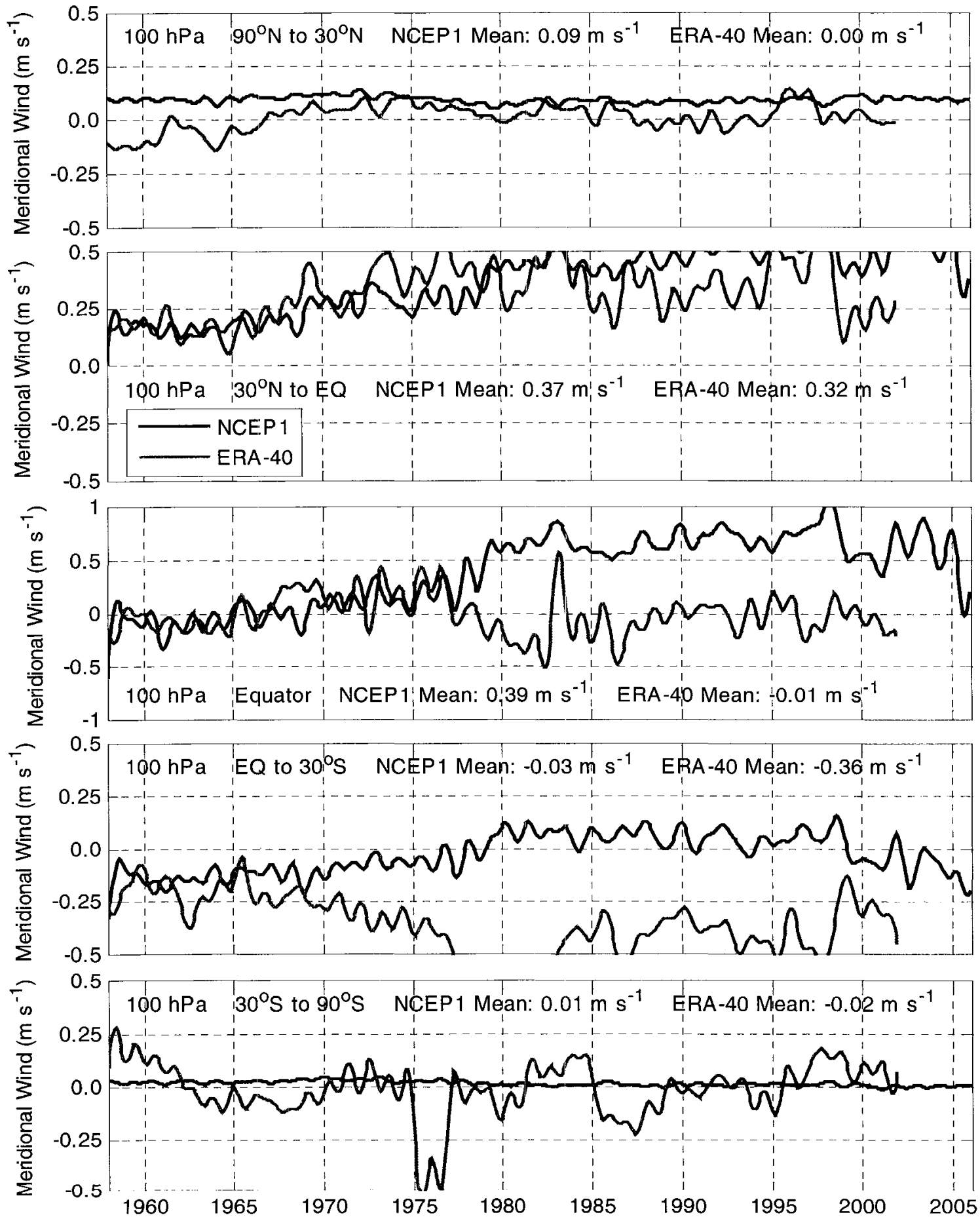

Fig. 6-5. Time series of monthly-and zonally-averaged meridional wind speed (all variance with period less than 13 months removed by frequency-domain filtering): [lon: mean of $0^{\circ}-357.5^{\circ}$; lat: as stated; press: $100 \mathrm{hPa}$; time: Jan 1958-Dec 2005 (NCEP1), Jan 1958-Dec 2001 (ERA-40)]. 


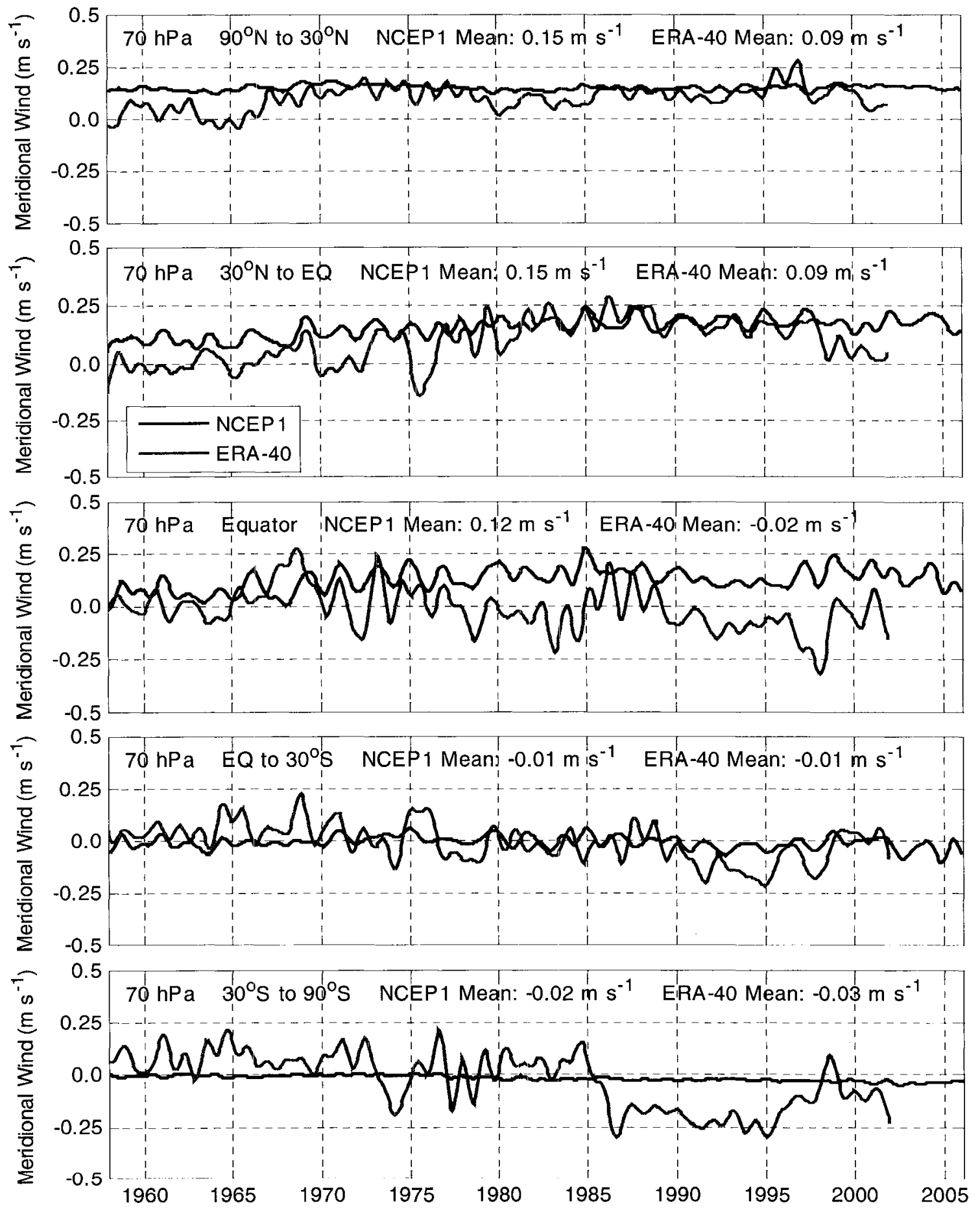

Fig. 6-6. Same as Fig. 6-5 except press: $70 \mathrm{hPa}$. 

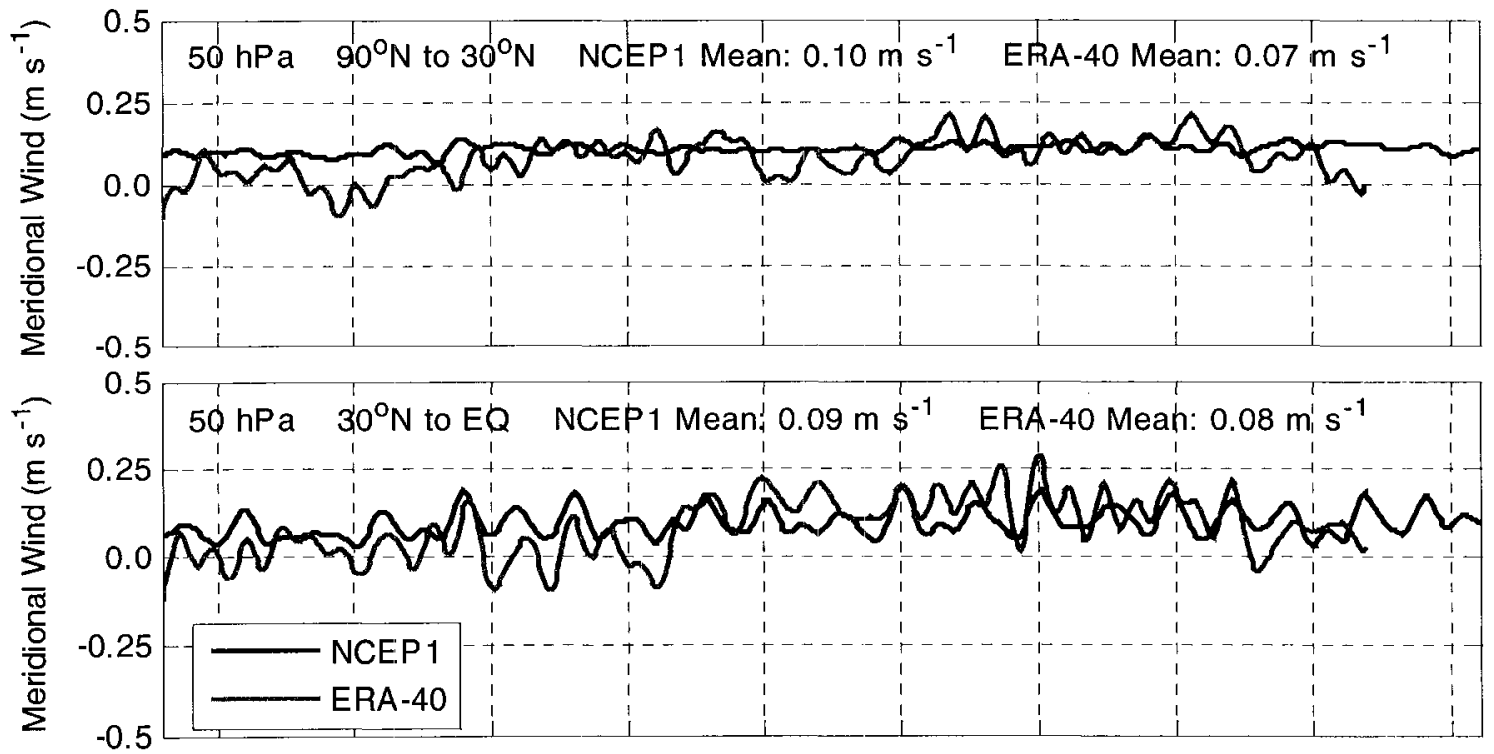

票

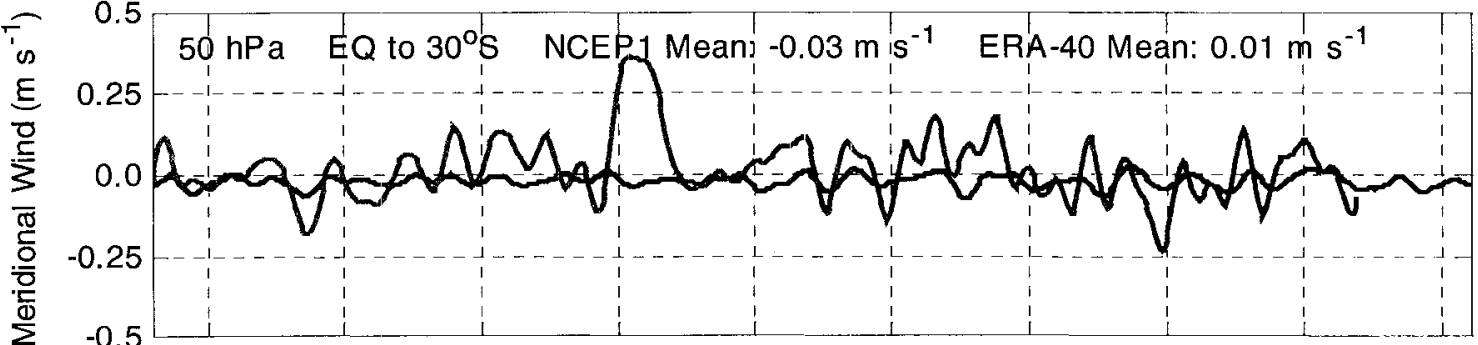

is $\quad 0.5 \quad 50 \mathrm{hPa} \quad 30^{\circ} \mathrm{S}$ to $90^{\circ} \mathrm{S}$ NCEP1 Mean: $-0.01 \mathrm{~m} \mathrm{~s}^{-1}$ ERA-40 Mean: $-0.00 \mathrm{~m} \mathrm{~s}^{-1}$

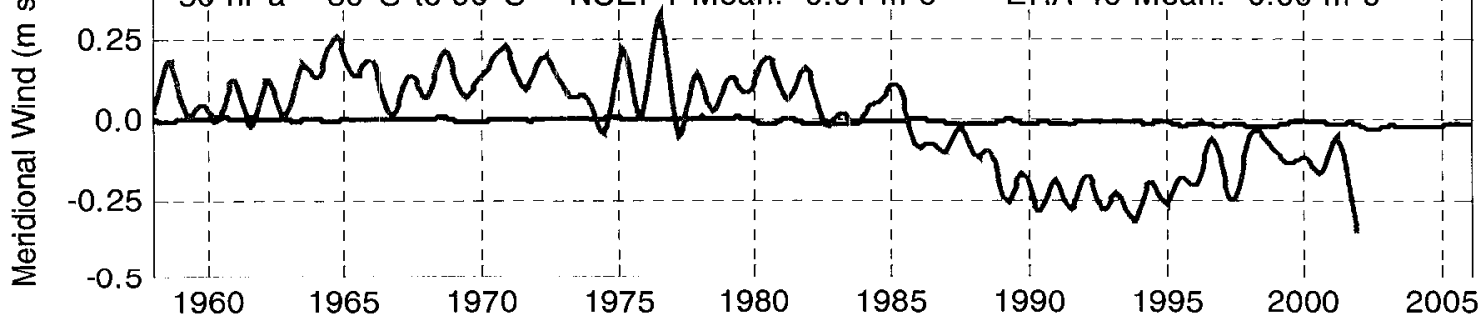

Fig. 6-7. Same as Fig. 6-5 except press: $50 \mathrm{hPa}$. 

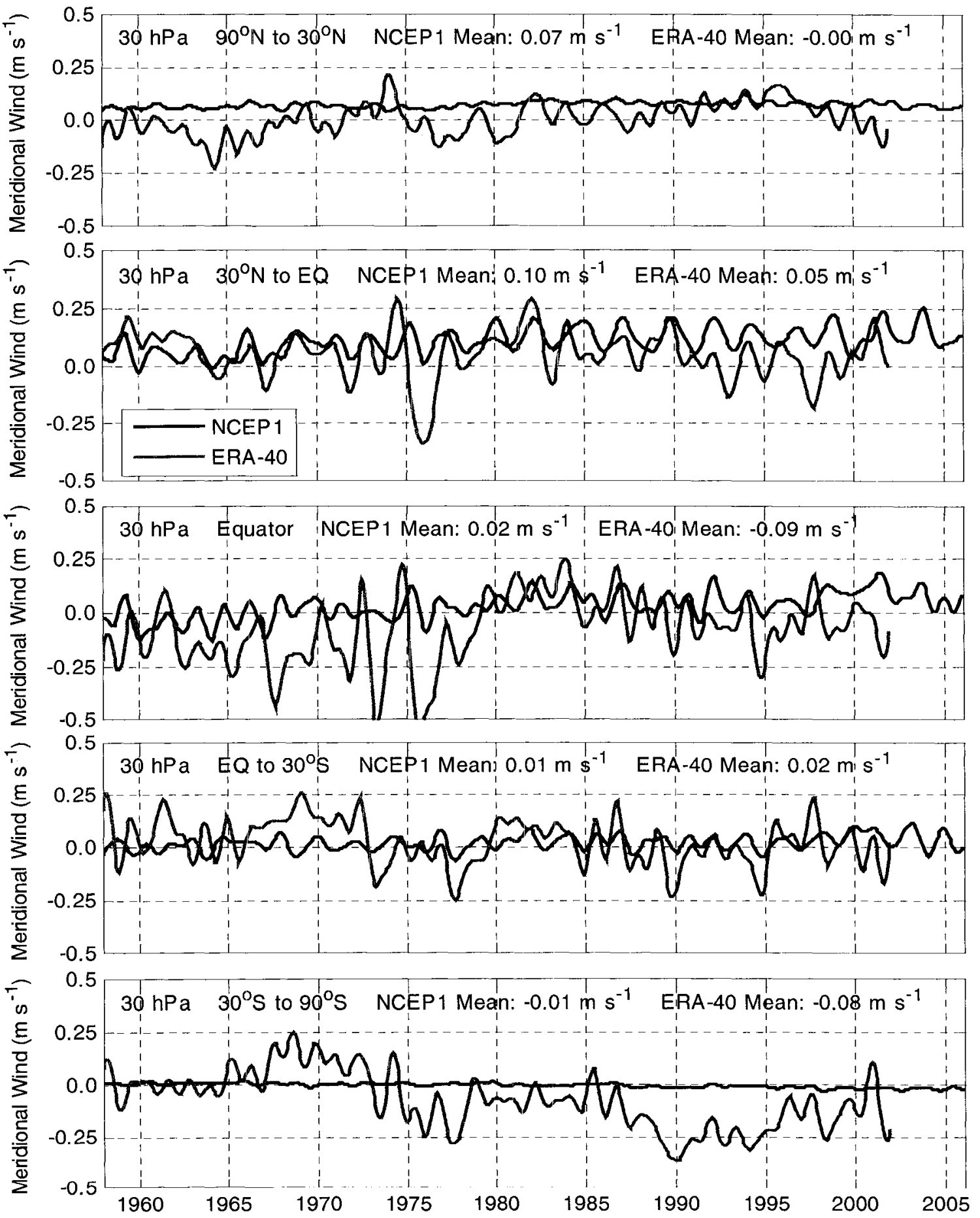

Fig. 6-8. Same as Fig. 6-5 except press: $30 \mathrm{hPa}$. 


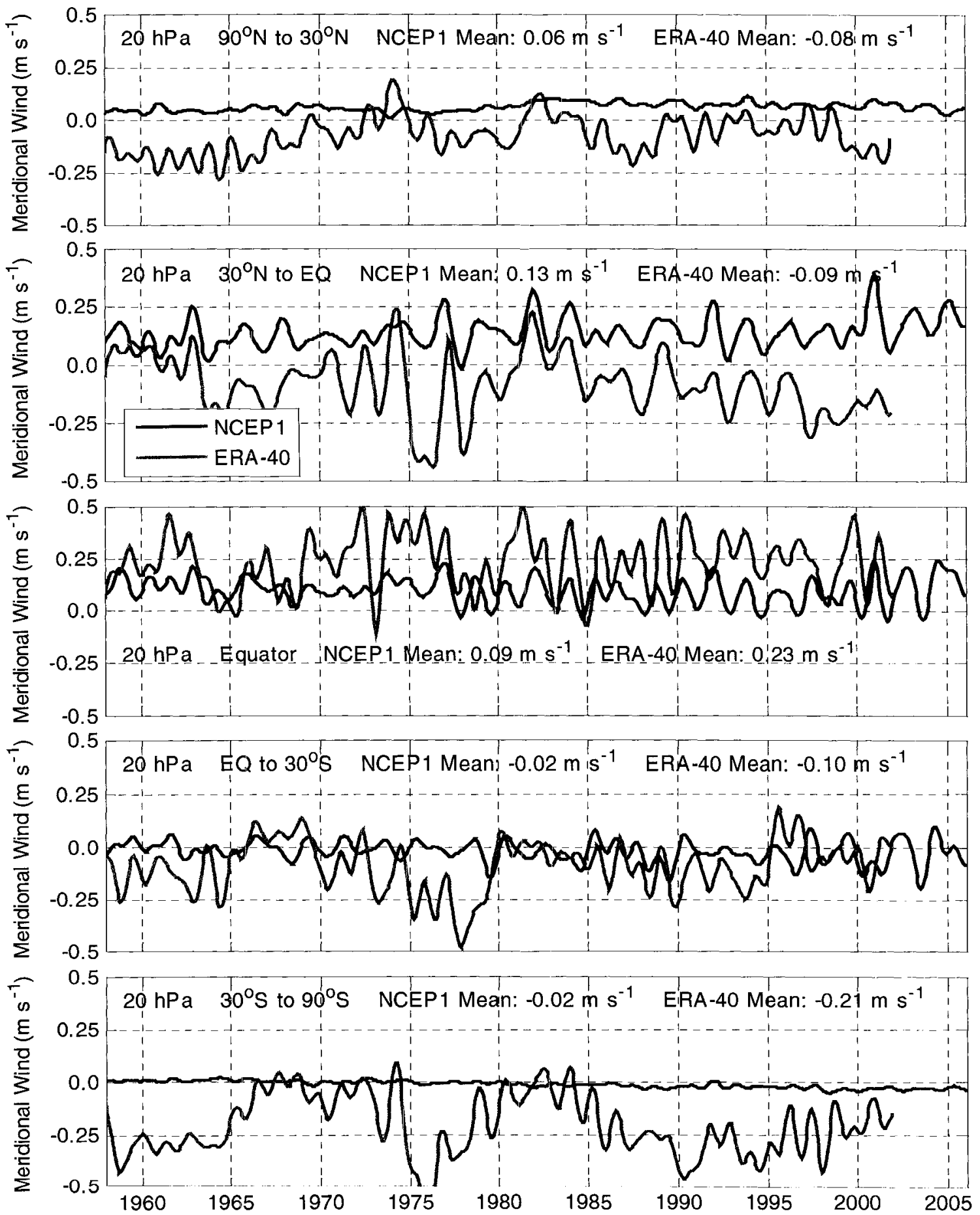

Fig. 6-9. Same as Fig. 6-5 except press: $20 \mathrm{hPa}$. 


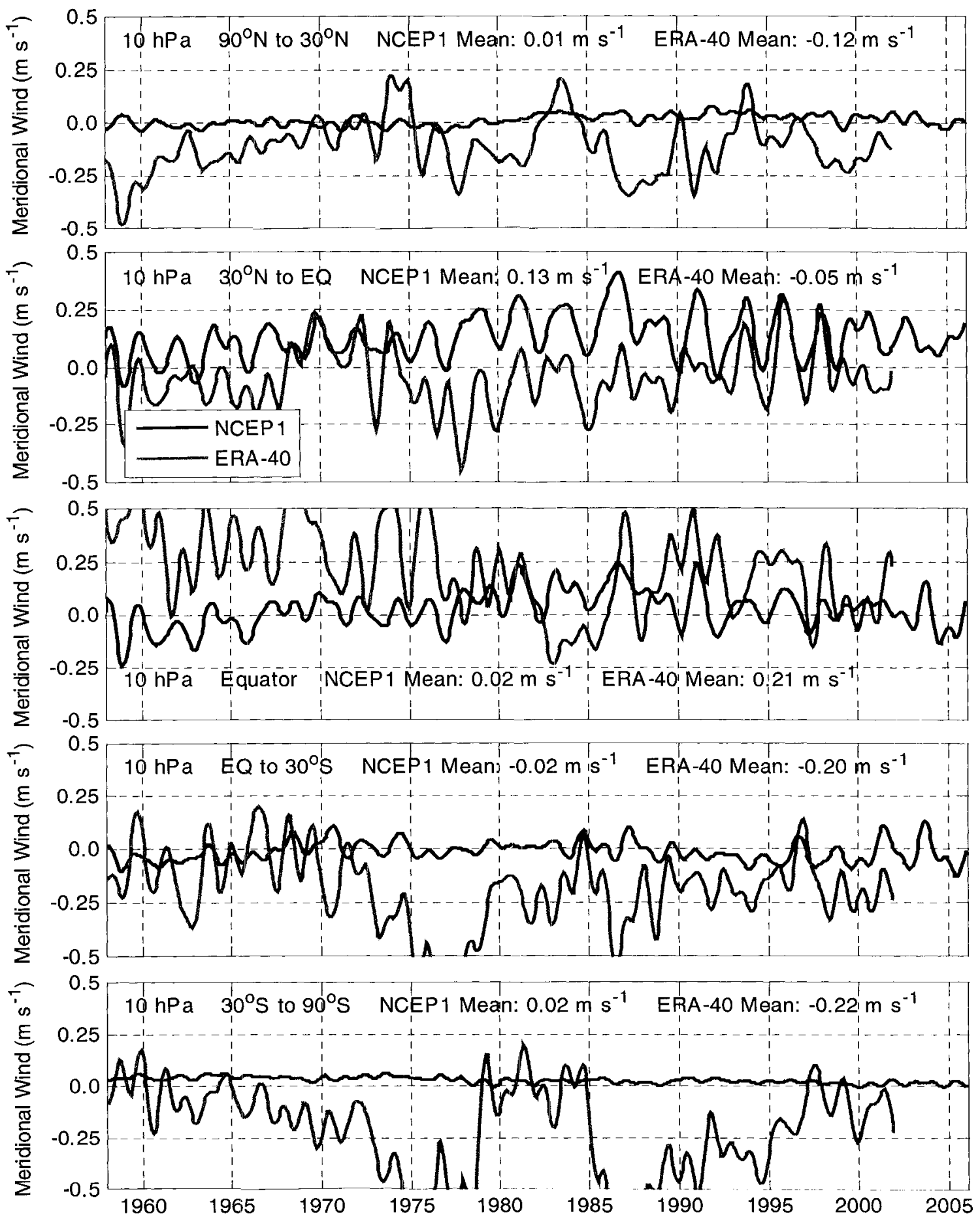

Fig. 6-10. Same as Fig. 6-5 except press: $10 \mathrm{hPa}$. 


\subsection{Meridional wind speed time-latitude structure}

This section compares NCEP1 and ERA-40 annually- and zonally-averaged meridional wind speeds in the time-latitude domain over all latitudes for their full 48- and 44-year durations. This approach is similar to that of Pawson and Fiorino (1998a), who plotted 15-year mean tropical meridional wind speeds in their Fig. 7. As Sections 6.1 and 6.2 demonstrate, standard deviations of meridional wind speeds are of the same magnitude as their means. Comparison of the six figures of this section, Figs. 6-11 through 6-16, which present the time-latitude structures for pressure levels 100 through $10 \mathrm{hPa}$, with the corresponding time-series of Figs. 6-5 through 6-10, will provide a more complete view of the reanalyzed meridional wind speeds.

Figure 6-11 plots the NCEP1 and ERA-40 meridional wind speed contours at $100 \mathrm{hPa}$. NCEP1 winds show little annual variation except at the equatorial division between southerly and northerly flow. This division drifts from $10^{\circ} \mathrm{N}$ in 1958 to $\sim 15^{\circ} \mathrm{N}$ by 1980 where it remains until 2000 when it again moves toward the Equator. In 1979 the northerly equatorial flow intensifies, peaking at approximately one $\mathrm{m} \mathrm{s}^{-1}$, the strongest meridional wind speed, driven by the steepest meridional gradients, in the NCEP1 stratospheric reanalysis. It is this strong, extended northerly flow and its implied mean mass transport from Southern Hemisphere to Northern Hemisphere that is the unexpected result discussed in Section 6.1 and by Pawson and Fiorino (1998a). The ERA-40 Northern Hemisphere meridional wind speeds are generally temporally consistent. However in the southern Tropics (Equator to $30^{\circ} \mathrm{S}$ ), winds begin to increase in 1970 and plateau in 1980. The 1975-77 anomaly caused by VTPR calibration 
problems (Section 2.2.4) and a possible anomaly during 1985-89 distort a rather steadystate, zero-mean pattern. The consistent, broad in latitude, with northerly and southerly flow northward and southward, respectively, of a constant, near-equatorial, dividing line pattern occurs in the ERA-40 reanalysis only at $100 \mathrm{hPa}$. These strong outward flows on both sides of the equatorial region imply significant vertical flow.

Figure 6-12 plots the NCEP1 and ERA-40 meridional wind speed contours at $70 \mathrm{hPa}$. NCEP1 wind speeds are extremely consistent over the entire 48-year data set. Again, a consistent northerly flow extending from $\sim 15^{\circ} \mathrm{S}$ to the North Pole for the 48-year duration of the reanalysis is unexpected. ERA-40 wind speeds at all latitudes possess considerably more structure than NCEP1; as in Figs. 6-2 and 6-6, the standard deviation of these speeds is of the same order as their mean magnitude. The transient structure at $20-60^{\circ} \mathrm{S}$ at the same latitude and time as those at 100 and $50 \mathrm{hPa}$ during 1976 is yet another manifestation of the VTPR calibration problem.

At $50 \mathrm{hPa}$, Fig. 6-13 plots another temporally consistent NCEP1 wind speed contour. The Northern Hemisphere northerly flow is weaker than at $70 \mathrm{hPa}$ and is found from the Equator to $90^{\circ} \mathrm{N}$. Measures of mean and rms differences between NCEP1 and ERA-40 in the five latitude bands of the time series are very similar at 50 and $70 \mathrm{hPa}$ (Tables 6-1 and 6-2, respectively); thus the description of more structure with winds on the same order of magnitude applies again. However, the anomalous peaks in the ERA-40 northerly flow centered at $20^{\circ} \mathrm{S}$ and $60^{\circ} \mathrm{S}$ during 1976 are located directly above one of the southerly flow anomalies at $100 \mathrm{hPa}$. 
The NCEP1 northerly flow at $30 \mathrm{hPa}$ in Fig. 6-14 ranges from $20^{\circ} \mathrm{S}$ to $\sim 50^{\circ} \mathrm{N}$. ERA-40 variance continues to increase from its minimum at $70 \mathrm{hPa}$ with notable transients of $0.5 \mathrm{~m} \mathrm{~s}^{-1}$ at $25^{\circ} \mathrm{S}$ in 1969 (northerly flow), $0^{\circ}$ to $30^{\circ} \mathrm{N}$ in 1973 (both northerly and southerly flows), and $70^{\circ} \mathrm{S}$ in 1989 (southerly flow).

Figure 6-15 plots the NCEP1 and ERA-40 meridional wind speed contours at $20 \mathrm{hPa}$. The NCEP1 northerly flow at this level extends from $10^{\circ} \mathrm{S}$ to $50^{\circ} \mathrm{N}$. ERA-40 possible southerly flow anomalies exist at $25^{\circ} \mathrm{N}$ and $30^{\circ} \mathrm{S}$ in 1976 and $40^{\circ} \mathrm{S}$ during 1990-96. The $25^{\circ} \mathrm{S}$ anomaly is in vertical alignment with similar behavior at 100 and $50 \mathrm{hPa}$ and possibly similar behavior at $70 \mathrm{hPa}$.

At $10 \mathrm{hPa}$, Fig. 6-16 shows the NCEP1 northerly flow to have a minimum width of $0^{\circ}$ to $50^{\circ} \mathrm{N}$. Stronger southerly and northerly flows than at lower levels in the northern and southern extratropics, respectively, are also present. ERA-40 at $10 \mathrm{hPa}$ is dominated by four major flow systems. A narrow equatorial band of strong northerly flow exceeding $0.5 \mathrm{~m} \mathrm{~s}^{-1}$ runs almost continuously for the entire length of the reanalysis. To its south a second, wider $\left(60^{\circ}-40^{\circ} \mathrm{S}\right.$ to $10^{\circ} \mathrm{S}$ with core at $\left.30^{\circ} \mathrm{S}\right)$, almost continuous region of southerly flow reaching maxima of $1.5 \mathrm{~m} \mathrm{~s}^{-1}$ in 1976 and $1.0 \mathrm{~m} \mathrm{~s}^{-1}$ in 1994 also runs for the duration of the reanalysis. Gradients on the southerly side of this interface are extremely strong, consistent with Fig. 6-3. As with NCEP1, ERA-40 exhibits stronger southerly and northerly flows than at lower levels, and much stronger ERA-40 flows than those of NCEP1 exist in the northern and southern extratropics, respectively. 


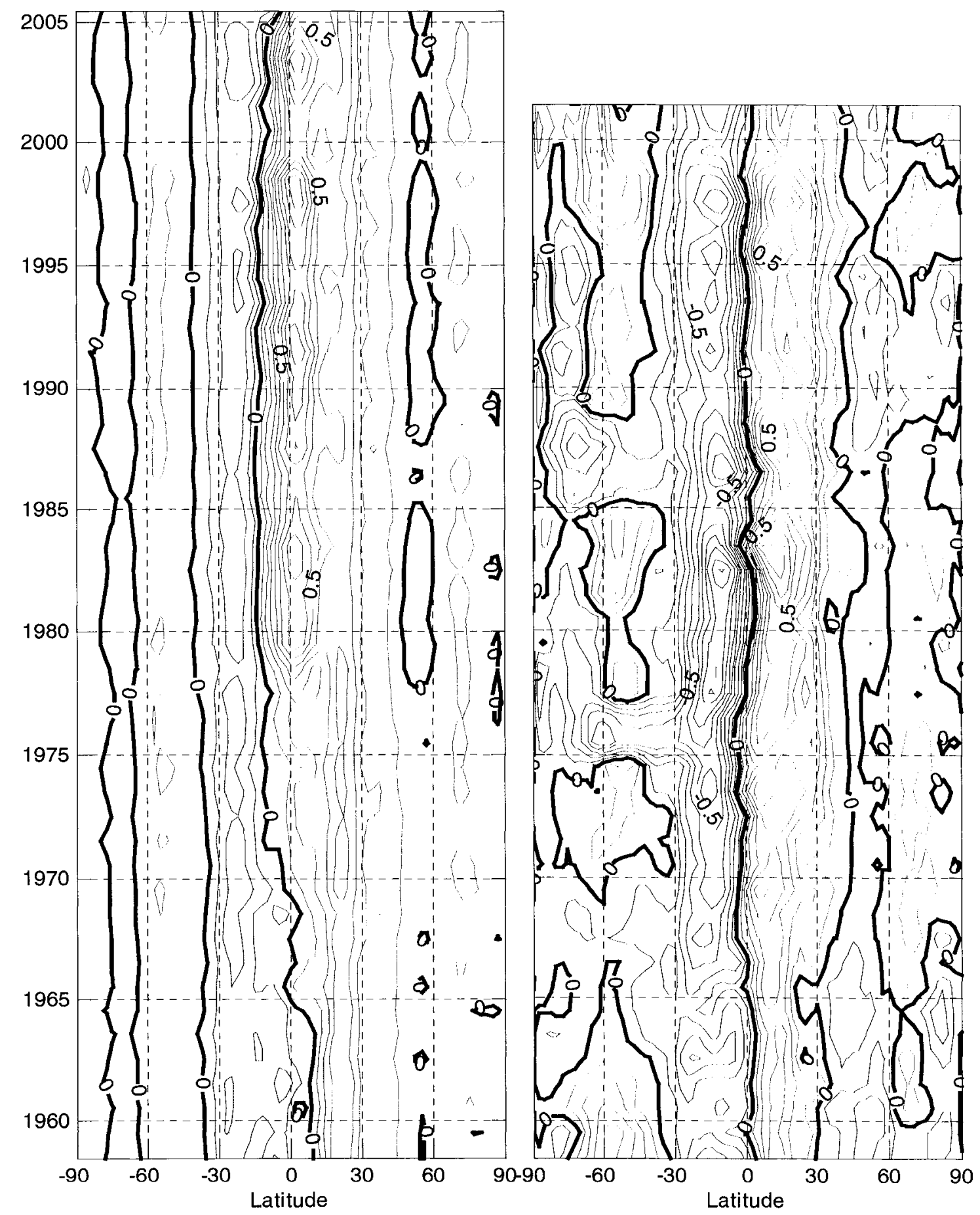

Fig. 6-11. Time-latitude structure of monthly-and zonally-averaged of meridional wind speed for NCEPl (left) and ERA-40 (right) Contours $0.1 \mathrm{~m} \mathrm{~s}^{-1}$ : [lon: mean of $0^{\circ}-357.5^{\circ}$; lat: as stated; press: $100 \mathrm{hPa}$; time: Jan 1958-Dec 2005 (NCEP1), Jan 1958-Dec 2001 (ERA-40)]. 


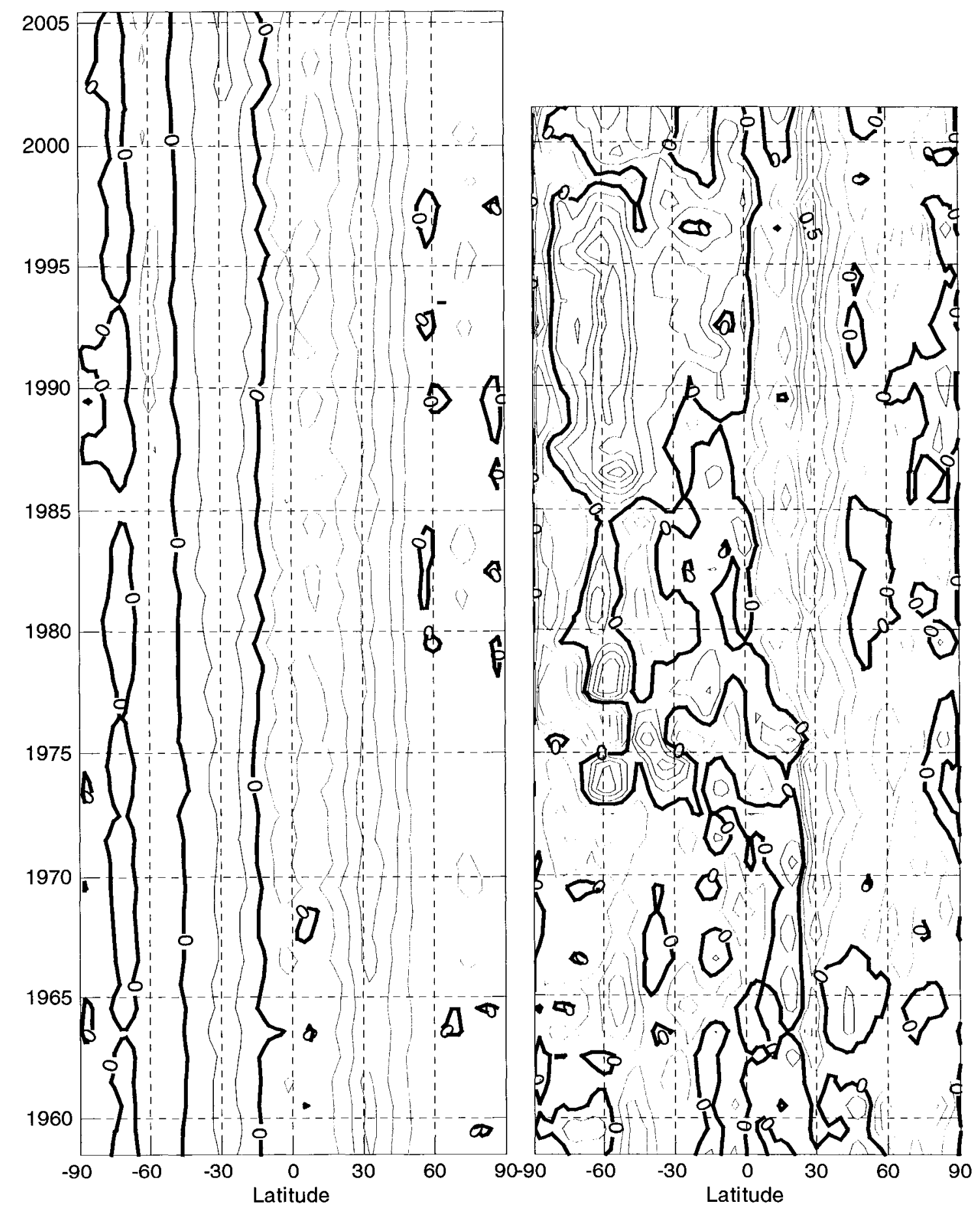

Fig. 6-12. Same as Fig. 6-11 except press: $70 \mathrm{hPa}$. 


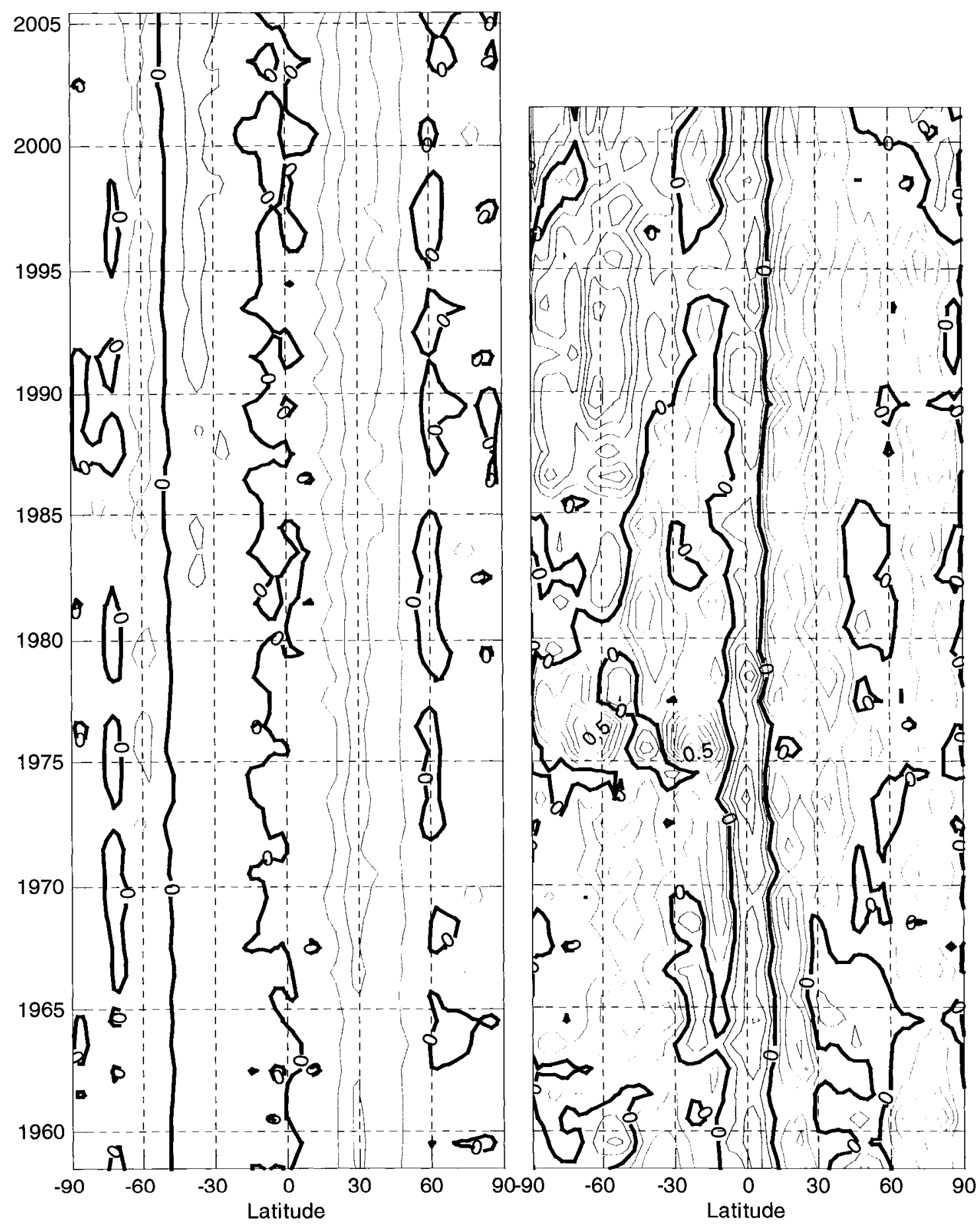

Fig. 6-13. Same as Fig. 6-11 except press: $50 \mathrm{hPa}$. 


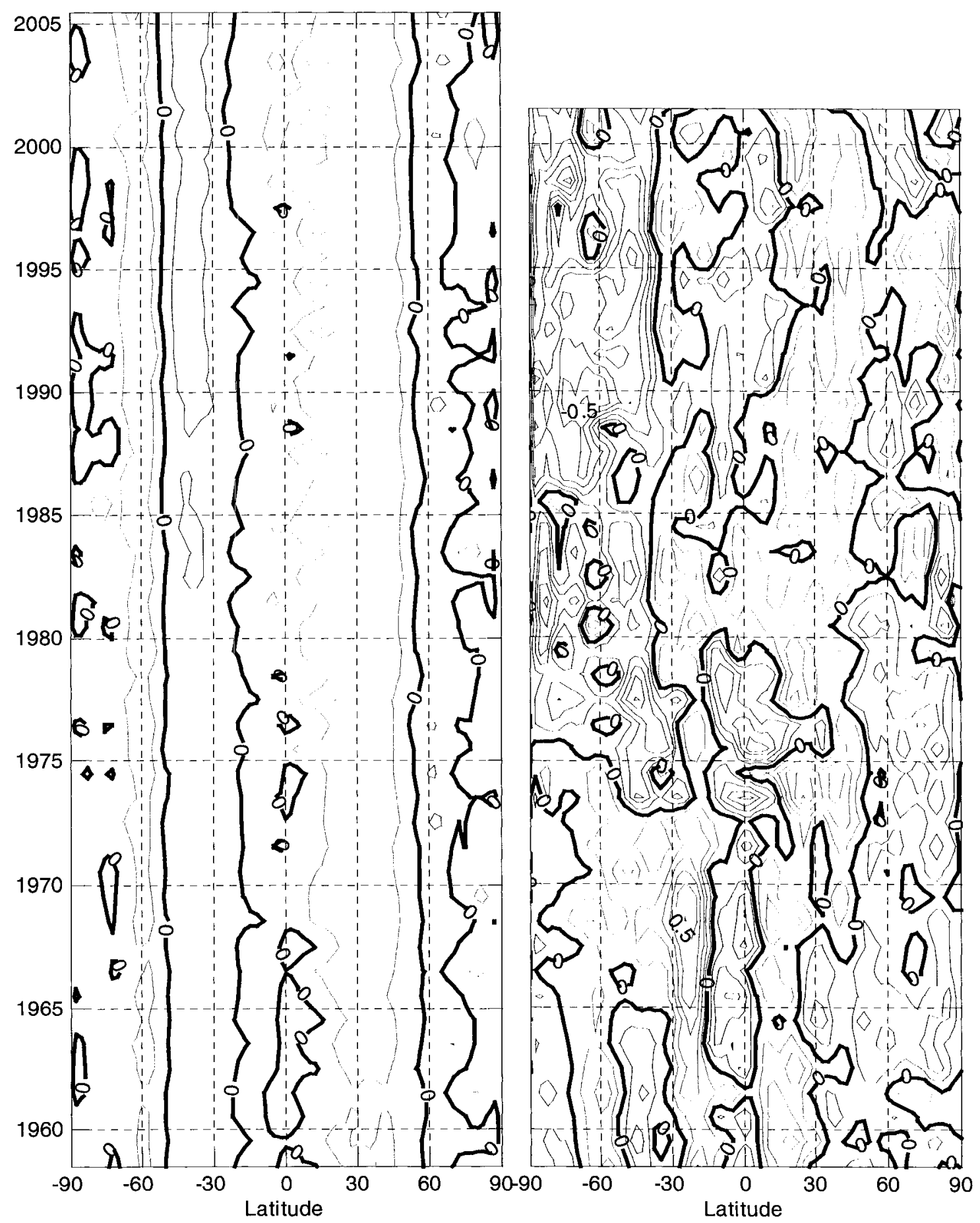

Fig. 6-14. Same as Fig. 6-11 except press: 30 hPa. 


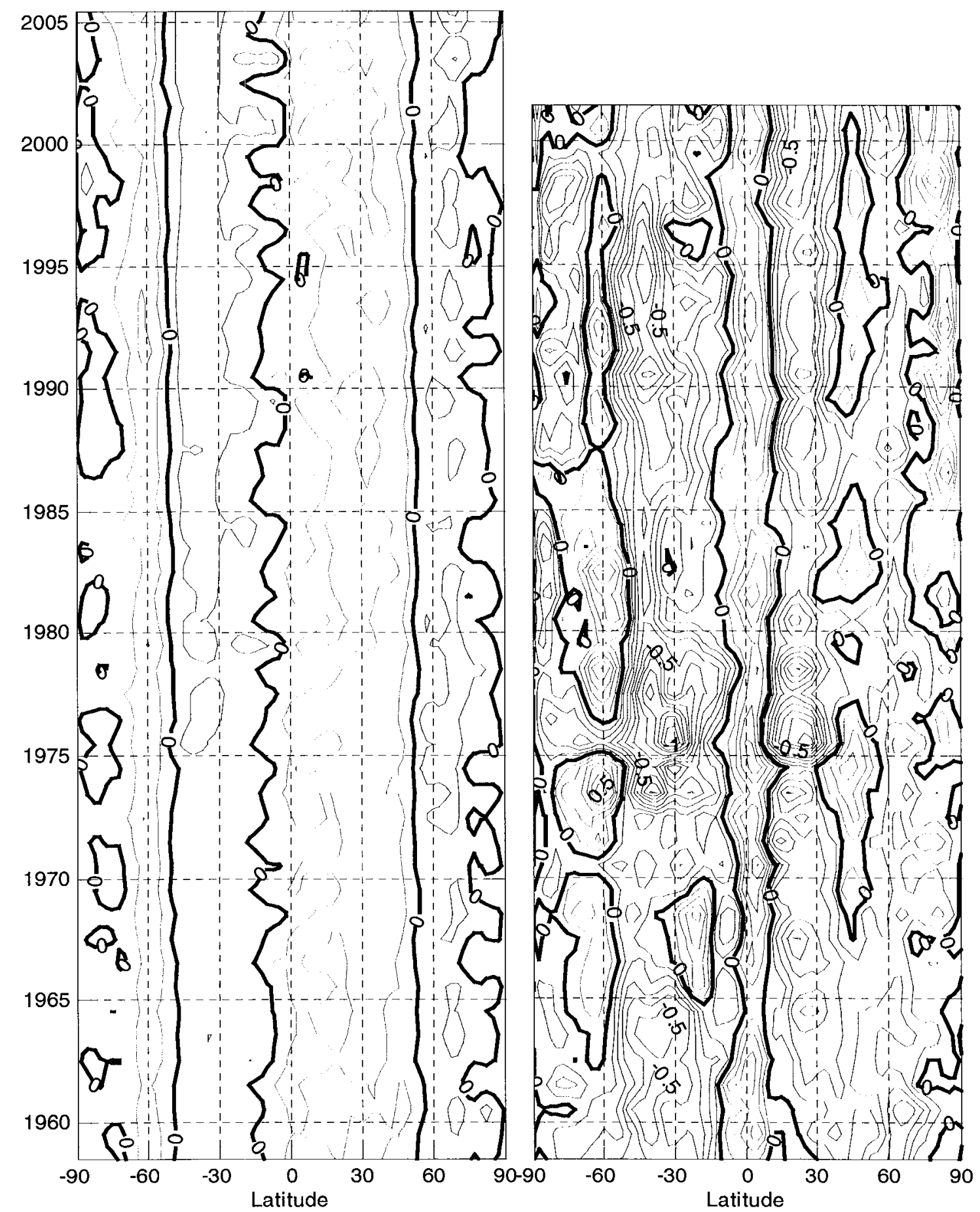

Fig. 6-15. Same as Fig. 6-11 except press: $20 \mathrm{hPa}$. 


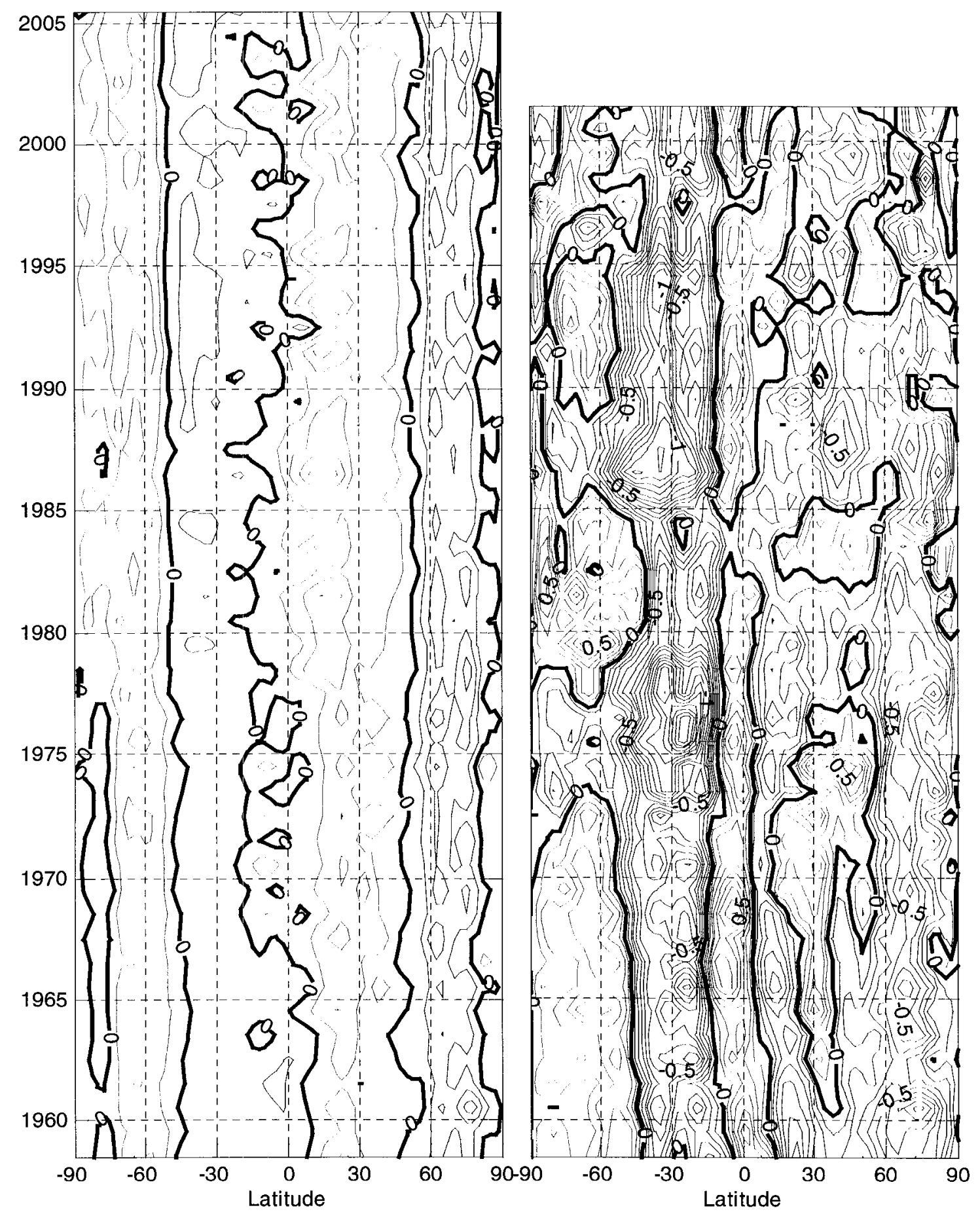

Fig. 6-16. Same as Fig. 6-11 except press: $10 \mathrm{hPa}$. 


\subsection{Summary}

The NCEP1 and ERA-40 reanalyses offer dramatically differing interpretations of the nature of 100 through $10 \mathrm{hPa}$ stratospheric meridional wind speeds. Figure 6-3 instantly illustrates the differences that Section 6 substantiates.

Height vs. latitude projections of NCEP1 meridional wind speed contours are generally vertically stratified at all levels. This reanalysis is also characterized by low wind speeds; at $70 \mathrm{hPa}$ and above no monthly- and zonally-averaged wind speed is greater than $0.2 \mathrm{~m} \mathrm{~s}^{-1}$. At $100 \mathrm{hPa}$ at the Equator a maximum of approximately one $\mathrm{m} \mathrm{s}^{-1}$ exists. At all levels, wind speed contours in time-latitude projections are very temporally consistent over the entire 48-year reanalysis. The only long-term exception is the 1979-2005 intensification of the northerly flow, peaking at approximately one $\mathrm{m} \mathrm{s}^{-1}$ to become the strongest meridional wind speed driven by the steepest meridional gradients in the NCEP1 stratospheric reanalysis. At all pressure levels for all years of the reanalysis, an annually-averaged northerly flow of broad latitudinal extent, $0^{\circ}$ to $50^{\circ} \mathrm{N}$ at its minimum, exists. However, this strong, extended, consistent northerly flow and its implied mean mass transport from Southern Hemisphere to Northern is an unexpected result and introduces serious questions as to the validity of the NCEP1 lower stratospheric meridional wind speed reanalysis.

The ERA-40 lower stratospheric meridional wind speeds exhibit significantly more complex structure in height vs. latitude and time vs. latitude projections. At $70 \mathrm{hPa}$ and above, zonally-averaged wind speeds are generally significantly greater than those of 
NCEP1, reaching $0.92 \mathrm{~m} \mathrm{~s}^{-1}$ southerly at $10 \mathrm{hPa}$. Numerous transient wind speed excursions, possibly anomalous, exist in both projections.

This section demonstrates that the following monthly- and zonally-averaged measures of meridional wind speed as described by both reanalyses:

- Means

- Standard deviations

- Means of the NCEP1 minus ERA-40 difference

- Standard deviations of the NCEP1 minus ERA-40 difference are of the same order of magnitude.

Thus without a third, independent, commensurate data set, it is not possible to objectively describe a meridional wind speed climatology, much less select the most "accurate" reanalysis. ERA-40 might appear to be the preferred reanalysis because of its higher model height $(0.1 \mathrm{hPa}$ vs. $10 \mathrm{hPa})$, its better horizontal resolution, and its use of pressure-level model coordinates in the stratosphere, and NCEP1's northerly mass transport defect. However, ERA-40 suffers from a too strong Brewer-Dobson circulation (Uppala et al. 2005) and from the numerous, possibly anomalous, transients illustrated in this section. 


\subsection{THE FUTURE OF REANALYSIS}

Current reanalysis results give a generally consistent description of the atmosphere over the last 27 years and, with reduced confidence, over the last 49 .

Increased computational speeds and capacities, meteorological research, and operational experience have fostered improvements in forecast models and refinements in assimilation technology. The observational database is becoming more and more accurate and robust. Reanalysis state of the art is maturing but is not yet mature. With the experience gained through the projects discussed in Sections 2.2.1 through 2.2.5 it would seem the time to begin the next-generation of reanalysis.

Kalnay et al. (1996) confidently predicted,

this first phase of reanalysis will be followed by a second phase in which a 1998 state-of-the-art system will be used for a second reanalysis. NCEP plans currently call for an updated reanalysis every 5 years or so. The successive reanalyses will be made easier by the availability of the comprehensive database ... generated by the present reanalysis.

The next year, the "preliminary plans" of Kalnay et al. (1997) were presented at the First WCRP International Conference on Reanalyses. These plans included "Data reprocessing using the first Reanalysis (advanced QC using the metadata collected in ... their archive) and preparation of [a] new global assimilation system" and a "second global reanalysis during 2002-2004." At the same conference Kanamitsu et al. (1997b) discussed at length problems in reanalyses including the "ultimate question": "Is the data assimilation used in NWP best suited for climate analysis?" They also listed the reasons for waiting for "another 4 - 5 years."

- The delay would allow adequate time for user comments and criticism. 
- Another reanalysis with finer resolution, improved physics, and error removal might not provide new information.

- An expectation that the next "drastic improvement" in reanalysis will come from the use of "unconventional data" such as precipitation and 4DVAR assimilation.

The following prediction by Kistler et al. (2001) after the first milestone proposed by Kalnay et al. (1996) had passed was more cautious with an expanded timeline.

NCEP's future reanalysis plans, if supported, call for an updated global reanalysis using a state-of-the-art system every 10 years or so, and a maintenance of a CDAS allowing analysis of current climate anomalies. Within this timescale major improvements in the operational global system should take place, making the previous reanalysis further away from the state of the art, and justifying such major effort. Future reanalyses will be greatly facilitated by the quality-controlled, comprehensive observational database created by the present reanalysis, so that the development and execution of new global reanalyses should be completed in four to five years.

Using as a reference the beginning of NCEP1 production in April 1994,

- the first prediction is now nine years overdue (Kalnay et al. 1996),

- the second, three to five years overdue (Kalnay et al. 1997),

- the third, four to six years overdue (Kanamitsu et al. 1997b).

In October 2006 Dr. Masao Kanamitsu stated that he believed that neither NCEP nor the National Weather Service were currently "interested in performing any form of reanalysis." Dr. Wesley Ebisuzaki, whom Kanamitsu believes "is responsible for the continuation of the two reanalyses [NCEP1 and NCEP2 ] to the present time, but he is not managing the program," pointed out that NCEP1, NCEP2, and the North American Regional Reanalysis (NARR), discussed in Section 2.2.8, were all funded with "soft money" and internal support. Ebisuzaki also stated that in the future "People want to get away from the 3-5 year special funding model into a line-item budget. With a line-item 
approach, the funding would be stable and more basic research and development could be attempted." However, Kanamitsu felt that such a potential national reanalysis project combining NOAA, NASA, National Science Foundation (NSF), and DOE and headed by Dr. Philip Arkin of the Earth System Science Interdisciplinary Center of the University of Maryland "was not going anywhere at present." Arkin said "there is some planning at NOAA. However, there isn't anything published or formal." (M. Kanamitsu 2006; W. Ebisuzaki 2006; P. Arkin 2006; personal communications). The summary and recommendations of a workshop on "a U. S. National Program for Ongoing Analysis of the Climate System" sponsored by NOAA, NASA, and NSF in 2003 provided an overview of a possible national program. Included in the workshop report are recommendations for continuing reanalyses (Arkin et al. 2006).

It appears obvious to the author of this thesis that the Republican administration in the United States that began in 2001 did not and does not look with favor on a new, state-of-the-art reanalysis by NCEP, which could be employed to further analyze and quantify global climate change. Until it recently reversed its position, the Republican administration insisted that global climate change was not real. In such an environment even the unbelievable drive and focus of Eugenia Kalnay was not able to prevail.

This author, having personally sailed through the Pacific doldrums twice and able to recognize "a state of dead calms and light fluctuating breezes" when he sees them, has the sense that the state of multi-decade global reanalysis in the United States is in the doldrums. Having been carried to the Intertropical Convergence Zone (ITCZ) by brisk trade winds of enthusiasm from 1988 to 2000 , the future of reanalysis now seems adrift. 
However, while the United States in general and NCEP in particular suffers though a long dark night, reanalysis is alive and well in the European Community. The comprehensive ERA-40 overview of Uppala et al. (2005) concludes with a concise paragraph describing ECMWF's plans for future reanalyses. The ERA-40 reanalysis will

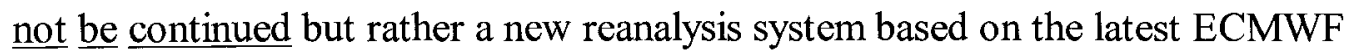
operational assimilation system will be developed. Initial tests of this new system show that several ERA-40 problems have been significantly reduced or eliminated. This new reanalysis system will be used as a test bed for testing new versions of the reanalysis system, adding new atmospheric constituents, and supporting work with older observational data. An "interim" reanalysis from 1989 onward in near real time, promised by Uppala et al. (2005), had "just started" in October 2006, with completion anticipated "late next year" (A. J. Simmons 2006, personal communication). Uppala et al. (2005) predict that the research phase "should lead to a capability for an extended re-analysis to succeed ERA-40 toward the end of this decade." 


\subsection{CONCLUSIONS}

This thesis identified five major global reanalyses: NCEP1, NCEP2, ERA-15, ERA-40, and JRA-25. NCEP2 is a modestly updated and corrected version of NCEP1, which corrected a number of NCEP1 problems, primarily in the boundary layer, but added others, especially in the stratosphere. ERA-15 covers only the years from 1979 through 1993. JRA-25's output was not available in time for use in this thesis.

Thus only two of these reanalyses are considered in this thesis: NCEP1 (January 1948 through the present) and ERA-40 (September 1957 through August 2002). These two have reanalyzed 44 calendar years in common. Comparison of only two reanalyses required commensurate comparison data from METO, FUB, RATPAC, and TLS.

NCEP1 was a ground-breaking project. It produced what today might be considered a pedestrian reanalysis. NCEP1 represents 1994 state of the forecast model and assimilation art, constrained by early 1990's "made in USA" computer capabilities.

ERA-40, in contrast, was a true second-generation project. It benefited from seven years of meteorological research and operational experience, from a seven-year growth in computational capability, the lessons of NCEP1 and NCEP2, and the in-house experience of ECMWF's first-generation pilot project, ERA-15. Many of the forecast model advancements of the early 2000 s contributed to improved characterization of the stratosphere. It would seem that ECMWF “did everything right." However, some stumbles, minor in scope but major in consequence, accompanied this great leap forward. Because of three of the "largest apparent biases" identified by Randel et al. (2002, 2004): "warm tropical tropopause" and the "satellite data discontinuity across 1978-79" 
(NCEP1), and "oscillatory vertical structure in temperature, especially large over Antarctica" (ERA-40), temperature data from both reanalyses must be treated with caution. These behaviors have repeatedly been identified in the different views of this thesis; care must be exercised in comparing GCM temperature output with either reanalysis.

Table 8-1 contains the recommendations of the author of this thesis for application of NCEP1 and ERA-40 temperature reanalysis data as a function of pressure level and latitude. The $\mathbf{N}^{\star *}$ limitations at $100 \mathrm{hPa}$ result from the NCEP1 "warm tropical tropopause" and "satellite data discontinuity across 1978-1979" (Randel et al. 2002, 2004) which, as Fig. 4-25 shows, persist until 2000. Absence of satellite radiance data prior to the modern satellite era in a region of minimal radiosonde coverage contributes to the $\mathbf{N}^{*}$ (NCEP1) limitation south of $30^{\circ} \mathrm{N}$ at $10 \mathrm{hPa}$ and the $\mathbf{E}^{*}$ (ERA-40) restrictions in latitudes $90^{\circ} \mathrm{S}-30^{\circ} \mathrm{S}$. The ERA- $4090^{\circ} \mathrm{S}-30^{\circ} \mathrm{S} X$ is a result of the "oscillatory vertical structure in temperature, especially large over Antarctica" (Randel et al. 2002, 2004). 
Table 8-1. Recommended reanalysis temperature usage.

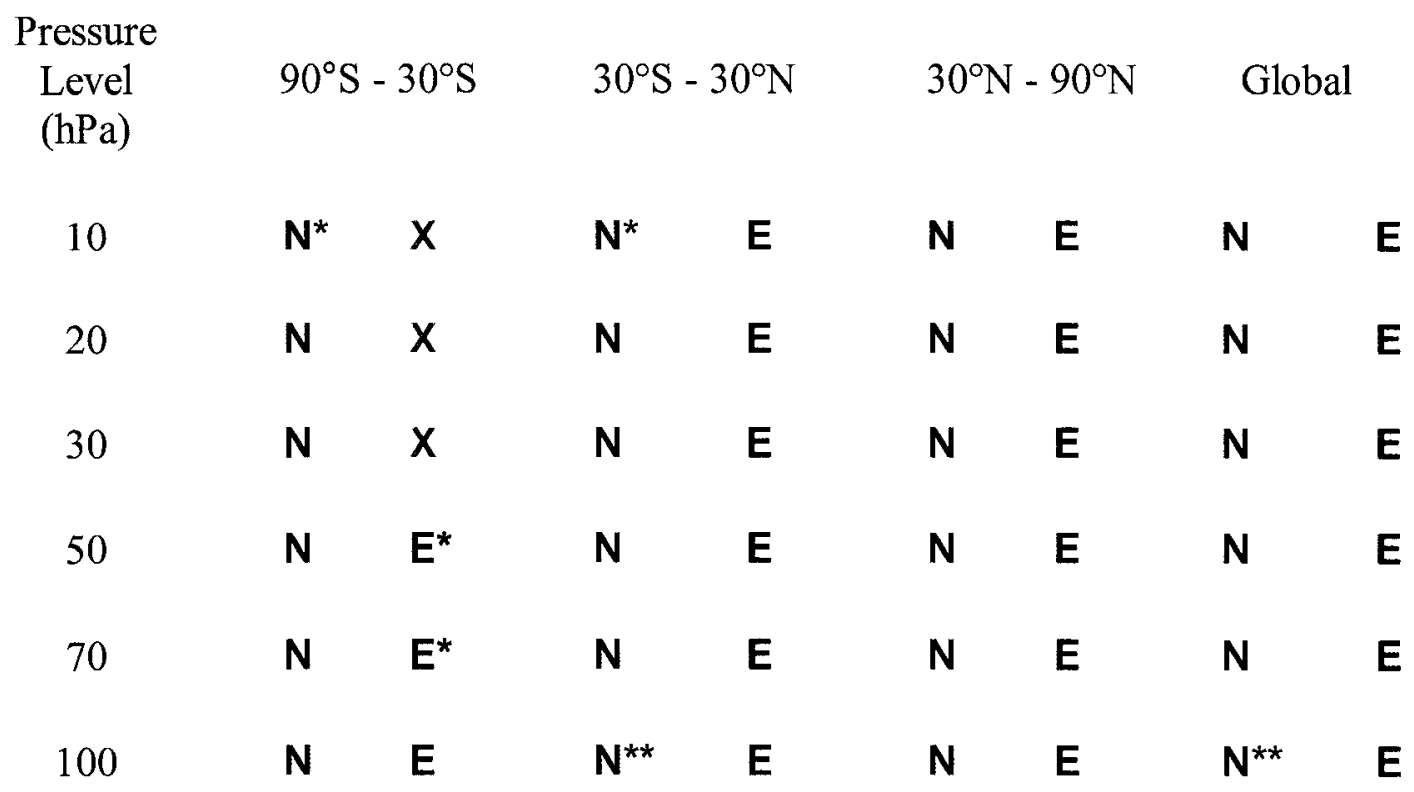
X ERA-40 not recommended
N NCEP1 recommended
E ERA-40 recommended
$\mathbf{N}^{*} \quad$ NCEP1 use with caution before 1980
$E^{*} \quad$ ERA-40 do not use before 1975
$\mathbf{N}^{\star *} \quad$ NCEP1 do not use from 1978-2000

Agreement between NCEP1 and ERA-40 zonal wind speeds is good in 1958-78 and excellent in 1979-2001 except in the Tropics. For lower stratospheric climatology, either reanalysis is acceptable. ERA-40's characterization of the QBO is much closer to FUB radiosonde data than NCEP1, so although most GCMs do not spontaneously generate a $\mathrm{QBO}$, this fidelity is of value in evaluating QBO simulations in those GCM's that do.

Temperature and zonal wind speed trends in polar stratospheric regions during the modern satellite era of 1979-2001 are almost everywhere statistically insignificant 
because of their small (23-year) sample size and the large variance in reanalysis data. In the vicinity of the Northern Hemisphere polar vortex core, cooling trends exist except for a strong warming trend in December; at the Southern Hemisphere core, cooling trends also predominate except for slight, localized warming trends in August through October. Again in the vicinity of the Northern Hemisphere polar vortex core, increasing zonal winds speed trends are slightly positive in October, strongly positive in February and March, and moderately negative from November through January. At the Southern Hemisphere core, considerable inconsistency exists between NCEP1 and ERA-40; a slightly decreasing zonal wind speed trend in June is the only consistent result.

The application of a simple temperature reversal algorithm demonstrates the efficacy of employing monthly-averaged data to detect occurrences of stratospheric sudden warmings (SSWs), phenomena whose existence is often measured in days. This technique could be applied to the monthly output of a GCM. Because they result from displacement and not splitting, Canadian warmings are detected with a $25-30 \%$ success rate; major midwinter warmings a $75-81 \%$ rate, final warmings a $100 \%$ rate, but $26-30 \%$ of "detections" prove to be false. Daily zonal wind speed and temperature data from GCMs must be analyzed to determine if the formal SSW criteria of the WMO are met.

Considerable differences exist between the two reanalyses of meridional wind speed. NCEP1 exhibits a mean mass transport problem from Southern to Northern Hemisphere throughout the year in the entire stratosphere, replicating the results of Pawson and Fiorino (1998a). ERA-40 shows better transport balance, much more structure in pressure level vs. latitude and time vs. latitude plots, but a known, too strong 
Brewer-Dobson circulation. Further analysis including comparisons with METO and JRA-25 may lead to a definitive climatology.

GCM vs. reanalysis comparisons that differ by amounts shown in Tables 4-2 (temperature) and 5-2 (zonal wind speed) may be considered consistent with the two reanalyses. The most accurate comparisons with reanalysis data may be obtained by repeating the analyses of this thesis, in particular those of Sections 4.1,4.2, 5.1, 5.2, and 5.4, comparing the GCM with both NCEP1 and ERA-40.

Future work with existing reanalysis and comparison data could include:

- Comparison of NCEP1 and ERA-40 stratospheric temperature, zonal and meridional wind speeds, and geopotential heights with those of JRA-25, a full set of METO data, and ERA-Interim. This comparison should extend to the highest levels available, currently $0.3 \mathrm{hPa}$.

- Investigations of annular mode analyses, including the Northern and Southern (Hemisphere) Annular Modes (NAM and SAM) with NCEP1, ERA-40, JRA-25, METO, and ERA-Interim.

- Exploration of stratospheric to tropospheric forcing in ERA-40, JRA-25, METO data, and ERA-Interim.

When the ERA-Interim reanalysis becomes available, its results can be included in the three areas outlined above. Completion is expected late in 2007.

For the San Jose State University Meteorology Department to become a center of middle atmosphere research, one or more courses in this area must be created and offered. The critical mass for such a course or courses currently exists in the faculty, graduate student body, and current research. 


\section{REFERENCES}

Andersson, E., and Coauthors, 1998: The ECMWF implementation of three-dimensional variational assimilation (3D-Var). III: Experimental results. Quart. J. Roy. Meteor. Soc., 124, 1831-1860.

Arkin, P. E., E. Kalnay, J. Laver, S. Schubert, and K. Trenberth, cited 2006: Ongoing analysis of the climate system: A workshop report. NASA, NOAA, and NSF, $48 \mathrm{pp}$. [Available online at www.ofps.ucar.edu/joss_psg/meetings/climatesystem/FinalWorkshopReport.pdf.]

Baldwin, M. P., and T. J. Dunkerton, 1999: Propagation of the Arctic Oscillation from the stratosphere to the troposphere. J. Geophys. Res., 104, 30 937-30946.

$\longrightarrow$, and _ 2001: Stratospheric harbingers of anomalous weather regimes. Science, 294, 581-584.

- - and L. J. Gray, 2005: Tropical stratosphere zonal winds in ECMWF ERA-40 reanalysis, rocketsonde data, and rawinsonde data. Geophys. Res. Lett., 32, L09806, doi:10.1029/2004GL022328.

— , and Coauthors, 2001: The quasi-biennial oscillation. Rev. Geophys., 39, 179-229.

—, T. Hirooko, A. ONeill, S. Yoden, A. J. Charlton, Y. Hio, W. A. Lahoz, and A. Mori, cited 2006: Major stratospheric warming in the Southern Hemisphere in 2002: Dynamical aspects of the ozone hole split. [Available online at http://aero.jussieu.fr/ sparc/News20/20_Baldwin.]

Bengtsson, L., and J. Shukla, 1988: Integration of space and in situ observations to study global climate change. Bull. Amer. Meteor. Soc, 69, 1130-1143.

Black, R. X., 2002: Stratospheric forcing of surface climate in the Arctic Oscillation. J. Climate, 15, 268-277.

Bromwich, D. H., and R. L. Fogt, 2004: Strong trends in the skill of the ERA-40 and NCEP-NCAR reanalyses in the high and midlatitudes of the Southern Hemisphere, 1958-2001. J. Climate, 17, 4603-4619.

Brooks, F. P., Jr., 1975: The Mythical Man Month. Addison Wesley, 195 pp.

Chan, J. C. L., 1995: Tropical cyclone activity in the western North Pacific in relation to the stratospheric quasi-biennial oscillation. Mon. Wea. Rev., 123, 2567-2571.

Charlton, A. J., and L. M. Polvani, 2007: A new look at stratospheric sudden warmings. Part I: Climatology and modeling benchmarks. J. Climate, 20, 449-469.

- A. O'Neill, W. A. Lahoz, P. Berrisford, 2005: The splitting of the stratospheric polar vortex in the Southern Hemisphere, September 2002: Dynamical evolution. J. Atmos. Sci., 62, 590-602. 
Coughlin, K., and K. -K. Tung, 2001: QBO signal found at the extratropical surface through Northern Annular modes. Geophys. Res. Lett., 28, 4563-4566.

Courtier, P., 1998: The ECMWF implementation of the three-dimensional variational assimilation (3D-Var) I: Formulation. Quart. J. Roy. Meteor. Soc., 124, 1783-1808.

Dethof, A., and E. Hólm, cited 2006: ERA-40 Project Report Series: 4. Ozone in ERA-40: 1991-1996. [Available online at http://www.ecmwf.int/publications.]

Dubois, P., 1993: Das observatorium Lindenberg in seinen ersten 50 jahren 1905-1955 (The Lindenberg observatory in its first 50 years 1905-1955). Geschichte der Meteorologie in Deutschland, $\mathbf{1}$, Selbstverlag des Deutschen Wetterdienstes.

Duchon, C. E., 1979: Lanczos filtering in one and two dimensions. J. Appl. Meteor., 18, 1016-1022.

Durre, I., R. Vose, and D. Wertz, 2004: Overview of the Integrated Global Radiosonde Archive. J. Climate, 19, 53-68.

Ebisuzaki, W., and R. Kistler, 1999: An examination of a data-constrained assimilation. Proc. of the Second WCRP International Conf. on Reanalyses 23-28 August 1999, Wokefield Park, Reading, UK, World Climate Research Programme, 14-17.

ECMWF, cited 2006: Userguide to ECMWF forecast products, Appendix A: 4DVAR an elementary introduction. [Available online at http:/www/ecmwf.int/products/forecasts/guide/index.html.]

Eskridge, R. E., O. A. Alduchov, I. V. Chernykh, P. Zhai, A. C. Polansky, and S. R. Doty, 1995: A Comprehensive Aerological Reference Data Set (CARDS): Rough and systematic errors. Bull. Amer. Meteor. Soc., 76, 1759-1775.

Free, M., D. J. Seidel, J. K. Angell, J. Lanzante, I. Durre, T. C. Peterson, 2005: Radiosonde Atmospheric Temperature Products for Assessing Climate (RATPAC): A new dataset of large-area anomaly time series. J. Geophys. Res., 110, D22101, doi:10.1029/2005JD006169.

Gibson, J. K., P. Kållberg, S. Uppala, A. Hernandez, A. Nomura, E. Serrano, cited 2006: ECMWF Re-Analysis Project report series: 1. ERA-15 description (Version 2 May 1999). [Available online at http://www.ecmwf.int/publications.]

Gillett, N. P., M. P. Baldwin, D. W. J. Thompson, E. F. Shuckburgh, W. A. Norton, and J. L. Neu, cited 2006: Report of SPARC Workshop on the role of the stratosphere in tropospheric climate. [Available online at http://www.atmosp.physics.utoronto.ca/SPARC/News21/21_Gillett.html.]

Hamilton, K., A. Hertzog, F. Vial, and G. Stenchikov, 2004: Longitudinal variation of the stratospheric quasi-biennial oscillation. J. Atmos. Sci., 61, 383-402.

Haynes, P., cited 2006: Stratosphere-Troposphere dynamical coupling. [Available online at http://www.atmosp.physics.utoronto.ca/SPARC/Newsletter25.pdf.] 
Holton, J. R., 2004: An Introduction to Dynamic Meteorology. 4th ed., Elsevier Academic Press, 529 pp.

- and H. -C. Tan, 1980: The influence of the equatorial quasi-biennial oscillation on the global circulation at $50 \mathrm{mb}$. J. Atmos. Sci., 37, 2200-2208.

Hsu, K. J., and Y. L. Yung, 1999: Ozone trend over Taiwan from TOMS data. Terrestrial and Oceanic Sciences, 10, 619-632.

Huesmann, A. S., and M. H. Hitchman, 2003: The 1978 shift in the NCEP reanalysis stratospheric quasi-biennial oscillation. Geophys. Res. Lett., 30(2), 1048, doi:10.1029/2002GL016323.

IPCC, cited 2006: Climate Change 2001: Working Group 1: The Scientific Basis. [Available on line at http://groupsites.ius.edu/physics/ kyle/P310/[PCCSum2001.pdf.]

JRA-25, cited 2006: Preliminary categories and elements of our products. [Available online at http://cdp1.kishu.go.jp/Exp/dat category e.html.]

JRA-25 Working Group, cited 2006: Japanese 25-year reanalysis plan (2001). [Available online at http://www.jreap.org/publications/plan/jra25plan_e.pdf.]

Kållberg, P., A. Simmons, S. Uppala, and M. Fuentes, cited 2006: ERA-40 Project Report Series: 17. The ERA-40 archive. [Available online at http://www.ecmwf.int/publications.]

Kalnay, E., and R. Jenne, 1991: Summary of the NMC/NCAR reanalysis workshop of April 1991. Bull. Amer. Meteor. Soc., 72, 1897-1904.

- , and Coauthors, 1996: The NCEP/NCAR 40-year reanalysis project. Bull. Amer. Meteor. Soc., 77, 437-471.

- R. Kistler, and M. Kanamitsu, 1997: NCEP/NCAR 40-year reanalysis overview. Proc. of the First WCRP International Conf. on Reanalyses 27-31 October 1997, Silver Springs, MD, World Climate Research Programme, 1-7.

Kanamitsu, M., 1989: Description of the NMC global data assimilation and forecast system. Wea. Forecasting, 4, 335-342.

—, cited 2006: NCEP reanalysis status and US national reanalysis program plan. ECMWF Workshop on Re-analysis 5-9 November 2001, Reading, UK, ECMWF, 423-427. [Available online at www.ecmwf.int/publications/library/ecpublications/proceedings/ERA40reanalysis_workshop/index.html.]

- , R. E. Kistler, and R. W. Reynolds, 1997a: NCEP/NCAR reanalysis and the use of satellite data. Adv. Space Res., 19, 481-489. 
- E. Kalnay, R. Kistler, J. Woollen, W. Ebisuzaki, G. Potter, and M. Fiorino, 1997b: The NCEP/NCAR reanalysis - future plans. Proc. of the First WCRP International Conf. on Reanalyses 27-31 October 1997, Silver Springs, MD, World Climate Research Programme, 410-413.

, W. Ebisuzaki, J. Woollen, J. Potter, and M. Fiorino, 1999: Overview of NCEP/DOE reanalysis-2. Proc. of the Second WCRP International Conf. on Reanalyses 23-28 August 1999, Wokefield Park, Reading, UK, World Climate Research Programme, 1-4.

—, W. Ebisuzaki, J. Woollen, S. K. Yang, J. J. Hnilo, M. Fiorino, and G. L. Potter, 2002: NCEP-DOE AMIP-II reanalysis (R-2). Bull. Amer. Meteor. Soc., 83, $1631-1643$.

Karl, T. R., and Coauthors, 1995: Critical issues for long-term climate monitoring. Climate Change, 31, 185-221.

Kidder, S. Q., and T. H. Vonder Haar, 1995: Satellite Meteorology: An Introduction. Academic Press, 466 pp.

Kistler, R., and Coauthors, 2001: The NCEP-NCAR 50-Year Reanalysis: Monthly means CD-ROM and documentation. Bull. Amer. Meteor. Soc., 82, 247-267.

Labitzke, K. G., and H. van Loon, 1999: The Stratosphere. Springer-Verlag, 169 pp.

— [Available online at http//www.atmosp.utoronto.ca/SPARC/News 15/15Labitzke.html.]

- , and Coauthors, cited 2006: The Berlin stratospheric data series. [Available online at http://strat27.met.fu-berlin.de/products/cdrom/documents/BerlinDataSeries.pdf.]

Lanzante, J. R., 1996: Resistant, robust, and non-parametric techniques for the analysis of climate data: Theory and examples, including applications to historical radiosonde station data. Int. J. Climatol., 16, 1197-1226.

$\longrightarrow$, S. A. Klein, and D. J. Seidel, 2003a: Temporal homogenization of monthly radiosonde data. Part I: Methodology. J. Climate, 16, 224-239.

—, S. A. Klein, and D. J. Seidel, 2003b: Temporal homogenization of monthly radiosonde data. Part II: Trends, sensitivities, and MSU comparison. J. Climate, 16, 241-262.

Li, X., S. Uppala, J. K. Gibson, and R. W. Saunders, 1999: The use of VTPR-1c data in ERA-40, Part 1: Pre-processing. Proc. of the Second WCRP International Conf. on Reanalyses 23-28 August 1999, Wokefield Park, Reading, UK, World Climate Research Programme, 69-72.

Lorenc, A. C., and Coauthors, 2000: The Met. Office global three-dimensional variational data assimilation scheme. Quart. J. Roy. Meteor. Soc., 126, 2991-3012. 
Manney, G. L., J. L. Sabutis, S. Pawson, M. L. Santee, B. Naujokat, R. Swinbank, M. E. Gelman, and W. Ebisuzaki, 2003: Lower stratospheric temperature differences between meteorological analyses in two cold Arctic winters and their impact on polar processing studies. J. Geophys. Res., 108, 8328, doi:10.1029/2001JD001149.

- and Coauthors, 2005: Diagnostic comparison of meteorological analyses during the 2002 Antarctic winter. Mon. Wea. Rev., 133, 1261-1278.

Marshall, G. J., 2002: Trends in Antarctic geopotential height and temperature: A comparison between radiosonde and NCEP-NCAR reanalysis data. J. Climate, 15, $659-674$.

McNally, A. P., cited 2006: The assimilation of stratospheric satellite data at ECMWF. Proceedings of ECMWF/SPARC Workshop on Modelling and Assimilation for the Stratosphere and Tropopause, June 2003, Reading, UK, ECMWF, 103-106. [Available online at http://www.ecmwf.int/publications/library/ecpublications/_pdf/workshop/2003/ Stratosphere/ws_stratosphere_monally.pdf.]

McPherson, R. D., 1994: The National Centers for Environmental Prediction: Operational climate, ocean, and weather prediction for the 21 st century. Bull. Amer. Meteor. Soc., 75, 363-373.

MERRA, cited 2006: Introduction to MERRA. [Available online at http://gmao.gsfc.nasa.gov/research/merra/intro.php.]

Mesinger, F., and Coauthors, cited 2006: NCEP regional reanalysis. NARR Users Workshop 11 January 2005, San Diego, CA. [Available online at http://wwwt.emc.ncep.noaa.gov/mmb/rreanl/narr/ptt.]

Mo, K. C., X. L. Wang, R. Kistler, M. Kanamitsu, and E. Kalnay, 1995: Impact of satellite data on the CDAS-Reanalysis system. Mon. Wea. Rev., 123, 124-139.

Nathan, T. R., and E. C. Cordero, 2007: An ozone-modified refractive index for vertically propagating planetary waves. $J$. Geophys Res., 112, D02105, doi:10.1029/2006JD007357.

National Research Council, 1999: Adequacy of Climate Observing Systems. National Academy Press, $51 \mathrm{pp}$.

Naujokat, B., 1986: An update of the observed quasi-biennial oscillation of the stratospheric winds over the tropics. J. Atmos. Sci., 43, 1873-1877.

NCAR/MMM, cited 2006: Overview of NCAR/MMM's 3DVAR system. [Available online at http://www.mmm.ucar.edu/mm53dvar/docs/3dvar_overview.htm.]

Palmer, T. N., 1981: Diagnostic study of a wavenumber-2 stratospheric sudden warming in a transformed Eulerian-mean formalism. J. Atmos. Sci., 38, 844-855. 
Pascoe, C. L., L. J. Gray, S. A. Crooks, M. N. Juckes, and M. P. Baldwin, 2005: The quasi-biennial oscillation: Analysis using ERA-40 data. J. Geophys. Res., 110, D08105, doi:10.1029/2004JD004941.

Pawson, S., 2000: The GCM-Reality Intercomparison Project for SPARC (GRIPS): Scientific issues and initial results. Bull. Amer. Meteor. Soc., 81, 781-796.

- and M. Fiorino, 1998a: A comparison of reanalyses in the tropical stratosphere. Part 1: Thermal structure and the annual cycle. Clim. Dyn., 14, 631-644.

and - 1998b: A comparison of reanalyses in the tropical stratosphere. Part 2: The quasi-biennial oscillation. Clim. Dyn., 14, 645-658.

—_ and _ 1999: A comparison of reanalyses in the tropical stratosphere. Part 3: Inclusion of the pre-satellite data era. Clim. Dyn., 15, 241-250.

Randel, W. J., and F. Wu, 1999: Cooling of the Arctic and Antarctic polar stratospheres due to ozone depletion. J. Climate, 12, 1467-1479.

$\longrightarrow$, and —-, 2006: Biases in stratospheric temperature trends derived from historical radiosonde data. $J$. Climate, 19, 2094-2104.

—, M.-L. Chanin, and C. Michaut, 2002: SPARC intercomparison of middle atmosphere climatologies, SPARC Report, WCRP 116, WMO/TD No. 1142, 96 pp.

—_, and Coauthors, 2004: The SPARC intercomparison of middle-atmosphere climatologies. J. Climate, 17, 986-1003.

Remote Sensing Systems, cited 2006: Description of MSU and AMSU data products. [Available online at http://www.remss.com.]

Rood, R. B., A. Y. Hou, S. Pawson, and S. D. Schubert, cited 2006: Reanalysis efforts in the United States: NASA and national reanalysis program. ECMWF Workshop on Reanalysis 5-9 November 2001, Reading, UK, ECMWF, 437-443. [Available online at www.ecmwf.int/publications/library/ecpublications/proceedings/

ERA-40reanalysis_workshop/index.html.]

Sakamoto, M., S. Kobayashi, K. Kato, T. Matsumoto, H. Koide, K. Onogi, T. Ose, and H. Hatsushika, cited 2006a: Ongoing Japanese long-term reanalysis project (JRA-25); assimilation of NOAA polar-orbiter satellite sounder data. [Available online at http://ams.confex.com/ams.pdfpapers/84537.pdf.]

long-term reanalysis project (JRA-25); assimilation of NOAA polar-orbiter satellite sounder data. Abstract, AMS Symp. on Integrated Observing and Assimilation Systems for Atmosphere, Oceans, and Land Surface, San Diego, CA, Amer. Meteor. Soc., Poster Session 1. 
Santer, B. D., T. M. L. Wigley, J. S. Boyle, D. J. Gadden, J. J. Hnilo, D. Nychka, D. E. Parker, and K. E. Taylor, 2000: Statistical significance of trends and trend differences in layer-average atmospheric temperature time series. J. Geophys. Res., 105, 7337-7356.

Schoeberl, M. R., A. R. Douglass, Z. Zhu, S. Pawson, 2003: A comparison of the lower stratospheric age spectra derived from a global circulation model and two data assimilation systems. J. Geophys. Res., 108(D3), doi:10.1029/2002JD002652.

Schubert, S. D., 1997: The GEOS-1 Reanalysis: Overview. Proc. of the First WCRP International Conf. on Reanalyses 27-31 October 1997, Silver Springs, MD, World Climate Research Programme, 8-11.

Overview and results. NASA Tech. Rep. series on global modelling and data assimilation, Nr. 6. Ed. M J. Suarez. NASA, Goddard Flight Center, Greenbelt, MD, $201 \mathrm{pp}$.

Seaman, R., and T. Hart, 2003: The history of PAOBs in the Bureau of Meteorology. Aust. Meteor. Mag., 52, 241-250.

Seidel, D. J., and Coauthors, 2004: Uncertainty in signals of large-scale climate variations in radiosonde and satellite upper-air temperature datasets. J. Climate, 17, $2225-2240$.

Simmons, A. J., cited 2006a: Development of the ERA-40 data assimilation system. ECMWF Workshop on Re-analysis 5-9 November 2001, Reading, UK, ECMWF, 11-30. [Available online at www.ecmwf.int/publications/library/ecpublications/proceedings/ ERA-40reanalysis_workshop/index.html.]

- cited 2006b: Representation of the stratosphere in ECMWF operations and ERA-40. Proceedings of ECMWF/SPARC Workshop on Modelling and Assimilation for the Stratosphere and Tropopause, June 2003, Reading, UK, ECMWF, 1-17. [Available online at http://www.ecmwf.int/publications/library/ecpublications/_pdf/workshop/2003/ Stratosphere/ws_stratosphere_simmons.pdf.]

—, and J K. Gibson, cited 2006: ERA-40 Project Report Series: 1. The ERA-40 Project Plan. [Available online at http://www.ecmwf.int/publications.]

Sterl, A., 2004: On the (in)homogeneity of reanalysis products. J. Climate, 17, 3866-3873.

Thompson, D. W. J., and S. Solomon, 2002: Interpretation of recent Southern Hemisphere climate change. Science, 296, 895-899.

- M. P. Baldwin, and J. M. Wallace, 2002: Stratospheric connection to the Northern Hemisphere wintertime weather: Implications for prediction. J. Climate, 15, 1421-1428. 
Trenberth, K. E., and J. G. Olson: 1988: An evaluation and intercomparison of global analyses from the National Meteorological Center and the European Centre for Medium Range Weather Forecasts. Bull. Amer. Meteor. Soc., 69, 1047-1057.

—, and D. P. Stepaniak, 2002: A pathological problem with NCEP reanalysis in the stratosphere. J. Climate, 15, 690-695.

UK Met Office, cited 2006: Stratospheric Analysis. [Available at http://www.metoffice.com/research/stratosphere/modelling/analysis.html.]

U. S. Government Printing Office, 1976: U. S. Standard Atmosphere, 1976. U. S. Government Printing Office, $227 \mathrm{pp}$.

Uppala, S., J. K. Gibson, M. Fiorino, A. Hernandez, P. Kållberg, X. Li, K. Onogi, and S. Sardine, 1999: ECMWF second generation reanalysis - ERA-40. Proc. of the Second WCRP International Conf. on Reanalyses 23-28 August 1999, Wokefield Park, Reading, UK, World Climate Research Programme, 9-13.

- and Coauthors, 2005: The ERA-40 re-analysis. Quart. J. Roy. Meteor. Soc., 131, 2961-3012.

Waugh, D. W., 1997: Elliptical diagnostics of stratospheric polar vortices. Quart. J. Roy. Meteor. Soc., 123, 1725-1784.

Waugh, D. W., and W. J. Randel, 1999: Climatology of Arctic and Antarctic polar vortices using elliptical diagnostics. J. Atmos. Sci., 56, 1594-1613.

Wilks, D. S., 1995: Statistical Methods in the Atmospheric Sciences. Academic Press, $467 \mathrm{pp}$.

Woollen, J. S., and Y. Zhu, 1997: The NCEP/NCAR observation archive, 1957-1997. Proc. of the First WCRP International Conf. on Reanalyses 27-31 October 1997, Silver Springs, MD, World Climate Research Programme, 402-405. 
APPENDICES

\section{Appendix A Acronyms}

3DVAR

4DVAR

AMIP

AMS

AMSU

AMV

ANOVA

ATOVS

BAMS

BSDS

CARDS

CDAS

CFC

CLIVAR

CMAP

CMV

CMW

COADS

COBE

COLA

$\mathrm{CPC}$

CRIEPI

DAO

DJF

DOE

ECMWF

EDAS

EMC

EP

EQ

ERA-15

ERA-40
Three-dimensional variational assimilation

Four-dimensional variational assimilation

Atmospheric Model Intercomparison Project

American Meteorological Society

Advanced Microwave Sounding Unit

Atmospheric Motion Vector

ANalysis Of VAriance

Advanced TIROS Operational Vertical Sounder

Bulletin of the American Meteorological Society

Berlin Stratospheric Data Series

Comprehensive Aerological Reference Data Set

Climate Data Assimilation System

Chlorofluorocarbon

CLImate VARiability and Predictability

CPC Merged Analysis of Precipitation

Cloud Motion Vector

Cloud Motion Wind

Comprehensive Ocean Atmosphere Data Set

Centennial in-situ Observation-Based Estimates

Center for Ocean-Land-Atmosphere Interactions

Climate Prediction Center (of NCEP)

Central Research Institute of Electrical Power Industry (Japan)

Data Assimilation Office (of NASA)

December, January, and February

Department of Energy

European Centre for Medium-Range Weather Forecasts

Eta Data Assimilation System

Environmental Modeling Center (of NCEP)

Eliassen-Palm

Equator

ECMWF 15-year (1979-93) reanalysis

ECMWF 45-year (1958-2002) reanalysis 
ERS

ESE

ESSIC

FD

FFT

FGAT

FGGE

FUB

GARP

GASP

GCM

GDAS

GEOS

GMAO

GMS

GOES

GPS

GRIPS

GTS

IDL

IFFT

IGRA

IPCC

ITCZ

JJA

JMA

JRA-25

LKS

LLNL

LTS

MAM

MATLAB

MERRA

METEOSAT

METO
Earth Resources Satellite

Extreme Stratospheric Event

Earth System Science Interdisciplinary Center (Univ. of Maryland)

First Difference

Fast Fourier Transform

First Guess at Appropriate Time

First GARP Global Experiment

Institut für Meteorologie, Freie Universität Berlin, Germany

Global Atmospheric Research Program

Global Atmospheric Sampling Program

Global Climate Model

Global Data Assimilation System

Goddard Earth Observing System (NASA)

Global Modeling and Assimilation Office (of NASA)

Geostationary Meteorological Satellite (Japan)

Geostationary Operational Environmental Satellite

Global Positioning System

GCM-Reality Intercomparison Project for SPARC

Global Telecommunications System (of the WMO)

Interactive Development Language

Inverse Fast Fourier Transform

Integrated Global Radiosonde Archive

Intergovernmental Panel on Climate Change

InterTropical Convergence Zone

June, July, and August

Japan Metrological Agency

Japanese 25-year (1979-2004) reanalysis

Lanzante Klein Seidel

Lawrence Livermore National Laboratory

Longitude-time series

March, April, and May

MATrix LABoratory - A matrix-based interactive development environment.

Modern Era Retrospective-analysis for Research and Applications

European Space Agency geostationary spacecraft

United Kingdom Meteorological Office stratospheric analysis 
MRF

MSU

NAM

NARR

NASA

NCAR

NCDC

NCEP

NCEP1

NCEP2

NESDIS

netCDF

NMC

NOAA

NSF

NWP

NWS

MMM

OI

PAOB

PCMDI

PNJ

QBO

QC

RATPAC

RMS

RSS

SAM

$\mathrm{SH}$

SJSU

SON

SPARC

SSI
Medium Range Forecast (model)

Microwave Sounding Unit

Northern (Hemisphere) Annular Mode

North American Regional Reanalysis

National Aeronautics and Space Administration

National Center for Atmospheric Research (Sponsored by the National Academy of Sciences)

National Climatic Data Center

National Centers for Environmental Prediction (of NWS)

NCEP-NCAR 59-year reanalysis

NCEP-DOE AMIP-II Reanalysis (R-2)

National Environmental Satellite Data Information Service network Common Data Form

National Meteorological Center (after fall 1995, the Climate Prediction Center (CPC) of NCEP)

National Oceanic and Atmospheric Administration

National Science Foundation

Numerical Weather Prediction

National Weather Service (of NOAA)

Mesoscale and Microscale Meteorology (division of NCAR)

Optimal Interpolation

PAid (meaning "fitted") OBservations (Australia)

Program for Climate Model Diagnosis and Intercomparison (of LLNL)

Polar Night Jet

Quasi-biennial Oscillation

Quality Control

Radiosonde Atmospheric Temperature Products for Assessing Climate

Root Mean Square

Remote Sensing Systems

Southern (Hemisphere) Annular Mode

Southern Hemisphere

San Jose State University

September, October, and November

Stratospheric Processes And their Role in Climate

Spectral Statistical Interpolation 
SSM/I

SSU

SSW

TIROS

TLS

TOMS

TOVS

UAH

UARS

UCSD

UKMO

USGS

UTC

VTPR

WCRP

WMO

ZTS
Special Sensor Microwave/Imager

Stratospheric Sounding Unit

Stratospheric Sudden Warming

Television Infrared Observation Satellite

Temperature Lower Stratosphere

Total Ozone Mapping Spectrometer

TIROS Operational Vertical Sounder

University of Alabama, Huntsville

Upper Atmosphere Research Satellite

University of California, San Diego

United Kingdom Meteorological Office

United States Geological Survey

Universal Coordinated Time

Vertical Temperature Profile Radiometer

World Climate Research Programme

World Meteorological Organization

Zonally-averaged time series 


\section{Appendix B Data sources}

- NCEP1 monthly temperature, u-wind, and v-wind data were obtained from the NOAA-CIRES Climate Diagnostic Center, Boulder, Colorado, USA, from http://www.cdc.noaa.gov/ on 5 February 2006.

- "ECMWF ERA-40 data used in this study/project have been provided by ECMWF and have been obtained from the ECMWF data server." ERA-40 monthly temperature, $\mathrm{u}$-wind, and v-wind were downloaded from http://data.ecmwf.int/data/d/era40_mnth on 4 August 2005.

- RATPAC data was downloaded from http://www.ncdc.noaa.gov/oa/cab/ratpac/index.php on 10 February 2006.

- MSU/AMSU data are produced by Remote Sensing Systems and sponsored by the NOAA Climate and Global Change Program. TLS data and weighting functions were downloaded from www.remss.com on 9 February 2006.

- UK Meteorological Office (UKMO) stratospheric analyses, referred to herein as "METO" for consistency with Randel et al. (2002, 2004), were downloaded from the SPARC data center, http://sparc.sunysb.edu, on 4 September 2005. Bill Randel and Fei Wu provided this data set. The late Petra Udelhofen reformatted the data into netCDF and provided the "ctl" files. Their contributions are gratefully acknowledged.

- QBO data was downloaded from http://badc.nerc.ac.uk/cgi-

bin/data_browser/data_browser/badc/CDs/berlin_strat/data/qbo/qbo_53-01.dat on 22 Aügust 2005. It is an online portion of the Institut für Meteorologie of the Freie Universität Berlin Stratospheric Data Series, and the work of K. Labitzke and her collaborators is hereby acknowledged. 


\section{Appendix C Data flow}

Figure C-1 illustrates the flow of netCDF-formatted NCEP1 and ERA-40

reanalysis data from the data source sites of Appendix B to the binary-formatted

zonally-averaged and pressure level files used in the MATLAB analysis programs of this thesis. 


\section{Reanalysis Data Source Sites:}

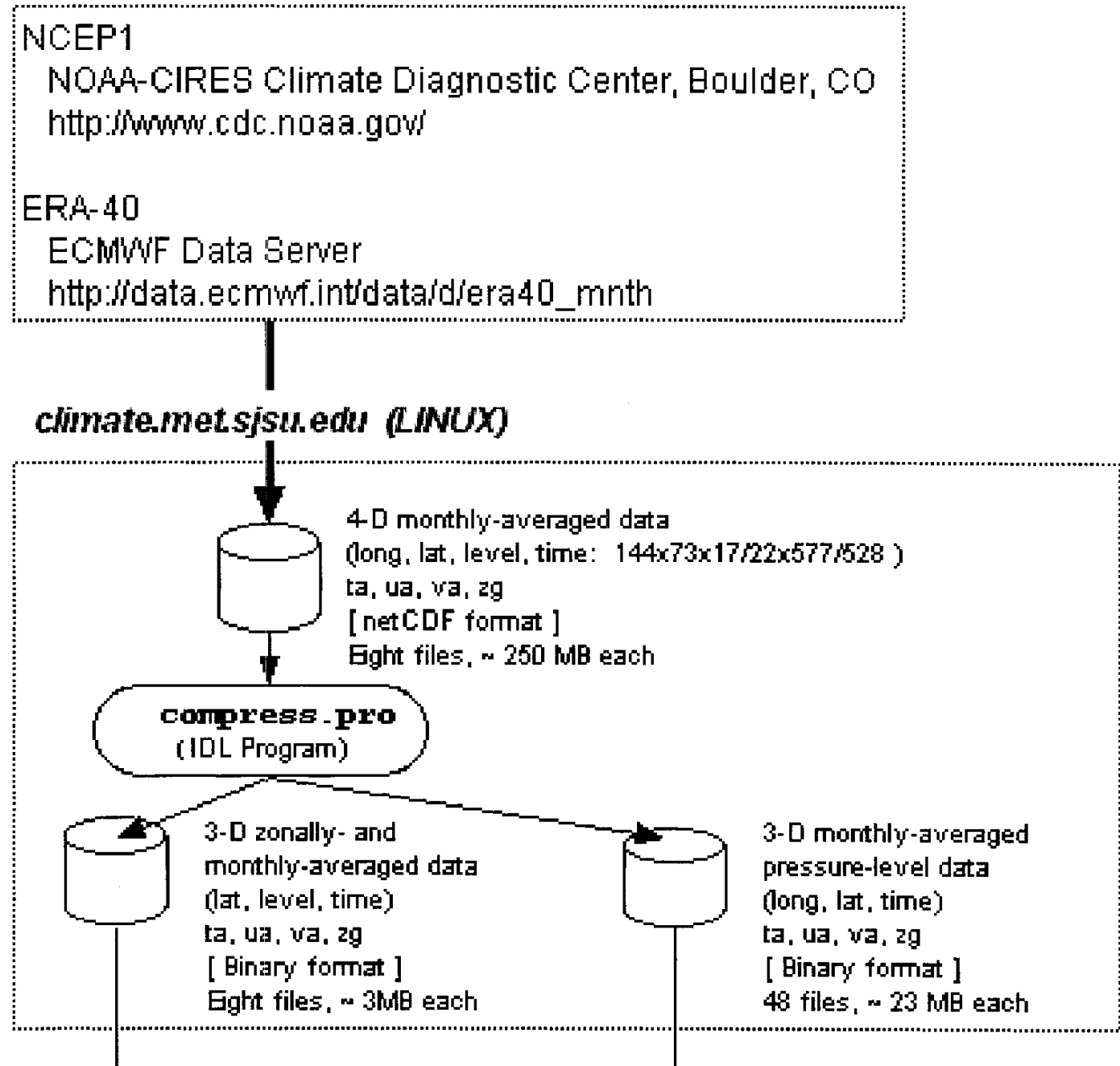

MS Windows workstations and laptops:

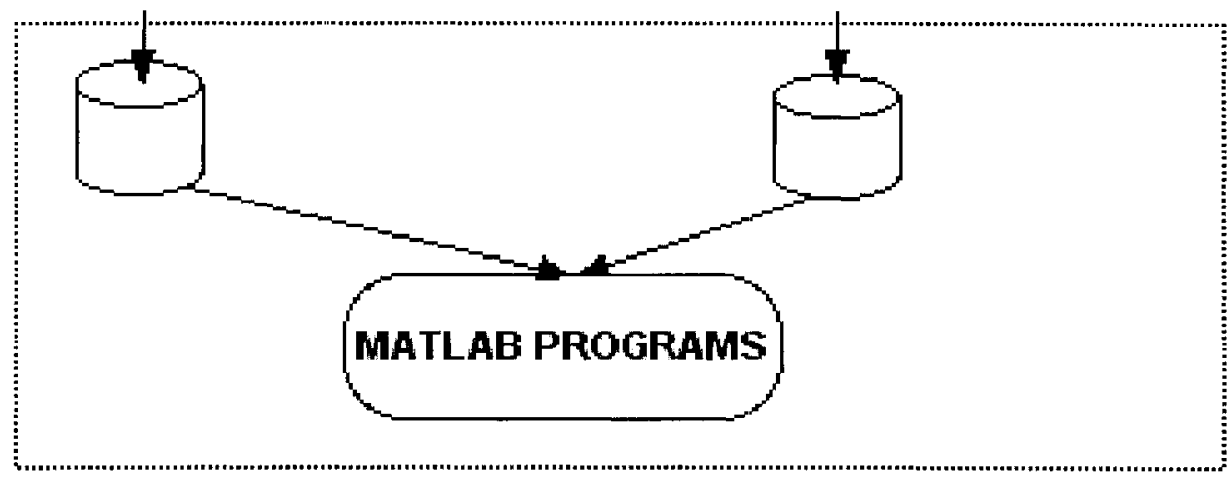

Fig. C-1. Data Flow 


\section{Appendix D Longitude-time series}

As illustrated in Fig. C-1, NCEP1 and ERA-40 reanalysis data are downloaded from their sources as a single netCDF file for each atmospheric variable. The netCDF unpacking process produces a conventional four-dimensional (longitude, latitude, pressure level, and time) data file. For the purposes of this Appendix, only the stratospheric pressure levels $(100,70,50,30,20$, and $10 \mathrm{hPa})$ and the common NCEP1 ERA-40 time span (January 1958 - December 2001) of such a file are considered. Such a file then has the attributes of:

- Variable: This file contains the data of a directly imported atmospheric variable

- Longitude: 144 values from $0^{\circ}$ to $357.5^{\circ}\left(2.5^{\circ}\right.$ spacing)

- Latitude: 73 values from $90^{\circ} \mathrm{S}$ to $90^{\circ} \mathrm{N}\left(2.5^{\circ} \mathrm{spacing}\right)$

- Pressure levels: 6 values of $100,70,50,30,20,10 \mathrm{hPa}$

- Time: 528 values (months) from January 1958 to December 2001

- Size: 33,302,016 elements (33.3 mega-elements)

No graphical representation of this file type is provided.

Figure C-1 also shows data from the netCDF files compressed by zonal averaging. With the longitude dimension removed, the example data consists of $73 \times 6 \times 528=231,264$ elements. Figure D-1 is a representation of this zonally-averaged data set. 


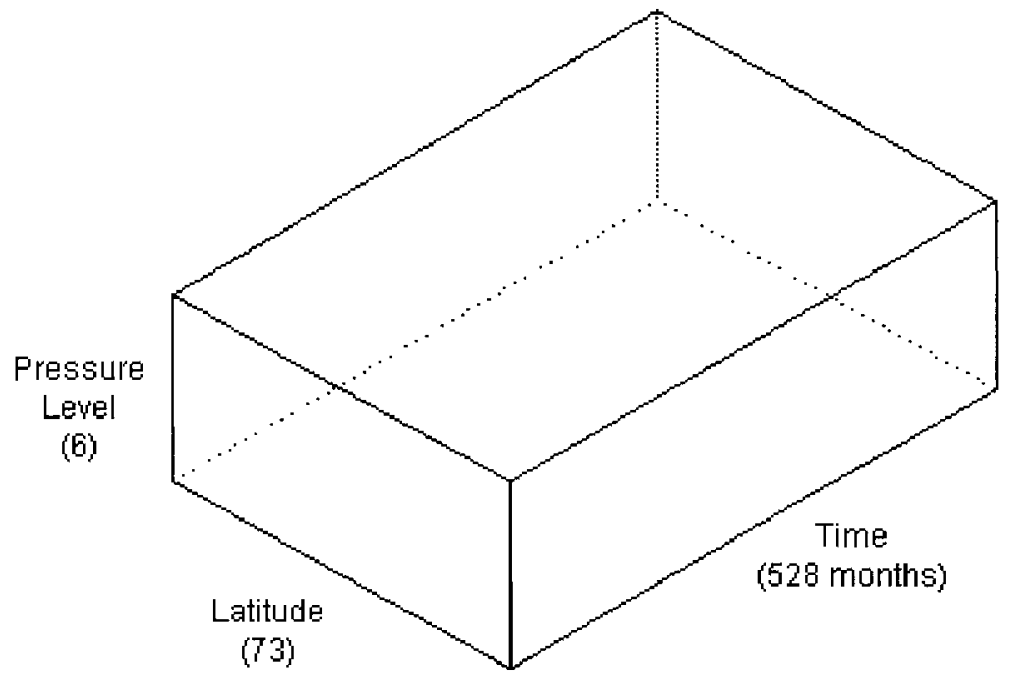

Fig. D-1. Three-dimensional zonally-averaged data set.

Statistics, such as the means, standard deviations, rms, and maxima/minima displayed in this thesis, may be performed on the time series of this data. The data of Fig. D- 1 contain $73 \times 6=438$ time series. Figure D- 2 schematically illustrates a single time series for a single point in pressure-level - latitude space.

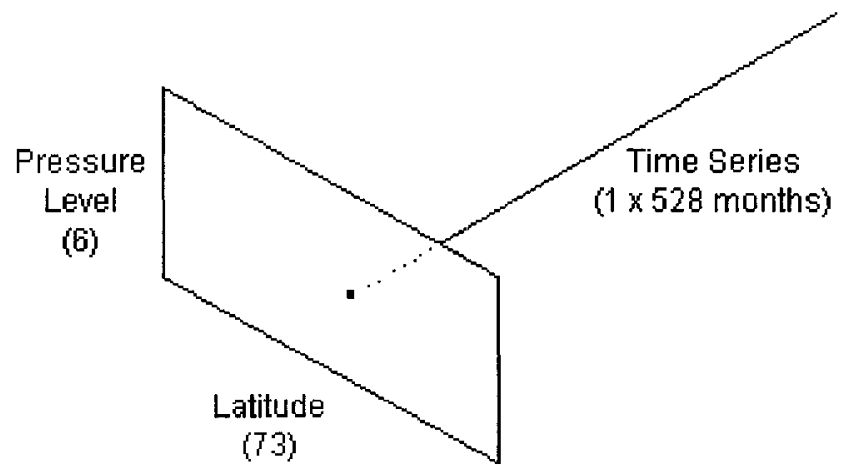

Fig. D-2. Time series for one pressure-level - latitude point in a three-dimensional zonally-averaged data set.

However, in the interest of providing a two-dimensional display of statistical results, the zonal averaging process removes all longitudinal variance from the statistics listed above. The solution to this loss is the inclusion of the time series for all longitudes at each point in pressure-level - latitude space. This produces the two-dimensional 
longitude-time array, shaded, of dimension and size 144 x $528=76,032$, seen in Fig. D-3. Computationally, the data in the longitude vs. time array associated with each latitude pressure-level coordinate may be "reshaped" into a linear vector of length 76,032 elements and treated, without loss of generality in this application since time order is not relevant, as a simple time series, referred to in the body of this thesis as a "longitude-time series." Statistics performed on this extended time series contain the variance lost in a simple zonally-averaged time series.

Means of the conventional time series (Fig. D-2) and the longitude-time series (Fig. D-3) are equal. The remaining statistical measures (standard deviations, rms, and maxima/minima) will have larger values at corresponding latitude - pressure-level coordinates for the longitude-time series than for the conventional time series.

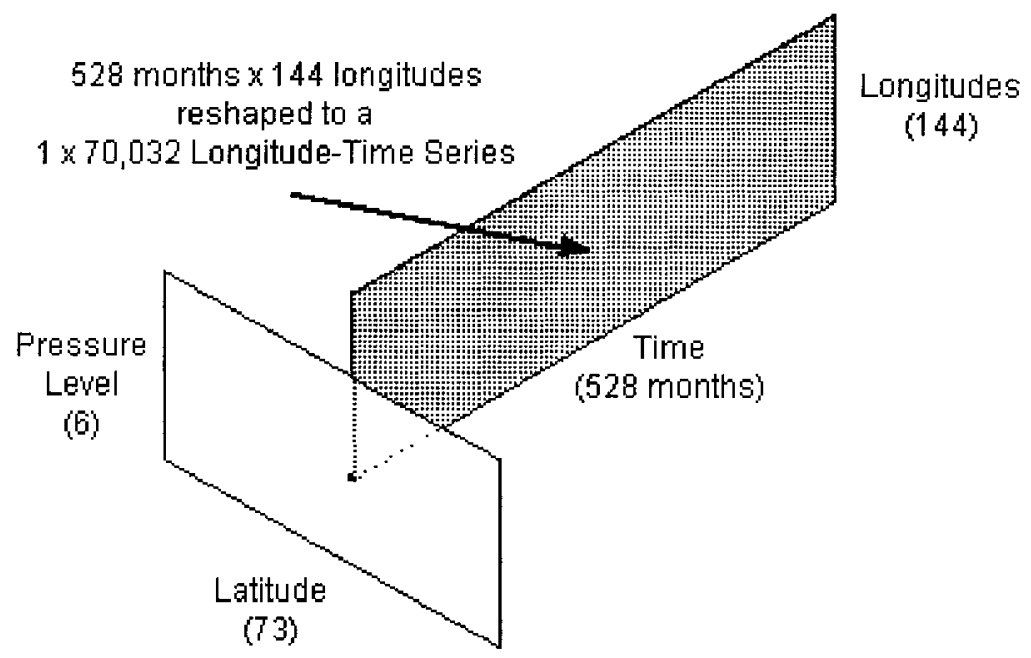

Fig. D-3. Longitude-time series for one pressure level - latitude point in a threedimensional data set. 


\section{Appendix E Frequency-domain filtering of time series}

Stratospheric time series of the three variables studied in this thesis (temperature, zonal wind, and meridional wind speed) all exhibit large annual variations in magnitude that are typically significantly larger (from two to ten or more times) than the underlying longer wavelength variation that is of interest in a comparison. Figure E-1, a plot of the NCEP1 monthly temperature anomaly at $10 \mathrm{hPa}$ averaged from $90^{\circ} \mathrm{S}$ to $30^{\circ} \mathrm{S}$, illustrates this situation. The annual average for each calendar year is also plotted at the center of that year as an indicator of the "underlying data" in this case. The peak-to-peak variation of the annual average waveform is about two $\mathrm{K}$. The reanalysis time series varies $30 \mathrm{~K}$ peak-to-peak. Thus longer wavelength information must be extracted from a signal with a magnitude 15 times larger. 


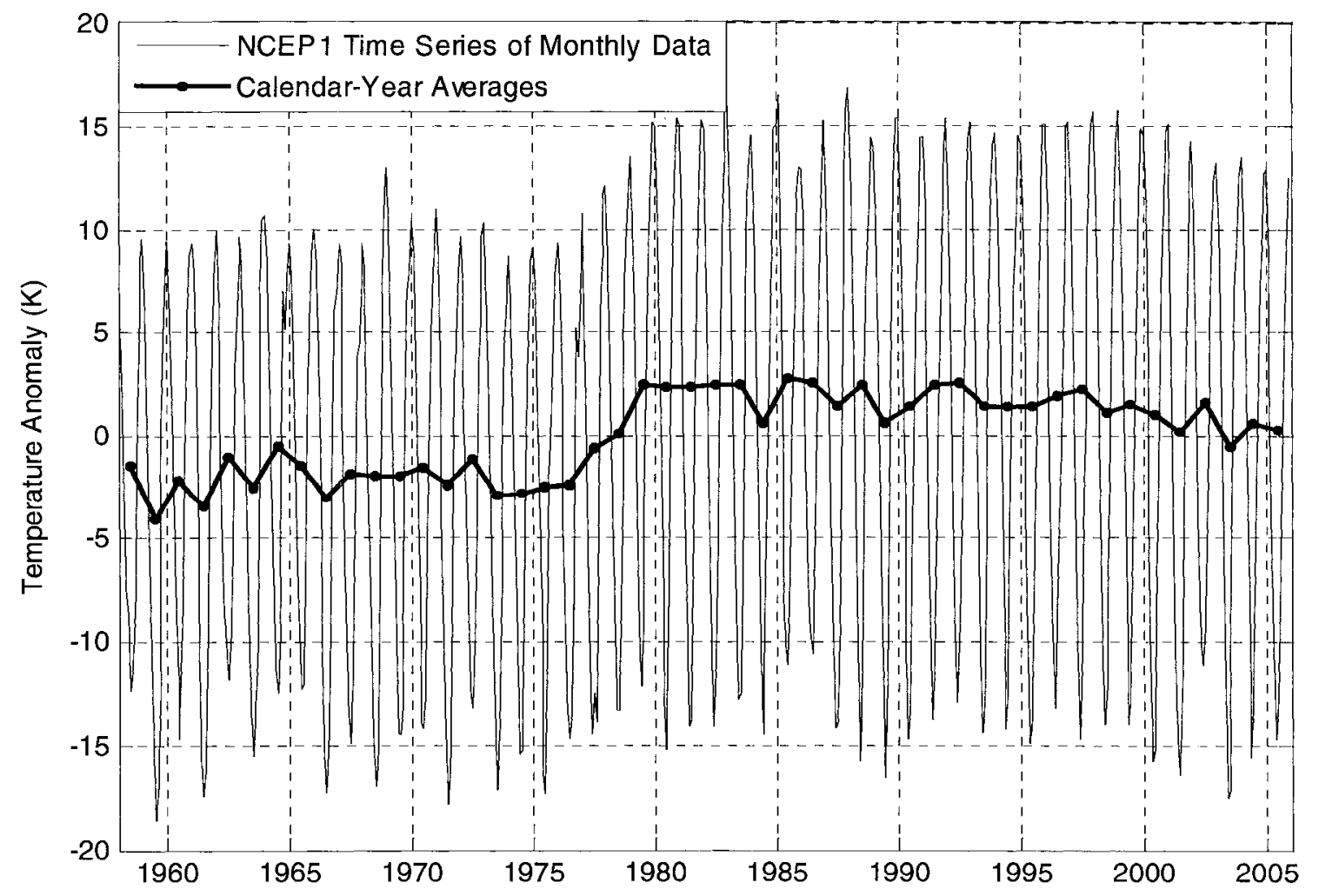

Fig. E-1. NCEPl monthly temperature anomaly time series at $10 \mathrm{hPa}$, latitude-weighted from $90^{\circ} \mathrm{S}$ to $30^{\circ} \mathrm{S}$ and corresponding annual calendar-year averages.

A large number of data filtering techniques exist. Several of the most common

are:

- Moving averages or medians, linear (as in Fig. E-1) or weighted

- Deseasonalization, wherein averages are computed for each month of a time series and then subtracted from the series

- Time-domain filters such as simple linear filters, Chebyshev, and the more laborintensive Lanczos method (Duchon 1979)

- Frequency domain filtering, wherein time series are fast Fourier transformed (FFT) to the frequency domain, unwanted frequency components removed, and the altered data inverse transformed (IFFT) back to the time domain 
Deseasonalization is the most common method used in atmospheric science to remove the annual cycle. Frequency domain filtering is employed in this thesis because of its flexibility, simplicity, and to demonstrate its efficacy in the applications of this thesis. As illustrated in this Appendix, frequency domain filtering may be employed to remove only the annual cycle and its harmonics or to remove all variance less than a given period. Further, it does not remove the long-term mean as does deseasonalization. Frequency domain filtering may be used to remove a selected range of frequencies; this feature allows removal of QBO frequencies while retaining lower and higher frequencies.

In the remainder of this appendix, the terms frequency and its reciprocal, period, are both employed. In this thesis, the frequency domain is typically graphed on the abscissa with units of period (years or months).

Display of time series transformed to the frequency domain provides a graphic representation of the frequencies present in the time series and their relationship. Fig. E-2, the frequency domain transform of the time series of Fig. E-1, demonstrates that the annually varying waveform and its harmonics are fixed in frequency, narrow in bandwidth, and separated in frequency from the lower frequencies, or longer periods, of interest. This signature exists in all the variables considered in this thesis; to remove the annual variation and its harmonics while preserving the underlying data, frequency domain filtering is employed. As with many elegant techniques, the essence of its implementation, consisting of only 21 lines of MATLAB code, is extremely simple. Frequency domain filtering is applied in two ways in this thesis: 
- Notch filtering to remove the variance at the annual frequency with a period of 12 months and its three harmonics with periods of six, four, and three months as in Trenberth and Olsen (1989) and Randel and Wu (1999)

- Low-pass filtering to remove the variance at all periods of less than 13 months (filter characteristics are defined in terms of frequency - a "low-pass" filter passes lower frequencies or longer periods)

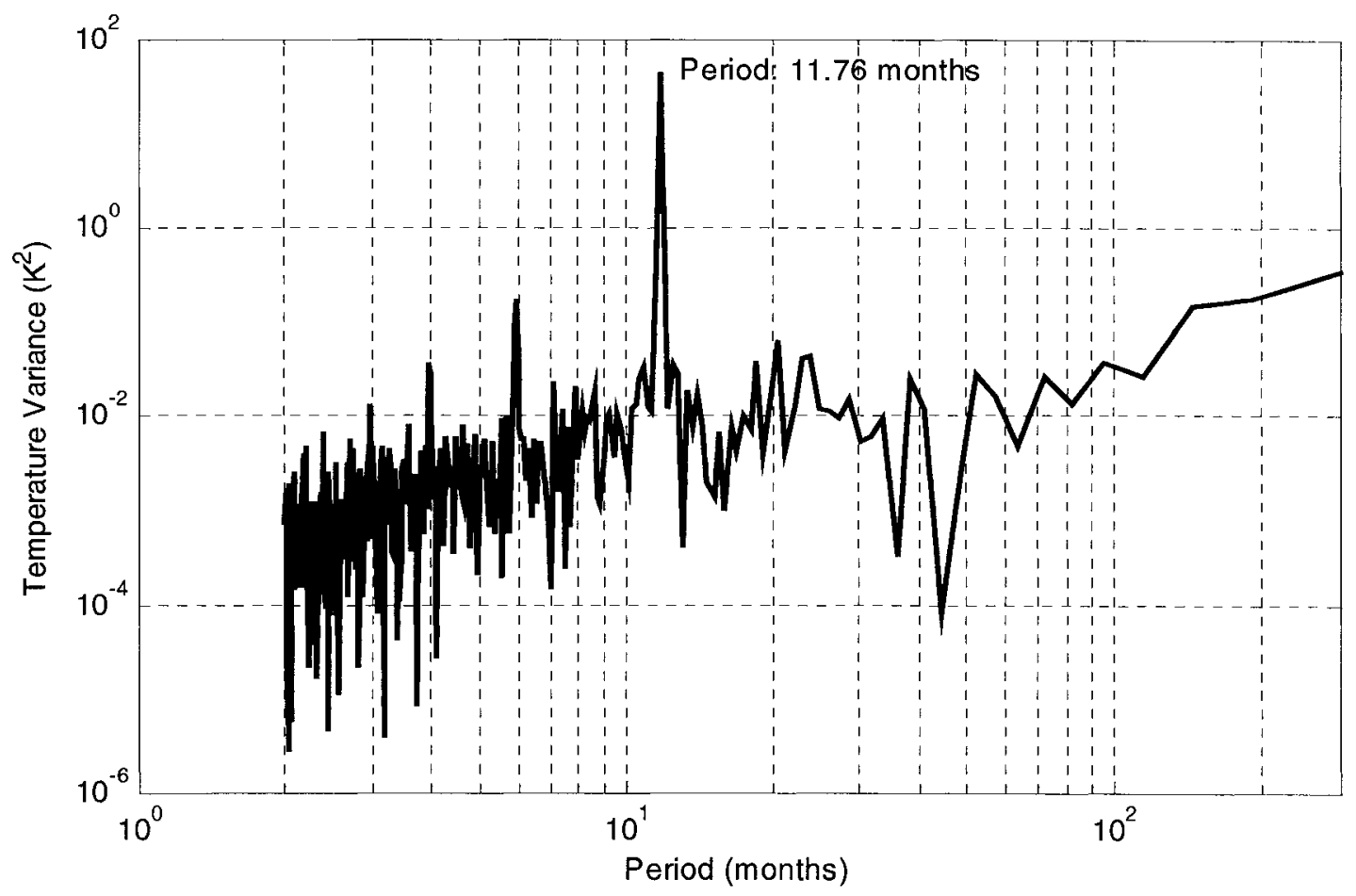

Fig. E-2. Variance spectrum of NCEPI monthly temperature anomaly time series of Fig. E-1.

Figure E-3 shows the frequency domain behavior of these two approaches. In the upper plot, all data with period within $5 \%$ of the fundamental (12 months) and the first harmonic (6 months) and $2.5 \%$ of the second and third harmonics (4 and 3 months, respectively) have been removed. All data with period less than 13 months have been removed in the lower plot. 

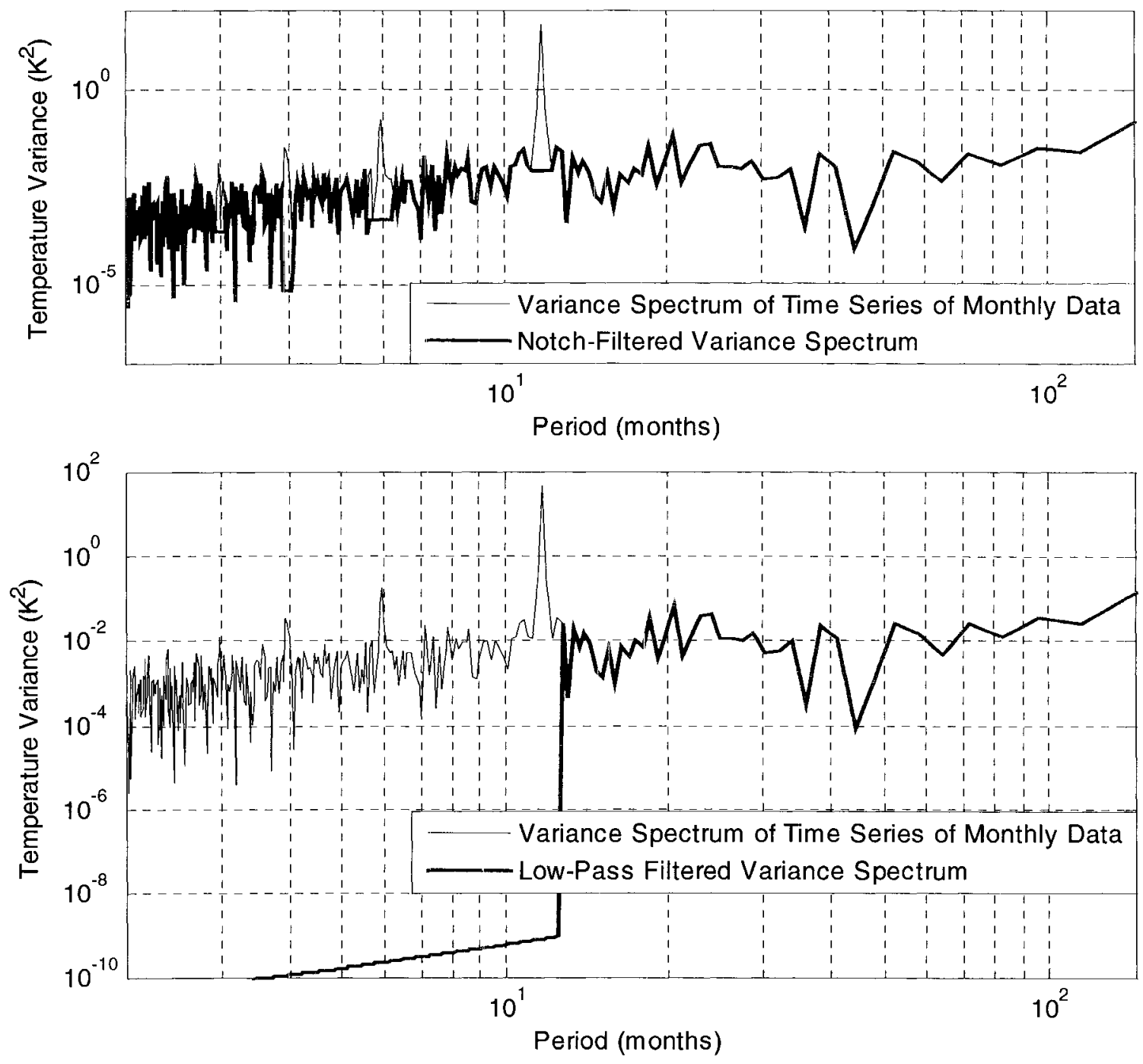

Fig. E-3. Variance spectrum of NCEPI monthly temperature anomaly time series of Fig. E-1 and spectrum after notch filtering at 12, 6, 4, and 3 month periods (upper) and after removal of periods less than 13 months (lower).

Figure E-4 plots the time series produced by the two approaches. Each of these two time series is compared with the annual average for the calendar year, which is plotted at the center of that year. The notch filter approach leaves almost all high frequency variance and is used where the full bandwidth of the time series, without the annual signal and its harmonics, is required. In this case, if the annual variation were 
present, it would produce an inappropriately large standard deviation. The 13-month low-pass filter retains much more of the variation above the cutoff period than the annual average, without the high frequency variability of the notch filter, and is the approach chosen for clarity when plotting multiple time series on a common axis as in Figs. 4-25 through 4-30 and similar plots throughout the thesis. Both filtered time series plots contain much more information than the calendar-year average.
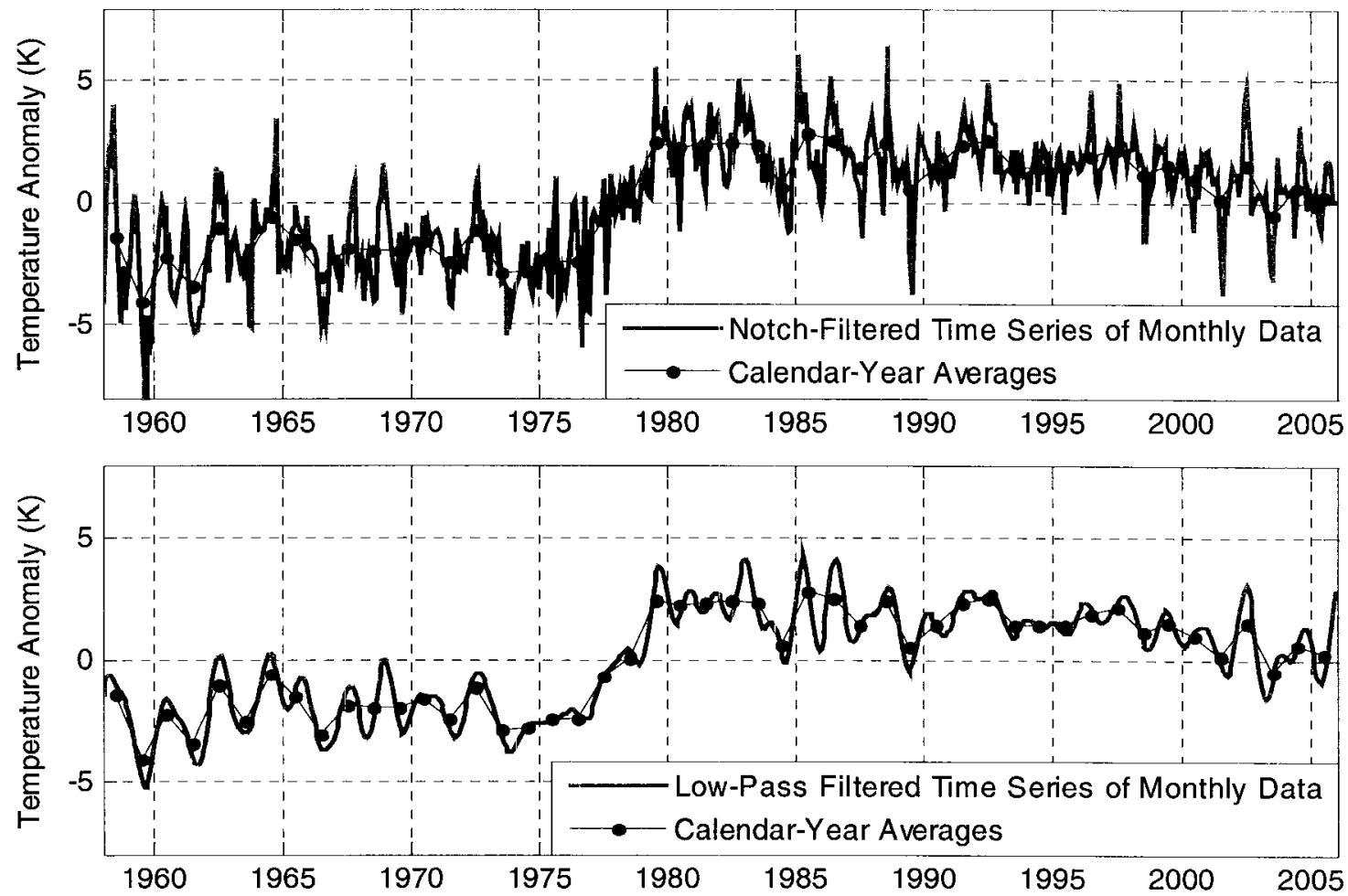

Fig. E-4. Time series from notch (upper) and low-pass (lower) filtering (Fig. E-2) and annual calendar-year averages from Fig. E-1. 
Variance spectra may used to compare two or more time series. The variance spectra of two cases of time series and the ratios and differences of their spectra are plotted in Figs. E-5 and E-6. The subject data sets are NCEP1 and ERA-40 monthly- and zonally-averaged temperatures from January 1958 to December 2001.

The first pair of data sets is latitude-averaged from $\underline{90^{\circ} \mathrm{S}}$ to $\underline{30^{\circ} \mathrm{S}}$ at $10 \mathrm{hPa}$; their time series are shown in Fig. 4-30. The NCEP1 time series of this case is plotted in Fig. E-1. Figure 4-31 lists a 9.2 K maximum difference between these two time series with an average rms difference of $3.2 \mathrm{~K}$. The second pair of data sets is latitude-averaged from to $\underline{30^{\circ} \mathrm{N}}$ to $\underline{90^{\circ} \mathrm{N}}$ at $\underline{100 \mathrm{hPa}}$; their time series are shown in Fig. 4-25. Figure 4-32 lists a $-1.1 \mathrm{~K}$ maximum difference between these two time series with an average rms difference of $0.3 \mathrm{~K}$. These statistics of the differences of the two cases of time series and a visual comparison of Figs. 4-30 and 4-25 reveal that the NCEP1 minus ERA-40 temperature differences at $100 \mathrm{hPa}$ are significantly less than those at $10 \mathrm{hPa}$.

Figure E-5 displays:

- The variance spectra of the $10 \mathrm{hPa}$ NCEP1 and ERA-40 data (NCEP1 data is shifted upward in period one percent and the ERA-40 data shifted downward one percent for clarity)

- The ratio, NCEP1 / ERA-40

- The difference, NCEP1 minus ERA-40

Figure E-6 repeats this format for the $100 \mathrm{hPa}$ data.

Comparison of the two figures shows that while the ratio and difference of the temperatures at $10 \mathrm{hPa}$ are large over all periods from 2 to 264 months, the $100 \mathrm{hPa}$ ratio 
exceeds a factor of two (or one-half) at 70 months and this ratio and accompanying difference never reach the magnitude of those at $10 \mathrm{hPa}$. 

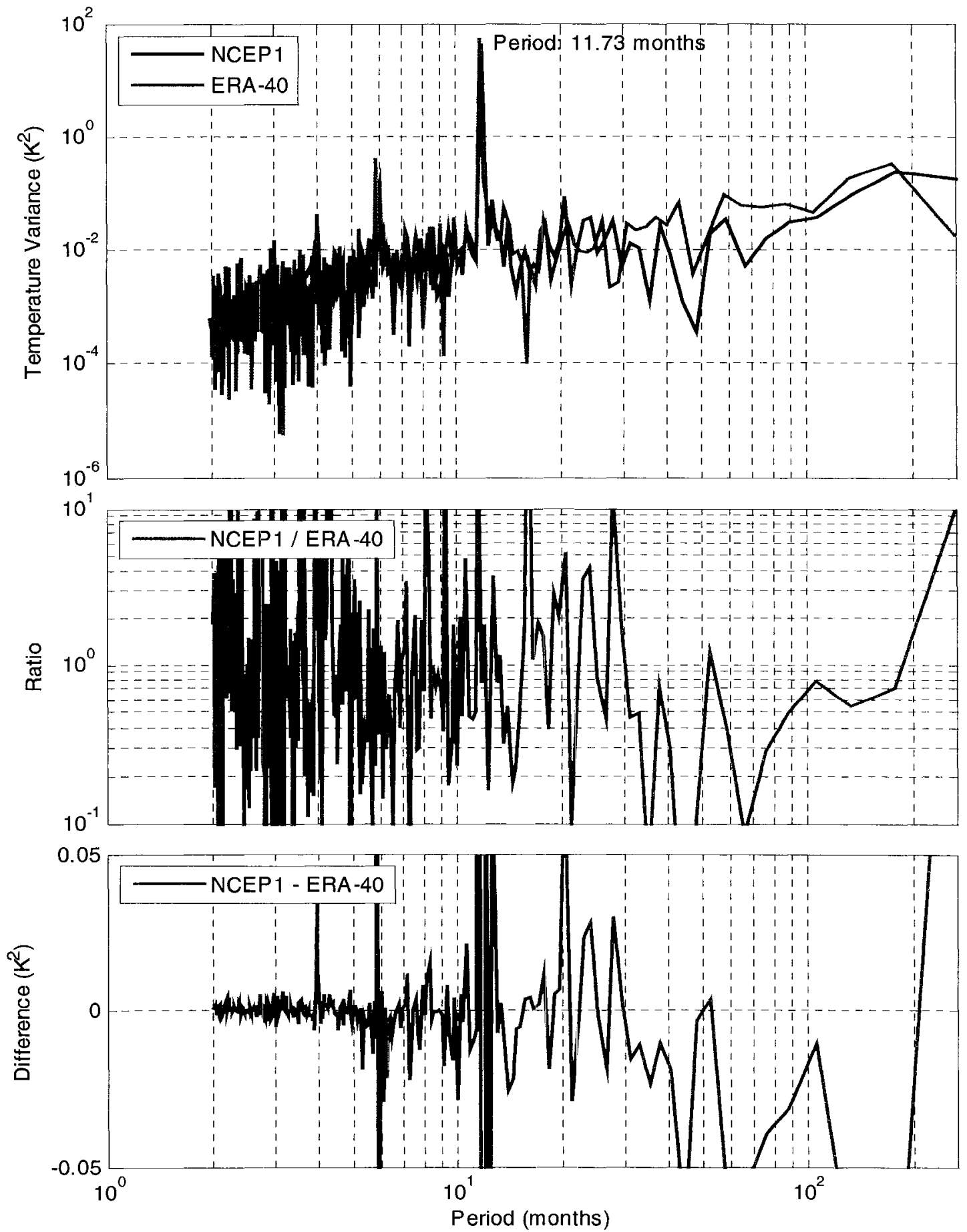

Fig. E-5. Variance spectra of anomaly time series of zonally-and monthly-averaged temperatures: NCEPI and ERA-40 (upper), ratios (middle), and differences (lower) of their spectra [lon: mean of $0^{\circ}$ to $357.5^{\circ}$; lat: $90^{\circ} \mathrm{S}$ to $30^{\circ} \mathrm{S}$; press: $10 \mathrm{hPa}$; time: Jan 1958 to Dec 2001]. 

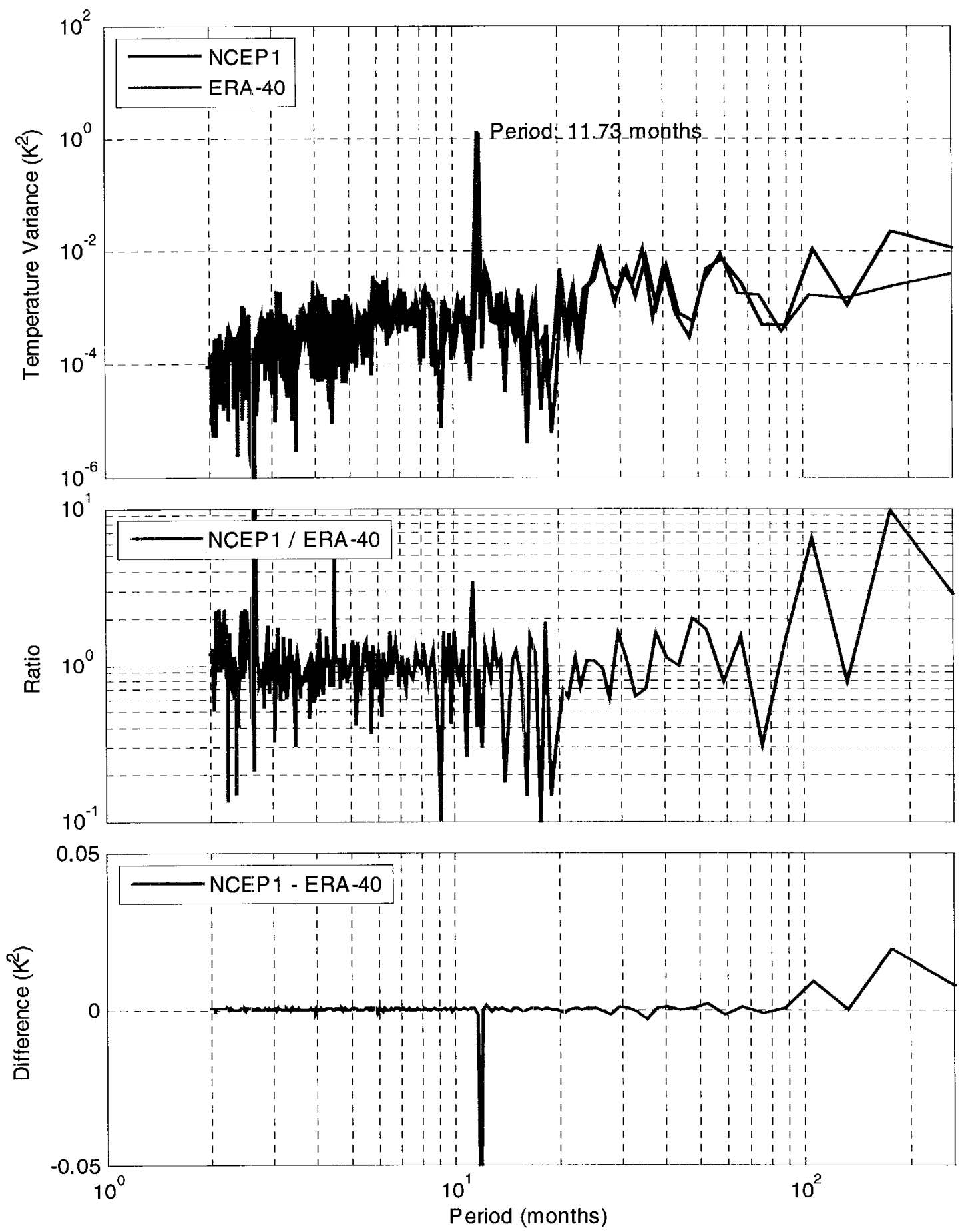

Fig. E-6. Variance spectra of anomaly time series of monthly-and zonally-averaged temperatures: NCEPI and ERA-40 (upper), ratios (middle), and differences (lower) of their spectra [lon: mean of $0^{\circ}$ to $357.5^{\circ}$; lat: $30^{\circ} \mathrm{N}$ to $90^{\circ} \mathrm{N} ;$ press: $100 \mathrm{hPa}$; time: Jan 1958 to Dec 2001]. 
The "fourfilt" (Fourier Filter) MATLAB function was provided by Dr. Larry Breaker of Moss Landing Marine Laboratories. It was written by Jeff List and slightly modified by Rich Signell, both of the United States Geological Survey (USGS). Notch filtering and further functional modifications were added by the author of this thesis. 